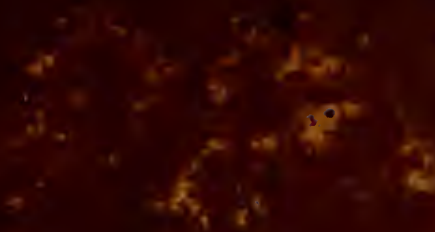

2.1.
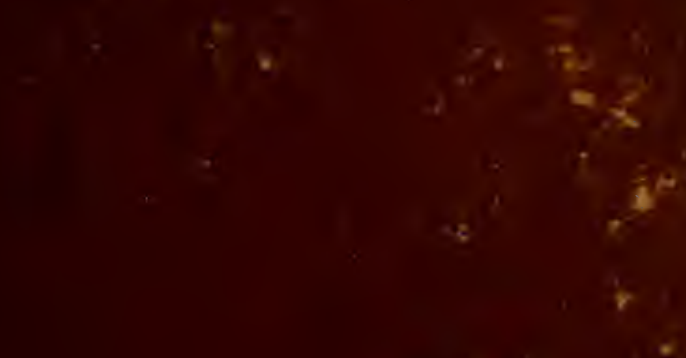

I

ti

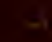

$\%$

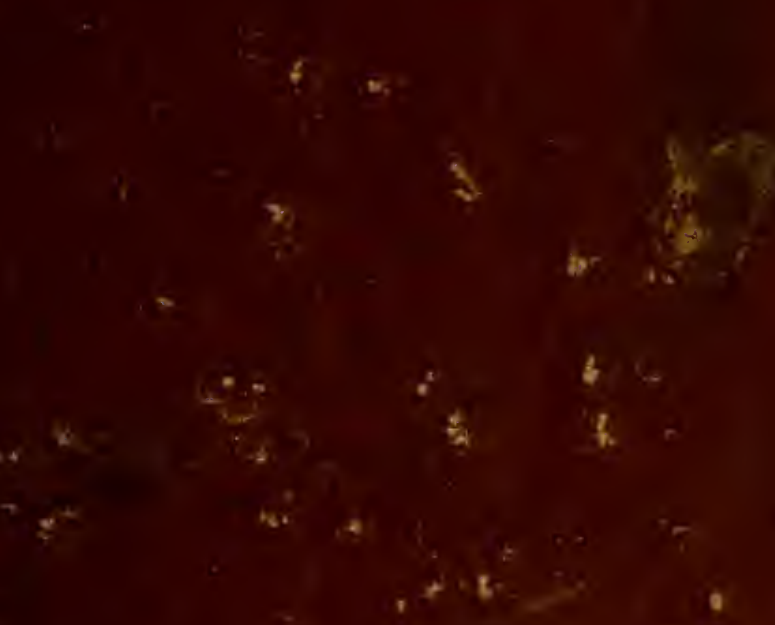




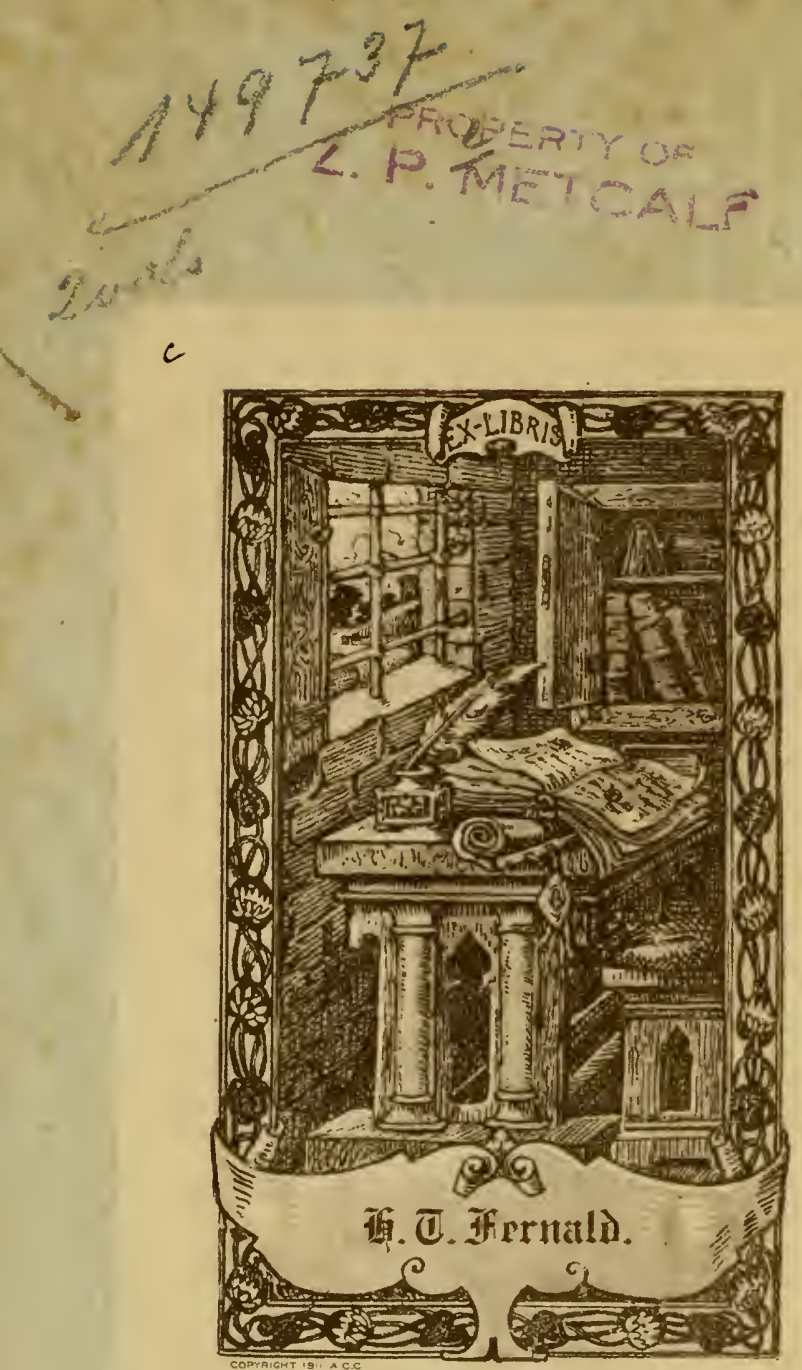


. 



\section{FAVNA ETRVSCA}

S I S T E N S

I N S E C

Q VAE

IN PROVINCIIS FLORENTINA ET PISANA

PRAESERTIM COLLEGIT

PETRV, R OS I V S

IN REGIO PISANO ATHENAEO PVBL. IROF. ET SOC. ITAL.

TOMVS PRIMVS.

c $\checkmark \mathrm{M} X I$ TAB.

MANTISSAE PRIORE PARTE ADIECTA,

ITERVM EDITA

ETA ANOTATS RER E E T I S AVCTA

A

D. IOH. CHRIST. LVD. HELLWIG

PVERORYM ROELLIVM IN AVLA RRYNSVICENSI PRAEFECTO MATHEMAT. ET MISTORIAE NATVRALIS PLOFESSORE SOCIETATYM NATYRAE CVRIOSORVM, BEROLINENSIS, FRANCOEVRTENSIS, GOETTINGENSIS, HALENSIS ET IENENŚ́S socro.

HELMSTADII

ร. ITERIS C. G. F LE CKEISEN

MDCCIXXXXV.

1745 
cenciarty 


\section{(p. 102.)}

CLASSIS SECVNDA.

\section{VLONATA.}

\section{MANTIS.}

225. II. Rofia baculiformis aptera. Fn. Etr. d. 322.636 .

Variat magnitudine minori, et colore toto ferrugineo - Ifufio, antennis longioribus, articulis 16, vitimo longiore. Mas.

Iconem exhibet etiam D. Cyrilius in Fin. Neapolitana fib nomine $M$. filiformis $F$. - Hab. in fepibus ad folfas aquarum, et in Ericetis frequens.

*226. MT. Spatlanzania viridis, thorace laeui, elytris, alisque breuiffimis, alis externe rubris, interne violaceis.

Statura M. oratoriae, cluits pupa primo intuitu videtur, at fextuplo minor. Tota viridis, immaculata. Caput magnum. Frons punctis duobus impreffis. Antennae fetaceae, mediocres. Oculi magni oblongi. Thorax laeuis. Abdomen latum reflexum. Elytra breuifima rudimentis tantum alarum breuifimis, fupra dimidiato-ferrugineis; fubtus exteriore parte rubris, interiore violaceis. Femora antica valde incraffata. Incedit pectore erecto, abchomineque reflexo; et ponit oua congefta, agglutinata, quemadmodum $I$. oratoria; v. Roef. Inf. 4. t. I2. f. I. 2. 5. e quibus anno poFf lteriore 
fteriore menf. Aug. laruae mihi domi exclufae, capite maximo, fronte lata, concaua, femoribus albo obfolete annulatis. Specimen miffum etiam ad Cl. virum D. Giorna Taurin. Entomologum magni nominis, cui naturalis hiltoria amplificata plurimum debet. Hab. prope aquas in filuis, rara.

\section{(p. 103.) \\ XCVI. LOCVSTA.}

227. L. tuberculata flano-viridis, capitis vertice tuberculato laeui, elytris elongatis, maxillis Hlanis. Ful. Etr. I. 33 I. 65 I.

Statma fere $L$. Viridiffmae, at dimidio minor. Caput flauo-viride, vertice leuiter tuberculato. Antennae flauae, elytro paulo minores. Oculi cinerei, fafcia fer lineola longitudinali nigra. Maxillae flanae. Thorax planiufcus, flauo-viridis. Elytra flauo - viridia, angufta, abdomine duplo longiora, apice obtufo. Alae fubuirides, lyalinae, reticulatae, elytro aequales. Abdomen flano - viride. Cauda enfifera recta, biualuis, longitudine abdominis, flanefcens, cofta media fufca. Pedes flauo - virides, antici, et fecundi paris breues; poftici longiffimi, et elytris fubaequales. Tarfi omnes in viuo pallidiores, et fere cinerei. Habitat in pratis, fed frequens etiani in hortis. 


\section{NOTANDA ETADDENDA.}

(quo hanc primam partem fpectant.)

Haec notanda volumini pcfteriori huius operis in calce adiecta erant, hinc a me ferius obferuata funt, quam quod is fuo loco vti potniffem. Cum tamen haec notanda, ex confilio operis, integra feruanda effent, de eo tantum venia a lectore petenda eft, quod, licet paucis, emendatis non vterer. Ea, quae ipfe adhuc adnotanda habebam, fuo loco iuferui, et, vt per totum librum fuit, minoribus litteris diftinxi. $\mathrm{Hg}$.

Pag. 13. \$. 26. Sc. valgus Linnaei an vere refpondet Sc. Ptinifico. F, Sp. 27, I20? Dubium fuboritur, confer Linn. adnotationem pag. 559. Syft. Nat. confer defcriptionem $L$. interrupti pag. 560. demum Roefelii figuram a Fubricio citatam in $\mathrm{Sc}_{0}$ Panifco.

Linnaeus 1. c. ait, Luc. interruptus ct Sc. valgus adeo inter fe aftines, vt demtis antennis non de genere, fed fpecie etiam difputaretur." - Sufpicio nobis fuboritur, cui tamen vix fides habenda eft, acutiffimum Linnaeum, eundem, qui $\mathrm{Hy}$ dram Hamburgenfem (Seb. thef. I. t. IO2. f. I.) primo ftatim afpectu verum ar'efactum agnofceret, et probaret, antennis falfis deceptum effe in determinando S. valgo; fed qui artem nouit, qua plures mutilata infecta ințegerrima reftituere valent, idem cum nobis fentiet. — Id certe fatis perfpectum habemus, Sc. valgum Linn. et $P a-$ nifcum Fabr, nullo modo eandem fpeciem effe.

$\mathrm{Hg}$.

-32. $\int .7 \mathrm{~T}$, adiiciatur citatım: Hifler minutus. Herbft. K. t. 36 . f. 4 . Hg. 
P. 35. 5. 79. affinis $D$. violaceo, at fpecie videtur diftinctus. Etfi raro inuentus in floribus mox tamen lecti quamplures in fuilla falita fub Iulii finem.

P. 37. \$.82. De genere adhuc haereo; Antennae thorace longiores. Palpi quatuor fubaequales, clauati, clana nigra, compreffa, et oblique truncata. Magnit. cedit figurae quam exhib. Tab. III. Hab. in ligno antiquo.

H. 1. adiiciendum eft citatum Tillns ferraticornis. $F$. E. S. I. 2. 78. 3. Equidem hoc infectum numquam in Tillor

P. 40. \$. 92. Bofrichus bidentatus? Long. 3 . lat. $x$.

Niger, colecptris teftaceis apice retufis bihamatis. F. Mant. $37 \cdot 8$ ?

Antennae nigrae, claua perfoliata. Caput et thorax antice pilis flauefcentibus longioribus hifpida, fcabra nigra immaculata, quae nihil differunt a capise et thorace $B$. capucini. Elytia punctulata, obfcure teftacea, poftice retufa denticulo elongato nigro ad futuram, aliisque in fingulo tribus lateralibus ohfoletis. Subtus niger magisque villofus digitis obfcure teftaceis. Magnit. non variat. Hab. in truncis fere exficcatis vitis viniferae eos rotundis foraminibus terebrans. Saepe lectus menf. April.

Specimen modo a Dom. Langio mecum communicatum, $2 \frac{1}{2}$ lin. longum, vix tamen latitudini $B$. Capucini cedebat. Coleopteron fingulum poftice trihamatum erat, fpina vna fuperiore minore, duabus reliquis inferioribus, paullulum oblique pofitis, 
pofitis, ita, vt exterior, minor, aitior eflet; qua nota praecipue ab alio affini differt, quem, paullo maiorem, e Gallia accepi. Hg.

f. 43. Adnotatum ad $\int .95$. deleatur. Byrrhus Rhenii Schneid. nunc declaratus eft Dorcatama Diesdenfis Herbft. K. tom. 4. p. I04. t. 39. f. 8. Dermeftes ferra. Fabr. E. S. I. 2. $234 \cdot 40$. Hg.

p. 54. \$. 127 . Auctor nofter recte monet, fe non probarc hoc infectum eidem cum priore generi annumeratum effe. Ne antennae quidem fatis conueninnt, reliqua ftatura vero omnino recedit. Equidem hunc Ptilinum, vt nunc res eft, cum. Derm. dentato n. 82 . coniunetum, aut peculiare genus confituere, aut Melyrium gentem fubire iuberem. $\mathrm{Hg}$.

p, 55. J. $x=8$. Linnaeus S. N. I. 2. 603.2. atque Fabricius patriam Barbariam dicunt, fed vtriusque deferiptio congruit, nifi guod Linnaeus Hifpae fuae eandem cum $H$. atra magnitudinem tribuat, qua duplo maior eft. Spinae bafi eundem cum corpore colorem rufo - teftaceum habent, apice vero nigráe funt. $\mathrm{Hg}$.

p. 60. n. x39. Citata e Fabricio et Herbftio omnino delenda funt. Opatrum, quod Dn. Roffius nomine O. Agricoiac exhibet, nobiscum a Dn. Langio communicatum, idem fere eft, guod in multis mufeis Germaniae nomine $O$. arennrit occurrit, licet Germaniae indigenum a vero $O$, arenario $F_{\text {. }}$ multum difcrepet. Habitus fere idem, qui $O$. Cabulofo, fed dimidio minus eft, elytris punctatoftriatis, interftitiis vix eleuatis.

o. Agricolat (quod nomen, licet in Agaricola mutetur, tamen falfum eft, quia hoc infectum numquam in Agaricis, fed in Boletis occurrit) mihi nouum genus conftituit, quod, victu, antennis et habitu ab Opatris F. admodum alienum, BoliFf 3 tophagus 
tophagus mihi audit (a $\beta \omega \lambda$ rrns, Boletus, et $\varphi a^{\prime} y^{(s)}$, comedo.). E reliquis mihi notis $O$. crenatum $F$. illi adiunxi, quamuis Fabricius dicat, id in arenofis occurrere. $\mathrm{Hg}$.

p. 66. 5. I54. Coccinella 9-punctata Anctoris fane alia ef, quam fic nominata Fabricii, Linnaei et Udmanni, quod verba pauca illa Fabricii probant: puncta 2. 2. oblique pofita, communi maiori. Nec Auctoris, nec Geoffroyi defcriptiones huic conneniunt. $\mathrm{Hg}$.

P. 74. §. r 72. Add. Frons punctis duobus minutiffimis albis notata. Variat elytris quadrimaculatis, plerumque femina. Frequens in Mai. in iisdem plantis cum C.bipunctata, quae quiden habet puncta duo alba in fronte, fed paullo maiora, quam in C. jexpuftulata.

P. 85. \.204. De Chryf. aucta vix dubito, quamuis mea fpecimina cedant magnitudine C. marginatae praefertim Degeerianae.

P. 88. \$. 2 I2. Add. * Aliam Chryfomelam habeo ftatura et magnitudine huic omnino fimilem, at diftinctam nempe: Fufco-aenea; nitida, antennis craffiufculis, thorace laeui, elytris punctatoftriatis; fubtis tota fufco - aenea femoribus pofticis non incraffătis. Lecta in pratis filuaticis $\mathrm{m}$. Mai.

P. 96. \$. 236. Cryptoc. Bucephalus. Long. $2 \frac{1}{3}$. lat. $\mathrm{I} \frac{1}{5}$

Cyaneus ore, thoracis marginibus, pedibusque rubris. F. Mant. 82. 4I.

Chryf. bucepiraliz. AEt. Hall. I. 276.

of La Melolonte motthe. 'Geoff. Inf. I. x97. 5 .

Maxillae 
Maxillae in altero fexu magnae incraffatae forcipatae rubrac, apice nigrae. F. Caput magmun cyaneum ore antemnisq̨ue bafi rufis, a!lice nigris. Pedes rufi femoribus bafi, plantisque fiperne cyaneis. Hab. Florentiae non frequens.

De citato a me adieço vide, qque in Fn. Etr. x, 87. 2 ro. ad Chryfom. collorem monui. Hg.

P. 98. \$. 239. Add. Hoc infectum etfi connenire etiam videatur cum figura a Sihaeffiro allata 1. c. forte fpecies eft diftincta a $C$. mar ginato $F$. ideoque interea referendatum ad C. flartilabrem, cum quo copula imctum in mea collectione videre eft, fiquiden de hoc etiam in indicando non fallor.

Confer 1.'c. a me monita. C. janilabris Anctor. ad C. marginatum referendus eft, minime autem vice verfa, vt Auctor vult. Hg.

p. Ir 4. $\$ .274$. Lagriae hirtc aç pubefcens magna copia menf. Ang. in eodem loco captae funt; hinc nulli dubio fubiectum eft, quod diu ian fufpicabar, illas fexu tantum diue:fas efle. L. pubefiens f. mas, praeter ea, quae 1. c. diximus, antennarum quoque articulo vltimo longiore a $L$. hivta differt; quod antennis Dermeftidum Pellionis, vndulati, quodammodo refpondet. $\mathrm{Hg}$.

p. II5. 5. 279. Adde citatum:

Cantharis fufca, elyeris antice, thoraceque elongato rubris. $L_{i} \mathrm{C}$. foumi. Geoffr. Inf. $\mathrm{x}$. 344. 8 .

Vide Mantifla n. I I 4. adnot. $\mathrm{Hg}$.

p. $x 36 . \$ 3=6$. Idem eft cum

Curculione diffintio. Fabr. E. S. I. 2. 459. $272 .-\mathrm{Hg}$.

P. $165 \cdot \$ \cdot 376$. Pupa alba quiefcens in ligno excauato, alis, antennis, pedibusque inflexis, $\mathrm{m}$. 
Mai. metamorplioin fubitura, fenfim fufca euadit; nigredo ab oculis maxillis alis ac geniculis incipit, et fpatio quatuor aut quingue dierum rupta, et depofita funica completa imagu prodit antemis longitudine corporis, thorace punctis octo, elytro fingulo fex nigris: color ftatim cinerco - fufcus, mox fit fubuiridis, et pubes demum cinerea antennas, pedesque nigros obducit.

p. I7 4. $\$ \cdot 390$. Callid. Arietis cum femina maiore, at eodem modo formata ac fignata, copula iunetum repertum eft d. I 2 . Iulii I 794 ; id, quod differentiam fpeciei huius et Gazellae, de quo mihi ian dudum nullum dubium reftiterat, aperte probat. Si illae fpecies tanto dubio fubiectae erant, quantamne dubitationem duae aliae, his modo dictis fimillimae, et tamen dinerfae fpecies excitabunt, quas Halie Saxonum detectas, amicus meus. Hiibneris, benigniter mecum communicanit?

$\mathrm{Hg}$.

P. I 79. Callidiorum generi inferatur: * $C$. unifaficitum. Long. 4. lat. I. vix. Thorace rotundato rufo, elytris bafi rufis, apice nigris; fafcia vnica aiba.

Statura C. Alni, fed paullo maius. Primo intuitu fi elytra tantum infpiciantur Clero vnifafciato fimile. Totum pilis canis breubus hirtum. Palpi quatuor clauati. Antennae mediocres fubrufae pilofae." Caput, exceptis oculis nigris rufum. Thorax rotundatus rufus. Elytra bafi rufa, apice nigra, in nedio fafcia vinica alba ex inaculis duabus lunatis compofita, et ad futuram vix interupta. Femora omnia clauata nigra. 'Tibiae et tarfi fubrufi. Alae et abdomen nigra. Hab. in filuis Pifanis. 
p. I8S. \$. 4I ב. Iycum vidi, quem $I$. Auroram habeo, aegre a $L$. minuto diftinguendum. Eiusden fere magnitudinis erat. Thorax niger, parum rufo-hirtus vt ruber dici potuifiet. In vtroque Lyco thoracis pars longitudinalis media eleuata, quafi maiginata eft; in L. Aurorr fere fexangularis erat, in L. minnto autem antice magis deprefla et admodum dilatata poftice attenuata, vt fere campanae figuram fiftat. Thorax prioris, refpectu elytrorum, brenior. Elytra minuti breuiora. Elytra Anrorac, computato margine ac futura, lineas fex eleuatas habent, lineis transuerfis intermediis denfis ita coniunctas, vt elytra denfe cancellata fiant. In L. minuto totidem lineae longitudinales funt, fed in medio inter duas, linea alia parum eleuata decurrit, et inter hanc lineam minorem et illam maiorem vtrinque puncta profunda feriem confituunt. Antennae $L$. aurorae vnicolores, nigrae; L. minuti autem, apice anrantiacae funt.

Haec comparatio feftinanter foribenda erat, cum per pauca tantum temporis momenta vfus illius $L$. aurorae fpeciminis mihi permifius efiet. $\mathrm{Hg}$.

P. 22 I. $\$ .473$. comige: Thorax marginatus vtrinque depreffus puictis binis excauatis. Elytra integra, fubinarginata; varietas: $a b$ aliis deinde lectis certior factus, affero.

P. 224. Cicindelis adiungatur: * Cic.4-punctato. Long. 6. lat. $2 \frac{1}{4}$. Supra obfcure viridis, fubtus cyanca elytris bafi et apice lunula, medio punctis quatuci albis.

Statura C. hybridae. Caput fufcum ante, et circuin oculos viride. Labium fiperius album. Maxillae nigrae bafi albidae. Thorax magis latus, 
quam longus, et fulco longitudinali in duos lobos diuifus. Elytra fufco-viridia fiugulis lunula bafeos ad marginem exteriorem, punctisque quatuor mediis, quorum duo marginalia; tum lunula altera apicis albis. Abdomen laete cyaneum. Antennae infra medium, pectoris latera, femora tibiaeque rubro aurato colore nitentes. Habit. in fabulofis rara.

C, littoralis. F. E. S. I. I, I72. I3., praecipue ibidem adnotata varietas, optime hnic modo defcripto convenit, fed thoracem in duos lobos diuifum non dicerem. $\mathrm{Hg}$.

P. 238. inferatur: C. violacens, apterus, aeneo-. niger, thorace fubuiolaceo, elytris punctis minutiffimis excauatis triplici ordine. De Geer. Inf. 4.89. Schaeff. Ic. t. 98. f, I.

Hab. in filuis Pifanis rarior.

C. violuceum Degeer, auctor in Mantiffa $n$. I 70 . in Paykullii veftigium, ad $C$. horten $\mathrm{em}$ refert, Schueff. Ic. t. 88. f̣. x. ad C. putrpurafcentem ibid. n. 169. translocat, nulla huins Appendicis mentione facta; utrum itaque hic Carabus Violacens, an Purpurafiens fit, decernere nequeo. Hg.

P. 270 . inferatur: C. bigutitatus. Long. $I_{\frac{2}{3}}$. lat. $\frac{2}{3}$. Thorace rotundito aeneo, elytris nigris macula apicis pallida. F. Sp. 3 I 3.83 ?

Statura et magnitudo fere $C$. 4 -maczilati, fed in hoc elytra minus obtufa, et manifefte ftriata, macula ante apicem pallida. Thorax rotundatus paullo latior quam in C.4-maculato. Pedes toti pallidi. Color nigro - aeneus nitens. Hab. in arenofis.

P.292. \$.594. Ad infectum quod attinet, a me fub nomine $L$. Afrac relatum, et ad $M$. tefacean $F$. forte 
forte amandandum; cum ex obferuationibus nuper factis, mihi nunc conftet de copula etian inter indiuidua eiusdem coloris in elytris, nempe teftaftaceis apicibus nigris (quae apuả nos frequentiora funt); concludi poffe videtur vnam effe fpeciem, eandemque in mafculis interdum variare elytris totis nigris. Occurrit in Carduis menf. Iun. et Iul. frequens.

P. 295. $\$ .596$. Adde: Hab. Pifis; in floribus Scabiofae inuentae plures etiam in hortis.

P.322. \$.636. Adde: Sexus in feminis diftat ab ano, fitus fub abdomine inter duas valuas ante fegmentum penultimum. Oua fubrotunda figura, et magnitudine feminis Milii, nigra, operculata. 
CORRIGENDA.

Pag.6. lin.6. ab infima, a loerio lege e Voetio. p. 14.1. 5. polt sininime del. conmatis fign: 1. 18. aliis deefl, aut ininimum leg. diti soon, ant vix. p. 34.1.22. caute leg. canere. p. 44. 1. 23. minor leg. minzus. 1.28. poft teflacei infer. fign. (:). p. 45. 1.19. apicem lege apice. p.57.1.20. referenda lege referenda. p.59.1.15. prutito firiata lege punitato-fiviata. 1.22.óbliqua lege oblique. p. 6o. 1.4. punet. poft rugofus mut. in comma. P.77.1.13.6ispu/hilatu leg. bisbipuflutata. p. 87. 1.13. 14. veltituin leg. reflitutum. p. 88.1.15. ferere conditzm leg. fere recondintun. p. 95. 1. 4. ab int: wtubro freques:s leg. nubo non frequess. p. 97. 1. 8.9. ab inf. verus leg. vera. p. 98.1.1.2. ab inf. perfundius leg. profundius. p. $x 00$. 1. 20. obferatefet leg. obfernaret. p.110. 1. 5. fepararit leg. Separiait. p. 129. 1. 24. displo leg. dupla. p.134-1.21. mutta leg. mutto. p. 146. 1. 2. rubra leg. nigra. p. 164. 1. 12. poffe leg. potuife. p. 170. 1. 6. licitatus leg. liciatus. p. I90. poft 1. 17. haec anktoris omiffa verba inferenda funt: Antennarum bafis, harunque articuli 2,3 , 4, caput infra antennas, thorax (excepta macula nigra) et abdomen lateribus tantum fului coloris; fufca vero alia omuia. Vis riat thorace rubro immaculato; et rarius $\beta$. femoribus tibiisque fuluis. --., Hab. fepes: vere et ineunte aefate frequens praefertim in floribus vmbellatis, ad mufcas aliaque Infecta praedanda, animal rapacifinum. Long. 7. lat. 2. p. ¿so. lin. . poft asitor punct. mut. in colon. p. 205.1 . 19 . poft quidem punct. mut. in colon. p. 215. 1. 22. foret leg. foret. p. $=16.1$. 8. poft gut adiiciatur etmi. p. 217. 1.27. Chryflofligma leg. Chryfoligma. p. 232.1.28. poft fe inferatur illim. p. 244.1.25. 280 . leg. 200 . p. 248.1.25. ojtino leg. optime. p. $250.1 . \mathrm{Ir} . T_{a b} V$. leg. Tab.IV. p. 293.1.23.

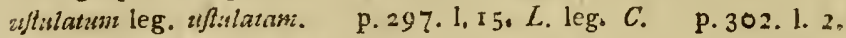
ab inf. congruenti leg. congruentibus. p. 398.1 . 9. C. leg. $N_{\text {s }}$

Letiora beneuolus iple lector emendet. 


\section{PRAEFATIO.}

Dill eft ex quo renum naturalium fitudiofi infecta beatae illius Italiae accuratius cognofcere concupinerunt. Vifis enim nonnullis ex hac animantium claffe fpeciebus fub felici illo coelo natis, collectionem eorum omnium fyftematico ordine digeftam, enixe vtique exoptabant. Primus fere fuit $R o \int f$, qui, faltem refpectu eius Italiae partis, quae ipfi notior erat, huic defiderio fatisfaceret. Viri celeberrimi opus *), cuius priorem partem lectoribus tradimus, et cuius pofteriorem breui fecuturam fpondemus, luculento teftimonio demonftrat, non nimiam fuiffe de diuitiis Italiae exfpectationem. Exhibentur in eo non folum pllrimae nouae infectorum fpecics, fed etiam illtftrationes exoptatifimae et non nifi in Italia parabiles, quikus fpecies hucusque non fatis notae melius

*) Fauna Etrufa etc. Liburni, typis Tliome Mafi et Sociorum, Praefidum facultate MIDCCXC. $\approx \mathrm{T} a$ mi. 4 to. 
melius innotefunt. Confilium auctor fecutus eft illud, omnia infecta in vicinia Florentiae et Pifae reperta defcribendi, fynonymisque, fi aderant, inftruendi. Cum in tali opere, entomologis omnibus neceffario, fpectanda fit inprimis communis vtilitas, cuitis in editione Italica haud habita eft ratio, libri pretio propter chartae fplendorem et typorum amplitudinem nimis auto, rem gratam equidem entomologis me facturum putaui, fi illis hoc opus traderem multo minore fumtu, fed haud diminuta vtilitate. In hac autem opella non fubfiftendum ratus, cum librum et accuratiore exainine indigere, et permulta infecta auctori haud vifa effe animaduerterem, quae oculis fubiecta habuerit neceffe eft is, qui illuftrare plura, quam turbare mauult, omnia equidem diligenti inueftigatione retractaui. Numerus collectorum infectorum, quae ipfe poffideo, fatis amplus, amica voluntas Dn. Langii, Auguftae Vindelicorum rei entomologicae fudentis, beneuolentia denique illuftris viri de Paykull, qui complura infecta Itala, ab ipfo celeb. Ro/ja accepta, mecum communicauit, copiam mihi fecerunt, infpiciendi et cunn aliis comparandi pleraque infecta in hoc opere defcripta. Quo factum eft, vt ampla mihi luppeditaretur 
ditaretur materia, qua non folum ad augendam operis Rofliani vtilitatem, fed et ad amplificandum vniuerfae fcientiae entomologicae ambitum, me vfum effe, fpero. Adnotata mea typis minutioribus diftincta, a reliquis infuper fubfcriptis litteris $\mathrm{Hg}$. differunt, in quibus id potiffimum egi, vt et obferuata entomologis haud inaccepta diuulgarem, et fynonymiam grauiffimi praecipue operis amici mei, celeberrimi Fabricii, quantum in me effet, emendarem. Neque minore ftudio refcindenda videbantur fuperuacanea quaedam. Itaque omifi defcriptiones omnes, quas feruatis quoque isdem verbis e Fabricii operibus transfcripfit auctor, appofita a me vbiuis nota: Fabr. defcriy) fit vel defcriptio Fabr. Deinde, quibus locis laudatum erat aliquid Fabricii opus, is citata a Fabricio iam exhibita repetere nolui. Citatis Fabricianis, a noftro auctore omiffis praepofui notam $\odot$; additis ab eo, fignum $D$; et adiectis a me, notam q. Hoc modo ct repetitionem inutilem enitaffe, et fatis perfpicue, quid auctori noftro debeatur, indicaffe mihi videor. Cum vero paginae editionis noftrae non refpondeant paginis Italicae, numeris paginarum prioris editionis fuo loco appofitis, citata facilius inuenienda

reddidi, 
reddidi, fi forte ad paginas editionis Italicae facta occurrerent. Sic e. g. pag. 57. noftrae editionis linea 13 inuenias vncis inclufum numerum 53 , qui de paginis Italae editionis intelligendus eft:

Anno MDCCXCII. Ro/fi edidit Mantiffam maiori operi accommodatam, et tractationis genere prorfus fimilem; cuius illam partem, quae primum hocce volumen refpicit, ei adiungere e re vifum eft, eoden modo, quo ipfum opus, retractatam. Omifi igitur et ill ea characteres genericos inutiliter repetitos, defcriptionesque, e Fabricio et Paykullii Monographiis verbotenus transfcriptas. Ad calcem huius voluminis collocata videbis, benenole lector, additamenta $a b$ auctore fecundo volumini annexa, et mihi ferius animaduerfa, cui fecundum volumen non fatim cum primo in munus venerat. Hac opportunitate vfus fum, quaedam infuper addenda fuis locis inferendi.

Imagines aeri incifae prorfus caedem funt, quae in editione Itala, contracta quidem chartae, forma, fed haud diminuto figurarum modulo. Quae in ornata eius editionis fronte, in qua et dedicatio operis continetur, depicta funt infecta, 
in Tabula noftra A. exhibentur. Ornamenta vero ifta neglecta effe neque entomologi nec elegantiores talium iudices aegre ferent.

Satisfaciendum denique eft animi grati officio et publice commemorandum auxilium, quod. mili lubentiffime impertiit $I$. C. IV. Illiger, optimae fjei iuuenis, in Collegio Carolino apud Brunfuicenfes, liberalium artium ftudiolis, et in perfcrutanda hiftoria naturali curae meae alumnus. Cuins egregia in me voluntas eo erat exoptatior, quo moleftior milhi crạt oculorum per. illud tempus, voi editionem moliebar, infirmitas, propter quam, nifi ille mihi adfuiflet, a nonnul-: lis laboribus huic operi perficiendo neceflariis, fuiffet abltinendum. Neque dubito fore, quin praeclare de hiftoria naturali mereatur is iunenis, in quo felici quódam confortio, eximiae ingenii dotes, varia doctrina iaborisque afiiduitas mature coniunguntur cum fernido illius fcientiae amore. Faciunt huic fpei fidem fpecimina ab ipfo in Schncideri actis entomologicis edita, in quibus notras quasdam elentheratorum fpecies, e mea collectione, depromtas, diligenter defcripfit.

Ipfe vero, cum nil humani a me alienum effe, vt qui maxime fentiam, ingenue profiteor, 
me gratifimo animo accepturum, quidquid viri docti monuerint, ad rectius conftituenda ea, in quibus errauerim, vel ad amplificandam vniuerfam rem entomologicam mihi iucundifimam et gaudiorum mihi impertitorum largo prouentu locupletem. Iudicaui quidem faepius de laboribus virorum doctorum, fimili ftudio hanc fcientiam amplectentium, quibus fi libertatem iudicii excufandam crederem, verendum effet, ne aut rem, prorfus fuperuacaneam agerem, aut animos eorum offenderem. Facile enim fentient, quae dixerim, e veritatis amore, non vero ex reprehendendi libidine profecta effe. Scripfi Brunfuigae, menfe Aprili 1 ?95.

Hellwig. 


\section{LECT OR I.}

\section{$\mathbf{P}$}

aucis te moror, Amice Lector; non is enim fum, qui de vtilitate Scientiae Infectorum, iam a tot praeclariffmis viris fufe, fapienterque illuftrata, commenta repetere velim. Id vinum te fcire cupio, Entomologiam, quae Rhedianis innixa experimentis, fub hoc coelo ad nouam veluti lucem reuxit, dum ingenio fudioque muitorum, temporibus hisce noftris, apud exteras gentes in vegetiorem aetatem florere admiraremus, domi, nefcio quo fato pene neglectam iacere dolebamus, quUIn PETRYS LEOPOLDVS animum aeftimationemque fuam fatis aperte oftendens, ditando, ea qua folet magnificentia, Regium Sutm Mufeum rara, et nultiplici Infectorum Collectione, aditumque omnibus illuc patefaciendo, amorem in nobis ea cognofcendi excitare vifus eft: quo factum, vt ego, non vltimus, incredibili eorum defiderio inflammatus fiterim. Etfi autem naxima animi alacritate ftudium hoc fufcepiffem, omnesque horas meas fubfeciuas in eo excolendo impendiffem, cum tamen omnia Marte noftro, absque vllo duce, aut alieno confilio, nulla librorum fupellectili, et in paupere re conficienda forent, vix dici poteft, quanta opus fucrit patientia, induftriaque, vt fructum aliquem laborum meorum retuliffe mihi viderer. Hic certe exiguus, at qualemcumque offero nunc in animi mei erga Patriam gratum teftimonium, non quod aliquod operae pretium me fecifie arbitrer, fed quod alios, poft me, maximum facere poffe confidam. 
Praeterea dicendum, me in diftributione elegiffe Methodum Fabricianam vti accuratiorem et recentiorem, atque in defcribendis Infectis femper praepofuife eius diagnofiu. Cum autem multis de caufis impoffibile mihi fuerit, habere ante oculos opera omnia huius generis, quae in lucem prodemt in diem, (atque hic liceat mihi occáfionem arripere publice profitendi me gratum Nobiliffimo Viro Sigismundo Conịti de Hochenwart, qui in fuppeditandis rariffmis; et pretiofifimis libris - mihi adiutor fuit, ) confultum duxi notare afterifco ommes detectas frecies, quas Clariffimus Fabricius non habet, nouae fint, aut non; defcriptae ab aliis, aut indefcriptae, Experientia vero edoctus, quantum incertitudinis, atque laboris Entomologiae ftudiofis afferre foleat breuitas, plerumque manca, in defcribendis Infectis, praefertim nouis, non nimus damnandam verbofitatem, quam pauciloquium effugere ftudui. Reliquum quod praeftiti, ex opere ipfo deprehendes. Vale.

$$
\begin{gathered}
\text { Dab. Pifis Kal. Sept. } \\
1-89 .
\end{gathered}
$$




\section{- INDEX \\ GENERVM ET SPECIERVH.}

Aflerifous notat fpeciem aut nourm offe, aut a Clariffino Fabritio indefcriptom.

\section{LVCANVS.}

I. Ceruu's.

2. Capreolus?

3. Parallelepipedus.

4. Caraboides.

II. SCARABAEVS.

5. Naficornis.

6. Difpar.

7. Typhoeus.

8. Silenus.

9. Subterraneus.

ro. Fimlatarius.

Ir. Bimaculatus.

I2. Erraticus.

I3. Confpurcatus.

I4. Luridus.

I5. Granarins.

I6. Irroratus. *

I7. Stercorarius.

I8. Vernalis.

9. Punciuintus. *

20. Quadrimaculatus.

$2 \mathrm{I}$. Porcatus.

22. Afper.

23. Quisquilius.

24. Lumaris.

25. Bifon.

26. Hifpanus.

27. Taurus.

28. Vacca.
29. Nuchicornis.

30. Caprie.

$3 \mathrm{r}$. Furcatus.

32. Sacer. -

33. Laticollis.

34. Schaefferi.

35. Geoffroare. *

Sc: Pilularius. F.

36 Schreberi.

37. Flanipes.

38. Onatus.

\section{TROX.}

39. Luridus.

\section{MELOLONTHA.}

40. Fullo.

4. r. Vulgaris.

42. Solftitialis.

43. Oblonga.

44. Denota. *

45. Agricola.

46. Horticola.

47. Ruricola.

48. Argentea.

49. Pulnerulenta.

50. Brunnea.

$5 \mathrm{r}$. Frifchii.

52. Vitis.

53. Nitidula. * 
VI. TRICHIVS. 5. Fafciatus. 55. Hemipterus.

VII. CETONIA.

56. Aurata.

57. Speciofiffima.*

58. Morio.

59. Eremita.

6o. Hirta.

6r. StiEtica.

\section{HISTER。}

62. Vnicolor.

63. Laeutis. ${ }^{\text {*ै }}$

64. Quadrimaculatus.

65. Aeneus.

66. Brunneus.

67. Detritus.

63. Depreffus. .

69. Abbreuiatus.

7o. Sulcatqs. *

7. Minimus. *

72. Bimaculatus,

X. DERMESTES.

73. Iardarius.

74. Murinus.

75. Pellio.

76. Tellellatus.

77. Cadauerinus.

78. Violaceus.

79. Rufipes.

80. Sanguinicollis.

8r. 20-guttatus.
82. Dentatus.

83. Tomentofus.

34. Fumatus.

85. Scaber.

XI. MELYRIS.

86. Bimaculatus. *

XII. BOSTRICHVS.

87. Carucinus.

88. Typographus.

89. Micrographus.

90. Piniperda.

9. Scolytus.

92. Bidentatus.

93. Billchoides. *

XIII. BYRRHVS.

94. Pilula.

95. Niger.

XIV. ANTHRENVS.

96. Pimpinellae.

97. Scrophulariae.

98. Mufeorum.

99. Verbafci.

XV. ANOBIVM.

roo. Pertinax.

IOI. Thoracicun. *

I02. Molle.

I03. Minutum.

I04. Serraticorne. * 
XVI. PTINVS.

I05. Fur.

106. Germanus.

I07. Scotias.

XVII. BRVCHVS。

ro\&. Pifi.

I09. Granarius.

I I 0 . Seminarius.

I I r. Cifti.

I I2. Bipunetatus.

XVIII. EL.OPHORVS.

II3. Aquaticus.

XIX. SPHAERIDIVM.

I 14. Scarabaeoïdes.

I 15 . Melanocephalum.

I I 6. Haemorrhoidale.

I 7 . Atomarium.

I 8 . Immaculatun. *

XX. TRITOMA.

I 1 . Incerta. *

\section{IPS.}

I20. Quadripuftulata.

I2I. Crenata.

I22. Taxicornis.

123. Linearis. *

I24. Pufilla. *

\section{HISPA.}

I 25. Flabellicornis. r26. Pectinicornis. 127. Flanefcens. ".
128. Teftacea:

I29. Atra.

I30. Ponaticornis. *

XXIII. NICROPHORVS.

I3 1 . Vefpillo.

\section{XXIV: SILPHA.}

I32. Littoralis.

I33. Atrata.

134. Rugora.

I35. Sinuata.

r36. Ruffica.

XXV. OPATRVM.

137. Sabulofum.

x38. Grifeum.

I39. Agricola.

\section{NITIDVLA.}

I 40. Bipuftulata.

I 4 I. Obfcura.

I42. Fiano - maculata."

I3. Punctata.*

I44. Varia.

I 45. Hirta.

146. Aeftiua.

147. Veris. *

I 48. Pedicularia.

XXVII. COCCINELLA.

I49. Impunctata.

I50. Bipunetata.

I5 I. Tripunctata.

I52. Quinquepunctata.

I53. 7 - punctata.

b 
I54. 9 - punctata.

55. Nigro-fafcicta. *

I.56. I $x$-punctata.

I5.. I3-punctata.

I 58. I 4-punctata.

I59. I9-punetata.

160 . Conglomorata.

I6r. Conglobata.

I62. 2 2 -punetata.

I63. 24 -punctata. I64. 25-punctata. I6.5. $x_{3}$-maculata. x66. Pallida. I67. Detrita. I68. I0-guttata. I69. Bipuftalata. I o. Similis. *

r7т. 4-puftulata. I 72.6 - puftulata.

I73. I 2 - puftulata.

$x\rceil 4$. Variabilis.

I75. Io-pufulati.

I 6 . Pantherina.

I7. 20 - guttata. ч 8 . Minutifjima. * I79. 4- maculat!. *

XXVIII. CASSIDA.

Iso. Viridis.

I 81 . Murraea.

I82. Nobilis.

I83. Nebulofa.

I84. Ferruginea.

I85. Limbata.
XXX. CHRYSOMELA. I 86. Tenebricola. I 87. Haemoptera. I88. Bankii. I 89. Ruftica. r90. Tanaceti. I9x. Populi. I92. Staphylaea. I 93. Polita:

194. Sanguinolenta. I95. Graminis. I96. Aenea. I97. Speciofa. x 9 . Boleti. 199. Alni. 200. Vitellinae. 201 . Betulae. 202. Coccinea. 203. Obfcura. 204 . Narginata. 205. 20 - punctata. 206. Cerealis. 20\%. Americana. 208. Dytiscoides. * 209. Polygoni. 210. Collaris. 2I I. Cerafi.

212. Metullica. *

213. Hyofcyami.

2 I 4. Oleracea.

2I 5. Erythrocephala. 216. Helxines. 2 I 7 . Rufipes. 218. Fufcipes. 219. Nitidula. 220. Exoleta. 
22 I. Atra.

222. Teftacea.

223. Hemifphaerica.

224. Tabida.

225. Linerta. *

226. Lurida. "*

227. Tiuncatr."

XXXI. CRYPTOCEPHALVS.

228. I.ongipes.

229. Quadripunctatus.

230. Bipunctatus.

231. Bimaculatus.

232. Scopolinus.

233. Cordigrer.

234. Tridentatus.

235. Moraei.

236. Auritus.

237 . Sericeus.

238. Nitens.

239. Marginatus.

240. Flauilabris.

24I. Cyaneus.

242. Sexpunctatus.

243. Flauipes.

244. Labiatus.

245 . Oftomaculatus. "

246. Trimaculatus. *

247 . Bipuftulatus.

248. Sexpuftulatus."

249 . Hiftrio.

25 . I o-punetatus.

25 I. Coryli.

252. Vitis.

253. Quadripuftulatus.
254. Pilofus. *

255. Pufillus.

\section{CISTERA.}

256. Gigas.

257. Ceramboides.

258. Ceruina.

259. Teftacea.

260. Murina.

$26 \mathrm{r}$. Pallida.

262. Sulphurea.

$26_{3}$. Lepturoïdes.

XXXIII. CRIOCERIS.

264. Calmarienfis. 265. Merdigera. 266. I 2-punetata. 267. Sanguinea. 268. Tenella. 269. Cyanella. 270. Melanopa. 27 I. Flauipes. 272 . Phellandrii. 273. Campeftris.

XXXV. LAGRIA.

274. Hirta. 275 , Caerulea. 276 . Bifafciata. 277. Nigra. 278. Antherina. 279. Floralis.

\section{GVRCVIIO.}

280. Colon.

$28 \mathrm{I}$. Bilineatus. 
282. Bimaculatus.

283. Puluevulcntus. *

284. Cardui. *

285. Afcanii.

286. Bacchus.

287 . Betulae.

288. Campanulae.

289. Alliariae.

290. Cyaneus.

291. Flanipes.

292. Nigriroftris.

293. Rufiroftris.

294. Pericarpius.

295. Paraplecticus.

296. Abbreuiatus.

297. Peud'acovi. *

298. Paftinacae.*

299. Pini.

300. Curuiroftris.

30 I. Frumentarius.

302. Granarius.

3०3. Sirpi. *

304 . Acridulus. .

3०5. Filiformis.

3०6. Quinquemaculatus.*

307 . Cerafi.

3०8. Faber. *

309. Vilis, *

310. Scrophulariae.

3 II. Abietis.

3 2. Fatuus.

3'3. Statua.

3 4 . Nucum.

3 5 . Tortrix.

3 I0. Druparum.

31 . Fumofus. *
318. Cinctus. *

319. Violaceus.

320. Salicis.

32 . Alni.

322 . Viminalis.

323 . Barbarus.

324. Nebulofas.

32.5. Sulciroftris.

326. Ophthalmicus. :

327. Affinis. ?

328 . Viridis.

329. Verecundus.

330. Grifeus.

33 I. Lineatus.

332. C. nigrum.*

333. Hifpidulus.

334. Scabriculus.

33.5. Puneratus.

336. Punćtatulus.

337. Senex. *

338. Afpcrfus.

339. Latiroftris.

340. Nigrita.

34 I. Pyri.

342. Oblongus.

343. Ouatus.

344. Argentatus.

345. Nubilus.

346. Fullo. *

XLI. RHINOMACER.

347. Brentoides. *

XLII. ATTELABVS.

348. Coryli.

349. Curculionoides. 
350. Pubefcens.

\section{CLERVS.}

35 r. Formicarius.

3.52. Vnifafciatus.

353. A piarins.

XLIV. NOTOXVS.

3.54. Monoceros.

355. Schucdia."

XLVIT. CERAMBYX.

356. Nofchatus.

357. Cerdo.

358. Heros.

359. Nebulofus.

з 60. Varius.

36 I. Hifpidus.

362. Quercus. *

XLVIII. LAMIA.

363. Textor.

364 . Sutor.

365 . Kaehleri.

366. Funefta.

$36_{7}$. Nebulofa.

369. Pedeftris.

360 . Molitor.

370. Vnifafciata. *

XLIX. STENOCORVS.

37 I. Lamed.

\section{CALOPVS.}

372. Serraticornis.
LI. RHAGIVM.

373. Etrufcum."

LII. SAPERDA.

374. Cylindrica.

375. Cardui.

376 . Punctata.

$37 \overline{7}$. Populnea.

378. Cateruleficns. *

379. Suturalis.

330. Filum. *

LIII. CALIIDIVM.

38 I. Baiulus.

382. Holofericenn. *

383. Onega. *

384. Fennicum.

385. Vivlaceum.

386. Sanguineum.

387. Teltaceum.

388. Arcuatum.

389. Florale,

390. Arietis.

39 1. Verbafci.

392. Trifafciatum.

393. Luridum.

394. Alni.

395. Plebeinm.

\section{IIV. DONACIA.}

396. Craffipes.

397. Simplex.

LV. LEPTVRA.

398. Melanura.

399. Linida.

b 3

400. 
400. Villica.

40 I. Quadrifafciata.

402. Villofa. *

403. Sexguttata.

404. Scutellata.

405. Atra.

406. Nigra.

40\%. Attenuata.

403. Ruficornis.

409. Abbréniata.

LVI. LAMPYRIS.

410. Italica.

41 I. Splendidula.

LVII. PYROCHROA.

4I2. Sanguinea.

LXI. CVCVIVS.

413. Flauipes.

LXII. CANTHARIS.

4r4. Fufca.

4 I 5 . Liuida.

416. Obfcura.

4 I 7 . Pallipes.

4I8. Nigripes.

4 I. . Ruficollis.

420. Aelanura.

421. Biguttata.

422. Minima.

LXIIT. MALACHIVS.

423. Aeneus.

424 . Eipufulatus.

42.5. Sanguinolentus.
42. Pedicularius.

LXIV. NECIDALIS.

427. Viridifima.

423. Caerulefcens.

429. Ruficollis.

430. Atra.

43 I. Rufa.

432 . Podagraria.

433. Caerulea.

434 . Humeralis.

LXV. EIATER.

435. Aterrimus.

436. Obfcurus.

437. Sputator.

438. Filiformis.

439. Niger.

440. Carbonaritis. *

44 I. Murinus.

442. Tefiellatus.

4.3. Sanguineus.

$4+4$. Balteatus.

445. Ruticollis.

$4+6$. Ferrugineas.

$4+7$. Prolifericornis. *

448. Rufipes. "*

449. Minutus.

450. Marginatus.

45 r. Pulchellus.

452. Maculatus. *

453. Binuculatus. *

454. Crucifer. is

455. Bipuftulatus. 
LXVI. BVPRESTIS. 4.56. Mariana. 457. Cuprea. 45S. Ruftica. 459. Tenebrionis. 460. Faftidiofa.* 46 I. Aruminata. 462. Aurulenta. * $463 \cdot 9$-maculata. 464. Qundrifafciata. * 465. Chryfoútigma. 466. Manca. $46 \overline{6}$. Rubi. 468. Viridis 469. Cyanea. 470. Nitidula.

47. I. Salicis. 47 ¿. Minuta. 473. Bignttata. 474. Taeniata.

LXVII. CICINDELA. 475. Campeltris. 476. Hybrida.

LXVJIL. ELAPHRVS. 477. Riparius. 478 . Aquaticus. $4 \div 9$. Semipunctatus. 4So. Flauipes.

LXIX. HYDROPHILVS.

48 I. Piceus. 48 2. Caraboides. 48.3. Scarabreoïdes. 434. Luridus.
485. Minutus. 486. Coicinelloides. * LXX. DYTISCVS. 487. Punctulatus. 488. Marginalis. 480. Difpar. * 490. Cinereus.491. Sulcatus. 492. Bipuftulatus. 493. Feneftratus. $49+$ Striatus. 495. Ieander. * 4.6. Ouatus. 497. Planús. 498. Erythrocephalus. 499. Lituratus. 500. Fermiginens. * 50 I. Paluftris. 502. Pufillus. 5०3. Granularis.

LXXI. GYRINVS. 504. Natator.

LXXII. CARABVS, 505. Coriaceus. 506. Hortenfis. 507 . Clath:atus. 508. Gramulatus. 509. Sycophanta. 5 ro. Caliginofus. 5 I I. Leucophthalmus. 5 I2. Arenarius. 5I3. Ruficornis. 5 I 4 . Objoletus. * b 4 
5 I5. Vulgaris.

5 I6. Caerulefcens.

5 ? . Lepidus.

5 I 8 . Cupreus.

5 I 9 . Latus.

520. Interruptus.

5 I. Ferrugineus.

522. Germanus.

523 Cinctus.

524 . Marginatus.

525. Limbatus.

526. Puluerulentus. *

$52 \%$. Madidus.

528. Oblongo-punetatus,

529. Dimidiatus." *

530. Puntulatus. *

53 1. Pilicornis.

532. Silphoides. *ै

533. Carboncrins."

534. Complanatus.

535. Crepitans.

536. Crux-maior.

537. Olens. *

538 . Lunatus.

539. Spinibarbis.

540. Cyanocephalus.

541. Melanocephalus.

542. Turcicus.

543. Viridanus.

544. Chry focephailus. *

545. Prafinus.

546. Teftaceus.

547. Crux - minor.

548. Andreae.

549. Cycithiger. *
550. Azureus.

55 I. Dentatiss. *

552. Mendax.*

553. Fafiolatus. *

554. Meridianus.

555. Truncatellus. *

556. Atricapillus.

557. Pedefuis. *

553. Quadriguttatus.

559. Jeuipes. *

560. Quadrimaculatus.

$56 \mathrm{I}$. Bipunctatus.

562. Exfonguis. "*

563. Minutus. *

56.1. Sigma."

565. Pufillus. *

566. Non plus ultra. *

LXXIII. SCARITES。

567. Gigas.

568. Thoracicus. *

569. Arenarius.

570. Ciypeatus. *

57 1. Calydonius. "*

572. Kufus. *

LXXV. PIMELIA.

573. Muricata.

574. Carinaza.

575. Rellexa.

LXXVIII BLAPS.

576. Mortifaga.

LXXIX. 'TENEBRIO.

577. Molitor. 
578. Affinis. *

579. Mauritcuicus. *

580. Culinaris.

58 r. Fufcus. *

582. Chryfomelinus. *

583. Dubitus. *

LXXX: HELOPS.

584. Lanipes.

585. Dentipes.

586. Triftis: *

587 . Chalybarens. *

588. Quifquilius.

589. Quntuts. *

LXXXIT. MELOE.

590. Profcarabaeus.

591. Tuccia. *

LXXXIII. LYTTA.

592. Veficatoria.

593. Erythrocephala.

594. Atra.

LXXXIV. MYLABRIS.

595. Cichorei. 596. Algirica.

LXXXV. CEROCOMA. 597. Schaefferị.

LXXX'I. MORDELI.A.

593. Aculeata.

599. Fafciata.

600. Sexpunitata. * 601. 12-puntata. *
602 . Flabellata.

603. Paradoxa.

604 . Flama.

60.5. Bimaculata.

LXXXVII. STAPHYLI= NVS.

606. Politus.

607 . Maxillofus.

608 . Marinus.

609. Erythropterus.

610. Ferrugineus: *

61 1. Vlmi.

612. Cuprelss. *

6 I 3 . Aeneus. *

6 I4. Morfitans.

6I5. Saratilis. *

6I 6. Chryfomelinus.

617 . Subterraneus, *

6 I 8 . Rufipes.

6 r9. Fufcipes.

620. Bipuftulatus,

621 . Obtufus.

622. Picens.

0:3. Buphthalmus. *

1.XXXVIII. OXYPORVS.

624 . Rufus.

625. Bicolor. *

I.XXXIX. PAEDERVS.

6=6. Piparius.

62 \%. Impreffus.

XC. FOrTICVLA.

628 . Auricularia.

b 5

629. 
XXII INDEX GENERVM ET SPECIERVM.

629. Minor.

XCI. BLATTA

630. Americana. 63 I. Orientalis. 632. Lapponica. 633. Marginata, XCII. MANTIS.

634. Religiofa. 63.5. Pauperata. 636. Roffica. * 637. Pagana.

XCIII. ACRYDIVM.

638. Bipunctatum.

639. Subulatum.

XCIV. TRVXALIS.

640. Nafutns.

61. Turritus.

XCV. ACHETA.
643. Domeftica.

544. Campeftris.

645. Italica.

\section{LOCVSTA.}

646. Viridifima.

647. Verruciuora.

648. Varia.

649. Perforata. *

650. Falcala."

65 I. Tuberculata. *

XCVII. GRYLLVS,

${ }^{6} 52$. Lineola.

653 . Flauus.

654. Thalafinus.

655. Italicus.

656. Caerulefcens.

6 57. Caerulans.

658 . Viridulus.

659. Rufus.

642. Gryllotalpa. 


\title{
INDEX ALTER
}

INSECTORVM IN MANTISSA ADIECTA CONTENTORVM.

\author{
Quadd locce volumen fipectat.
}

Aflerifio wotata ab Ausfore prorfus nouta exhibitn funt; litteris miszoribus diftinati: in Opere ipfo nonsum occisrumt.

\section{LVCANVS, I6. Squamosa.}

I. L? Virefcens.

III. SCARABAEVS.

2. Punctulatus.

3. Folfor.

4. Sordidus.

5. Rubidius.

6. Irroratus.

7. Hurcifer. *

8. Rufipes.

9. Plagiatits.

10. Fufcus. *

Ix. Lunaris.

IV. TROX.

12 Niger.

V. MELOLONTHA.

13. Rnficonis.

4. Abdominalis.

I5. Argentea.
I7. Dubia. *

VIII. HISTER.

I8. Macios:

19. Cyanells.

20. Maculatus. *

2 I. Minor. *

.22. Decemftriatus. *

23. Duodecimftriatus? *

24. Sulcatus.

25. Paruulus. *

26. Pufillus. "

27. Atomos. *

$\mathrm{X}$. DERMESTES.

29. Vulpinus?

29. Trifafcintus.

30. D? Dentatus.

3I. Fornicatus.

32. Fuliginofus. *

33. Bicolor. 
34. Dubius.

XII. BOSTRICHVS.

35. Chatcographus.

36. Pygmaeus.

37. Crenatus.

38. Ligniperda.

39. Varius.

40. Ater. *

$\mathrm{XV}$, ANOBIVM.

4I. Punctatuin.

XVI. ETIN়VS.

42. Longipes. *

43. Variegatus. *

44. Latro.

XIX. SPHAERIDIVM.

45. Fimetarium.

45. Pulicarizm.

47. Luteum.

XX. TRITOMA.

48. Bipufrulata.

49. Sericea.

XXI. IPS.

50. Fallax.

5. Teítacea. *

52. Atra. *

XXIV. SILPHA.

53. Laeuigata.
XXVI. NITIDVLA.

54. Maculatą. *

55. Strigata.

56. Difcoidea.

57. Chryiomeloïdes. *

58 Hoemorhoidalis?

\section{XXVII, COCCINELLA.}

59. Tripunctata.

60. Nigrofafciata.

6 I. 23 - pun Eีata.

62. $12-p$ unatata.

63. 14-maculata.

64. 14-guttata.

65. 14-puftulata.

XXVIII. CASSIDA.

66. Margariacea.

XXX. CHRYSOMELA.

67. Goettingenfis.

68. Faftuofia.

69. Violacea. *

70. Nigricornis. *

7. Gloriofa.

72. Gemellata. *

73. Vulgatiffma.

74. Armoraciae.

75. Pyritofa. *

76. Ruficollis.

77. Nenorum.

78. Pulex. *

79. Violaceo-punctata.

80. Dorfalis. * 
XXXI. CRYPTOCE- I05. Breniroftris. * PHALVS.

8r. Moriei.

82. Tricolor.

XXXII. CISTELA

CEBRIO.

83 . I ongicorinis: 106. Linofus. 107. Vifus. * Iôs. Armadillo. * 109. Sulcatus.

\section{RHINONACER.}

i I o. Brentoides.

XIII. ATTELABVYS.

XXXIIT. CRIOCERIS

85. Caprene.

XLIII. CLERVS.

XXXV. LAGRIA

86. Acnea.

87. Viridis" *

XLIV. NOTOKY'S.

II 3. Trifafciatuś. *

XI. CVRCVLIO.

88. Bardanae. II 4. Pedeftris.

I 5 . Cinetellus. I 6 . Hifpidus. * i1 7 . Myrmicocephalus. " I 8 . Hifpidulus. * Irg. Non plus vitra. * 120. Tenuicollis. * I 2 r. 4 -guttatus. * 122. Bifafciatus. * 95. Infidus. 95. Infidus. XLVII. CERAMBIX. I23. Fof ciculatus.

XLVIII. LAMIA.

124. Vnifaficata.

LII. SAPERDA.

I25. Erythrocephala. 126. Linearis. 
127. Liñeola.

LIII. CALLIDIVM. $\mathbf{I} 23$. Salicis. I20. Clauipes. I30 Variabite. I.3 Gibbofum? I32. Puflium.

\section{LEPTVRA.}

I33 Elongata.

LVII. PYROCHROA.

I34. Coccinea.

LXI. CVCVIV́S. 335. Tejiacens.

IXII. CANTHARIS. I36. Pallida. I37. Plambea.

IXIV. NECYDALIS。 I38. Hybrida. * I 39. Flanefichs.

\section{ELATEY.}

1 40. Litigiofus. *

I4 I. Lineatus.

I 42. Cylindricus. *

143. Thoracicus. I44. Binotatus.
LXVI. BVPRESTIS.

I45. Nouemmaculata. I 46 . Cyanicornis. * 147. Deaurata. * If8. Ritilans. I49. Elata. I50. Iacta. I 5 r. Caerulea. * 152. Pygmaer. I53. Minutid. I54. Nitida. *

\section{ELAPHRVS.}

I55. Caraboides. I56. Nebulofus. I57. Pyritofus. *

LXIX. HYDROPHILVS,

I 58. Caraboïdes. I59. Orbiculatus. I60. Pallidus. *

\section{DYTISCVS.}

161. Diffimilis. 162. Bipufulatus. 163. Transuerfalis. I64. Puftulatus. * 165. Hybucri. I66. Taeniatus. I67. Bipundictus? 167. ${ }^{2}$ Vliginofus. I68. Minutus. 
INSEGTOR. IN MANTISSA ADIECTA CONTENTOR. XXVII

LXXII. CARABVS. 202. Velocipes.

I 69. Purpurafiens. 203 . Rufives. 204. Dorfilis. Iтo. Horten?is. I 7 i. Conmexis. 205. Erratns, 施

I 72 . Paykullii, * I 73. Granulatus. I74. Leucophthalmus. I 75. Aurcpunetatus. LXXIII. SCARITES. 206. Dama. I76. Terricola. I77. Punitulatus. LXXV. TENEBRIO. I 78. Asvictus. I 79. Cinstus. 180. Ruficornis. I8I. Véftitus. I8?. Spoliatus. * I83. Nigricornis. I84. Rufus. * 185. Humerclis. I 86 . Smaragdulus. * 187. Sexpunfiatus. 188. Tenebrioides. I89. Dentatus. 90. Diftinçus. I9I. Germanus. I92. Exhalans. * I93. Pammodes, * I94. Aterrimus. I95. Prote:s. 196 Apjimilis. I97. Obfenus. I98. Infidus. I90. Agilis. 200. Ouatus. $20 \mathrm{I}$. Votiuus.

20\%. Cornifrons. * 208. Mauritanicus.

\section{MYLABRIS.}

209. Teftacea. 2 I0. Fulua. *

LXXXVI. MORDELIA.

21 I. Abdominalis.

212. Frontalis.

213. Nigra. *

LXXXVII. STAPHYLLNVS.

$214 . H i v l$ ls.

2I5. Cigcineats.

216 . Bicinctus.

217. Pubefcens.

2 I 8. Tomentofus.

aI9. Canaliculatus. 
XXVIII INDEX ALTER INSECTORVM IN MANTISSA etC.

220. Clavicornis.

XCII. MANTIS.

22 I Pallidus. *

222. Orbiculatus.

223. St? Sanguineus.

225. Roffia.

226. Spallanzania. *

LXXXIX. PAEDERVS.

24. Elongatus.

XĆVI. LOCVSTA.

227. Tuberculata. 


\title{
ENTOMOLOGIA ETRVSCA.
}

\author{
CLASSIS PRIMA. \\ ELEVTHER A T A.
}

Os palpis quatuor, aut fex. Maxilla inferior nuda liber't.

\section{LVCANVS.}

\section{(p. I.)}

Penicilli duo fub labio palpigeri. Antennae pectina. to - fifilles.

ז. L. Ceruus mandibulis exfertis, vnidentatis, apice bifurcatis, labio deflexo, ruga transterfali eleuata. F. Sp. I. I. 2.

$\odot$ Bergftr. Olear. Voet. Schluga.

D Müller. Z. D. 4\{3. Schrank. E. I. 32.

\& F. Ent. fyft. I. 2.236.2. Herbft. K. 3.28\%. t. 33 .

f. I. 2 .

Habitat filuas et praecipue Tlices fat frequens. Variat inagnitudine minori. Long. 23. lat. 8. Platyc. fufc. Geofi. Inf. I, 60. 2. nil nifi femina, mandibulis haud exfertis. Copula iunctam cum praece. denti vidi. 


\section{$(p, 2$.}

2. L. Crpicoins? mandibulis exfertis, apice tantum bifucatis, labio ruga transuctia elcuata interrupta. F. Sp. I. I. 4.

D) Merian. Europ. 168 .

1. Ceruo fimilimus, fed ab eo capitis thoracisque forma praefertim diftincus; cum non defit omni110 dens lateralis mandibuarum, nec femora, vti notat Degeer, arantiaca fint, de identitate cum L. Capreolo afferere muime placuit; mihi nona fpecies. Hab. cum praecedenti rarior. Long. 10 . lat. 6.

L. Cipreoluin a me fecundum defcriptionem Degerii pro vero habitum Dn. Herbf. Ton. III. NaturSyft. der Köf. p. 302. t. 34. f. 2. 3. fecundum fpecimina Mufei mei, ex America boreali mihi allata ipfe, deferipfit iconibusque illufranit accuratiflimis. Vide etiam iconem optimam Panzer. Beytrïge $J$. t. I. pag. 1. Lucanum D. Rofif vero, a mulcis Lutomologis pro varietate L. Cerni declaratum, p. 299. t. 33. f. 4. 5. Herbft. 1. c. fub nomine L. Hirci inuenies. Hg.

3. L. parailelenipedus mandibulis dente laterali elenato, corpore depreflo. F. Sp. I. 2., 6.

$\odot$ Pontopp., Voet., Bergitr., Deg.

D Inüller. Z. D. $4 \frac{16}{6}$. Laich. 5. 3.

? F. E.S. I. 2.239. I I. Herbit. K. t. 34. f. 5. Panz. F. I. G. II. I. 4 .

Variat magnitudine. Puncta feu potius tubercula Geoffroyana in capitis vertice optime feminam a mafculo diftinguunt, character conftans. Habit. in filuis Pifanis frequens. Lecti faepe plures congregati fub corticibus arborum, et in truncis cario. fis menl. Febr. et Mart. Long. 9. lat. 4. 


$$
\text { (p. 3.) }
$$

4. L. Caraboldes cactulefcens, mandibulis lunatis, thorace marginato. F. Sp. 1. 3. 9.

○ Scop., Voet., Schaeff. Ic, t. 75 . f. 7 .

q F. Ent. fyft. I 2. 23y. I 4. Herbft. K. t. 34. f. 6. 7. Variat colore fupene viridi, infeme fufco. Habit. easdem arbores non frequens. Long. 5. lat. 2.

Varietas notata eft L. ruffipcs. Herbft. K t. 3. p. 3 I 1 .

t. 34. f. 8. mea quoque obferuatione nil nifi mera varietas L. Caraboïdis. Hg.

\section{SCARABAEVS.}

Palpi quatuor filiformes. Jabium enrarginatum apicibus elongatis palpigeris. Antennate lamellatae.

a. Scutellati thorace cormuto.

5. S. maficomis feuteliatus, thorace prominentia triplici, capitis cornu recuruo; elytris lacubus. F. Sp. I. I I. 33 .

$\odot$ Voct., Frifth., Drury, Olear.

D Scop. E. C. I 4. Schrank. E. I. 2.

\& F. Ent. f.ft. 1. I. I 4.38 .

Hab. in ligno putrido, vaporariis, fimeto frequens. Long. 16. lat. 9.

6. S. difper futellatus, thoincis cornu fubulato protenfo, capitis fubulato fubrecuruo, fcutello cordato. F. Sp. I. 5. \%.

$\odot$ Pall.

O F. Ent. fytt. I. I. 5. \%०

Hab. in ftercore bouino non raitts. Long. Io. lat. $4 \frac{\pi}{3} n$

7. S. Typhoous fcitellatus, thotace tricorni; inrermedio minori, lateralibus porrectis longitudin capitis mutici. F. Sp. I. Io. 30. 
$\odot$ Ray., Mouf., Bergftr., Petiv., Voet.

\& F. E. S. I. I. I2. 34. Herbft. Käf. t. 6. f. I. 2. Panz. F. I. G. II. 23 . m. et f.

Hab. fub fercore humano, profundos cuniculos fodiens;' fupra terram plerumquic occurit menfe Maio. Long. 6-8. lat. 4-4!

Citatum Fabricii malum ex Iconibus Schaefferi in opus fuum transtulit Roni. Figura enin prima t. 5. non feminam huius, fed S. ftercorarium, tefte quoque Harrero, absque vllo dubio defignat. Huic citato, f. 9. t. 23. fubftituendam efie putat Schneiderus, N. Mag. d. Entom. 3. B. 2. St. p. I00. fed ex iconibus, quales Schaefferus exhibet, de infectis non multum a fe abhorrentibus nil certi determinari poteft. Hg.

$$
\text { (p. 5.) }
$$

b. Scutellati, thorace inermi, capite cormuto.

\$. S. Silenus futellatus, thorace inermi, antice excauato; capitis comu recuruo, elytris lacuiffmis. F. Sp. I. I3. 44.

$\odot$ Voet.

D Scop. Del. Fl. et Fn. Inf. p. T. t. 2 I. f. C.

Q F. Ent. fyft. I. I. x8. 5 I. Herbft. K. t. Io. f. 2. Alter fexus thoracem habet puluinatum non excauatum, caput muticum conuexun. Hab. ruri. Iul. menf. in arenofis faepe mortuus reperitur, fortafle folis aeftu peremtus, cuiminus, quam alii fuac gentis refiftere videtur. Long: 9. lat. 4.

Voet. t. xg. f. IIg. bene praetermifit Fabricins in Ent. fyft. cum huius Scarabaei nomen Voetianum indicet, eum adlatum effe ex India, et cum e defcriptione addita nimis breui non liqueat, illum effe eundem cum Italico. $\mathrm{Hg}$.

9. S. fubterraneus fcutellatus, thorace inermi capite tuberculis tribus, elytris ftriis crenatis. F.Sp. I. 15.58 .

Schrank. 
D. Schrank. E. I. 7 .

O. F. E.S. I. I. 23 , 70.

In meis fueciminibus tubercula capitis tria. Hab. in ftercore paffim, primo vere frequens. Long. $2 \frac{1}{3}$. lat. I⿳亠口冋.

Cum Schrank. 1. c. Scarabaeo fuo fubterraneo vnicum tantum frontis tuberculum tribuat, atque elytra eius ad lentem taritum punctato - ftriata commemoret, auctor illum allegare non debuiffet. Nobis inter tòt huius Scarabaei fpecimina nullus vnico tantum tuberculo oblatus eft. Werofimile mihi porro, Sc. Julcatum F. E. S. 24. 74. cuius defcriptio exactiffime defignat fubterraneum, meram eius. dem effe varietatem. $\mathrm{Hg}$.

10. S. finetarius fcutellatus, thorace inermi, ater, capite tuberculato, elytris rubris. F. Sp. I. I6. 64 .

(2) Voet., Frifch., Scop., Ray.

D) Roef. Inf. 2. Sc. A. f. 3 .

을. F. Ent. S. I. I. $27 \cdot 84$. Herbf. K. t. I2. f. $4^{\circ}$ Sexus alter nulla habet tubercula. Abundat in bouinis faecibus. Long. $3 \frac{1}{\frac{1}{2}}$. lat. 2.

Scar. foetens. F. Mant. I. 8. 63. E. S. I. $24.75 \circ$ Herbft. K. 2. I73. et Sc. vaccinarius. Herbft. K. 2. I38. t. I2. f. 5. fecundum obferuationems meam nil, nifi varietates Sc. finetarii. $\mathrm{Hg}$.

\section{(p. 6.)}

II. S. bimaculaitis fcutellatus, thorace inermi, capite fubtrituberculato, elytris ftriatis, macula bafcos rubra. F. Mant. I. 8. 67.

오 F. E.S. I. I. 26. 82. Herbft. K. t. I2. f. I4. Panz. Naturf. 24. 3. t. I. f. 2.

Hab. in cadaucribus et ftercore bouino. Long. $24^{\circ}$ lat. $I_{4}^{\prime}$. 
Sc. terreftrem, haud dubio S. bimaculati varietatem, miramur ab auctore non effe repertum, atque hac re ducimur ad coniiciendum, S. fubterraneum aum ctoris forfan effe S. terreftrem, qui non raro ab auctoribus confundi folent. $\mathrm{Hg}$.

12. S. erraticus fcutellatus, thorace laeui, capite tuberculo vnico, clytiis fuccis. F.Sp. I. I . 66. 아 F. E. S. I. I. 27.86 .

Hab. in ftercore, riis. Long. $3 \frac{r}{2}$. lat. 2 .

Citatum ex Sch. Iconib. t. 26. f. 9. non huic, fed potius $S$, merdario adiungendum eft, quamuis figura et hunc haud optime repraefentet. Hg.

I3. S. confpurcatus thorace marginibus latera. libus albidis, capite tuberculato; elytris grifeis fufco-maculatis. I. Sp. r. $17.6 \%$.

(1) Ray., Pontopp.

\& F. Ent. S. I. I. 2 \&. 8\%. Herbft. K. t. I2. f. \&. $\mathrm{Hab}$. cum congeneribus in ftercoratis. Long. $2 \frac{\pi}{3}$. lat. It:

Cum Linnaeana defcriptio Sc. confurcati ad plures Scarabaeos referri pofit, et genuinus Sc. confpurcativ a caeteris diffingui exacte nequeat, fuadendum eft, vt fecundim ea, quae Herbfius 1. c. fcripfit, eius Scarabaeum pro Linnaeano habeamus. Cui fententiae acceffit 'etiam Fabricius inferta in Enton. fyft. obferuatione illa Herbftii. Quod fi probaierimus, citata a Voetio et Schaeffero, in Spec. et Eiit. fylt. exhibita, omittenda funt, ad Scaraaaeum !wridmin pertinentia. $\mathrm{Hg}$.

\section{(p. 7.)}

I4. S. luridus ater, capite tuberculato, elytris grifeis, nigro-friatis. F. Sp. I. I 7.60.

․․․ Ent. fyft. I. I. 29. $9 I_{0}$ Voet. Scar, t. 2I. f. $x+4$. Scháeff، 
Schneff. I. t. 26 . f. 8. Sc. interpunitatus. Fuesl. A. H. 4. 8. t. I9. a. f. Ix. Herbft. K. t. I8. f. 3. Hab. bouinas facces. Long. $3 \frac{1}{\frac{1}{2}}$. lat. 2 .

dilerae varietates $S_{i}$. lividi, ex obferuatione mea nulli dubio fubiectac, funt: Sc. variegntus. Fuesl. A. 4. g. t. Ig. a. f. r.z. Herbft. K. t. IS. f. 4. Sc. arator. liuesl. A. 4. 9. Herbft. K. t. I9. f. 3. Sc. rufipes. Herbft. K. 2. 282 . Sc. nigripes. F. E. S. I. I. 35. III. Hg.

15. S. gramarius nigèr, clypeo tubcrculo folitario, clytris poftice teftaceis. $F$. Sp: I: I 7.00.

D) Schaeff. Ic. t. I44. f. 5 .

?. F. Ent. fyft. I. I. 29. 92 .

Totus ater, lacuis. Elytra ftris excanatis, poftice verius marginem rufefcentia. Pedes et femora obfene rufa. Hab. in ligno putrido fub macérie. Long. I. lat. 紊.

Fatricius, quem auctor nofier fecutus eft, Sc. granae rium perperam confundit cum Sc. hacmorrhoidali, in Spec. Inf. In Ent. fyft. crrorem correxit, Vir celeberrimus duas inde feparatas fpecies efiiciens. $\mathrm{Hg}$.

*.15. S. irroratus futellatus, thorace foffula imprefia, capite fubcomuto, elytios finlcatis, nigris, rufo-maculatis.

Sc. Moeris. Pall. Tc. p. 3. f. 2.

?. Nant. Rofii. p. 7 . n. 6.

Clypeus marginatris, riga elenata laterali, et in nedio comiculo breni armatus. Antennae nigrae. Thorax rugofus margine cienulato. Scutellum patuxm. Elytra maculis plurimis obfoletis rufis, vix diftinguendis, adfperfa. Tibiae anticae quadridentatae. Hab. Pifis, non frequens. Long. S.lat. 4.

Sc. Mocris nullo iure a Fabricio in Ent, fyft. apud

Sc. teftudinarium allegatur. $\mathrm{Hg}$.

c. Sirs 
c. Scutellati mutici, capite thoraceque inermi. (ip. 8.)

I 7. S.fercorarius fcutellatus, muticus ater, clypeo rlombeo, vertice prominulo, elytris fulcatis. F. Sp.' 1. 18. ?4.

$\odot$ Ray., Lifter., Frifch,, Voet.

D Goetze. E. B. r. 26. 42. Scop. E. C. 26. Schrank. E. I. 23 .

\$ Herbft. K. 2. p. XVI. praef. p. 254. text. t. A. Fab.E. S. I. I. 30. 97. Schaeft. Ic, t. 5. f. I. t. I3. f. $x$.

Saepe ita infeftatur ab Acaro Coleoptratorum, ve procedere nequeat. Colore et magnitudine admodum variat. Var. maior, colore fubtus femper laete violaceo, tibiis anticis faepius fexdentatis, elytris ftriatis, femoribus pofticis latere interiore facpe bidentatis, praefertim in feminis. Var. $\beta$. minor, colore plerumque fubtus aeneo, elytris laeuibus, aut vix ftriatis refpectu maioris. Duo etiam ininora fpecimina poffideo, vmum nitidifimum elytris laenibus, aut vix ftriatis colore vbique violaceo; alterum videtur effe varietas a Geofroyo nutata. Hab. in ftercore bouino. Long. ro. lat. 5 .

De noua et quafi intermedia fpecie inter Sc. ftercorarium et Sc. vernalem. vide Scriba Journ. f. d. Liebh. d. Ent. 3. St. x79 T, p. 250 . vbi Sc. fercoratus vocatur. Ipfe faepius in filuarum fungis Sc. fterioFrariun ręprehendi, folito minorem, et mihi diffe:re vifum. $\mathrm{Hg}$.

$$
\text { (p. 2.) }
$$

I8. S. vernalis foutellatus muticus elytris glabris laeuiffmis, clypeo rhombeo, vertice promimulo. F. Sp. I. I9. 75 .

(-) Voet. 
I Schaef. Ic, t. 222 , f. 3. Schrank. E. I. 24. 9. F. E. S. I. I. 3r. 9s. Herbit. K. t. I8. f. r. Thorax fere acquat magnitudine abdomen. Var riat magnitudine et colore caerulef́cente et obfcuro. Mofchim olet. Mas duplo minor femina. Hab. paffin in ftercore, agris. Long. 7. lat. 4\%.

Scarabacos fcutellatos pro thoracis et abdominis proportione diuidere licet in eos, quorum thorax longitudine sequat abdomen, et eos, quorum thorax abcomine brevior. Prioribus aunumerandus eft Sc. vernalis. Recte igitur auctor non adduxit effigiem Voetianam, yuae, quod ad hanc proportionem pertinet, prorfus inaccurata eft. Neque Schatiferianam citafiemus, in qua elytra fulcata funt. Hs.

* I 9. S. puncuilutus, lcutellatus; mandibulis laciniatis, clypeo triangulari, thorace puncato, cl)tinis fublineatis, punctisfue mainsculis inordinate excauntis.

S Sc. Monodon. F. E. S. I. I. 20, 5-? Sc. Idiotri. Herbft. K. t. I \% . . 4.? Mant. Roffi, p. 5. 2 .

Totus ater, lubtus hirtums, cly seo pene angulato, mga transuerla in nedio diufa. Tibiae anticale fexdentatae. Mandibulae exterius trilaciniatae. Labium inferius integrum linguiforme. Thorax conuexus rotindatus. Elytra late lineata lir.cis lacuibus, et vix eleuatis. Tab. I. f. I. Fab. filuas frequens. Long. 9. lat. 5 .

20. S. 4. maculatus, fcutcllatus, muticus oblongus, nigei, elytris maculis duabus rubris. F. Sp. r. 21. 86.

\% He: bft. K. t. I8. f. Io. S. 4. puftulatus, F. E.S. I. I. $36.1 \mathrm{I} 6$.

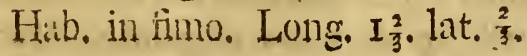




\section{(p. To.)}

2. S. porcaius fcutellatus, muticus, fufcus, elytris porcatis. F. Sp. r. 21. 92.

O. F. L. S. I. 1. 38. 126.

Tibiae anticae tridentatae. Thorax comexus lateribus vix marginatus. Hab. in plantis putridis. Inucnti pluies in curie flicis d. 22. Decembr. Long. $I_{\frac{\pi}{3}}$. lat. I.

22. S. afiper. fcutellatus, muticus, capite thoraceque transuerim fulcatis, elytris friatis. F. Sp. 22. 99.

QE. E. S. I. I. 39. I=8. Herbft. K. t. I8. f. I4. Hab. in plantis putrinis. Long. I'. lat.

23. S. quisquilius fcutellatus muticus ater, elytris linidis. F.Sp. 2I. 9 I.

Caput, thorax punculatus. Scutellumpartumi. Corpus nigrum. Elytra caftanea, fubpunctata, obfolete ftriata. ß. Variat eiytris teltaccis futura signa, nempe S. merdarius. F. Sp. 21.90. Hitb. in ftercorce Long. $I_{\frac{1}{5}}$ lat.

Prinum commemoiatur Sc, quisquilius a Linnaeo in Fn. Su., qui ei faciem Scarabei tribuit, eiusque habitationem in quisquiliis efie dicit. Linneus dubitaffe vicletur, an pro vero Scarabneo habendus fit. Inde receptus eft in einsd. Syftema Natirae, adiecto Synonymo Sc. minimi. Scop. qui eitus habitationem in folis plantarum, quod infelitum eft Scatabueis, elle confirmat. Receptus poitea a liabricio in Syftema Entomologicum. afhinis efie dicitur S. merdario, ibidem commemorato. nil quod huic futura elytiorum nigra fit. Quacfiuerunt Entomologi Scarabaeos, fuic deferiptioni refpondentes. Sc. merdarium cito fe reperiffe vifi funt, cum omnes eum intelligcrent, cuins effigiem exhibet

Herbitius 
Herbftius in N. S. d. Kïf. t. I8. f. 5. Citatus Sc: minimus Scopolit induxit Entomologos, vt crederent, marem Sc. vnipunctati, puncto elytrorum communi carentem, verum effe $S_{i}$. quisquilium. Videatur T. 19. a. f. 8. Fuesl. Arch. et Herbftii N. Syft. d. Käf. t. I8. f. I5. Ante editam hane eftigiem Herbftianam Fabricius in Mantiffa fra Se. merdarism pro mera varietate $S c$. quisquilii declasatum, prorfus omiferat. In Entom. Syft. ambas effigies modo laudatas citat, hand dubio maren Sphaeridii vnipunctati fui (antea Scarabaei unipunEtati) repraefentantes. Inde colligo, Fatricium aut non vidiffe illum Scarabneum, quem efle putant Fabricii merdarivm, aut exlibuifie el:m in Ent. Syftem. fub nomine mihi incornito. Alterutrum fi fratuimus. ex sute dictis fequi puto, vel verum Sc. quisquilinm nondum cogniem efic, vel efie eundem cum nure Sph. vaipunctu. Quisquid horum placeat, nomen 'je. quisquilii omnino delendum eflè victur in Fabricii vilemate et c. quisquilius Herbftii pro sph. mipunetuto Fabr. ha$\mathrm{b}$ endis, it tandem controverfiae, que fcientiam Entomologicam minime intat, finis imponatur.

$\mathrm{Hg}$.

d. Exfcutcllati, thorgce cormito.

$$
\text { (P. II.) }
$$

24. S. Iunaris exfcutellatus, thorace tricorni, com intermedio obtufo bifido, capitis corm erecto, dypeo emarginato. F. S?. I. 24. 108.

( ) Linn. Fn. Suec. Ray., Frifch., Petiv., Bergftr., Voet.

D Schrank. E. I. I. Degeer 4. t. Io. f. I.

옥 F. E. J. I. I. 46. I50. Sc emarginatus ib. 15 I. Herbft. I. 2. 39: t. 8. f. 7. 8.9. Mant. Rofii. p. S. II. : 
Caput planum, antice roundatum, crenulatum, bifidum, vertice coinuto. Elytra ftriata, vel putius fulcata. Niger totus, verticicornis, thorace ad vtrumque latus hemifphaerico, fcalpello veluti excifo, ita vt dens vnus, vel cornu vtrinque in margine thoracis fit, dum fulco medio in duas partes aecquales diuilus eft. Antice truncatus apparet, et in medio partis ábfiffae capuct verfus, tubercula duo parua acuta vtrinque adfunt. Scntellum nullum. Elytra fulcata fulcis in fingulis oćto. Mas.

Fenina differt thorace magis rotundo, antice minus retufo, et abscue dentibus lateralibus, aut vix eminentibus. Capitis vero comu erectum, hlnulatum, bifidum, triplo minus quam in mafculo; cuius cormu acutum et integrum eft. Reliqua in vtroque fexu fimilia, fed magnitudo admodum variat. Apud nos adeo copiofus, vt habuerim innumeros, et vna vice laepius plus quam viginti inuenti funt in eodem loco. Inter tot fuecimina a me examinata duas tantum feminas, vidi capitis comu non lumulato, led integro et minori. Specimen a Schrankio obferuatum huic varictati finile fuife videtur, quod dubium ab eo indicatim refoluit. Variant figura faepe etiam comua, vel dentes laterales thoracis. Habit. faeces bouinas. Long. 8 . lat. 5 .

e. Exfcutellati, thorace inermi capite cormuto. (p. 12.)

25. S. Bifon exfentellatus, thorace antice nucronato, capite cornubus duobus lunaribus. F.Sp. I. 26 . II 5 .

옥.F. E: S. I.' I. 50. I64. Hab. in fiercore bouno frequens. Long, $\eta_{2}^{x}$. lat. 4 . 
26. S. Hifpnnus exfortellatis, thorace inermi retufo, clypco comuto, cmarginato, elytris triatis. F. Sp. 1. 12.26.

$\odot$ Linn. Niuf. Lud. Vlr.; Roef.

Doet. Sc. t. $24 \cdot$ f. 13.

S. F. E. S. I. x. 57. I88.

Statura S. heneris, at paullo maior. Clypeus emarginatus. Thorax antice retufus. Elytra trriata, integra, in S. panifco len Vulgo Limn. breuia. Hab. Florentiae. Long. 9. lat. 6.

27. S. Teurus exfcutellatus, thorace incmi, occipite, comubus duobus reclinatis, arcuatis. F. Sp. 28. 125.

() Ray., Schreb., Schaeff.

D Schaeff. Ic. t. 63. f. 4 .

P. E. S. I. I. 5 t. xา8. eiusd. S. Capra ibid. p. 55. n. 180. S. reficornis Leske Iter, p. 45. t. A. f. S. 9. Panz. Fn. Inf. Germ. XII. 3.

Hab. in ftercore equino pafcuorum, primo vere fiequens. Long. $3 \frac{1}{2}$. Iat. 2 .

Permulta huius Scarabei a me collecta, eaq̣ite gradatim inter fe variantia, fpecimina docuerunt me, Se. Capram $F$. et rceticornem Leskii meras efle varietates Sc. Tauri, quae feminte huius Scarabaei (cuins clypeus capitis inermis et transtierim fulcatus) fenfim appropinquant, adeo, vt vterque. fexus vix dignofei poffit. $\mathrm{Hg}$.

\section{(p.13.)}

28. S. Vacca exfcutellatus, thorace inemi retufo, occipite fpina erecta gemina. F. Sp. 1.28. I26.

$\odot$ Schroet.

S F. E. S. I. T. 55. I79. Panz. F. I. G. XII. 4. Hab. bouinas faeces non rarus. Long. 4. lat. $z_{2}^{\mathrm{r}}$.

Citatura 
Citatum Schroeter. M7ifi. 1. t. 3. f. 5. a Fabricio etiam in Entomel. fyltemat. repetitum, ad S. Bontfum F. fpectat. Cititum Sihacf. Ic.t. $7 \hat{3} \cdot f \cdot 4 \cdot 5 \cdot$ a Fabricio in Ent. fyft. adductum monente Harrero S. Vaccam minime repraefentat. fid nouam fpeciem, quam cum Preislero Cent. I. Inf. Bohem. S. fraEicornem vocat. Cacterum mirmur, aticorem non commemorafie marem huius Sirrabaei, cuius occiput, vt mafculi S. nuchicornis, fpina erećta armatum elt. $\mathrm{Hig}$.

29. S. muchicomis exfcutellatus, thorace rotundato inermi, occipite finina eiecta armato. F. Sp; I. 30.132 .

( Voet., Riy.. It. Gott. Linn.

D Schaeff. Ic. t. 73 . f. 2.3 . Schrank. E. I. 3 .

O. F.E.S. I I. 58. r92. Preisi. Livf. Buhiem. Cent. Ta

t. .. f. to. A. B. Panz. Fn. Inf Gerrr. IV. T.

Inter mea fpecimina alia habent fininam, aliis deeft, aut minimum, rigis duabus elenatis transucrfis, femina. Frab. frequens in ftercore bouno. Long. $2 \frac{1}{2}-3 \frac{1}{2}$. lat. $1 \frac{x}{3}-2$.

S. pianicomis Herbft. K. t. I4. f. I3. mera varietas' minor s. nuchicornis efle videtur. $\mathrm{Hg}$.

3o. S. Capra exfcutellatus, thorace inermi, occipite fpina erecta gemina, corpore nigio obfcuro. F. Mant. I. 15. 144.

9. F.E. S. I. 1. 55. 180. Scriba Ephem. Entom. x. 52. An femina S. Tauri?

Hab. in ftercore bouino. Long. $3:$ lat. 2.

Mera varietas S. Tauri. Vide aduotata ad $S$. Tuinmum.

$\mathrm{Hg}$. 


\section{(\%. I.4.)}

3. S. furcatus exfontellitus, thorace inemi rotundato, capite combus tribus erectis approximatis, intemedio brenion. F. Sp. I. 30 . I34,

D S. Vitulus Laich. Inf. t. I. f. 20.

Q. F. E. S.I. т.60. I98. P'unz. I. I. G.XII. 5.

Cormin capitis apice ubtufa. Thorax fubpubefens. Elytra fulca obolete punctato - ftriata. Femina inermis linea transuerfit eleuata. Habit. in ftercore bouno rarius. Long. 2. lat. $I \frac{T}{4}$.

\section{f. Exfutellati mutici.}

32. S. facer exfoutellatus, clypeo fexdentato, thorace inemi crenulato, tibis polticis ciliatis, elytris lacuibus. F. Sp. I. 3 x. 39 .

$\odot$ Linn. Mur. Lud. Vir, Amqun. Acad, Osb. It. Loefling. It., Voet.

D Schaef. Ic. t. 20I. f. 3 .

\& F. E. S. I. I. 62. 205.

Hab. Florentiae et Pifis fieçuons, praefertim in filua dicta St. Petri. Long. I 4. lat. 5 .

33. S. laticollis exfoutellatus, muticus, niger, clypeo fexdentato, clytris lulcatis. F. Sp. I. 3 I. I 40 .

○ Ray.

D Petagna Inf. Cal. n. 4. defcriptione optima.

P. F. E.S. I. I. $6 \geq .=06$.

Inter mea fpecimina duas habeo varietates, S. ficro dinidio ninores. Altera raxiat thorace punctis maiusculis excauato; altera clytris late ful. catis, clypeo absque comn nudinento, quod in $S$. facro obferuatur, at in cateris omnibus nimis illi fimiles et affines videntur. Hab. cum praecederti rarior. Long. ?. lat. 5 . 


\section{(p. 15.$)$}

34. S. Schaeffiri cxicuteliatus, inuticus, thorace rotundato, clypeo emarginato, clytris triangulis, femoribus pofticis elongatis, dentatis. F. Sp. I. 32. 148.

q F. E.S. I. I. 66.220 .

Capitis clypeus emarginatus, antice bidentatus. Fcmora poftica lata compreffa, Habit rarius cum aliis. Long. 4. lat. $2 !$

* 35. S. Geoffroyae exfcutellatus niger, capite clypeato, elytris nargine exteriore finuatis. Geofir. Ini. I. g. . .8.

Sulz. Hift is t. I. f ?. Goetze E. B. I. 74. I5. Schneff Ic. $t 3 \mathrm{f} z$.

Sc. pilularius $F$ Mant $\pm ? \pm 70$ ?

qF. E.S.I I 67222 . Vuet Col.t. $26 \mathrm{f} 28$. Herbft. K. 2.3 II. t. 20 f. 5. Preisl. Inf. Boh. Cent. I. 4038 .

Totus niger, etiam inferne, elytris laeuibus poft bafin ad litera contractis, finuatis. Femora et ti. biae pofticae elongatae. Nottra fpecimina contreniunt ommino cum defcriptione Geoffroyi et figuia Schaefferi. Hab. bouinas faeces frequens. Long. $5 \frac{1}{2}$. lat. 3 .

Citata Herbftii e fcriptis Degeerii, Drury et Voet. t. 27. f 37 . apud sc. pilularium, qui Sc. Geoffroyge auctoris eft. in N. S.d K. adducta, et ad $S_{c}$. volquntem F. E. S. z2 I. pertinentia, delenda funt.

$\mathrm{Hg}$.

\section{(p.16.)}

36. S. Schreberi exícutellatus, muticus, ater, glaber, elytris maculis duabus rubris. F. Sp. I. 33. $95 \mathrm{r}$.

\& F.E.S. I. I. 68.225

Macula 
Macula ad bafin elytri oblonga, altera ad apicem fere rotunda. Hab. bcuinas faeces non rarus. Long.' 3. lat. I ${ }_{4}^{3}$.

Maior, thorace quadrituberculato, Fabricio a Daldorfio miflus, certiflime nil nifi mera varietas Sc. Schreberi. Hg.

37. S. flauipes cxfcutellatuts muticus nigricans, thoracis marginibus, clytris pedibusque flaudicentibus. F. Sp. App. 495.

D Geoffir. Inf. I. po. 6? Sc. thoricocircularis. Laich. 24, i 7 . Schaefl. Ic. t. 74. f. 6.

오 F. E. S. I. I. 70. 233.

Magnitudo Si. funetarii. Lividus; thurax circularis, fubacneus, funeola latcrali, ant potins puncto nigro. Clypeus antice fubmarginatus. Elytra fíbrugofi. Tibiae anticae cuadridentatae. Habit. in ftercore bonino rarior. Long. $33^{\mathrm{T}}$. lat. 2.

Voet. t. 28. f. 48. ad exoticum perinet, et hune Scarabaeum male repracfentat; citari ergo, vt a nonnullis Entomologis factum eft, eo minus debuifet. $\mathrm{H}_{3}$.

$$
\text { (p. IT.) }
$$

38. S. ouatus exfcutellatus, muticus, niger, thorace rotundato, fubaeneo, elytris abbreuiatis. F. Sp. I. 34. I 5 S.

ㅇ F. E. S. I. I. 70. 23\%. Preisl. Inf. Boh.C. I. 46. 49. Hab. in ftercore bouino infrequens. Long. $I_{\frac{2}{3}}$. lat. I.

\section{TROX.}

Palpi quatuor capitati. Maxilla bifida. Antemae clauato - lamellatae.

39. T. huridus? niger, thorace marginato, inaequali, elytris ftriato $\sim$ fpinofis. F. App. Sp. 496 . $3-4 . ?$ 
\&F. E. S. I. 1. 87. 6. Mant. Roffi 9. x2.

Onmia, vt in defcriptione Fabricii, fed in fingula elytro ftriae tantum quinque eleuato-fpinofae. Hab. Florentiae. Long. $3{ }_{3}$. lat. vix 2.

\section{MELOLONTHA.}

Maxilla breuis, comea; apice multidentata. Antennce lamellatae.

40. M. Fullo teftacea, albo-maculata, fcutello macula duplici, antennis heptaphyllis. F. Sp. 35. r.

$\odot$ Ray., Frifch., Ephemerid. N. C., Aubent., Sulz. Hoefin', Voet.

D Degeer. Inf. 4. t. Io. f. I3.

\$ F. E. S. I. 2. I54. I. Herbft. K. t. 22. f. I. 2. In femina antennarum lanellae exiguae, rotundae, tibiaeque anticae magis dentatae. Plcrumque magnitudine mafculo aequalis, hoc eft longa lin. I3, at vnam habco valde maiorem, quae longa eft a capite ad anum $\mathrm{I}_{\boldsymbol{\zeta}}$ lin. Latitudo $?$ lin.

Semel inuenta volitans fub vefperum in horto nienf. Iul. In locis montofis filuaticis Etruriae non rara, vti in valle vulgo dicta $V$ mbro/in, et alibi. Florentiae et Pifis rara; Florentiae tainen hofpitatur in horto Pagninio, ubi fingulis annis reperitur in planta quadam exotica Pinu filueftri Iun. et Inl. .

$$
\text { (p. 18.) }
$$

4r. M. vulgaris teftacea, thorace villofo, incifuris abdominis albis. F. Sp. 35. 3 .

$\odot$ Ray., Lift., Petiv., Voet.

D Schrank. E. I. Ir.

\& F. E. S. I. 2. I55. 3. Herbit. K. t. 22. f. 6.7. Antennae articulatae verfus bafin clauam exfe- 
zunt oblongo - planam, fexlamellatam, longiffimam, ad angulum acutum, cum articulis bafis. Long. I2. lat. 6. Hab. apud nos rarus.

$$
\text { (p. 10.) }
$$

42. Mt. Solftiticlis teltacea, thorace villofo, elytris luteo-pallidis, lineis tribus pallidioribus. F. Sp. 37. ?.

$\odot$ Ray., Mouf., Petiv., Voet.

우 F. E. S. I. 2. r57.xx. Herbft. K. t. 22,9 .

M. vulgari fimilis, fed dimidio minor, et villofior cauda non elongata, lineae albidae in mortuo vix apparentes. Long. 7. lat. $3 \frac{\mathrm{T}}{2}$. Hab. Florentiae in arboretis ad folftitium aeftiuum. Pifis rara.

43. MI. oblonga, glabra nigra, elytris obfolete friatis. F. Sp. 37. 9.

$\odot$ Scop.

\& F. E. S. I. 2. I59. I8.

Iunior colore violaceo, linidoque variat. Long. 5. lat. $2 \frac{1}{3}$. Hab. in filueftribus frequens.

AuEtor citatum Fabricii e Scopolio bene omifit, qui Scarabaeus veriftmillime $S c$. tufipes F. Ent. fyft. eft. Hg.

* 44. M. deuota villofa, atrouiolacea, elytris laeniter fulcatis, apice pallidis.

Villofa pilis albidis: Antennae rufae. Macula flaua vtrimque ad bafin abdominis ab elytris occultata. Long. 5. lat. $3 \frac{1}{5}$.

Forte M. abdominalis F.Sp. App. 496 ? Hab. in locis apricis. Semel imuenta.

Ne fit $M$. abdominalis, auctori infi perfuafum eft. Vid, Rofi. Mant. Io. I4. M. abdominalis, Hg... 
45. M. Agricolc thorace villofo, elytris liuidis limbo fafciaciue nigris, clypeo apice reflexo. F. Mant. 23. 5?.

(-) Poda, Schaefi., Voet.

- D Schaeff. Ic. t. 23 . f. 8. t. 63. f. x. Schrank. E. I. I7. Goetze E. B. I. 35. 58 .

ㄱ. F. E. S. I. I. I73. 74. Herbit. K. t. 24. f. ro. Fabricii diagnofis optima. Variat magnitudine et macula elytrorum nunc quadrata, nunc cyathiformi. Margo elytronmin non femper niger. Femora poftica crafla. Clypens capitis apice femper reflexus. M. fruticola 1 . Mant. 23. 56. nihi videtur mera varietas. Long. $3^{\frac{1}{2}}, 5$. lat. $2,3 \frac{1}{4}$. Hab. fiequens in Cerealibus.

Citatum Fabricianum e Schaeffero, erroneum, bene emendauit auctor. Hg.

46. MT. horticola capite thoraceque caeruleopilofis, elytris teftaceis, pedibus nigris. F. Sp. 42. 4.

(-) Lift. , Schaeff.

D) Schaeff. Ic. t. 53. f. 4 .

O F. E. S. I. 2. I I I. 68. Herbft. K. t. 25. f. I. Antennae nfae clatia nigta. Tibiae anticae bidentatae. Long. 4. lat. $2 i^{\prime}$. Hab. in hortis frequens.

47. M. ruricola atra fericea, elytris rufis, margine nigio. F. Sp. 43. 45 .

오 F. E. S. I. 2. x73. 75. Herbft. K. t. 25. f. 2 . Tota nigra exceptis elytris croceis, nigro vndique

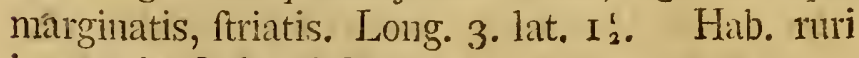
in graminofis haud frequens. 


\section{$(p .2 \pi$.}

4S. Th. argentea clypeo marginato nigra, fubtuśs argenteo nitcns, elytris teftaceis. F.Sp, 44.98.

- C Voet.

o Rofi. Niant. p. xo. n. I 5. Herbft. K. t. 25 . f. 6 : Fabriciana et Scopolina defcriptio exacte congrnit cumnoftris, magnitudo, habitatio omnia conueniunt. Pedes poftici vero parum elongati funt, et tefacei coloris.

- I7. farinofa, quae dicitul Lugdunenfos, l'Ecaillew violet, hac maior atque nitidior elt, et colore fipra caeruleo, nitidiffmo, nec apud nos occurrit. Hab. in pl:ntis et floribus frequens. Long. 3. lat. I'.

Maxima fane confutio intcr hanic et fimiles latet MeJolonthas, quan extricatum iri, vix fperari potelt. Attamen anctor Fubricii $M$. fquanofan K. S. I. 2. э - +. 78 . habuifie videtur. cuius coniecturae Scopolii defrriptio. altabre facta, et a Rorfio iamian laudata, certiorem me facit. Mantifia denique auEoris magis id nilhi perfuafit. Fabricii enim angenteci vera, eft illa, quan Herbft. fub nomine Plit linthus K. 3. p. II9. n. - 2. t. 25 . f. 4. defcriplit, ac depingi curatit. Ceterum in Fabr. E. S.

1. c. deleatur citatum: M. mimzsa, Panzer. quia malum. Perperam deinde Fabricius in eodem loco. Scopolii Sc. argentenm apud H. argentean fuam allegat, qui fine dubio ad M: fquanofan referendus eft.

Pedum color in' $M$. argentea, vel potius $\int q u a m o f a$ valiat, nunc niger, nunc teftaceus, nee notam praebet firmam. Hg.

49. M. puluerulenta corpore polline virefcenti argented, elytris, pedibusque teftaceis. F. Sp. 45 . 56.

$\odot$ Sulzer. 
옫. E. S. I. 2. I 78 . n. 93. exclufo fynonyme Sulzeri, quod ad M. argenteam F. pertinet. Herbft. K. t. 25. f. 7 .

Statura omnino M. argentcae, et apud nos eiusdem ferne magnitudinis. Defcribit Fabricius. Pedes in meis nigri: poftici elongati; thorax pilofus.

M. frinofa, argentect, ct puluerulenta adeo confufae a defcriptoribus, it absque omnium notitia, et comparatione dificillimum fit, rnan pro altera non affumere. Hab. in locis apricis filuaticis rarior. Long. 3. lat. $I \frac{1}{3}$.

$$
\text { (p. 22.) }
$$

50. M. brumea glabra, teftacea, elytris friatis, thorace utrinque puncto notato. F. Sp. 39.26.

$\odot$ Voet.

P F.E.S.I. 2. 165. 42. exclufo fynonymo Sulzeri, quod ad M.variabilem F.E.S. I. 2.I80. To r. pertinet. Herbft. K. t. 24 . f. 3 .

Antennarum lamellac longiufculae. Defcr. Geoffroycur optima. Hab. in plantis et arbuftis non frequens. Long. 4 . lat. $I_{f}^{3}$.

$5^{\mathrm{r}}$. M. Frifchii nigro aenea, elytris teftaceis. F. Sp. 4. 33 .

$\because$ ○ Voet.

D) Degeer. I. 4. t. I0. f. x6. Schaeff. Ic. t. 23. f. 3. 7 .

q F.E.S.I. 2. I67. 53. et M. Iulii ibidem n. 5 I. Herbft. K. t. 25. f. 8. et 9. M. dusia.

Caput et thorax viridi-aenea. Elytra viridi - flaua. Tibiae anticae bidentatae. Hab. in Vite. Long. $6 \frac{\mathrm{x}}{\mathrm{i}}$. liat. 4 .

M. dutic Herbftii et M. Frifchii nil, nifi varietates, inde etiam Fabricii $M$. Iutii ac Frifchii. Eig. 
52. M. Vitis viridis, thoracis lateribus flauis. E. Sp. 4 x. 34 .

$\odot$ Voet.

D Goctze E. B. I. 104. I70. Schaeff. Ic. t. 259. f. 6.

\% F. E. S. I. 2. $167 \cdot 54$

Elytra abdomine breniora, colore fuperius viridi obfcuro nitente, inferius nigro, corpore ounto. Hab. in arnis frequens. Long. 5. lat. $3 \frac{1}{2}$.

M. Vitis quandam, a $M$. Frifchii fpecie diftinctam, equidem nondum vidi. Illam tamen exfiftere, in verba tam multorum Entomologorum credo. Varietatem autem $M$. Frifchii, margine thoracis pallido, ipfe noui. Qui margo cum fola differentiae nota fit, hanc emendari debere, omnis mecum fentiet. $\mathrm{Hg}$.

\section{(p. 23.)}

* 53. II. nitidula fulco-grifea, pubefcens, capite nigro, elycrorum maigine clliato.

Tota nigra, tomento flauo cinereo holofericeo pubefcens. Clypeus apice reflexus. Pedes fulci. Hab. in floribus rara. Long. 2. lat. I.

\section{TRICHIVS.}

Palpi quatuor filiformes; maxilla bifida; antemate lamellatae.

54. T. fut ciatus niger, tomentofo - flauus, elytris fufciis tribus nigris, abbreniatis. F. Sp. 48. I.

(c) Drury, Ait. Nidr., Voet.

D scop. E. C. 5. Schrank. E. I. I6.

q F. E. S. I. 2. II9. 4. Herbft. K. t. 2ヶ. f. 10: Melolontha fafciata.

Voet., Panz. tom. I. Icon. titul. f. .2. 
A me numquam inuentus. Florentiae tamen habitare dicitur in Rubo. Long. $4 \frac{\frac{1}{2}}{2}$ lat. 3 .

Variat tomento flauo et albo. Mihi differentiam fexus indicare videțur. Hg.

55. T. hemipterus thorace tomentofo, rugis diuabus longitudinalibus, elytris abbreuiatis. F. Sp. 48. 4 .

๑) Bergftr.

D Sc. fquamulattis, Müller. Z. D. P. 472 . Schaeff. Ic. t. 46. f. I0. Ir. mas.

P. F.E.S.I 2. I2I. 9. Herbft. K. t. 27. f. I3. I4. Knccí. Beytr. II. t. 7. f. II. I2, piçura fumma diligentia elaborata.

Tibiae anticae quadridentatae. Elytra colore variant, modo nigerrimo, modo fufco, modo cinereo. Ani regio faepe cana. Fenina actheo ani rigido inftrueta. Habit. frequens in varis pluntis, et floribus Comi, Chacrophylli fihncitris, ct in ligno, putrido tempore hiberno, Long. 3. lat. $I_{4}^{3}$.$$
\text { (p.:24) }
$$ \\ VII. CETONIA.}

IItaxilla apice fetofi, Iabium coriaceum, emarginatum. Antemnce lamellatae.

56. C. murata, aurata, fegmento abdominis primo lateribus vnidentatis, elytris albo-maculatis, F. Sp. 50.4 .

() Frifch., Ray, Schaeff. Ic. t. 50, f. 8. 9. Voet. Drury.

욱 F. E. S. I. 2. x22. 8. Herbft. K. t..29, f. 2: Magnitudine et colore admodum variat: Habit. paffim in floribus; larua faepe in formicarmm aceruis. 
nis. Interdun gregatin conueniunt in arboribus e cortice humorem fillantibus, vi ctian formicae inicniuntur. Long. 8. lat. 4\%

$$
\text { (p.25.) }
$$

* 57. C. Jpeciofejgina lcutellata, aurato-viriass, laeuis, tibiis interno latere rufo ciliatis, pofticis edentatis, bifuinofis.

Scrop. Del. Fl, et Fn, Inf. p. r. t. XXI. f. A.

q C. faftuofi. F. E. S. I. 2. I27. 9. Herbf. K. t. 20..f, $x$.

Thorax puluinatus, laeuis, absque fouedis. Elytia plmćculata prope bafin lateris extemi obioleto fini excauata, jotuberantia media verifus ajicen linguli clytri. Pedes clytris concolores mfis vitlis ciliati, tridentiti. Dinerla a $C$. nurata, colore vtringue aurato - viriai fplendentifimo, fegnento primo abdominis absque lateribus rnidentatis, fne foncis in thcrace, nec abdomine poftice punctato. Schaef. Icon, t. 66. f. 4. quoraninodo conuenit. Hab, in Salice, rara. Long. 14. lat. 8.

58. C. Morio nigra, obfena, corpore fubtus nitidiore. F. Sp. 5 I. 5 .

D) Sc. fuliginoíus. Scop. Del. Fl. et Fn. Inf. p. I.

\& F.E. J. I z, J 209. r5. Herbft. K. t. 29. f. II.

Hanc accepit ab Allionio Pabricins. Caput clypéa tetragono, punculato. Os inteme villofm. Thoras pulunatus.- Scutellum conicum. Elytra vix fulcata, prominentia prope fingulum apicem, et anterius appendiculata; character commun aliis Celoniis L. Variat quandoque elytris puctis minutifimis albis afueris ; varictas a Fabricio determinata nomine C. fumeftue App. Spec. 5 I. 2. P.

$$
\text { B } 5
$$

Varie- 
Varictatem aliam etiam poffideo, quae duplo maicr elt, et fuperne nitidior, hoc eft non atro fnligine cbducta, vt aliae apparent. $\delta$. C. quedripunitata $F$. quam quidem habeo, mera forte huius varietas eft. 'Hab. paffim. Long. 8. lat. 4!.

\section{$(p .20$.}

59. C. Eremita aeneo - atra, thorace inaequali, fcutello fulco longitudinali. F. Sp. 53. I5.

$\odot$ Berofttr.

\% Trichius Eremita. F. E. S. I. 2. Ir8. I. Melol. Eremita. Herbft. K. t. 27 . f. 9.

Pifis femcl inuentum cadauer in finu excauato arboris antiquae; Florentiae non rara. Long. I4. lat. 8 .

6o. C. hirta nigricans, hirta, elytris pallido. maculatis, thorace carinato. F. Sp. $59 \cdot 5^{\circ}$.

D Schaeff. Ic. t. rog. f. 8.

of F. E. S. I. 2. I 48.8 I. Herbft. K. t. 30. f. 6. Panz.. Voet. Icon. titul. I. f. 8 .

B. Sc. liqualidus. Linu. S. N. 565.68 .

Hab. flores vbique. Long. $4 \frac{1}{2}$. lat. 3 .

- Sc. fqualidus. L. ab anctore pro C. hirtae varietate habitus, fpecie differt tefte de Villers. Hg.

61. C. ftitica clypeo emarginato nigra, albomaculata, abdomine fubtus punctis quatuor albis. F. Sp. $59.5 \mathrm{r}$.

$\odot$ Ray.

온. E.S. I. 2. I 49. 83. Panz. F. I. G. I. 4.

ß. Sc. funefus. Scop. E. C. 7. et Schrank. E. I. 20. foretne alter fexus Sc. fictici ? Certe fimillimi fint, et faepe copula imnctos vidi. Huic defunt fub abdomine puncta quatuor alba. Hofpitantur tamen eodem tempore in floribus, et apud 
nos ita promifui, et frequentes, vt deftruant flojes omes in hortis, praelertim rofas menf. Maii et Yunii. Long. 4. lat. 3 .

Hic Sc. funeflus lenis tantutn varietas, punctis quatuor abdominis detricis. Hg.

\section{(p. 27.) \\ VIII. HISTER.}

ATaxilla videntata. Labium comeum, cylindricum, integrim. Alitemua clauatae.

62. H. vnicolor ater, elytris oblique ftriatis. $\mathrm{F}$. fyft. Ent. 5 2.2. Sp. 60. 2.

$\odot$ Ray., Sulzer., Act. Nidror.

\& F. E. S. I I. 72. n. 2. Schaeff. Ic. t. 208. r. 5 . Herbft. K. t. 35. f. I. A. Panz. F. I. G. IV. 2. Hoc genus differentias, atque notas fpecificas in hominulis adeo obfcuras, et indiffinctas exhibet, it dificillimum fit; fpecies determinare absque erroris periculo.

In hac fpecie iudicra res obferuata eff. Cum duos haberem magnitudine diuerfos, contigit videre faepius minorem confanter veitigiis maioris inhacrentem, vt tandem dentibus arriveret pedem ilizis pofticum ad tibiae bafin, vbi exilior eft: quo facto ducem paffibus non aequis, nec facile dinellendus, quocumque traheret, anxie fequebatur. Hab. in ftercore, arena frequens. Long. 7 . lat. $2 \frac{1}{2}$.

Citatum Schaeff. Ic. t. 42. f. ro. a D. Rofiio quoque adduetum, et ad Hifermin pygmaeum $F$. tefte Harrero pertinens, delendum eft. H. vnicolor Scop. $\mathrm{ab}$ auctore, vti a Fabricio in Spec. citatus, fed in Ent. fyft. recte omifius, ob elytra apice punctata - Juc non pertinet. Hg. 


\section{(p.28.)}

$\therefore$ *63. H. laemus ouatus, ater, glaber, elytris margine ftriatis, thorace antice vtrinque foucola impreflo, mandibula finifua longiori.

Haec fpecies, quae apud nos frequens occurit, tota atra eft, et finilima H.4. macuínto. Plenmque longa 7 lin. et lata 4 lin. Geoffroy, Scopoli, alique finut maximam longitudinen $I$. vnicoloris ad 4. lin.. Schronkius ad 3 lin, non computato cajite. Neque dubitari polle videtul hanc effe fpecien IVacorem Fab., etii in alis collectionibus detowninatam hoc nomine viderin, nan thoracis margines numpuam pilis ferrigineis ciliatos in hac obferure mini thtum eft. Fornam babet ointan, rotundatan, fibdepreflam. Cajut prominens labio fmpcrion clongato, maxillis validis, exfertis, longis, finifira multo longioni, finfun areuata. Thorax planus, glaber, nitens, pofice trurcatus, et antice vtringue foncola imprelius. Scutellum nullum, Elvtra abdominc nuito broniora, fylendida, ad marginem lineis quingtue exarata. Alae atrae. Nardibula finitra raiue longiot dextra in fpecininibus maioribus (character conftans,) vbifoucola lateralis thoracis; thoraxque mumquan marginibus ciliatis optine difingunt ab aliis. Táb. II. f. I. 2. Thab. in ftercore bouino frequens.

In figura noftra vicio chalcographico mandibula dextra Jongior finifira $\in$ ft, quod autem in tabulis auctoris ipfius recte, exhibitum eft. $\mathrm{Hg}$.

64. H. 4. maculatus ater, elytris bimaculatis. F. Sp. \%1. S.

$\odot$ Gad. Sat.

Schrank. 
D Schrank. E. I. 66. Scop. An. nat. 86́. 30. Schaeff. Ic. t. 3.f. 9 .

9. F.E.S.I. I. 70. I8. Herbfe. K. t. 35 . f. 2.

Hab. in tercore bouno frequens. Long. 4. lat. $2 \frac{2}{3}$.

In E. S. F. 1. c. Synonymum Voct. transferatinr in H. finucatunl, vbi etiam fabricius iterata exhibuit vice. Citatum anctoris Schneff. Ic. t. 3. f. 9. ad H. finetarin Herbft, vel H. binuculatun Fabr. E.S. I. $x .76 . x ?$. pertinet, qui bimainatans ininime autem Linnaeanus $\mathrm{eft}$. Hg.

\section{(P. 20.)}

65. H. aenens, acneus, elytris bafi friatis, apice punctatis. H. Sp. 62.9.

오.F. E. S. I. I. 76. I\%. Herbft. K. t. 35. f. 5 . Elytra breniora, bafi furata, apice punctata, colore aenco. Abdomen in mafculo magis oblongum, quam in femina. Variat colore nigro minus nitido. Hab. cum praecedenti in ftercore, rarior. Long. 3. lat. 2.

66. H. brumeus ferrugineus, elytris fubftriatis. F. Sp. 61. 4.

․ F. E. L.I.T. 74. 8 .

Sinillimus H. vnicolori, at color totus brumeus. Elytra fubitriata friis decem rectis, aut faltem mimus obliquis, et a bafi ad apicem ductis. Hab. cum praecedenti. Long. 2. lat. $2 \frac{1}{2}$.

Varietas $H$. vinicoloris exfifit quaedam, colore brunneo, quae autem diuerfa ab $H$. brinneo, quem Herbftius K. t. 35. f. Io. depingi curauit. Auctoris autem $\mathrm{H}$. brunneus mera varietas $\mathrm{H}$. vnicoloris mihi videtur. Hg.

67. H. detritus ater, nitens, elytris piceis, apice obfcuris. F. Sp. 62. 10. 
Totus ater. Caput intra thotacen non retractum, labio fuperiore non attenuato. Maxillae panum prominent. Thoras et elytia in medio tantum fulendida, lateribus, et apice obfcura, punctata, et quafi detrita. Variat magnitudine minori. Striae omnino defunt. Hab. bouinas faeces. Long: 23 . lat. 2.

Hifter, a Fabricio defcriptus, Nonae Hollandiae indigenus eft, et quanquam ambae regiones eandem infectorum fpeciem habere poffint, attamen equidem pro mea parte cenfeam, nullum ex folis defcriptionibus, fed ex bene comparatis fpeciminibus ipfis, ita indicare debere, praecipue quidem, cum delcriptio haud omnibus conueniat notis, it h. 1 . vbi ftriae, a Fabricio commemoratae, plane defunt. Qua de canfia ab auctore Hiftero Italico nomen męlius nouum inditum, et Fabricii citatum, vt incertum, figno interrogationis exhibitum fuifeet. $\mathrm{Hg}$.

68. H. depreffus, depreflus, ater, nitidiffmus, elytris fubfriatis. F. Mant. 32.8 .

\& F. E. S. I. I. 74. I0. Herbft. K. t. 35 . f. 9. Corpus oblongiufculum, depreflum, nitidum. Ca. put intra thoracem reconditum, maxilis tantum exfertis. Thorax fubquadratus. Elytra ad narginem fubfriata, intus lacuinma. Habit. fub corticibus Betulae Alni frequens. Long. I $\frac{1}{4}$. lat. ${ }_{4}^{3}$,

$$
\text { (p.to.) }
$$

69. H. abbieuiatus ater, elytris crenato-friatis, friis interioribus abbreuiatis. F. Sp. 61.6.

Statura omnino H. pygmazi; ater, nitidus. Elytra friis quatuor ad latera integris, et in medio parum abpreuiatis, minusque confpicuis. Corpus yloa 
globofum. Hab. fub arborum cortice, et in ligno putrido, non rarus. Long. I. lat. $\frac{3}{4}$.

Hic quoque idem, quod fupra n. 67, adnotandum eft, cum Fabricii Abbrenticus ex America boreali oriundus fit. Praecipue in Hifterorum, genere canendum eft, cuius fpecies tam difficulter verbis diftingui a fe innicem poffunt. Hg.

* 7o. H. fulcatus ater, thorace, elytrisque punEtato-fulcatis, apice retufis. D. L-Sintic. S. Limo II. filcututs.

An $I$. friatus, thorace, elytrisque ftriis elena. tis. Forfter. N. 1. C. I I.?

옥.L.S. I. x. 74. I2. H. firiatus. Herbf. K. t. 36. f. I. a. b. H.

Totus ater, corpore fúcglobofo. Antennae longiufculae bafi crafla, clana folida. Caput nigofum intra thoracem retractilc. Thorax breuis, latus, punctatus, fulcatusque lineis elcuatis in medio quatuor, ad vtraque latera duabus. Elytra integra, margine fubreflexo, apice angultata, retufa, et lincis eleuatis, interiectis fpatiis punctatis, exarata. in elytro fingulo lineae quinque integrae omnes, minimeque abbreuiatae. Alite hyalinae corpore duplo longiores Táb. II. f. 3. Hab. in ftercore equino pafcuorum primo vere. Long, I, vix, lat. I. vix.

Nihil prohibere videtur, quo minus $H$. fulcatum $F$. eundem cum $H$. fulcato h. 1. defcripto, habeam. Omnia enim conueniunt, fed thoracis defcriptio nimis obfcura eft, quamquam mihi auctor eadem dicturus videatur. Lineas enim eleuatas quinque video: vnam in medio, binas ad vtrunque latus integerrimas. Interfitia inter lineas elevatas elytrorum fulcata funt fulcis haud profundis. Ibi, 
'vi fulci lineis eleuatis appropinquant, linea punctorum eleuatorum recta defcendit, $v t$ in fingulo interftitio duae tales lineae fint. Differt itaque nofter ab $H$. Sulcato anctoris thoracis lineis elenatis quinque, media abbreuiata, nec quatuor, fpatiis elytrorum, lineis eleuatis interiectis, fulcatis, nqc punctatis. H. Julcatus Moll. neues Ent. Magnz. v. Fuesly. II. p. I72, n. I05. huic contenire videtur. $\mathrm{Hg}$.

* 7'. II. minntus ater, elytris la euiflimis nitidis antennis claua ferruginea.

Magnitudo vix feminis Nilii, qua nota praefertim dilinçus ab H. pygmaeo videtur: Caput omnino reconditum, antemnis tantum confpicuis clatia ferruginea. Elytra laeuifina, partun abbreuiata, et quafi truncéta. Corpus rotundum, globofum. Hab. intra corticein et ligntum, in arborum carie. Long. $\frac{1}{4}$.

$$
\text { (p.3t.) }
$$

72. H. bimaculatus ater, elytris poftice rubris. F. Sp. 6x. 7 .

( V) Vdr., Degeer., Schreft., Voet.

Q Herbft. K. t. 36. f 8 .

$\mathrm{Hab}$. in ftercore bounio rarior. Long. 2. lat. $\mathbf{I}_{2}^{\mathbf{k}}$,

Fabricii H. binaculatum, ob citata e Schaeffero et Voetio, allegare nequeo. $\mathrm{Hg}$.

\section{DEERMESTES.}

Palpi inaequales filiformes. Maxilla bifida. Labium comeun, obtufum, integrum. Anternae claua perfoliata.

73. D. Intdarius niger, elytris antice cinereis. F. Sp. 63. I. 
$\odot$ Ray., Schaeff. Elem., Frifch.

D Scop. E. C. 34. Schrank. E.I. 40. Goed. t. 2: t. 4 I.

아. F. E. S. I. T. 227. I. Herbft.K. t. 40. f. I0: Preisl. Bühm. Inf. I. $\mathbf{I}_{3} \cdot X$.

Hab. in animalibus afferuatis, lardo, carnibus. Long. 3 : lat. 2.

Preislertm allegaui, quia in 1. c. monftratur via, quo. modo illa arte, qua D. Jardarius fceleta fabricat, bene vti poflimus. $\mathrm{Hg}$.

$$
\text { (p. 3.). }
$$

74. D. murinus oblongus, tomentofus, nigro alboque nebulofus, abdomine niuco. F. Sp. 6, 9.

$\odot$ Voet.

D Scop. E. C. 35 . Schrank. E. I. $4 I_{\text {. }}$

\& Herbft. K. t. 40. f. 2 .

Caput pilis fuluis pubefcens. Abdomen fubtus albicans. Larua oblonga brunuea, ore atro. Hab. fub cortice, et in cadaueribus. Long. 2,3 . lat. I, I $\frac{1}{2}$ Diagnofis, a Fabricio data, poting D. Cattae Panz. Naturf. 24., quem etia:p Herbfitus K. t. 40, f. 4-' fub eodem nomine depictum exhibet, conuenit. $\mathrm{E}$ citatis a Fabricio factis, fufpicarer, Illum D. mnrinim et $D$. Caltam varietates tantun credere, cui tamen affentire non poffun:. D. murinum énim creberrime in cadaueribus reprehendi, numquam autem in focietate cum $D$. Cotta, cuius aliquando duo fpecimina in carduo, et pofter nonnulla in are boribus emortuis, cepi. Dingnofi Fabriciana $D_{2}$ ' teffeltori E. S. I. I. $23^{\circ}$. $x 5^{\circ} \cdot$ cuin Linmaeana $D_{0}$ onurini comparata, ambo eadem videntur fpecies effe. Adnotata Fabricii in Syft, Ent. apud D. $t e j$ fellatum id mihiperfuaderent, niti abdomen fufcum illi tribuifiet, quod nec in D. Catta, nec in $D_{4}$ mutino videre pollum.

$$
\mathrm{C}^{\mathrm{Hg}}
$$


75. D. Pellio niger, elytris puncto albo. F. Sp. 63.5 .

$\odot$ Ray.

2. F F.E.S.I.r: 228.5 . Herbft. K. t. 40. f. 8 .

Elytra, nunc fufco-caftanea, nunc fufca, nunc denique nigra. Puncta alba aliquando defunt. Larua oblonga, cauda fetofa mihi vixit integrum annum lanam comedens, deinde fe declarauit menf. Iun. Hab. paffim in lardo, pellibus, veltimentis, quae inftrata Papyri folia praferuant. Frifch. 5 . p. 33. Long. 2. lat. I $I_{\mathfrak{a}}$.

Experientia quoque mea me dòcuit, antennarum ftructuram interdum variare. Mlegatomam Schaefferi, atram et brenicornem Herbft. K. t. 39. f. I. 2.3. varietatès $D$. Pellionis, et forte alterum fexum coniicio, quod experientiae plures nos edocebunt. In plantis vmbellatis faepius Dermeftem reperi, $D$. Pellioni fimillimum, vt diftinguere non poffem. Qui fi diuerfa effet fpecies, quod ob victum diuer. fum verofimile eft, iterum documento effet, quam valde aliquem in iudicio de identitate infectorum fibi cauete debere. . Hg.

76. D. teffellatus oblongus, tomentofus fufcocinereoque nebulofus, abdomine fufco. F. Sp. 65.

\section{I.}

(2) Geoffroy.

Hab. cadauera, et exefas arbores. Long. 2.

$$
\text { (p.33.) }
$$

77. D. cadculerinus niger, ore ferrugineo. F. Sp: $63 \cdot 3$.

D. Schaeff. Ic. t. II8. f. I.

\& Conferat D. vulpinus? Mant. Rofi. I6. 28 . Antennae fufcac claua fermginea. Thorax niger lateribus grifeo-villofis. Abdomen fubtus aitbum. $\rightarrow$ s 
Elytra tota nigra. Pedes nigri. Valde affinis $D$. carmiuoro. Hab. cadauera. Long. 4: lat. $2 \frac{1}{2}$.

Fabricius $D$. cadauerinum fuum Infulam Helenam habitare dicit, qua de caufla valde dubito, eundem cum auctoris' cadanesino efle, licet Harreins in Defcr. Ic. Schaeft. 4 to. p. 5 r. figuram Schatfieri, ab auctore citatam, ad D. cadaucrinum $F$. pertinere, exhibeat. $\mathrm{Hg}$.

78. D. violaceus nigro - caerulefcens, thorace villofo pedibus nigris. F. Sp. 65 . I3.

$\odot$ Ray., Scop.

D Schaeff. Ic. t. I66. f. 4. Scop. E. C. 5 r. Schrank. E. I. 45 .

q F. E. S. I. I. 230. I6. Panz. F. I. G. V. 6.

Corynetes violaceus. Herbft. K. t. 4 I. f. 8 .

Attelabus Geoftroyanus, Laich. Tyrol. Inf. I, 247. Caput deflexum. Antennae bafi atro-violaceae, villofulae. Thorax cum elytris villofis. Corpus totum atro-caeruleum. Hab. flores 110 n rarus. Long. $I \frac{1}{2}, 2$. lat. $\frac{2}{3}, \mathrm{I}$.

Mirum eft, quod auctor Schaeff. Ic. t. I66. f. 4. apud $D$. violaceum alleget, cum $D$. mifipedem quoque exhibeat, cui haec citata figura potius contenit. Sed h.l. vt alibi, Harrerum'fecutus efie videtur:quem, cum aliis Entomologis, hunc et fequentem ccnfudifie puto. In noftris autem regionibus D. violaceus in offibus vulgaris, interdum etiam in floribus occurrit, numquam autem pedibus rufis variantem vidi; quam ob rem $D$. rufipes, etiam fi alias haud computaremus notas, fpecies diftincta eft, nec confundenda cum violaceo. $\mathrm{Hg}$.

79. D. rufipes nigro-caerulefcens, thorace villofo, pedibus rufis. F. Sp. 65.14 .

$D$ Anobium rufipes. Thunb. n. fp. Io.

Degeer. Inf. 5. 165. t. I5. f. 4 . 
Affinis: $D$. violaceo; facie et habitu accedit ad $C_{t-}$ ros. Hab. flores rarior. Long. $2 \frac{1}{2}$. lat. $I_{4}^{1}$.

Ob diueram patriam $D$. mfipedis $F$. de identitate amborum dubius haereo, et id furpicionem mean aliget, quod Fabricins in Ent. fyft. emend. alkforem haud alleganit. $\mathrm{Hg}$.

$$
\text { (p. 34.) }
$$

o. D. Junguicollis elongatus, -initus, violaceus, thorace, abdomineque nifis. F. Mant. 35 - I8.

of F E.S.I.,. 23ㄷ. xo.

Corynetes fanguinicollis. Herbft. K. t. 4 I. f. Io. Echaeff. Ic. t. 220. f. 4 ?

Antennae perioliatae, fufcae, ad bafin fenugineae. Caput nigrum. Thcrax hirtus, rufus, immaculatus. Elytra cyanea, nitida, fubpunctata, fubhirta. Abdumen rufum. Pedes nigri plantis rufis. Semel lectus ruri. Long. $3 \frac{1}{3}$. lat. I. et vltra.

Figuram Schaefferi, quamuis depictum ibi infeetum optime cum D. Janguinicolli conueniat, vt incertam exhibeo, cum Harrerus in Defcript. Ic. Schaeff. 8uo. p.' I 45. duorum capitis corruum mentionem faciat, quibus infectum, a quo icon defumta eft, inftructum fit; id, quod efficit, vt plures eandem figuram ad Ipem' haemorhhoidalem F. E. S. I. 2. 5 3 . Ir. referant, cui opinioni corpus magis elongatum $D$. fanguinicollis, deinde elytra ab Harrero commemorata, pilofa et in icone fic apparentia, contradicunt, qualia minime 1. huzemorrhoidatis, omnino autem $D$. finguinicollis habet. Hg.

8I. D. 20 -guttatils oblongus, ater, punctis viginti albis. F. Sp. 64. ?:

9. F. E. S. I. I. 229. 8. Herbft. K. t. 40. f. Io. Statura omnino et magnitudo $D$. undati. Corpus atrum, glabrum, laeue. Punctum maiusculum e pilis 
pilis albis vtrinque ad bafin thoracis, et nonen in fingalo elytro. Abdomen nigrum certo fita albicans. Variat interdum punctis in fingulo elytro decen. Inueni faepius in floribus Crataegi Monogynae. Long. $\mathrm{I}_{\frac{1}{3}}$. lat. $\mathrm{x}$.

* \$2. D. dentatus elongatus, hirtus, niger, elytris teftaccis.

? liofil Mant. 16.30 .

Facies omnino Lagriae hirtae, fed dimidio minor; ct antennae luius generis, nigrae, articulis quatuor, primo longifimo filiformi; fecundo et terio profunde dentato, vltimo rotundo extrorfum craffioribus. Totus niger, pilofius, exceptis elytris teltaceis. Tab. III. f. 2. Hab. in ficribus rarus. Long. $2_{4}^{3}$. lat. $x$.

83. D. tomentofus oblongus, villofus, 'grifeus, capite punctis duobus fufcis. F. Sp. 66. I 7 .

S.E.S. I. I. 232.24 .

Elytra punctato-friata friis deccm. Hab. flores. Long. 2. lat. I.

\section{(p. 35.)}

84. D. fimatus oblongus, teftaceus, oculis nigris. . F. Sp. 66. 16.

(c) Scop., Degeer.

J) Scop. E C. 39 .

F. E. S.I. I. 23 I. 23 .

Hab. in floribus rofac, fcabiofac, et in fimo. Loing. $\frac{2}{3}$.

85. D. Caber grifeus, thorace elytrisque fcabris. F.Sp. 66. 20.

Defcriptio Fabr. in Syft. Ent. data, hic repetita eft. Hab. fub arborum cortice rarus. Long. $2 \%$ lat. $I_{4}$.

$\mathrm{C}_{3}$

$D$. 
$D$. Scaber $F$. in noua Zelandia habitat, inde dubium meum de identitate vtriusque. Hg.

\section{NVELYRIS.}

Palpi filiformes. Maxilla vnidentata, acuta. Labium clauatum, emarginatum. Antennae totae perfoliatac.

* 86. M. bimaculatus niger, villofulus, elytris bafi macula fanguinea.

An hic H. bipuftulata F. Sp. 82. 8. quae mihi ignota?

Niger, pilofis, corpore cylindrico. Antemae totae perfoliatae. Palpi filiformes. Caput nigrum. Thorax niger marginatus, poftice latiur. Elytra nigra fubpunctata, marginata fingulo elytro ad bafin macula laterali fanguinea. Subtus totus niger. Huius, generis videtur. Tab. VII. f. I4. Hab. in floribus paffim. Long. $2 \frac{2}{3}$. lat. $I_{4}^{1}$.

Nil obftat, quo minus Hifpa bipuffulata $F$. eflet, fub quo nomine etiam infectum hocce a celeberrimo Paykull mihi miffum eft. Sed perfuafum habeo, illud nec Hifporum, nec Melyrium gyeneri inre adnumerari pofie. Equidem itaque inter Lagrias, L. metallicae fimiles, retuli, quarum habitus fingularis, et antennae ferratae (quas auctor haud optime totas perfoliatas vocat) peculiare genus bene conftituere videntur. $\mathrm{Hg}$.

\section{(p. 36.) \\ XII. BOSTRICHVS.}

Mlaxilla comea. Labium cylindricum integrum, apice palpigerum. Antennaé claua folida. 
87. B. capucinus niger, elytris abdomineque ru. bris, thorace emarginato, retufo: F. Sp. 67. 1.

$\odot$ Sulz. et quidem fummo iure.

D Schrank. E. I. 38. Schaeff. Ic. t. I89. f. x.

\& Apate capucinus. F. E. S. I. $2.36=7$.

Ligniperda Capucinus. Herbft. K. t. 46. f. $z$.

Herbft. Kenntn. d. Infekt. I. t. I 2. f. 3 .

Reliquis noftratibus maior. Caput paruum, angufuin, atrum, fere totum retractum intra thoracem. Antennae nigrae. Thorax connexus, gibbus, fcaber, antice retufus. Elytra rubra punctis excauatis. $\beta$. Variat elytris etiam totis nigris, varietas a nemine, quod fciam, obfenata. Inueni plures totos nigros (abdomine excepto in nonnullis rufo) vna cum aliis iam cognitis in trunco quercus entortuae, menf. April. t. V. f. 3. - Hab. in truncis exficcatis rarus. Long. $4 \frac{3}{3}$. lat. 2 .

Fabricius hunc Boftrichum ad Apates genus bene einendauit, fed caue, ne eundem cum $A$ capucino Mlant. 33. 5. credas, quae inter Sinodendra. relata eft, vbi autem errore quodam Boftrichus capucinus loco $A$. Capucini, citatus eft. Valde tamen dubito, infectum, qrod antea generi Apates annumeratum fuit, Sinodendris adiungi poffe. Hg.

88. B. typographus teftaceus, pilofus, elytris ftriatis, retufis, pracmorfo-dentatis. F.Sp. 67.3.

$\odot$ Ray., Sutz. Act. Holm.

D Schaeff. 1c. t. 259. f. 3. a.

o F. E. S. I. $2 \cdot 365 \cdot 3$. Herbft. K. t. 48 . f. r. a. Variat colore teftaceo et fufco. Hab. intra cortices arborum, praefertim coniferarum, labyrinthos efformans. Long. $2 \frac{1}{3}$. lat. I. 


\section{(p. 3r.)}

89. B. micrographus ferrugineus, elytris integris teftaceis. F. Mant. 37.7 .

D Degeer. Inf. 5. 195.4 .

S. F. E. S. I. 2.366 .8 .

Paruus. Thorax fibferrugineus. Elytra teftacea.

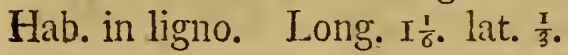

96. B.pimiperta niger, fubuillofus, elytris piceis integris, plantis rufis. F. Sp. 68. ?.

오․ E. S. I. 2.367. 17. Herbft. K. t. 48 . f. 4. d. Hab. frequens in ranulis inferioribus pini, quos perforat, exficcat. Long. $2 \frac{1}{2}$. lat. $2 \frac{1}{4}$.

Variat colore toto teftaceo, et nigro, exceptis antennis plantisque teftaceis. Elytra punctata, leuiflime fulcata, ftriis punctatis. Si B. teftaceus Fabr. diuerfa fpecies, cauendum, nę cum varietate teftacea $\mathrm{B}$. Piniperdae confundatur. $\mathrm{Hg}$.

9r. B. Scolytus giaber, elytris truncatis, integris, abdomine retrfo. F. Sp. 68. 6.

오. E. S. I. 2.366 .9 .

Eccoptogafter fcolytus. Herbft. K. t. 49. f. x. a. Defcriptio Fabricii optima; facile dignofcitur. Hab. fub Vlmi corticibus, apud nos rarus. Long. $I_{\frac{1}{3}}$. lat. $\frac{2}{3}$.

Iam Herbftius 1. c. adnotauit, Schaeff. Elem. tabulam Ir2, minime autem Schaeff. Iconum, citandam effe. Figura Sulzeri potins ad $B$. qy gincen $F$. quam ad hunc referri poffe videtur. Specimina mea minima 2 lin., folita autem 23, imo 3 lin. longa. et I lin. lata funt. $\mathrm{Hg}$.

$$
\text { (F, } 38.3
$$

Q2. B. bidentatus niger, coleoptris teitaccis, spice retufis, bihnmatis. E. Mhat. 37.4.

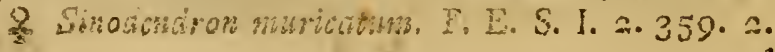


Antenne nigrae, claua perfoliata, articulis tribus vitinis extrorfum craffioribus. Caput et thorax rigra, immaculata, quae nihil differint a capite et thorace B. capucini. Elytra obfcure teftacen, poftice retula denticulo elongato, hamato; nigro ad futuram. Pedes teltacei. Lectus fub cortice vitis. Long: 3. lat. 1 .

Citatum Herbft. Arch. 24. 6. t. ro. f. Io, quod auEtor exhibet, Fabricium fecutus, ab hoc celeberrimo Viro in Ent. S. bene omiffum eft, cum ad Boftrichum pertineat, vix lineae menfuram fuperantem, et a vero B. biduntato F. Niant. vel Sinodendro muricato F. E. S. valde difcrepantem. Videtur autem Fabricii B. bidens E. S. I. 2. 368.24. cfie. Data auctoris menfura et defcriptio certum me faciunt, illum nec $B$. bidentatkin Ferbfii, nec Fabricii nouiffe, quem Linnaeus optime defcripfit. Statura fingularis, et maxime $A$. capucino fimilis eft, cui Apates generi equidem libenter annumerarem. $\mathrm{Hg}$.

- 93. B. bruchoides niger, thorace comprero, elytris itriato-punctatis pilofis.

Facies Bruchi, fed character huius generis. $\mathrm{Ca}$ put elongatum, compreffum. 'Antennae clauatae, nigrae. Thorax compreffus, obfolete rugofus lineis tribus, et punctis pluribus pilorum rufonm. Elytra decemtriata, ftriis punctatis. Maculae rufopilofae marmoratae. Subtus niger, fubpilofus. Pedes picei. D. d.Antic Soc. Linn. Hab. in Etruria et Parifiis. Reperitur primo vere fub cortibe cupreffi faftigiati. Long. $2 \frac{1}{2}$. lat. $I_{\frac{1}{4}}^{\frac{1}{*}}$

\section{$\mathrm{C}_{5}$}

XiII. 


\section{XIII: BYRRHVS.}

Palpi aequales, fubclauati. Maxilla bifida. Labiun bifidum. Antenuae claua perfoliata.

94. B. pilula fufcus, elytris ftriatis atris interruptis. F. Sp. 69. I.

D Ciffela fafciata. Forfer. N. T. C. x. 12. Ciftela Forfteri. Fues!. Verz. Schw. Inf. 66. Ciftela fafciata. Goetze L. B. I. I52. 2. Schaeff. Ic. t. 95. f. 3. Elem. t. 45. f. 2.3. melior.

o F. E. S. I. I. 84. 2. Panz. F. I. G. IV. 3 . Curpus obouatum, globofim. Palpi quatuor fubclauati. Antennae thorace breuiores. Caput parum, rotundum, retractile. Thorax comexus, conicus, tectus pilis mis, naculis nigricantibus fparfis. Scutellum nigrum. Elytra fufca ftriis quatuor longitudinalibus interruptis, obfoletis, pilis nigris crectulculis nigram maculam conftituentibus. Pedes nigri. Fenora tibiaeque ommes comprelfae, incuruae, latere extemo parum femlatae. Abdomen fubtus nigrum. Inceffus grauis. Taćtus, aut panens (Ciftelarim more) fe contrihit modo fingulari, et caput, antennas, pedesciue applicat in canis abdominalibus, adeo recipiendis partibus accommodatis, vt corpus fphaericum continuatum euadat. laene, et nullo modo amplius pro infecto dignofcendum. Magnitudine variat. Quoad genera Byrrhi, et Cifteice, confer quid habeat Forfterus Inf. p. I3. - Hab. in campis filuaticis arcuofis rarus. Long. 4 . lat. $2 \frac{2}{3}$.

$$
\text { (p.39.) }
$$

* 95. B. niger ater, nitens antennis ferrugineis, elytronm futura prominula. 
Proxinus Dernoftict Anthreno, etforte Anthrenus. Magnitudo Anthr. forophalarine. Ater, nitidus glaber, immaculatus. Artennae ferrugineae clatia noninihil perioliata. Palpi ferruginei. Thorax conicus, libmarginatus angulis pofticis acutiuiculis. Elytra fubpunctata, ad bafin latiora duplicatura laterali, fituraque prominula. Hab. in floribus Crataesi Monogynac.

Quodammodo cum B. Rhenii Schneideri conuenire videtur. Hg.

\section{(p.40.) \\ XIV. ANTHRENVS.}

Palpi inaequales, filiformes. Maxilla membranacea, linearis, bifida. Labium corneum, integrum. Antennae claua folida.

96. A. pimpincllase niger, elytris fafcia alba, apice ferrugineis litura alba. F. Sp. 7o. I.

\& F.E.S.I. I. 263 . I.

Abdomen etiam fubtus albidum. Hab. in floribus. Long. I. lat. ${ }_{4}^{3}$.

97. A. Scrophularine niger, elytris albo-maculatis, futura fangunea. F. S sp. 70.2.

○ Ray., Degeer., Sulz., Berģftr.

D Dermeftes variegatus. Scop. E. C. 4 I. Schrank. E. I. 73.

o F. E. S. I. I. $26+3$. Panz. F. I. G. III. I J. Corpus fubrotundum. Thorax latus. Elytra nigricantia, albedine adfperla. Hab. in fcrophularis et in floribus pomonae, fambuci frequens, copiofus. Long. I $\frac{1}{4}$. lat. $\frac{2}{3}$.

98. A.mufeorum nebulofuis, elytris fubnebulofis. F. Sp. ๆ०. 3. F. 
P. E. S. I. I. $264 \cdot 4 \cdot$

Habit. in mufeis. Long. $\frac{r}{3}$.

$$
\text { (p. 4I.) }
$$

99. A. verbafci niger, elytris fafciis tribus vndatis albis. F. Sp: 70. 4 .

(c) Bergft:

? F.E.S.I. $x .^{\prime}=64.5$.

Habit. in verbafco frequens. Long. $\frac{2}{3}$. lat. $\frac{\pi}{2}$.

\section{ANOBIVM.}

Palpi clauati. Maxille obtufe dentata. Labium integrum. Alitennae filiformes.

1oo. A. pertinax fufcum, immaculatum. F. Sp. 21. $\mathrm{r}$.

$\odot$ Geoffr.

울 F.E.S. I. I. 23\% 5. Herbft. K. t. 47. f. 3 .

Captum fe contrahit immobile. Hab. ligneas fitpellectiles, in ligno quercino frequens. Long. $2 \frac{1}{3}$. lat. $\mathrm{I}$.

*' Iог. A. thoracicim nigrum, thorace marginato, elytris teftaceis lacuifimis pilofis. $D$. d Antic. Soc. Limn.

Statira A. mollis, at panllo minor. Caput nigrum. Antcnnac teftaceae, tres articuli vltimi maiures. Thorax villolus, niger, antice eleuatus, latcribus marginatis. Elytra villofa, teftacea, leuiffima. Abdomennigmm. Femora nigra. Tibiae teitaceae. Tarfi teitacei articuli quinque. Differt ab A. molli thorace magis marginato, elytris laenifimis, et colore capitis, thoracis, abdominis, pedumque. Hab. in ligtio putrido. Long. 2. lat. $\frac{3}{3}$. 


\section{(p. 42.)}

102. A. molle tefaceum, oculis nigris. F. Sp. 7.1. 2.

오. I. E. S. T. I. 237 . 7. Herbft. K. t. 47 . f. 9. Hab. in quisquilis in Iul. frequens. Long. 2. lat. $\frac{3}{4}$.

103. A. mimutum fubuillolum, nigriun, thorace, elvtrisque cynueis. F. S1. 72. 8. Lagria minuta. I. Sp. App. 3 . 14.

9. Jagr. minuta. F.E. S. I. 2. 8 r. 17. Hab. in Horibus. Long. $1_{4}^{1}$. Lat. '.

Etiam h. l. identitas hairs et Tabriciani, ob patriam tam diverfan, et ob deferiptionem Fabricii nimis breuem, mihi dubia ct. Caeterum, quomodo Anobium, Lagriarum genus intrare pofit, mihi haud rite notum efi. Hig.

* Iof. A. Jerraticome nigrum, antemis ferratis, elytris friatis, pedibus teftaceis. D. d Antic:

Caput nigum. Ilandibulac exfertae piceae. Antennae nigrae, lematas; apicen parum craflori. Thurax niger, fubplaus. Elytu picea, ftriata. Subtus nigmom. Pedes tefracei. Variat colore toto nigro, vel piceo. Infectun!, quod accedere videtur ad genera Dermofisis, Boptrichi et Anobii, fed magis vitimo proxinum. Hab. Pifis. Long. $I \frac{T}{2}$. lat. $\frac{2}{3}$.

\section{$(p, 43$.}

\section{PTINVS.}

Palpi filiformes. IVaxilla bifida. Labium bifdum. Antennae filiformes.

105. P. fur teftaceus, thorace quadridentato, elytris fafciis duabus albis. F. Sp. 73. 4.

$\odot$ Sulz., Frifch., Schaeff, Elem.

Schrank. 
D Schrank. E. I. 63. Schaeff. Ic, t. I 55. f. 3. a. b. S F. E. S. I. I. 239.4.

Medius quafi eft inter Dermeftes et Cerambyces, cum caput fub collo retrahat, et antenias gerat Cram'bycum.' Variat I) colore ferrugineo, absque fafciis et tuberculis, 2) fafciis elytri albis. Specimen habeo, quod accedere videtur ad $P$. longicornem $\mathrm{F}$. Fere duplo maius $P$. fure antennis filiformibus, corpore longioribus. Hab. fub arborum cortice atque in herbariis et collectionibus infectorum. Long. $I_{\frac{1}{2}}$. lat. $\frac{2}{3}$.

P. latro, qui differt ftatura anguftiore, nec fubglobo$f a$, alter $P$. furis fexus milhi videtur, cum femper in iisdem cum illo locis occurrat. Hg.

ro6. P. Germanus fufcus, thorace quadridentato, elytris villofis, cinereo - variis. F. Sp. 72. 2.

o F.E.S.I. I. 239.2. Schaeff. Ic. t. 155 . f. 4 a. a. Maior P. fure. Totus fufcus. Thorax eleuatus, dentibus quatuor. Elytra punctato-ftriata, villofa, cinereo - varia. Pedes fubferruginei. Hab. lub cortice, in lignis cariofis frequens. Long. 2. lat. $\frac{2}{3}$. vix.

\section{(p. 44.)}

107. $P$. fotias thorace laeui, piceo; elytris. connatis, fufco teftaceis, nitidis. F. Sp. 74. 8 .

D Schaeff. Ic. t. I55. f. 5. a. 5. b.

P. Seminvilum. Schrank. E. I. 65.

\& F.E.S. I. I. 24 I. I2. Panz. F. I. G. V. 8.

Schrankii defcriptio 1. c. itcrata. Hab.in aedibus: lecti plures in pariete domeftico. Long. I. lat. $\frac{2}{3}$. 
XVIT. BRVCHVS.

Palpi acquales, filiformes. Aaxillir membranacea, bifida. Labinn acuminatim. Antennae filiformes.

Ios. B. Pif elytris nigris, albomaculatis; po: dice albo: punctis binis nigris. F. Sp. 74.2.

$\odot$ Linn. Mur., Kalm, Linn. Amoen.; Lederm.

D Schrank. E. I. Igo. :Schaeffi. lc. t. 2I3. f. 4. a: b. Rhinomacer.

O. F.E. S. I. $2,370.5$.

Hab. Legumina palfim. Long. 2. lat. I.

$$
(p .45 .) 3
$$

I00. B. granarius elytris nigris, atomis albis, fenoribus pofticis viidentatis. F. Sp. ?6. I I.

을. E.S. I.' $2 \cdot 3 \div 2.15$.

Antemna bafi teftaceae. Thorax fitura alba. Elytra ftriata abdumine bręuiora. Peles antici rufi. communes etian B. Pifi. Hab. in feminibus plantarum frequeus. Long. $\mathrm{I}_{2}^{\mathrm{T}}$. lat. $\frac{2}{3}$.

- Ipfe in $B . P i f i$ pedes anticos totos rufos video, femorum bafi fola obfolete nigricante. $\mathrm{Hg}$.

I 10. B. Jeminarius ater, antennarum bafi, pedibusque anticis teftaceis, femoribus muticis. F.Sp. 76. 14.

\section{\& F. E.S. I: 2. 373. I9.}

Caput prominens, antennae pedesque nigri inermes. Hab. flores. Long. I. lat. ${ }_{2}^{3}$.

I I r. B. Ciffi ater, immaculatus, femoribus muticis. F. Sp. 76. 12.

O F. E. S. 1. 2. 372.16. Hab. in floribus. Long. $\frac{1}{2}$.

Abdomep hịto-cinereum. Hg. 


\section{(p. 46.)}

I. B. bipunitatus cinereus, elytris fufcis, puncto bafens occllari atro. F. Sp. 75. Io?

$\odot$ Sulz.

Cinereus, elytris fufcis,' puncto ad bafin vtrinque nigro.

Ne fpecies multiplicarem, ad hanc Fúvicii fpeciem noftrum infectum relerre malui, quod differt tamen pumcto bafeos non ocellari. Hab. in plantis. Long. $\frac{2}{3}$. lat. $\frac{\text { ? }}{2}$.

Equidem punctum ocellare et non ocellare haud leve fane difcrimen praébere credu, et illi, qui fpecies, his notis differentes, exhibet nouns, multiplicationem fpecierum fuperuacaneam exprobrare minime fas eft. Alins enim confufio multiplicatur. Hg:

\section{ELOPHORVS.}

Maxilla cornea, apice membranacea. Labium corneum, quadratum.' Antennize claua folida.

II3. E. aquaticus fufcus, thorace rugofo, elytrisque fufco-aeneis. F. Sp. 77. I.

I F. E. S. I. I. 204.1 .

Hab. in aquis fagnantibus, in Conferua, et Potamogeto frequens, victitans laruis infectorum. Long. $I \frac{2}{3}, 3 \frac{1}{2}$. lat. $\frac{2}{3}, I_{4}^{\frac{3}{4}}$.

Errore quodam calami auetor Degeer. 1. 105. 15 . pro Degeer. 4. 379.5 . exhibet, diagnofi adiecta Creoffroyana. - Caeterum valde dubium infectum eft; cum fpecimina nunc 3 , nunc 2 , nunc $i_{4}^{-1}$ lin. longa habeam, quorum differentiam aliam detegere nequeo. E. flanipes Herbft. K. t. 49. f. 6 . e insximorum numero fpecimen videtur. $\mathrm{Hg}$. 


\section{SPHAERIDIVM.}

Paliji quatuor filiformes. Labium quadratum, emarginatum. Antemnae perfoliatae.

$$
(1 \cdot 4 \pi)
$$

I 4. S. Scarabacoütes onatum, atrum, elytris maculis duabus ferrugincis. F. Sp. 78. II.

D Dermefeses 4-maculatus. Schrank. E. I. 43.

o F. E. S. I. I. 77. I. Herbff. K. t. 37\% f. I. A. Voet. t. 32. f. I. Panz. F. I. G. VI. 2.

Habitus Chrifomelace Corpus ouatum. Thorax fubmarginatus. Color ater, nitidus. Elytra fingula in difco macula rubra, in apice macula, alia maiori fernginea. Pedes fetis rigidis fpinofi. Variat magritudine, et colore interdum toto nigro nitente, varietas, S. fimetarii nomirie a Fib. indicata. Hab. in ftercore bouino frequens. Long. 3 . lat. I $\frac{3}{4}$.

Sph. bipuffulatum. Herbft. K. t. 37. f. 2. et marginatum f. 3. nil nifi merae varietates S. fcarabøeoïdis funt. Haud fupernacaneum effe puto, fi omnes a me obferuatas varietates enumero: $a_{\text {. }}$ maculis duabus ferrugineis in fingulo elytro: $S$. fcarabaeoïdes. f. macula ferruginea elytrorum difci ferme extincta. $\gamma$. absque vilo maculae difci rudimento: S. 2. puffulatun Herbfi. et lunatum $F: \delta$. absque macula apicis. $\varepsilon$. absque maculis et difci et apicis; elytrorum margine folo tenuifitime ferrugineo: S. marginatum F. - Scopolii citatum a Fabricio et anctore exlibitum, ad Nitidulani bipuffulatam pertinere videtur; certe mihi perfuafum eft, eiusdem $D$. fcarabcioïdem nullo modo huc referri poffe. Hg.

I I5. S. melanocephalum atrum, glabrum, elytris grifeis, macula communibafeos nigra. F.Sp. 78.6. 
오 F. E.S.I. I. 80. I4. Herbf. K. t. 37. f. I0. K. L. M.

Hab. in ftercore. Long. $\frac{2}{3}$

Varietates, excepta magnitudine, quam valde mutabilem obferyani, enumero fequentes: a. elytris grifeis nacula communi bafeos nigra; $F$. elytris grifeis absque macula; $\gamma$ - elytris cbfcure cultaneis; $\delta$. elytris rufo nigroque variis; $\varepsilon$. elytris nigris, apice ferrugineo: Sph. haemorrhoïdile. - C. Caeterum figurae Herbfti i l. c. haud optimae funt, et f. 1o. L. potius $S$. xanthopterum Laichar't vel marem $S$. vnipunEutii repraefentare videcur. Hg.

I 6. S. hamorrhoidule atrum, elytris apice rufis, pedibus nigris. F. 'Sp. 79. ?.

of F. E. S. I. I. 8 I. I8. Herbft. K. t. 37. f. 9. I. Hab. in fercore bouino rarius. Long. I. lat. $\frac{r}{2}$.

Nil, nifi mera varietas praecedentis, vide ibi adnotata. Hg.

\section{(p.48.)}

I 7 . S. atomarium laeue, atrum, elytris crenato - ftriatis, pedibus palidis. F. Sp. 78. 5 .

‥F. E. S. I. 1. 80. 13.

Hab. in aquis ftagnantibus. Long. I. lat. $\frac{x}{2}$.

Nullo modo verum Sphaeridium auctor habuic, cuim habitationem tam diuerfam, et Sphaeridio minine conuenientc exhibeat. Potius Elophori aut Dytifci fpecier huc retulit. $\mathrm{Hg}$.

* I IS. S. inmaculatum atrum, immaculatım, thorace elytrisque leuiffmis nitidis.

Diuerfim a $\bar{S}$. fintetario, at nulla alia nota, nifi forma, et magnitudine diftinguendum. Corpus ouatum. Statura admodum conuexa, hemifphaerica. Tab. III. f. 5 . Hab. in ftercore rarius. Long. $\mathbf{I}_{4}^{\frac{3}{}}$. lat. I. et vitra. 


\section{TRITOMA.}

Palpi anteriores fecuriformes. Labiun emarginatum. Antemae claua perfoliata.

* I Io. T. incerta atra, elgtris macula laterali rubra, fronte ferruginea.

\section{An T. bipuftulata. F. Sp. 80. I?}

I T. bipufiwlata. Roffi Mant.

F. E. S. I. 2. 505. I08. Herbft. K. t. 43. f. Ix.

1. L.

Tritoma Geoffi. Inf. I. 335. I. t. 6. f. 2. ciuerfa videtur quoad magnitudinem et macularum figuram. Corpus magnitudine Coc. 7- punctatae, fed magis oblogum, atrum, glabrum, lacue, nitidum. Autennae brenes, claua ferruginea. Thorax ater contexus. Elytra fubmarginata nigra, bafi macula magna rubra laterali trapeziformi futuram non attingente. Pades nigri plantis lubrufis. Raro inuenta in fiuis fub cortice arborum. Long. $I_{3}^{2}$. lat. I.

Citatum Geoffroyi nullo modo huc referri poteft. Omnes ibi datae notae, figura optime exprefia, thoracis, eiytrorum, antennarum forma optime $\mathrm{N} / y c e$ top'ugo 4-maculato conueniunt, nifi tarfi 3 -a:ticulați ibi exhiberentur, qui in Mycetophago 4articulati funt, articulo tertio, a bafi nnmerato, minimo, et quarto multo minori. $\mathrm{Hg}$.

$$
\begin{gathered}
\text { (p. 49.) } \\
\text { XXI. IPS. }
\end{gathered}
$$

Palpi breniffimi aequales, articulo vltimo obtufo. Maxilla bifida. Labium breue, emarginatum. Antennae perfoliatae.

120. I. quadripuftulata oblonga, nigra, elytris puncts duubus fermuineis. F. Sp. 80.2. 
○ Geoffr., Frifch.

7) Schieff. Ic. t. 189. f. 3 .

o F.E.S.I.2. $5 \times 2$. 6. Herbft. K. t. 42. f. I. a. A. Geofir. Inf. I. I06. I? ?

Defcriptio Linnaei in Fn. Sv. 446 . exacte conuenit cum noftris. Hab. cortices cariofos in filuis non frequens. Long. $1 \frac{1}{4}$. lat. $\frac{1}{4}$.

I2 I. I. crenata oblonga, nigra, thorace rugofo, elytris friato-cronatis, maculis duabus rubris. F. Sp. 81. 5 .

I Lyctus crenatus. F. E. S. I. 2. 504. 9. Bitoma crenata. Herbft. K. t. 46. f. 6. f. F.

Synchita crenata. Fiellw. ap. Schneid. Ent. Mag. IV. $405 \cdot 7$.

Corpus anguftum, depreflum magnitudine dupla pediculi. Antennae perioliatae. - Capiut bipunctitum. Thorax longitudinaliter rugofus, obfure niger. Elytra ftriis crenatis nigra, bafi apiceque - macula magna ferruginea. Abdomen et pedes nigri. Hab. fub arborum cortice frequens in filuis. Variat colore toto teitaceo.

Antea, quam Fabricius genus $L_{2 y}$ Ii conderet, primus huc pertinentes fpecies, paucis aliis adiectis, fub nomine Synchitae coniunxi, vt videre licet in Schneid. Ent. Mlagazin 1. c. De hoc nominelubenter autem cedo, cum Fabricio, viro celeberrimo, aliud nomen indere placuit. Fig.

* I22. I. taxicornis fulua, nitens, corpore angufto cylindrico, thorace elytrisque laeuifimis, fubmarginatis.

An Córticens vnicolor. Piller. It. per Pofeg. Scl. p. 87.t. IX. f. 3 ?

\$ Hypophloens caftanews. F. E. S. I. 2. 500. I. 
Color teftudineus, nitens. Antennae perfoliatae. Thorax longus, elytris obfcurior, fubnarginatus, laeuis. Elytra laeuifima, cylindrica, rotunGatil, fubmarginata, tefudinea. Abdomen et pedes concoloria. Alae nägnae albae. Variat maguitudinc. Tab. IV.f. 2. Hab. in filuis fub vImi. cortice tempore hiemali. Long. 3. lat. $\frac{8}{3}$.

Equidem fub cortice gutercus et figi femper reperi. Variat colore teftaceo, vel caftaneo diluto Oculi nigri funt. Hg.

\section{(p.50.)}

* I23. I. linearis atra, nitens, corpore anguito cylindrico, thorace elytrisque profinde fulcatis.

of Colydium clongatum. F. E. S. I. 2. 495. 2. Panz. F. I. G. IIT. r7. optime.

Synchita elongatc. Hellw. ap. Schneid. Ent. MIag. IV. 406 . Io.

Mignitudo et fatura praecedentis. Tota atra, nitens practer pedes, qui fului funt. Antemiä perfoliatic articulis vitimis extrorfum crafioribus. Thorax ater, longus, laenis, fubmarginatus, et in medio tantum fulcatus. Elyira cylindrica, fubmarginata, fulcis quatuor profunde exaratis, fpatiisque intericctis punctatis. 'Tab. II. f. 4. 5. Hab. in querculun truncis cariofis.

- Variat elytris coltaneis, vti etian in Poraeri $F$ Gun

Germanica depicta eft. Solitus auten color nitidifime niger. Colyd. filiforme. F. E.S. I. 2. 496. 3. mera varietas. Hy.

* I24. 1. pufilla oblonga, nigm, nitens, glabia, elytris ancice dimidiato - rufis.

울 Hypophloeus fafciatus. F. E. S. I. 2. 50 r. 3.

Panz. F. I. G. VI. I 7 .

Panlio minor $I$. crenata. Antennae, caput et thoD 3 . rax 
rax nigra. Pedes ruf. Hab. fub arborum cortice fiequens tempore hiberno.

\section{HISPA.}

Palpi aequales medio crafiores, fufformes. $\quad M I a-$ xilla bifida. Labium comoum, integrum. Antennaze cylindricae.

125. H. flabellicornis antennis pectinatis, elytris laeuibus, atris. F. Sp. 8I. 2.

\& Melafis flibellicomis. F. E. S. I. I. 244. I.

Herbft. K. t. 47. f. x. a. Panz. F. I. G. III. 9.

Corpus totim cylindricum, atrum. Caput globofum antennis vno latere valde pectinatis nigris. Thorax cylindricus, bafi vtrinque terminatus dente valido acnto. Elytra fcabra fufca. Capta Pifis in horto. Long. $I_{3}^{\frac{2}{3}}$. lat. $\frac{2}{3}$.

Elytra fcabra, ftriata. Hs.

$$
\text { (1).5I.) }
$$

I26. H. pectinicomis antennis pectinatis, fufca, peribus luteis. F. Sp. 82. 3 .

D-Schrank. E. I. 6. . Schaeff. Elem. App. t. I36. Icon. t. 2 I 5 . f. 3. a. 3. b.

I Ptilinus petinicornis. F. E.S. I. I. $243 \cdot 3$.

... Panz. F. I. G. III. 7 .

Ligniperaia paqinicornis. Herbft. K. t. 46. f. II. h. H.

Antennae flauae, vtrinque pinnatae. Pedes flati. Corpus cylindricum nigricans, minime nitidum. Hab. vetuftas: falices, quas rotundis foraminibus larua terebrat. Long. 2. lat. I.

* 1.27. H.? flaue (cens.

Plilimus niger, fubuillofus, thorace plano, margi- 
nato, elytris flauis mollioribus. Geoffi. Inf. I. 66 . 2. t. I. f. 2.

If Ptilinus flawefcens. F. E. S.I. I. $243 \cdot 3$.

Panz. F. I. G. III. 8.

Antemae nigrae vno latere valde pectinatae, articulis vndecim, primis dnobus vix apparentibus, vltimo longiore. Thorax marginatus niger. Elytra fibuillofa, minime ftriata.

Huc refero infectum, quod venia Cl. Fabricii, nec in Sy/tem. Ent. nec in Spec. defcriptum inuenire potui. Libenter amandaffem ad alind genus, nifi me comenientia Geoffroycun clum praecedenti, et animus imminuendi difficultates potins, quam augendi nonitate, retimuiffet. Defcriptio Canthari-

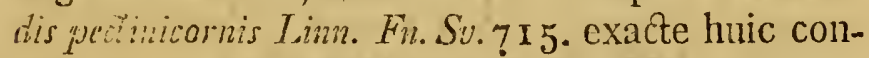
uenit, iti etian animaduertit Geoffroy, led defectus veficul riam abdominalium a Cantharidun geinere remonere videtur. Hab. flores non frequens. Long. 2: lat. I.

$$
\text { (p.52.) }
$$

128. H. teftacea antennis fufiformibus, corpore tefacev, antemis, aculeisque nigris. F. Sp. 83 . I I.

q F. E.S. I. $2.7 \hat{\mathrm{T}} \cdot 2$.

Totä teitacea, praeter antennas et aculeos elytrorum qui nigri funt. 'Antennae porrectae. Hab. in quisquiliis rara. Long. 2. lat. I.

129. H. atra antennis fufformibus, thorace, elyrisque lininofis, corpore atro. F.Sp.S3. 10.

$\odot$ Act. foc. Berol.

? F. E. S. I. 2. 70. I. H. ífracea fere dimidio minor. Tota atra, antennis tantum apice cinereis, quas porrectas gerit, et

$$
\text { D } 4
$$

fimul 
fimul iunctas. Hab. in pratis ad graminum radices. Long. $I_{\frac{1}{2}}^{\mathrm{r}}$, lat. $\frac{3}{3}$.

* 130. H: pomaticornis antennis clauatis, teftacea, thorace fufco, elytris frriatis fubpilofis.

Antennae longitudine thoracis, diftantes, porrectae, clauatae, teftaceae, articulis octo approximatis et nonnihil pilofis. Caput parum exfertum. Thorax rotundatus, cylindricus inaequalis, fubniger. Elytra teitacea ftriata Itriis clenato - pilofis. Abdomen pedesque teftacei. Hab. intra corticem et lignum arboris putrefcentis. Long. $I_{\frac{1}{3}}^{\frac{x}{3}}$. lat. $\frac{1}{2}$. vix.

\section{(p.5.) \\ XXIII. NICROPHORVS.}

Labiun cordatum, emarginatum. Antennae perfoliatae.

I3I. N. Vefpillo ater, elytris fafcia duplici fernuginea. F. Sp. 82. 2.

○ Ray., Lifter., Frifch., Mouffet, Voet., Bergftr. Petiv.

D Scop. E. C. 33 . Schrank. E. I. 74.

of N. Vefpillo. F. E. S. I. I. $247.4 \cdot$

Panz. F. I. G. 2. 2 I. Herbft. K. t. 50. f. 4. 5 .

In nonnuliis fafcia media interdum interrupta conftans punctis tribus diftinctis. Deferiptio Scopolina optima. Mofchun fpirat, ocyfime volitat, elytris erectis approximatis. Acaris infeftatur. Hab.cadauera. Long. 9. lat. 4.

XXIV. SILPHA.

Maxilla vnidentata. Labiun dilatatum, bifidum. Alitennae perfoliatae. 
132. S. littoralis atra, elytris lacuibus, lineis elcuatis tribus, thorace orbiculato, nitido. "F.Sp. S5. 2.

(.) Frifch., Sulz., Bergftr. x. t. Ir. f. 3 .

D) Schrank. E. I. 75.

Q F.E.S. I. I. 249. 2. Herbít. K. t. 50. f. 7. 8.

Figura Schaefteri a Cl. Fabricio in Mant. citata, conueniretne potius $N$. Germanico?

Atra, lacuis, nitida, oblonga. Antennae nigrae cliua elongata articulis tribus ferruorineis compofita diftinctis, vltimo conico. Thorax orbiculatus. Scutellum magnum. Elytitum punctatum lineis tribus eleuatis, prima et fecunda gibbofitate connexis, cium tertia, futurac proxima, a baii ad apicem recta decurrit. Mofchum olet. Fab. in cadanoribus inxta aquas, non frequons. Long. $8:$ lat. $3 \frac{2}{3}$.

Cuod ad quaeftionem auctoris attinet, figura hacc Schaefferi Ic. t. $2 x 8$. haud quidem $\backslash$ pertinet ad S. littoralem, contra tamen non ad N. germanicum, fed ad N. humatorem F. E. S. referenda eit. Caeterum S. littoralis varietas fiepins occurrit, femoribus pofticis incraflatis, quae tamen certifime haud peculiarem conftituit fpeciem. $\mathrm{Hg}$.

\section{(p.54.)}

133. S. atrata atra, elytris punctacis, lineis eleuatis tribus laeuibus, thorace integro. F. Sp. 87. IO.

D Scop. E. C. 56 . Schrank. E. I. 80.

q F. E. S. I. r. 25 I. Ir. Herbf. K. t. 5 r. f. r. Hab. in riis ct hortis. B. Var. B. (Gecfr. Inf. I. I 8. I.) fpatio interiecto punctato, thorace lacui, vbique aequali. Dubito, $S$ obfuram et $S$ opacanis huc elle referendas. J.Var.C. (Geonir. l.c.) lpa. 
tio interiecto punctato, thorace lacui, punctis duobus impreflis. In Ciuis et agris rarior. Long. 4 , 5,6 . lat. $2,3,4$.

Nefcio, quid de varietatibus his enumeratis dicere pofien. Equidem Geoffroyum valde fufpectum habeo, ne Silphas obfcuram atque opacam, atratamque, (affines quidem primo afpecțu, fed attentiore oculo lufratas, vilcie dinerfas, mifcuerit. Secundum meam experientiam ex plurimis lectis fpeciminibus defumtam, nulia alia datur varietas, quan colore brunneo, loco nigri, vt in nuper declaratis fieri folet. $\mathrm{Hg}$.

\section{(p.55)}

134. S. rugofi nigricans, elytris iugofis, lineis cleuatis tribus, thorace ragofo, poftice finuato. F. Su. 86. 9.

○ Degeer. 4. I8z. 7.; Bergftr., Ray.

P F. E. S. I. I. 25 I. 10. Herbft. K. t. 50 . f. $x 2$.

S. fcabra. Scop. F. C. 59.

Panz. F. I. G. V. 9.

$\mathrm{Hab}$. in cadaueribus frequens. Capta humorem foetidifimum enomit. Long. 5. lat. $2 \frac{1}{2}$.

Auktor citat 'segreer. Inf. 5. 43. 5. t. 2. f. 2 r. et alterum Fauricii citatum 4. I82. $\%$. omittit. Sed comparata vtraque deferiptione id nouifimum foJum verum, et Tenehr. rugofus Degeer. $5 \div 3 \cdot 5$. t. 2. aì Opatrum labulofim pertinet. H.s.

135. S. fonuate thorilce emarginato, icabro, elytris lineis elenatis tribus, apice finuatis. F. Sp. 88.16.

D) S. icabra. Siop. E. C. 59?

S F.E.S.I. I. 252 . т8. Herbft. K. t. 5 T. f. 7 . Thorax antice cmarginatus, fufcus, tomento albo, r ciuti fplesdens. Elytra nigra apice finu rotundo exarata. Hab. cadumera rarior. Long. 5. lat, $2 \frac{1}{2}$. 
Qunedam fpecimina appendiculo elytrorum carent, et cum appendictidatis minores fint, mafulos coniicere licet. Horum ;arietatem fufcam nóliúco.

$$
\text { Hig: }
$$

136. S. Puffacioblonga, 1ufa, antennis elytris pertoreque nigris. F. Sp. 85.5 .

$\odot$ Vadm.

o Ips nigripennis. F. E. S. I. 2. 5I3. Io.

Statura differt a pracedentibus oblonga, glabra, nitida. Anternae nigrae fere longitudine theracis, clauta perfolinta articulis tribus vltimis craflioribus, cxtimo maicre rotundo. Palpi antici lecuriformes, pofici clauati. Caput et thorax rufa. Thorax latus, conuexus, marginatus. Elytra caeruleonigra, laeuia, fibtilifime puncto friata, marginata. Peftis, pedes et abdominis pars poftica rufa. Tarf quatuor. In ftatu vix declarato tota rufa. Figuram exhibet tab. A. f. 6 . Hab. fub arborum cortice, in ligno putrefcente hiberno tempore frequens.

\section{(p. 56.) \\ XXV. OPATRVM.}

Palpi anteriores clanati, claua obliqua truncata, polteriores filiformes. Labium fubemarginatum. Anteance moniliformes extrorfum craffiores.

I37. O. fabulofum fufcum, elytris lineis elcuntis tribus dentatis, thorace emarginato. F.Sp. 89. 2.

$\odot \mathrm{Vdm}$.

올. F. E. S. I. I. 89. 3 .

Tenebrio rugofus. Degeer. V. 43. 5. t. 2. f. 2 x. 22 .

Herbft: K. t. I. II. f. 5. Panz. F. I. G. IIf. 2.

Color: 
Color caftaneo-niger. Palpi clanati. Antemne parmm pilofae articulis 9 ant 10 grlobofis, vitimo maiore. Caput intra thoracem, clypeo fcabro pundato. Ocnli exigui. Thorax mgofus. punctatus, colore magis opaco, nargine laterali dilatato, prominulo, anitice emarginatus. Elytra connata nargine eleuato, acuto, fubreflexo; in medio carinata, carina dentata irregulari fobra villofa, apicem non attingente; in medio ad bafin alia brenior linea cleunta; fiatium omine inter lincas tuberculis fpinofis cxafperatum, praefertim ad apicem elytrotum aduninatum. Pedes fuci. Abdomen fibturs pilis lauis pubcicens. Hab. in campis fabulofis rarum. Long. 4. lat. 2 .

\section{I1 - (p. 57.)}

38. O. grifen cirereum, thorace plano, mar. ginato, elytris friis tribus eleuatis, pofice dentatis. F. Sp. 80. I.

1) An Geofti. Inf. I. 347.2 . t. 6. f. 6 ?

\& F. E. S. I. x. 88. $x$.

Defriptum habes in Fabr. 1. c. Thorax planus margine dilatato, fod minus elenato, gnam in $O$. fabulofo. Elytra marginata, margine fibreflexo, ftriis tribus elcuatis. Tibiae apice bilpinofic antice latere exteriore ctiain acute angulato. Hab. in locis arenofis; Hlorentiac non rarum. Long. 8. lat. 4.

139. O. agricola nigrum, thorace lacui, elywis trintis. F. Mant. 5 r. 8?

I F. E. S. I. x. 9r. I4. Herbit. K. t. 52 . f. g. figura hand optima!

Thorax Laevis fubmarginatus. Elytra friato - puncata fubrigola. Corpus parmm, totum obfourc nigrmm. 
nig̈um. Hab. in ligno putrefcente, et fub arbórum cortice tempore hibemo. Long. $22^{3}$. Tat. I t.

Variat colore teftacco et rufo, habitat creberrime in Buleto luteo Linis. $\mathrm{Hg}$.

\section{NITIDVLA.}

Patpiflifomes Ihtaxilla cylindrica, membranacea. Labinin cylindricum, integrum. Antemate clata folida.

\section{(}

140. N. bipufutata ouata, nigra, elytris puncto Inbro. F. Sp. 91. I.

D) Oftoma $=$-puftulata. Laich. I. I06. 3 .

q F. E. S. I. T. 255 . I. Panz. F. I. G. "III. Io. Herbft. K. V. t. 53. f. т. A.

Hab. cadauera putrida. Long. $1 \frac{2}{3}$. lat. $\frac{2}{3}$.

14I. N. obfura onata, nigira, obfcura, pedibus piceis. F. Sp. 91.3.

q F. E. S. I. I. 255. 3. Herbft. K. t. 53. f. 2. b. B. Plana, laenifima, opaca. Antennae fermgineae. clatia nigra. Elytra flexilia abdomine paullo breniora. Pedes fubferruginei. Alac albae. Hab. in cadaucribus. Jong. I $\frac{2}{3}$. lat. I.

* I42. N. flawomaculata ouata, nigra, elytris laenibus, flanomaculatis.

Praecedenti finillima, et in eodern loco reperta. Differt tantum elytris bafi, et iin, medio prope fituram colore palcaceo, feu obfolete aureo-maculatis. Magnitudine etiam admodum rariat. Tab. III. f. 8. Imeni plures cum praecedenti, et Scarab. 2 -maculato in Cameli cadauere in fepulto. Long. 2. lat. I. 
Equidem, excepta fignatura elytrorum, et thoracis margine laterali eiusdem, vt elytrorum maculae, coloris luteo - ferruginei, differentiam inter hanc et praecedentem detegere nequeo. N. obfcuram attamen femper eandem vidi in noftris regionibus, nec variantem. $\mathrm{Hg}$.

* I43. N. pundtata cblonga, nigia, elytris puncto bilobo dorfali cinereo.

Accedere videtur ad Derm. lunatum. F. Mant. App. Is - I 9 .

Statura N. obfcurae, fed corpus minus depreflum. Antemae claua ferruginea. Thorax fubmarginatus, fubpubefcens, niger, linea laterali eleuata. Elytra punctato - ftriata, nigra, puncto ad firtiram bilobo dorfali cinereo. Abdomen nigrum pube albida. Pedes ferruginei. Hab. fub arboruin cortice in filuis. Long. $\mathbf{I} \frac{\mathrm{T}}{5}$.

\section{(p. 50.)}

144. N. varia ouata, thorace elytrisque nigroferrugineopue variis. F.Sp. 92. 7 .

오․ E.S. I. I. 258 . I2. Herbft. K. t. 53. f. 4. d. D. Deficriptum vides a Fabricio 1. c. Hab. in truncis exficcatis, cariofis. Long. $\mathbf{I} \frac{3}{4}$. lat. I.

145. N. hirta? atra, thorace elytrisque pubefcentibus. F. Sp. 93. I5.

Statura praecedentis, fed corpore magis elongato, nigro, fufco. Antennae ferrugineo-fufcae. Caput, thorax et elytra pilis breuibus, erectis, ferrugineis adlperia. Thorax marginatus. Elytra punctato-friata: Pedes fufci. Tab. III. f. 9. Hab. cum praecedenti. Long. 2. lat. $\frac{2}{3}$. 
I46. N. arfika tefacea, fabtomentola, thorace emarginato, octilis niguis. F.Sp. 9 r. 5 .

8. F. E.S.I. I. 25 6. 6. Herbit. Arch. t. 20. f. 24 ? Hab. in flurious. Long. I. lat. $\frac{1}{2}$.

Herbltii deferiptio 1. c. cum N. fermginea $F$. valde conuenit. $\mathrm{Hg}$.

$$
\text { (p. со.) }
$$

* I4:. N veris teftacea, fubtomentofa, corpore connevo, antennis apice nigris.

\& Spidatidium luleum. Rofii Mant. 2x. 4\%. F. E: S. I. . . 79. 6.

Strongylus luteus. Herbft. K. t. 43 . f. 3. c. C. $N$. aeftita maior, et corpore magis conucxo. Tota teftacca, holofericea, clytris marginatis, oculis, antennarumçe apicibus nigris. Tab. II. f.' I2. Hab. medio veri in floribus Crataegi. Long. 2. lat. $I \frac{t}{5}$.

I48. N. pedicularia nigra, elytris lacuibus, tho-, race marginato. F. Sp. .92. I2.

P. F. E. S. I. I. 260.20.

Hab. flores.

\section{XXVIT. COCCINELLA.}

Palpi anteriores fecuriformes, poticriores filiformes. Labium cylindricum. Antennae claua folida.

Coccinellarum fpecies, quod ad colores, tam elytrorum, quam thoracis, attinet, valde mutabiles efie, ian dudum notum eft. Cuique enim, qui vera di- ligentia earundem fpecimina colligit, de hoc perfuafum erit. Iure itaque mirari videmur, cum auctorem huius rei quafi haud informatum videmus. Ne autem adnotata noftra in hoc Coccinellaruan genere mole fia opus granent, deinde, ne iam, et melius, 
lius, dicaa iteremus, Herbftii celeberrimi Coleoptratolum fyftematis Tomum V. luc fpectantem, praccipue autem tractatum illum de Cuccinellarum genere, in N. Magaz. d. Entom. Fol. Il. acutifimi Scimeideri, ct eiusdem recentionem Anonymi cuiusdam in Allgem. Littesat. Zeit. quae Iencie prodit, Anni ${ }^{793}$. Nr. 15. commendare iuuat. $\mathrm{Hg}$.

149. C. impunctata coleoptris rubris, puncto nul10. F.Sp. 93. a.

\& F.E.S.I. $2.267 \cdot$ 9. Herbft. K. t. 48 . f. I7.

Schneid. Mag. II. I 49. I4. C. globofa var. a. Rubra fint thorax, elytra, pedes antici: reliqua fufca, alis cratilore margine nebulofis. Habit. in hortis. Long. $I_{3}^{2}$ lat. I.

Mera varietas $C$. 24-punctatae n. I63. efr, puncetis nigris nondum declaratis. $\mathrm{Hg}$.

150. C. bipunitata coleoptris rubris, punctis nigris duobus. F. Sp. 94. I0.

() Lifter., Ray., Petiv., Frifch., Sulz., Act. Nidr.

D Scop. E. C. $23 \%$ Schrank. E. I. 94.

o F.E.S.I. I. 270.2 I. Herbft. K.t. 58 . f. I-9. I0?

C. difpar. Schneid. Mag. II. $173 \cdot 32$.

Macula inagna alba ad thoracis marginem, bafi. puncta duo, et apice lineola et punctis duiobus alba. Hab. paffim in hortis, victitans Aphidibus. Long. $2 \frac{1}{2}$. lat. I'.

C. 4-pufulata n. I7x. et C.6-puftulata n. I72. va= rietates huius bipunciatae funt. $\mathrm{Hg}$.

\section{(p. or.)}

* C. triplinitata coleoptris rubris, punctis nigris tribus. Linn. Fn. Sv. 472.

ㅇ Rofii Mant.25. 59 .

Defcriptio Linnaci iterum exhibita eft. Forte fequentis mera varietas eft? Hab. frequens in plantis. Long. 2. lat. I. 
In Mautiffa pro variętate certa 5 -punftatae declarata eft, adiecta obferuatione, in Fauna Etrufca pro qraecedentis effe legendum fequentis, quod fatim correxi. Si autem ibidem dicit auctor, punctum elytri pofticum matginale $C .5$-punfiatae fortuito tantum deefic, id defcriptioni Linnaei, quae caeterum optime conuenit, repugnat. Hic enim puncti verfus apicem mentionem ficit, quod autem in 5-puntiatu, fub illa conditione, in medio dorfo, prope futuram, apparet. Ideo Linnaei tripunfat aut varietas 5-punitatue eft, punctis duobus'dorfalibus deficientibus, fed punctis illis duobus marginalibus integris, aut nobis nondum nota fpecies. Priori coniećturae id obftare videtur, quod puncta dorfalia marginalibus triplo, imo quadruplo maiora, hand tam leuiter deeffe folito more pofiunt, quam marginalia. - Ne citem Fabricii C. tripuntatam, prohibent illa, quae Schneiderus habet N. Enta Mlag. II. I 73-322. Hg.

152. C. quinquepunctata coleoptris fanguineis punctis nigiis quinque. F. Sp. 86. 17.

D Sclrank. E. I. 96.

오 F. E. S. I. I. 273. 36. Herbft. K. t. 58. f. Ir. Elytia rubra, fingula punctis duobus, et vno comnumi ad bafin. Thoraxangulis anticis albus. Habitat in hortis paffim., Long. 2. lat. $\Sigma_{\frac{2}{3}}$.

153. C. Septempunätata coleoptris rubris, punctis nigris feptem. F. Sp. 96.21.

○ Petiv., Ray., Liffer., Roefel, Hollar.

D Scop. E. C. 235 . Schrank. E. I. 98.

o F. E. S. I. I. 274 . 4I. Herbft. K. t. 57. f. 8. Puncta elytrorum in triangulum pofita. Hab. vbi. que in plantis. Long. $3 \frac{1}{4}$. lat. $2 \frac{1}{2}$. 
I54. C. nouempanctatc coleoptris nubris punctis nigris nonem. F. Sp. 97. 27 .

$\odot$ Vdm.

定 F. E.S. I. T. 276.48 .

Puncta 2. 2. ublique pofita, communi maiori. F. Prope bafin finguli elytri puncum minutum nigrum, et tria in trianguli foman difpofita vltra medium maiora. Corpus inferne nigrum, vtrinque ad latera puncta cino alba. Hab. in plantis frequens. Long. 2'. lat. 2.

A quibusdam Coccinella, quam Herbftius nomine 9notatae defcriptam, et t. 55 . .8 . bene depicam habet, C. 9-pounquatct Linn. creditur, cui autem et thoracis pietura, et patria, dum ex America boreal's affertur, repugnant. Hg.

* I55. C. nigrofafciatca coleoptris rubris, punctis nigris octo, filicia media atra.

\%. Roffi Mant. 25. 60.

Omnino fimilima antecedenti. Differt tantum quod habet vlterius fafciam atram fiexuofam, in medio elytrorum a puncto communi bafeos enatam. Hab. in hortis cum priori, cuius forte varietaś eft. Long. 2 r. lat. 2.

Nefcio, quomodo auctor tam leuem varietatem, quae in Coccinellarum genere, vbi eiusmodi mutationes femper occurrunt, nulli momenti eft, vt fpecie diuerfam enumerare potuit, praecipue cun fimul cum priore cepit. $\mathrm{Hg}$.

$$
\text { (ip. } 3_{3} \text { ) }
$$

* I56. C. II-puntata coleoptris mbris, puncis rigris vndecim. Linn. Syft. Nat. 58 1. I8.

\& F. E.S. I. I. $277 \cdot 53$. Herblt. K. t. 58. f. I3. Arch. t. 43. f. I5.

Thorax 
Thorax niger punctis duobus albis. Magnitudine variat, fed inter fipecies minores numcrari poteft. Hab. in plastis. Long. $\mathbf{I}, \mathbf{I} \div$. Jat. $\mathbf{I}, \mathbf{I} \frac{1}{2}$.

Fabricii citatum Gioffr. I. 324. 8. quod auctor dubium exhibet, omnino delendum eft. Ne minima quidem nota, vndecim exceptis punceis, conuenit. $\mathrm{Hg}$.

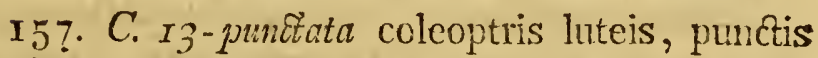
nigris tredecim, corpore oblongo. F. Sp. 99. 38 .

D Scop. E. C. 238. Schrank. E. I. I02. $\beta$.

I F. E. S. I. I. 279.6 r. Herbft. K. t. 57 . f. II. Schneid. Mag. II. I 39.8 .

Puncta elytrorum I. 2. 2. I. et communi bafeos. Hab. in hortis. Long. $2 \frac{1}{2}$. lat. 2.

159. C. 17 -punciata coleoptris flauis, punctis nigris quatuordecim, quibusdam contiguis. F. Sp. 99. 39 .

D Geofir. Inf x. 328. $x$ ? Schrank. E. I. x०4.

\&. C. teffellata vair. b. Schneid. Ent. Mag. II. I58: I9. b.

Herbft. K. t. 57 . f. I6-I's.

Thorax flauus, poftice macula magna nigra tecraloba. Elytra flaua, punctis angulatis, plerisque contiguis, non tamen connexis. Pedes flaui. Hab. in lalice, et vrtica dioica non frequens. Long. 2 . lat. I.

Citatum e Gecffroyo bonum eft. Varietates innumeras iam Geoffroyus obferuauit, vide etiam Schneiderum 1. c. - C. conglomeratar n. I60. et 12 pujtulata n. I73. varietates quoque huius funt.

Hg.

$$
\text { (p.64.) }
$$

159. C. I9-punetata coleoptris flauis, punctis nigris nouemdecim. F. Sp. 100. 43. 
오 F. E.S.I. I. 280.67. Herbf. K. t. 57. f. I7. Puncta elytronam 2.3. 3. I. et vnico conmuni. Corpus parum. Thorax flaum, punctis fex nigris, quorum duo pofteriora. Elytra flaua, fingula punctis nouem nigris. Hab.in plantis non frequens. Long. $1 \frac{2}{3}$. lat. 1 .

Puncta in meis, futellari communi excepto, hoc ordine pofita obferuo: I. (maximo) 2.3. (intermedio parum promoto, vt haec puncta tria trianguluin deprefium fiftant) 2. I. $\mathrm{Hg}$.

I6́. C. conglomerata coleoptris flauefcentibus punctis nigris plurimis contiguis. F. Sp. Ior. 49.

(- Gecffr. I. 316. I 2. Ray., Lifter., Frifch.

D Geoffr. Inf. I. 326.12 ? Conf. Schaeff. p. 30 . I63. Inf. Rat.

요 F. E.S.I. I. 282.75 .

Schneid. Mag. II. I 59, C. teffellain var. $c$.

Dezeerius defcribit optime. Habit. in hortis. Long. $2 \frac{\mathrm{T}}{3}$. lat. I $\frac{\mathrm{t}}{2}$.

Fabricii C. conglomercta nil, nifi mera varietas C. 14maculatae, vi puncta, quae in conglom. pafim confluunt, diftincta funt. Caeterum, citatum Geoffroyi, errore typographico 316 . in 326 . emendato, ad conglobatam pertinet. Linnaei conglomerata alia videtur. $\mathrm{Hg}$.

I6 I. C. conglobata coleoptris rubris, punctis nigris plurimis fubcontiguis. F. Sp. 102. 49. $\beta$.

ㅇ F.E. S.I. 2. 282. 76. Schneid. Mag. II. I56. I8. Herbft. K. t. 58. f. I4.

Species valde diuerfa a praecedenti. Thorax punctis nigris feptem diffinctis. Elytra in infecto viuo rofea, punctis in fungulo octo nigris, quorum par primum futurae propius, par fecundum margi- 
ni exteriori, vnum iterun anteriori, et par tertium cxteriori: punctum denique vltimum iterum margini interiori adiacet.

In hac puncta non tetragona, it in praecedenti. Hab. in arboribus inter Aphiảes. Long. $2 \frac{1}{4}$. lat. I Nullo modo cum conglomerata coniungi poteft, cum etiam ftatura longiore differat. Eadem autem eft cum 15 -maculata $F$. Variat thoracis pictura, quit tandem totus nigger euadit, macula laterali excepta flaticante, quae punctum nigrum medium circumdat. Hanc varietatem excipit alia, cui nomen $C_{\text {. }}$. impuffulatae a Linnaeo et Fabricio inditum eft, et. quam cum C. conglobata copula iunctam reperi., Vide Herbft. K. V. p 356. t. 58. f. I 5. In his eascem faepius thoracis fignaturas, quas in conglobatu $L$. et autiorum, idere licet, interdum antem thorax totus niger, margine laterali obfolete rufestente. $\mathrm{Hg}$.

\section{(p. 65.)}

I61. C. $22-$ punctatu coltuptris flauis, punctis nigris riginti diobus. F. SP. IOI. 45 .

○ Ray.

D) Geoffr. Inf. I. 329. I7.

T C. 20 -puntata. F. E.'S. I. I. 28 I. 69.

Herbft. K. t. 57 . f. I 4 .

Subtus infpecta obferuatur punetum nignum in margine elytrorun, quod complet numerum 22 punctorum, exclufis punctis quinque thoracis. Hab. apud nos frequentiffma. Long. $I \frac{1}{2}, 2$. lat. I, $I_{\frac{1}{4}}$. Punctum marginis minimum in nonnullis etiam fuperne confpici poteft. AuEtorem Linnaei 22 - punctatam, et Fabricii 20 -punciatam, quae eadem eft, habuiffe, e defcriptione adiecta et citato Geoffroyano, fit apparet. Caeterum citatum Schaefferi Ic. t. 30. f. I0. quod auctor dubium exhibet, ad $C$. 
14-p11fulatcim aperte referendum eft. Rayum auctor numquam citauit, quia non poffediffe videtur. Sed comparatia etiam defcriptione Rayi al hanc Coccinellam, equidem vitere nequeo, quid emolumenti ta'e citatum, ne futis certum quidem, praebere por- . fit? $\mathrm{Hg}$.

163. C. 24-punciata coleoptris rubris, punctis nigris viginti-quatuor. F. Sp. ror. 47.

\& F. E S I I. 28 T. 72. Herbf. K. t. 98. f. I $7-20$. C. globofar. Schneid. E. M. II. I5r. I4, d.

Elytra rubra, fingula punceis duodecim nigris, tribus in bafi disinctis, quatuor intermediis, duobus comnexis, dein tribus, denique in apice duobus vix disiunctis. - Hab. frutices. Long. $I_{2}^{\mathrm{I}}$. lat. I.

Varietates innumerae huins Coccinellae reperiuntur, quarum inam C. impnicîatan fupra n. $1 \div 9$, aliam nomine C. 25-pruntatae n. 16 4. exhibet auctor.

$\mathrm{Hg}$.

\section{(p.66.)}

* I64: C. 25-punctatu coleoptris rubris: punEtis nigris viginti quinque. Limn. Fn. 488. Syft. Nat. 583. 29 .

C. 22 -puizitata. Degeer. 5.380. 13 .

Magnitudo antecedentis, cui eft finillima, magis tanen obfcure rufa, et punctis magis connexis et confufis. Habit. inter Aphides minus frequtiens.

Auctoris Cuccinella mera tantum praecedentis varietas eft, num etiam Linnei, et Degeerii? De Linnaeana valde dubito, cum puncta prioris tam inconfrantia in duobus fpeciminibus fint, vt punctum vnicum Linnaeo tam acuto et praecauto, caufam fnfficientem haud pra buifit, nouam inde conftituendi fpeciem. Deinde nihil de affinitate cum 24 - puntata dixit. $\mathrm{Hg}$. 
I65. C. I3-maculata coleoptris flanis, punctis nigris tredecim, corpore orbiculato. K.Sp. $99 \cdot 3 \%$. 온. F. S. I. T. 279.60.

C ro-puntate vir. 9. Herbfr. K. V. P. $347^{\circ}$

$C$ variabilis var. $K$. Schneid. Mag. II. I65. 24 .

Thorax albus maculis quatuor nigris. Coleoptra flaur punctis nigris tredecim, némpe I. 3 . 2. et communi. Hab. arbores rara. Long. $2 \frac{1}{2}$. lat. $I \frac{1}{2}$. Punet. quatuor tantum thoracis equidcm nondum obferuani. Inter varietates huius Coccinellae, C. лоpufiulatam quoque referri debere, experientia mea me nondum docuit, cum nec tranfitus pofideam, nec in codem reperi loco. Sed fi ita fibi haberet, $C$. zo-prufu! lata peculiarem fpeciem nullo modo conftituere debet, vti Celeberr. Schneiderus 1. c. innuit, quamquam de identitate ipfi perfuafum fit.

Hg.

* 166. C. palliail oblonga, coleoptris pallide luteis, punctis fex nigris obfoletis, et ad futuram pofitis.

Tota pallide lutea. Caput albicans, puncto nigro ad bafm.' Ocnli nigri, qui lunati videntur ob cumexionem cun punco capitis. Thorax luteus monctis fex nigris, quorm do pofteriora funt. Elytia margine albicante, fingulum punctis tribus longitudinaliter ad fituram pofitis. Pedes flaui. Cunpus atrum. Hab. frutices rara. Long. $2 \frac{1}{2}$ lat. I. frum varietas praecedentis? Certe variabili cuidam fpeciei annumeranda videtur. $\mathrm{Hg}$.

$$
\text { (p. 67.) }
$$

167. C. detrita? flaua, coleoptris immaculatis, thorace albo-maculato. F. Sp. IO2. 50.

Corpus totum flauefcens. Caput punctis duobus albis. Thorax vtrinque margine puncto, lineis dua- 
bus flexuofis, lineaque dorfali albis. Elytra immaculata. Forte incompleta? - Semel capta ruri. Long. 2. lat. I.

Quomodo anctor in Coccinellarum genere, fpecimen incompletum, quod fine dubio haec C. detrita eft, idem cum Coccinella credere poteft, quae Nonae Hollandiae indigena eft? Hg.

I68. C. Io-guttata coleoptris luteis, punctis albis decem. F. Sp. I02. $5 \mathrm{I}$.

\& F. E. S. I. I. 284.82 . Herbf. K. t. 59. f. I. Puncta elytrorum 2.,2. I. Habit. in fluis rara. Long. I $\frac{\mathrm{I}}{2}$. lat. $\mathrm{I}$.

169. C. bipuftulata coleoptris nigris, punctis rubris duobus compofitis, abdomine fanguineo. $\mathrm{F}$. Sp. I04. 6r.

\section{$\odot$ Frifch.}

\& F. E. S. I. x. $287 \cdot$ roo. Schneid. Mag. II. 283. 40.

C. fafciata. Herbft. K. t. 59. f. I I.

Tota atra, nitens, margine prominulo. In medio elytrorum puncta tria transuerfim pofita, coccinea, faepe confluentia. Abdomen rufelcit. Hab. cortices arborum; frequens in hortis. Varietas Degeriana tamen inuenta tantum in filuis: anme diuerfa? - Long. $1 \frac{\pi}{2}$. lat. $\frac{x}{4}$.

Maxima confufio iterum h. 1. videnda eft. Duae enim Coccinellae, primo intuitu fimillimae, pro vin fpecie habitae funt. C. a-puftulata Linnaei et Fabricii enedem mihi videntur, cum vterque inaculäe elytrorum transuerfalis, et e tribus interdum conflatae, inentionem faciat, quamuis Celeberr. Fabricins, cui ambas Coccinellas miferam, certiorem me fecit, C. bipuftulatcm Degeerii, etiam fuam bipuftulatam effe, et fafciatam Herbfiii varietatem tan- 
tum videri, fed appofita in Entom. Syftem. verba contrarium edocent. Niliil etiam prohibet, quo minus Herbtti fof ciata efiet, quam quod caput rufum tacet linnaeus, quod tamen femper praefens obferuaui. Ex citatis Fabricii et auctoris Degcerius et Roefolizs itaque tollendi funt; peruliarem fpeciem defiribentes, quam Schneiderus C. renigufuliticn, et Heroftiw's K. t. 59. f. I I. bipuftulatcin vocauere. Ne fit varietas, vt Roffius dubitat, prohibent habitatio dinerfa, magnitudo duplo maior, caput haud rufum, praecipue autem punctum femper intererrimum, fubrotundum, numquam e pluribus concretum. Hg.

\section{(p. 68.)}

* I 7o. C. fmilis coleoptris marginatis, nitidis, atris, macula folitaiia nors, fubrotunda vtrinque. Gazcph. p. I7 I. 60 9.

Coccinellan Cadi Pihis habitare, prorfus inuerifimile videri debet: ideoque habenda modo eft haec noftra pro C. bipujututác fortuito Americanae afinis faciem mentiente. Cun autem in hac defcribenda potifintum forniendum fit veritati, nullis profecto aliis verbis fleri poffe id accuratins videtur, quam is a Grononio adhibitis ad C. Cacti indicandam.

Eit e minoribus. Thorax, caput atque elytra laeuia, glaberrima, hemiiphaerica, marginata, connexa, atra. Elytra in centro vtrinque maculam gerunt infignen!! coccineam rotundam integerrimam, neque e tribus compofitam, qua nota a $C$. bipriftulata differt. Abdomen coccineum. Gron. 1. c.

$$
\text { E } 5 \quad \text { Differt }
$$


Differt a C. bipufulatac varietate (conf. Degeer. Inf. 5. t. 10. f. 25.) macula non reniformi, led rotunda integra, et maiori Tab. VII. f. 4 . Lecta in Alno Pifani nemoris d. 23. Mai.

Nefcio, quid de hac Coccinella dicam. Ne fit C.ronipufullata $S$. adnotata audoris ad antecedentem impediunt. Vide etiam, quae Schneiderus habet. Mag. II. I84. $4 \mathrm{I}$. Hg.

I I. C. 4-puflulata colcoptris nigris, punctis rubris quatuor, interioribus longioribus. F. Sp. 10.4. 6.3 .

() Bergftr.

은 4-virucata. F. E. S. I. I. 2S8. 104.

Herbft. K. t. 59. t. I2.

Onati, nitida, atra. Thorax ater, immaculatus. Elytra atra, maculis in fingulis duabus haigumeis, rna prope bafin reniformi, altera in medio ad marginem interioren rotunda minori. Anus riber.Fabricius hanc cum C. 2 -pundata copula jinctam faepe vidit. Hub. in vrtica, rubo, aliisque plantis. Long. $I_{\frac{1}{2}}$. lat. $1 \frac{x}{2}$.

Fabricius varietatem fexpuffulatae vel potius bipunGiatae, nullo modo autem veram 4-pufulatam cum bipmatata copula ianctan videre potuit. Haec enim eaden cum bipuftulata fratura gaudet, quain Geoffroyns voce toriue bene exprimit. Ad hanc 4-pufulitam, vel 4-vervicatan.F. E. S. citata omnia, in fpecieb. exhibita, pertinent, excepto Sihaefferiono, quod aperte ad illam bipuntiatae varietatem referendum eft. $\mathrm{Hg}$.

$$
\text { (p. og.) }
$$

1 22. C. $\sigma$-puftulata coleoptris nigtis, punctis rubris. F. Sp. 105.64 .

$\odot$ Rav. 


\section{Scop. E C. 245. Scimmk. E. I. I I \\ 을.E.S. I. . . 2üg. roz. et quadripuffulata. n. Iоз. \\ C. bipuncintae variett. Herbh. K. t. 58. f. I-9.

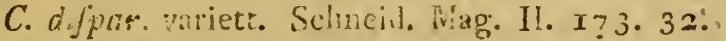

Elytra atra, filigula maculis tribus coccincis notata, carnin plinia magna ad baíin maroinis exterioris; alteril in medio elytri; tcria ad apicen prope inarginem exteriorem. $\quad \beta$. variat thorace toto atro, elytris guadrinaculatis. $\delta$. puncto minutifimo ad futuram rubro. Hab. in hortis.

I73. C. I2-puftulata coleoptris nigris, puncis duodecim albis, exterioricus margine connexis. F. Sp. Iо6.'6\%.

\section{\& F.E.S.I.r. 290 . IIO.}

C. teffellater var. d. Schneid. Mag. II. p. I60. Thorax antice aibis, pofice niger. Hab. in plane tis fat frequens.

Nil, nili varietas C. 17 -maculatae. Hg.

I 74. C. variabilis coleoptris nigris, punctis duobus nibris, orbiculatis, fubmaiginatis, corpore oblongo. F. Sp. I0 7. 62 .

\& F. E.S. I. I. $28 \%$. TOI.

C. vaifafciatr. Schneid. Mag. II. I79. $3^{6 .}$

C. bipunfiatis. vas. II. Herbft. K. t. 58. f. ro.

Variat élytris luteicentibus, atçe aetate elytra nigra, et puncta rubra euadunt. Noftrum fiecimen habet latera thoracis alba. Hab. in flinis rarifima. Loing: $2 \frac{1}{2}$. lat. 2 .

Herbftus etiam hanc, $C$. bipunatatae crcdit varietatem. C. biguttatn Herbft. K. t. 59. f. 8. Haud diftincta videtur. ' $\mathrm{Hg}$. 


\section{(p.70.)}

I75. C. 10-payftilatiz coleoptris nigris, punctis fuluis decem. F. Sp. I05. 66.

() Schaeff. Ic. t. 30 . f. ro.

9. F. E. S. I. x. 239. xo9. Herbit. K. t. 59. f. 16 .

Schneid. Mag. II. x68. 25.

Variat punctis flamis, et rubris. Puncta elytrorum 2. 2. I. duo baleos lunata, interdum connata. Pedes fului. Hab. in hortis. Long. $2 \%$ lat. $I_{2}$.

I76. C.pontherina coleoptis nigris, punctis lauis octo. F. Sp. 106. $7 \mathrm{r}$.

TF.E.S. I. I. 29 r. II 4.

Cf. Schneid. Marg. II. I8 I. 37.

Degerius 1. c. defcrint optime. Hab. raro apud nos. Long. $2 \frac{1}{2}$. lat. 2.

I77. C. $20-$ guttata coleoptris mbris, punctis albis viginti. F.Sp. $103 \cdot 56$.

D Goetze, E. B. 229: 37. Schaeff. Ic. t. 9. f. x2.

\& F. E. S. I. I. 285.90 . et cigrina 1. II8.

C. tigrina. Herbft. K. t. 59. f. I8. Schneid. Mag.

II. $x 83 \cdot 390^{\circ}$

'Puncta elytronum 2. 2. 3. 2. I. anticis oblin̨uis. Varietatem C. tigrinae credit Degent, qui dat optimam deforiptionem. Hab, in arus noin frequens. Long. $2 \frac{\mathrm{T}}{4}$. lat. $I \frac{\mathrm{I}}{2}$.

$$
\text { (p. } ; . r .)
$$

* I78. C. minuiffrma fubuiliola, nigra, fafciis duabus transueris rubris: Geoffr. Inf. 1. 332.2 I.

Tota atra, tomentofa. Elytra maculis duabus rubris, quae fafciam formare videntur. Thorax vtrinque fabmaculatus. Hab. in Pomonae arboribus. Long. I. lat. $\frac{1}{3}$. 
* r 9 . C. 4-maculata fubuillofa, nigra, punatis quature hitco-aturis. Greoft. Inf. I. 332. 22.

Subuillofa: Caput nigrinen. Thotax niger margine rtringue rufo, punctito. Elytra nigra, nitida in fingulo ciytro punfa cho tentra rotunda primo - mainsculo in medio, altero minori ad apicem. Tibiace tibrufae. Groffrogus dubitat, effe varictatem praccedentis.

Hilec a me raro inuenta hiberno tempore fub. arborum cortice; illa frequcus per aeftatem occurrit. Long. I这. lat. $\frac{2}{3}$.

Fabricius idem Geoffroyi citatum apud $C$. frontcalcm fuam allegat, quae cum bispuffulata dubium infectum manet. Hg.

\section{CASSIDA.}

Palpi anteriores clauati, pofteriores filiformes. Labium elongatum, integrum. Antennae moniliformes.

\section{(p.72.)}

I80. C. viridis viridis coipore nigro. F. Sp. 107.1 .

$\odot$ Ray., Goedart., Merian.

D Schrank. E. I. 92.

Q. F. E. S. I. I. 292. I.

Pupam habui, a quo exiit Ichneumon. Variat magnitudine. Hab. in carduis, in Cynara Scolymo frequens. Long. 3,4 . lat. $2,2 \frac{1}{3}, 3$.

I8I. C. Murraea nigra, clypeo rubro, elytris fanguineis punctis nigris fparfis. F. Sp. I08. 6 . O F. E. S. I. I. 294. 9. Antennae bafi rufae, apice nigrae. 
Hanc differre a $C$. viridi-maculata, aetate tantum pronectiori credit Geoffroy. Habui equidem fpecimina, elytris fitura dorfali confertius nigromacriatis; at mihi difincta fpecies.

Hob. in hortis rarior. Long. 3. lat. $2 \%$.

Geoffroyn Fabricius mecum confentit. Habitat Brun.

fuigae frequens in Inula dy fenterica. Hg.

182. C: nobilis grifea, elytris linea caerulea nitidifinna. F. Sp. I IO. I7.

O Ray., Suhaeff.

F. E.S. I. I. $297 \cdot 24$.

Hab. plantas verticiliatas, et carduos frequens. Long. I $I_{4}^{3}$ lat. I

Citatum Schaeff. Ic. t. 96. f. 6. fane errore quodam omiffum, cum bonum. I'g.

$$
\text { (p. } 73 .)
$$

183. C. nebuífa pallido-nebulofa, fufco-punEtata. F. Sp. $10 \% \cdot 5 \cdot$
() Ray., Frifch., Schaeff.
D Schrank. E. I. $9 x$, Schueff. Ic. t. 40. f. 5. 6.
? F. E. S. T. I. 293. ?.

Hab. in hortis frequens. Long. 2, 3. lat. $I_{2}^{\frac{x}{2}}$.

I84. C. ferruginea nigra, thorace elytrisque ferrugineis immaculatis. F. Sp. Io8. I.

() Schrank.

Schaef. Ic. t. I 4 2.f. 4. a. b. F. E.S. I. I. 294 IO. Cl. Fabricius varietatem efie praecedentis credit, cui libenter affentior. Habit. in hortis paffm. Long. 2. Iat. $I_{2}^{1}$.

Equidem hanc a C. murraen, quam Fabricius huic praecedentem habet in fcriptis fuis, manifefte differre contendo, et colore conftanter eodem, et fculptura elytrorum, et habitatione. Sic etiam a C. nebulofa differt. $\mathrm{Hg}$. 
I S5. C. limbata capite, thoraceque pubefcentibus obfure aeneis margine pubefcente. F. Mant. 63.10.

Q F.E.S.I. r. $294 . \mathrm{rr}$.

Hab. Pifis non rara. Long. $2 \%$ lat. 2.

Diagnofis in Mantifia crrore typographico value corrupta cf, et ideo illa Entomologiae iyftemat. etc. confulenda eft. $\mathrm{Hg}$.

\section{(p.7+.) \\ XXIX. CHRYSOMELA.}

palpi fex extrotiun crafiores. Libium cornemm, integrum. Antennae moniliformes.

I86. C. tencbricofa aptera, ouata', atra, antennis pedibusque violaceis. F. Sp. II 6 . I.

D Schaeff. Ic. t. $x 26$. f. x. Elem. t. x. f. 6.

o F. E. S. I. I. $308 \cdot 3 \cdot$

Acu transfixa hunorem effundit fanguineum, qui ad tincturam videtnr idoneus, et fi viuens carboni accenfo admoucatur, colorem induit magis purpureum. Habit. filuas, in locis apricis frequens. Long. $5,6,7$. lat. $3,3 \frac{5}{2}, 4$.

I87. C. hamoptera onata, violacea, plantis, alisque rubris. F. Sp. IIS. I8.

$\odot$ Pod.

D Schaeff. Ic. t. 65. f. 8 .

o. F. E. S. I. I. $315 \cdot 37$.

Elytra punctata. Antennac nigrae capite thoraceque longiores. Alae fanguineae. Corpus fub elytris flauo-1ufum. Hab. in hyperico perforato frequens. Long. $3^{\frac{\pi}{3}}$. lat. 3 .

Veram $C$, haemopteram $F$. nondum noffe videmur, punc hottentottam, nunc Hyperici Degeer, $5.3 \mathrm{r}=$. 
20. (C. varians. F. E. S. I. I. 3 T5. 3\%) nunc violaceam Schaller. Act. Hallenf. I. p. $27^{\circ}$. coniicimus illam. De hac nonifuma Cel. Fabricius ipfe in litteris certicrem me facit, eandem effe cum C. haemoptera fur; fed nec plantae, nec antennarum articulus primus ruti, et deinde in Entom. emend. 1. 3I5. 39. C. violacecm iftam habet. AuEtor, qui Degeer. citatum adhuc exhibuit, $C$. vavicntem $\mathrm{F}$. habuife videtur, quae et Brunfuigae habitat, in hyperico perforato. $\mathrm{Hg}$.

\section{(p.45.)}

I88. C. Bankii ouata, fupra aenea, fubtus teltacea. F. Sp. I I ? ?

온 F. E. S. I. I. 310.16.

Defcripfit F. Syit. Ent. 95. 4. Thorax margine laterali craffufculo. Hab. ruri, frequens etiam in hortis. Lollg. 4. lat. $2 \frac{3}{4}$.

I 89. C. ruftica oblonga, nigra, thorace elytrisque grifeis. F. Mant. 74. 95.

1 Galeruca ruftica. F. E. S. I. 2. I5. I I.

Geoffr. I. 252. I. Schaeff. Ic. t. 65. f. Io.

Hab. ruri frequens. Long. $3 \frac{2}{3}$. lat. $2 \frac{x}{2}$.

I 9o. C. Tanaceti oblonga, nigra, punctata, elytris coriaceis. F. Sp. I 28. 20.

D Scop. E. C. 256 . Tenebrio triftis.

Schrank. E. I. 1 39. Roef. Inf. t. 2. Scar. t. 5.

q F. E. S.I.2. I5. I0. Galeruca Tanaceti.

Hab. in Tanaceto. Long. $3 \frac{\mathrm{T}}{2}$. lat.' $2 \frac{1}{2}$.

Auktor haud bene Geoffr. I. 252. 1. citat, vbi G. ru-

fticam defcriptam inuenimus. $\mathrm{Hg}$.

Igr. C. populi ouata, thorace caerulefcente, elytris rubris apice nigris. F. Sp. IIg. 20.

- Merian., Lifter.

D Schrank. E. I. I23.

I F. E.S.I. I. 3 I6. $44^{\circ}$

Saepe 
Sacpe variat elytris apice concoloribus, et magnitudine minori. Forte C. tremuli. Mant. 60. 28. Hab. in Populo tremula, Salice frequens, copiofa. Long. .5. lat. 3 .

192. C. ftajsilaed ouata, obfura teftacea. F.Sp. II9. 2 I.

$\odot$ Geoffr.

D Linn. Act. Vpfal. I 736 . I 7 . 3 .

Schrank. E. I. I29. Miull. Z. D. Pr. 88 r.

Schaeff. Ic. t. 2 I. f. I 2.

\& F. I.S. I. I. $317: 4 \%^{\circ}$

Synonymon Geofir. I. 263. I5. a Degeer. et Fabr. citatim aliam Chryfomelam indicare videtur. :

C. polita maior, et a Iimmeo in Fn. Suec. 5 I8: optime defcripta. Inter mea fpecimina vnum habeo ftatura praecedenti fimillimum, quod tamen differt magnitudine multo minori, et colore nempe teftaceo, fed magis nitido, magisque flauefcente, fcrte referendum ad $C$. patlidam. Linn. Syft. Nat. 589. 25. Hab. in filueftribus rara. Long. 4. lat. 2 ?

Illi, quod auctor de citato Geoffroyano dicit, lubens affentio, cum nec capitis, nec corporis inferioris, nec elytrorm thoracisque ipforum defcriptio con. gruat. Hg.

$$
\text { (p. } 78 .)
$$

193. C. polita ouata, thorace amato, elytris teftaceis. F. Sp. I I 9. 23.

$\odot$ Scop.

\& F. E. S. I. I. 3 I 7: 49.

$\mathrm{Hab}$. in lalice frequens. Long. 3. lat. 2.

Citatum Schaeff. Ic. t. 65. f. 9. D. Roffi dubium habet; et quiden iure, quia ad C. cupream $F$. pertinet. $\mathrm{Hg}$.

I04. C. fanguinolenta ouata, atra, elytris mar. gine exteriore fanguineis. F. Sp. 125.53. 


\section{$\odot$ Sulzer. \\ I) Schrank. E. I. 133 . \\ ? F.E. S. I. I. $325 \cdot 90$}

Nigra, nitida, inferins caenlea. Thorax majginatus. Elytra margine exteriore fanguinco. Habit. falices. Long. $2 \frac{2}{2}$. lat. $2 \frac{1}{2}$.

Iam Laicharting Inf. Tyrol. p. I59. demonftrauit, $B_{l l}$ preft. Sanguinolcntan Scop., ab anetore noltro cum Fabricio huc irelatain, ad Cryptoiep?. linbotum fum p. I8 1 ., noftra experientia meram varietatem Crypt. bipunctati Fabr., pertinere. Hg.

-i 95. C. griminis ouata, viridi-caenulea, nitida, anternis peäibusque concoloribus. F. Sp. I I S. I6,

1. D Schrank. E. I. I32:

․ F. E. S. I. I. 3 I $4 \cdot 33$. Schaeft. Ic. t. 65. f. ? ? Antennae atticulis quatuor vltinis fufcis. Elytra admodum conuexa. Pedes virides. Hab. in gramine. Long. 4. lat. 3 .

\section{(p.78.)}

I96. C. aenea oblonga, viridis, nitida, elytris aeneis. F. Sp. 130.80.

D Geoffr. Inf. I. 26 I. I. Schrank. E. I. I3I, Scliaeft. Ic. t. 2 I. f. $3-6$.

o. F. E. S. I. I. 329. 109.

Hab. in alno. Long. 2. lat. $I \frac{\pi}{2}$.

Citatum e Geoffroyo, a noftro ançtore exhibitum, nullo i. modo tuc referri poteft, fed ad Chr, faftuofam, pertinet. Auczor ipfe fic etiam in Mantifia fua 28. 68. adn. emendauit. Hg.

197. (*) C.curat́a ouata, viridi-fericea, elytris lineis duabus aureis. F. Sp. 124. 49.

○ Hollar.

D Schaeff. Ic, t. 2 I. f. 8 .

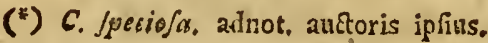


오 C. Speciofa. F. E. S. I, I. 324.83.

Hab. in filuis rara. Long. $4 \frac{1}{2}$ lat. 3 .

Nefcio, quomodo et cur anctor C. fpeciofae, cuius et characterem et citata Fabriciona addidit, nomen dedit $C$. auratae, quae tamen alia eft, ac nullo modo cum hac confundi poteft. ,Cactérum citatum Linnaeanum neutiquam adhiberi poteft, cum ipfe dits cat, colpus dimidio minus effe $C$. Jegetali, quo nomine haud dubio C. cercalem indicare voluit.

\section{$\mathrm{Hg}$.}

198. C. Boleti ounata. nigra, elytris fafciis tribus flauis repandis. F. Sp. I20. 25 .

D Schaeft. Elem. Vdinamn.

ㄱ. F. E.S.1. 2. 5 16. I. Dirperis Boleti.

Hab. in boletis arborum frequens. Long. $3 \frac{t}{2}$. lat. $2 \frac{r}{2}$.

Optime Fabricius nounm ex hac et D. violacea, condidit genus, Geoffroyum fecutus. Hg.

$$
\text { (p. } 29 .)
$$

199. C. Alni oblonga, violacea, elytris vage punctatis, pedibus, antennisque nigris. F.Sp. I28. 72.

$\odot$ Frifch., Sulzer.

D Müller. Z. D. Pr. 9ז I. Schaefi. Ic. t. 65. f. 6.

9 Galevuca Ami. F. E. S. I. I. 2. I6. I3. Tota laete violacea, nitida; folae antemae et pedes nigra. Elytra puncis minutifimis, Thorax anguftatus. Habit. Alnum frequens. Long. $3 \frac{\text { to }}{4}$ lat. $1 \frac{1}{2}$.

Citatum e Sulzero D. Rofi bene emendauit. $\mathrm{Hg}$.

200. C. vitellinae oblonga, viridi-aenea. F. Sp. 130.78.

$\odot$ Scop. Carii. 224.

\& Galeruca vitellinas. F. E. S. I. 2. 18.22.

$$
\mathrm{F} 2 \text { Corpus }
$$


Corpus onato-oblongum. Elytra ftriato-punctata. Hab. in falice frequens. Long. 2!. lat I I $_{4}$

Citatum e Scopolio cuin anctore omittendum eft, cum elytra tantum punctata dicat, nec punctato - ftriata. Fabricius in nouo fuo fyftemate hanc cum vulgatifima fua eandem putat, cui plures affentiunt. Omnia enim eadem, colore caeruleo excepto, in G. vulgatifima funt, etiam locus. Hg.

\section{(p.so.)}

201. C. Betulue oblongo - violacea, elytris punEato-1triatis. F. Sp. 129. 76.

$\odot$ Roef.

․ F. E. S. I. 2. I\%. I9. Galeruca Betulae.

Corpus fubglobofum. Elytra punctis excanatis fparfis. Hab. in Alno, et Salice frequens. Long. 2. lat. $\mathrm{I} \frac{\mathrm{x}}{2}$.

Negligentia hatd leuis fane in hac Chryfomela patet, cum anctor ipfe contra diagnofin, elytra dicat punetata punctis vagis. Qua de caufia veram C. Betulue habere non potuit. Sed illis, qui habent, citata Scop. E. C. 221 . et Degeer. 5. 318.24. emendanda funt, eandern ob rem. Caeterum Roefel. 1. c. C. vitellinae repraefentauit, et hoc citatum bene omifit Roffus. $\mathrm{Hg}$.

202. C. coccinea oblonga, thorace marginato - fanguineo, macula nigra; elytris fanguineis maculis duabus nigris. F.Sp. I3 I. 83 .

o Galeruca coccines. F. E. S. I. 2. 20. 3 I.

$\mathrm{Hab}$. in Vlmo rarior. Inuentae plures fub cortice

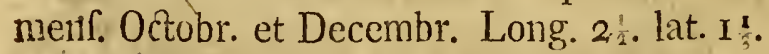

Haec et affines: $G$. cruciata, et $G$. Bouiftae, iam diu Entomologos dubitantes reddidere, cui annumerarentur generi. Fabricius Galerucarum, alii Tenebrionum genus aptifimum putant, multis autem 
nouum confituunt genus, cui primus nomen Endomychus indere audeo, quod verbum, vt fieri folet, a Graeca lingua ortuin ducit, $a b$ sidov (intus)

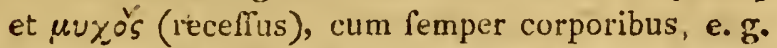
Lycop. Bouifta inclufi fint, et lucem fugere videantur. $\mathrm{Hg}$.

$$
\text { (p.8r.) }
$$

203. C. obfrura ouata, nigra, thorace rotundato angutiori, pedibus ponticis elongatis. F. Sp. 127. 63.

D Goetze E. B. I. 289.96. Schaeff. Ic. t. 102. f. 5 . $\$$ Cryptoceph. obfcurus. F.E.S.I.2.60.35. Panz. F. I. G. V. 12.

Thorax valde gibbus, elytris anguftior, rotundatus. Abdomen inforne globofum, elytris fubquadratis corpore paullo breuioribus. Pedes poftici et fecundi paris longi tibiis arcuatis. Hab. in filuis rava. Long. $2 \frac{1}{2}$. lat. $x_{5}^{\prime}$.

Tránfitum quidem a chryfomelis ad cryptocephalos facic, fed vera chryomela eft, et ftaturam et antennas fi refpiciamus. $\mathrm{Hg}$.

204. C. marginata onața, nigro-anea, elytris margine luteis. F. Sp. $125 \cdot 54$.

○ Sulz. Hift.

D) Schrank. E. I. I 70.

\& F. E. S. I. 1.325 . gr.

Elytra ftriato - punctata. Thorax totus nigro - aeneus, nec luteo - marginatus. Hab. in pratis apricis. Long. 2. lat. 1.

Citatum Sulz. Hift. t. 3. f. Io. bene omifit auctor, cum ad C. fanguinolentan pertineat. $\mathrm{Hg}$.

205. C. 20 -punctata oblonga, viridi-aenea, thoracis marginibus albis, elytris albis, maculis decom acneis. F. Sp. r3 1 . 86.

Petiv: 


\section{- Petiv.}

D Scop. E. C. 242. Schrank. E. I. I37. Schaeff. Ic. t. 3 . f. 4 .

9 Panz, F. I, G. VI. Iо.

Galeruca $20-$ punatate. F. E. S. I. 2.2 I. 36.

Schrankius 1. c. optine defcripfit. Hab. Florentiae in agro Fefulano; Pilis marior. Long. 3\%. lat. 2. Mihi Chryfomela, nec Galeruca, eft. Hg.

\section{(1). 82.)}

206. C. cerealis onata, aurata, thorace lineis tribus, colcoptrisque quinque caeruleis. F. Sp. 124. 45 .

() Goetze E. B. I. 258 . I7.

ㅇ F. E. S. I. I. 323. 76 .

Variat coloro purpureo loco årei. Hab. in fegetibus, et fpartio fcopario rara. Long. $3 \frac{1}{2}$. lat. $2 \frac{\pi}{2}$.

207. C. Americanc onata, viridi-aenen, elytris ftriis quinque fanguineis. F. Sp. I24. 46.

오 F. E. S. I. I. 323 . 78 .

Ouata. Antennae bafi ferrugineae, breuiores, quam in $C$. cereali, in qua totae nigrae. Abdomen viridiaeneum. Elytra in $C$. cereali punctis inordinatis excauta, in hac ftriis punctorum duplici ferie ordinatis. Caput aenem macula retricis coccinea. Habit. in agro Florentino, loco vulgo dicto le Cam. pora. Inuentae plures fimul congregatae in Roremarino menf. Octobr. Long. $3 \frac{\%}{\%}$. lat. $2 \frac{1}{2}$.

$$
\text { (p.83.) }
$$

* 208. C. dytifcoilics oulata, violaceo-acnea, nitida, thorace lacto, elytris punctatis.

C. Alni maior. Facies Dytifci fcarabacoïdis. Ouata, comexa, fupra violaceo-aenca, fubtus nigra. Antenae brenes, nigrae. Elytra ftriato - puncta- 
ta. Pedes nigri plantis fufcis. Alae albae. Tab. II. f. 6. Tab.IV. f. 33. Hab. in filuis, non rara Pifis in Vlmo. Long. 4. lat. 2.

209. C. polygoni outat, cacrulea, thorace, femoribus, anoque rufis. F. Sp. 123. 43.

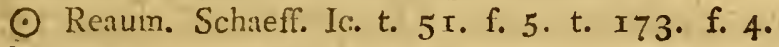

D) Bupreftis falicing. Scop. E. C. 199.

Schrank. E. 1. 142 .

․․ F. E.S. I. I. 322. 73 .

Hab. in Polygono. Long. $I \frac{2}{3}$. lat. $\frac{2}{3}$.

Figurae tabularum Schaeff. Ic. t. 5 r. f. 5. t. I73. f. 4. bene $a b$ auctore omiflae funt, cum minime huc referri debeant. Citatum e Geofï̀oy porro, reftitum eft verum 263 . $x 6$. pro falfo 283 . 4. Fabricii, et 242. 4. Limnei. De citato Scopoliano adluc haereo, cum Cryptocephalos F. hoc genere habeat Scopolius. Hg.

210. C. colleris onata, violacea, thioracis marginibus albis, puncto nigio. F. Sp. I2I. 29.

D) Geofir. Inf: 1. 15\%. 5. Meloluntha. ete.

P. F. E.S. I. I. 3 I9. 56 . Schael, Ic. t. I 73. f. 4 . a. b?

Hab. in falice. Long. 2. lat. $1 \frac{1}{2}$.

Geoffroyi Melolontha ab auctore citata, nullo modo huc referri poteft, cum ne generis, nedum fpeciei characteres coniceniant. Quomodo Geofiroyus, tim oculatus, Chryfumelam veram cum Clytra (vt nomine, a Laichartingio bene Vielolontliarum Geofir. generi artributo, vtaii) vel Cryptocephalo confundere poffet, qui ipfe primus fere difinxit? Elt autem Cryptocephalus bucephalus F. vel potius Clytra bucephalus. - Schaefieri fig. xx. 12. tab. 52. Ic. Inf, vulgo citatae, minime hanc, fed Cryptocephalum auritum livel melius Clytram auritam re$\mathrm{F} 4$ prae- 
praefentant. In figura autem a me citata, Chryfomelam agnofco, cui autem marginis thoracis punctum nigrum deef, quod etiam in quibusdam meorum fpẻciminum obferuaui. $\mathrm{Hg}$.

\section{(F. 84.)}

* 2 r 1. C. Cerafi outata, liuida, antenuarum apicibus, oculisque nigris Lim. Sylt. Nat. 588. 13.

C. Padi. Degecr. Inf. 5. 30I. I r. Hab. etiam in falice. Long. $I \frac{1}{2}$. lat. $I \frac{t}{4}$.

* 212. C. metallica fubrotunda, cuprea, lacuis, foueola frontis impreffa.

Non confundenda cum $C$. metallica Laichart. Verz. I. 44. 3. quac conuenit cum $C$. cuprea $F$.

Ouato - globofa, fupra tota cuprea, nitens, fubtus fufca. Antennae thorace longiores, ad bain rufae. Caput intra thoracem ferere conditum. Thorax antice anguftatus, poftice transuerfus. Elytra punctis vix oculo armato confpicuis excauata. Pedes omnes ferrugineo-íufici femoribus pofticis fubclauatis. Tab. III. f. I I. Hib. in filuis non rara. Long. $1 \frac{1}{5}$. lat. $\frac{2}{3}$.

Inter minores itaque referenda, nec cum C. metallicas Laich. et Fabr. confundi poteft, quas easdem coniicerem, nif in Fabriciana margo thoracis lateralis haud incrafiatus effet. Caetera, etiam magnitudo, et ftatura conueniunt. Perperam antem $C$. cupream $F$. eandem cum C. metalicc Laich. putat auctor. Chryf. auct. a Fabr. apud C. aerugineam E. S. 330. II6. citatur. Hg.

Alticae faitatoriae, fenoribus pofticis increffatis.

213. C. Hyofcyami faltatoria, viridis, nitida, pedibus teftaceis, femoribus polticis violaceis. F. Sp. I33. 97 . 
$\odot$ Ray.

9. Galonuca Hyofiyami. F. E. S. I. =. 29. I9.

Ouata, virefcens, elytris ftriis excauato - punctatis. Femora crafla nigra. Tibiae ferrugineae, femoribus pofticis viridi-aeneis. Habit. in Hyofcyamo et in Braffica frequens. Long. I. lat. $\frac{x}{2}$.

I'Altife dit choux. Geofir. 248. II. ab anetore cum Fabricio aliisque citata, probe feparanda eft, quia valde differt a vera $A$. Hyofcyami, faltem ab illa, quam ob defcriptionem optime conuenientem, et ob locum, vbi cepi, $A$. Hyofcycimii credo. Differt autem ftatura oblohga, nec poftice attenuata. vel ouata, vt in $C$. $H_{y}$ ofoys $: m i$, dein colore viuacifimo viridi, interdum caeruleo, ftriarum punctis profundioribus, antennis totis luteo-rufis, femoribus pofticis haud craffiffmis, et metallicis, fed atris, interdum apicibus rufefcentibus. Hg.

$$
\text { (p. 8.5.) }
$$

214. C. olercicen faltatoria, viridi caenulea. F. Sp. I 33. 94 .

$\odot$ Scop.

2 Galerica oleracca. F. E. S. I. 2. 28. 74.

$\mathrm{Hab}$. in olcribus paffin. Long. $1 \frac{1}{3}$ lat. $\frac{1}{2}$. ct vitra.

G. Erucae. F. E. S. 1. c. 23. 75 . nil, nifi mera varietas, colore tantum caeruleo differens. Hg.

215. C. erythrocephala fattatoria, atro-cacrulea, capite geniculisque pedum mis. F.Sp. 134. 103. 9. F. E. S. I. 2. 31. 87. Galeruca evythrocephala. Hab. in plantis. Long. I. lat. $\frac{x}{2}$.

216. C. Helxines faltatoria, viridi-aenea, antennis fufcis, pecibus teftaceis. F. Sp. I3a. 100.

$\odot$ Sulz.

\& F.E.S. I. 2. 30. 82. Golcrucc Helxines. Hab. in plantis variis frequens. Long. $I_{\ddagger}$. lat. $\frac{x}{2}$. 
(1).85.) race, pedibus antemnarumque bafi rufis. F.Sp. I35. Ios.

I G. rufipes. F. E. S.I. 2.32. 94.

Elytra frriata, ftriis decem punctatis. Cum mea fpecimina elytra habeant friata, pertinere videntur ad C. caeruleo-ftrintum. Degeer. 5. 34.3. 48.

Hab. in plantis non rara. Long. I $\frac{1}{3}$. latt $: \frac{3}{4}$.

Schaeff. Ic. t. I66. f. 5. ad C. nitidulam pertinet, vbi etiam auctor labet. Cur autem h. l. iternm exhibuit? Caeterum minime veram C. rufipedem habuifle videtur. cum elvtra in füis fint ftriata, quae punctata, nitida in noftris funt. Forte habet Geoffioyi A!ticam tertiam, qui illam etiam cum Altica fua fecunda, vel $A$. ruffipcre confundit. $\mathrm{Hg}$.

218. C. fufcipes faltatoria, violacea, capite, thoraceque rufis, pedibus nigris. F. Sp. I35.I09.

9. Galenta fufcipes. F. E. S. I. 2: 32.95 .

Forte varictas C. ruffipedis, ait Fabricius, fed elytra apice magis rotunda, quam in praecedenti.

Hab. in hortis rarior. Long. I . lat. $\frac{3}{4}$.

219. C. nitidula faltatoria, viridis, capite, thoraceque aureis, pedibus ferrugineis. F. Sp. I34. 99.

() Echaeff. Ic. t. 8 - . f. 5 .

D Schrank. E. I. I6́3. Schaeff. Ic. t. ז6ú. f. 5. a. b.

of Gal. nitidula. F. E. S. I. 2. 30. 8 I.

Antennae bafi ferrugineac. Variat interdum colore. Hab. ficquens in Malua arborca. Long. $I_{2}^{\frac{Z}{2}}$. lat. $\frac{2}{3}$.

$$
\text { (p.85.) }
$$

220. 'C. cxoleta faltatoria, ferruginea, elytris Itriatis. F. Sp. 136. IIt. 
○ Linn. Syft., Nat., Geofir.

o. Gah cxoleta. F. E.S.I. 2. 33. roo. Herbft. Aich. t. 44. f. 7.1 . m.

Tota teftacea, oculis nigris. Elytra, amatis oculis, furiato - punctata. Femora poltica crafiora. Forte Alt. flunu. Geofli. Inf. I. 250. I8? quae, ni fallor, rariat in Alt. elytris ftriatis, et in Alt. absque pedibus faltatoriis, varietates omnes, cuas habes. Hab. in plantis non frequens. Long. $\frac{1}{3}$. lat. I.

Citatum Fabricii Geoffir. I. 250 . I6. optimum eft. Nam n. I8. alia Chryfomela, vel melius Altica, et quidem Altica tabidia $F$. eft. Degeeriana et Linnaeana Chryfomela etiam aliae videntur. $\mathrm{Hg}$.

2zI. C. atra faltatoria, atra, nitida, antennarum bafi, plantisque piceis. F. Sp. I.3\% I I 5 .

Q Gal. atra. F. E.S. I. 2. 34.105. Hab. in fruticibus. Long. $\frac{2}{3}$. lat. $\frac{1}{3}$.

\section{(p.s8.)}

26.2. C. teftacen faltatoria, teftacea, gibba, elytris licuifmis. F. Sp.' 136. I 10.

운 Gal. teftacer. F. E.S. I. 2. 33. 99: Hab. in hortis liequens. Long. $\frac{1}{3}$. lat. I.

223. C. hemifphacrica faltatoria fuborbiculata, deprella, nigra. F. Sp. I3\%. II

운 Gal. hemifpinatrica. F. E. S. I. 2. 34. 107. Elytra lacuia. Pedes fubrufi. Hab. Muri frequens. Loing, $\frac{2}{3}$. lat, $\frac{2}{2}$.

II 3 .

224. C.tabida pallida, oculis nigris. F. Sp, 136 .

of Gal. tabida. F. E. S. I. 2. 33. 102.

LiAttife jaune. Geofir. Inf. x. 250 . I 8 .

Statura C. exoletae, at minor, et pallidior, imma. culata, 
culata, oculis folis nigris prominulis. Antennae longiufculae apice nigrae. Elytra laeuia. Hab. frequens in floribus Verbafci Thapfi. Long. $1 \frac{1}{4}$. lat. $\frac{x}{2}$.

Veram Alt. tabidam, quam Fabricius ipfe ita mihi determinauit, femper in floribus Scnecionis Iacobaene reperi. Srd defcriptio h. l. data bene congruit, itaque auktor, aut in Verbafco cepit, aut veram habitationem oblitus eft. In Verbafco autem aliam cepi, cui color qqui flauicans in tabida, ) fupra fuluo-cinereus eft, fed abdomen, femoraque poftica brunnea funt, quam rabricius veram fuam G. Sifymbrii efie, litteris certiorem me fecit, fed diagnofis ac defcriptio hand conuenire videntur.

$\mathrm{Hg}$.

* 225. C. lineata faltatoria, oblonga, zibba, teftaceo - flaua, elytris linea duplici nigra interrnpta. Antennae nigrae, capite, thoraceque longiores, bafi mafae. Thorax foucola vtrincue excauatus. Flytra ftriata linea duplici nigra longitudinali interrupta. Forte C. litura F. Sp. $\mathbf{0} \approx .57$ ?

Hab. in filuis. Long. $I \frac{1}{2}$. lat. I.

C. Litura F. mera Chryfomela, nec Alticae fpeciom prae fe fert. $\mathrm{Hg}$.

$$
\text { (p.89.) }
$$

* 226. C. Lurida fufco - rufa, abdomine, oculisque nigris. Scop. E. C. 2 I 9.

Magnitudo Ch. nemorum, habitus $C$. atricillae, fed caput et pedes ommes, vina cum thorace, ely- trisque concolores. Elytrum punctatim, fubpelJucens. Hab. in Saluia Sclarea et Verbafco. Long. $\frac{3}{4}$. lat. $\frac{1}{2}$. 
227. C. truncata nigra, elytris truncatis, apice fermgineis; pedibus antemisque rufis. Scop. E. C. 218 .

Ouata, elytris nec punctatis, nec ftriatis. Hab. fub arborum cortice. Long. $\frac{3}{4}$. lit. $\frac{1}{3}$.

\section{CRYPTOCEPHALVS.}

Palpi filiformes. IItaxilla vnidentata. Labinm corncum, integrini. Antennce filiformes.

228. C. longives niger, obfurus, elytris pallidis, maculis tribus nigris, pedibus anticis clongatis. F. Sip. I37. 1.

I) Scop. E. C. 285 . Bupreft. б-punfata. Laichart. Verz. I. 166. I. Chytra longipes.

f. F. L. S. I. 2. 52, I.

Antennae brenes, ferntac, articulis 8 -dentatis, vltimo quali rotundo. Thorax violaceo - niger fubpubefcens. Elytra pallica punetis 3, nigris, vnico ad bafin, et binis in medio. Pedes thoraci atque abdomini concolores, antici femper elongati. Thorax marginatus, fcutello thoraci annexo. Hab. in hortis frequens. Long. $4 \frac{2}{3}$. lat. 2.

\section{(p. 20.)}

229. C. 7-punctatus niger, elytris rubris punctis duobus nigris, antennis breuibus ferratis. $F$. Sp. I 33. 3 .

○ Schaeff. Elem. t. 83, f. I.

D Schrank. F. I. 164.

\& F. E. S. I. $2.54 \cdot 6$.

In fingulo elytro puncta duo nigra, quorum prius magis rotundum ad bafin, polterius, maius, transuerfum. Hab. in plantis ruri. Long. $4 \frac{T}{2}$. lat. 2. 
230. C. 2-punctutus niger, nitidus, elytris rubris punctis duobus nigris, antemuis longitudine compris. F.Sp. IfI. I 9 .

○ Vdm., Fetiv.

D) Schaeff. Ic. t. $7 \%$. f. 8 .

\% F. E. S. I. 2. 59. 29. et

C. lincola. 59.-30. nil nifi varietas.

Variat rarius elytris mácula vnica oblonga nigra elytris partlo breuioni, margine omni elytrorum nigro. Hab. 1it quercu. Long. $2 \%$ lat. $\mathrm{I}_{3}^{\mathrm{T}}$.

- Fabr. errore typographico exhibet Schaef. Ic. t. $s$.

f. 8. prot. 86. f. 8. Auctor nofter citat t. $7 \%$

f. 8. quáe varietas ab Harrero, vocata eft Co obiong go-guttatus, a Fabricio C. lineola, a Laichartingio C. limbatus, nil tamen eft, nifi mera varietas ${ }^{\circ}$. $2-p$ unitati. Rarius elytrorum color totus ferme niger euatlit, marginibus tantum obfolete ferrugineis. Hg:

\section{(p. pr.)}

23I. C. bimcuculatus niger, obfcurts, thorace fuluo, elytris teftaceis, punctis duobus nigris. $F$. Sp. 14 I.20. Mant. 1. 80. 24.

D Schaeff, Ic. t. 264 , f. 3. a. b.

O F. E. S. I. 2. $59 \cdot 3$ I.

Caput nigrum. Os flaum. Antennae fubpectiratae, nigrae, bafi flatae. Elytra linido - fluma punctis duobus obfoletis nigris. Abdonten obfcure acneum. Pedes fraui, cxteritis nigri, antici longiffmi fenoribus nontihil incraflatis. Alae fufcefcentes. Variat elytris vnipunctatis et immaculatis. Hab. Florentiae. Long. $3 \frac{1}{3}$. lat. $I_{\frac{1}{4}}$.

232. C. Scopolinus niger, thorace rufo, elytris rufis, fafciis duabus cyaneis. F. Sp. I 45. 44.

D $B v: p n$. 
D Bupi. 1-fufrint. Scop. E. C. 205.

\& F. E. S. I. 2. 68.29.

Antemae fertatae articulis primis duobus fubrufis. Color fafcianum caerulens, quae nec marginem, nec futuram attingunt. Femorun apices et tibiae filuac. Tarfi nigri, vel potins atro-cactu1ei. Hub. Salices et flores Danci Carotae frequens. Long. $2 \frac{1}{5}$. lat. $1 \frac{1}{3}$.

$$
\text { (p. ap.) }
$$

233. C. cordiger thorace variegato, elytris rubris, punetis duobus nigris. F. Sp. I4I. 21 .

$\odot$ Vdm.

Q F. E.S. I. 2. 59.32

Geoffr. Inf. I. 235.7 .

Hab. in falice rafus. Long. $2 \frac{2}{3}$. lat. $1 \frac{2}{3}$.

Fabricius illum, quem Farrerns in edit. II. defcriptionis Iconum Schaeferi C. ofropipunetiatum vocat, dum citat Schaeff. t.. 30. f. I. quam figuram omnes ferme anctores apud hunc allegant, cum hoc con; fundit. In mufeis antem-rub nomine C. variegatus Hiibneri affer natur. Differc vero et thoracis, et elytrorum notis, ab Harrero exhibitis, et fatura angultiore. $\mathrm{Hg}$.

234. C. tridentatus caerulefcens, elytris teftaceis. F. Sp. 139. 8.

D Schrank. E. I. r 44. Petagna Sp. Cal. f. VIII. Bupr. caevulefiens. Scop. E. C. 200 . Schaeff. Ir, t. 36. f. I3.

ㅇ F. E. S. T. 2. $55 \cdot$ I 4 .

Variat colore aeneo - viridi. Pedes antici in altero fexu elongati. Hab. in filuis. Lectus in rubro frequens. Long. $3 \frac{1}{4} \cdot$ lat. $I \frac{2}{2}$.

In omnibus fpeciminibus meis punetum bafeos elytrorum cyaneum, cuius mentionem Fabricius facit, 
confpicere nequeo. Dom. Schneiderus autem alium, huic fimillinum, mifit fub nomine $C$. humeralis, cui hoc punctum ineft. Diferepat hic autem nulio modo colore, qui viridi - aeneus eft, elytris totis pallidis, puncto tantim humerali illo cyaneo excepto; fed thorace antice minus angufato, et eiusdem ferme latitudinis, atque pro longicuaine elytrorum longiore, quam in $C$. 3 -dentrio, dcinde capite mulco latione, mandibulisque duplo ferme maioribus; fpecies itaque flint' piane difinctae. Hg.

23. C. Nortaei ater, elytris maculis duabus flanis marginalibtis. F. Sp. 14.4. 37.

○ Vdm., Schaeff. Ic. t. 30. f. 5 .

D) Schaeff. Ic. t. 238 . f. 2 .

8. Roffi Mant. p. 33 . n. S r. F. E. S. I. 2. $66.67 \%$ Variat elytris apice, et macula marginali rubris flauisue, maculae in mafculis flanac, in feminis ntbrae. Thoracis margo anticus in femina plenin-, que immaculatus. In vtroque thoracis anguli poftici albidi. Hab. in pratis apricis non frequens. Long. $I_{2}^{\frac{2}{2}}$, 2. lat. ${ }_{4}^{3}$.

\section{(p. 93.)}

236. C. auritus ater, thorace vtrinque macula lutea; tibiis flanis. F. Sp. I39. I 2.

D B. Lunata. Scop. E. C. 204 . Schrank. E. I. 172 . Schaeff. Ic. t. 52 , f. II. I2.

? F. E. S. II. $57 \cdot=0$.

Macula lutea, rotunda, ad thoracis latera. Elytra immaculata. Pedes rufi. Femora antica craffiora. Hab. in falicetis. Long. I $\frac{2}{3}$. lat. $r$.

Nec Scopolium, nec Schrankium quisquis iure citare potéft. Scopolii defcriptio nedurn minimis conuenit, et Schrankii, excepto Linnaeano citato, nullam praebet notam conuenientem. Caeterum menfura, 
ab auctore exhibita, nimis parra eff. Equidem longitudinem lin. 3 , latitudinem lin. $\mathrm{I}_{4}^{3}$ menfus fum. Saepius hunc defcetum obfervavi, et femper ferme $a b$ alio atctore menfuran defumtam fenfi. H. 1 . Schankii menfura exlibita eft. - In noftris regionibus alinm, C. anvito fimilimum inueni, cui nomen $C$. affinis indidi. Differt primum, quod duplo minor fit, deinde colore elytrorum craneo, pedibusque totis, nec tibiis tarfisque folis, flauis. Longit. 2. lat. I. lin. $\mathrm{Hg}$.

237. C. fericeus viridi-cacruleus, antennis nigris. F. Sp. 143. 32.

D Schreff. Ic. t. $8 \%$ f. 5. Schrank. E. I. 174.

Q. F. E. S. I. $2.63 \cdot 56$.

Blipr. Syngenefine. Scop. E. C. I93.

Caput intra thoracen reconditum., Elytra punctata, punctis contiguis fabriuscula, pone dehifcentia. Alac, pedes, antennae nigricantes. Hab. in lalice. Long. 3 . lat. $I \frac{I}{2}$.

Variat ftatura duplo minore, colore cyaneo, cupreo, et obfcure viridi. Foueolas thoracis nunc adefe, - nunc videri non pofle, obferuaui. Cltatum e Scopolio certo huc referendum eft, cum omnes notale $C$. Sericeo $F$. et $L$. congruant. Maximam autem Harrerns in Deicr. 1c. Schaeff. confufionem induxit. Euus enim $C_{r}$. Hypocharidis, nil nifi verus $C$. Sericen $L$. eft; et eiustiein $C$. fericeus, mera varietas $C$. Seviceae $\mathrm{L}$. videtur.' $\mathrm{Hg}$.

\section{(p. 97.)}

239. C. nitens viridis, nitens, ore, pedibusque teftaceis. F. Sp. I44. 33.

S. F. E.S. I. $2.64 \cdot 57 \cdot$

Colore variat nunc viridi, nunc caeruleo. Pedes in nofris fpeciminibus elytris concolores. Os 
tamen fuluum. Forte Chr. Hypochacridis. Linn. fylt. 589.2 I. Bupr. Synegcnefiae. Scop. I. C. 193? Hab. in plantis frequens. Long. $1 \frac{2}{3}$. lat. $x$. Quid huic auctoris conlecturae refpondeam, vide in snnotatis ad praecedentem. Hg.

239. C. marginatus nigro-aeneus, elytris flanis, margine nigro. F. Sp. I40. I ?.

$$
\begin{aligned}
& \text { I Schneff. Ic. t. } 87 \text {. f. } 6 . \\
& \text { O. F. E. S. I. } 2.58 .27
\end{aligned}
$$

Corpus totmu nigro-aeneum. Elytra flana margine omni nigro. Fabricius dubitat, hunc effe varietatem C. longimani; nithi omnino dituncus. Statura et habitus $C$. mitentis. Corpus totum atroaeneum, nitens. Antennae longinsculae. Elytra flaua, laenter puncato - frriata, fitnra et margine nigro. Feminam C. flauilahris. F. Mant. 84. 67. cum hoc femper reperi, et copula iunctam afieruo. Hab, apnd nos frequens. Long. 2. lat. I.

Celeberrimus Fabricius, vt aliis mecum videtur, Cryptocephalos fuos, fi non genere, tamen certe familia, bene feparaffet in illos, quibus antenne funt Serratae (Clytras Laich. Mclolonthas. Geofir.) et in illos antemis non Jerratis (Crypiocephalos. Laich. Geoffr.) Semper cnim dubii de C. marginato reftaremus, cum Fabricius, a C. Ionginano, qui Clytrae generi annumerari debet, haud multum differre contendat, nifi in Ent. fyft. fua C. phaleratan Act. Hall. citafiet, vbi Cryptocephalus nofter bene defcriptus eft. Huins varietatem in copula cum C. marginato a me quoque captam acfideo ipfe, omnibris notis C. flanilabri fimillimam, attamen colore obfeuriore, elytrorum apicis macula obfoleta lutea, praecipue autem elytris perfundius fulcatis diftinctum a vero $C$. flanilabri $\mathrm{F}$. 
cui color laete caeruleus eft, quem autem numquam in codem loco cepi. Verofimile itaque videtur, auctorem illam C. marginati varietatem vel noftra expericntia marem, pro vero $C$. flauilabri habuilie. $\mathrm{Hg}$.

$$
\text { (p. 2.5.) }
$$

240. C. flaulabris obleuro-acneus, nitidus, ore lutcfeente, antemis pedibusque nigris. F. Mant. 84. 67.

D) Chryfornela nigro-caerulea. Goetze E. B. I. 320: 25 .

Schaef. Ic. t. 65 . f. 5 .

i F. E. S. I. 2.65.63.

Caeruleo-riolacens. Os flaucfens. Antennac longiusculae, bafi lutefcente. Elytra frriato-punctata. Hab. cum praccedenti. Long. 2. vix. lat. I. Vid. annot. ad antecedentem. $\mathrm{Hg}$.

24I. C. cyaneus, cyaneus, thorace, pedibusque rufis.. F. Sp. I43. 30 .

D) Schaefl. Ic. t. 5 I. f. 5 .

으. F. S. I. $2.63 \cdot 52$.

Hab. in filuis rarior. Long. I $\frac{1}{2}$. lat. $\frac{3}{4}$.

Haec data menfura, e Geoffroyo defumta, nimis parua eft. Longus enim eft 3 vel $2 \frac{2}{3}$, latus autem $I_{3}^{\frac{1}{*}}$. Caterum Geoffroyi defcriptio congruit. $\mathrm{Hg}$.

242. C. Sexpruftatus niger, thorace variegato, elytris rubris, maculis tribus nigris. F. Sp. 142. 27.

(C) Scop.. Sulzer. Schaeff. Ic. t. 30. f. I-3.

D Schaefi. Ic. t. 3 'o. f. 2. 3 .

Bupr. 5-punctata. Scop. Carn. 207.

Schrank. E. 1. 167.

Geoffr. Inf. $x \cdot 235 \cdot \%$

\& F. E. S. I. 2. 62. 44 . 
Notae fpecificae in hac fpecic admodum inconftantes. Characier fpecificus confitit plerumcue in geniculis pureto albo notatis. Hab. in Alno. Long. $2 \div$. lat. I 2 .

Charaeter ab auctore datus etiam $C$. oflopt:naficto Harreri conuenit. Caeterum citatum e Scopolio Fabricianum n. 208. ad C. longipedem pertinet. AuEtor B. 5 - punditutum Scop. n. 20\% allegat, quam Harrer. defer. Ic. Schreff. 4 to F. I33. n. I 49. fub nomine $C .5$-qunfati habet, delcriptione accuratifima coniuneta. Nil autem, quam mera C. 6 punciati $F$. varietas eft, quae tautum maculis duabus, futurae approximatis, confuentibus a folito differt. Statura, charaeteres caetcri, imo habitatio endem. In fpeciminibus meis nonnullis macula poftica elytrorum nigra in fafciam mutata eft, adnotante etiam Fabricio Mant. 8I. 34. Scopolius fpecinen haud nultum a folito $C .6$ - punสato difcrepans habuifie videtur, cum vnum tantum punctum commune obferuafiet. - Citatum auctoris e Gecffroyo nullo modo tolerari poteft. Eit autem $C$. cordiger. vide fupra n. 233 . Hg.

$$
\text { (p. 96.) }
$$

243. C. flauipes ater, nitidus, capite, pedibusque luteis. F.Sp. 146. 50 .

․․ F. E.S. I. 2. 65. 64. Schaeff. Ic. t. I02. f. 5 .

C. parenthefis. Schrank. in Fuesly N. Ent. Mag. p. I53. I70. antea C. marginata. E. I. I70.

Hab. in falice. Long. 2. lat. I.

Interdum femora poftica nigrefcunt. $\mathrm{Hg}$.

244. C. lobicatus ater, nitidus, ore, pedibus, bafique antennarum litefcentibus. F. Sp. 146. 49. ㅇ F.E. S. I. 2. 65.62 .

Elytra ftriato - punctata. Hab. in hortis. Long. $\frac{2}{3}$. lat. $\frac{x}{3}$. 
* 245. C. 8 -maculatus tefacens, thorace duo. bus, elytris punćlis quatuor nigris.

Statura et habitis C. fericci. Totus tam inferne, quam fupeme teftacens. Caput intra thoracen reconditum. Oculi nigri lunati. Antemnae longae articulis tribus vltimis nigris. Thorax pulnimatus, ad latera puncto vnico minutiffumo nigro. " In fingulo elytro puncta quatzor pama inftar quadati transuerfim pofita. Pedes rufi. Alae fufcae. Hab. in populo rartis. Long. $2 \frac{1}{4}$. lat. $I_{\frac{1}{4}}^{r}$.

* 23,6. C. trimaculatus niger, thorace atro immachlato, clytris rubris, punctis tribus nigris, anteminis longis.

An idem, quem Cl. Petagna placuit referre ad C. 6 -punitatam?

Statua C. bipunardati. Antennae longae, nigrae, bafi mface. Caput et thorax atra nitida, inmaculata. Elytra rubra puncto rnico ad bafin, et drobus in medio nigris. Subtus totus niger. Differt a C. bipmatiato litu punctormm diverfo, et defectu marginis nigri clytronm. Tab. IV. f. I2. Habb. in pratis filuaticis. Long. $2 \frac{1}{2}$. lat. $1 \frac{1}{3}$.

\section{(p. 27.)}

247. C. bipuffulatus ater, elytris macula apicis rifi. F. Sp. I $45 \cdot 4$.

2 Fipr. biguttati. Scop. E. C. 20 r.

C. férrugata. Schrank. E. I. 169 .

C. Podae. Laicls. Verz. I. I7 ? ?.

Schaeff. Ic. t. 30. f. 4. 5. t. 77. f. 7.

o F.E.S. I. $2.67 \cdot 74$.

Mea fpecimina variant interdum thorace in medio flauo - lineato, macula apicis elytrorum flana, 
et caput verfus tricufpiciata. Antennae thorace duplo longiores. An C. flnuo-guttatus Schrankii et Schaefferi?

f. Varietatem etiam poffideo, paullo maiorem, cui color eft atro-violaceus, nitidifimus, os luteum, antennae longae bafi flanefcentes; thorax. ad latera flano-marginatus, vti etian elytra, tamen vsque ad dimidiun, apicibus flano-macilatis. Pedes etiam flaui femoribus nigris; antici toti flani. Cum praecedenti coniunxinus, milhi enim merac varietates.

Hab. in Chryfanthemo coronario. Long. $I_{3}^{\frac{\pi}{3}}$. lat. $\frac{\mathrm{r}}{2}$. Iterum menfura nimis parua. Verus enim $C . \Sigma-p u$ fulatus ftaturam et magnitudinem C. vitis habet, fed illa longitudo, quam illi tribuit anctor, conuenit potius cum $C$. Hiibneri $F$, vel $C$. biguttata Schalleri in Act. Hallenf. p. I. qui autem triplo, ino quadruplo minor eft. Secundum Harierum fig. Schaeff. Ic. 7. tab. 77. nouam confituit fpeciem, cui nomen $C$. Schaefferi dedit, et comparata a efcriptione, valde differt. Idem celebb. vir fufpicatur, t. 3o. f. 5 . fupra dictum C. Hübucri repraefentatum efic, de quo ob male expreffam iconem judicare nequeo. - Varietates, ab auctore enumeratas taceo, cum fpecimina fatis multa mili defint. $\mathrm{Hg}$.

* 24.8. C. fexpufulatus ater nitens, elytris fuicis, maculisque tribus flauis.

An C. 8-gluttatlis. F. Mant. 82. 50?

Statura C. Morclei. Caput ore, punctoque in fronte flauis. Antenuae longac nigrae, bafi flauefcentes. Thorax ater nitidus, laenis, antice et ad latera flauo - marginatus. Elytra punctato - ftriata, atril, 
atra, fingulo macula bafeos arcuata fluma. Maculac duac, fubrotundae flauae in imedio, aliaque fimillima ad apicem. Pedes nigri, tibiis femoribusque anticis flauis. Variat capite thóraceque totis nigris. Tab. II. f. ?. Hab. in Fraxino et Alno ron rarus. Long. $I_{\frac{2}{2}}^{2}$. lat. I.

\section{(p.98.)}

249. C. Fiftrio nigcr, thorace elytrisque ferruginea-variegatis, pedibus fermgineis, geniculis nigris. F. Sp. I $45 \cdot 43$.

온 F.E.S.I. 2. 68. 78.

paruus. Caput nigrum linea ante oculos flaua. Thorax antice tantum et lateribus flavo-marginatus, lineolis tribus abbreviatis femgineis. Elytra punctato-ftriata, bali flato-marginata, puncis fere in fingulo vindecim oblongis flauis adfperfa, quibustam comexis, apiceque flat:o-maculato, macula lineari ramofa. Pedes flaui femoribus naxima parte nigris, pofticisque ad apicem extcritis albopmotatis. Hab. in pratis filuaticis. Long. $\frac{1}{3}$. lat. $\frac{3}{3}$.

250. C. 10-punftatus capite, thoraceque variegatis, colcoptris flauis, punctis decem nigris. F. S. 145.39 .

I) Schiafti. Ic. t. II 4. f. 6. a. b?

P. F. S. I. 2. 66. द T. Herbft. Arch. t. 45. f. 4 . Parnis, fatura pracedentis. Deícriptio labrici Syft. Ent. I I I. 27. affabre facta. Elytron color in lioc magis flauus, fere fuluus, et puncta nigra maiora 2. 2. I. interdum connatil. Hab. in Salice, Alno, Populo non frequens. Long. I I $_{3}$. lat. ${ }^{3}$.

Schaefferi figura, $a b$ auktore citata, aperte ad C. hicroglyphicrnn F. referenda eft. Fabricius in Ent. fyit. 
1. c. male citat C. licroglyphicum Herbot. Arch t. 23. $\mathrm{f}$. 26 . quae figura quidem mala, attamen, defcriptione quoque comparata, C. ro-pnnfiato nullo modo conueniens eft. Caeterum magna confufio inter hos ambos Cryptocepinalos eft. Laichartingii $C$. hieroglyplicus ftatura quidem valde affinis C. Io-punctato, fed tamen differt: punctis, ftriarum elytrorum impreffis, magis promifcuis, et profundioribus, quae autem in $C$. so-punifato ftrias plane difinctas conftituunt. Color et fignatura femper abhorret, nec inter millia vnins alteriusue harum fpecierum fpecimina rnicum vidi, quod transgrefium inter ambos pararet. Id autem certum me fecit, dinerfas.efle fpecies, quod in valde difantibus locis vnam illarum fpeciem folam, nec alteri immiftam, reprehenderim, et quidem copia abundante. C. ro-plunetatus variat maculis maioribus, femper antem rotundis, interdum etian colore toto niggro, nacula excepta frontali emarginata, thoracisgue lineis tribus longitudinalibus luteis, quae varictas fpeciei, nec fexus, (cum nunc mares, nunc feminas eiusdem coloris vidiflem, videtur Laichartingii $C$. frenatus. Verz. I83. IO. In Fabricii fpecieb. pro Lim. 597. 79. lese 599. 93. Eodem loco errore alio calami diagnofis Faunae Suecicae, nec fyftematis ipfius exhibita eft, quod vitium auctor nofter, numeris fatis bene mutatis, iterum exhibuit, adiefto rurfus alio errore $\mathrm{Fn}$. Su. 56 I. pro 560. $\mathrm{Hg}$.

\section{(p. 29.)}

$25 \mathrm{I}$. C. coryli niger, thorace, elytrisque teftaceis immaculatis. F. Sp. I42. 24.

D Schrank. E. I. I68. Schaeff. Ic. t. I87. f. 4 . f F. E. S. I. 260.38 .

Supra rufus, fubtus niger. Caput nigrum puncto Rauo ấté oculos. Scutellum nigrum. Hab. in Corylo raxus. Long. 2? lat. Ix. 
252. C. vitis niger, glaber, elytuis rufis. F. Sup. I42. 23 .

. …… I. 2. 60. 35́.

Cilput nigrum. Antennae nigrae, longae, filifor-: mes. "ihomx niger, nitiỏs, gibbis. Elytra rufa, fubpilofa. Subtus totus niger, peảbus longiusculis. A C. cory/i fat difinous. Hab. in Vite vinifcra. Long. 2. lat. I.

Variat interdum elytrorm punctis"quatuor nigris, vno minore humerali, altero maiore pone medium, vt in C. bipunfiato. $\mathrm{Hg}$.

253. C. 4-puffulatur niger, elytris unfo-bimaculatis. F.Sp. $145 \cdot 42$.

$\therefore$ F. E. S. I. 2. 63.76 . Schaef. Ic. t. 30. f. 6. Antennae nigrae, articnlis primis et apice rufac. Caput ferrngincum. Thorax niger punctis duobus imprefris. Elytra nigra, vix fubtriati, macula ad bain maiore rufa, alia ante apicem ninore nifa. Abdomen et pedes ferruginei. Tlab. fub cortice rarus. Long. 2. 1it. $\frac{3}{4}$;

$$
\text { (j). } 700 .)
$$

* 25a. C. pilofus totus oblcure aenelis, elytris poftice punctis villoris aibidis.

Totus rnicolor. Thorax ct elytra nunculata fafciculis pilonm alborm, poftice pralertim, afperfi. Hab. fub ulmi cortice frequens. Long. I $\frac{1}{3}$. lat. $\frac{2}{3}$.

255. C. pmeflut thorace fulno, elytris teftaceis nigro-maculatis. I. Sp. ra6. 48 .

\% F. E. S. I. 2.169 .86$.

Chryfonela minuta. Herbft. Arch. III..5 $5 \mathrm{j}^{\mathrm{r}}$. t. 23. f. I9.

G 5 Thorax 
Thorax plerumque antice niger. Elytra ftriata, pallide flaua, lingulo lineola media nigra longitudinali, quac non femper apicem attingit. Abdomen nigium. Pedes tefacei. Hab. in graminofis frequéns. Long. $\frac{1}{2}$. lat. $\frac{1}{4}$.

Cryptocephalus hicce, vt eiusmodi fpecies folent, mire variat, vt illi, qui laud ipfe magna copia collegit fpecimina, fpecies diftinctae videantur, quae tamen varietates funt. Harum quas pofídeo, enumerare. liceat. a) elytris rnicoloribus grifeo-teftaceis $(C$. minutus. F. E. S. I. 2. 70. 87. An Le gribourg fauve. Geuffr. I. 237. I2?) $\beta$ ) puncto humcrali parto, r) puneto humerali et fafcia verfus apicem nizricante obfcleta, quaie $\delta$ f fafeia lata fit, et diftinctior, ( $C$. pufullus $F$. et auctoris), $\varepsilon$ ) dimidium pone, occupat elytrum, apice folo excepto, imo ?) totum ferme tegit. bafi exemta, vbi color teftaceus in nigricantem vergit, puncto hamerali dilatato; margo tamen femper teftacens reftat. ク) Elytra fufco - nigra funt, margine exteriore toto apiceque tefraceis, Э) tandem tota nigra enadunt," margine exteriore bafi tantum fauicánti albo: (le gribon moir ì corcelet rouge Geofit. I. 236. II. C. gracilis. F. E. S. I. 2. 70. 88.)

In haec itaque verba in fyftemate difingui pofie videtur: C. antennis flunis, ver uns apicem nigris, copite thoraceque fuluis nitiais, periore ablomineque nigris. padibus pallidis.

Caeterum ric citato Herbftii nullus dubitabit, miratus, quomodio Vir celeberrimus Cryptocephalum verum Chryfomelarum gencre exhibuifiet, fi defcriptionem affabre factam, iconemque bonam adiectum comparauerit. Hg. 


\section{CISTELA.}

Palpi filiformes. Intaxilla vinidentata. Labum membranaceun, bifidum. Antemac filiformes.

256. C. Gigas villofa fuca, clytris abdomine, - femoribusque tefaccis. Fo Nant. 84. I.

D Olinier. n. 3 I. pl. r. f. I. b.

\& Rofii Mant. $34 \cdot 8.3$.

Cebrio Gigas. F. E. S. I. 2. 4r. I.

Panz. F. I. G. V. To.

Infectum forte noui generis, Elateri proximnm. Ann. I 787 . a me transiniflim ad Scopolium; Wic, non dubitauit afferere $E$. flinjyedm elle Fabricii. - Caput exfertnm nigrum, oculis magnis atris prominulis. Nandibulae porrectac fimplices. Antemae nigrae, filiomes, feratac, fubpilofac, elytro paullo breuiores, articulo vlumo breuifino. acnto. Palvi quatuor (mihi) flifomes, inaequales, longiusculac. Thona niger, fubpunctatus, tomento rufeicente pubcfcens. Elytra fubmarginata, mollia, faturate teftacea, vix friata. 'Femoia teftacea, pofticaque trochantere femquali nuodanmodo initucta. Tibiae brevies, comprefile, fuinefac. Tarfi quingne longi fulci. Abdomen pallide rufumpilofm. Ams acuminatus. Alae albac bafi flaua nigricante. Variat clytris mis et fulcis. Etf habeat thoriaccn fibtus mucione donatum, et ad datera acuminatum, lapfus tancn nullus: volat volatu breni impetuofo etiam noctu.

Cum pridem Kal. Septembr. ruri offem loco proninciae Pifanae vulgo dicto Fauglia, contigit mili cocnanti fub incdian nocten; vt plures per 
fencftras ingreffi coenaculum, impetu quodam ac fufurro in candelas ardentes irruerent, mihique et conuiuae moleftiam non mediocrem afferrent. Notatu dignum id enenife poft ingentem fluniam primum in illa aeftate e coelo delapfam. Poftridie viif non pauci in campis et viis ad fepes volitantes, ct identidem impetu quodam coeco me vrgentes. Ian captum alias in plantis filune Pifanae m. Septembr. Tab. VIl. f. 9. Lotig. ?. lat. $2{ }_{4}^{\text {T. }}$

\section{(p. ror.)}

- 257 . C. ceranboildes atra, eiytris ftriatis, teftaceis. F. Sp. 147. 4.

"S F. E. S. I. 2. 42.4.

Subtomeñitofa. Anteinne nigrae, forratac. Thorax marginatus, antice anguftatus, poftice trans-i nerfus ad latera angulattis, niger. Elytra teftacea, mollia, friata. Variat thorace interdum teftaceo. Hab. in filuis. Long. 5. lat. 2.

\section{(1. i02.)}

258. C. ceruina linida, pedibus fufcis. F. Sp. I $46 . \mathrm{I}$.

D) Geoffr. Inf. I. 355.5 .

S F. E. S. I. 2. 42 . I.

Balis antenuatun, et tibiae ferrugineac. Elytra parum marginatr. Habit. cum pracecedente frequcns. Long. 4. Lat. $I_{\frac{2}{3}}$.

De citato Genfroyano dubius adhuc hacreo. Antenhue dimidium corpus haud fuperant, totaeque ferrugineac, nec bail flaure fint. Strias elytrorum 'etfi levifimas afpicere nequeo. Adiecta auctoris

- verba, hocque citatum coniuncum, dubitantem me reddunt, an veram Ciftelam habuerit. $\mathrm{Hg}$. 
250. C. tefincea nigra, thorace, elytris, abdomiveque tefaceis. F. Si. 147. 5.

\section{‥ T. I. S. I. 2. 43.6.}

Antennat nigrat, articulis conicis. Caput, pectus, pedesque nig.a. Hab. inplantis. Long. $3 \frac{1}{4}$. lat. $1 \frac{1}{2}$.

26́o. C. muinin nigra, elytris pedibusque tefaceis. F. Sp. I4\%. 9 .

\% F.E S. I. 2.44 . I6.

Habit. in piantis variis. Long. 3 . lat. $1 \frac{1}{3}$.

261. C. palliria capite elytrorumque apicibus fifcis. F.Sp. 14S. I 1 .

\section{?. F.E. S. I. $2.46,26$.}

Antennac tenues. Capit deflexm. Oculi nigri. Thorax antice anguftatus, poltice dilatatus, emarginatus. Elytra lacuia. Pedes pallidi. Hab. in plantis. Long. $2_{+}^{i}$. lat. 1 .

262. C. Sulphnrea flaua, elytris fulphureis. F.Sp. 147.6 .

\& F.E.S. I. 2.43 .8 .

Tenebrio flauns. Scop. E. C. 260.

Antenmae apice fufcae, articulis elongatis. Alac nigrae. Deferiptio Geofroyana I. 35 I. I I. accuratifimn. 'Hab. in floribus vmbellatarum rara.

- Long. $3 \frac{x}{3^{*}}$ lat. $\frac{1}{4}$.

2.63. C. lepturoides atra, thorace quadrato, elytris friatis teftaceis. F. Niant. 85.6 .

D) Cantharis fraxinea. Scop. E. C. I34.

9. F.E. S. I. 2. 43 . 5. Panz. F. I. G. V. IT. Antennae nigrae, mediocres. Palpi quadriarticulati. Caput et thonax nigra: hic vero transuerfus, fubquadratus, margine laterali prominulo. Elytra fubftriata. Hab. in hortis frequens. Long. 6. lat. $2^{\mathrm{r}}$. 


\section{CRIOCERIS.}

Palpi filiomes. Illaxillit bifida. Labiun corneum, integrum. Antemate filiformes.

Fabricius in noua fyfematis fai editione Crioceridum genus bene fepararit, et anter immiftas, v. c. $C$.

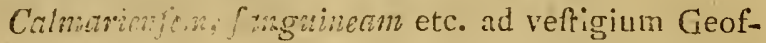
froyi nonum ow is Galerucarum conftituere itffit, cui optime Chy. rr.ficcom, Tonaceti, Alni annumerauit. Sul dubio Entomologorum fubiectum manet, an Vir celeberrimus genus Alticarum et oblonģas quasdam Chryfomelas e. g. vulgatifjmann etc. huic generí rite annumeranerit. Hg.

264. C. Calmarienfis ouata, cincrea: elytra vitá, lineolaque bafeos nigra. F. Sp. I50. 6 .

I C. ronthomelaena. Schrank. E. I. I 45. cum optima detipripione.

9 Galevica Catmarienfis. F. E. S. I. 2, 23. 46. Hab. in Vlmo, Salice, Aino frequens. In agro Senenfi aciate 1788 copiofinma. Long. $2 \frac{1}{2}$. lat. I $\frac{1}{3}$. In fpecininibus meis thorax lineola tantum vnica longitudinali notatus eft, nec elytrorum bafeos lineulam afpicere poffum. - - Variat interdum tota grifea, absque vitta, antennisque totis ferme rutis, apice excepto, quae in folitis nigrae funt, bafi fola rufa. $\mathrm{Hg}$.

$$
\text { (p. I0.4.) }
$$

265. C. Merdigera mibra, thorace cylindrico vtrimque impreffo. F. Sp. 152. I9.

() Sulz. Hif., Reanm. Merian.

D Sulrank. E. I. 182.

O. F. E. S. I. 2. 6. I9.

Hab. in Lilio. Long. 4. lat. $2 \frac{\mathrm{T}}{3}$.

C. vufipes, vel C. conucillaviaze Harrer. femper dinerfa ab illa, quam in Liliis reperimus, pedibus capiteque rubiis. 
repris. Color femper idem, et habitatio numquam, mutati, ditlerfis fuecies elle, mihi perfuadent, quanquan alias, quam coloris, notas detegere non polim. Hg.

266. C. I2-qunatata thorace cylindrico, rubra, elytris punctis fex nigiis. F. Sp. I 53. 20.

$\odot$ Frifch.

D) Schrank. E. T. I\$3.

O F. E. S I. 2. 2. 20 .

Hab. in Alparago frequens. Long. $2 \frac{x}{2}$. lat. I

\section{(p.105.)}

267. C. funguined ouata, rufa, antounis oculisque nigris. F. Sp. 15 I. 9.

D Geoffr. Int: r. 253 . 2. Schaefr. Ic. t. 2 I6. f. 4 . a. b.

을 Galeruca fanguinea. F. E. S. I. 2. $23 \cdot 49$.

Noltra fpecinina connenint cum defcriptione Geofir. 1. c. Stitura onmino C. camene, fubtris tota nigra, fupra rufa, punculati. Antennae nigrac primis articulis nufis. Oculi nigri. Fenona tibiaeque fubcomprella. Variare videtur magnitudine minore, elytris interdum nigro bilineatis, pedibus rufis: forte fpecies diftinca? - Tab. miri, faepe inuenta ctiam in horti parietc. Long. 2. lat. I.

Virietates' plures eiusmodi huius greneris infectorum obferuare licet, nec coiores fuli, praecipue tam incerti, characteres differentise fpeciei luppeditant. C. fanguinea et apud nos diuerfimode variat. $\mathrm{Hg}$.

268. C. teneila fermginea, thomace, clytromumque nurgine flauis. F. Sp. I 5 \%. Io.

\& Galeruca tenella. F. E. S. I. 2. 23. 50. Statura E. Calmarienfis, fed dimidio minor. Antemnae flanefcentes. Pedes flani. Abdomen fuicum. Habit. in filuefribus. Long. I $\frac{T}{2}$. lat. $\frac{3}{4}$. 
260. C. czancllid caerulea, thorace cylindrico lateribus gibbis. F. Sp. 154.30 .

올. E. S. I. 2. 9. 35.

Tota caerulca nitida. Antenna folae nigtac. Hab. in plantis. Long. 2. lat. $\frac{2}{3}$.

\section{(p. 106.)}

270. C. melunom oblongi, caerulea, thorace. pedibusque rufis. F. Sp. $155.3 \mathrm{r}$.

D) Reaum. Inf. t. 3. t. I7. f. 15 . Geoffit. Inf. I. 242.4 .

O F.E.S.I. 2.

Oblonga, caenulea, nitidinima. Differt a praecedente thorace ferrigineo. Elytra firiata. Femora ferruginea. Plantae nigrae. Hab. in Quercu. Long. 2. $\mathrm{I}$. lat. $I$.

27 I. C. flanipes oblonga, nigra, thorace pedibusque flauis. F. Sp. I55. 32 .

S.F. E. S.I. 2. I0. 37.

Praccedenti fimillina et elytta nigra. Thorax angufus, marginatus, (in hac flaus, in illa fuluus.) Elytra non friatae. Antennac, quae in $C$. melanopu nigrae funt, in lac fubferrugineae. Hab. in Anygdalo Perfica. Long. 2. lat. $\frac{2}{3}$.-

Similitudo huins et prioris, colore excepto, mihi certe tam exigna eft, vt C. flatipedenn ad grenus Galerucae referre mallem. Caeterun C.mufipes F. E. S. I. 2. ro. 39. nil, nifi varietas fexus, et quidem femina, eft, thorace nigro. Hg.

272. C. Phellandrii oblonga, nigra, thorace, elytrisque lincis duabus flatuis. F. Sp. I56.36.

S F.E.S. I. 2. II. 43 .

Oblonga. Antennac apice crafliores, nigrac, thoracis margine laterali flauo, tibiis femoribusque 
parte interiore flauis. Apex elytrorum flauus. Hab. Phellandrii caules. Long. 2. lat. ${ }^{2}$.

De genere valde dubito. Cum Geoffroyo optime inter Chryfomelis reponi potelt. Hg.

$$
\text { (p. ro r.) }
$$

273. C. campeftris oblonga, nigro - caenlefcens, thorace limbo nigro, elytris punctis tribus pofticis margine flauo connatis. F. Mant. 90. 47.

$\odot$ Vallisn.

(D) Schneff. Ic. t. 52 . f. 9. To.

\& F. E. S. I. 2. 1 I. 44 .

Habitus et magnitudo fere $C$. melanopae. Caput nigro-acneum, punctulatum.' Antennae nigrae, porrectac, craffiulculae.' Thorax nigro - aeneus margine antico et poltico rufo, anguftior, et magis cylindricus, quam in C. Phellandrii, *) cui primo intuitu fimilis. Elytra nigro - acnea, punctatoftriata, limbo externo, et apice rufis. In fingulo linea media longitudinalis flaua, apicem nọn attingens; quae in nonnullis interdum punctis nigris interrupta occurrit. Pedes pallidi nigro-aerei, varii. - Margo elytrorum in cadauere flauus euadit. Admodum variare videtur. Hab. in Afparago filueftri. Long. $2 \frac{1}{4}$ lat. $\frac{2}{3}$.

An varietas tantum $C$. Afparagi? Haec fane valde variat. In icone Schaefferi citata nil nifi $C$. Afpovìgi cognofcere poffum, quod etiam de Panz. F. I, G. III. I 2. dicere libet. C. rampeftris certo nobis nondum nota eft, praecipue, cum in Barbaric habitet. Hg.

XXXIIT.

") Notae collatitiae cum aliis fpeciebus eitusdem generis, cognitionem infectorum facilius eruere, ac fiftere videntur, cum Ico. uum defectui quodammodo fuppetant. Adnot. ault, ips. 


\section{LAGRIA.}

Palpi quatuor, anteriores fecutiformes, pofteriores extrorfiun craffiores. Labium membranaccum, integum., Antennae filiformes.

274. L. hirta villofa, nigra, thorace tereti; elytris teftaccis. F. Sp. I60. I I:

() Scop. E. C. $13 \%$.

온 F.E.S. I. 2. $79 \cdot 4$.

Antemae nigrae, paullo thorace longiores. Sub. - tus töta nigra. Thorax cylindricus, teres, niger, emarginatus. Elytra pubefcentia pallide teftacea. Alae nyalinae. Hab. in floribus trequens. Long. $2 \frac{2}{3}$. lat. $\mathrm{I} \frac{2}{3}$.

.. L. pubefcens $F$. an fexus tantum varietas? Differt thorace anguftiore, corpore longiore, elytrisque magis linearibus, quae in L. hivta ab ortu fenfim latiores defcendunt. Habitat cum L. hirta. $\mathrm{Hg}$.

275. L. caerulea fubuillofa, cyanea, antennis nigris. F. Mant. 94. 22.

Anobitin cyaneum. F. Sp. 72. \%

D Geoffr. Inf. 1. I77. 14 .

\& F.E. S. I. 2. 8 I. 14.

Antennae breues. Thorax marginatus, teres. Elytra fubpunctata. Colore variat. Hab. in floribus. Long. 3 . lat. 1 .

* 276 . L? bifafciata atra, elytris fafciis duabus flauis, duabusque nigris alternis.

Caput, corpus, thorax atra, laeuia, immaculata. Elytra fafciis duabus flauis, atrisque altemis, e quibus flaua bafeos, atra apicis. Pedes flaui. Hab. in plantis non frequens. Long. $1 \frac{1}{2}$ lat. $\frac{x}{3}$. 
277. L. nigra pilofa, nigra, elytris molliusculis. F. Sp. App. 498. 13 - I 4.

D Petagna. Sp. Inf. Cal. fig. IX.

\& F.E.S. I. 2. 80. Ir.

Nigra, pilis vbique pubefcens. Antennae elytro breuiores, fubdentatae, articulo vltimo ouato. Habit. in floribus. Long. $2 \frac{\mathrm{r}}{4}$. lat. ${ }_{2}^{\mathrm{T}}$.

278. L. antherina nigra, elytris fafciis duabus ferrugineis. F. Sp. I60. I4.

2. Notoxus antherinus. F. E. S. I. 2. 2 I2. 9. Hab. in floribus.

279. L. floralis nigra, thorace ferrugineo. F. Sp. I 6 I. I5.

\& Notoxus floralis. F.E.S.I. 2. 212 . IO.

Priori fimillima. Statura eadem. Elytra fufca, maculis duabus ouatis transuerfis pallidis. Hab. in floribus primo vere frequens.

\section{XL, CVRCVLIO.}

Palpi quatuor filiformes. Maxilla cylindrica, vnidentata. Labium integrum. Antemae infiden- tes roftio corneo.

\section{(p. 110.$)$}

* Longiroftres fenoribus fimplicibus.

280. C. Colon longiruftris, grifeus, elytris puncto albo. F. Sp. I64. I 6.

$\odot$ Schaeff. Ic. t. I55. f. 2.

D C. paluftris. Scop. E. C. I 0.4. Schrank. E. I. 2o\$. Schaeff. Ic. t. 62. f. Io. t. I60. f. 6 .

\& F. E. S. I. 2. 401.29.

Roftrum thorace longius, apice dilatatim. Thorax elytris obfcurior linea laterali albida. Elytra fria- 
ta, puncto albo in medio notata. Hab. in pratis paludofis. Long. a bafi roftri ad anum $4 \frac{\mathrm{T}}{2}$. lat. 2 . In fpecininibus meis elytrorum duo punita media, transuerfim oblique pofita obferuo, quorum alterum luturae propius manifeftum, alterum autem obfcurum, cohaerere videtur cum illo. - Schneiderus Schaeff. Ir. t. 160. f. 6. ad alium referre mauult Curculionem, quanuis Harrerus apud hune alleget, qui t. I55. f. 2. ad bilineatum Laich. pertinere contendit. $\mathrm{Hg}$.

23 r. C. bilineatus? longiroftris, fufcus, elytris lineis duabus punctorum alborum. F.Sp. I64. 18. - o C. coftatus. Rolli Mant. 4I. IO2.

Roftum breuius, craffusculum. Thorax niger lineis quatuor albis, duabus inmedio, et binis marginalibus. Elytra friata, punctata, nigra, lineis duabus e maculis quadratis albis, quarum altera ad marginem, altera ad futuram. Abdomen fubtus cinereum nigro - punctulatum. Pedes fimplices cinereo-fufci. .Háb. in pratis. Long. 5. lat. $1 \frac{3}{4}$.

- Hic Curculio iterum documento eft, quam timide auEtor ante fe dicta legat, cum h.l. Curculionem breuiroftrem in familiam longiroftrum transtúlit, quia paullulum cum aliquo conuenit iam defcripto. In Mantifra fua $C$. coflatum $F$. vocat, fed milhi diftincta fpecies. Ipfe iam antea pofledi, et poftea a D. de Paykull, cri a D. Roflio miffus erat, accepi. Statura quafi intermedia inter coftatum et obliqunm $F$. eft. Thorax haud carinatus, vt in coftato, fed aequalis; interdum in medio linea longitudinali imprefla. Elytra in coftato in medio gibba vel conuexa obferuantur, et eiusdem, vt thoracis lineae, coloris luteo-cinerei; in hoc autem planiufcula, minime conuexa, cinerea difco folo nigro maculis fparfis cinereis. C. alternanten vocaut. $\mathrm{Hg}$. 
282. C. bimaculatus longiroftris, futcus, elytris punito cinereo; roltro, pedibusque atris. F. Mant. 9S. 21 .

P F. E. S. I. $2.40 \mathrm{r} \cdot 30$.

Defcribit Fabricius l. c. Color fufcus vergit in teftaceum. Hab. in filueftribus frequens. Long. $4 \frac{r}{3}$. lat. I.

An idem cum C. Semicolo Herbit. Arch. IV. 69:4? Cui coniceturae id autem repugnat, quod Herbftius 1. c. varietatem putat $C$. coli, a quo tamen praecipue thorace fubglobofo, nec conico, differt. Variat magnitudine. .. $\mathrm{Hg}$.

\section{(p. III.)}

* 283. C. mulucrulentus longiroftris, thorace punctis proninulis, elytris transuerfim rugofis. Scop. Ent. Carn. 75.

\& C. angufatus. F. E. S. I. 2. 4I8. 106.

Cylindricus. Cum flores continuo frequentet, vbique polline antherarum afperfis apparet plerunıque colore flauo - viridi. Roftrm teres. Antennae fiubrufae. Femora mutica. Hab. in plantis variis frequens. Long. 5. lat. 2.

Diagnofis in Fabricii fyftemate data, nimis laxa eft, et pluribus conuenit Curculionibús. Schacff. Ic. t. 79. f. x. qui Curculio fecundum Harrerum elytra ftriato - punctata, nec rugofa, gerit, h.l. citare nequeo. Mirum eft, quod Scopolius 1. c. nullam ftaturaé cylindricae mentionem fecit. $\mathrm{Hg}$.

* 284. C. Cardui longiroftris niger, polline flano adfperfus, elytris punctato-frriatis, femoribus muticis.

An C.triangularis. Petagna Sp. I. C. 7 I ?

\& C. Cynarae. F. E. S. I. 2. 40 I. 28. C. Latus. Herbft. Arch. IV. 7I. 2I. t. 24. f. 9., 
Statura C. colon, fed triplo fere maior. Corpus totum polline flauo irroratum, vti in C. puluesulento Scopoli. Antennae fractae, fufcae, clana cinerea. Roftrum craffiusculum teres, fubcarinatum. Thorax planus, fcaber, poftice et fcutelli loco obfolete angulatus. Elytra non transuerfim rugofa vti in C. puluerulento, fed pun Eato - ftriata, ftriis in fingulo decem. Abdomen;pedesque flauo-villofi. Alae fufcae. Mihi vifus longiroftris. Tab. V. f. I 1, Hab. Florentiae, frequens in floribus Cardui Inutantis. Long. 8\% lat. 4 .

Huic Curculioni plures valde fimiles vidi, quorum differentiam eruere, difficillimum eft. Diagnofin hujus $C$. Cyniarae itaque his verbis bene expreflam credo: longiroftris, niger, viresienti-irroratus, roftro thoraceque carinatis. Alium, ftatura fimillimum poffideo, quem Schallerus Act. Hallenf. I. p. 280. Sturnum vocat. Differt, praeter magnitudinem femper duplo, interdum: quadruplo, imo quinquies minorem, etian roftro thoraceque haud carinatis. C. Iacea F. E. S. I. 2. 400. 24: ab hoc tamen differre videtur, quamquam fub hoc nomine, idem Sturntts mihi mifius fit. Alium denique haben, qui ab illis duobus femper latis ac depreffis folo corpore cylindrico, caeterum fimillimo, diuerfus eft. Huic nomen C. tereti tribui. Hg.

285. C. Afcanii longiroftris, cylindricus, ater, lateribus fubcaerulefcentibus. F. Sp. I 73.67.

\& F. E. S. I. 2. 4 18. 108.

Corpus tota finctura $C$. paraplectici, fed apex elytrorum acutus, non mucronatus. Totus ater, linea canefcente marginali ab oculis vsque ad elytroium apicem dicta. Subtus totus canefcens.

- Habit. ruri frequens. Long. 4. lat. I. 


\section{(p. 122.)}

2\$6. C. Bacchus longiroitris, aureus, roftro, plantisque nigris. F. Sp. I $65: 22$.

○ Sulz.

D Schrank. E T. I99. Degreer. 5. 366. 38 .

O Attclabus Bachus. F. E. S. I. 2. 387 . 15 .

Rubro-aureus villolulus. Thorax femper muticus. Roltrum es tibiae nigrae. Cháracter fgniti a Lirnaco datis $\bar{C}$. Populi, huic magis contenire videtur. Hab. in lortis.' Long. 2. lat. I."

Menfura data nimis parua eft. ${ }^{2}$ Ef enim longis, so apice thoracis'ad anum usque $2^{3}$, computato ia tem roftro $4 \frac{1}{\frac{1}{2}}$ lin., latus $I \frac{1}{2}$ lin. Citatum Geofrer. 270. 4. $a b$ auctore cum Fabricio exhibitum, ad A. arquatum $F$. pertinet.

Fabricius in Entomologia fyftematica fua Curculiones iftos, Bacho fimiles, ris, quibus antennae rectae funt, quique ftatura pyriformi gandent, e. g. frumentario, Craccac etc. athibitis, ad genus Attelabi amandauit. 'Equidem pro mea parte illos pofteriores: Frumentariann etc. aut in Curculionum genere retinerem, aut noứm ac peculiare genus confituere iuberem. Attelabis haec tantum annumerarem mihi nota infecta: $A$. Coryil, Auellanae, curculionöldem, et, vt mihi videtur, nondum publicatam fpeciem, intermedian. Iilos denique C. Bachim, Betulae, cupreun, acquatum, ruficollem Herbte. et alios, quos' nominari locus non finit, in feparato ac diftincto genere, fub nomine Rlynnchites coniungerem. Hg.

287. C. Betulae longiroltris, corpore viridi - aurato, fubtus concolore. F. Sp. 165.23.

() Erifch., Sulz., Schaefi. Ic. t. 6. f. 4 .

D Scop. E. C. 77. Schrank. E. I. 197. Schaeff. Ic. t. IO4. f. 4 . 
\& Attelabus Betuleti. F. E. S. I. 2. 38\% 16. Variat faepius colore omnino caeruleo; alter fexus, qui tinoracen antrorfum fpinofum gerit: $R \bar{h} i-$ nonacer bifpinus Scaeff. Ic. t. 6. f. 4. Müll. Z. D. Pr. 1023, C. violaceus. Scop. E. C. 28.

Habui quamplures eductos omnes e foliis Vitis contortis. Nonnulli habebant thoracem anguftiorem inermem, et probofcidem breuiorem. Alii thoracem latiorem fuinis duabus armatum, et probofciden longiorem; huiusmodi characteres notaui conftantcr tam in iis, qui corpore viridi - aurato fubtus etc. quam in is, qui corpus habent caenleum a quibusdain (vid. Geoffir. pag. 27 1.4.) pro feminis habiti: quare thorax, fpinae, probofcis, non color, vt optime etiam animaduertit $\mathrm{Fa}$ bricius, fexum diftingumt, fed fpinas thoracis, quae iuxta Linnaeum maribus competunt, in feminis Geoffroyanis occurrere tantum obferuauimus. Long. $2 \frac{1}{4}$. lat. $I_{4}^{\mathbf{r}}$.

Equidem etiam in mare nonnumquam fpinas thoracis vidi, aliquando antem marem feminamque copula iunctos reperi, ambos fpinis thoracis carentes, et quorum mas duplo minor, obfcure caeruleus, femina autem, maicr, viridi - aurata erat. Interdum vierque caeruleus, aut viridis eft. $\mathrm{Hg}$.

\section{(p.713.)}

288. C. Campunulae longiroftris, niger, ouatus, elytris friatis obtufis. F. Sp. I67. 3 I.

우F. E. S. I. 2. 406.55.

Hab. in pericarpiis Campanulae. Long. $\frac{\mathrm{r}}{3}$.

289. C. Alliariae longiroltris, violaceus totus. F. Sp. 168.40.

(6) extifts.

Geoffr. 
D Geoffr. I. 27 I. 5. C. Icofindriae. Scop. E. C. 85 .

of Attelabus Alliariae. F. E. S. I. 2. 390. 2z.

Villolilus, nitisis. . Caput, thorax, femora nigra cacnilcícentia. Elytra ftriata. Colore et magnitudine admodum variat. Hab. in Eryfuno Alliaria. Long. I $\mathbf{I}_{2}^{\mathrm{y}}$. lat. '.

Cititum e Geoffroyo, ab Auctore, cum Degeerio, exhibitum, bonum videtur. Niea fpecimina autem conftantia obferuaui, nec magnitudine infolita; nec colore variantia. $\mathrm{Hg}$.

\section{(p. 114.)}

290. C. cyaneus longiroltris, ater, elytris violaceis. F. Sp. I 68. 41 .

of Attelabus cyineus. F. E. S. I. 2. 39 r. 30 . Elytra violacea. Pedes ferruginei. Hab. in Salice. Long. $I_{4}^{\prime}$. lat. $\frac{t}{2}$.

29I. C. flaipipes longiroftris, ater, femoribus luteis. F. Sp. 169.47 .

of Attelabus flauipes. F. E. S. I. 2. 39r. 33. Totus ater, femoribus folis liteis. Elytra ftriata. Hab. frequens in locis apricis. Long. $\frac{2}{3}$. lat. $\frac{1}{3}$.

292. C. nigriroflris longiroftris, viridis, roftro atro. F. Sp. $167 \cdot 33$.

옥 F. E. S. I. 2. $407 \cdot 56$. et variabilis. n. 57 . Caput fufcum, roftro cylindrico, atro. Thorax gibbus, rotundatus, riridis, lineis duabus dorfalibus fufcis, vix confipicuis. Elytra tomentofa, viridia, immaculata. Pedes fufci, femoribus fimplicibus. Hab. etiam in hortis. Long. $\frac{3}{4}, 1$. lat. ${ }_{2}^{1}$.

Fabricii $C$. variabilis nil nifi mera huius varietas eft, tomento virefcenti detrito, vnde color teftaceus apparet. In eodem cum nigriroftri loco femper cepi, praecipue in pratorun floribus autumnali tempore. $\mathrm{Hg}$. 
293. C. rufiroftris longiroftris niger, roftro dimidiato, pedibusque rufis. F. Sp. I67. 35 .

올 Aitelibus rufirofiris. F. E. S. I. $2: 390.26$. Roftum elongatum a medio ad apicem rufun puncto nigro. Elytra nigra, ftriata. Thoriax niger. Pedes rufi fimplices femoribus anticis bafi nigra. Lectus in filuis; fub arborum cortice. Long. $I \frac{1}{2}$. lat. $\frac{\pi}{3}$.

-.In Fabricii Entom. Syftem. 1. c. errore quodam defcriptio C. nigrirofiris p. 407. n. 56 . iterata eft, loco defcripionis in Syftemate Entomolog. 132. 25. datae. $\mathrm{Hg}$.

\section{(p. r15.)}

294. C. pericarpius longirotris, fubglobofus, nebulofus, elytrorum futura bafi alba. F. Sp. I67. 37 .

D Geoff. Inf. I. 298.46.

\& F. E. S. I. 2.408 .63 .

Statura C. Campanulae; totus fupra obfcure fufcus, fubtus cinereus. Roftrum tenue, longuni. Femora clauata mutica. Pedes picei. Hab. in Scrophulariis. Long. I. lat. $\frac{1}{2}$.

Geoffroyns l. c. Curculioni fro femora dentata tribuit, quae tamen mutica funt. Roftrum hăd elongatum in meis, qua de caufla Auctor C. pericrspium Schrank. E. I. r09. 209. habuifle videtur, qui differt a meo $C$. pericarpio, quem verum Linnaeanum et Fabricianum habeo, praecipue rofiro tenui, elongato, thoracisque futura antica valde elevata ac reflexa. Hg.

295. C. paraplecticus longiroftris, cylindricus, fubcincreus, elytris mucronatis. F. Sp. 1 72.62.

$\odot$ Schreb. Sulz.

D Goetze. E. E. I. 348. 34 . 
․ F. E. S. I. 2. 4 I $\{\cdot 9$ I. Panz. F. I. G. VI. I5. Degeerii defcriptio accuratifima cit. Non cubitaudum de C. nemorep Scop. E. C. Ior. Habitat larua intra caulem Plellandrii. Equis paroplegiam couffiri creditur. Linn. at reuocat in dubium. Long. 7. lat. $1:$.

Magnitudine mire variat. Citatum e Scopolio mihi femper dubium remanet, et ob alas in meis praefentes, et ob deferiptionem nimis abhorrentem, et ob habitationem tam diuerfan. Geoffroy ths certifime autem e citatis tollendus eft $\mathrm{Hg}$.

\section{(p. Is6.)}

296. C. abbreuiatus longiroftris, ater, thorace plano, prnctito, elytris abbreuiatis. F. Mont. 99. 25 .

을. E. S. I. 2. $403 \cdot 37$.

C. mutilatus. Laich. Tyrol. 2 I6. I2.

Totus ater, picens. Roftrum elongatum, incuruun. Antemae fractae nigrae claua cinerea. Thorax planis, punctatus, fubcarinatus. Elytra fubitriata, abdomine bremiora. Alac margine crafiore flaucfcente. Hab. filuas in Quercu Robore frequens. Long. $5^{\frac{1}{2}}$. lat. 2 .

* 297. C. Pfcut-Acori longiroftris, corpore ouato, cincreo, thorace clytrisque nigris friatis, puncto ad futuram albo.

C. Puntum album Fuefly. Arch, t. 24. f. IT.

S. F. E. S. I. 2. 408.61 .

Statura C. Scrophliturine, fed maior. Antennae fractae cinereae claua nigra. Roftrum fubulatum bafi cincreum, apice nigrum, quod fub ventre inflectit. Caput rufo - cinererim. Thorax niger poftice dilatatus fulco medio dorfali impreflo, et late- 
lateribus cinereus. Elytra nigra, gibba, ftriata, abdomine paullo breuiora, punćto pone futellum albo ad futuram communi. Abdomen fubtus cinereum. Pedes fimplices latere externo cinerei, interno nigri. Variat interdum colore elytrormm fubteftaceo fufco. Hab. in floribus Ireos PfeudAcori menf. Mai. frequens. Long. $2 \frac{r}{5}$ lat. I

* 298. C. Paftinacac longiroftris, grifeo-flauus, muticus, roftro nigro, elytris apice nigro'-punctatis.

Grifeo-flauus, villofus. Roftrum deflexum, nigrum, pilofum, antemis apice nigris. Thorax vix nigro-inaculatus. Elytra verfus apicem lineis tribus e punctis nigris diftinctis ftriata, quarum vna ad marginem, altcra ad futuram, et tertia in medio. Hab. in umbellis Paftinacae fatiuae, vbi nidulatur, pedunculos intemos contorquens, crifpans. Long. 2. lat. $\frac{\hat{3}}{3}$.

\section{(f.TiY.)}

299. C. Pini longiroftris, elytris rufefcentibus, fafciis nebulofis. F. Sp. 163. I3.

$\odot$ Frifch.

\& F. E. S. I. 2. 399. 2 I.

Thorax punctis duobus, fcutelloque albis, fafcia clytrorum ex maculis fermgineis. Fenora grifeomaculata. - Limmeus in Fn. Sv. dedit huius defcriptionem fub $C$. Abietis. Confer Scirank $C$. Pini. 195. - Habit. in Pini filueftris cortice. Long. $3_{i}^{\mathrm{i}}$. lat. $\mathbf{I} \frac{1}{3}$.

De Curculionibus Pini et Abietis femper aliquid incerti reftat, quod ad Linnaeum attinet. Aut Linnens errauit, $C$. Pini duplo maiorem efle $C$. Abietis, dicens, aut C. Abiktis $L$. alius, quam vulgo putatus, eft. Hg. 
300. C. curuiroftris longiroftris, ater, nitidus, roltio arcuato. F. Sp. 166. 29.

Corpus totum atrum, immaculatum, nitidum. Roftrum elongatum, cylindricum, arcuatum. Thorax punctatus. Elytra fubftriata. Femora mutica. Proximus C. Campanulae. Hab. ruri frequens. L.ong. 2. lat. $\frac{3}{4}$.

Fabricii Curuiroftris e Noua Hollandia allatus eft. Hinc Curniroftris Auctoris valde dubius fit, praecipue fi tam innumeras Curculionum fpecies, quas brenibus verbis diftinguere difficillimum eft, refpicius. $\mathrm{Hg}$.

30I. C. frumentarius longiroftris, fanguineus. F. Sp. 169. 49.

$\odot$ Act. Nitr., Act. Holm., Lenwenh.

\& Attelabus frumentarius F. E. S. I. 2.392. 35. Roftrum teíme. Elytra friata. Hab. in frumento diutius afferuato. Long. $\frac{3}{4}$. lat. $\frac{1}{2}$.

Curculionem habeo, qui defcriptioni a Paykullio aliiss que datae, omnino conuenit, quem autem numquam in frumento, fed femper in Rumice aquatico reperi. Videtur Scopolii C. purpureus. 86. Alium, a priore ne minimis quidem, habitatione fola, et magnitudine triplo minore excepta, diuerfun, numquam in eodem loco, fed in viis ad terran cepi. $\mathrm{Hg}$.

$$
\text { ( } p . \text {. } 18 .)
$$

302. C. granarius longiroftris, piceus, thorace punctato, longitudine elytrorum. F. Sp. I 7 I. 56 .

$\odot$ Rai., Ioblot.

D Scop. E. C. 89. Schrank. E. I. 207. Schaeff. Ic. t. 174 . f. $5 \cdot$ a. 5. b. Leuwenh. lettre du 6. Aout. I $687 \cdot$. p. 74.83 .

ㅇ F. E. S. I. 2. 4 I 4.88. 
Hab. in frumento diutius afleruato. Pellitur aqua calcis. Long. $I \frac{1}{2}$. lat. $\frac{\pi}{2}$.

* 303. C. Scirpi longiroftris, femoribus fimplicibus; niger, thorace fubcarinato, elytris piceis, vilis cinereis afperfis, puncto cinereo.

Statura et facies $C$. Equifeti, at paullo maior. Caput nigrum. Oculi picei. Roftrum arcuatum; cylindricum, friatum, nigrum, thorace longius. Antennae tefraceae, fractac. Articulus primus aequat longitudinem omuium caeterorum. Thorax piceus, fubglobofus, punctato - excauatus, dorfo fubcarinatus, lateribus macula cinerea. Elytra comata, fubporcata, fubrugofa, picea, pilis rufo-cinereis afperfa, ct macula in fingulorum medio verfus futuram. Abdomen nigrum, lateribus fupra fericeum. Pedes teftacei, pilofi; inermes. Differt a $C$. equifeti elytris non muricatis, et non maculatis in apice, et a $C$. maculato, elytris fibrngofis, et pedibus teftaceis. D'Antic. Act. Soc. Linn.

Habit. in paludibus, an in Scirpo maritimo? , Ego faepius inueni fub arborun cortice in locis paludofis.

304. C. acridulus longiroftris piceus, abdomine ouato. F. Sp. 169. 46.

\& F. E. S. I. 2.411 .75 .

Elytra ftriato-punctata. Hab. in floribus. Long. I $\frac{1}{4}$. lat. $\frac{1}{2}$.

$\therefore$ Errur typographicus in citato e Linnaeo irrepfit, Syft. Nat. 133. 32., pro 60\%. I3. exhibens. Citatum e Geoffroyo 290.32 ., quod Fabricius in Entont. Syftem. fua bene omifit, nullo modo I tolerari poteft, quia Curculionem defignat, frumen- 
mcntario fatura et autennis reitis fimilem, colore autem caeruleo-chalybace. Hinc menfura quoque, e Geoflioyo defumta, nimis parua eft. Longus enim eft $a b$ apice thoracis ad anum $2 \uparrow$, computatu roftro $3 \frac{r}{5}$. lin. latus $\tau$. lin. Variat autem duplo minor, 3. lin. longitud, et $\frac{3}{4}$. laticud. Hg.

$$
\text { (p. } 1 \text { I\% ) }
$$

305. C. filiforinis longiroltris; cylindricus; fubcinerens, thorice lineis tribus futcis. F. Sp. i 72.63 .4

q F. E. S. I. 2. $41 \%$. 102.

Statura C. parruplectici, at minor. Roitrum cylindricuin, fuifum. Thorax cinerens lincis tribus fufcis. Elytra cincrea, immaculata, minine punctata, obtufa. Pedes fufci. Hab. in locis apricis; humentibus. Long. 3. lat. $\frac{\mathrm{T}}{2}$.

*306. C. quinquemaculatus longiroftris, grifeus, colcoptris miculis quinque albidis. Lini. Fn., Sv. 6o I. Sylt. Nat. 609. 30 .

Corpus ouatun, nigricans, fronte alba. Roftri apex ferruginens, glaber. Thurax et clytra fubfermginea. Macula alba in pofica thoracis. Elytronm lintma longitudinalis alba. Macula alba ad bafin, et alia verfis apicen cuinsuis elytri. Subtus cinereus. Hab. in filuis. Long. I $\frac{1}{3}$. lat. $\frac{3}{3}$.

C. quinquepunfictus valde affinis videtur, fod in cinnibus tpeciminibus meis femora dentata funt, dente valido. Hg.

307. C. Cerafi? longiroftris, fomoribus dentatis, ater, elytris opacis, oblongis. F. Sp. I ?9. 104.

$\odot$ Geoffr. r. 299. 48., Frifch.

D Geoffi. Int. I. 288. 23? Schrink. E. I. 204. 
Totus ater; elytra ftriis decem. Pedes fimplices. Hab. in Pruno Cerafo. Tong. x. lat, $\frac{1}{3}$.

Auctorem verum $C$. Cercfi. $F$. ignoraffe, ex citatis et deferiptione additis fatis apparet. Verus enim C. Cerafi. F. in Paijkullii Monogr. Curc. 3. 7 . bene defcriptus eft. Nullo modo autem hic idem cum Linnaeano videtur, cum femcra mutica, imo defcriptio tota, ab illo commemorata, abhorreant. Auctoris Curculio idem cum C. Cerafi Schrankii videtur. Vterque Geoffroyum citat, cuius Choranfon Satin - gris equidem in Antirrhini Linariae. L. floribus reperi. Idemque a $D$. de Paykult nomine C. Antivrlinin ' ' $p$. mihi miffus eft.

Iure autem Auctori exprobrari licet, quo modo Curculionem fuum eundem cam Fabriciano credere potuit, cum nec familiae, nec defcriptionis characteres conueniant? At Fabricius Linnaeum eodem modo citat. Sed errores, quos Celeberrimus quisque committere poteft, haud augere fas eft. $\mathrm{Hg}$.

\section{(p. 120.)}

* 308. C. Faber longiroftris, oblongus, muticus, elytris caftaneo-nigris fulcatis, thorace plano, obfcuriore.

Totus fubniger. Roftrum elongatum, tenue, deflexum. Antennae fractae. Thorax planus, niger, vix punctatus. Elytra fufca, profunde ftriata, abdomine paullo bretiora. Tibiae fubferrugineae. Hab. in pratis fluaticis. Long. $3 \frac{\dot{1}}{2}$. lat. $\frac{T}{3}$.

* 309. C. vilis longiroftris', cylindricus, obfcurus, niger, roftro fubcarinato, elytris punctato - friatis. 
Fufco-niger, muticus. Antennae breues, fractae. Roftum craffiufculum fubcarinatum, longitudine thoracis. Elytra obtufa. Femora antica crafliora. Hab. in hortis incultis. Long. 3 . lat. $\frac{2}{3}$.

* Longiroftres, femoribus dentatis.

310. C. Scrophulariae longiroftris, femoribus dentatis, coleoptris maculis duabus dorfalibus atris. F. Sp. I77. 95.

$\odot$ Lif.

\& F. E. S. I. 2. $434 \cdot 167$.

Etfi vere niger fit, totus grifeus apparet, eo, quod tomento cinereo - virefcenti contectus.

Habitat in Scrophularis, faepe etiam in Verbafco Thapfo. Larua mollis, veluti gutta gluti'nis metamorphofin fibitura, in foliis confiftit, gluten exficcatur, fit folliculus, deindeque fpatio vnius hebdomadis, Curculio exit declaratus. Videtur Limaeus vidiffe capfinlan iam formatam. In Verbafco habitat Curculio fmilis praccedenti, at magis fufco-grifeus, immaculatus, tomento non adeo deprefio, thorace latiore, roftro nigro, quod non inflectit fub ventrein viti prior: corte noua fpecies, quae differt etiam marnitudine dupla maiore, nifi forma et colore a Curculione habitare folito in floribus Antirhini Linariae. Long. 2. lat. $I \frac{1}{3}$.

Dubium adhuc remanet, num C. affimitis Harrer. Defcr. Schaeff. Ic. tab. 232. f. 8. pag. 184.299., qui etian C. Thepfus F. E. S. I. 2. 434. I68. et Geofir. Inf. I. 297 . videtur, varietas tantum $C$. Sirophnelariae fit? Detritis pilis pollinofis, num- 
quam tamen eandem figuram $C$. Scrophularicie repraefentare mihi vifus eft. Sed alium dillerentia charaterem detegere nequeo. Hinc cundem putare licet. Huius certe fidem do, me $C$. affinilem migna copia in floribus Verbaci Thapfi reperilie, nullo autem fpecimine $C$. Scrophularlae immilto. - Id atem mihi perfuafum habeo, Fabricii C. Verbafci, 1. c. I69. nil nifi varietatem, ftepius occurrentem, $C$. Scroplublirice effe.

Auctor nouae et incognitae fpeciei mentionem facit, quam cur feparato loco, et nomine nouo indito, haud exhibuerit, ignoro. Ipfe in floribus Verbafii nigri Curculionem, his commemoratis finilem, fed diftinctum, reperi, quem paucis verbis indicandi, locus hicce aptus vilus eft. $C$. Setofin vucaui, et in haec verba fyftemati inferi pofie putaui: C. longiroftris, femoribus dentatis, obfurus, hirfutus; elytris friatis: interftitiis fetis fafciculatis. Magnitudine C. Blattariae F. circiter aequat. Hg.

\section{(ti. $12 \pi$.}

$3^{r}$ r. C. Abietis longrofris, femoribus dentatis, elvt... fufcis, fafciis duabus linearibus interuptis abidis. F. Sp. 175. 84.

(C) Linn. It., Petiv.

Schrank. E. I. 2 I $\mathrm{r}$.

F. E. S. I. 2. 428 . I 44 .

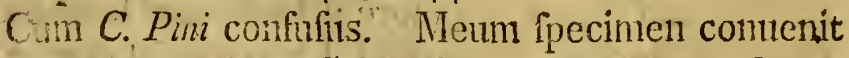
cum decriptione Schrankii 1. c. et cum figura Schazfi. Ic. t. 25. f. I. Spinae apicis tibiarum incrise character a Schrankio in hoc Curculione ntatus, communis eft etiam aliis. Lectus in arbore trunca cariofa. Long 5 . lat. $2 \frac{x}{4}$.

Vide adnotata mea ad C. Pini fupra n. 299. Variat culore teftaceo-brunneo. $\mathrm{Hg}$. 


\section{(p. 222.)}

* 3r2. C. fatuus longiroftris, femoribus dentatis, feringineo-furcus, elytris punctato-ftriatis, atomis lparlis cinereis.

Paullo minor praecedenti. Totus ferrugineofufcus, glaber, fubnitidus. Roftrum apice incraffatum thorace paullo longius: Antennae fractae fufcre claula fubcinerea. Thorax rotundatus punCtis clenatis fiaber. Elytra ftriato - punctata punctis ad lentem rufo-tomentofis fplendidis, atomisque nunc cinereis, modo flauis alperla; in fingulo nonem antice, pofticeque partitis. Pedes ferruginci lemoribus ommibus apice nigris, clauatis, dentatis, tibiisque fubdentatis fpina apicis incurua. Elytrorum apex nomnihil angutatus.

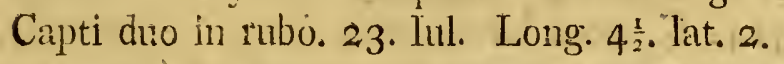

* 3 I 3 C. Statua longiroftris, fenioribus tibiisque dentatis, ater, fcutello, fafciaque elytrorum poltica albis.

Singularis prae caeteris omnibus noftratibus. Fufco - niger. Roftrum fubferrugineum, bafi et apice nigrum. Antennae fractae, brenes, nigrae. Thorax punctis eleuatis numerofis fcaber, niger, atonis aliquot indiftinctis albis. Loco fcutelli punctum albidum. Elytra punctato-ftriata, triis prominulis acutis, apiceque obtufa, atra, fed ad bafu vinque macula fubfcrruginea, punctisque binis (quae non femper adfint,) in medio contiguis, et pone fafcia transuerfa alba marginem non attingente. Pedes omnes fufci, cinereonigroque fafciati. Femora acute dentata, ad bafin attenuata, ad apicem craffiora, incurua, ac 
veluti exarticulata. Tibiae omnes latere interiore dentatac, 'breues, ' compreflac, pofticac etian incurnatae, et exterius gibbae. Pectus profimide canaliculatum, pro receptione capitis, et roftri. Abdonnen fubtus fordide flanefcens. Accedere videtur ad C. ftolidum. F. Sp. 176. S9. Infectum timidifimae indolis. Vtcumque tactum motu timorem exprimente, contractis ad femora tibiis mortuum le effingit, immoilitatem induens fingularem, quam non deferit, nifi elapfo temporis fpatio non breni. Acu transfixum ftridet agitando abdominis apicem, attritu elytrorum ad anum. Tab. V. f. 4. 8. Capti tres inter corticem et lignum quercus in filuis d. 9. Mart. Long. $3 \frac{1}{4}$. lat. I.

\section{(p. 123.)}

314. C. ancum longiroftris, femoribus dentatis, corpore grifeo, longitudine roitri. F. Sp. I 79. Io6.

( ) Vdin., Roef., Schaeff. Elem., Sulz.

D Scop. E. C. I05. Schrank. E. I. 2I 3.

온. E. S. I. 2. 440.192.

Specimen vnum habeo exiniae magnitudinis, hoc eft longum 4. lin. latum 2. 1. Antennae lincis 5 . longiores, articulis nouem, computata clami, fcutello minime albo, elytris concolori: affinitatem C. probofcidei forte confirmat. Hab. larua in Nucibus Anellanis. Long. $2 \frac{2}{3}$. lat. $I_{\frac{1}{4}}$.

Infectum, quod ad magnitudinem, roftri longitıdinem, atque colorem attinet, mire variabile. Citatum e Sulzeri Hiftoria falfum eft. Forfan aliud opus eiusdem Auctoris fpectat Fabricius. Hg.

$$
\text { (p. 124.) }
$$

315. C. tortrix longiroftris, femoribus dentatis, cor- 
corpore teftaceo, pectore fufco. F. Sp. I8I. IIf.

운. F. E. S. I. $2.444 \cdot 2 \times \mathrm{r}$.

Flano - teftaccus, pectore fifen, oculis nigris, ely, tris fitriatis, roftro temui longiufculo. Hab. in Po. pulo tremula, folia contorquens. Long. $2 \frac{1}{4}$. lat. $\frac{2}{3}$.

316. C. druparun longiroftris, femoribus dentatis, clytris tefaceis, obfolcte fafciatis. F. Sp. ISI. II 3 .

○ Sulz.

\& F. F. S. I. 2. 443.205 .

Hab. in Cerafi Padi nucleis. Long. 2. lat. $\frac{2}{3}$.

- De citato Sulzeri rid. Adn. ad 3 I 4. Hg.

* 3 г - C. fimo/us longiroftris, femoribus dentatis, thorace breni rotmudo, elytris grifeis nigronebricfis.

Statura C. toitricis, at paullo maior. Roftruni elongatum, tenue, deflexum. Antemnae ferruginene. Thorax fulcus. Elytra fubftriata, grifea, nigris obfolctis maculis variegatta. Hab. in aruis. Long. $2_{4}^{3}$. lit. ${ }_{4}^{3}$.

\section{(p. $x=5$.}

* 3 I8. C. cinctus longirottis, femoribus dentatis, niger, thorace poltice fuluo-marginatò.

Totus niger glaber, thorace excepto poftice fuluo-marginato. Elytra ftriato-punctata. Lechus fub arbonum cortice. Long. $I \frac{2}{2}$. lat. $\frac{I}{2}$.

319. C. violaceus longiroftris, femoribus dentatis, violaceus totus. F. Sp. I79. 105 .

$\odot$ Bergftr.

\& F. E.S. I. 2. 440 . I9I.

Elytra punctis excaulatis ftriata. Hab. in Pinu. Long. 2. lat. $\frac{1}{2}$. 
- Statura tota C. Cerafi. F., hinc verus Curculio. Citatum e Geoffroyo, ab Auctore cum Fabricio exhibitum, fupra iam ad C. Alliaviae, n. 289. relatum videmus. Certe veru Rhynchiti, nec Curculioni annumerandum eft. $\mathrm{Hg}$.

\section{* * Longiroftres femoribus pofticis incraffatis filtatoriis.}

320 C. Salicis longiroftris, pedibus faltatoriis, elytris atris, fafciis duabus albis. F.Sp. 183.125.

of F. E. S. I. 2. $445 \cdot 216$.

Elytra fafcia latiori alba vndata ad bafin, et alia in medio lineari transuerfa. Hab. in Salicis floribus. Long. $\frac{x}{3}$. lat. $\frac{1}{4}$.

\section{(p.126.)}

321. C. Alni longiroftris, pedibus faltatoriis niger, colcoptris teftaceis, maculis duabus obfcuris. F. Sp. I83. I23.

온 F. E. S. I. 2. $445 \cdot 216$.

Saltator. Rufa funt thorax, elytra, antennae, pedes. Nigra: caput, roftrum, pectus, tibiarum apices. Elytra ftriata maculis quatuor nigris; in fingulo elytro duae, quarum poftica multa maior. Hab. in Alno et Vlmo. Long. $1 \frac{x}{3}$. lat. $\frac{1}{2}$.

322. C. viminalis longiroftris, pedibus faltatoriis, corpoic teltaceo. F. Sp. 184. 126.

? F. E. S. I. 2. 447.223.

C. rufus. Schrank. E. I. 220.

Roftrum tenue, thorace longius. Elytra punctatoftriata, femoribus pofticis incraflatis fpinofis. Hab. in Salice, Qnercu, Vlmo. Long. $\frac{1}{2}$. lat. $\frac{x}{3}$.

Omnino dinerfus a praecedenti eft, cuius magnitudinem aequat. Citatum e Linnaeo C. Quercus. a 
Fabricio et Auctore huc relatum, alium defignat Curculionem, nec vllo modo hic pertinet. Fabricius quoque iterum in Entom. Syftem. apud $C$. Quevils fuum n. 79. allegat, vbi pro $L$. $S y j$. Nat. 614. legatur 609. $25 . \mathrm{Hg}$.

**** Breuiroftres, femoribus muticis.

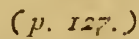

323. C. Barbarus breunoltris, thorace fubfipinolo, elytris angulo duplici crifiato. F. Sp. I94. I0I.

S Brathycerts Barbarus. F. E. S. T. 2. 38 I. S. Nlas liger, femina cincrea. Oculi a capfula e capite pruminente marginati. Pupillae parum conuexac. Hab. in locis arenofis frequens. Long. 4, 4\% lat. 2, 3 .

324. C. mbulofus breniroftris, canus, elytris falcis oblumis nigtis. F.S. 186. 142.

( ) Iritih., Schatf. Ic. t. 25. f. 3 .

D C. iners. Scop. E. C. 63. Schaeff. Ic. t. 25. f. 9. 온. E. S. 1. 2. $457 \cdot 265$.

Cincresis. Ruftrum rufum fulcis duobus in medio carinam habentibus. Ocnlorum cuafi palpebrae flauae. Thorax rugis cleuatis longitudinalibus pallidioribus quinque. Elytra albida maculis quibuscam, filciisque oblique trunsueris obfurioribus diabus, punctatoltriata. Subtus cinereus. Hab. in campis. Long. $5 \frac{1}{2}$ lat. $2 \frac{2}{3}$.

325 . C. fulciroflris brcuiroltris, oblongus, cinerens, lubnebulolis, roltro triinlcato. F.Sp. $18 \%$ 143.

D Degeer. Inf. 5. 240. 26. Schrank. E. I. 228. Schaeff. Ic. t. 25. f. 3. 10. t. 163. f. I. 
9 F. E S. I. 2. 453.268

Knoch. Beytr. I. t. 6. f. 8-II. icones cesarbtiffimas.

Maior C. nebulofo. Roftrum trifulcatum. Hab. in Caricibus. Long. 6. lat. 3 .

\section{(p. 128.$)$}

* 326. C. ophthalmhirus breniroftris, oblongus, cincreo-nigiogue varius, elytris punceato-1triatis, dorfo macula vtrinque difincta cinerea ocellata.

Statura C.fulciroffris, fed maior. Roftrum breue, crafium, bifulcatum, fulcis grifco-cinereis. Antemnae apice cinercac. Thorax planus cinereofufcus. Elytra coadunata, fere coalita, punctis minutifimis ftriata, cinerea, praefertim ad bafin: pone medium in dorfo punctum vtrinque maiufulum diftinctum, cincreum, ocellatum, alterum. yue ad apicem minutum. Corpus nigrum tomento fordide cincreo tectum. Femora antica clanata, exterius cinerea, intene nigra. Tibide, tarfiqne cinerej. Mas nigrior eft, led color magis ninusue cinereus $a b$ aetate pendet. Tab. V. f. I $\approx$. mas. Tab. I. f. 12. fim. Hab. in pratis filuaticis arenolis humentibis frequens. Long. 7. lat. $3 \frac{1}{*}$.

Hinc inde in Germaniae mufeis fub nomine $C$. occhotus Schmederi occurit. Ipfe ex Italia mifum accepi. Statura crafia. Hg.

* 327. C. affinis breuiroftris, cblongus, niger, elytris cinereo - variis, roftri carina bifurca. Schrank. E. I. 230 . Schiaefi. Ic. t. 34 . f. $\%$.

8. C. clbidus. F. E. S. I. 2.460 .275

Totus liiger, fed thorax lateribus, et elytra per totwim cinereo-variegata. Roftrum fulcis duobus. Thorax 
Thnrax punctis elenatis confluentibus fcaber. Elytia fingila ltris decem ex punctis. Hab. in filuis. Long. 4. lat. $1 \frac{1}{3}$.

328. C. virilis breniroftris, virefcens, thoracis, elverumgue latcribus flauis. F. Sp. 185. I39.

$\odot$ Suiz.

D Pod. Muf. Gr. 30. 1 r. Schrank. 233.

\& F. E.S. I. 2.454 .254 . Schaef. Ic. t. 7 6. f. 1.2.3. Dilute viridis, non nitens, frriatus. Subtus flauoviridis. Hab. in filuis et hortis. Long.4\% lat. 2. Antemae fubrectae funt. Variat elytris fufcis margine flauis. $\mathrm{Hg}$.

\section{(p. 329.$)$}

* 329. C. veresundus breuiroftris, fupra teftaceoruftis, fubtus viridiargenteus, thorace lineis tribus aurintiacis.

Oblonglis. Caput rufum roftro breninimo, crilli, excaunto, antennis fractis, teftaceis, apice nigitis. Thorax rufus lineis longitudinalibus aurantiacis in medio, et lateraliter notatus. Elytra et pedes rufa. Abdomen fubtus colore riridi -argenteo, femoribus anticis craflioribus. Hab. in aridis incultis. Long. $I \frac{1}{2}$. lat. $\frac{x}{2}$.

330. C. grifeils breuiroftris, fupra grifeo-fufcus, fubtus cinereus, roltro canaliculato. F. Sp. I 88. 152.

P.F. E. S. I. 2. $464 \cdot 292$. Statura $C$. lineati, at paullo longior. Roftum cylindricum fulco longitudinali excauatum. In thurace lineola alba dorlalis a roftri bafi duzta ad foutelum. Fenora fimplicia. Hab. in hortis. Loing. 3. lat. I.

$$
I 5-331 \text {. }
$$


33 r. C. lineatus breniroftris, fufcus, thorace friis tribus pallidioribus. F. Sp. I89. 155.

D Schrank. E. I. 242.

S F. E. S. I. 2. 466.302.

Hab. in variis plantis. Long. $I \frac{1}{2}$. lat. $\frac{r}{2}$.

$$
\text { (p. 130.) }
$$

* 332. C. C. nigrum breniroftris, cinereo, nigroque varius, fingulo elytro lateraliter facia femicirculari alba, ponticeque lumula nigra,

Onatus. Roftrum craflium, breue, deflexum. Elytra $C$. nigro-notata, et punctis nigris afperfa, ftriis interioctis altemis. Pedes nigro-fafciati. Femora mutica. Videtur diuerfus a $C$. luiato $F$. Hab. in locis paludofis non fequens. Long. 3. lat. $\mathrm{I}_{3}^{\frac{2}{3}}$.

Secundum defcriptionem vix C. Innato. F. fimilis efle. poteft. $\mathrm{Hg}$.

333. C. hiffuidulus breniroftris fufcus, thorace cinereo lincato, elytris hifpidis, punctis obicurioribus ftriatis. E. Sp. 189.158.

SF.E.S. I. 2. 468. 3 Ir.

Thorax in noftro fpecimine niger, fortaffe attritu fquamilis villofis fpoliatus. Hibit. in fepibus. Long. 2 \%. lat. 1 .

334. C. Aabriculus brenirotris, corpore cincreo hifpicio. F. Sp. Is9. I $5 \%$.

S. F. E. S. I. 2. 469. 3 3 . Herbft. Airch 8. p. I6?. t. +5. f. ?.

Totus cincreus. Thorax punctis elcuatis faber. Elytra friata hifpida. Habit. in locis fabulofis. Lons. 3. lat. I:

C. Vinerra. Herbft. Arclis 4. p. 83. 27. idem videtur, 
tur, quamquam C. fcabriculus, eodem loco iterum 1. c. exhibitus, et bene depistus fit. Hg.

\section{(p.ris.)}

335. C. punctatus breuiroftris fufcus, elytris punctis hololericeis, eleuatis, margineque flauo. F. Sp. I 90.166.

D Schaefi. Ic. t. 25, f. 6.

안. E. S I. 2.472 .329 .

Ouatus. Roftrum breuifimum. Thorax glaber. Elytra Itriato - punctata, punctis elenatis holofericeis atris, albisque alternis adiperfa. Habit. in filuis non trequens. Long. 3 ?. lat. 2 .

Schaefleri figura, ab Auctore cum Harrero citata, nullo modo cum hoc Curculione conpenit. $\mathrm{Hg}$.

336. C. puncuatulus breuroftris, niger, elytris punctis nigris albisque altemis holofericeis ftriatis. F. Sp. I87. 146.

Habitat ruri.

Vide adnotata ad C.curnirofircm 300 . Hicce Punfiatulus enim Terrae Nouae Americes indigenus eft.

$\mathrm{Hg}$.

* 337. C. Senex. fufcus, apterus, elytris rugrifo-ftriatis. Geofir. lnf. 1.293.37. Le charanfon Cartifunne.

Breniroftris, fupra obfcurus, fubtus ater, thorace amplo fubcarinato, elytris lineis, et puntis elcuatis feabris.

Reftrum brene, craftum, apice dilatatum, antemnis fractis fufcis, et veluti ab ore deriuatis. Thorax planus humeris rotundatis, in medio linea eleuara dorfali, punctis paffim confluentibus $\mathrm{fca}$ bor. Elytro fingulo lineis tribus eleuatis puncisque prominulis exáfperato. Abdomen fubtus nigrum 
nigrum, quod magnam partem elytra tegunt connata, et poftice acuminata femoribus muticis fubclauatis. Alae nullice. Variat magnitudine. Hab. in: Pinetis non rarns. Long. $4 \%$. lat. 2.

\section{(j). 132.$)$}

338. C. afperfus? breniroftris, ater, elytris politice albo-maculatis F. Sp. I90. I65?

Ouatus. Reftrum breue, obtufum. Elytra crenato-ftriata, pofice inprinis albo-maculata, nacula magna triangulari vtrinque ad nharginem. Habit. in filuis. Long. $I_{*}^{\prime}$. lat. $\frac{2}{3}$.

Vide adnotata ad $C$. catrivioftren. 300. Ho.

339. C. latiroftris brcinofuis, roftro latifimo, plano, elytris apice albis, punctis cinobus nigris. F. Sp. I 0 O. IS I. Mant. 118.237.

of Anthribus latirofiris. F. E. S. 1. 2.376 .2 .

e. Violaceus, ater. Caput fupra cincrenur ct veluti limo exiccato obtecum, apice niggrum, planum, conprefum, clongatm, naxillis validis. Antennae rofto infientes clumatae, clana ex articulis tribus compofiza. Oculi prominuli nigri punctis certa luce, et ad lunen candelac nicantibus, etian in nortuo. Thoms astice et joftice angutatus, dorfo fcabro concaro, cminentia laterili. Elyta fufco-violacea fafcis tribus uleginter cinereo - pietis, et nigto mitrgine laciniatis, apice cincrea, punctis diobus nigris 10n fumer pracentibus. Abdomen fubtus cinereum lateribus nigris. Pedes nigri cincreo-ammlati tarfis tribus fpongiofis. Alac albac. Tab. V. .

B. Variat interdum, fed rarius corpore toto nigro elytrorum tantiun apice cincico; varietas a Geoffroyo obicuata.

Habi- 
Habitat in Fraxino et Alno cmortua non rarus menif. lamiar. C. Albinus mumquam inuentus. Long. 5. lat. $2 \frac{\pi}{4}$.

Varietas $\beta$. vberius occurrit, quam fpecies $\alpha$. ipfit. Inter fpccimina mea vnum tantum obferuo, hic defcripto conuenians, capite et roftro fupra pilis grifeis obtecto, elytrisque fafciis tribns obfulatis apiceque grifeo-pilofis. At color in mortuo minime violaceus, fed fufco-holofericeus eft. Pedes annulis multis cinereis vndulatis, anguftis.

Fabricins hunc et fimiles in feparatum genus bene transtulit, cui nomen, a Geoffroyo vitatum, Anthribus dedit. Sed ifte etiam illos Curculiones, quos fub generc Atteliabi nunc habet Celeberrimus Fabricius, ibi coniungit. Hinc nomen characterifticum Platyfiomos (a miarv' latum et $50 \mu \dot{s}^{\prime}$, os), a me antea huic generi inditum, magis placet. $\mathrm{Hg}$.

***** Breuirofires femoribus dentatis.

$$
\text { (y. I } 33 .)
$$

370. C. Nigrita bieuiroftris, femoribus dentatis, niger, obfcurus, thorace fcabro, elytris crenato-ftriatis. T. Sp. I9?.211.

S. F. E.S. I. $2.485 \cdot 38 \mathrm{r}$.

Corpus onato-globofun. Antennac articulo primo praclongo. Elytra cum fint in foueolis cirereotomentola, apparent pulucrulenta. Hab. in filuis fiequens, Long. $+\frac{1}{2}$. lat. $=$ T.

37r. C. Pyri breuiroftris femoribus dentatis, acneo - fufcus. F. Sip. 193. 2 I \%

$\odot$ Linn. It., Sulz.

F F. E.S. I. $2.487 \cdot 390$.

Oblongus. Roftrun brene, defexum. Thorax punctatus. Elytra crenato-friata, aenco-fuica, pedi- 
pedibus antennisque rufis. Variat pedibus nigris. Femina mafculo fure duplo maior, et magis nitens. Hab. in Pyro. Long. 4. lat. I

- Citatum Schaeft. Ic. t. 2. II. ad mubilum pertinens,

Fabricius in Ent. Sy/t. bene umifit. Caetcrum equidem magnitudinem conftantem ferme eandem, nec tanto, ve Auctor dicit, difcrimine variantem cbferuaui. Sufpicari licet, Ainctorem ant alium pro vero C. pyri habrifie, aut plures fpecies confudifie, cum eiusmodi viridium Curculiopum magna copia c:ftet, primo intuitu fimilium, fed fi attentiore oculo luftraueris, valde diuerfuorum. $\mathrm{Hg}$.

342. C. oblongus bretiroftris, femoribus dentatis, antemis elytris pedibusque fermgineis. $F$. Sp. 199.220.

D) C. pruni Scop. E. C. 95. Sclurank. E. I. 224 Schaefl. Ic. t. 563 . f. 6.

오. F. E. S. I. 2. $489 \cdot 400$.

$\mathrm{Hab}$. intra corticem et lignum. Long. ${ }^{t}$.

Wire, variat. Varietates a me vifie funt féquentes: $\alpha$. totus niger, pedibus antennisque pallidis, flauoluteis. B. niger, antennis pedibusque flaucicentibus, elytris cultaneis: margine exteriore fuperius nigro. $\gamma$. omnia eadem, fed elytra pedibus concolora pallide teftacea, margine illo nigro anguftiore. $\delta$...- margine nirgro deficiente. $\varepsilon$. totus fubcaftineus; quae varietas iam a Geoffroyo obferuata eft.

$\mathrm{Hg}$.

\section{(p. 134.)}

343. C. ouatus breniroitris, femoribus dentatis, corpore nigro, pedibus antemisque rufis. F. Sp. 199. 22 I.

\& F. E. S. I. 2. 490, 402. Herbft. Arch. t. 24. f. 36 . 
Roftrum brenifimum. Antennac longiufculate, fractac. Abdomen valde contuexum. Habit. in hortis frequens. Long. $2 \frac{3}{3}$. lat. $\mathrm{I}_{2}^{\frac{1}{2}}$.

344. Co argentutuls brenirofuis, femoribus dentatis, corpore viridi-argenteo. F.Sp. 198.218.

$\odot$ Sulz.

\& F. E. S. I. $2.489 \cdot 399$.

a. Variat pelibus antennisque rufis. C. argyreus. F. Poll. W. G. p. 30.

ß. Colore vinidi argenteo.

$\gamma$. Colore viridi aurato. Scop. E. C. gr. Schrank. E. 1. 223 . Schiaft. Io. t. I zo. f. I.

Hab. in varis jlantis, frecrens in Iafmino officinali, et in Populo trumta. Long. $2^{3}$. lat. 3.

Conferitur adnúatum al $C$. pyrri. n. 34 I. Hg.

345. C. nubilus brenirofris, femoribus dentatis, grifeus, elytris punctis obictinioribus nunerofis. F. Sp. 197.210?

q F. E. S. I. 2. $485 \cdot 380$.

Grifeus, corpore onato-globofo. Roftrum brene, apice non incrafiato. Elytra cronato - ftriata, punctis muncrofis obfeuris afpera, et magnam abdominis partem fubtus tegentia. Femora dentata. Hab. in filuis. Long. 3\%. lat. 2.

\section{(p., 555 :)}

* 346. C. Fullo breuirottris, femoribus omibus dentatis, elytris nigris aibopunctatis. Sciurank E. J. 22 I.

Abdomen ouatum. Color niger, fed elytra albo macnlata, vti in Sc.Fullone, etiam thorax, fed punctis mininis. Pedes fufci, femora omnia dentata, primi paris clauata. Roftrum valde breue, craffiml.

Differt 
Differt a $C$. gemmato Scop. et Fizbr. antennis breuiffinis non villofis, et roftro apice non dilatato. Habit. in locis filuaticis non rartis. Long. $3 \frac{\pi}{2}$. lat. $I_{\frac{1}{2}}$.

\section{XLT. RHINOMACER.}

Palpi quatuor extrorfum crafliores, articulo vitimo truncato.' Antennae fetaceae roftro infidentes.

* 347. R. Brentoides glaber, piceus, thorace punctato, elytris punctato-ftriatis. D. D.Antic. Soc. 'Linn.

P. Pimelia anguftatc. F. E. S. I. I. Í5. 32. Inlectum apud nos frequens. A me an. If89. cum aliis multis raris et indefcriptis transminium Parifios ad D. D'Antic., qui refpondit, hoc habitare etiam Monfpeliae, et defiriptionen aftibre facuin conceffit, quae fequens cft:

Patullo maiór C. gronario. Caput ellipticim, deprefium, thorace paullo mains, punctatum, fubmarginatum, fubuillofum, piceum. Oculi nigri -nedio pofiti. Antennae monilifornes, crafae, vix capite longiores, hirtae, nigrae, ante (ct fubtus oculos pofitae. Palpi fermginei. Thoras elongatus, marginatus, punciatus, glabci, piceus, paullo minor capite. Elytra elliptica commta, margine reflexa, punctato-itriata, pical. Abdo. men piccum. Pedes picei. Tarfi villofi articulis quatuor?, Tab. III. f. I4. "Hub. fub arbwinn cortice. Long. I $\frac{2}{3}$. lat. $\frac{1}{3}$.

Specimen meun longum eft $2 !$. lin. eiustem iconis longitudinis. Fabricius inter Pimeliss loc inlictum retulit. 
rétulit, quod tamen minime eandem ftaturam gerere videtur. Forte plures, huic fimiles, nouum genus conftituere poffent. $\mathrm{Hg}$.

\section{( $p ., 3,6$. \\ XLII. ATTELABVS.}

Palpi filiformes. Maxilla bifida. Labium corneum palpos tegens. Antennae moniliformes infidentes roitro.

De hoc genere vide quae fupra dicta funt ad $C$. Bachum 286 . $\mathrm{Hg}$.

348. A. Coryli niger, elytris rufis, reticulatis. F. Sp. I 99. I.

$\odot$ Linn. It., Sulz., Pontop., Schaeff. Ic. t. 75. f. 8. f. F.E. S. I. 2. 384. I.

Defcriptio Linuaei in Fn. Sv. 638. optima. In meis fpeciminibus tamen femora femper rubra. Hab. in Coryli foliis, quae inuoluit in cylindrum vtrinque claufum. Long. $3 \frac{1}{2}$. lat. $I_{3}^{2}$.

Ille, quem vulgo $A$. Coryli $L$ putamus, minime verus eft, cum thorax totus ruber fit, quem Linnaeus in $A$. Coryli antice atrum, uti caput, poftice vero rubrum, ut elytra, commemorat. Porro femora nigre dicit. D. de Paykull fub nomine C. Auellanae verum $A$. Coryli $L$. defcribit. A. Coryli, vulgo fic diEtus, autem C. Auellance Linn. Syft. Nat. p. 6 Ig. n. I. eft, vbi id tantum adnotandum, qnod pro thorax et pedes exceptis geniculis nigva aperte legendum eft rubri. Deinde Linnaeus ibidem $\boldsymbol{C}$. collarem Scop., qui ad C. Auellanae referatur, et Sulz. Hift. t. 4. f. I2. quod citatum ad $A$. Curctlionö̈dem pertinet, perperam citat. Sed vtrum $A$. Coryli $L$. diffincta fpecies, an varietas fola fit, equidem difcernere non audeo. In omnibus enim 
fpeciminibus meis femora fanguinea, bafi apiceque rubra funt, etiam in varietate quadam, vbi thorax futura antica et macula dorfali cum futura, cohaerente nigris, notatus eft. Caeterum variat: totus pallide teitaceus, vbi ruber efle folet, interdum capite infuper rufefcente. $\mathrm{Hg}$.

349. A. Currulionoïdes niger, thorace elytrisque rubris. F. Sp. 200. 3 .

$\odot$ Sulz.

D Schrani. E. I. I93.

Q. F. E. S. I. 2. 386 . I 2.

Totus niger, thorace, et elytris rubris. Habit. in Corylo, Salice et Alno frequens. Long. 3. lat. $\frac{1}{2}$.

Variat pallide teftaceus, vbi ruber effe folet. Nouam et quafi intermediam inter $A$. Auellanae $L$. et Curculionoïdem fpeciem brenibus verbis indicare iuuat, cui nomen $A$. intermedius dedi, et ita diftinxi: A. niger, elytris rubris, nitidis, punctato - friatis. Statura A. Auellanab, at duplo minor. Totns niger, nitidus, elytris folis rubris, nitidis, punctatoftriatis, ut in $A$. Coryli $F$. nec obfcure ftriato- punctatis, vti in $A$. Curculionö̈de. Species plane diftineta, quam tamen Fabricius in adnotato ad $A$. Curculionoïdem l. c. indicaturus videtur. Habitat in Corylo. Hg.

\section{(p.137.)}

350. A. pubefcens violaceus, hirtus, roftro atro. F. Sp. 200. 5 .

. F. E. S. I. 2. $387 \cdot 14$.

Fucies C. Bachi et Betulae, fed paullo minor. Rofiru longitudine thoracis, atrum, fulcatum. Thorax cylindricus, gibbus. Elytra violacea, punctatu-friata, pilis fufcis hirta. Hab. in plantis non frequens. Long. $\mathrm{I}_{2}^{\frac{1}{2}}$ lat. $\frac{3}{4}$. 


\section{CLER VS.}

Palpi quatuor, anteriores filiformes, pofteriores longiores fecuriformes. Antennate monili-, formes.

35 I. C. formicarius niger, elytris fafcia duplici alba. F. Sp. 20 I. 4.

$\odot$ Ray., Sulz.

D Attelabus formicarins. Scop. E. C. IIт. Dermeftes formicarius. Schirank. E. I. 35. Schaeff. Ic. t. 186. f. 4. ' E'em. 137.

o F. E. S. I. 2. 207. 5. Panz. F. I. G. IV. 8. Ruber, capite, thoracisque antica parte nigris, elytris nigris bafi rubra, poftquam. interiecto fpatio "nigro fafcia alba, tum fpatium alterum nigrum maius, et alia fafcia alba vltra elytrorum mediunn. Pedes colore variant, modo tcti mubri, modo femoribus tibiisque nigris. Hab. vetuftas arbores; innentus etiam in Alno. Long." 4. lat. $1 \frac{\mathrm{T}}{2}$.

\section{(p. $\left.{ }^{2} 38.\right)$}

352. C. vnifuficiatus niger, elytris fafcia alba bafi rubris. F. M. 125.8 .

Niger, nitens, thorace nigro, elytris bafi rubris, apice nigris, in medio fafcia vnica alba.

I F. E. S. I. 2.207 .8 .

Similis C. formicario, fed minor, et elegantior. Caput et thorax nigra, villofa. Thorax pottice angufitatus. Elytra ftriata, bafi rubra, ante medium vsque ad apicem nigra. Fafcia alba tranfit per nigredinem. Subtus totus niger. Inuentus in arbore trunca 111. Septembris. Long. 3. lat. $\frac{\mathrm{T}}{2}$.

353. C. apiarins elytris rubris, fafciis tribus cacrulefcentibus. F. Sp: 202.9. 
$\odot$ Sulz.

D Reaum. Inf. 6. t. 8. f. 9. Io. Scop. E. C. IIo. Schrank. E. I. 36.

․ F. E. S. I. 2. 208: I4.

Trichodes apiarius. Herbft. K.IV. $x_{56}$. I. t. 4 T.

f. $x \mathrm{I}$.

Si vera eft opinio ingeniofa Cl. Schrankii de laruae domicilio, quod Schaefforus ponit in aluearibus Apum, ille vero in floribus; cur numquam laruae ab ouis, exclufae hofitantes in floribus reperiuntur? - Hab. paffin in tloribus. Lung. max. 6. 1at. 2 .

-Duae ab auctoribus confufae funt fpecies probe difinguendae, quas Clariff. Fabricius'bene feparatas babet, $C$. apiarium. L. et aluearinm. Ad hunc pofteriorem citata pertinent fequentia: Geofli., Inl. I. 304. 1. vbi defcriptio optima eft, in figura autem t. 5. f. 4 . fafcia poftica elytrormm caerulea haud, diftincta eft, fed vt in apiavio apicem tegit, absque margine rubrc, id, quod omnino vitic chal. cographico irrepfit. Porro: Schaeff. Elem. t. 46. Icon. t. 48. f. II. Haud bene itaque Fabricius Geoffroyum ad ambos refert Cleros, cum ad $C$. ath nearium folum pertineat. Clariflimus Herbft. eandem adhuc putauit fpeciem. Sunt quidem primo intuitu fimiles, fed differentiae fatis reftat. Thorax in $C$. alueario longior, pone minus anguftatus; obfcurior, magis conuexo-punctatus, pilis, etiam capitis, longioribus ac denfioribus. Antennie vnicolores. nigrae; in apicirio bafi rufae funt. Elytra in alueario profundius punctata, numquam nitida funt, fafciaque prima pone bafin, a margine exteriore oblique defcendit, ad futuram declinans; fafcia tertia et vltima angufta eft, nec marginem attingit. Apex elytrorum ruber; inde cum rubedine 
dine fpatii inter fufciam z. ct 3. cohaeret; in topia*io apex femper cyaneo-niger eft. C. apiarius in regionibus noftris rulgaris, in floribus occurrit, fed C. aluetrium nunquari repréhendi. Hinc habitatio mihi-ignota eft. Quem itaque habuerit auEtor, extricare nequeo, cum deforiptionem haud adiecit. . Hg.

\section{(p. 239.) \\ XLIV. NOTOXVIS:}

Palpi quatuor fecuriformes. Intilla vnidentata. Antenane monilifórmés.

354. $N$. Monoceros thorace in cormu fupra caput protenfo. F. Sp. 203. 2.

DSchrank. E. I. 42 I. Schaeff. Ic. t. I88. f. 3.

q F. E. S.I:I2. 211.6.

Elytra teftacea fafcia poftica nigra, punctoque ansice nigro. Non frequens.

ß. Variat magnitudine, et numero fafciarum in clytris, mihi fuecies diftincta, ct primum capta menf. Iun. in filiqua ficca Nerii Oleandri; poftea inteni faepe multos per totum Imii menfem in foliis Nucis Iuglandis rcgiae velociflme curfitantes, qui differunt a fpecic Limaeảna; nam pro puncto habent fafcias tres nigras, et cednnt magnitudine. Etiam Scirankius varietatem adnotanit; fed mea fpecimina maiora duas lineas tantum attingunt. Tab. II. โ. 14.

\& N. trifufciatus. Rofii Mant. 45. I13. N. cornutus. F. E. S. I. 2. 2 I I. . .

Habitat Florentiae et Pifis.

Nefcio, qua de caufia Roffius hunc Notoxum, qui fpecies plane diftincta eft, ftatim haud feparauit, K 3

cum 
cum fibi tainen differentiam fpecificam perfuafam haberet. In Mantifia quidem vt, nouam exhibet fpeciem. Color in fpecinine meo haud flauus, fed flauus in album vergens. - In N. Monocero punctum marginale elytrorum interdum deeft. $\mathrm{Hg}$.

* 355. N. Schaedia totus fubflauus, villofus, thoracis dorfo fcabrofitate nigra cornea notato.

Statura et facies N. mollis. Palpi quatuor fecuriformes. Elytramollia flexilia. Scabrofitas apparet quafi fruftulum ligni cuti adliaereat. Lectus, fi. bene recordor, in floribus Scabiofae. Long. $3 \frac{x}{3}$. lat. $\frac{2}{5}$.

\section{CERAMBYX.}

Palpi filiformes. Maxilla obtala nidentata. Labiunn bifidum. Antennae fetaceac.

356. C. mo fchatus thorace fpinofo viridis nitens, antennis cyaneis mediocribus. F. Sp. 210.9.

-O Ray., Lifter., Frifch., Mouff., Bergftr.

D Scop. E. C. I05. Schrank. E. I. 249 . Schaeff. Ic." t. II. f. 7 .

i F.E.S.I. 2. 25 I. I.

Odorem. fpargit gratum, et foporiferum. 'Hab. in Salice pafiim. Long. max. 17. lat. 4.

Variat colore rubro-aeneo. Hg.

357. C. Cerdo thorace fpinofo rugofo niger, elytris rotundatis, antennis longis. F. Mant. $\mathbf{3} 32.2 \mathrm{r}$. Sp. 212.18:

$\odot$ Bergftr., Frifch., Mouff., Drury.

D Degeer. Inf. 5. 69. 6 .

C. picens. Fourcroy. Ent. Parif. 74. 2.

Schaeffi. Ic. t. I 4. f. 8. 
q F. E.S. I. 2.255 . I 4 .

Antemae corpore longiores, in mafculis, et ad: apicem cinerco-lucidae, articulis quinque vltimis compreffis fpinaque terninatis. "Hab. in filuis. Saepe lectus in Thalictro pafcuorum. Long. 10:" lat. 3 .

Auctor cum Fabricio perperam citat Linnaei $C$. Cer ionem, qui aperte ad fequentem referéndus eft.' Linnaeus ipfe $C$. Cerdonen $F$. haud fatis noffe vi-i detur, cum marem $C$. Hevois $F$. coniiceret; figno quidem interrogationis adiecto. Ideo auctores $C$. Cerdonem $F$. a Linnaeo quafi rarietatem exhibitum, haud bene citant. Mas C: Cerdozis, "ivt in plerisque huius gentis, antennis longioribus diftinExus eft.

ir In omnibus Bergftrnefleri tabulis et figuris, a Fabricio citatis, nil nif $C$. Heroem $F$. agnofco, quia elyrra apice femper picea funt, id quod, numquam in C. Cerdone $F$. obferuaui. Hinc einsdem figurae praeter neceffitatem multiplicatae funt. Tain paruum autem Heroem, vt t. Ix, f. 6 . habet, havd vmquam vidi. - Citatum Degeerii, ab auctore addictum, dubios nos relinquit, vtrum $C$. Cerdonene $F$. an Heroem $F$. habuerit. Hg.

\section{(p. $x+1$.}

358. C. Heros thorace finiofo rugofo niger, elytris apice fubfpinofis teftaceis, antennis longis. F. Mant. 132. 22. Sp. Cerdo. 18. $\beta$.

q F. E. S. I. 2. $255 \cdot$ r.

Magnitudo, infra indicata, apud nos communis et conftans. Antennae plerumque corpore duplo longiores, fed interdum breuiores, et vix corpori aequales. Reliqua vt in diagnofi Fabricii. Sane pro fpecie, a C. Cerdo diftincta, habendus, etfi diffe- 
rentias omnes in vno tantum fpecimine a Scopoli adnotatas, miki in plurimis obferuare non datum. Occurrunt alii etiam, led rarius, antennis, corpore, elytrisque totis teftaceis mollioribus; qui veterem confufionem difcuffam in animis renouare non debent, nam varietas haec repetenda efie videtur a ftatu eorum recens declarato. Háb. in filuis fiequentifimus, et inuentus etiam in horto academico in caudice Salicjs Babylonicae. Long. 20. lat. 6. Specimina mea ferme ' $17 \frac{1}{2}$ lin. longa, et 5 lata funt latitudine bafeos elytrorum fumta. Antennae in maribus duplo longiores funt, quam in feminis. Thoracis rugae inordinatae, nunc obliquae, nunc transuerfales, nitnc longitudinales, atque hinc inde interruptae, in $C$. Cerdone $F$. femper transuerfales funt. Caeterum vide adnotata ad priorem. $\mathrm{Hg}$.

$$
\text { (p. 142.) }
$$

359. C. vebutofus thorace fpinofo, elytris punctis fafciisque nigris, antennis longionibus. F. Sp. 215.26.

$\odot$ Sulz.

D Schrank. E. I. 246 . Schaeff. Ic. t. 55. f. 4, 5 .

q Cerambyx nebulofus. F. E. S. I. 2. 26I. 35:

Panz. F. I. G. XIV. I3.

Hab. in Salice, Pinu. Long. $3 \frac{\mathrm{T}}{\mathrm{r}}$. lat. $\mathrm{t}$.

- Citatum Geoffroyi excludi debet, quia ad Lam. nebu lofam $F$. pertinet. Schaefi. Ic. t. I4. f. 9. citanda videtur, quia id citátum, ab aučore exhibitum, ad eandem L. nebulofam F. referti oportet. Laichartingius fub hoc nomine Lam. variam nec verum C. nebulofun défcripfit. Fabricii C. nebutofus idem cum illis mihi videtur, /quos Schaeff. 1. c. et Voet. II. t. 4. f. 4. depictos habent, hinc ille Cerambyx, qui in nonnullis Germaniae mufeis tomine C. mutio Panzbri occurrit, eadem fpecies 
eft. In hoc elytra luteo-cinerea funt, femoraque bafi gracilia, in clauam definunt. Degeerii citatum dubium remanet. $\mathrm{Hg}$.

360. C. varius thorace fpinofo tuberculatoque, corpore nigro cinereoque variegato, femoribus clauatis, antennis mediocribus. F. Mant. 130.2.

An C. clauipes. Schrank. E. 1. 256 ? Schaeff. Icon. t. $203, \mathrm{t}, \mathrm{5}$.

\& Lamia varia, F. E. S. I. 2,27 r. 18.

Lamia nebuloja. Laich. Tyr, 25, 6 .

Defcriptio Fabricii l. c. iterata.

Habit. in locis filuaticis minime rarus. Long. 5 , $5 \frac{1}{3}$. lat. $I_{3}^{\frac{2}{3}}, 2$.

Equidem de citato Schrankii hand dubito. Caeterum confufio in hoc et fimilibus maxinia efto. $\mathrm{Hg}$.

\section{$(p$, 24.3.)}

36r. C. hifpidus thorace finofo, elytris apice bidentatis, antennis mediocribus hirtis. F.Sp. 215.27 .

(.) Schaeff. Ic. t. I 4. f. 9 . Frifch., Rai.

D Sclirank. E. I. 248 . Scliaeff Ic. t. 176 . f. 5

\& Panz. Naturf. XXIV. 26.35 . t. 1. 35 . Eiusd. F. I. G. XiV. I6.

Feinora variegata, elytrorum colore, in mafcuils clanata. Habit. in pomariis, et pratis. Long. $2 \frac{1}{12}$. lat. $\mathrm{t}$.

Schaeff. Ic. t. 14. f. 9. , ad C. nebrilofut m referatur. Degeerii citatum. V. 7I. 9. t. 3. f. I 7 . quod et Fabricius in Ent. fyft. iterun exhibet, aperte ad C. fafciculatum $\mathrm{F}$. pertinet, cum ipfe adnotet, elytra apice minime dentata, fed parum truncata, et integra ac rotundata effe. Coleb. Fabricius in Ent. fyit? I. 2. 262. 41. C. pilofum habet, a D. Hübnero illi miffum, a quo ipfe quoque accepi. 
Hinc pro mea parte contendo, omnia citata fub C. hispido (Degeerii, ad C. falciculat. pertinente, excepto,) quae Auctor nofter exhibuit Fabricium fecutus, ad $C$. pilofum referri debere. Linnaei, Geoffroyi, Schrankii deferiptiones in hunc omnino. competunt. Fabricii $C$. hifpidus omnino alius videtur. Cum Laichartingius Tyrol.' II. $27.7 \cdot$ Geoffroyi citatum dubium habeat, quia fpinas, nec. fafciculos pilorum videt, refpondere liceat, tubercula illa elytrorum tria interdum fpinarum inftar indiuifa efle, -vti ipfe obfernauit, interdiom a autem diffindi, et fetofa apparere, quod in aetate pros vectioribus fieri videtur. Forte etiam álium habuit Cerambycem, quam noftrum. $\mathrm{Hg}$.

* 362. C. Quercus thorace fpinolo, elytris integris fufcis pilofis, fafcia pone medium viidulata nigra, antennis longióribus' hirtis.

Accedere videtir ad $C$. fafciculatum $\mathrm{F}$. mant. 134. 37., at dinerfus. Statira fere $C$. nebulofi. Antennae fufco-nigrae, corpore paullo longiores, graues, pilis teriuifimin longis quait ciliatae, artieulis vndecim, quorum primus craftus, fecundus brenis, tertius longiufculus, reliqui fenfim minores, extimo oblongo. Caput et thorax fufca.: Thorax vtrinque fpina acuta inftrnctus. Elytra, grifea fufca integra, pilis raris nigris longis vndi-: que afperfa, bafi ad latera nigricante, et fafcia pone medium transterfa vidulata nigra. Pedes fufici. Femora craffuscula. Habit. in Quercu. Long. $3 \frac{5}{6}$. lat. I. 


\section{LAMIA.}

\section{(p. 244.)}

Palpi quatuor filiformes. Maxilla cornea bifida. Labiun corneum, bifidum. 'Antemnat fetaceae.

363. L. Textor thorace fpinofo, elytris conuexis atris, antennis medioctibus. F.Sp. 21 ? ?.

, $\odot$ Bergftr.

D Schrank. E. I. 255 .

o F. E. S. I. $2=268.8$.

Variat adinodum magnitıdine." Aptera, rudimentis tantum alarum. Defcriptio Scopolina optima. In meis fpeciminibus fane multis, elytra femper punictis nigris elenatis, et antennae in nomnullis brcuifitimae; in aliis corpore duplo longiores. Stridet, vt maxima pars huius generis, non retrahendo caput in thoracem, vti alferitur, fecd attritu thoracis eo loco, quo fuperinis báfin elytrorum tegit: fi cultro ea pars tantillam abraditur, ftridor ceffat. Hab. in ligno putrido, in tiluis, in hortis vbique. Long. I 3. lat. 5 .

In permultis fpeciminibus antennas fenper corpore paullo breuiores vidi, et colorem haud variantém, fed fufco nigrum. Scopolii E. C. I6 4 , varietas L. triftis $F$. eft. Laichartingius etiam ita fufpicatur; fi autem contra $C$. afperum Sulz. Hift. Inf. t. 5. f. 3. varietatem coniicit, valde erat. Hanc Italiae idigenam Lamiam, quae eadem cum L. Latgubri F. E. S. I. 2.283.63. eft, miramur, cur auctor non reperit. - Figura Schaefferi mala eft, et ftaturam, et maculas elytrorum fufcas fi refpiciamus, quas ferme inuifibiles femper, obfertani. Bergftr. Nom. t. I. f. 8. melius congruit. $\mathrm{Hg}$. 364. L. Sutor thorace fyinofo, elytris atris , ferru- 
ferrugineo-maculatis, fcutello liteo, antennis longiffmis. F. Sp. $218.15 \cdot$

○.Vdm., Sulz.', Scop.

Dechaeff. Ic. t. 65: for.

\& F. E. S. I. $2.27 \%$. 4 I. -

Voet. Coleopt. II. t. V. f. 6.7 .

Elytra fufco-aenea, ferngineis vel albidis atomis maculata. Scutellum luteun. Habiti apud nos rarior. Long. 9. '̉at. $2 \frac{\mathrm{T}}{2}$.

Elytra nonnumquam minus maculáta, quod in mafculo obferuaui, "vbi tibiae anticae" antennaéque valdé elongataé, atque elytra pone magis attentata funt, quod in mafculis huiùs gentís fieri fólet.: Mafculus talis mihi eft, totus fufco-aeneins, fcu-? ... tello folo luteo, et ftatura minore, quam in reli-t quis, fimillimus iconi Voet. II. t. V. f. 6.5 Inde? L. Sirtor F: 1. c. 278, 42. mera tantum yarietas videtur, elytrorum maculis detritis. Hg.

\section{(p. 24.5)}

365. L. Kachleri thorace fpinofo nigra, clytris fanguineis, macula nigra, F. Sp. I. Ig. 20.

○ Scop.

D. Lamia Hungarica. Herbft. Arch.

I Cerambyx Kaehleri F. E. S. I. 2., $257 \cdot 24$;

Veet. tom. II. t. xr. f. 48 .

Thorax variat, immaculatus, et macula latcrali rufa; forte fexus diucritas? ... Elytra quoque nunc immaculata, n!!!c macula nigra; apice emarginata. Specimen habeo cijtris fanguineis, fingulo macula vnica nigra rotunda in medio pofita. In omnibus alae femper atro-cacruleae. Hab. in filuis, fed inuenta etiam in horto. Long. 2, 9. lat. $2 \frac{\bar{z}}{3}, 3$. 
Linnaci L. Kalhleri thoracem macula laterali rufa habet, hinc alia, quan Fabricii L. Kachleri eft, fi alias Schneiderum fequimur, qui diuerfas fpecies credit. Mihi tamen vtraque eadem fpecies videtur. Attancn maculas thoracis minime differentiam fexus indicare, mihi perfuafum eft, cum $L$. Kathieri tiurace inmaculato, marem feminamque polidea:n. "Huius fpecimen inicum elytro finiftro, maculae communis dimidia parte excepta, aliam infuper maculam transuęrfalem gerit nigram, quod etiam variabilein colo:un indolem prodere videtul. - Faud bene autem auctor L. Hungarican Herbft. Arch. t. 25. f. 6 . citat, quae fane diverfa eft, ftatura multo anguftiore, thorace aut lateribus funguineo, aut maculis lateralibus ita confinentibus, vt falciam conftituant, et macula communi elytrorum in medio incipiente, fenfim dilatata, et elytrorum npicem obtegente, $i d$, quod numquam in T.. Kaellevi vidi.

Caeterum haec L. Kaehlevi in Brunsvigae regionibus ráriflimum animal, circa Nagdeburgum autem hand raro occurrit, numquam tamen, quod firam, macula thoracis laterali fanguinea. $\mathrm{Hg}$.

366. L. funefta thorace fpinofo fufca, elytris laeuibus: maculis duabus atris; antennis breuibus. F. Mant. I39.38.

- 온 F. E.S. I. $2.294 \cdot 65$.

Coramb. puluerulentus. Scop. Ann. V.95.55. Affinis L. trifti, at minor, et antennae breuiores. Tota fupra terrei coloris. Antennae brenes, nigrofufcae. Thoracis margo anticus pilis fubalbidis ciliatus, mucrone craffo. Elytra haud fcabra: fingulum maculis duabus tomentofis, irregularibus, quae laepe figura variant. Corpus et pedies nigro- 
nigro-fufca tibiis mediis appendiculatis, vel dente notatis: character Scopolinus aliis huius generis communis. 'Habit. apud nos frequens in filuis. Long. 9. lat. 3 .

Citatum e Scopolio celeberr. Panzer Naturf. XXIV. 26. 36 , vbi figura huins infecti elegantiflima datur, iam exhibet. Linnaei $C$. triftis num haecce funefta? Certe elytra ab illo' commemorita fufca in hanc potius, quam in L. triftem. F. quadrant. $\mathrm{Hg}$.

\section{(p. 146.)}

367. L. nebulofi thurace inermi ferrugineo, nigro-lineato, elytris fufco ferrugineoque variis, macula marginali cinerea, antemis mediocribus. F. Sp. 213.13 .

P.F. E. S. I. $2.277 \cdot 38$.

Defcriptam habes a Fabricio 1. c. Hab. in filuis. Long. 5. lat. $2 \frac{2}{3}$.

368. I. pedeftris thorace fpinolo, atra, vitta integra alba, antennis mediocribus. F. Sp. 223. 41 .

D Sclirank. E. I. 26r. Schaeff. Ic. t. 55. f. 3.

Q. F. E. S. I. 2. 288.82.

Nigra, aptera, capite et thorace linea media longitudinali alba, quae per futuram decurrit isque ad apicem elytronm. Antennae brenes, bafi fulua, vti etiam femora et tibiac. Pedes vero plerumque fufci. Tibiae mediae ad latus externum appendiculatac. Elytra nigra albo marginata. Corpus fupcrne opacum, fubtus cinereo polline adfperfum. Occurrit frequens varietas minor, quae differre videtur ab hac, elytris pracfertim punctatis, et nitidioribus, non referenda 
neque ad $L$. Morionem, neque ad $L$. lineatam $\mathrm{F}$. Mant. Forte mas praecedentis? - Hab. ruri in locis, et viis arenofis frequens. Long. $6 \frac{2}{3}$. latt. 2 ;.

Variat colore, tomento elytra et corpus tegente nunc fufco-nigro, nunc aterrimo. Fabricius in Entom. fy.tt. L. vufipcdem habet, vbi referatur Schaeff. Ic. t. 55. f. 3. fecundum Harreri defcriptionem; Schrank. E. I. 26 I.; L. arenaria Laich. Tyr. II. 20. 4. Vuet. II. t. 9. f. 3I., quam Scopolius varietatem tantum putat, vti et Auctur nofter. L. pedeftris Herbft. Arch. IV. 9r. 9. t. 25 . f. 9. alia eft, quod ex icone adiecta apparet. Hg.

\section{(p. $14 \%$.)}

369. L. Molitor Etriffca thorace fpinofo, oleagineo-fufca, futura, rittisque tribus àlbis a capite ad apicem elytrorum ductis.

Huc referre maluimus, at differre in multis videtur a L. Molitoire F. Sp. 224. 42.

Facies L. pedeftris, at corpore magis globofo. Tota fuperne tomento decumbente fufco fubuiridi holofericeo nitens, antennis mediocribus fufcis. Vittae tres albidae latae practer futuram albam, a fronte per fingulum elytrum excurrunt ad apicent, quarum maior marginalis eft. Pedes fufci. Hab. liluas. Long. $\zeta \frac{1}{2}$. lat. 3 .

Ipfe Lanias, h. 1. defcriptis fimiles, poffideo, quas olim fub nomine L. molitoris F. miflas accepi, a qua autem et vittarum figura et patria differunt, cun Fabricii Lamia Indiae indigena fit, noftra autem ex Hungaria nobis allata eft. Herbftii $L$. pecieftris l. c. eadem mihi vifa eft, fed color ibi depictuc niger, in meis oleagineo-fufcus eft. Omnibus ferme notis cum. anetoris defcriptione conmeniunt, fed defcriptio breuis adiecta difcernat. Eadem 
Eatem capitis thoracisque pictura, vt L. pedefris, colure atro excepto. Elytrorum futura et margo. alba funt. Superne ad carinah elytrorum laterilem vitta alba decurrit in medio elytro ferme fita, quae, ftatim, vbi excurtit, finditur, vt linea fufca huic vittae intermedia iacsat. Verfus apicem vittae brachia rurfus connecuntur, eodem modo, it in L. lineata F. vel L. Scopoli. Herbf. Arch.t. 25. f. Ix. Haec vitta elytro inprella eft, vt fpatium lineare intermedium emineat, quod praecipue in varietate quadam apparet, vbi tomentum vittae album detritum eft. Color fufcus etiam a tomenta depreflo originem ducit. Pedes antennaeque fufci interdum nigricant. $\mathrm{Hg}$.

* 370 L. unifafiata thorace inermi, onato, fordide nigro, elytris fufcis nigro-punctutis, falcia media atra flexuofa flauo-marginata.

- S Rolii. Mant. 50. I 24.

Corpus onatum. Antennae longitudine corporis articulis fubferugineis, apice pallidis, tertio et quiarto prialongis, arcuatis. Caput, et thorax fufco-nigra. Elytra obtufa punctis nigris raris excauata, fafcia lata nigra margine vtrinque flexuofo angulato flawo, quae magniam partem abdominis tegunt. Femora fufca tibiis apice albido. Tab. I. f. 5. Hab. fub arborum cortice in filuis. Lecta $\mathrm{m}$. Dec. et Feb. Long. 4. lat. $1 \frac{\mathrm{r}}{3}$.

\section{STENOCORVS.}

Palpi quatuor. anteriores filiformes, pofteriores clauati. Antennae fetaceae.

37 I S. Lamed thorace fpinofo pubefcente, elytris faftigiatis liuidis taenia obfcura longitudinali finuata. F. Sp. 226.3. 
I) Leptura hafarta. F. Mant. r $_{5}$. 2. Petagna. fp. I. C. f. XVII.

o Leptura hiaftata. F. E. S. I. 2. 339. 2.

Gcofroyana defcriptio noftrum infectum optime defignat thonace non fpinofo, et macula haftata in elytris. Totus fubpubefcens. Caput nigrum, palpis anterioribus porrectis triarticulatis, antennis nigris. Thorax niger rotundus, dorlo plano punctato, lateribus tubcrculufis, antice, et poftice 'marginatus. Scutellum nigrum. Elytra mbefceltia faltigiata, praemorfa, apice nigro, et corpore panilo breniora, macula haftata nigra medictate liturae. Subtus totus niger corpore tomentofo albo lucido. Pedes longi. Hab. flores praefertim Rubi et Scabiofa pafin. Long. ó. Iat. $I_{3}^{2}$.

Auctorem $C$. Lomcd I. haud novifie, ex. his iamian dictis fatis apparet. Quyomoto autem ob citatum falfum credere potnit, Celeperrimum. Fabricium idem infectum bis et in diuerfis generibus defcripfiffe, quem fcimus nullas in fyltema excipere fpecies quas Ipfe haud vidit? Qua deinde ratione thoraccm exprefe dictum frinofum in dingnofi, negligere, eandemque diagnofin in Lepturam thorace inermi, transferre potuit? - Citatum Sulzeri, Petagnae et Geoffrovi ad $L$. haftatam $F$. pertinet, vbi etiam Fabricius' in Ent. r ft: fua habet, quamuis id nouifimum iterum fub $S t$. $L a$ med exhibeat, fane er:ore quodam calami.

St. 'Lnmed femper e Suecia accepi. 'Statura eadem, quae Lept. 4-maculatace, elytraque eiusdem linido-teftacei coloris. C:rput nigrum iniquum, oculis brunneis. Antennae dimidia corporis longitudine. Thiorax niger," pubefcens, punctatns, antice anguftatus: futura ibi capitis collum arcte cingente; in dorfo linea longitudinalis im 
preffa eft ; latera fpina valida armata funt. Scute!lum mediucre, atrum. Elytra liuido-teftacea, pone attenuata, vt in congeneribus, excauatopunctata. A humero fafcia decurrit verfus futuram obliqua, finuata, Hebraileum 7 (Limed) tamquam referens, nigra. Verfus apicem macula nigra eft, fubconica, fupra fubemarginata. Corpus fubtus cum pedibus nigrum ac pubefcens eft. Magnitudo variat. Longus 8 , nonnumquam Ix. lin. et. $3-4$ latus, latitudine bafeos elytrorum computata.

Pauca de genere Stenocori addere licent. Fabricius in Entom. fyftem. fua hoc genere plura coniungit coleoptera, quae feparanda, et fingula fuis generibus annumeranda videntul v. c. St. Marylandicus verum Callidium, vel potius Clytus (genus noumm, a Laichartingio conditum, bonum,) eft.' St. Feftiuus Prioni genus fubiret, vbi $P$. depsavium ftatura fibi fimilem habet. Leptura quadrimaculata contra inre huic generi adiungenda videtur, et totum habitum, et thoracis fpinas fi refpiciamus. $\mathrm{Hg}$.

\section{CALOPVS.}

Palpi quatuor antici clauati, poftici filiformes. Labium membranaceum bifidum. Antennae filiformes.

372. C. Serraticornis fufcus, antennis contpreflis. F. Syft. Ent. I82.

P. F. E. S. I. 2. 303. I. Panz. F. I. G. III. I5. Antennae mediocres, compreflae, ferratae. Palpi - antici longiores clauati, ante mandibulas porrecti, poftici filiformes. Labium membranaceum, apice fubpilofim. Oculi magni nigri. Caput pone elon- 
selongatum; et thorax conformata omnino, vti in Attelabo Coryli. Lecti duo e terrae filliura exeuntes in loco montuofo inxta truncum Quercus Siberis d." 22. Aug. in tatu nomnihil incompleto, nempe elytris mollioribus abbreuiatis. Lorg: 8 , 9. lat. $2,2 \stackrel{5}{ }$.

Antenne maris admodum compreffie, latae: articulis latere exteriore rcctis, interiore aútẹn, prominentibus fubfinuatis; antenuarum feminae articuli ferme lineares, parum comprefii ferratique. Haec longa eft 9 , ille 7 . lin. hic lisus $x$. illa 2 ? . lin. Caeterum: miror, hoc pulcherrimum infectum, quod ex Suecia femper accepi, ; etiam in Italia occurrere, quamuis milni notum eft. in Germania quoque repcrtun efle (vid. Celeb. Panz. 1. c). Forte noula fpecies, praecipue cum fatus tam iuuenis ac fingularis, quen in einsmodi infectis nondum obferuaui, determinationem certam fallere poflet. Hg.

\section{(p. 147)}

\section{RHAGIVIT.}

* 373. R. Etrifcum thorace obtufe fininolo rutum, elytris violaceis.

- Pallas Ic. InI. t. F.f. I 7. exhibet Iconem quodam modo fimilem, fed paitlo minorm.

9 Callidinni Salicis. Rołfi. Mant. 5x, 12. 8.

F. E. S. I. 2. 329. 5 I.

Cerambyx bicolor: Schrank. E. I. 25 I.

Rhagium Schrankii. Laich. Tyrol. II. Iт8. 2.

Geoffr. Inf., I. $224 \cdot 4$.

Statura $R$. Inquifitoris, fed maius. Antennae breues ante oculos pofitae, nigrae, quatuor primis articulis rufis. Palpi quatuor capitati, ruî.

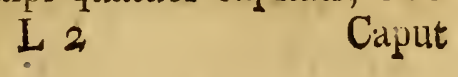


Caput rufum.) Oculi nigri. Thorax rufus, vtrinque - fpina obtufifima, feu potius tuberculo inftructus. - Elytra violacea nitida. Abdomen, pedesque rufi, tarfis quatuor fufcefcentibus. Pectus nigrum. Alae nigrae. Variat antemis totis rufis. Defcriptum non imeni. Tab. I. f. 4.

2: Hab: Florentiae rnmm. Long. 10. lat. 3...

- H Mairis St. Inquifitore, et anguftius, nec pone attenustum. Elytra nigro-cnerulea, margine prope bafin flauicante. Variat elytris grifeo-teftaceis : varictas aetatis widetur. Anctorem noftrum defcriptum inueniré non poffe, id inde deducendum videtur, quod a Fabricio ad Callidia relatum fit. Mihi - cum multis aliis Entomologis verum Rhagium eft. $\mathrm{Hg}$.

\section{(p. 150.)}

\section{S A PERDA.}

Palpi filiformes. Maxilla membranacea, bifida. Labiun cordatum, truncatum. Antennue fetaceae.

374. S. cylindrica cylindrica, nigra, pedibus anticis luteis. F. Sp. 232. ₹.

D) Schaeff. Ic. t. 48 . f. 4 .

\&. F. E. S. I. 2.3 I0. 14 .

Villofula. Caput inclinatum. Antennae corpori aequales. Elytra punctata femoribus rufis bafi nigris:" Habitat in Corylo, etiam in floribus non frequens. Long. 4. lat. $I$.

Citatum Geoffroyi I. 208. 2. optime conuenit, fed nihil de pedibus anticis medio flanis dicit, quos tamen femper praefentes obferuo. Ne fit $S$. dubic Lach prohibent praecipue illa verba: on voit fur les étuis deux raies longitudinales plus élevées, quod exacte cum elytris $S$. cylindricae, fed etiam 
S. crevulefcentis. n. 378. quadrat. Hinc pedum colorem aut tranfiit Geoffroyus, aut varietatem. quamdam pedibus: omnibus vnicoloribus, aut $S$. caevulefcenten defcripfit, vbi autem color haud bene nigro-cacrulens vocatus effet. Cuins linea thoracis medii alba, quae nonnumquam paullo fcutellum vltra extenditur, faepius deleta eft. Citatum Roefel. Inf. 2. Scar. 2. t. 3. aperte ad S. lineareme $F$. referri debet. $\mathrm{Hg}$.

375. S. Cardui fufca, thorace lineato, fcutello flauo, antennis longis. F. Sp. $233 . .16$.

\& S. nigricomis. F. E. S. I. $2.3 \mathrm{I}+3^{\mathrm{I}}$.

Cerambyx Cardui. Schrank. E. I. $x_{40 .} 265$.

Corpus tomentolum flauefcens, thorace fufco lincis tribus longitudinalibus flauis, quarum vna in medio, et rna ad fingula latera. Scutellum flaum. Antennarum primus articulus ater, reliqui cincrei apicibus nigris. . Variat admodum magnitudine. Habit. ruri in Carduis frequens. Long. ?. lat. 2.

Iinnacus lineam longitudinalem a capite per thoracem atque elytra ductan efle memorat, cuius tamen in elytris imo rudimentum detegere nequeo. Magnitudo tam variabilis eft, vt faepius triplo minor occurrat. pili flauicantes, qui animalculum in fatu integro viridiflauum reddunt. maculis immittis raris nigris, vbi tomentum deficit, interdum ferme toti detriti, hinc nonnumquam nigricans apparet. $\mathrm{Hg}$.

376. S. puntata thorace cylindrico viridis, punctis nigris numerofis, antennis mediocribus. $F_{\text {r }}$. Sp. 234.20.

D Schaeff. Ic. t. 20I. f. 4.

오․ E. S. I. 2. 3I 5.39 . 
Femina multo maior mafculo. Antennae et pedes elytris concolora, at facile nigra euadunt ob iacturam tomenti. In feminis abdomen ctiam faepe nigro - punctatum. Hab. in fepibus non frequens. Long. 5 , $7 \frac{1}{2}$. lat. $I_{\dot{T}}^{\mathrm{T}}, \mathrm{I}_{2}^{\mathrm{T}}$.

Auctor cum Fabricio Schaeff. Ic. t. Ior. f. I., vbi $S$. tremula $F$. bene repraefentata eft, citat. $S$. punctatum Lin. et Fabr. me nondum nouife, confiteor. Hinc nefcio, vtrum icon Sulz. Hift. tab. 5. f. Io., quam Auctor figno interrogationis adiecto exhibet, an Schaeft. Ic. t. 20 I. f. 4. vera fit. Sulzeri figuram Dns. Herbit. (Fuesl. Arch. IV. 94. 7.) apud $S$. punfiatam $F$, quam ofto. punctatum vocat, allegat. Ex ibi adnotato, licet. breuiffimo, concludere poffumus, illum veran S. tremulam $F$. habuiffe, et Sulzeri figuram perperain allegaffe. $\mathrm{Hg}$ :

377. S. populner thorace flauo-lineato, elytris punctis quatuor flauis, antennis mediocribus. F. Sp. 234.18 .

$\odot$ Gadd:

D Schrank. E. I. 266.

Q F. E. S. 1. 2. $3^{\mathrm{I}} 5 \cdot 3 \%$.

Mea fpecimina thoracem habent lateribus tantum

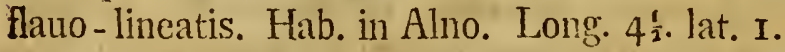

Bergftraefr. Nomencl. t. 2. f. 4. huc referenda eft. - Magnitudo quidem variat, fed haud infolito modo. Linea thoracis intermedia faepius detrita eft; rarius integra, fed paullulum interrupta, confpici folet. Maculas elytrorum quinque, hand endem directione difpofitas, numero, quas tamen inconftantes obferuo: nunc albicantes minores, nunc maiores, ac luteas. Q Quarum pofteriorum mache iae quafi confuentes e duabus minoribus apparent, 
vt fafciae obliquae fpeciem prae fe ferant, quod Bergftraeflerus l. c. fpectare videtur. Hg.

\section{(p. 152.)}

* 378. S. cacrulefiens thorace mutico cylindrica, albo-cacrulea, lineis tribus thoracis, fcutelloque pallidioribus. Schrank. E. I. 27 r.

Leptura caeruleficus. Scop. E. C. I60. Fuesly. Arch. Ent. t. 26. f. 5. Petagn. Inf. Cal. f. 18.

q Laichart. Tyrol. II. 45. 9 .

S. virefiens. F. E. S. I. 2. 3I 5. 40 ?

Thorax lineis tribus albicantibus, vti etiam elytrontun futura. Antennac albo nigroque maculatae corpore longiores. Tuta tomentofa albocaenulea. Habit. flores. Long. $4 \frac{1}{2}$. lat. $1 \frac{1}{3}$.

Citatum e Scopolio femper obfcurum manebit. Omnes illae Saperdae, quas Celeb. Herbltius ac Panzerus (Naturf. XXIV. t. I. f. 37.) depictas exhibent, antennas vnicolores habent, futuram als bam infuper in fpeciminibus meis confpicere nequen, nec Schrankium eiusdem mentionem feciffe, video. Hinc Auctoris Saperda noua ac diftincta videtur fpecies. 'Noftram paucis defcribere iumat.' Elytra eiusdem cum $S$. cylindrica fabricae. Qua ratione Geoffr. I. 208. 2. Lept ardoifée haec S. caerilefcens efle poffet. Vid. Adn. ad S cylindricam. n. 374 . Eadem Atriata, punctis, quamuis diffitis, rocat Schrank. 1. c. fed punctata appellare mallem, punctis quidem, fed admodum îregulariter, in ftrias approximatas, difpofitis. Aliquando fub nomine $S$. nigricornis. $F$. mihi miffa eft. Locus idoneus vifus eft, breuia do translatione cuadam nominum $h .1$. adiicere. In Entom. fyftem. Fabr. nempe $S$. nigricornis eft $S$. Cardui Mant., dein S. Yuturalis: S. nigricomin, 
ac denique S. amulata: olim fic dicta $S$. fotura lis. $\mathrm{Hg}$.

379. S. Suturalis thorace rotundato, lineato, virefcens, elytris acuminatis: futura alba. F. Mant. I 49. 25.

o $S$ annulata. F. E. S. I. 2. 3 T 4.33. Statura S. Cardui. Magnitudo S. lineiris. Antennae longitudine corporis bafi aibae, apice nigrae, primo articulo toto nigro. Thurax rotindatus, fufco-viridis, lincis tribus flanefccntibus. Elytra glabra fufco-viridia, fubmarginata, apice acuta. futura prominula alba, punctata, fod punctis vix sudo oculo confpicuis. Hab. in plantis ninime. frequens. Long. 5. lat. $2 \frac{1}{5}$.

Ex Italia Saperda mihi mifia eft, quam futuralen puto, cui autem color fufco-niger, elytraque. aperte punctata funt. Caetera defcriptioni conueniunt. $\mathrm{Hg}$.

* 330. S. Filum thorace cylindrico, nigrofufca, antennis nigris corpore duplo loigioribus. \& S. hirte. E. S. I. 2. 3 : $7.5 \mathrm{I}$.

Facies, et color S. yomeliuede, fed triplo minor, et immaculata, vix cinereo-pubelcens. Caput, thorax, elytra punctnlata vnicoloria. Pedes nigri plautis alibidis. Tab. 5 . f. ıo. Lecta in prato filueftri. Long. 3. lat. $\frac{x}{3}$.

Citatum e Fabricio, auctoritate Celeb. Viri, éxhibeo. $\mathrm{Hg}$.$$
\text { (p.T53.) }
$$ \\ LIII. CALLIDIVM.}

Palpi quatuor clauati. Marxilla membranacea bifida. Labinin bifidum laciniis tenuifimis acutis. Anternae fetacene.

38r. C. Baintus thorace villofo tuberculis duoous, corpore infco. F.Sp.236.I.

Sulp., 
(- Sulz., Frifch.

D Geoffr. Inf. I. z I 8. I. Schrank. E. I. 2\&I. I ?. S. F. E. S. I. 2.3 I8: 2 .

Totum nigricans, capite, et thorace praefertim villofo, tuberculis duobus lucidis atris. Elyera punctis prominulis fcalbra. Femina caudata clytris pallidioribus. Variat magnitudine minori. Lim. confer in Fn. Sv. - Habit. in arborum truncis. Long. 7. lat. 3 .

Nas duplo minor femina. C. Linneianun Laich. Tyrol. II. 69. 6. varietas tantum viletur, cum magnitudinem, et elytiorum maculurum numerum inconttantem obferuarem. Si deinde Geoffroyi inféctum ad $C$. Linneignum frum, hec at $C$. Batuhnil referre maunlt, idem, quod apud $C$. Baiulum, obftat fecundum opinionem Laichartingyi , thoracen vtriusque hatud rhomboïdalem efle; fed verba Geoffrovi tam anxie legere, haud bene factum milli videtur. $\mathrm{Hg}$.

* 382. C. holofericeum thorace nutico rotundato globofo, corpore elytrisque cinereis lolofericeis.

An C. Jericelum. F. Mant. I 52. I 4?

Statura et magnitudine proximum C. mftico, fed totum tomento cinereo obtccum. Difiert etiam oculis magis lnulatis, thorace punctis eleuatis non concauis, femoribus crafioribus. Pedes, antennacque cincreae pilis adfuerlac. Tab. I. f. 6.

Repertum pluries in horti pariete. Long. 9. lat. $3{ }^{\mathrm{r}}$.

Fabricius in Ent. fyft. I. 2. 322. I7. apud C. Seriсенm fuum allegat. $\mathrm{Hg}$.

$$
\text { (p. 154.) }
$$

383. C. Omega thorace rotundato, nigroJ. 5

fufcum, 
fufcum, elytris punctis quatuor transuerfis fafciaque arcuata cincreis.

Dificrt a C. Baizlo magnitudine paullo minori, ét thorace magis rotmudato, minus depreflo, nequé villofo, noquic tuberculofo.

- An C. licitatus. Limn. Sylt. Nat. 636. 78?

Antennae nigize, brenes. Caput fizb oculis. cincrco-tomentofum, linea nigra lucida inter antemas ad os decurrente. 'Thorax vix pubefocns, miger, fufcis, punctis giifeis farfis. Elytra nigra, oblcura, punctata, pone merium punctis quatuor trinsucrlis cinereis, vno vtrinque marginali, altero ad futuram trilobo. Mox fafcia angufta, arcuata, obfelete cincrea. Apexquoque cinereoalbidus. Pedes nigri, femora fubclauata longa, ad bafin exilia, fubrufa.

- Reperi in Populo tremula. Long 6. lat. $\mathrm{I}_{\frac{2}{2}}$.

C. Onrga Auctoris C. licintum L. effe, libenter equidem credo, cum raro fpecimina thoracis figmatura integra occurrant, qualia Panz. Naturf. XXIV. t. I. f. 38 . exhibet. Elytrorum qunque punćta albida villofa fiepius deteruntur. Tale fpecimen detritum Celeberimus Fabricius ipfe pro C. atomario fuo determinauit, quamuis bene fciam $C$. liciatum. $L$. ab eodem apud $C$. Hafnienfe fuum allegatum efie. Anne' itnque ' $C$. atomarimm $F$. varietas tantum detrita C. Hafmequ/is? Hg.

3S4. C. Fenicum thorace tuberculato, rufo, elytris violaccis, antemis mediocribus. F. Sp. 236.2 .

(2) Uim.

D Schrank. E. I. 28z. Schaeff. Ic. t. 4. f. $x 2$.

-9.F. E. S. I. 2. 319. 3.

Lectum in filuis fub Julii finem. Long. $4 \frac{3}{4}$. lat. $I \frac{1}{2}$.

Con- 
Confufo in hoc et variabili maxima eft, quam extricuri, vix fperare licet. Specimina mea cum def riptione Schrankii exacte congrunnt. Plures Entomolegi vtrumque Caliidium idem putant, milji tameu femper aliquid incerti reftat, praecipue de citato Limueano, quia incredibile videtur, Linnaeun tam leues, ac primo intuitu recognitas varietates ad fpeciem vnam liand reuocaniffe. Geofiroyi citatum dubium habet AuEtor, nii tamen impedit, quo minus $n .18,19,20$. hoc referantur.

Fabriçins in Ent. fytt. apud C. variabile fuum (32г. т6.) C. cienentm (id. quod legendum pro $C$. Hungaricum) Herbit.Arch. t.26. f. I 2. allegat, quod errore quodam factum effe putaremus, mifi Ipfe, idem $C$. ancum Herbftii, (quod C. Cencum Degeerio quoque audit,) $C$. variabile funm efle, nos certiores fecifiet. Qua de caufa citata Linnaei, et Schaeff. Ic. t. 4. f. 12. delenntur; C. Fenmicuns vero Fabricii, C. variabilis nomen optime meretur, com numquam idem reperiatur. $\mathrm{Hg}$.

385. C. violacen thorace fubpubefcente, corpore viclaceo, antemis breuibus. I.Sp. $237 \cdot 5$.

$\odot$ Gadd., Frifch.

D Pod. M. G. $3^{6}$. I8. Schrank. E. 1. 277. Schaeff. I. t. 4. f. $x_{3}$. t. 234 . f. 1 .

q F. E. S. I. 2. 320.9.

Abdomen pedesque atro - violacea. Elytra pone rotundata. Hab. in hortis. Long. 4. lat. $I_{3}^{\frac{1}{3}}$.

$$
\text { (p. 155.) }
$$

386. C. fangumenn thorace fubtuberculato, elytrisque fanguineis, antennis mediocribus. F. Sp. 238. 16.

( ) Schiaeff. Ic. t. 6 \% f. r.

D Pod. M. G. 36. Schtank. E. I. 284 . Schaeff. Ic. t. $\sigma_{4}$. f. 7 . 
- ․ F. E. S. I. 2. 326.35 .

Supra fericeo-fanguneum, fubtus totun nigium. Hab. in filuis. Inuchtum etiam in pariete citcrno. Lol:g. 5. lat. 2.

- 387. C. teftaccum thorace fubtuberculato, corpore teraceo, antemis mediocribus fufcis. F. Sp. 239. 17.

. 2 F. E. S. I. $2.3=6.36$.

Habit. flores rmbellatos. Long. $4 \frac{3}{4}$. lat. $I \frac{r}{2}$.

Figuram Schaeff. Ic. to 64 . f. 6 . Harrerus 1. c. apud C. Fennicum allegat. $\mathrm{Hg}$.

$$
\text { (p. } 1.56 .)
$$

388. C. arcugtun tinorace rotundato, clytris fafciis quatuor flauis prima intermpta, reliquis retrorfun arcuatis. F. Sp. $24 \mathrm{r} .35$.

(1) Vdm., Frilch., Leche., Rai., Petiv., Schaeff. Ic. t. $107 . \mathrm{f} .2 .3$.

D) Sciratis. E. I. 308 .

? F. E. S. I. 2.333 .64$.

Sclrankii defcriptio iterum exhibctux.

Hab. in floribus obuinm. Long. 5. lat. $I \frac{7}{3}$.

Sclirankii ceferiptio, quam Auctor optimam vocat, haud omnibus conuenit notis. et Callidinn aliud defignat, forte fequens C. florcle. cum et minimus quisque character illi congruat. C. arcuati defcriptionem adiiciam, tt comparate liceat. Magnitudine $C$. Arietis $F$. fuperat, cui fratura fimilis eft. Color toto corpore nirger. Antennna rufae funt. Caput, pone antennas, annulo cinģitur, qui, vti et frons, luteus eft. Thorax baft interdum flano-marginatus eit, fed infuper pone medium ab itroque hatere maculam transuerfalem, anguftam, (rariflime ac ne vix quidem couiunctam,) flatar gerit, qune, vti futura antica, punçumq̀ue 
laterale fubtus ad pedem, flauum, nmmquam deeft. Fafciam bafeos elytrorum haud vmquam vidi, fed, f́cutello computato, puncta quatuor laua it; difpofita lunt, vt rhombiom conftituant, cuius apex alter fcutcllum, alter huic oppeftus, maculitifuralis communis eft. Ad humeros macula alia haua confpicitur. Fafciae tunc quatuor flauae numerantur; prima angufta, nec marginem, nec futurim ittingens, fimuta in figuram ferme litterne $\boldsymbol{C}$ iacentis. Scionda ac teitia, futuram tantuin attingunt, ibique coniunguntur, et ita curtatae funt, vt indlexura elytrorum bafin, finus atutem apicem fpecter. Apex quartan atque infiman habet, extremo tamen margine nigro. Abdoninis fegmentolum margines, pcÉtorisquie latera, lutefcunt. . I'édeš rufefcentes. Femora cluata funt, clanis, in primis parium anteriorum, nigricantibus.

Schaeff. Ic. t. Io?. f. 2. omnino hục pertinct, eiusdem tabulae autem tiģrara 3. ad. C. Gazcllam F. 1. c. 11, 66. Stituran thomeis pofticam interdum flauicare, iam fupra notatum eft, atque $\mathrm{Ge}$ offroyus. 212. Io. ita defuripfit. Varietates, $a b$ eodem, énmeratie, mullo mudo huc fpectunt, priol C. astritzm, pofterior Callidium fiftit, quod nondum noui. $\mathrm{Hg}$.

3S9. C. florale thorace globofo, albo-fafciato, elytris nigris, fafciis quinque albis. F. Sp. 24I. 33 .

F F.E.S. I. 2. 332. 6I.

Clytus aulicuis. Laich. Tyrol. II. 103. 5 . Leptutra arcuata. Sclirank. E. I. 308 ?

Habit. in floribus. Lung. $5 \%$. lat. $\mathbf{I}_{3}^{1}$.

Pallas, quem Auctor cum Fabricio citat, C. floralem fuum maiorem effe $C$. arcuato dicit, et D. Rofins menfuram h. 1. exhibet $C$. arcuati menfutam finpe- 
fuperantem. Specinsen meum inaximum, quod et magnitudini adichartingio, "et iterbftio, datae çonuenit, 5. lin., C. arüctum vero 7. lin. longiim eft: De citato Schrunliji vide, quae fupra ad C. arcsatnin atnotaui. Hy.

$$
\text { (p. }
$$

300. C. Arietis thorace rotundato, nigro, elytris nigris, fafcis flauis, fecuida antrorfun arcuata, pedibus formginiois. F. Sp. 242.36 .

$\odot$ Petiv., Frifch.

D Schrank. E. I. 307 Schaef. Ic. t. 38. f. 8. Defcriptio Linnasi in. Fis. Sv. optima, cui addi potef, thorax etiam margine poftico flutus. Mag-nit. variat. Hab. in floribus; apud nos primo vero iam declaratum.' Lon!g. j: lat. $\frac{2}{3}$.

Duae ab atictoribus confufac futut plane difinetne fpecies, quas Selineiderus primus fere reparauit. Fabricius quoque in Eut. fyft. I. 2. 333. C arietis (n. 65. ) et $C:$ Gazellam in. 65.) habet, fed citata, ibiłem exhibita, difponi neceffe eft: Apnd $C$. Arietis F. 1. c: n. 65. citata: Linnaens, Oliuier, Geoffroyus, Schaeft: Ic. t. I07. f. 3. ad $C$. $G a-$ zellom n, 66. pertinent, quibus adiungantur: Clytus Arietis. Laichart. Tyrol. II. 92: 2. ; Schaefo. Ic. t. 38. f. 8. et Panz. F. I. G. IV. I5. Equi$\operatorname{dem} C$. Arielis $F$. diftinctam fpecien habeo, cum femper confans fit, ac notae haud varient. Si enim thoracis lineam cafu detritam putaremus, cur idem cafus in C. Guzella $\vec{F}$. vel $C$. Arietis $L$. nondum apparuit; cur femper huic cum antenna-. rum apice nigricante, utque fafcia fecunda elytrorum fatim ad futuram coniuncta, nec verfus fcutellum afcendente, lineae thoracis interruptae propriae funt? At alii infuper characteres adfunt. In $C$. Arietis $F$. inter oculos lineae duae longitudinales 
dinales a fronte ad os defcendutut, flet thorix elytris latior, et verfus bafin angulattis eft; in $C$. Avictis $L$. antem globofus atque aequalis. Ely. trotum balis in $C$. Arietis $F$. detrita atque ftefea effe 'olet. Hg.

391. C. Feruagei thorace rotundato nigro-maculato, clytris fubuirefcentibus, falciis, tribus nigris, prima lumari, $F$. Sp; $244 \cdot 43$.

( Geoffr. Inf. I.izI6. I4. Schrank. E. I. 309. Schaefi. Ic. $t .=03$. f. 4 .

\& C. onctum. F. E. S. I. 2. 336: 77 .

Color glanco - cinereus, vel pallide fulphureus. Thorax globofus, tomentofus, fafcia media ingra. Elytro fingulo prope bafin fafcia literam C. referens, ita pofita, it comexa pars alterius oppofitac comexam pariter partem refpiciat. - Tum fafciae duae communes nigrie. Antennae pedesque nigri coloris, tomento glauco cinereo. Vumm mihi prodiic declaratum e fedili faginco domeftico, quod diem noctemque erodendo tandem terebranit. Stenócorus C. duplex Scop. Fi. et Fir. InI. p. 2. f. 5. videtur huic nimis affinis? Hab. paffim in floribus. Long. 5 . lat. $\mathrm{I}_{4}^{\mathrm{r}}$.

Duae iternin fpecies inter fefe confufae funt ab anctoribns, quas differre, nunc demum nobis perfuafum eft. Fabricius C. Verbafic (1.. c. n. Z6.) et C. ornatum (11. $77^{\circ}$ ) vocanit, fed haud quaeque citata fuae attribuit fpeciei. Sulz. Hitt. Inf. t. 5. f. I2. ad C. ornatum pertinet, cui, cum Auctore noftro, adiingantur: Clytus Veibafci Laichart. Tyrol. II. 105. 6. Geoffr. Inf. I. 216. 14. et Schaeff. Ic. t. 203 . f. 4. Imo. Linnaeus ipfe ibidem allegari debet, cum nuliam fafciarum interruptarum mentionem faciat, quod tamen haud 
leuis momenti cft; cum vero rnicum tantum $\boldsymbol{C}$. ornati fpecimen mili fit, difcernere nequeo: vtrum fafcia thoracis transuerfa nigra, interdum e punctis tribus connata fit, an non. Illam priorem notam Linnetus, quem Sclirank. 1. c. fequutus eft, dedit. Olinier haec C. Vévofci atque ornatun meras putat varietates, cui tamen aflentife non pofium. "Hinc in Ent. fyft alterum Olinievii citstum ad $C$. ometum referatur, 'vtrum, difcernant, qui habent eiusden opus. i Deferiptionem in compara. tione nifan adiicere placet; atque pro breui expreffione $C$. Verbafii F. iittera $A$. ; C. omitum contra, littera $B$ defiguati:

A. elongatum eft, ac proportione gracilius. Longum e. $\mathrm{g}$. 0 . lin. latum $\mathrm{I} \frac{1}{2}$. vix. $B$. autem incraflatum, ac breuius. long. 5. lin. lat. I vitra. A. Thorax brenior, ciobofior, in medio fafcian transuerfam sat integram, ferme linearem. $B$. dorfo maculam magnam, latereque fingulo punctum habet multo minus. A. Elytrum maculam humeralem, aliamque lruic oppofitam, inflexam, finuque humerum fpectantem, nec cum illa cohaerentem, habet. $B$. Signatura in eadem loco adeft vnica, litteran $G$. reuerfam (D) repraefentans. $A$. In fingulo elytro dein maculae duae fequuntur, nec marginem; nec futuram attingentes, plerumque fubrotundae: altera in medio, altera verfus apicem. $B$. Macularum loco fafciae duae, cum iisdem elytri alterius fafciis coninnetae, confpiciuntur anguftae, quarum pofterior, latior, marginem quoque occupat. - Onines fignaturac defectu tomenti luteo-virefcentis quod totum corpus obducit, 1) efficiuntur, atque nigrae funt. Icones in Arch. $\therefore$ d. Inf. a D. Herbft exhibitae, adhiberi poffunt bonae. : $\mathrm{Hg}$.

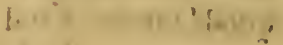




\section{(p. 158.$)$}

392. C. trifufciatum thorace globofo ferrugineo, elytris nigris, fafciis tribus albis, prima annulari. F. Sp. 244. 42.

ㅇ F. E. S. I. 2. 336,75 .

Defcribit. F. l. c.

Habit. Fiorentiac rarum. Long. 3. lat. I.

393. C. luridum thorace fubtuberculato nudo, niginum, elytris teftaceis. F. Sp. 239.18.

\& F. E. S. I. $2.32 \% \cdot 4 \mathrm{I}$.

Simillimum C: Batulo, fed triplo minus, inmaculatum. Thorax nudus, fubtuberculatus. Elytra lurida. Inueni quatuor in pariete horti, Long. 4. lat. 1 .

Pédes nunc nigri, nunc caftanei funt. Variat colore toto nigro, pedibus non exceptis; interdum vero nigrum eft pedibus obfcure caftaneis. $\mathrm{Hg}$.

394. C. Alni thorace rotundato nigrum, elytris fafciis duabus albis, elytrorum bafi, antennis, tibiisque fertugineis. F.Sp. 245.46.

D Stenocorus Alni. Scop. Del. Fl. et Fn. Inf. t. XX. f. 3. A:

Totum fubnigrum, villofulum: Antennae iufac, elytris longiores. Thorax rotundatus. Elytra bafi rufa fafciolis duabus albis fubobliquis. Abdomen nigrum. Pedes ferruginei femoribus clauatis. Hab. in Alno non frequens. . Long. 3. lat. $\frac{2}{3}$.

$$
\text { (p. i.5.) }
$$

395. C. plebeium thorace globofo, immaculato, elytris nigris, fafciis linearibus albis. F: $\mathrm{Sp}_{\mathrm{s}}$ 243. 3\%.
(1) Rai., Petiv.
\& F.E. S.I. 2. 334.67 .

Herbft. Arch, V. p. 98: n. I5; 
Clytus funebris. Laichart. Tyrol. II. I I I. 8. Nigrum, tomentolum. Scutellum abum. Elytra fritigiata, vnidentata, fafcia vna alteraue lineari abida vndulata. In pectore antice pofticeque lineae albae vtrinque vti et in primo abdoninis fegmento. Hab. etiam in hortis. Long. $3^{\frac{1}{4}}$. lat. I.

Deferiptio anctoris nimis laxa eft, nec noftrum defignare videtur infectum. L. rufico Linn. iam diu mihi fufpesta fuit, atque $C$. atomarinz $F$.-coniicieham; fed, ob deforiptionem datam nimis brenem, coniecturam, meam publici iuris facere haud aufus eflem, nifi Olinier in Encyclopaediae methodicae libro, qui Pariliis prodit, Hift. Nat. Inf. Tom. V. p. 266. n. 75. contenderet; fe in Mufeo Linnaei fub nomine $L$. ruficce verum $C$. liciatuin L. vidifle. 'Idem auctor contendit: citatum Géofr. Inf. I. 2 I5. Iz: ad C. Majfilienje fuum, quod Fabricitis in Entom. fyftem. 1. c. n. 6s. excepit, pertinere, cuil libenter affentio. Hoc $C$. Maffilienfe etian in Germania occurrit, et a Schneidero Chytus fichillecie vocatum elt. Harrerus in Defer. Echaeff. Icon. 8vo p. 2 I5 355. verum C. plebeium fub nomine $L$. lamdae defuribit, $C$. plebeium autem fub L. rufica. 35 4: citat, quae tamen figura Callidium defignare videtur, quod Herbft. C. hieroglyphicin vocat: Arch. t. 26. f. 20 . et quod certo nil, nifi C. myftici varietas eft, cui color bafeos elytrorum ruber deeft.

Antea, quam genus Callidii mittam, pauca de bona Laichartingii diftributione in genera: Callidium et C'ytum addere liceat. Illis Callidia $F$. annunerat, quae thorace depieflo-globofo gaudent, quaeque Linnaeus genere Cerambycum comprehendebat. Clytus autem illa Callidia $F$. continet, quibus thorax fubglobofus eft, et quae tin- 
naeus Lepturis annumerabat. Tranfitus ab altero genere in alterum quidem haud deelt. $\mathrm{Hg}$.

\section{DONACIA.}

Palpi filiformes. Mlaxilla vinidentata. Labium membranaceun, integrum. Antennae fetaceae.

396. D. craffipes femoribus pofticis clanatis, dentatis. F. Sp. 245.

O Rai., Frifich.

D Scop. E. C. I 47. Schrank. E. I. 29.r. Pod. M. G. 3 6. I. Schieff. Ic. t. 84. f. $1-4$.

Variat magnitudine, colore, fenoribis pofticis clauatis, muticis. Antcmae elytris breuiores. Elytra ftriato-punctata, interdun rugulis transnerfis crifpata: hinc natae varietates $L$. confimilis, et a/finilis Schrankii.E. I. 292. 93. Confer etiam De Geer. 5. I40. 80. - Habit. in locis paludofis frequens. Inuenta ctiam in hortis in Portulaca, et fub arborum cortice in filuis, hieme. Long. 4. lat. $I_{40}^{\prime}$

$$
\text { ( } p .160 .)
$$

397. D. fimplex fenoribus fimplicibus. $\mathrm{F}$. Sp. 245.2.

D Schaeff. Ic. t. I 6 . f. $\%$.

Nimis affinis priori. Habitat cum praecedenti.

Auctor cum multis aliis Entomologis magnam fpecie. rum multitudinem confundit, quas tamen differre, mihi perfuafum eft.: Quomodo enim ftatura abhorrens, elytrorumque fculptura atque iniquitas, quae ditterfae in diuerfis reperiuntur, varietates folas ftatuere poffunt? Plures copula iunetas reperi, et in mufeo afferuo, femper tamen eidem peciei ob notas, fupra memoratas, easdem, annu-

$M 2$ meraxy 
merandae erant, quamuis faepius colore differrent. Bene itaque Fabricius in Entom. - fyft. I. 2. p. I I5. fpecies antea confufas feparauit. $\mathrm{Hg}$.

\section{LEPTVRA.}

Palpi filiformes. Maxilla vnidentata, apice fetofa. Labiun membranaceum, bifidun. Antennae fetaceae.

398. L. melanura (a) nigra, elytris rubefcentibus linidisne fittura, apice nigris. F. Sp.245.2.

$\odot$ Gadd., Rai.

D Scop. E. C. $x_{49}$. Pod. M. G. p. 37.

ㄱ F. E.S I. 2.340 .5 .

L. Sutura nigra. Degeer. V. I38. I5.

Hab. in floribus paffim. Long. 5 . lat. $\frac{1}{2}$.

Infectum valde dubium eft. Citata admodum multa exhibentur, fed pauca certo huc referri pofiunt. Degeerius 5. 137. 14. fane aliam, et, vt mihi videtur, $L$. finguinotentan $F$. defcripfit; eiusdein enim L. Sutura. nigra. 1. c. huc pertinet, vti defcriptio bona edocet; citatum ibidem, et ab. aliis, exhibitum: Geoffr. r. 226. 6. L. haffatce. F. annumerandum eft. Geoffr. I. 226.7 . t. 4. f. r. etiam dubius eft, forte quoque ad L. fangninolentam amandandus. Schaeff. Ic. t. 39. f. 4., ab Auctore cum Fabricio citata, varietatem fiftit, quąm Schrankius (E. I. 298.) L. bifaficiatano vocat, et quae faepius cum $L$. hufftata. $F$. confufa eft. Scopolius (E."C. I49.) L. Janguinolentue marem defcripfifie videtur, eiusdem vero L. dubia. I $^{\text {T. apid }}$ L. melanuram potius produci poteft, aut varietatem rariflimam $L$. Songuinolentae. defignat, cuiuss elytri margo externus

cum

(a) Lo haftata vid. Stenoccrus Adnot. Audtor, infius. 
eum apice, vittaque longitudinali, futuram legente, niger $e$ ft.

Mas, duplo minor femina, elytra pallidiora, fummo apice futuraque intima folis nigris, gerit, faepius copula iurctus inuentus cum fenina multo maiore, ac crafliore, elytris obfcure fanguincis futura apiceque late atris. Marso externus interdum quoque nigrefcit:. L. bifusciata Schrankii l.c. minime mas, fed varictas fortuita, eft.

In Lepturarum şenere talis differentia fexus faepius occurrit. Mares feminis graciliures, et multo minores effe folent. Color fi feminis ruber, his teftaceus eft. Praecipue id in L. mbra et teftacea F. E. S. I. 2. 342. I3. I4., apparet, quas aegre adhuc fpecies diftinetas exhibitas video, etfi Fabricius earum fexus folam diuerfitatem perfuafum fibi habeat. $\mathrm{Hg}$.

$$
\text { (p. } 16 x_{\text {. }} \text { ) }
$$

399. L. linida nigra, elytris teftaceis, immaculatis, pedibus nigris. F. Sp. 246. 3 .

오 F. E. S. I. 2. 3 +0. ?.

Similis praecedenti, at plas dimidio minor, elytris integris fordide flauis inmaculatis. Hab. in floribus. Long. $2_{+}^{1}$. lat. $\mathbf{~}$.

Similitudo inter hanc et praecedentem, quod ad ftaturam attinet, fane exigua eft. Haec enim pro portione longitudinis crafia, atque ferme linearis eft, illa contra tenuis, et pone admodam anguftata. Elytra in L. lintida paullulum truncata, angulis rotundatis, in $L$. melantivi vero acute oblique truncata funt, angulo externo longiore acuminato. Hg.

400. L. villica ferruginea, antemis elytrisque fufcis. F. Sp. 246.5 . 
오 F. E. S. I. 2. 34I. I2.

L. reneftita. Iinn. Syft, Nat. I. 2. 638.6.

Laicharting. Tyrol. II. I53. xo.

Antennà nigrae, longitudine corporis, bafi nufae. Caput rufum oculis nigris. Thorax rufus angulis pofticis acutis fubfuinoris. Pectus nignum. Abdomen et pedes fermginca tarfis pofticis nigris. Habit. in Populo tremula. Long. 5 . lat. $I_{3}^{\frac{1}{3}}$.

Fabricius in Ent. fyft. 1., c. citat: Schaeff. Ic. t. 69. f. I, et quidem, vti videtur, optime. Nil enim, nifi antennae totae rufae obftant, quas tamen in iconibus, quales Schaefferus exhibuit, haud refpicere licet. Harrerus l.' c. ad C. Fennicum alle. gat. Nefcio quidem, cur Schaefferus Lepturamo thorace plano, et non conico, vti folebat, rocare maluit. $\mathrm{Hg}$.

401. I. 4-fafciata nigra, elytris teftaceis, fafciis quatuor dentatis nigris. F. Sp. 248. I9.

D Schrank. E. I. 294.

Haec admodum variare videtur. Habeo, cuae variant a defcriptione Linnaei, thorace antice et poftice flauo-marginato, elytrisque apice truncato, vnidentato; a defcriptione rero Scopolina antennarum articulis totis fuluis, tibiisque omnibus, non dente, fed fpinis duabus apice inftructis, palpis totis flanis, et corpore fupra rufo, fubtus nigro lncido quatuor vltimis legmentis. flauo - fubmarginatis. Figura Schaefieri citata quodammodo conuenit, fed differt pedibus nigris, thorace immaculato, et fafciis paullulum diuerfimode figuratis. Tn meis fpeciminibus pedes femver ful:u, femoribus bafi nigris; antennae modo fuluae, modo totae nigrae. Habitat, in 
pratis fillaticis, in Altheae floribus. Long. $7 \frac{1}{2} \cdot$ lat. $2{ }_{4}^{\mathrm{T}}$.

Iternm vltro examinandi locus datus eft, cum plures dinerfas fpecies confufas videamus. Id fatis perw fpecum habeo, auktorem veram $L$. 4-fofciatch $L$. et $F$. non nouifie.

Fabricius, Degeerius, Linnaeus eandum habue re Lepturam, quam Schaefferus Ic. t. 59. f. 6. repraefentat. C. fafciatus. Scop. E. C. $17=$. idem cum L. Scopoliana Laich. Tyrol. II. I 45.7 . et L. elongata. Rofii. Mant. 54. I33., vbi citatas bene adhibita, vltro conferantur. Fabricii $L$. cals carata. E. S. I. $2.347 \cdot 4 \mathrm{r}$. quoque hue fpectat, citato Geoffroy. Inf. I. 226 . I. in Inf. I. 224. 5. emendato. L. avmata. Herbft. Aach. t. $26 . f_{0} 24$. etiam ad eandem peitinet. Spinae enim tibiarum pofticarum maribus tantum propriae funt, quod et Geoffroyus notauit; hinc differentia in defcriptio. nibus nata eft, et hinc concludi poteft $L$. Jubfpinofam. F. E. S. I. 2. $347 \cdot 42$. feminam defignare.

Auctor pedes fuluos femoribus bafi nigris; and tennas nunc fuluas, nunc nigras obferuavit, quae nota cum $L$. arcuata mea, quam Celcb. Panzerus F. I. G. VIII. I2. optime depingi curauit, cum margine thoracis antico pofticoque aureo-flato conuenit. Fabricii. 1. c. 348. 45. L. aurulenter, quae huic affinis videtur, an eadem cum Leptura Auctoris? $\mathrm{Hg}$.

\section{(p. 162.)}

402. L. villofa nigra villofo - flaua maculis duabus in elytro fingulo glabris, nigris. Geoffroy. InI. I. 2 I I. S. La lepture velours janne?

Tota colore fubuiridi flauefcente, et macnlis plerumque fex nigris non margini annexis, fed diftin- 
ctis rotundis, in fingulo elytro tribus longitudinaliter pofitis. Elytra apice truncata. Pedes nigri. Statura $L$. arcuatre: at magnit: variat. Habit. etiam in hortis non rara. Long. $4,4 \frac{\pi}{2}$. lat. $\mathbf{I}, \mathbf{I} \frac{\mathbf{x}}{3}$.

Geoffroyi citatum minime huc referri poteft, cum, ob defcriptionem haud quadrantem, tum ob genus atque familiam, vbi infectum fuum habet. Einsdem enim Lepturac thorace globofo, Fabricii Callidiomum diuifionem iftam defignant, cui Laichartingius Clyti nomen dedit. $\mathrm{Hg}$.

403. L. Sexguttata nigra, elytris maculis tribus flauis. F. Sp. 249. 21 .

\section{와 F E S. I. 2. $349 \cdot 47$.}

Tota nigra, fingulo elytro maculis tribus flauis obliquis, anteriore bafeos minori, fubrotunda. Hab. in floribus rarior. Long. $3 !$ lat. I.

Harrerus Defcr. Schaeff. Ic. $8^{\text {vo }} 225$. 375. citat t. 39. f. 10 fignran certe male exprefiam, dein nomine L. V Jdmannianae n. 376 . tab. 39. f. II. ct t. 182. f. 4. , varietates prioris, maculis elytrorum in vnicam aut duas coadunatis, fufpicans. Ne fexus varietas fit, vti cum Vddmanno. Differt.

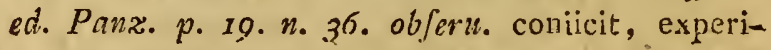
entia mea, de hac Lepcura facta, prohibet. Eft enim varietas quaedam fortuita. In vno fpecimine integerrino maculae elytrorum optime cońfpici poffunt, ita politae, vt duae ad bafin reliquis magis appropinquatae fibi fint, fubrotundae; binaie, dein fequentes, remotiores, ante medium pofitne fribobliquae; pone medium vero duae aliae transuerfules. Quae maculae interdum tam exigwae decrefcunt, vt vix confpici queant; hinc nata illa varietas, quae Schaeff. Ic. t. I82. f. 4. depicta eft. Nonnumquam autem maculae illae poft riores vtrinque in lunulam conflatae funt, cuius finus mara 
marginem. flexura vero futuram fpectat. Haec eft. L. Vddmannicina Harr. 1. c. atque L. Exclamationis. F. E. S. I 2. 343. 20 . fecundum fpecimen a Paykullio celeb. ipfo, a quo Fabricius quoque acceperat, mili miflium. Hg.

$$
\left(\mu . x \sigma_{3}\right)
$$

404. L. Scutellata nigra, fcutello albo. F. Sp. 247: 10.

S F. E. S. I. 2. 343. 22.

Defcribit Fabricius 1. c. Hab. in pratis filuaticis minime frequens. Long. 6. lat. $I_{-4}^{3}$.

Laicharting. Tyrol. II. 140. L. notiis fuam eandem cum L. Siutellate $F$. credit, cui tamen permulta refiftunt; praecipue thorax tuberculatus, lateribus fpinofis, elytra haud excauato-punctata. etc. Hanc L. nofitis Stenocoris annumerare mallem; Fabricius inter Rhagia locum ilii dedit; mihi Stenoc. (Rhag. F.) Ciw foris maris varietas videtur. Hg.

405. L. atra corpore toto nigro. F. Sp. $247 \cdot$ ?.

\section{\& F. E. S. I. 2. 342 . 18 .}

Stenocorus totus niger. Geoffr. Inf. I. 228. Io. varietas. Abdomen ferico albo nitet. Habit. in filueftribus frequens. Long. 3. lat. 1 .

L. atra. Laichart. Tyrol. II. I 48. 8. eadem cum $L$. Melanaria. Herbft. Arch. V. Ior. 8. eft, numquam tamen variat pedibus rutis, hinc alia, quam Fabricii, videtur. $\mathrm{Hg}$.

406. L. nigra elytris attenuatis, corpore nigro, nitido, abdomine rubro. F. Sp. 247. 13.

\& F. E. S. I. 2. 344. 27 .

Praecedentibus minor. Thorax in hac lateribus M 5 pofticis 
pofticis magis acutis. Abdomen infum. Hab. in florbus, Rubo froquens. Long. 3. lat.

Abdomen bafi faepilis rigricat. Hg.

407. L. attemuata elytris attemiatis flauis, fafciis qunituor nigris, pedibus teflaceis. F. Sp. 248. I8.

- $\odot$ Scop., Ann.

D Cerambyx fafciatus. Scop. E. C. I72. mas. Schrank. E. I. 295. Schąeff. Ié. t. 65. f. ${ }^{\prime}$ I I. Q. F. E. S. I. 2.346 .40$.

Caprt ét thorax nigra. Elytra flaua attenuata, apice fafcisque tribus atris. Fafcio anterior variat, iam integra, iam intemuta, interdum omnino deeft. Mlarem $L$. 4-fifcatae ele monet Scopoli; fed haec numquam imenta Pifis.

Habitat Florentiae. Long. $5 \frac{\mathrm{T}}{\mathrm{T}}$. lat. $\mathrm{I}_{4}^{\mathrm{t}}$.

Citatum e Scopolio ad hanc minime pertinet. vid. adnot. ad L. 4 -fafciatcim. n. $40 x$. Fafcias numquam interruptas vidi. Hg.

$$
\text { (p. 167.) }
$$

408. L. raficomin nigra, antennis pedibusque 1ufis. F. Sp. 24?. Ix.

2. F. E. S.I. $2.34+25$.

L. pumiliz. Schall. AEt. Soc. Hall. I. p. 299. Defcripfit Fabricius l. c. Abdomen fubtus albidum. Habit. flores in filuis non infrequens. Long. 3 . lat. $\frac{1}{2}$. vix.

Autennáe rufae, articúlis, primo excepto, apice nigricantibus. Femora pedum anteriorum fupra nigrefcunt, quae nigredo in fecundo pari dimidiam clavae partem tegit et circumdat, in poftico vero totam femorum clauam occupat. Hg. 4.09. L. aubrewiata elytris dimidiatis forrugineis 
gineis immaculatis, antemnis breuibus. F. Sp. 250 . 25 .

o Molorchus abbrevinatus. F. E S. I. 2.356 . I. Habitare dicitur Florentiae Long. Ir. lat. 2.

\section{LAMPYRIS.}

Petpi quatuor anteriores fubclanati, pofteriores fecuriformes. Mutaxilla bifida. Labbiun comeum. Anterinate filiformes.

4IO. L. Italica elytris fuficis, thorace transuerfo rufo. F. Sp. 253. 16.

$\odot$ Sulz.

\& F. E.S. I. 2. 102. 20.

Antemae nigrae. Cculi magni, atri, nitentes. Thorax transuerfus, omniro tinfis, immaculatus, vtrinque marginatus, fentello puriter rufo. Elytra fulca, mollia, fibmargimatil. Pectus, pecescule thorici concoloria: Abdomen nigrum vitimis duobus anmulis albis phofphoreis. Alae fuicae. Femina aptera, nigra, finguio fegmento macula vtrinque marginali flanefcente. Hab. vbique monf. Iun. Long. $4 \frac{1}{4}$. lat. I.

\section{(p. 165.)}

4. I. L. fiplendidula oblonga, fufca, clypeo apice hyalino. F. Sp. 25 I. 2.
( ) Rai, Column., Bradl.
D) Schaeff. Wicm. t. 74 . Icon, t. 268 . f. 4.
\% F. E. S. I. 2. 98.2 .

Antcnnae apice attenuatae fufcae articulis videcim. Palpi quatuor, antici longiores fubclauati. Oculi nigri. Thoracis clypeus fipra vtrumque oculum pellucidus, pallidus, antice rotundatus, 
pone truncatus inacula media poftica nigra. Elytra fuca, flexilia, lineis tribus elcuatis. Abdomen luride flaum fogmentis ad latera dentatis, poftrenis duobus albis licem in tenebris enittentibus. Pedes abdomini cuncolores. Fenina aptera frequens. Mas declaratus raro inuentus menf. Iul. Habit, in agris. Long. $5 \frac{1}{2}$. lat. I⿳亠丷厂

\section{(p.x60.) \\ LVII. PYROCHROA.}

Palpi quatuor fubfiliformes. Maxilla integra. Labiun comeum, lincarc, integram. Antennae filiformes.

412. P. funguinea nigra thoracis lateribus, elytrisque fanguincis. F. Sp. 254.2.

(c) Rai., Suhaeff Ic. t. $7 . f .$, .

D Schaefi. Ic. t. 24 . f. $r$ ?

올 Lycus fonguineus. F. E. S. I. 2. 108. 10. In meis difus thoracis, fcutellnm attingens, femper ater. Hab. in fepibus rara. Long. $3 \frac{\mathbf{x}}{2}$. lat. I.

Valde variat, magnitudinem quod attinet, longus interdum 4.3 . lin. et latus ferme 2. nonnumquam vero longitudo 3 . lineas latitudo antem I.' lin. vix aequat.

Fabricius in Ent. fyft. I. 2. 108. I 1. L. Auroram habet, valde affinem $L$. fonguineo, vti ait, elytris folis reticnlatis diuerfun. Hinc concludere fas eft, Linnei ét Degeerii Lamp. Janghineams nullum alium, quam hunc $L$. Auroram defignare, quia vterque elytrorum reticulatorum mentionem facit. Verus $L$. fonguneus $F$. elytra leuiter ftriata habet, et Geoffroyi Inf. I. I68. 3. de- 
fcriptio optime quadrat. Sulzerus Hif. Inf. abbr. t. 6. f. 4. effigien exhibuit, cuibs thomax omnino ruber eft, quod negrligentia factum, ex iliis Sulzeri iconibus coniicere licet; eiustem ve:o defcriptio, quae, ex, opere alius Auctoris defumta videtur, $L$. Aurovae F. congruit. Num P. Aurora. Herbft. Arcil. V. IO5. t. eadem cum Lyco Fabriciano fic vocato? Scopolii Caffid Janguinerm Ent. Carn. I I9., quam D. Rufí cum Fabricio apud hunc, allegauit, eandem cum $P$. Auroro Herbftii: 1. c. efle, éx defcriptione data vtriusque fatis patet; id quod etim de'I. fonguindc. Schrank. E. I. 322 . concludi poteft. Cuius adnotatum de elytris col lentem reticulatis, coniecturam de identitate cum $L$. Aurora. F. vel $L$ fanguinec. L. confirmat. Talis elytrorum fculptura in L. minuto F. 1. c. $\dot{r}_{3}$. confpicitur. Voetius t. 46. f. 8. I. Janguineun Fabr.; t. 47. f. 9. L. Auroram einsdom repraefentanit, tefie Pánzero, qui ambos poffidet.

Celeb: Fabricius a Pyrochroae genere Lycosm genus, in Oliuierii veftigium, optime feparanit, cum, excepto colore, nulla conueniens nota, "coniunctionem vtriusque gentis, iufferat. $\mathrm{Hg}$.

\section{C V C VIVS.}

Palpi acquales articulo vltimo truncato, crafiori. Labium breue, bifidüm laciniis linearibus diftantibus. Antennae moniliformes.

4I3. C. flauipes thorace denticulato nigro, pedibus flanefcentibus, antennis filiformibus, longitudine corporis, F. Mant. 166.6.

ㅇ F. E. S. I. 2. 95. 8.

C. plapatus. Herbft. Arch. II. 7. t. 7. f. 7.8.

Totus 
Totus fufco-nigcr., Corpus deprefim. Antennae fetaceae villofulac longitudine corporis, articulo primo longifino." "lhorax margine vtrinque fcabro; mucronato. Eiytra ftriata, ftria laterali elcuata. Hab.in finis intra corticem. Long. $34^{\mathbf{x}}$. lat. $\frac{1}{2}$.

Variat corpore toto flauo-teftaceo. Hg.

\section{(y. 707.$)$ \\ "LXII. CANTHARIS.}

Palpi fecuriformes. Maxilia:bifida. Labium integrum. Antenue filifomes.

4.4. C. fufa thorace niarginato rubro macula nigra, elytirs fufcis. F. Sp. 257. 1.

(C) Rai,, Frifch., Sulz., Schaeit. Ic. t. 26. f. 9- I2.

D. Scop. E. C. I20. Schrank. E. I. 325. Schaef. Ic. t. I6. f. IO. II.

Q F. E. S. I. I. $2 x_{3}$. I.

Varietates mihi notas huius fpeciei enumerare placet:

a. C. fufca proprie fic dicta.

ß. thorace ab-que macula nigta, femoribus paris anterioris totis rufefcentibus, fine nigredinis rudimento.

a, thorace è rufo nigricante.

b, thorace fuluo - rufo.

I. femoribus fecunti paris rufis, aut fummo tautum apice nigricantibus.

2. apice, nigro.

$\gamma$ elytris liuidis, thorace rifefcente.

a. macula frontis angufta nigra.

b. macula frontis deficiente, pedibusque omnibus absque vlla ferme nigri coloris tinczura.

Varie-, 
Varietates $\beta$. et $\gamma$. coniunctas, Geoftroyus $a_{\gamma} C_{8}$ frt $c a$ feparaut et Int: I. I I I. 2, vt difinctam exhibuit fpecien. C. difpa: F.E. S. I. 2. $213 \cdot 3$. quoque hui pertinere videtur. 'Varietas \%. $C$. Iirida. F. et Atuloris eft. Invicem inter féfe copula iunęue reperiuntur, - E Schaeferi Iconib. t. x́́. f. 9.10 .1 I. I2. allego adieda f. I 4. fecrndum Harrerum varietm. $\gamma$. C. lizidam reprefentante.

$\mathrm{Hg}$.

4I5. C. limid thoriace marginato, tota teftacea. F. Sp. 258.2 .

○ Rai.

\& F. E. S. I. 2: 2 I3. 2.

Forte habenda pro varietate C. Fufcae it aiunt, fed mihi diucrfa. Habit. in plantis mimus frequens. Long. $4 \frac{1}{2}$. lat. $\mathrm{r}$.

Vide adnotata ad priorem. n. 4r4. Hg.

(p. 1.68.$)$

416. C. obfuic thorace marginato, marginibus rubris comore nigro. F. Sp. 253.3.

$\odot$ Act. Nidrof.

D Goetze. Ent. Beytr. x. 230. 5. Schraik. E. I. 335.

2. F. E.S. I. I. $214 \cdot 6$.

Variat thoracis margine rubro, et pallide furco; pedibus rubris et nigris. Habitat in floribus. Long. $2 \frac{2}{5}$. lat. I.

In omnibus fpeciminibus meis pedes nigri funt. Aliam pofideo huic fimillimam ex America feptentrionali allatam, cuins characterem fpecificum vix, ac ne vix quidem, eruere potui. Margo thoracis vndique flauus; in noftrite autem thoracis macula nigra cum futura antica etiam pofticam occupat, lateribus folis flauis aut luteis. Haec fola differentiae 
rentine nota, patria tam remota computata, fuffuciens videtur. $\mathrm{Hg}$.

4I 7. C. pallipes thorace marginato atra, elytris pallidis apice fuccis. F. Sp. 259. 10.

\section{\& F. E. S. I. I. 216.14 .}

Delcripta eft a Fabricio 1. c. Habit. in hortis non frequens. Long. $3 \div$. lat. I.

Variat elytris apice concoloribus, quae varietas fae. pius occurrit, quam illa elytris apice nigricante. Fabricii $C$. pallida. 1. c. I 6 . eadem cum hac varietate videtur, praecipue cum citet Geoffroy. Inf. $I$. I73. 6. qund citatum iterum ad C. teftaiean. 1 c. 220. 33. allegat. Geoffroyus nempe varietates credidit, cui tamen affentire nequeo, cum tho. racis forma dinerfa fit. Deinde $C$ : pallipes. $F$. gracilior, et pro portione longior eft $C$. teftacea $F$. 'Hg.

4IS. C. nigripes thorace rotundato lutea, abdonine, pedibus elytrorumque apicibus atris. F. Sip. 260.15.

Caput et thorax lutea. Antennae fufrae. Elytra pallide teftacen, bafl et apice nigra. Abdo. men et pedes atra. Similis. C. Melanurae. An eaden quae C. fulut. Scop. E. C. 224 ? - Hab. ruil. Long. 3i. lat. I!

Auctor minime veram $C$. nigripedem. $F$. habuit, quam ipfe a Dn. Lund, a quó et Fabricio miffa erat, accepi. Habitus illan prorfus a Cantharidum genere remouet, et mihi vera Necydalis videtur, ubi $N$. vfulatom fibi valde fimilem habet. Defriptionem adiicere placet.

Caput cum thorace aritennisque luteuin eft, mandibularum apice oculisque, atque interdum macula frontis obfoleta, nigris. Thorax capitis 
latitudinis, deprefficfeulus, fubcordatus, inmarginatis, punctulatus, nonnumquam rnaculis quibusdam obfoletis ac fere exftinctis nigricantibus. Elytra mollia. punctata, pone acumisata, e luteo colore in liuidum vergunt, apice atro. Striae duae in medio carinulam eleuatam gerentes defcendunt parum vifibiles. Marginem ftria infculpta fimplex legit. Alae fufcefcunt. Abdomen pedesque nigricant, ano geniculisque rufe. fcentibus. Magnitudo et fratura $N_{e c y d}$. uftulatae.

Scopoiii C. fulua eadem cum AuEtoris C. nigripede effe poteft, fed nullo modo ad Fabricianam C. nigripedem pertinet. $\mathrm{Hg}$.

419. C. ruficollis? atra, thorace abdomineque xufis. F. Sylt. Ent. 206. ?•

\& F. E. S. I. I. 2I T. I?.

Facies Cantharidis. Antennae filiformes nigrae, bafi rufae fere longitudinc corporis, pilofie, articulis vndecim, fecundo breuiflimo. Caput nigrum mandibulis palpisque quatuor rufis, aequalibus, articulo vltimo craffiori oblique truncato. Thom rax rufus, plamus, tuberculofus, marginatus. Elytra nigra. Abdomen rufum. Pectus nigrum. Pedes rufi, tarfis quatuor fulcis. Variare videtur thorace lateribus denticulato. Hab. ir: floribus. Long. $3 \frac{1}{4}$. lat. I.

420. C. Melanura thorace rotundato corpore luteo, elytris apice luteis. F. Sp.260. 16.

○ Rai.

D Goetze. E. B. I 538 2\%. Schaeff. Ic t. 58. f. ๆ०

q F. E. S. I. I. 2 I\%. 26 .

Antennae nigrae bafi lutea, longiores quam in praecedenti: reliqua omnia lutea, exceptis ely- 
trorum apicibus nigris. Hab. paffim in floribus praefertim Dauci Carotae, et Athamantae Oreofelini, ad minora infecta praedanda.

, Thorax minus recte rotundatus vocatur, eft enim fubquadratus, antice paulluhm anguftatus, vndique vero marginatus. Schaeff. Ic. t. I G. f. I 4 . auctor incertam habet; Harrerus, ad $C$. liuidam pertinere, ait. T. 58. f. 7. mihi tamen dubia eft, licet Harrerus iple fic habeat. Necydalis fpeciem prae fe fert, et forte $N$. vftulata eft. $\mathrm{Hg}$.

$42 \mathrm{I}$. C. biguttati thorace marginato medio atro, elytris abbreuiatis nigris apice flauis. F. Sp. 26 I. 20.

I. Sela aff Ic t. I8. f. I2, I3.

f F E. S. I T. 22 T. 34. Panz F. I. G. XI. 15.

Co ut et thurax ommino atra. Elytra nigra fumnits apicibus flauis. Hab. in plantis. Long. $I^{\frac{1}{2}}$. lat. $\frac{\mathrm{r}}{\mathrm{a}}$.

Duas habeo Cantharides, fibi fimiles, fed diligentiore intuitu admodum dinerfas. Altera noftra $C$. $b i$ guttata eft, vti Panzerus 1. c. depinxit, quae variat thorace luteo - marginato, et toto nigro. Altera longior, elytris liuido-nigricantibus, apice, corpore reliquo, pedibus, ore antennarumque bafi flauis. thorace flauorufo dorfo atro, ftria longitudinali media flaua; capite rero praecipue diftincta, nigro, thorace latiore, poftice valde attenuato, vt in Attelalis. An Fabricii $C$ minima? Linnaei Cantharis fic rocata, Malachii fpecies videtur.

- Citatum: Schaefferi Icon. t. I8. f. x2. I3. quod Auctor cum Fabricio habet, nullo modo ad hanc pertinet, fed ad $M$. aeneum, ipfo quoque confentiente Auçore. vid. n. 423. Citatum: Geoffr. Inf. I. I 7 6. I I. a Fabricio in Ent. fyft. omiffum eft, et Malachii fpeciem defignare videtur, 
tur, forte $M$ pedicularim. In huius locum Fabricius Geoflr 372. r. t. 7. f 2. fubftiuit. E yora vero corpore haud breuiora funt, qued tamen Geoffi:uyus et defcriprione et figuru expr. fir, et turfos $v t$ in Cantharide efle folent, aficio. Hocce citatum Fabricius ad C. minimam fuam iterum refert, vbi etiam Auctor habet. $\mathrm{Hg}$.

$$
\text { (p. 170.) }
$$

422. C. minima thorace marginató info, macula nigra, corpore fufco, elyiris apice flauis. F. Sp. 160. Is.

\& F. E. S. I. I. 220.32 .

Ancenae fubienatie. Thora marginatus rufus, mucula nigra. Hab. in hortis. Loing. I

De citato Geoffr. Inf. I. 372. I. vide adnctuta ad priorem. $\mathrm{Hg}$.

\section{MALACHIVS.}

Fotpi fliliormes, articulo vitimo fetaceo. Maxilla vucicntata. Iablum rotundatum, membranaccum. Antennate filiones.

4=3. M. aenens corpore viridi-aeneo, elytris fanguneis. F. Sp. 26I. I.

() Rii., Schaeffi, Mon., Sulz.

D Scop. E. C. 126. Schrant. E. I. $3 \approx 9$.

O F. E. S. I. I. 22 I. Panz. F. I. G. X. 2.

Defribitui a Limaco in Fn. Su. 708. Hab. in variis plantis. Long. 3. lat. I $\frac{1}{3}$.

Variat interdum elytris fere tutis rufis, macula fcutellari fola viridi - aenea.

Rilum citare haud iunat, quia defcriptiones eius raro ad infectum cognofeendum fufficiunt. Citatum huc a Fabricio reiatum 77. 12. ad $M$. 
fanguinolentum pertinet. Snlzer Hift. t. 6. f. 5. a Fabricio citaca, ad $M$. bipuftulatmm referatur. $\mathrm{Hg}$.

\section{(p. 17r.)}

424. $M$. bipuftulatus aeneo viridis, elytris apice rubris. F. S: 262.2.

$\odot$ Schaeff. Ic. t. I9. f. I 4 .

D Scop. E. C. r27. Schrant. E. T. 230.

†. F. E S. I. I. 222. 2. Schaeff. Ic. t. IS. f. I4. Sulz. Hift. Inf. t. 6. f. 4 .

Defcriptus elt a Limnaco in Fn. Sr. 209. Os flaumm. Habitat in variis plantis, frequens in Auena fatiua victitans rapina minimorum infectosum. Long. $2 \frac{2}{3}$. lat. I $\frac{1}{4}$.

Antennarum formae fingularis plures iam mentionem fecere. Sed laud in omnibus iftos appendiculos videre licet. Etiom in $M$. aeneo interdum a dunt.

De citato e Sulzero vide adnotata ad praecedentem. Citatun Schaeff. Ic. t. I9. f. I4. in Entom. fytem. 1. c. deleatur, errore calami pro t. I 8. f. I4. fcriptum. Hg.

$$
\text { (p. 172.) \% }
$$

425. M. Janguinoientus nigro-aenens thoracis margine, elytrisune fanguineis. F. Mant. $169 \cdot 4$.

\& F. E. S. I. I. 223. 6. Rai. Inf. 7\%. 12.

M. rifus Herbit. Arch. V. 108.5 .

Defcribit Fabricius. 1. c. Hab. in lloribus frequens. Long. 2. lat. $\frac{1}{2}$.

Aliquando in Arundine copiofinime captus eft. $\mathrm{Hg}$. 426. M. pedicularius ater, elytris apice rubris. F. Si. 202.3 . \& F. E. S. T. I. 224 . II. Hab. in hurtis meni. ius. Long. $x_{2}^{\prime}$. lat. ${ }_{4}^{3}$. 


\section{NECYDALIS.}

Palpi fubsiliformes. Maxilla vnidentata. Labiums meinbranaccum, late emarginatum. Antennae filiformes.

42\%. N. viritifima thorace teretiufculo, corpore cacruleo fibopaco. F. Sp. 263. I.

$\odot$ Degeer., Gieoffir.

Q F. E. S. I. 2. 350. 2. Schaeff. Ic. t. 94. f. 7. Hab. in floribus frequens. Lonig. 3. lat. I.

Thelephorum glaucefcentem Harrer. 1. c. 84. I35. quoque citarem, nifi defcriptio nimis breuis prohiberet. Geoffroyi Infectum, a Fabricio huc relar tum, minime huc fpectare videtur. Hinc citatum 2b Auctore bene oriifium eft. Fabricius pedum anticorum flauefientium mentionem facit, quos in figura Schaefferi citata bene contpicere licet. Sed nec Linnueus, nec Degeerius, nec Auctor ipfe pedes anticos flauos commemorauere. Inde colligere fas eft, diuerfas fpecies defcriptas effe $a b$ vtraque parte.

Thorax, in Fabriciana, teretiufculus, inaequalis. Elytia frriata funt: in fingulo ftriis eleuatis, praeter marginalem et foturalem, duabus, quarum altera, futurae propior, abbreuiata eft, et medium ron attingit, altera vero integra, et per totum decurrens e'ytrum. Femora poftica nunc clauata, clauis craffis, fubincuruis, nunc fimplicia. Color pedum anteriorum luteo - flauus, quatuor vero pofticorum viridi - aeneus eft. $\mathrm{Hg}$.

428. $N$. caerulefcens thorace teretiufculo, corpore caerileo fibopaco. F. Sp. 263.2.

을. E. S. I. 2. 35 a. 3. Similis $N$. viridiffmat, at paullo maior, er minus nitens. Hab. in floribus. Long. $3^{\frac{1}{2}}$. lat. $x$. 
429. $N$. ruficollis tiorace teretiufculo, abdomineque rufis, capite, elytrisque viridi-aeneis. F. Sp. $263 \cdot 3$.

․ F. E. S. I. 2. 352.9 .

Fabricius 1. c. defcripfit. Habit. ruri in floribus frequens. Long. $3 \frac{\hat{z}}{3}$. lat. $\mathbf{I}$.

430. $N$. atra elytris fubulatis nigra, femoribus ominbus clanatis. F. Sp. 263. 4.

운 F. E. S. I. 2.352 . I 1 .

Tota nigra fcutello excepto, et fafciis tribus albis, quae ad latera cingunt abdomen. Antennae elytro fubaequales. Thorax trituberculatus. Elytra fubulata marginibus, lineagque media elenatis. Clauae femoram quatuor anticorum fubrotundae, pofticorum vero magis elongatae. Interdum fafciae abdominales latera non attingunt, ab elytris occiltatae. Hanc cum $N$. rufa fenper copula iunctam vidi. Habit. frequens in Vmbellatis. Long. 4. lat. I.

Hinc eadem ofpecies cum $N$. rufa eft. Eiusmodi enim fexus varietates in hoc genere femper ferme occurrunt, $\mathrm{Hg}$.

431. N. mf $_{\mathrm{f}}$, clytris fubulatis, nigra, femoribus ommibus clatatis, elytris antenisque rufis. F. Sp. 263.6 .

D Schrank. E. I. 3 II. Goetze. E. B. I. 5 12. 6.

S. F. S. I. 2. 353 I ?.

Antentiae fubrufie bafi nigra. Caput nigrum. Thurax niger tubcrculis tribus lucidis, margine antico et poftico quafi femper bimarulato luteis vel albialis pilis. Scutellum luteum vel albidum. 
Humeri nigri. Elytra attenuata rufa, marginibus lineaque media prominulis. Abdomen ad latera faepe luteis rel albis fafciis cinctum. Fenorum bafis, tibiae et tarfi pleruncque rufi. Clanae yedum quatuor anticorum nigrine et fubrotundae, poiticorum rufae, apice nigro, et magis elongatae. In nomullis tamen etiam clavae pofteriores nigrae funt. Ihas $N$. atrae. Habiat cum priori fiequens. Long. $3 \frac{3}{3}, 4$. lat. 3 ,

Harrerus, praeter Schaeff. Icon. t. 94. f. 8., etiam t. 85 f. 7. citat, de quo ob defectum copiae fpeciminum fufficient s itdicare nequeo. Thoracis forma infoliti Geoffroyum fatis excufat, quod hoc infectum Lepturis potius annúmeraret. $\mathrm{Hg}$.

432. N. Podagrariae elytris fubulatis teftaceis, corpore nigro, femoribus pofticis clauntis bafi teftaceis. F. Sp. 264.8.

() Schaeff. Ic. t. $85:$ f. 7 ?

f F. E.S. I. 2. 354.20 .

Specimina habeo, quae variant thorace etiam teftaceo, puncto vtrinque imprefio, an Canih. flanefcens fubuillofa elytris attennatis. Geofir. Inf. I. 343. 5? N. flauefcens $F$. et Limn.

Feniora poftica cuafifima, gibba, arcuata, nigra, vti in $N$. caenulea. Femora primi et lecundi paris tefareá, vti tibiarm bafis: digiti vero nigri. Alae fucae. Occurrit etiam alia miti varietas praeccientis, pedibus finplicibus, thorace, elytris, abdomineque tefaceis; in omnibus, tamen elytrorum color magis flauefcens, quam. teftaceus. Habit. in filuis minus frequens. Long. 4. lat. I. 
Confer, quae habet Auctor. N. flauefcens. Mant. 56. 139 .

Femora poftica crafifima, flauo-teftacea, apice nigra. Elytra teftacea, margine exteriore apiceque nigris. Deferiptio Geoffr. Inf. 3+3. 4. nimis breuis eft, et hoc infectum non fatis defignare videtur, id quod etiam de 343.5. dicere licet, quod AuEtor apud varietatem allegat. Ipfe varietatem puto, fed $N$. flculefcentem $L$. alian effe, milhi perfuafum hábeo. Videtur potius $N$. pratescana. Schrank. E. T. 3 I G. femoribus pofticis fimplicibus, apice atris. Differt praeterea elytris minus fubulatis, vnicoloribus. C. femorati. Scop. E. C. 145. ab Auctore, Fabricium fecuto, citata, alia eft, et praecipue pedibus omnibus nigris differt. $\mathrm{Hg}$.

\section{(p. 175.$)$}

433. N. caerulea elytris fubulatis, caerulea, feinoribus pofticis clauatis, arcuatis. F. Sp. 264. 7 .

$\odot$ Sulzer.

\& F. E. S. I. 2.354 . 19.

Schaefferi Icon. t. 94. f. 7. mala eft. Colore riridi-caeruleo nitens, villofula. Antennae nigrae, elytro fubaequales, attenuatae. Palpi triarticulati. Thorax cylindricus, tuberculofus, in medio coarctatus, antice et poftice fubmarginatus. Elytra fubulata, modo viridia, modo cacrulea, linea media eleuata apicem non attingente. Femora poftica globola, plerumque cacrulea. Femora, tibiaeque primi et fecundi paris concoloria. Abdomen viride, at tarfi fufci. Variat colore toto viridi, et pedibus omnibus fimplicibus, vti et magnitudine. Femora clauata in hoc 
genere, nec fexum, fod varictatem tantum indicare videntur. Habitat in hortis frequentifima, tam femoribus poiticis fimplicibus, quam globofis. Long. 4 . lat. I.

Schaefferi Iconum figuram citatam ad $N$. viridifjimam. n. 427. pertinere, ibidem adnotatum eft.

$\mathrm{Hg}$.

434. $N$. humeralis elytris fubulatis nigris, bafi flauis. F. Sp. $263 \cdot 5$.

\&. F. E. S. I. $2 \cdot 352$. I2.

Primo intuitu, fi caput inficiatur, videtur Meloe, aut Lytta. Tota nigra practer humeros, qui fuhui funt. Elytra fubulata absque lineis. Pedes fimplices, fufici. Curpus molle. Alae nigrae. Habit. fuper herbam in filueftribus rara. Long. 5 . lat. $\mathrm{I} \frac{2}{3}$.

\section{(p. 176.$)$ \\ LXV. ELATER.}

Palpi fecuriformes. Maxilla vnidentata, obtufa. Labium bifidum. Antennae filiformes.

435. E. aterrimis thorace atro nitido, elytris Ariatis nigris. F. Sp. 267 . I4.

\& F. E. S. I. 2. 221.24 .

Corpus totum aterrimum, villofulum. Thorax punctatıs. Elytra ftriata. Hab. in hortis frequens. Long. $6 \frac{1}{4}$. lat. I 1 .

Flaterum fpecies difficillime verbis diftinguuntur, hinc confufio in his maxima eft. Defcriptiones breuiores effe folent, et ratio partium inter fele, $\mathrm{ab}$ auctoribus rarifime obferuata eft, quae tamen praecipue diftinctionem fpecierum inuat. Anctor nofter defcriptiones aut nullas, aut breuiinimas 
adiecit; difcernere itaque nequeo, an verum femper habuerit Elaterem. $\mathrm{Hg}$.

436. E. obfcurus piccus, thorace elytrisque ubfcure nigris. F. Sn. 260.26.

D Scop. E. C. 284 . Schrank. E. I. 347 . Schaeff. lc. t. I9. f. 2 . 6 .

Q F. E. S. I. 2.226 .44$.

Habitat faepe in tloribus Paftinacae. Long. 5. lat. $\mathrm{I}_{2}^{\mathrm{I}}$.

Elater Linnaci diuerfus ab Elatcre Fabricii mihi vide. tur. Schaefferi figurae citatae, quas et Celeb. Schneiderus in recenfione fua (N. Migaz. d. Entomol. v. Fuefsly. III. 2. I3I. 260) approbat; Elaterem aenei coloris repraefentare videntur, hinc nullo modo tolerari poftunt, cum nec Fabricius, nec Linnaers talis coloris mentionem faciant. $\mathrm{Hg}$.

\section{( $\mu . x=5$.}

437. E. fiutator thorace fufco-nitido, elytris cinereis, corpore nigro. F. Sp. 272.39.

( Schaefi. Ic. t. I\%. f. I I.

D) Schaeff. Ic. t. I90, f. 8 .

․․ F. E.S I. 2. 230.62 .

Antennae ruiace Thorax fuicus, nitidus. Elytra rufo-fufa. Pedes ferruginej. Hab. in Salice. Long. $4 \frac{1}{2}$. lat. $I_{+}^{\mathrm{T}}$.

Etí pro Schaff. Ic. t. In. f. I I., vti Fubricius habet, Jergeres f. 2., tarien Sputator ibidem non repraeCantatus eft; lisume 3 . et 5 . citusiem tabulae potius allegari pofient. Figura \&. tabulae syg, quam apud hunc exhibent, alium repratentare videtur, quem ipfe poffideo, cuius elytra apicem verfus nigrefcunt. Paullo longior eft illo, quem verum E. Spulatorem habeo, duplo vero craffior.

$\mathrm{Hg}$ : 
43S. E. filiformis cinereo-fufcus, immaculatus. F. Sp. 270.28 .

\& F. E. S. I. $2.227 \cdot 48$.

Cinerco-fufcus inmaculatus; antennae longitudine thoracis. Hab. in filuis. Long. 3 ; . lat. 1 .

* 439. E. niger niger, thorace laeui, elytris, pedibus, corporeque nigris. Limn. Sytt. Nat. 656 . 33. Fu. Sv. 743 .

Geofti. Inf. 1. 134.8 .

q F. E.S. I. 2.221 .25 .

Habit. in pafcuis. Lony. 5. lat. 2.

Geoffroyi citatum falfum effe, omnis mecum fentiet, qui oculo, et fugitino, eiusdem defcriptionem perluftrauerit. Fortafie ad E. murinum amandanduin eft: $\mathrm{Hg}$.

* 440. E. carbonarius atcr, atro - farinofus, punctis ninutiffmis albis interiectis, antennis ferratis. Schrank. E. 1. 343 .

Schrankii defcriptio 1. c. iterata. Variat magnitudine. Hab. fub arborun cortice frequens in filuis; at innentus femel ctiam in hortis fub cortice Populi trcmulae. Long. 8. Iat. $3 \underset{i}{\mathrm{x}}$.

Infectum fingulare, atque pulcherrimum eft. Magnitudo et ftatura eadem cum. E. fafcicto, fed thorax magis elongatus, et linearis, atque fupra inaequalis: foucola impreffa longitudinali obfoleta. Elytra thorace haud latiorn, nec duplo longiora funt. Caetera, vt in defcriptione Schrankii. Specimen meum, quod ex Italia accepi, cum menfura, ab Auctore data, conuenit; Schrankius $6 \frac{7}{2}$. lin. longum, et 2. lin. latum efie, dicit. $\mathrm{Hg}$.

\section{( $p .178$.}

44 I. E. minimus thorace obfcure cinereo, elytris cinereo-nebulofis, plantis rufis. F. Sp. 26 . 
ㅇ․ F. E. S. I. 2. 221.26 .

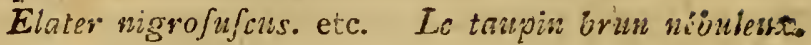

Geolr:. Inf. 1. I34. S. Schaeff. Ic. t. 45. f. 5 . Corpus nigro-cinereum, antennis plantisque ferrugineis. Hab. mivi frequens. Long. $4,5 \frac{7}{2}$. lat. $1:^{\circ}$

Citatum Geoffroy. I. I35. I0., quod auktor cum Fabricio ad hunc allegat, in Entomol. fyetem. bene - omiflum, et ad $E$. holofericeum. 222. 27. 1'elam tum eff. Mlihi Elater Geofiroyi, fupra citatus, et a Dn. Roflio ad nigrum relatus, huc fpectare videtur. Fabricii $E$. holofericens 1. c. an idem cum E. vndulato Herbftii Arch: V. I I . 8? Hg.

442. E. ieffellatus elytris aeneis, maculis pallidioribus confertis, vingubus rubris. F. Sp. 267. I6.

$\odot$ Gad.

옥 F. E. S. I. 2. 222. 28 .

Fufcus, maculis albidis variegatus. Confer Scogolizm. 1. c. Habitat in campis filnaticis. Long. 6. lat. 2.

Geoffoyi.citatum. T. 135.9. incertum habet auctor, et bene quidem, vt mihi videtur, nullam enim coloris cuprei mentionem facit. Item obftat, quo minus Scopolii E. thoracicus E. C. 282. ab Auctore cum Fabricio, adductus, imo E. teffillatus anctoris noftri ipfius, admitti poffit. Schaeff. Ic. t. 4. f. 7. ad alium amandare vult Herbftius, fed mihi noftrum Elaterem fatis bene repraefentare videtur.

$\mathrm{Hg}$.

\section{( $p$. 179.)}

443. E. fanguincus ater, elytris fanguineis. F. Sp. 271.36 .

$\odot$ Bergitr.

D Schrank. E. I. $34 \mathrm{I}$. 
\&F.E.S. I. 2.228 .55 .

Panz. F. I. G. V. 13. Schaeff. Ic. t. 3I. f. 2.

B. Variat rarius macula elytrorum magna communi atra.

E. fanguinolentus. Schrank. E. I. 34r. Beytr. 69. 16. t. 3. f. 15. Schaeff. Ic. t. 3I. f. 5 .

ㅇ E. cphippium. F. E. S. I. 2. 228. 56. Panz. F. I. G. V. I4.

Habitat fiis arborum cortice non rarus. Long. 5. lat. $I_{\frac{\pi}{2}}$.

Errore typographico fub $\alpha_{0}$ pro Schaeff. Ic. t. 3 I. f. \%., auktor habet f. 5 .

Elaterun fpecies colore admodum variare, iam dndum notum eft, inprimis autem in illis, quibus color ruber, linidus, auc aeneus eft, id obfernare licet. Variabilis color huius Elateris permultos induxit, vt fpecies haberent, quae merae varietates funt; imo de quibusdam adhuc incerti reftamus. Variat quidem.

I) magnitudine: Quaedam fpecimina longa funt $7 \frac{1}{3}$. lin. lata vero $2 \frac{x}{4}$; nonnulla contra longa $3 \frac{3}{4}$, lata $I_{4}^{\frac{\pi}{4}}$. lin.

2) colore.

a) elytris vnicoloribus.

a) laete rubris - $\beta$ ) obfeure rubris - $\gamma$ ) flauo-rubris, praecipue bafin verfus.

b) elytris rubris, nigro-pictis.

a) apice folo nigro (E. praeufutus. F. E. S. I. 2. 229.57. )

B) futura intima tantum nigra.

$\gamma)$ macula dorfali maiore, elliptica, nigra, nec bafin nec apicem attingente. (E. ephippi2um. $F$.)

-E. pomorum. Scheven. fpecies diffincta videtur, quamuis, elytrorum colore caftaneo - rufo excepto, cha. 
characterem alium detegere nequeam. IICdius eft, nec tanta magnitudinis varietate occurrit. E. glycereus cinsdem, minor eft, et eadem cum fpecininibus iftis paruis $E$. Sangumei magnitudine gaudet. Color elytrorum flauicans, apice nigro. Nil obfare videtur, quo minus $E$ niger, elytris flawis, Le taipin à étuis jaunes et corcelet liffe Gtoffroy. Inf. 1. I3 I. 3. idem cum $E$ pomorunz et glycereo, iamiam dictis, effe poffet, praecipue cum maximam E. Sanguinei affnitatem comme. mor ret Geoffroyus.

Varietas talis, qualem Schaefferus Ic. t. I I. f. 9. exhibet, et cuius mentionem Geoffroyus locis allegatis, facit, a me nondum vifa eft. $\mathrm{Hg}$.

447. E. balteatus elytris antice dimidiato-rufis, corpore nigro. F. Mant. I 74. 41. Sp. 27 I. 37 .

$\odot$ Rai.

․ F. E. S. I. 2. 229.59.

Hab. in pratis non frequens. Long. $2 \frac{\pi}{3}$. lat. ${ }_{4}^{3}$.

445. E. ruficollis niger, thorace poftice nubro, nitido. F. Sp. 270. 33 .

D Geoffr. Inf. I. I32. 5 .

S F. E. S. I. 2. 227.52 .

Antennae nigrae. Thorax antice niger, pofticeruber. Elytra atra, nitida, fubtiliffine furiata. Pedes rufi. Hab. intra corticem et lignum, rarus. Long. $2 \frac{3}{4}$. lat. $\frac{2}{3}$.

Geoffroyi citatum, aذ Auctore additum, falfum effe, et ad $E$. thoracicum pertinere, fatis perfpectum hateo. Ad eundem referatur $E$ gramineus. Scopolii E. C. 290. Sic et Auctor nofter in Mantiffa fua, ad E. thoracicum. p. 59. n. I 43. correxit. Schaeff. Ic. t. 3 l. f. 3 . ad eundem pertinet.

$\mathrm{Hg}$. 


\section{(p. 180.)}

446. E. ferrugineus thorace, elytrisque ferrugineis, corpore, thoracisque margine poftico nigris. F. Sp. 266. I I.

D Degeer. Int: $4 \cdot 87$. x. t. 5. f. r8.

o F. E. S. I. 2. 220 . I8. Panz. F. I. G. X. IO. Color aetate variat. Antennae nigrae valde ferratae. In adulto caput, et abdomen nigra, vti etiam elytrorum bafis, fcutellum, thoracisque dentes. Hab. in filuis; captus etiam in hortis in Malua arburea flore mbro. Long. $8_{\frac{2}{2}}$. lat. 3 .

Panzeri figura citata, quae arte folita elaborata eft, nimis paruium fpecimen repraefentat. Mea longitudinem I I lin. fuperant. E. rufus Degeerii, ab anctore citatus, idem cum E. rufo Fabr. E. S. I. 2. 3 I9. I 7. eft, quod figura, a Degeerio data, fatis oftendit. Quem Celeberr. Panzerus l. c. Ir. optime depinxit, hinc plura de differentia vtriusque: $E$. rufi et $E$. ferruginei, addere haud opus eft. $\mathrm{Hg}$.

* 447. E. prolifericornis olinaceo-nitens, pilofus, antennis duplicatis. D. D'Antic. Soc. Linn.

Dermeftes tentaculis etc. Geoffr. Inf. I. IO3. I 1. Le Dermefte à oreilles.

$\$$ Parnus prolifericornis. F. E. S. I. I. 245. I. Panz. F. I. G. XIII. I.

Caput fubretractile. Oculi nigri. Palpi pofteriores breuiffimi, anteriores triarticulati, articulo vltimo maiori apice accuminato. Antennae duplices fuperpofitae. Prior infidens capiti vniarticulata? breuis, lata, compreffa, triangularis, fubrecurua, pilofa, fufca. Pofterior inhaeret dorfo praecedentis in eleuatione verfus bafin, et mouetur independenter vniaiticulata, fubpuncta- 
ta, fubcompreffa, acuta ferruginea, pilofá, interne quatuor filla, vel pectinata. Thorax eleuatus, pilofus, vix punctatus, latera pofteriora acita, inferne medium uncronatum, calfiufculum. Elytra vix punctata pilofa. Abdomen fubfufum, pilofum. Pedes ferruginei fufci, tarfi articulis quinque vltimo maiori. D. D. Antic.

Habit. primo vere fuper plantis, et fub lapidibus, in locis aquaticis minime rarus. Long. 2. lat. $\frac{\mathrm{r}}{2}$.

Nec vietus, nec mores, nec ftatura Elaterem indicant: Grauiter incedit, dorfoque iacens, nullum, ac ne minimum quidem pulfum, vt Elaterum fpecies, habet. Antennae infuper diffimiles funt. 'Quid itaque auctorem, locum illi inter Elateres dandi, impulit? $\mathrm{Hg}$.

\section{(p. 881.$)$}

* 448. E. rufines niger, pedibus rufis. Geoffr. InI. 1. 136. 14. Le Taupin noir ì pattes fauves.

\& F.E.S. I. 2. 23 r. 70 .

Totus ater, nitens, thorace lucido, elytris ftriatis laeuibus, pedibus rufis. Habit. fub arborum cortice. Long. 3. lat. $\frac{2}{3}$.

449. E: minutus thorace atro, nutido, elytris nigris. F. Sp. 272.41 .

P. F. E. S. I. 2.23 I. 7 r.

Niger, nitidus, immaculatus. Elytra obfcure friata. Hab. in filuis. Long. $2 \frac{2}{3}$ lat. $x$.

450. E. marginatus thorace fulco, elytris teftaceis margine vndique nigro. F. Sp. 270. 30.

$\odot$ AEt. Nidrof.

D) Schrank. E. I. 352. Schaeff. Ic. t. I94. f. x.

I F. E. S. I. $2.227 \cdot 50$. 
Caput nigrum. Thorax fufcus lateribus ferrugineis. Elytra pallide teftacea margine nigra. Pedes rufi. Antennae fufco tèftaceae. Hab. in filuis. Long. 3. lat. $\frac{2}{3}$.

Schrankius 1. c. "magnitudine mire variare" dicit; vereor, ne plures confuderit fpecies, quas oculi bene quidem, minus antem verba diftinguere poffunt. Magnitudo enim huius fatis conftans eff. $\mathrm{Hg}$.

$$
\text { (p. 182.) }
$$

45I. E. pulchellus capite thoraceque atris, elytris nigris maculis flauicantibus, pedibus flauis. F. Sp. 272.44.

\section{ㅇ F. E. S. I. 2. 233.77 .}

Caput et thorax atra. Elytra nigra friata, antice fafcia fubinterrupta flaua, pone medium punctum flaum. Corpus nigrum. Pedes flaui. Hab. in ligno putrefcente. Long. $I_{4}^{\frac{1}{4}}$. lat. $\frac{\mathrm{I}}{3}$.

* 452. E. maculatus capite, thoraceque nigrofulcis, elytiis pallidis, nigro-maculatis.

Antennae brenes ferrugineae, apice nigrae. Caput nigrum. Thorax niger, minime nitens, conuexus, fubcarinatus, lateribus pofticis parum eleuatis, puncto vtrinque albo villofo obfoleto. Elytra friata, pallidá, macula nigra communi ad bafin, et punctis in fingulo tribus maiusculis oblongis nigris notata. Abdomen nigrum. Pedes ferruginei. Tab. V. f. 7. - Captus in Quercu d. 3. April. Long. $2 \frac{1}{4}$. lat. $\frac{3}{4}$.

* 453. E. bimaculatus thorace nigro, elytris antice dimidiato - fuluis poftice nigris bimaculatis.

\& F. E. S. I. 2. $233 \cdot 79$. 
Antennae longitudine thoracis rufae. Caput et thorax nigra, nitida, grifeo-tomentofa. Elytra fulua, feu potius in mortuo teftacea, ftriata, apice nigro maculis duabus pallide flauis. Pedes rufi. Tab. III. f. Iо. - Hab. fub cortice, et lignis exficcatis cariofis frequens. Long. $2 \frac{1}{3}$. lat. $\frac{1}{2}$.

$$
\text { (p.183.) }
$$

* 454. E. crucifer thorace teftaceo linea dorfali nigra, elytris concoloribus, cruce margineque nigris:

Facies E. cruciati, a quo tantum differt colore minus nitido, et magnitudine multo minori, in reliquis omnino illi fimilis. Hic tamen, etfi plures habuerim, numquam inuentus eft maior lineis duabus. Tab. V. f. 9. Hab. cum praecedentibus. "Long. $\mathbf{I}_{4}^{3}$. lat. $\frac{\mathrm{T}}{4}$.

455. E. bipuftulatus niger, nitidus, elytris puncto bafeos rubro. F. Sp. 273. 47.

\& F. E. S. I. 2. $235 \cdot 88$.

Pedes fului. Elytra ftriata. Hab. cum aliis rarior. Long. 3. lat. I.

\section{BVPRESTIS.}

Palpi filiformes articulo vitimo truncato obtulo. Maxilla vnidentata, obtufa. Labinm cylindricum acuminatum. Antennae filiformes ferratae.

456. B. Mariana elytris ferratis longitudinaliter rugofis, maculis duabus impreflis, thorace fiilcato. F. Sp. 2 \% 6.20.

D. Schrank. E. I. 362 .

\$ F. E. S. I. 2. I95. 4 r.

B. Hinlca. Pallas Ic. Inf. p. 68. t. D. f. S. 
Eft haec e maximis. Superne viridi-aenea rugis longitudinalibus impreffis irregularibus, cauitates cupreo - auratas efformantibus. Subtus fcabra, et aureo ignito colure diues: Antemnae nigrae. Elytra integra. Hab. in filuis. Long. I5. lat. 6 :

Variat colore nigro - cupreo, et nitidiore, absque ni-? gredinis mixtura,

Citatum Degeeer. Inf. IV. x28. I. t. 4. f. 8. omnino deleri, et $B$. calcaratae Schalleri Act. Hallenf. T. p. 3 Io. adfcribi debet, quae eadem eft cum $B$. oxyptera. Pallas Ic. p. zo. t. D. f. II. (quam figuram Fabricius perperam apud $\operatorname{lng}$ gbrem fuam citat. E. S. I. 2. 206 . 86.) B. Anftriaca Schrank. E. I. $364, B$. Berolinenf $i$ Herbftii, et B. plebeic Harrer. 1. c. p. 6r, n, 90. Schaeff. 1c. t. 35. f. 7 . vbi adnotare placet, $B$. plebeian $F$. nullo modo eandem cum Berolinen $/ 2$ Herbft. effe poffe. Priusquam hanc Bupreftin mittam, animaduertere liceat, fpinam tibiae, paris pedum intermedii, quam Schallerus primus et folus ferme commemoratus eft, maribus tantum propriam efle, cum in minoribus folis reprehendatur.

Illam veram $B$. marianam credo, quam Pallas 1. c. Herbftius Arch, t. 25. f. 4. Schaefferus Ic. t. 49. f. I. repraelentauere. Certe elytrorum puncta duo impreffa, vna cum rugis longitudinalibus, in nulla alia aequalis magnitudinis, confpicio, quamuis reliqua defcriptio Linnaei, haud bene conuenire videatur. Fabricius alian defcripfiffe videtur, et cum Linnaei diagnofin feruaret, haud bene difcernere poffumus, an eandem cum Linnaeo habuerit Bupreftin. Praecipue autem patria, a Fabricio commemorata, dubitantem me reddidit. Ipfe ex America boreali Bupreftin accepi, huic fimilli-,

$$
\text { O } 3 \mathrm{mam} \text {, }
$$


mam, coloris nigro-cuprei, elytris profundius infculptis, magis attenuatis, thorace aliter formato. Eft B. Virginenfis Goetze. Drury t. 30. f. 3. An haec Mariana Fabricii? Hg.

457. B. cuprea? elytris integerrimis, thoracis lateribus fcabris, fcutello tuberculari, corpore cupreo. F. Sp. 280.48.

$\odot$ Linn.

D Pod. M. G. 43 .

Mordella cuprea Scop. E. C. I9x.

Videtur differre a $B$. ciiprea Linnaei.

Caput aeneum, rugofo-punctatum. Thorax et elytra viridi - aenea, concolora. Thorax lateribus fcabriufculis. Elytrum margine externo ad bafin duplicatura anguftatum, bidentatum. Sub. tus tota cuprei pulcherrimi coloris, punctis variolofis maiusculis excauata. Hab. in filuis: inuentae tres etiam in Beta vulgari. Long. Io. lat. 3 .

Bupreftum fpecies difficulter extricari fecundum defcriptiones, absque iconibus adiectis, omni perfuafum eft, qui ipfe talem extricationem conatus eft. Inde dubitare licet, an veram Bupreftin habuerit auctor, cum Fabricius Americam patriam dicai, et cum defcriptio fupra data fpeciem defignet, $B$. act minatae aut Berolincnfi fimilem. $\mathrm{Hg}$.

458. B.ruftica elytris emarginatis ftriatis, ob. fcure aeneis. F. Sp. 279. 40.

D Schrank. E. I. 363 .

o F. E. S. I. 2. $205.8 \mathrm{r}$.

Viridi aurea, lateribus thoracis elytrorumque cupreis; maxillis, tarfis antennisque nigris. Ca- 
put et thorax pimctata. Elytra ftriata, apice oblique truncata: $\therefore$ Abdomen fupra profunde caeruleum, infra viridi - aureum. Antennae fere ferratae. Oculi obfcure nubri, a morte nigri. Elytra colore variait, Habit. in filuis. Long. $?_{\frac{\pi}{2}}^{\frac{\pi}{2}}$. lat. 3 .

Schaeff.' Ic. t. 2. f, x. ab auktore et multis aliis citata, colore nimis nigro tincta eft, et aliam repraefentare videtur; quam vero, difcernere nequeo.

$\mathrm{Hg}$.

$$
\text { (p. } 185 .)
$$

459. B. Tenebrionis atra, elytris integris truncatis, thorace variolofo dilatato.

$\therefore$ Fuesly Arch. t. 28. f. $x_{5}$.

Schaeff. Ic. t. 204 . f. 5 .

of F. E. S. I. 2. $20 \% \cdot 90$.

Tota nigra, thorace fcabro dilatato, fuperficie cicatricibus variolofis. 'Elytra fubtilifime lineatopunctata, apice obtufo, fed integro.

$\beta$. Occurrit alia in filuis, quae accedit ad $B$. Tenebrioidem. Pailaf. Rof. Ic. Inf. t. D. f. ?. Schaeff. Ic. t. 204. f. 5., adeo praecedenti fimilis, $\mathrm{v}_{\mathrm{t}}^{\mathrm{t}}$ absque diligenti infpectione eadem iudicetur; mihi tamen his notis diftincta fpecies: nempe non atra, fed aeneo-nigra, thorace minus vafiolofo, minusque dilatato, punctis faepe quatuor maiusculis nigris glabris lucidis. Elytra laeuiora quam in B. Tenebrionis, punctis impreffis minoribus, et 1101 raro cblcure aeneis. Corpus fubtus atrum, punctis minimis aeneis magis confpicuis adfperfum, dorfo fub elytris viridi; in praecedenti fufco. Praetereaque elytra in hac femper minus dura, et acu facilius transfigenda; in $B$. Tene- 
brionis duriffina, et aciculas obtundentia. Hab. in filuis frequens. Long. 9. lat. $3 \frac{\mathrm{r}^{2}}{2}$ :

Nefrio, quid ancturem impediit, quo minus illi Buprefti, quam fub $\beta$. defcripfit, et cuius differentiam fpecificam fibi perfuafam habet, fpeciei peculiaris iura concederet? Fabricius in Ent. fytt. in Oliuierii veftiginm, nomine $B$. tenebricofae (p. $207 . \mathrm{n}$. 89.) feparauit. Auctor recte iudicaffe videtur, Schaeff. Ic, $t$. 204 . f. 5. et Pallas Ic. t. D. f. 7 . ad hanc potius, quam ad $B$. Tenebrionis, 'referri poffe. Pallafius 1. c. f. 6. aliam repraefentat, cuius forma et defcriptio bene cum B. Tenebrionis congrueret, nifi ftafura gigantea illi attributa effet. Eadem cum B. cariofa. F. E. S. I. 2. 207 . 87. effe videtur. . Pallafius ibidem citatum ex̣ibet, quod bene allegari poffe videtur. Geoffr. Inf, I. I28. NB. Cucuius ater, thorace fcabro, entivere albicante confperfo, elytris obfolete friatis.

$\mathrm{Hg}$.

\section{$(p .186$.}

* 460. B. faftidiofa elytris ferrato-vnidentatis, aureo-viridibus, fafcia media lata longitudinali violacea.

Ad $B$. rufticam libenter retuliffem; fed nimia coloris diuerfitas, et apex elytrorum vnidentatus me ad nouam ftatuendam fpeciem induxit. $A b$ ea praeter antea dictas notas differre videtur etiam oculis obfcurioribus, fronte minus rugofa, et thorace in medio fulcato. Fafcia percurrit longitudinem elytri prope futuram. Hab. in filuis. Long. $9 \frac{1}{2}$. lat. $3 \frac{1}{2}$.

$46 \mathrm{r}$. $B$. acuminata elytris attenuato - acuminatis obfcuris, corpore cupreo, F. Mant. I8I. 59. \& F. E. S. I. 2. 206. 83 . 
Defcriptio Fabricii rurfus exhibetur. Hab. in filuis frequens. Long. 9. lat. 3 .

Fabricius in E. S. citatum: Pallas Ic. t. D. f. Io. apud $B$. Berolinenfen p. I88. I 2 . iterum exhibet, cum tamen ad hanc $B$. acuminatam pertineat, vbi ipfe habet. B. Berolinenfis Herbftii, qnam Fabricianam quoque credimus, fupra in adnotatis ad $B$. marianam n. 456 . commemorata, et fynonymis fuis aucta eft. $B$. acuminatore $F$. quidem fimilis eft, attamen fatis diftincta fatura latiore, thorace lateribus magis rotundato, nec ita angulato; elytris pone minus acuminatis, tum vero apice emarginatis, vt anguli promineant vtrinque acuti; porro fpina tibiae intermedii pedum paris, mafculis propria, vt taceam elytrorum fculpturam leuiorem et alia. $\mathrm{Hg}$.

* 462. B. aurulenta elytris faftigiatis bidentatis, viridibus, margine corporeque auratis, thorace fubpunctato. Lim. Syft. Nat.661. Io.

Schreff. Ic. t. 35 . f. 6 .

Anceps diu hacfi, et adhuc dubito, vtrum meum fpecimen foret huc referendum: a defcriptione iudicent cruditi. Caput viridi-aureo-nitens, punctulatum. Oculi a morte fufci. Antemae virides. Thorax viridi-aureus, rotmudatus, vix marginatus, obfolete punctatus, vtrinque foueola excauata. Elytra punćtato-ftriata, truncata, fubbidentata, vitta longitudinali caerulea futuram attingente, coloris eiusdem; reliqua pars elytri (margine quafi intacto) nitens eft aurea fafcia vsque ad apicem diducta. Subtus tota viridi-aulrata, laeuis, fubpunctata, fterno antice pofticeque emarginato. Defcriptio B. decorae Fab. Syft.

$$
05 \text { Ent. }
$$


Ent. 2I.7.6. quae mihi ignota; huic quodammodo competit. Hab. filuas rara. Long, max. 8. lat. 3 .

Linnaei $B$. aurulenta Carolinae indigena eft, et cum defcriptio eiusdem haud omnibus quadret, aliam credere licet. Fabricius in Ent. fyft. I. 2. 208 . 92. aurulentam habet, eiusdem magnitudinis et ftaturae cum $B$. manca. Hanc a D. Hübnero, qui circa Halam Saxonum detexerat, accepit, quae alltem nullo modo cadem cum Linnaeana eiusdem nominis eft, qucd tam $\in \mathbf{n}$ Fabricius innuere videtur, cum Oliuierium citet, qui veram fane habuit Linnaei aurillentam, quia fe illam e mufeo Dni. Smith, quem collecta a Linnaeo, emiffe fcimus, accepiffe afferit. $\mathrm{Hg}$.

\section{(p. I87.)}

463. B. 9-maculata elytris ferratis, nigra, fronte puncto vnico, thorace quatuor, elytris tribus flauis. F. Mant. I 79. 36 .

$\odot$ Sulz.

D Schaeff. Ic. t. 204. f. 4 ?

F. E. S. I. 2. I $98 \cdot 54$.

Rofli Mant. 60. $x 45$.

Tota nigra, fubtus nitidior. Frons flauo - maculata. Thorax vtrinque maculis duabus luteis ad bafin ouatis. Elytra vix ferrata, integerrima, fubftriata, maculis in fingulo tribus ad marginem exteriorem transuerfis luteis, quarum prior maxima furfum bifurca. Variat rarius capite thoraceque immaculatis: $B$. fexmaculati Sihaefferi. Hab. etiam in hortis non rara. Long. 5 . lat. $I_{\frac{3}{4}}$.

Auctor in Mantiffa fua afferit, fe thoracem maculis quatuor numquam notatum vidiffe, vti Fabricius dicit, quamuis fpecimina fatis multa habuifiet. 
Plures Entomologi huic affentiunt, ideo peculiaris fpecies effe videtur. Thorax et caput interdum immaculata funt, quod in fpecimine, reliquis duplo minore, obferuo.

B. 9-maculatam Linnuei aliam effe, et punfia quatuor flaua abdominis et latera thoracis flaua, cum maculae thoracis in noftra in dorfo poficae fint, et patria, quam Barbariam dicit, nobis fuadent. Hg.

* 464. B. 4-fafciato elytris integris, friatis, nitidis, atro-caeruleis, fafciis quatuor albis, primis duabus obliquis interruptis.

Statura et magnitudo fere B. o-inaculatoe. Antennae breues, nigrae, ferratae. Caput nigrum fronte albo-punctata. Thorax niger vix pubefcens, vtrinque lateribus albo-marginatis. Elytra frriata, fubpilofa, nitida, atro-caerulea fafciis quatuor transuerfis albis, prima antrorfum, altera retrorfum arcuata, interrupta.

Inter primam et fecundam punctum album marginale. Subtus tota atro-aenea. Color albus versit in flauum. Tab. IV. f. 6. et 7. - Hab. filvas in Plantagine rara. Long. 5. lat. I

B. vndatae. F. E. S. I. 2. $20 \%$. 88. affinis videtur.

$$
\text { (p. 188.) }
$$

$\mathrm{Hg}$.

465. B. Chryftoftigma elytris ferratis longitudinaliter fulcatis, maculis duabus aureis impreflis, thorace punctato. F. Sp. $277 \cdot 26$.

$\odot$ Degeer., Schaeff.

q F. E. S. I. 2. I99. 57.

Pallas Ic. Inf. t. D. f. $x 6$.

Caput, thorax, et elytra rubro-aenea fulcis quatuor vel quinque exarata: fungulo elytro pun- 
ćtis binis in figuram quadratam pofitis, impreffis, deauratis. Antennae fetaceae, breuifimae, aeneae. Oculi oblongi. Abdomen dorfo caeruleum. Subtus colore aeneo nitidior. Hab. in filuis rara. Long. 5. lat. $2 \frac{\pi}{5}$.

Thoracis forma in effigie, a Herbftio Arch. t. 28. f. 6. fuppl. exhibita, cum meis fpeciminibus haud congruit. Punetum tertium ante puneta bina cuiuscunque elytri, impreffum, magis minusue vifibile, $a b$ eodem celeberrimo Viro iam adnotatum eft. Degeerium e citatis omifit auč́or, eiusdem. defcriptio imo haud in omnibus conuenire videtur notis. Hg.

466: B. manca elytris integris, obfcuris, tho-: race aureo ftriis duabus nigris. F. Sp. 281. 52 .

D Mordella Hungarica. Scop. Ann. Hift. Nat. 5 . I0 4. 85 .

Schrank. E. I. $3^{6} 5$. B. elegantula, quam F. in Mant. remittit ad $B$. Salicis.

Schaefi. Ic. t. I 5 I. f. 5.6.

울 F. E. S. I. 2. 2II. I09.

Defcripfit Fabricius in Syft. Ent. fub nomine biftriatae. 222.37. Elytra punctata. Subtus tota aurea, dorfo fmaragdino colore nitens. Capt. Menf. Mai. Pifis in Rofae foliis. Long. 4. lat. $\mathbf{I}_{4}^{\frac{3}{4}}$.

Schaefferi Icon. figura citata noftrum infectum ant minime, aut peffime repraefentat. Priori conie. cturae potius affentio, cum Schaefferus ipfe Elateren vocet ibi repraefentatum Coleopteron. $B$. elegcntulan Schrankii, auctor, ficuti Oliuierius, huc refert, vt videtur, iure potiore, quam ad $B$. Salicis $F$. Nihil enim quam color, quem faturate viridem, rocat Schrankius, vibi fufcus eft, obftare videtur. 
videtur. M. Hungarica Scop. 1. c. quoque dubio fubiecta eft. $\mathrm{Hg}$.

$$
\text { (p. } 180 .)
$$

467. B. Rubi cylindrica nigra, elytris fafciis cinereis vndulatis. F. Sp. 280.44.

ㅇ. F. E. S. I. 2. 208. $9 \mathrm{I}$.

Nigro-aenea, fronte excaulata, thorace magis nitente in medio globofo, margine reflexo, pofticeque angulato, in bafi punctis quatuor impreffo. Elytra integra fafciis cinereis quatuor obfoletis, vndulatis. Antennae breues, ferratae. Hab. in Rubo frequens. Long. $4 \frac{1}{4}$. lat. $I_{\frac{1}{3}}$.

498. B.viridis elytris integerrimis, linearibus, punctatis; corpore viridi, elongato. F. Sp. 281.54.

( ) Scop.; Schaeff. Ic. t. 7 6. f. 9.

D Schrank. E. I. $3^{6} \%$. Schaeff. Ic. t. 53. f. 7. t. 67. f. 8.9 .

\& F. E. S. I. 2.2I3. II 4 .

Defcriptio Geoffroyana optima contenit exacte cum noftris. Hab. ruri fat frequens. Long. 3 . lat. $\frac{2}{3}$.

Magna bupreftum talium anguftarum copia exftat, quas vtrum fpecies peculiares, an varietates folas a fe inuicem, credere oporteat, nefcio. Color nunc fufcu-metallicus, nunc viridi - cupreus, nunc atrocaeruleus eft. $\mathrm{Hg}$.

469. B. cyanea elytris integerrimis rugofis, corpore cyaneo. F. Sp. 282.6.

\& F. E. S. I. 2. $215 \cdot 128$.

Praecedenti fimilis, at diftincta videtur. Tota caerulea corpore elongato. Antennae ferratae, thorace breuiores. Oculi nigri. Elytra in- 
tegra, ad bafin rugofa, punctata, margine, lineaque in medio prominulis. Thorax margine lateribus dilatato, pofticeque angulato, vtrinque ad bafin puncto impreffo. Hab. cum priori rarior. Long. $3 \frac{\mathrm{T}}{2}$. lat. $\frac{3}{4}$.

Caue, ne mifceas hanc et $B$. cyaneam. Herbft. Arch. t. 28. f. I6. quae corpore lato, ac depreffo, et eadem ftatura cum $B$. appendiculatc F. E. S. I. 2. 210. 102. gaudet. Haec enim Fabricii cyanea forte praecedentis mera varietas eft. $\mathrm{Hg}$.

\section{(p. xoo.),}

470. B. nitidula elytris integerrimis, thorace marginato, vtrinque depreffo, corpore viridi nitido. F. Sp. 282.58 .

D Schrank. E. I. 368 .

o F.E. S. I. 2. 214.123 .

Corpus viridiffimum. Thorax vtrinque depreffus. Antemae nigrae. Hab. flores paffim. Long. 2. lat. $\frac{2}{3}$.

B. laetam. F. E. S. I. 2. 2 I 4 . I24. varietatem huius effe, Linnaeo iam perfuafum erat, vti ex defcriptione fua in Fn. Sv. conftat. Iple copula iunctas reperi. Schaeff. Ic. t. 50. f. 7. t. 67. f. 5. 6. ob formam male expreflam, haud citarem. ' $\mathrm{Hg}$.

47 r. B. Salicis elytris integerrimis, viridis, nitens, coleoptris aureis bafi viridibus. F. Sp. 282. 60.

\& F. E. S. I. 2. 215.125 . Panz. F. I. G. I. I2. Elytra aureo-ignita, bafi viridia. Hab. in Salice. Long. 3. lat. 2.

Celeberrimus Panzerus 1. c. habet "Geoffroy. I. p. I26." fane, quod ex citato fequente: "C. rubinus. Fourcroy. E. P. x. 33. 4." concludimus, Geoffr. p. I27. n. 4. t. 2. f. 3. fpectaturus. Id autem fine 
fine dubio ad $B$. maniam $F$. pertinet. $B$. elegantulam Schrankii E. I. 365. iam fupra ad eandem Bupreftem retulit auctor; vide ibi adnotata n. 466 . Herbft. Arch. t. 4 6. f. 5. de qua icone Panzerus dubitat, huc tamen fpectare videtur. $\mathrm{Hg}$.

472. B. minuta elytris integerrimis transuerfe rugofis, thorace fubtrilobo laeni, corpore ouato nigro. F. Sp. 28 I. 53.

Linn. Sylt. Nat. 663. 23.

오 F. E. S. I. 2. 2 I2. III.

Colore fimilis $B$. Rubi. Non confundenda cum altera B. minuta. Corpus ouatum, obtufum, nitidum, fufco-aencum. Antenmae breuifinae. Frons impreffa. Hab. fub, et in plantis non frequens. Long. $I \frac{\mathrm{T}}{4}$. lat. $\frac{2}{3}$.

Haec altera $B$. minuta, cuius mentionem facit auctor, fine dubio B. minuta. Linn. Syft. Nat. 1. c. n. 24. eft, vbi confufio haud leuis eft. D. Roffi in Mantifla fua 63. r 53. B. minutam "alteram" defcribit, quae certo varietas mera eft, pilis albidis de. tritis. Hg.

$$
\text { (p. ror.) }
$$

473 . B. biguttate elytris integerrimis linearibus, viridibus, puncto albo, abdomine cyaneo punctis tribus albis. F. Sp. 281. 55 .

D Schaeff. Ic. t. 279 . f. 6. a. b.

\& F. E. S. I. 2.213 .115$.

Corpore elongato fufco - viridi. Caput viridefcens, ore oculisque nigris. Antemae breues, virides, ferratae. Thorax maryinatus, depreffus, et in medio punctis binis notatus. Elytra integerrima, marginata, apice acuminato. In fingulo elytro puncta duo excauata alba, vnum 
ad bafin, alterum pone medium ad futuram pofitum. Subtus caerulea nitens pectore vtrinque pilis albis pubefcente; et abdomine ad latera pariter tribus albis punctis maculato. Alii quoque fafciculi villofi albi ab elytris occultantur. Mas. Hab. in filuis rara. Long. 4. lat. $\frac{2}{3}$.

Variat colore fufco-aeneo, et caerulefcente. Thoracis puncta videre nequeo in meis. Punctum elytrorum bafeos album rarifime confpicitur obfoletum. Abdomen in medio elytris latius eft, et quafi margo prominet, puncto ibi imprefio albouillofo femper confpicuo. Elytra acuminata, apice obtufe rotundata, ferrata funt. Hg.

479. B. taeniata elytris ferratis, cinereo-villofa, elytris nigris: fafciis duabus ferrugineis. F. Mant. I80. 42.

An $B$. flauo-fafcicita elytris fafciis linearibus duabus vndatis, inauratis. Piller. It. per Pof: Scl. 84. t. 7. f. 12 ?

\& F. E. S. I. 2. 201. 62.

Facie fimillima $B$. Rubi, at paullo minor. Supra nigra, hirfuta villis certa luce canis: fubtus cinerea pubefcens. Caput antennaeque nigrae breuifimae ferratae. Frons villofa pilis albis. Thorax latus, magis hifpidus, margine reflexo. Elytra nigra, integra, ftriata, punctata, emarginata, fafciis duabus aureis vndatis futura interruptis. Corpus aureum pube decumbente cinerea obductum. Variat magnitudine. In nonnullis interdum afpicitur etiam punctum aureum, vel duo initio elytrorum ad margines, itemque vnum ad apicem. Color fafciarum certe auratus, non 
ferrigineus. Hab. in pratis filuaticis in Hyperici floribus frequens: Long. 2,4 . lat. $I_{\frac{1}{3}}^{\frac{1}{3}}$.

Defcriptio fatis bona. Elytra ferrata, punctato-ftriata, ftriis fetofis. In fpeciminibus meis emiortuis fafciae haud auratae, fed ferrugineo-teftaceae, nitidae funt: In Ent. fyft. in citato Oliuierii loco $B$. blanda legatur: B. taeniata. Olivierius fufpicatur B. hivtan Villers E. Fn. Sv. 338. 35. huc fpectare; figuia t. 2. f.42. quidem, quod ad formam attinet, bona, fed in illa, vt in defcriptione, nulla fafciarum mentio tit." Deinde latera thoracis, atque frons haud fquamis, fed pilis albidis teEta funt. Piller. It. per Pofeg. opui's non poffideo, hinc iconem adire non licuit, fed e defcriptionibus duabus datis, quas Borkhaufen in epitome (Scriba Ephem. Ent. I. p. 94.) feruauit, praecipue pofterior apta videtur. ${ }^{\prime} \mathrm{Hg}$.

\section{CICINDELA.}

Palpi fex filiformes, poftici pilofi. Labium corneum tridentatum. Antenade fetaceae.

475. C. campegeftris viridis, punctis quinque albis. F. Sp. 283. 3 .

(-) Mouff., Litt., Ionft., Bergitt., Sulz.

D Schrank. E. I. 35\%. Schaeff. Ic. t. 228. f. 3.

$\Varangle$ F. E. S. I. I. 170.9 .

Defcriptio Linnaei in Fanna Suecica affabre facta, fed Scopolina magis conuenire cum noftris videtur. Hab. in campis et viis arenofis frequens. Long. 6. lat. $2 \frac{1}{2}$.

Labium fuperius flauicans. Mandibulae etiam flauefcentes, apice dentibusque viridi-metallicis. Totum corpus, elytris abdomineque exceptis, pilis raa 
ris longis obfitum. Margo thoracis anticus et pofticus rubro-aureus. Elytra punctis quinque notantur, quatuor marginalibus, et vno pone medium in elytrorum difco, albicante, vt reliqua, fed limbo inaequali vfto circumdato. Punctum humerale femper adeft, fecundum vero marginale fiepius deficit, aut minimum eft. Apex elytrorum femper albicans. Margo elytrorum extimus non femper aureus, fed viridis interdum. Maculae valde variabiles funt. Specimina habeo, vbi punctum marginale tertium, lunulatum, cum puncto in elytroram difco fito cohaeret iflimo tenui ; in iisdem punctum marginale quartum cum macula apicis confluit. Sunt, qui habeant ante medium in fingulo elytro, iuxta futuram, punctum impreffum rotundum vfti coloris. Haec adiicere placuit, differentian in defcriptionibus, iamiam commemoratis, fublatura. $\mathrm{Hg}$.

476. C. hybrida fubpurpurafcens, elytris fafcia, lunulisque duabus albis." F. Sp. 283. 4.

- () Bergftr.

D Schrank. E. I. 355 .

\& F. E. S. I. I. I T T. Io.

Habitat in locis fabulofis filuaticis rarior. Long. $6 \frac{1}{2}$. lat. $2 \frac{1}{\frac{1}{5}}$.

Thorax pone minus anguftatus, quam in campefri, fed ferme quadratus. Liturae albae faepius obfcurae funt, vt vix confpiciantur. $\mathrm{Hg}$.

\section{(p.193.)}

\section{ELAPHRVS.}

Palpi fex filiformes. Labium rotundatum, acuminatum, integrum Antennae fetaceae. 477. E. riparius viridi -aeneus, elytris punctis latis excauatis. F. Sp. 287. I. 
$\odot$ Lift. Sulz.

D Pod. M. G. 42. 4. Schrank. E. I. 359.

Colore faepe variat. Nigro - cupreus, fubtus viridefcens. Oculi nigri prominentes. Thorax anguftus, teres. Elytra punctis latis excauata. Plantae nigrae. Facile dignolcitur. Hab. in locis humidis paludofis. Long. 3. lat. $\mathbf{I} \frac{1}{3}$.

Fabricius in Ent. fyft. alium habet, quem vliginofum vocat ( I 78. I.) ad hunc citatum Geuffroy I. I 56. 30, imo Linnaeus. Syft. Nat. 658. et Schrank. E. I. 359. pertinere videntur. Oliuierius verum Fabricii E. ripariun nomine $E$. paludofi t. I. f. 4. a. b. et $E$. vliginofum nomine $E$. riparii t. $I$. f. a. b. c. d. e. habet. Hg.

478. E. aquaticus aeneus, nitidus, capite ftriato. F. Sp. 288. 4.

$\odot$ Lift.

\& F.E. S. I. I. I79. 5 .

Hab. ad aquas. Long. $2 \frac{1}{3}$. lat. $\frac{2}{3}$.

$$
\text { (p. 194.) }
$$

479. E. Semipunciatus aeneus, nitidus, elytris punctatis dorfo glaberrimo. F. Sp. 288. 5 .

q F. E. S. I. I. I80. 6.

Infectum elegans, a Degerio cum praecedente confufum, et a Fabricio diftinctum.

Caput denfe frriatum. Elytra ad marginem craffiorem fubtiliffime ftriata, et ad futuram nitidiffima, laeuia. Hab. ad aquas in arena humente frequens. Long. 2. lat. $\frac{2}{3}$.

Diagnofis praecedentis fpeciei, a Fabricio data, etiam huic competit. Apex elytrorum interdum flauicat. Statura a reliquis Elaphrorum Speciebus recedit. Hg. 
480. E. flanipes obfcure aeneus, elytris fubnebulofis, pedíbus luteis. F. Sp: $287 \cdot 2$.

\section{q F. E. S. I. I. I.79:4.}

Statura et magnitudo fere $E$. Semipuncfati; at thorax anguftior." In fingulo elytro puncta duo imprefla prope futuram. Hab cum praecedente minus frequéns. Long. $I \frac{2}{3}$. lat. $\frac{1}{2}$.

Statüràm huius Elaphri equidem cum illa $E$. Semipan- Ctati non compararem, fed potius $E$. riparii habitum quodammodo fimilem haberem. $\mathrm{Hg}$.

\section{(p. 195.) \\ LXIX. HYDROPHILVS.}

Palpi filiformes. IVaxilla bifida. Labium corneum, fubmarginatum. Antennae claua perfoliata.

48 I. H. piceus niger fterno canaliculato, poftice fpinofo. F. Sp. 288. I.

○ Act. Berol., Frifch., Sulz., Bergftr.

D' Scop. E. C. 293.

q F. E. S. I. I. IS2. I.

Nocti volat, fortaffe aquae defectu ad meliorem fedem inueftigandam. Inter alios vnum habeo, qui aeftiua nocte ingreflus Coenobitae cubiculum, non fine quadam eius admiratione volauit ad lumen ardentis candelae. Hab. in lacubus, et pifcinis frequens. Long. 19. lat. 10.

Dyt. haftatum Act. Berol. Fabricius poftea e citatis ad H. olinaceum transtulit E. S. I. 2. IS2. 2. cuius femina ftatura maiore, curpore latiore, colore obfcuriore, elytrisque apice non truncatis, fed acutis differt, quodammodo $H$. piceo fimilis. $\mathrm{Hg}$. 
482. H. Caraboïdes niger, nitidus, elytris fubftriatis. F. Sp. 289. 3 .

$\odot$ Frifch., Bergftr., Sulz.

o F. E. S. I. I. 183.4 . Roffi Mant. 65. 558 .

Elytra fubftriata punctis ad marginem inordinate fparfis. Caput antice vtrinque linea punctorum recurua conftanter impreflum.

Specimen habeo longum $3 \frac{1}{2}$. praecedenti omnino fimile, et forte $D$. fufippes. Linn. Hydroph. Degeer. et Geoffr. InI. I. I 84. 4? - Hab. in aquis.

Variat elytrorum colore cyaneo - nigro. Hg.

483. H. Scarabaenïdes niger, elytris ftriatis, pedibus piceis. F. Sp. 289. 5 .

으․ E. S. J. x. $184 \cdot 8$.

Antennae omnino perfoliatae, at palpi prominentes filiformes, triarticulati, rufi. Hab. in aquis rarior. Long. $2 \frac{\mathrm{T}}{3}$. lat. $\mathrm{I}_{\frac{1}{4}}$.

Citatum Linnaei quomodo Fabricius exhibere potuit, nefcio, cum elytra laeuia, et magnitudinem eandem cum $H$. Caraboïde dicat Linnaeus. Hg.

484. Fr. luridus elytris ftriatis corpore cinereo fufco. F. Sp. 290.8.

온. E. S. I. ז. 186. 19.

Panz. F. I. G. VII. 3 .

Variat magnitıdine. Oculi nigri prominuli. Caput aeneo-nigrum, deflexum, thorace lateribus flauicante in medio nigro. Elytra ftriata lurida, punctis tribus nigris obfoletis. Inferne niger, pedibus elytro concoloribus. Hab. in aquis ftagnantibus, frequens in Conferua. Long. 2. lat. I. 


\section{(p. 197.)}

485. H. minutus ouatus, niger, elytris, pedibus thoracisque lateribus grifeis. F. Sp. 290. 9.

$\odot$ Forit.

† F. E. S. I. I. 186.20.

Noftrum infectum exacte congruit cum defcriptione Geoffroyana 1. c. at facies, palpi, tibiaeque anticae a Scarabaeis omnino remouere videntur. Hab. in aquis fagnantibus. Long. 2. lat. $\mathbf{I}_{4}^{\frac{1}{4}}$.

* 486. H. coccinelloildes antennis perfoliatis niger, thorace lateribus flauicante, elytris pallidis, punctato friatis. Schrank. E. I. 373 .

f H. bipuntiatus. F. E. S. I. 2. 186. 22?

(Dyt. marginalis. Herbft. Arch. I29. 29.)

Habitus Coccinellae, et ita conuexus. Subtus niger, pedibus pallide teftaceis. Caput nigrum ante acutum, vtrinque macula exigua flauefcente. Thorax niger lateribus flauicantibus. Scutellum nigrum. Elytra flanicantia, fed fingula ftriis r8. punctorum minutiffimorum nigrorum. Hab. cum priori in plantis aquaticis. Long. $\mathbf{I}$. lat. $\frac{2}{3}$.

Defcriptio hic data, comparatio ftaturae cum Coccinella, menfuraque, exacte cum Hydrophilo Fabriciano conuenire videntur, qui idem eft cum Dyt. marginello Herbft. 1. c. Punctum pofticum album raro confpicitur. Variat magnitudine. Citatum, a Linnaeo et Fabricio adhibitum: Geoffr. Inf. I. 184. 4. nullo modo tolerari poteft. $\mathrm{Hg}$. 


\section{DY' (p. Ig8.)}

Palpi fex filiformes. Labium coneum truncatum, integrum. Antemnae fetaceae.

487. D. punctulatus niger, clypeo, thoracis elytrorumque margine exteriore albis, elytris ftriis tribus punctatis. F. Sp. 292. 4.

() Bergitr.

\& F. E. S. I. x. 188. 4.

Femina fulcis decen dimidiato-elytrorum, vti in $D$. marginali, at paullo maior, et thorace tantum lateribus flano-marginato. A D. marginali recte diftinguit $D_{e}$ Geer. l. c., cuius optimae defcriptioni addenda, color elytrorum magis in hoc viridefcens, lunulaeque flauae in fronte defectus. Hab. in aquis frequens. Long. I5. lat. 8.

Color in meis obf:urior eft. quan in marginali. Lunu a porro in fronte etiam in hoc interdum vifibilis eft. Thoracis bafis paullulum lutefcit. Geoffroyi defcriptio hand bene quadrat, praecipue quod dicat, elytra fubtiliter transuerfe rugofa effe, cum tantum leuiter punctata fint. Inde Dyticus Geoffroyi forte ad D. frriaţum referendus effet. - Variat duplo minor, et corpore fubtus toto atro.

$\mathrm{Hg}$.

488. D. marginalis niger, thoracis marginibus omuibus, elytrorumque exteriore flauis. F. Sp. 29I. 3 .

○ Rai., Mouff., Lift., Sulz., Bergftr.

$\beta$. D. Semiffriatus fufcus, elytris fulcis dimidiatis decem. Syft. Ent. 231. 5 .

$\odot$ Rai., Frifch., Bradl., Bergftr.

D Schrank. E. I. 374.

\& F. E. S. I. x. 187. 3 .

$\mathrm{P}_{4} \quad$ Agilis 
Agilis in dorfo incubans, faltu fe reftituit; fi diu in licco detineantir, aegre fe immergunt. Maribus anteriorum pedum plantae hemifpharicae fcutatae reu patelliformes ad arctiorem copulam. Feminae femiltriatae. Oua oblonga, magna, alba. F.

Inter mea fpecimina vnum habeo, (ne dicam plures, ) in quo $D$. marginalis notae ab anctoribus indicatae tam manifefte apparent, vt euidenter pateat, bene fuife affumtum pro $D$. marginali; attamen in eo ftriae feminarum defunt, et defunt patellae pedum anticorum: ad quam igitur fpeciem reducendum, fi feminae D. latifjimi, pnnctulati et marginalis, aut fulcatae, aut femifriatae effe debent, et mares pedibus patellatis? Forte nequidem in his fpeciebus palmulae, et ftriae bene fexum diftinguunt? Hab. in foffis et pifcinis frequens. Long. I4. lat. 7.

Mafculos horum Dytifcorum, aliorumque, non femper patellis inftructos effe, nunc nobis perfuafum eit. Ipfe tale fpecimen poffideo, quod patellis caret. Hg.

\section{(p. pog.)}

* 489. D. difpar niger, depreffus, thoracis elytrorumque margine exteriore fermgineo flauefcente, elytris in feinina minus laeuibus, neque ftriato - punctatis.

'\$ D. diffimilis. Rofii Mant. 66. I6r.

D. Roefelii. F. E. S. I. I. I88. 5.

Statura D. punctulati, at corpore magis lato, et magis depreffo. Caput nigrum fafcia fupra os flaua, puncto vtrinque impreffo. Thoracis margo pofticus, et anticus haud flaui. 'Elytra obfcu- 
re nigra, fibrofa, minime nitida, absque ftriis, vel punctis, margine flano angutiore, quam in D. punctulato, et apicem non attingente. Femora, et tibiae flauae, turfi fufci. Femina. Mas autem vix differt a mafculo $D$. punctulati, neque vllas notas fpecificas inucnire potui, quibus ab eo diftinguerem: color tantum elytrorum et thoracis mimus viridefcens, et forma corporis paullulum diuerfa. Pedes antici patellati. Hab. in aquis non frequens. Long. $15^{\frac{1}{2}}$. lat. 8 .

Huius Dytifci feminae fpecimen folitarium poffideo, a D. Paykullio benigniter mecum comınunicatum, quod defcriptione breui magis diftinguere placet. Thorax lineis vndulatis intricatis inordinatis infculptus. Filytra lineis eisdem longitudinaliter decurrentibus intricatis confertis notata, futura poftice intima, apiceque elytrorum late glaberrimis, nec punctatis, nec ftriatis. Vitta marginalis lutefcens, pone anguftior fit, et narginem quidem legit, numquam autem ipfum tangit, et ante apicem iam definit. In apice margo externus lutefcit. Color viridi -obfcurus. De mafculo iudicare nequeo. Haec a me defcripta femina fimillima eft D. aciculato Herbitii, nec vllam fere differentiae notam detegere potui, futurae regione excepta, in $D$. aciculato tota glabra, nitida, quae tamen in noftro fimiliter ftrigata eft. In fpecimine meo $D$. aciculati vitta marginalis ad apicem vsque decurrit; color corporis fubtus, piceus eft, maculis in tribus abdominis fegmentis lateralibus ferrugineis folitariis, in fpecimine vero $D$. Roefelii color fubtus luteo-ferrugineus eft. Priusquam hos mittamus, coniecturae Celeberrimi Herbftii affentire liceat, qui $D$. limbatum $F$. marem $D$. aciculati putat, qnod etiam ex analogia tam affinis $D$. Roefelii fequi videtur. $\mathrm{Hg}$. 


\section{(p.200.)}

490. D. cinereus cinereus, elytrorum margine, thoracisque medietate flauis. F. Sp. 293. I I.

$\odot$ Hoefnag., Lift., Act. Nidr., Petiv.

D Schaeff. Elem. t. 59.

q F. E. S. I. I. I90. I 5 .

Omnes quot habui, plantis anticis patelliformibus. Marem fequentis fufpicatur Geoffroy, afferit Degeer, qui copula iunctos faepe vidit. Hab. in aquis ftagnantibus purioribus frequens. Long. 2. lat. 4 .

$D$. cinereus Linnaei aliorumque certifime mas $D$. fulcati eft, quod experientia tam multorum confirmatum fcimus. Fabricius, $D$. cinereum fuum omnino diftinctum effe a $D$. fulcati mare, contendit, cur autem $D$. cinereum non defcripfit, cur differentiam inter vtrumque nun monftrauit? Degeerius alium habet, quem bilineatum vocat, Inf. t. IV. p. 400 . 6. qui valde fimilis eft $D$. Sulcato mafculo, fed paullo minor, magis conuexus et fafcia thoracis transuerfali integra cum margine confluente; margine antico et poftico vero haud luteis. Hunc verum $D$. cinereum $F$. habeo, Oliuierii certe $D$. ci'nereus eft, vide eius Encycl. Method. VI. p. 309. n. ro. Ab eodem autem iterum nomine $D$. bilineati p. 3I9. I. fub illis exhibitus eft. quos haud ipfe vidit, fed ex aliorum auctoritate enumeranit, animaduerfione tamen adiecta, fe eundem cum $D$. cinereo fuo fufpicari. $\mathrm{Hg}$.

49 I. D. Julcatus coleoptris fulcis decem longitudinalibus villofis. F.Sp. 292. 6.

○ Rai., Frifch., Bradl., Bergftr.

q F. E. S. I. I. I 89.8 .

Mas elytris glabris omnino diftinctus a $D$. cinerpo F. Hab. cum praecedente. Long. 6. lat. 4. 
Conferantur, quae ad praecedentem adnotata funt. $\mathrm{Hg}$.

$$
\text { (p. 20r.) }
$$

492. D. bipuftulatus ater, laeuis, capite poftice punctis duobus rubris. F. Sp. 294. 15 .

$\odot$ Geoffr.

D D. immaculatus. Schrank. E. I. 377 ?

\& F. E. S. I. r. I9 I. 20. Schaeff. Ic. t. 8. f. 9.

Defcriptio Schrankii l. c. iterum exhibetur. Puncta rubra in mortuo non femper confpicua. Hab. ill aquis ftagnantibus frequens. Long. $4 \frac{2}{3}$. lat. $2 \frac{\mathrm{r}}{\mathrm{r}}$.

Geoffroyi citatum bonum videtur, quamuis difficillimum fit, e tanta fpecierum, fibi admodum fimilium, copia veram determinare, defcriptionibus breuibus congruentem. Olinierius l. c. p. 3 II. n. I9. Degeerii D. atrum Inf. IV. 40I. 8. citat, qui tamen alius eft, et fatura maiore ac colore toto atro differt. $\mathrm{Hg}$.

493. D. feneftratus? fubtus ferrugineus, fupra niger, elytris punctis duobus feneftratis. F. Sp. 294. I 7 .

\& F. E. S. I. I. I92. 23.

Defcriptio a Fabricio data repetita eft. Noftra fpecimina fupra fubaenea, fubtus nigra, in reliquis conueniunt. Hab. in foflis fat frequens. Lectus menf. Februar. Long. 3i. lat. 2.

494. D. friatus fufcus, elytris transuerfim fubtiliffime ftriatis. F. Sp. 293. 8 .

\& F. E. S. I. r. I89. ro.

D. fufcus, margine coleoptrorum thoracisque flauo. Geoffr. Inf. I. 185. I.?

Elytrorum ftriae transuerfae, tenues, confertiffimae facile diftinguunt $a b$ aliis. Noftra fpecimina 
mina tainen praeter has ftrias habent etiam in fingulo elytro duas lineas longitudinales puncrorum imprefforum aeque diftantium. Hab. in aquis. Long. 8. lat. 4 .

Geoffroyi Dyticus citatus quidem ad $D$. punctulatum refertur, et audere videor, cum ad hunc allegare mialo, fed defcriptio (confer. adnotata ad n. 487.) et menfura data, long. 8. lat. 4 , huic congruunt. $\mathrm{Hg}$.

\section{(p. 202.)}

* 495. D. Lecuder niger nitens, capite thoraceque rufis, elytris nigris ad marginem rufo-irroratis.

Affinis D. transuerfali. Caput rufum vertice nigro, antenis, palpis thoraceque rufis. Elytra nigra laeuifima, nitida, friis punctorum armato oculo vix confpicuis, et ad marginem verfus apicem rufo-variegata. Pectus fubflauum. Abdomen nigricans. Pedes ferruginei. Differt a $D$. transuerfali praefertim defectu falciae. Hab. in aquis ftagnantibus rarus. Long. $4 \frac{2}{3}$. lat. $2 \frac{1}{2}$.

496. D. ouatus ouatus, fufcus, capite thoraceque ferrugineis. F. Sp. 296. 30.

q F. E. S. I. I. 196.45 .

Habitat aquas ftagnantes. Long. 4. lat. $1 \frac{\mathbf{T}}{2}$.

Geoffroyi Inf. I. I9I. I0. noftrum infectum optime defignat, praecipue, cum dicat, colorem nitorem quemdam fericeum habere. Hac nota inprimis ab alin, caeteris fimillimo, differt, qui glaber, nitidus eft, thorace elytrisque punctatis, quibus autem ille nitor fericeus deeft. Huic nomen $D$. oualis dedi.

Hg.

497. D. planus ouato-oblongus, planus, niger, tibiis folis rufis. F. Sp. App. 50r. 24. 
․ F. E. S. I. I. I95. 36 .

Statura ommiuo D. erythrocephali; at caput nigrum. Corpus planum laeue, vix nitidum. : Tibiae fermugineae. Hab. in aquis ftagnantibus. Long. $I_{+}^{3}$. lat. $I \frac{1}{5}$.

$$
\text { (p. 203.) }
$$

498. D. eriythrocephalus ouato-oblongus, niger, capite pedibusque rufis. F. Sp.295. 24.

오 F. E. S. I. I. I94. 35 .

Habitat in aquis. Long. $I_{\frac{2}{3}}$. lat. 4 .

499. D. lituratus niger, elytris bafi, lituraque apicis pallidis. F. Sp. 296.31 .

\& F. E. S. I. I. I97 47 . Panz. F. I. G. XIV. 4?

Defcribit Fabricius 1. c. Caput nigrum. Habit. in riuulis et fagnis. Long. $\mathrm{I}_{2}^{\mathrm{I}}$. lat. $\frac{\mathrm{t}}{2}$.

Panzerus 1. c. Dytifcum Germanicum depinxit, et hunc allegat, mihi tamen dubius eft, praecipue cum defcriptio hand omnibus quadret, vti Panzerus ipfe monet. $\mathrm{Hg}$.

* 500. D. fermgineus ferrugineus totus. Linn. Sylt. Nat. 966. I6.

Magnitudine Cimicis, totus mfus feu ferrugineus. Confer Schrankium. E. I. 383.

Hab. in aquis.

501. D. paluftris laeuis, elytris lituris duabus lateralibus albis. F. Sp. 296. 29.

오 F. E. S. I. I. I96. 44.

Minor D. erythrocephalo. Thorax quandoque ferrugineus. Hab. in aquis.

502. D. pufillus ater, thorace elytrisque margine albis. F. Sp. 297.37.

q F. E. S. I. I. 201 . ?०. 
Corpus vix magnitudine pediculi nignum. Thorax ater, margine albo. Elytra nigra margine inprimis antico, et apicis albo. Facile dignofcitur. Hab. in Potamogeto, et plantis aquaticis frequens.

$$
\text { (p.204.) }
$$

-503. D.granularis niger, elytris lineis duabus flauefcentibus, pedibus rufis. F. Sp. 296. 33 .

q F. E. S. I. I. 998 . 54 .

Habitat in aquis Thermarum D. Iuliani; Pifis.

\section{GYRINVS.}

Palpi filiformes. Maxilla cornea, vnidentata, acutiffima. Labium emarginatum. Antennae cylindricae.

504. G. Natator fubftriatus. F. Sp. 297. I. $\odot$ Rai., Petiv., Merret, Sulz.

D Schrank. E. I. 72. Schaeff. Ic. t. I34. f. 5. a.

\& F. E. S. I. I. 202. I. Panz. F. I. G. III. 5.

Habitat in lacubus frequens; velociffime fuper aquam per orbes curfitans. Long. $2 \frac{x}{2}$. lat. $I_{\frac{1}{3}}$.

\section{CARABVS.}

Palpi fex, articulo vltimo obtufo. Labium integrum, truncatum. Antennae filiformes.

505. C. coriaceus apterus, ater, opacus, elytris connatis, punctis intricatis fubrugofis. F. Sp. 298. 2.

○ Pontop., Sulz., Bergttr.

D Schrank. E. I. 388 . Schaeff. Ic. t. I4 4 . f. I. femina.

\& F. E. S. I. I. I25. 3. Voet. t. 38. f. 43. 
Dum acu transfigitur tenuifimum e corpore humorem emittit, vrentem. Hab. in filuis frequens. Long. I6. lat. $5^{\frac{x}{2}}$.

Cum Fabricio aliisque auctor nofter citat $C$. coriaceum Scopolii E. C. 265 , qui absque dubio verum non habuit coriaceum $L$. Comparata defcriptio eiusdem euidenter probat, illum $C$. violaceum $F$. habuiffe, huic enim margo elytri violaceus competit. Praecipue autem ex illo patet, quod dicit: "Lin"naeus puncta excauata in C. coriaceo ponit, et "funt etiam, fi pro his fumantur fpatiola depreffa "punctulis eminentibus interiecta, alias minime." In $C$. córiaceo Linnaei excauata, in $C$. violaceo contra eminentia, fpatium maius occupant. In $C$. coriaceo rügae exftantes ne puntia quidem, minime vero punciula vocari poffent; in $C$. violaceo bene fic appellantur. Tandem menfura, a Scopolio data, coniecturam probat. Elytrorum longituidinem $8 \frac{1}{3}$ lin. fiftit; in C. violaceo imo nonnumquam longiora funt, in $C$. coriaceo vero lin. Ir. fuperant.

Omnes ferme Carabi, e maiorum praecipue numero, fupra ab auctore dictum humorem ex ore fpuunt, inprimis autem, dum aci transfixi igni apppropinquantur, vt citius mortem obeant. Cauie, ne oculis immittatur, quia maximos vrendo efficit dolores. $\mathrm{Hg}$.

\section{(p. 2p5.)}

506. C. hortengis apterus, niger, elytris laeuibus, punctis aeneis excauatis triplici ferie. F. Sp. 300. 10.

$\odot$ Rai., Lift., Stroem., Bergftr.

D Schrank. E. I. 389.

\& C. auropunctatus. Rofi Mant. 75. I75. 
Ater, nitidus. Antennae filiformes nigrae, apice fufcae. Thorax cordatus, bafi vtrinque impreffus. In vtroque elytro feries punctorum triplex longitudinalis. Puncta viridi - aurea etiam in margine elytrorum, fed vix oculo armato confpicua; in fingula autem ferie plerumque duodecim minutiffima. Mas habet tarfos pedum anticorum ampliores, quafi palmatos; character communis etiam aliis Carabis. Tab. I. f. 3. Placuit huius Carabi iconem cum aliis minus notis rurfus hic exhibere. Hab. in pratis filuaticis frequens menf. Iul. poft foenifecium. Long. max. I2. lat. 4 .

Verum C. hortenfem auctor in Mantiffa fua habet $7 \mathbf{I}$. $I \nmid 0$, animaduerfione ibidem adiecta, $C$. hurtenfem huncce eundem cum auropunitato efle, quod etiam ita fe habet. Variat elytrorum colore nigro et obfcure aeneo. $\mathrm{Hg}$.

507. C. clathratus apterus, nigricans, elytris aeneis, frriatis, interiectis punctis excauatis cupreis. F. Sp. 302. 18.

Q.F. E. S. I. T. 13 I. 29 .

Buprefit longus. Voet. t. 38. f. 40 ?

Statura $C_{0}$ hortenfis. Caput et thorax atra immaculata. Elytra ftriis plurimis exarata, punctisque mainsculis aeneis excauatis triplici ferie. In noftris puncta aurea, .vel aenea etiam in margine. Apex elytrorum retufus. Thorax valde marginatus, in medio fulcatus, et poftice foueolis excauatus. Puncta aenea aetate obfolefcunt. Varietás. Hab. in filuis prope aquas: faepe capitus circa cuniculum in terra effoffum. Long. I4. lat. $4 \frac{x}{2}$.

Fabri- 
Fabricius, in Paykullii veftigium optimum, C. aeneopunfatum Degeer. IV. 87. x. t. 3. f. I2. citat, cui adiungatur $C$. aeneopuenciatus Herbft. Arch. V. I3 x. I 4. vbi perperam Voet. I. t. 37. f. 35. citatum videmus, cuius t. 38. f. 40. potius addu. cendam credimus. Celeb. Schneiderus valde errat, C. aeneopunctatum Degeer. et Herbftii eundem cum $C$. gemmato $F$. coniiciens. (vid. N. Mag, d. Ent. v. Fuefsly. III. 2. p. I35.) Id quidem fin. gulare videtur, nec Degeerium, nec Herbftium, nec . Voetium, punctorum eleuatorum, puncta excauata coniungentium, mentionem feciffe.

Color variat, nunc cupreus, nunc ater. Eft e maximis, et $C$. violacei longitudinis, fed duplo fere latior. Interdum vero $C$. anrati ftaturae cedit.

Caeterum anctor, vt in aliis huius generis, in hoc quoque valde dubius mihi eft. Tacet enim et puncta eleuata, excauatis interiacentia, et carinas longitudinaliter decurrentes. Quem autem Carabum habuerit, ob defcriptionem breuiorem decernere nequeo. $\mathrm{Hg}$.

$$
\text { (p.206.) }
$$

508. C. granulatus apterus nigricans, elytris aeneis friatis interiectis punćtis eleuatis longitudinalibus. F. Sp. 30I. I \%.

$\odot$ Bergftr., Sulz.

D Scop. E. C. 263 . Schrank. E. I. 393. Schaeff. Ic. t. 18. f. 9 .

ㅇ Roffi Mant. 73. I73:

F. E. S. I. I. I30. 28.

Oliuier. Inf. t. 2. f, 20. a. b.

Bupreftis Chalybeus. Voet. t. 37. f. $3 \mathrm{x}$.

Variat admodum magnitudine, et colore etiam purpureo. Confer Scopolium, qui diftinguit $C$. granulatum a $C$. catenulato fuo, vel intricato Lin- 
naei. Hab. in filuis frequens fub cortice arborum antiquarum. Long. 8 , ro. lat. $3,3 \frac{1}{2}$.

Carabus hicce fingulariter variat:

a. Vulgariter occurrit $1 \circ \frac{1}{2}$ long. $3^{\frac{3}{4}}$ latus, ftatura deprefla, elytris quafi formae quadratae, antennis totis nigris.

ß. Raro autem II $\frac{\mathrm{r}}{2}$ long. $4 \frac{3}{4}$ latus reprehenditur, elytris conuexis, lateribus rotundatis, antennarum articulo primo rubro, thorace magis plano, magisque pone anguftato, foueolis in angulis pofticis leuioribus et obfoletioribus. Hanc varietatem, vulgaribus immiftam, rariffime vero reperi. Vtriusque feminae et mafculi occurrunt. Var. $\alpha$. femora rubra faepins habet, bafi apiceque nigra. Color viridi - et nigricanti - aeneus eft. Geoffroyus varietatem $\beta$. habuifie videtur, cum dicat, latiorem C. aurato tffe.

Alius infuper Carabus exftat, quem in Hungaria . et circa Berolinum reperere, varietati $\alpha_{\text {. }}$ colore antennarum toto nigro, $\beta$. vero, ftatura conuexa, ouata, admodum quidem maiore, fimilis. Quem C. morbillofum F. E. S. I. r. I30. 26. coniecere, et ita ipfe mihi determinauit celeb. Vir, fed l.c. dicit, fuum Mauritaniae indigenum effe.

Linnaei $C$. granulatus valde fufpectus mihi eft. Nullam enim linearum eleuatarum, ftriis punctorum interiętarum, mentionem facit, et eiusdem defcriptio data omnibus notis $C$. anlienfe competit, cum infuper colorem atrum praecipuum habeat, quod in $C$. amenfs bene ita fe labet, cuius fpecimina atra feu obfcure violacea frequenter, laete aenea verc, rarifime occurrunt. C. granuiatus $F$. femper ferme aeneus reprehenditur. Iam Scopolius E. C. 263. hunc defectum commernoratus eft. Auctor C. catenulatum Scop. (qui idem eft cum catenulato Fabricii) fane non nonit, eundem cum 
intricato Linn. et forte $C$. granulati $F$. varietatem coniiciens.

E Schaefferi figuris t. I8. f. 6. varietatem nom ftram $\beta$; t. I56. f. 4. varietates $\%$. illam mutationem femoribus rufis, repraefentat. T. I 8 . f. 9 . male picta eft, fed huc fpectare dicitur. $\mathrm{Hg}$.

509. C. Sycophanta alatus, violaceo-nitens, elytris ftriatis, aureis. F. Sp. 203. 25.

$\odot$ Bergftr., Rob., Sulz.

\& F. E. S. I. I. I49. I08.

Olivier. Inf. t. 3. f. $3^{\text {I. }} \quad$ Voet. Coleopt. t. 370 f. 32 .

In fingulo elytro ftriae fexdecim, quarta, octama, et duodecima, longitudinali ferie punctorum impreffa. Variat magnitudine. Larua nigra laruis Gloffatorum victitat. Hab. in arboribus; inter folia Vitis etiam inuentus. Long. I2. lat. 5 .

$$
\text { (p. 207.) }
$$

510. C.caliginofus ater, thorace quadrato, laeui, antennis piceis. F. Syft. Ent. 240. 25.

Defcriptio Fabricii iterum exhibetur. Maior C. Leucophthalmo. Lecus Florentiae. Long. $9 \frac{\frac{1}{3}}{3}$. lat. $3 \frac{2}{3}$.

Auctor Fabricianum Carabum non habuiffe videtur, qui Americae indigenus eft. Pluribus defcriptio Fabriciana competit.

In Ent. Syft. C. caliginofum reperire nequeo, quamuis in Mantifia adhuc exftaret, vtrum alio nomine exhibitus, an ad aliud genus redactus, an plane orniffus fit, nefcio. $\mathrm{Hg}$.

5 I I. C. Leucophthalmus alatus, ater, elytris ftriatis, thorace canaliculato. F. Sp. 304. 29.

$\odot$ Lift., Bergftr.

D Scop. E. C. 266 . Schrank. E. I. 396. 
9 Roffi Mant. 74. I74.

F. E. S. I. I. $13^{2} \cdot 3^{6 .}$

Variat magnitudine. Puncta quatuor Geoffroyana obferuata in elytris, optime diftinguunt $a b$ aliis. Caput punctis duobus impreffum. Hab. in liğno putrido, in campis. .Long. 7. lat. $3 \frac{\mathrm{T}}{2}$.

Caraborum nigrorum tanta copia exftat, vt omnes tantum, $a b$ vno eodèmque, impigro et indefeflo, defcripti, dubii efle ceffarent. Cuine autem fortuna contigit, modicum tantum illorum numerum cogendi? Tempus futurum forte hanc fecundam fecum ducet fortunam, et interim illam, quam licuit, incertitudinis partem tollamus.

E horum numero $C$. Lencophthalmis haud modico femper fubiectus fuit eritque dubio. Auctores citati aut prorfus tollendi funt, aut tam breui-, ter illum, quem fub oculis habuere Carabum defcripfere, vt nil certi de illis determinari queat.

C. Leucophthalmus Linn. absque vllo dubio haud idem eft cum Carabo fic vocato Fabricii et Paykullii. Magnitudinemilli $C$. hortenfis fimilem effe dicit, elytra laeuia ftriasque obfoletas vocat, thoracem ratione magnitudinis infecti, minorem, quam in reliquis, appellat. Ipfe Carabum pofideo, in quem omnia modo dicta competunt, cui Celeberr. Dn. de Paykull nomen $C$. Pinigero (Monogr. Carab. 43. XXV.) tribuit, quem Celeb. Fabricius C. planam (E. S. I. I. I33. 37.); nofter auctor vero $C$. obfoletum vocat (vid. 11. 5 I 4.) cuiusque iconem Panzerus nofter (F.I. G. XI. 4.) et Scriba (Beytr. Fafc. I. t. x. f. 4.) exhibent. Oliuierius quoque fub $C$. Leucophthalmo $F$. verum Linnaeanum defcribit. vid. Encycl. method. V. 334. 52 .

Degeerii Carabus alatus eft, et forte ad C. Frifchii Herbftii amandandus. Caeteri omnes nimis 
brenem defcriptionem dedere, quae tamen fatis probat, fe Linnaeanum C. Lelucophthalmum ignoraffe. Hg.

$$
\text { ( } p .208 .)
$$

512. C. arenarius? pallidus elytris maculis duabus dorfalibus atris. F. Sp. 305. 34 .

우 F. E. S. I. I. I33. 39 .

Totus pallidus, exceptis maculis dorfalibus atris, quas inter iacent fuatia quaedam pallida. Naxillae exfertae, pallidae apice nigrae. Palpi antennaeque pallidae. Caput pallidum ore fubtus pilofo, oculis teftaceis. Thorax obcordatus pallidus immaculatus. Elytra ftriata pallida maculis duabus communibus, angulatis, atris, nec marginem, nec apicem attingentibus. Pedes pallidi. Tab. III. f. 4. Hab. in arena maris Tufci. Captus d. 29. Mai. Long. S. lat. $34^{\prime}$.

- Nec Fabricii, nec auctoris C. arenarinn noui, fed recte dubitare videor, an eundem prae oculis habuerint. $\mathrm{Hg}$.

5I3. C. mficomis ater, elytris fulcatis laeuibus, antennis pedibusque rufis. F. Sp. 305. 35 .

() Lift.

D Schaeff. Ic. t. I8. f. 7 .

Similis C. Leucophthalmo, at paullo minor, et thorace magis obcordato, ftriisque fubpunctatis. Elytra interdum colore fugaci aureo nitent. Hab. in filuis frequens. Long. 5. lat. 2.

Auctor nofter in Mantiffa fua 77. I80. C. ruficornem $F$. iterum exhibet, et ibidem C. puluerulentum fuum, quem n. 526. fequentem habenus, citat. Nullam autem huius Carabi mentionem facit, hine nefcio, vtrum notae fpeciei annumerandus, an nouus habendus fit. $\mathrm{Hg}$. 


$$
\text { (p. 209.) }
$$

* 5 I 4. C. obfoletus alatus, nige:, elytris obfolete ftriatis, ftriis obfolete punctat:?

\& C. Leucophthalmus. Linn. Syft. Nat. 2.668. 4. Fn. Sv. 784. - Scriba Beytr. Ent. p. 7. n. 4. t. I. f. 4 .

Olivier. Encyclop. method. V. 334. 52.

C. ptonus. F. E. S. I. ז. I33. 37 .

Panz. F. I. G. XI. 4.

Ni agnitudo C.clathrati. Caput elongatim. Maxillae ferrugineae. Palpi picei. Antennae dimidiato-rufae. Thorax fubcordatus, antice et poftice margine ciliatus. Elytra laeuia nonem ftriis obfoletis, et in is puncta minima. D. D.Antic. Soc. Linn. Trochanter femoralis valde elongatus et acutus. Hab. fub faxis in hortis incultis. Long. II. lat. 4.

Color interdum brunneus, quod in nuper declaratis effe videtur. Caeterum vide adnotata ad $C$. lencophthalmum. n. $5 \mathrm{x}$. $\mathrm{Hg}$.

5I5. C. vulgaris nigro-aeneus, pedibus antennisque nigris. F. Sp. 308. 52 .

D Scop. E. C. 268 ?

오 F. E. S. I. I. I54. I28.

C. ouatus. Roffi Mant. 89. 280.

Alis femper deftitutum afferit Scopoli. An re. cte? - Hab. in filuis frequens fub arborum cortice. Long. 4. lat. $\mathrm{I}_{\frac{\pi}{3}}^{\mathrm{r}}$.

Confufio in his et fimilibus grauiffima eft. Equidem libenter contendam, C. ouato. Payk. Mon. Car. I32. 8I. quem eundem cum C. vulgari $F$. habet, etiam C. affinem Schrankii E. I. 399, quem idem celeb. Vir varietatem $C$. Protei fui 1. c. II5. 72 . exhibet, annumerari oportere. Carabus Scopolii citatus, 
citatus, quem apterum dicit, nullo modo h.1. allegari poteft, fed $C$. lepidus $F$. videtur.

Si $C$. valgaris $F$. idem cum $C$. olloto Payk. 1. c. eft, cui et auctor nofter in Mantiffa 1. c. affentit; citatum e Degeerio tolerari non poteft. Carabum enim defignat, quem $C$. lepidum aut illi admodum fimilem habeo. Citatum Geoffroyi pariter delendum eft. Schaeff. Ic. t. I3. f. 2. quoque ad alium transferatur Carabum. Hg.

5 I6. C. caerulefcens nigro - caerulefcens, elytris ftriatis. F. Sp. 308. 49.

D C. eleganis. Scop. E. C. 269.

\& F. E. S. I. I. I52. 123 .

Hab. cum priore. Long. 4. lat. 2.

Fabricius in Ent. Syft. 1. c. diagnofin Linnaei accepit, hinc verifimile videtur, illum $C$. cuprei varietatem defcripfifie, cui et Paykullium Mon. II3. 7r. affentientem video. Antea C. lepidi varietas pro Fabricii C. ccerulescente affumta fuit. Scopolii C. elegans huius varietatem indicare videretur, cum nullam antennarum primi articuli rufi mentionem faceret, nifi alatum dixiffet Carabum fuum。 $\mathrm{Hg}$.

$$
\text { (p. 2ro.) }
$$

$5 \mathrm{I}$. C. lepidus viridi-aeneus, thorace quadrato, poftice vtrinque biftriato, elytris ftriatis. F. Mant. 200.67.

오 F. E. S. I. I. 153. I 24 .

C. vulgaris. Scop. E. C. 268 ?

Defcriptio F. iterum exhibetur. Hab. fub corticibus tempore hiemali frequens. Long. 4. lat. 2. Variat colore obfcure cyaneo. Rationes, quas habeo, qua de cauffa $C$. vnlgarem Scop. $=68$. ad hunc $C$. lepidum referre mallem, enumerare inuat. Ne enim C. vulgaris $F$. (vel $C$. ouctus Payk. quem cuun Q 4 boc 
hoc celeb. Viro vulgarem $F$. habeo, fit, prohibent praecipue diagnofis Scopolinae verba: "Thoracis longitudo elytri latitudine maior. Idem antice paullo latior quam poftice." dein comparatio huius et fequentis $C$. elegantis, quem $C$. cuprei varietatem habere oportet. Haec, cum alarum defectu, optime in $C$. lepidum competunt. In C. lepido thoracis longitudo elytrorum latitudinem fuperat, et thorax ipfe antice latior eft. In C. cupreo thorax breuior, magisque linearis eft. $\mathrm{Hg}$.

518. C. cupreus cupreus, antennis bafi rubris. F. Sp. 308.50 .

D Schrank. E. I. 400. Schaeff. Ic. t. I8. f. 5.

\& F. E. S. I. I. I53. 126.

Variat admodum magnitudine et colore, at facile dignofcitur. Hab. filuas et arborum cortices frequens. Long. 5 . lat. 2.

C. caerulescentem $F$. et Linn. huius varietatem videri, iam fupra ad $C$. cuevulefcentem n. 5 r6. adnotatum eft. Vide etiam, quae de $C$. elegante Scop. E. C. 269. ibidem et in animaduerfis ad priorem n. $5^{I}$. monui. Hg.

510. C. latus niger, elytris ftriatis, crenatis; antennis pedibusque ferrugineis. F.Sp. 308. 54 .

D Schrank. E. I. 397.

\& F. E. S. I. I. I54. I 29 .

Totus ater. Thorax latitudine elytrorum, abdomini proximus. Elytra ftriata. Tibiae et antennae verfus bafin rufefcentes. Femora craffa. Punctum bafi thoracis vtrimque impreffum. Striae elytrorum octo, fecunda a margine interno obliqua abbreuiata. Variat magnitudine. Hab. in terra frequens. Long. $4 \frac{\pi}{2}$. lat. $2 \frac{1}{2}$. 
Carabus Fabricianus C. affini Schrank. fane fimilis eft, et defcriptio auctoris idem probat. Hinc C. Proteum Payk. Mon. Car. I 5. 72. citare nequeo, cum C. azureum $F$. inter varietates referat, quibus et C. latum $F$. annumerauit. Hg.

$$
\text { (p.2Ir.) }
$$

520. C. interruptus ater, thorace poftice rotumdato, abdomineque remotis. F.Sp.306.40. Fuesly Arch. t. 29. f. 4.

Magnitudine variat. Corpus totum atrum. Caput magnum vtrimque impreffum latitudine thoracis. Thorax antice emarginatus, poftice rotundatus, ab abdomine remotns. Elytra ftriata. Ad Scaritum genus accedit, facies, habitus idem; pedes antici tamen in hoc non palmato - digitati: antennae vix differunt, et reliqua aequalia. Alatus. Habitat in terra, et fub arborum cortice in filuis non rarus. Long. \%. lat. 2.

Auctor nec Fabricii, nec Herbftii Carabum habuit. Ille Orienti indigenus, hicce vero idem cum Scasite Subterraneo $F$. eft. Fabricius ipfe hoc citatum relegat, contra autem in E. S. I. I. I 44. 87. apud interruptum fuum citat Scaritem clypeatum auctoris noftri n. 570; fed idem, quod huic, obftat. Specimen habeo, quod celeb. Dno. de Paykull a D. Roffio ipfo miffum erat. Scaritum generi palpis, pedibus anticis, thorace etc. admodum accedit; ftatura autem conuexa, craffa eft, et verus Scarites non videtur. Conferantur, quae ad Sc. Calydonium n. 57 I. adnotata funt.

Antennae ferrugineae, fubtomęntofae, vndecim articulis. Palpi ferruginei, articulo vltimo haud la. tiore, compreffo, obtufo atque oblique truncato, vti in Carabis effe folet, fed ouatu. Oculi cinerei. 
Thorax antice parum emarginatus, fed potins truncatus, bafi angufta, truncata; vndique, apice excepto, marginatus. Glaberrimus et laeuiffimus eft, fulco leui longitudinali medio. Elytra aeque lata, ac thoracis prona pars, conuexa, rotundata, laeuia, friata, marginata, ad marginem pafiim punctata. Pectus et abdomen picea. Pedes atri, tibiis tarfisque fetofo-fpinofis ferrugineis, tibiis anticis apice fingulariter fiffis, fere palmatis, digitis nigris. $\mathrm{Hg}$.

521 . C. ferrugineus ferrugineus, elytris ftriatis obfcurioribus. F. Sp. 309.50.

D Schaeff. Ic. t. $13 \%$. f. 3 .

올. E. S. I. I. I55. I37•

Totus obfcure ferrugineus, pallidus, oculi tantum nigri, elytra punctato-friata. Habitat cum praecedentibus minus firequens. Long. $4 \frac{1}{2}$. lat. I $\frac{1}{3}$.

Duos Carabos poffideo, huc fpectantes, quos breuibus indicare iuuat verbis. Alter, maior, totus ferrugineus, thorace pone magis anguftato, elytris fimpliciter ftriatis. Alter, duplo fere minor, obfcurior, elytrorum nitore quodam orichalceo, qui etjam in varietate tota flauo-pallida apparet, ftriis in fundo punctatis. Prior C. ferrugineus Payk. Mon. I24. 76. videtur, quem Schaefferi figura citata optimo repraefentat. Pofterior eiusdem $C$. apricarius $\mathbf{2} \mathbf{2 5} .77$. effet, nifi varietas huius $\beta$. ibidem commernorata, a Viro celeberrimo beneuolenter mecum communicata, me edocuiffet, vtrimmque valde a fefe diuerfum effe. Paykullianus enim plus duplo minor eft, elytraque aperte punctato-ftriata gerit. Nofter vero numquam tam obfcurus occurrit, elytrorumque ftriarum puncta vix oculo armato confpici poffunt. Nomen illi dedi $C$. rufus.

De auctoribus citatis iudicare non audeo. Geoffroyi et Degeerii infecta a celeb. Paykullio ad apri- 
carium relata funt. Neuter nitoris illius orichalcei mentionem fecit. Geoffroyns 2 lin. longum vocat, quod cum illo fpecimine, a D. de Paykull. nifio, bene congruit. Fabricius, auctores modo dictos ad vtrumque retulit Carabum.

Forrugineus nofter nunc nitide, nunc lanģuide ferrugineus occurrit; ipfe totum pallidum, quam varietatem eandem cum $C$. pollente $F$. credunt, nonduin reprehendi; inde deriuandum duco, quial rarus in noftris occurrit regionibus. Rufum fiepius fic vidi, colore flauo - pallido toto, oculis vel cinereis, vel nigris, nitore orichalceo autem illo commemorato praefente. Striarum puncta in talibus bene confpici pofiunt, quod a colore fere hyalino deducendum videtur. $\mathrm{Hg}$.

$$
\text { (p. 212.) }
$$

522. C. Germanus cyaneus, capite, elytris, pedibusque teftaceis, elytris apice violaceis. F. Sp. 3I2. ? I.

D Scop. E. C. 273. Schrank. E. I. 403.

F. E. S. I. I. I62. I67.

Rofii Mant. 84. I9I.

Panz. F. I. G. XVI. 4.

Caput fulumm oculis nigris, antenuis rufis. Thorax atro-caeruleus, punctatus, villofulus. Elytra teftacea, ftriata. Corpus fubtus nigrum. Pedes elytris concolores. Variat capite nigro. Hab. in filuis frequens. Long. $3 \frac{\mathrm{r}}{2}$. lat. $I \frac{\mathrm{x}}{2}$.

Schaefi. Icon. t. 3 I. f. I3. citata, ad C. prafinum F.E.S. I. I. 163 . I73. pertinet, vbi Fabricius quoque iterum citare in animo habuit. Hinc in Harreri Defcr. Sch. Ic. p. I3 I. n. 209. omuia citata, atque diagnofis ipfa, ibi data, deleantur.

De varietate modo dicta, capite nigro, vide, quae habet auctor in Mantiffa fua l. c. Hg. 
52.3. C cinctus fufcus, capite thoraceque viridiaeneis, elytrorum margine pedibusque pallidis. F. Sp. 3 10. 62 .

옹 Roffi Mant. 77. 179 .

Fere duplo maior C. marginato. Caput et thorax viridi-aenea, glabra, nitida. Antennae palpique pallidi. Thorax canaliculatus, poftice vtrinque impreffus. Elytra fufca nitida, laeuia ftriis, feu potius fulcis fexdecim exarata, margine pallido. Corpus atrum, abdominis margine pallido. Femora et pedes toti pallidi. Alae albae. Tab. V. f. 9. Inuentus fub cortice Roboris menf. Febr. Long. $9 \frac{1}{2}$. Iat. $3 \frac{1}{2}$.

C. cinturs auctoris nullo modo idem cum Carabo fic vocato Fábricii eft, cuius patria ora Coromandeliana fertur. Defcriptiones quidem quadrare videntur, fed comparatio breuis adiecta nos docebit, ambos admodum differre a fefe. Specimen meum C. cintii Rolfiani e Sardinia mifium erat, vtrum ibi captum, an ex Italia continenti translatum, nefcio.

I. C. cinftus Roffii I I lin. longus, $4 \frac{\mathrm{J}}{\mathrm{T}}$ lin. latus eft; $C$. cincti $F$. fpecimen maius, vix 8 lin. longum, $3 \frac{\mathrm{r}}{2}$ vero latum erat. Hinc Carabus Italicus hunc valde fuperat magnitudine, et $C$. plani $F$. longitudinem aequat. Quod Dn. Roffius duplo tantum maiorem C. marginato vocet, inde deducendum eft, quia alium marginatum, folito multo maiorem, habuit.

2. Caput et thorax viridi - cyanea in $C$. cincto $R$. ; viridi - cuprea in Fabriciano.

3. Elytra laeviia, glabra, nigra, longiora funt ; in vero $C$. cincto autem punctulis prominulis afperfa, faepitus ferico obtecta, viridia, breuiura.

Conueniunt ftatura; thoracis capitisque forma; antennarum, pedum, marginisque, elytra abdomen. 
que cingentis, colore flauicante, elytrorum ftriis, et corpore fubtus nigro. $\mathrm{Hg}$.

524. C. marginatus viridis, elytris margine, tibiisque teftaceis. F. Sp. 310. 6I.

$\odot$ Pontop.

o. C'. velitus $\beta$. Roffi Mant. 78. I8r.

Caput et thorax viridi-aenea, antennis ferrugineis. Elytra viridia, pubefcentia, laeniter ftriata, margine exteriore flauo. Abdomen nigrum, nitens. Pedes pallidi. Apud nos occurrunt aliae duae forte fpecies diftinctae, huic quodam modo fimiles, et a nonnullis confufae cum $C$. marginato, quarum icones dedit Schaefferns t. 109. f. 5 . et Fuesly t. 29. f. 7. - Hab. íl filuis frequens. Long. 6. lat. $3 \frac{1}{4}$.

De varietatibus commemoratis conferatur Mantiffa auEtoris 1. r. - Schaefferi figura citata ne vllum quidem marginis rudimentum habet, Herbftii icon allegata vero $C$. cincium $F$. repraefentat, colore admodum obfcuro.

Hunc Carabum verum marginatum non effe, ipfe in Mantiffa dicit auctor. Hinc conferantur auctoris ibi dicta verba, noftraque et ad veftitum, et ad Spoliatum 79. IS2. adnotata. Hg.

$$
\text { (p. 213.) }
$$

325. C.limbatus fupra ferugineus, thorace nuacula, elytris fafciis vndatis, viridi - aeneis. F. Mant. 202. 87 .

\& Scolytus limbatus. F. E. S. I. I. I8I. 2. Panz. F. I. G. II. 9.

Facie dinerfus ab aliis. Statura Chryfonclae. Corpus glabrum, ouatum. Antennae filiformes, pallidae, thorace longiores. Palpi fex pallidi filiformes, 
formes, pilofi. NIandibulae acutae, nigae, fimplices. Labium integrum, edentatum. Caput pallidum, fronte ante oculos viridi nitente. Oculi pronimili, nigri. Thorax conuexus, punctulatus pallidus, lateribus fubmarginatis, macula medią bafeos viridi. Elytra gibba, punctato - ftriata, pallida, maculis anticis quatuor angulatis; pofticis duabus vndatis, viridibus, omnibus futurae viridi-connexis in medio cruciformiter bis dilatatae. Abdomen pallide ferrugineum. Pedes pallidi, tibiis ciliatis. Poftici longiufculi femoribus trochanthere infructis. Tarfi quinque. Alae albae. Tab. VI. f. 12. Hab. in locis arenofis, humentibus. Elytrorum fragmenta vifa etiam in ftercore, quo $\mathrm{Hi}$ rundines nidulantes domus meae feneftras Arni aquas refpicientes inquinant. Long. $2_{4}^{3}$. lat. $I^{\frac{T}{3}}$.

Saepius locus quidem praebitus erat, laborem, diligentiam fummamque elegantiam laudibus celebrandi, qua clariff. Panzerus nofter Fannam Infectorum Germaniae orditus eft, et in dies magis magisque auget. Icones ibi datae naturam religiofe legunt; colores aptiffimi, tam bene adhibiti funt, vt fculpturam (fic ftrias, puntta aliaque huc fpeetantia vocare iuuat,) quae fane maximi in infectis momenti eft, et quam artifex optima elaboratione repraefentauit, transparere finant, qui modus optimus eft in infectis depingendis, et quo neglecto opera quaedam gratiffima laborant. Comparatio huius $a b$ auctore datae effigiei, et iconis, in opere modo citato, exhibitae, laudes meritas confirmant.

Infectum hocce femper dubium fuit, et merito nomen $C$. dubii geflit. Caraborum genus minime aptum erat, hinc a nonnullis iure potiore Dytifcorum familiae illi annumeratum fuit, quae $C$. ona- 
tum et onclem comprehendit. Optime autem peculiare genus conftituit, fed nomen illi a Fabricio inditum Scolytus haud antum videtur, quia Geoffroyus eodem nomine ian vfus erat, ad defignandum genus, quod celeb. Herbftius poftea nomine Eccoptogufter defignauit. Hg.

* 526. C. puluerulentus nigger, fufcus, pedibus antennisque rufis, elytris tomentofis, friatis.

\& C. ruficornis. Roffi Mant. 77. 180.

F. E. S. I. 1. 134. 42 .

Caput nigro - fufcum, nitens, antennis, palpisque rufis, maxillis parun elongatis. Thorax fubquadratus, nigro - fulcus, antice laeuis glaber. Elytra nigro - fufca, friata tomento flauo adeo pubefcentia, vt transuerfim infpecta, appareant holofericea, et quafi flauicante puluere afperfa. Pedes toti rufi. Alae albae. Tab. III. f. 7 . Hab. in ligno putrefcente rarior. Long. 6. lat. $2 \frac{2}{3}$.

Citatum, quod Fabricius habet: Geoffr. I. I46. 8 . admitti non poteft, fed Paykullii: Geoffr. I. I60. 38. melius quadrat. Cacteru.n confer monita ad C. ruficomen n. 5I3. Hg.

\section{(p.214.)}

527. C. mudidus? thorace poftice vtrinque impreffo, niger, femoribus ruis. F. Sp. 3c6. 39 .

P F. E. S. I. I. .I 35. 48.

Corpus atrum, at vere quafi oleo inuncuim. Antemiae rufae, apice fufceícentes. Thorax obcordatus, bafi puncto maiori vtrinque excauatus. Elytra ftriata, parum compreffa. Pedes rufi. Hab. in filuis. Long. 6. lat. 2 .

C. madidus $F$. femper dubius fuit, eritque, ob deferiptionem breliorem, et ob copiam Caraborum atrorum nimis magnam. $\mathrm{Hg}_{\mathrm{s}}$. 
528. C. oblongopunctatus niger, elytris ftriatis: punctis dorfalibus plurimis impreflis. F. Mant. 202. 80.

\& C. aterrimius. Roffi Mant. 85. 194 .

F. E. S. I. I. 156.14 I.

Paullo minor C. Leucophthalmo. Caput et thorax atra; nitida, immaculata. Thorax rotundatus linea dorfali, punctoque vtrinque impreffis. Elytra atra, nitida, ftriata punctis dorfalibus tribus vtrinque manifefte impreffis, margine etiam punctato. Puncta marginalia ad bafin, et apicem conferta, in medio rariora. Subtus totus ater. Habit. cum aliis minus frequens. Long. 6. lat. $x_{4}^{3}$.

* 529. C. dimidiatus niger, thorace fubquadrato, antice laeuiori aeneo - nitido, elytris obfcurioribus.

Statura praecedentis. Antennạe nigrae, articulo primo rufo. Caput et thorax aeneo-nitida. Thorax fubquadratus, antice magis eleuatus, magis laeuis, magisque fempar nitidus, poltice punctulatus, fere fcaber, et caerulefcens. Elytra fufconigra, ftriata, obfcuriora. Subtus totus niger. $\mathrm{Ha}-$ bitat cum aliis rarior.

\section{(p. 215.)}

* 530. C. punctulatus niger, thorace quadrato, elytris ftriatis, punctis plurimis longitudinalibus impreffis.

9. Rofii Mant. 76. 17?.

Medius, et totus niger, glaber, laeuis. Antemnae fubferrugineae bafi rufa. Thorax latus, vix gibbus, canaliculatus, margine laterali antice deflexo. Elytra deprefla, ftriata, ftriis punctis raris pluri- 
plurimis obfoletis longitudinalibus imprefla. Differt a $C$. multipunitato $F$. punctis praefertim elytrorum non vagis. Hab. in filuis fub corticibus frequens. Long. 6. lat. $2 \frac{1}{2}$.

Idem Carabus eft, quem Panz. F. I. G. XI. I2. nomine $C$. ciftelödis Hellw. depinxit. Conferantur citata, ab auctore in Mantifia bene adhibita. Hg.

531. C. pilicomis? thorace rotundato, elytris ftriatis, punctisque impreflis, antennis pilofis. F. Sp. 30-. 48. Varietas?

\& C. infidus. Roffi Mant. 88. Ig8.

C. breuicollis. F. E. S. I. I. I50. $\mathrm{I}+3$ ?

(P.ınz. F. I. G. XI. 8.)

Facies $C$. Spinibarbis, fed fere duplo maior. Palpi, antennaeque ferrugineae pilis longioribus hirtae. Thorax rotundatus vti in $C$. Spimibarbi fufco-niger. Elytra fufco-nigra ftriata, vix gibba, pofticeque punctis tribus longitudinalibus impreffis. Pedes fubrufi; femora nigra. Colore variat. Hab. cum praecedentibus in locis iisdem minime rarus. Long. $5 \div$. lat. $2 \frac{1}{4}$.

C. pilicomem $F$. alium efe, quisquis e defcriptione atque e magnitudine concludet; Roflio quoque ipfi fic perfuafum eft in Mantiffa 1. c. Nil ferme obftare videtur, quo minus hic defcriptns idem cum $C$. brenicolli fit, defcriptione praecipue Mantiffae omnibus fere notis quadrante, quam hanc ob cauffam conferre fuadeo. Comparatio ftaturae, et magnitudinis duplo maioris $C$. Spinibarbis, thoracis elytrorumque defcriptio, puncta elytrorum ftriae fecundae imprefia quatuor maiora, eorumque forma fubdepreffa, color, locus; omnia imo congruunt.

$\mathrm{Hg}$. 
* 532. C. fylphoïdes ater, depreffus, thorace orbiculato, elytris apice finuatis, lineisque tribus eleuatis, fpatio interiecto punctato.

C. finuatus. D. D'Antic Soc. Linn.

\& F. E. S. I. I. I 48. 105 .

Statura C. Leucophthalmi, fed minus elongatus. Totus ater, non nitens. Caput punctatum. Thorax planus orbiculatus, in medio fulcatus, late marginatus. Elytra compreffa, abdomine paullo breuiora, apice fimato, lineis eleuatis, ac feriebus punctorum inter ftrias punctatas. Subtus nitidior. Alatus. Tab. I. f. ๆ. Hab. in filuis fub arborum cortice, primo vere frequens. Long. $5 \frac{\mathrm{T}}{2}$. lat. $2 \frac{3}{4}$.

$$
\text { (p.216.) }
$$

* 533. C. carbonarius niger, obfcurns, thorace orbiculato, elytris fubftriatis.

An C. holofericeus. F. Mant. 199. 52? Totus ater, fubpubefcens, et quafi puluere fuliginofo afperfus. Thorax antice et poftice rotundatus. Elytra parum depreffa, punctulata, ftriata. Pedes nigri. Mea fpecimina differunt capite minime aeneo. Hab. cum praecedentibus. Long. 5. lat. $2 \frac{1}{2}$.

Defcriptio nimis breuis eft, fed tamen hicce Carabus a $C$. holofericeo $F$. multum difcrepare videtur, imprimis, cum et varietates taceat, quibus $C$. holofericens abundat fingularibus. $\mathrm{Hg}$.

534. C. complanatus thorace orbiculato rufus, elytris fafciis duabus nigris. F. Sp. 306. $4 \mathrm{I}$.

$\odot$ Linn.

Statura et magnitudo C. crepitantis. Caput prominens cum antennis rufum. Thorax rotundatus, canaliculatus rufus immaculatus. Elytra ftria- 
ta, truncata, nfa fafciis duabus magnis vndulatis nigris. Fafciae in meo fpecimine connexae, Pedes rufi. Hab. in filuis rarus. Long. 3. lat. $1 \frac{1}{2}$.

Dubitare licet, num idem cum Fabriciano fit, qui infulae St. Domingo indigenus eft. $\mathrm{Hg}$.

535. C. crepitans thorace, capite, pedibusque ferrugineis, elytris nigris. F. Sp. $307 \cdot 44$.

$\odot$ Rol., Bergftr, , Voet.

+ F. E. S. I. I. $13^{6}$. 53 .

Elytra nigro - caerulea. Thorax longus anguftus. Maiores Carabos, qui eum venantur, crepitu ventris repellit. Hab. in filuis, frequens intra corticem et lignum. Long. $3 \frac{1}{3}$. lat. $I_{\frac{1}{3}}$.

$$
\text { (p. } 217 .)
$$

536. C. Crux maior thorace orbiculato rubro, coleoptris truncatis rubris cruce nigra. F. Sp. 311.67 .

\& F. E. S. I. I. I60. I58. Voet. t. 34, f. 7. Panz. F. I. G. XVI. I.

In noftris fane non paucis, thorax femper niger, imo totus Carabus niger, exceptis elytris rufis fafcia, bafi et apice nigris. Hab. fub cortice Quercus, Vlmi frequens. Long. 3. lat. I $\mathrm{I}_{4}^{\mathrm{T}}$.

Carabus hicce, cum C. Cruce minore, bipuffulato ac lunato admodum dubius fuit, nunc tandem extricatus. Auctor verum habuit Linnaei C. Crucems maiorem, quod ex animaduerfione breui adiecta apparet. Fabricii Carabus in Speciebus et in Mantiffa idem cum Carabo, ibidem vocato bipuftulato, fuit. In Entom. fyft., vbi Linnaei Carabi tandem fuis nominibus enumerati funt, 1 . c. citatum Geoffroy. I. I50. I 7. omifium, et ad C. Crucem minorem translatum eft, cum tamen optime huc fpectet. Hg.

$$
\mathrm{R} 2 \quad * 537 .
$$


* 537. C. olens rufus, capite nigro, elytris nigris macula bafeos rufa.

\& F. E. S. I. x. I39. 66.

Statura fere C. crepitantis. Antennae rufae articulo primo praelongo craffiufculo. Palpi rufi. Caput nigrum, collo fultentatun. Thorax obcordatus rufus. Elytra fufco-nigra, oblolete ftriata, macula ad bafin vtrinque rotunda, rufa, angulo. que apicis interno plerumque rufo. Inferne totus nufus. Tab. V. f. 2. Hab. fub cortice ad arborun radices rarior. Long. $4 \frac{\pi}{5}$. lat. $I_{4}^{\frac{T}{4}}$.

\section{(p.218.)}

538. C. lunatus thorace orbiculato rufo, elytris flauis maculis tribus nigris. F. Syft. Ent. 247. 60.

D C. Eques. Schrank. E. I. 405. cum accuratifima defcriptione Schaeff. Ic. t. 4I. f. 14.

q F. E. S. I. I. 163. 172 .

C. Crux minor. Sulz. Hift. Inf. t. ๆ. f. 6.

C. Sulzevi. Brahm. Infektenkalend. 246 .

Panz. F. I. G. XVI. 5 .

Infectum elegans omnino dinerfum a $C$. Cruce minori et optime exhibitum in icone Schaefferi l.c.

Mea fpecimina differunt tantum colore capitis viridi-aeneo nitente. Color thoracis, atque elytrorum faturate rufus, et fere fanguineus, qui in cadanere fubflauus enadit. Pedes pallidi femorum apice, digitisque nigris. Captus pluries fuper herbam in filuis menf. Mart. et April., fed inuentus etiam inter corticem et lignum Populi tremulae: rarus. Long. $2^{3}$. lat. $I^{\prime}$.

Citatum e Sulzero allegatum Fabricius in Ent. fyft. ad C. Crucem minorem contulit. Hg. 
539. C. fpinibarbis thorace orbiculato cyaneus, ore, antennis, tibiisque rufis. F. Sp. 307.47.

오 F. E. S. I. x. 137. 55 .

Corpus totum cyaneum. Caput prominens, ore antenuisque rufis. Maxilla fuperior bafi dilatata, inferior dorfo fpinis ciliato. Thorax orbiculatus. Elytra ftriata. Pedes fubrufi. Hab. in filuis fub arborum cortice frequens. Long. $3 \frac{1}{2}$. lat. $I \frac{r}{2}$.

Pedes nigri funt, in muper declaratis vero, cum colore totius corporis fufco-cyaneo, rufi.

C. breuicolli. F. E. S. I. I. I50. Ix 3. (qui idem videtur cum C. infido. Roffi Mant. vel C. pilicorui fupra memorato n. 53x.) ftatura thuracisque forma perquam fimilis eft, differt tantum magnitudine dinidio minore, et colore cyaneo. Mirum itaque eft, quomodo Fabricius in Ent. fy.t. fua $C$. Spinibarben familiae: thorace cordato, C. breuicollem contra familiae: thorace rotundato margine prominulo obtufo, annumerare, et ita tam fimiles Carabos familia feparare potuit. Vterque inter Carabos familiae pofterioris locum optime capit.

$\mathrm{Hg}$.

540. C. cyanoceplatus thorace pedibusque ferrugineis, elytris capiteque cyaneis. F.S.Sp. 310.65 .

( ) Ray.; Scliaeff. Ic. t. IO. f. I 4 .

D Schaeff. Ic. t. I r. f. I4.

\& F. E. S. I. I. I59. 154 .

Hab. in filuis fub arborum cortice frequens. Long. 3. lat. $I_{4}^{I}$.

Variat elytrorum colore nigro - et viridi - cyaneo: punctis in fpatiis, quae ftriis interiecta funt, magis minusue confpicuis: pedibus, tarfis folis nigris exceptis, rutis, vel geniculis quoque nigris. In Ent. fyft., quae erratis typógraphicis abundat,

Geoffr. 
Geoffr. Inf. I. I 40. legatur Geoffi. Inf. I. I 49. 40. et ibidem loco: Schaeff. Ic. t. Io. f. 4. legatur t. II. f. I4. Hg.

\section{(p. 219.)}

54 I. C. melanocephalus niger, thorace, pedibusque ferrugineis. F. Sp. 3 10. 64.

D Schaeff. Ic. t. 237 . f. 5 .

\& F. E.S. I. I. 159.153 .

Voet. Col. t. 35 . f. 15 .

Facies C. cyanocephali, fed caput nigrum. Elytra atra fubftriata. Thorax, pedes, antennae omnino vnicolores, ferrugineae. Sufpicio $D_{e}$ Geeriann hunc effe forte mafculum, minime improbanda: fub corticibus enim hieme inueniuntur faepins vna cum iis, álii fimillimi apteri, qui differunt tantum thorace elytris concolore; plerumque feminae. Hab. fub corticibus. Long. 3 . lat. it:

Faciei fimilitudo huius et praecedentis perquam exigua eft. Notis illis, cuique Carabo conuenientibus, exceptis, colorumque diftributione paullo fimili exèmta, nil ferme affinitatis reftat. $C$. melanocephalus ftatura fere eadem cum C. vaporariorum, cum quo et Linniaeus in $\mathrm{Fn}$. Sv. comparat, et cum punciulato Ro/fii n. 530. gaudet elliptica, thorace elytrorum pene latitudinis, antice caput arete cingens, quafi attenuatus, poftice elytrorum bafin denfiffime tegens, vt in C. pun fiulato $\boldsymbol{R}$. Elytra ouata, thorace haud latiora, interdum fufca euadunt, quod a ftatu nuper declarato deriuare mallem. Hg.

542. C. Turcicnis thorace orbiculato rufo, elytris nigris lunula bafeos pallida. F. Mant. 203. 96.

\& F.E.S.I. I. I6r. I6r. 
Defcribit Fabricius 1.c. Variat interdum elytris apice etiam rufis. Hab. intra corticen et lignum non infrequens. Long. 2. lat. 1 .

$$
\text { (p. 220.) }
$$

543. C. viridanus viridi-aeneus, elytris ferrugineis, macula comnuni nigro-aenea. F. Mant. 204. 105 .

\& C. prafinus. F. E. S. I. I. 163.173 . C. marclicus. Herbft. Arch. p. $\mathbf{r}_{3}$. n. 38 .

B. viridis, elytro fingulo ftriis oĩo, pedibus, elytrorum antica parte et margine fuluis. Geoffr. Inf. x. 148.13 .

Schaeft. Ic. t. 3r. F. I3.

Voet. t. 33. F. 5.

Panz. F.I. G. XVI. 6.

Dimidio minor C. Germano. Antennae nigrae articulo primo ferrugineo. Caput et thorax viridi - acnea, glabra, nitida. Elytra ftriata, teftacea, nucula magna communi nigro - aenea, a medio futırae ad apicem. Corpus nignum. Pedes rufi. Hab. in filuis non frequens.' Long. 3. lat. I.

Fabricii C. prafimus. IMant. 204. rog. idem eft cum C. viridano eius ibidem exhibito 1. c., vt Paykullius Mon. Car. 57. 34. citat, et Fabricius ipfe approbat. Thunbergii tractatum de nouis fpeciebus Infectorum, adire non licuit, defcriptione autem Oliuierii Encycl. method. V. 352. I46. comparata, patriaque, quam Caput Bonae Spei dicit, computata, eiusdem Carabus diuerfus videtur. - Geofiroyi citatum optimum eft, et ab Oliuierio 1. c. quoque probatum video.

Inter fpecimina fane multa nullum afpicio capite thoraceque nigris, quale Paykullius et Fabricius habuere. Hg.

$$
\mathrm{R} 4.544 \text {. }
$$


* 544. C. chryfocephalus alatus, capite thoraceque auratis, elytris obfcure viridibus, pedibus rufis.

Forte affinis C. amethyftino. F. Mant. 203. 92?

Statura C. Crucis maioris. Antennae fufcae bufi ferrugineae. Caput auratum, nitens ore fufco, palpis fubrufis. Thorax obcordatus aureus fulco medio longitudinali pofticeque puncto vtrinque impreffus. Elytra acneo- viridia, fubftriata, integra. Abdomen nigro-aeneum. Pedes ferruginei. T. II. f. 9. - Hab. in filuis fub arborum cortice frequens. Long. $3^{\frac{1}{\%}}$. lat. $1 \frac{1}{2}$.

C. amethyftinum $\mathrm{F}$. alium efie, iam patrin fuadet tam remota, Hg.

545. C. prafunus? niger, capite, thoraceque viridi-aeneis, elytris macula magna communi poftica nigra. F. Mant. 204. I O9.

Defcriptio Fabricii connenit omnino, fed antennae in meis fpeciminibus ferrugineae incertum tenent. Crederem $C$. dorfalem, nifi caput et thorax effent viridi-aenea, margine nullo modo pallefcente. Hab. cun aliis frequens. Long. 2. lat. $\frac{3}{4}$.

De $C$. prafino vide animadierfa ad C. viridanum. n. 543. Hg.

$$
\text { (p. s=r.) }
$$

546. C. teftuceus capite thoraceque ferrugineis, elytris teftaceis. F. Sp. 3 โ3. 84.

\& F. E. S. I. r. 166. I 86 .

Oliu. Encyclop. method. V. 355. I58.

Hab. in filuis. Long. $\mathrm{r}_{3}$. lat. $\frac{2}{3}$.

Oliuierium allegaui, quia optime annotat, citatum: Geoffr. Inf. x. I 53. 25. ad C'. atricapillum transferendum effe. Hig. 
547. C. Crux minor thorace orbiculato, rufo, coleoptris apice nigris, macula rufa. F. Sp. 3 I I. 69.

$\odot$ Schaeff. Ic. t. 4r. f. I3. Sulz.

D) Sclirank. E. I. 407.

\& F. E. S. I. I. '160. 1 59 ?

(Panz. F. I. G. XVI. 2.)

Caput nigrum palpis, antennisque rufis, apice nigris. Thorax tefaceus obcordatus. Elytra bafi nigra, tum macula magna flau, dein fafcia obliqua nigra, pofteaque ad apicem, macula altera flaua. Abdomen atrum. Pedes rufi. Variat elytris inacula magna nigra rotunda communi. . Hab. fub arborim cortice frequens. Long. $2 \frac{1}{\mathrm{i}}$. liat. $\mathrm{I}$.

Citata Fabricii e Sulzero. Hift. t. 7. f. 6. atque Schaeffero Ic. t. 4r. f. x $_{4}$. (ita legatur loco f. I3., ad C. Lunatum pertinent.' Fabricii C. cricem minorem Spec. et Mantiffae alium, quam Linnaei effe, ipfi Celeberrimo viro perfuafum eft, et in Ent. fyfr. bipuflulatum vocauit.

Anctorem verum $C$. Cnucem minovent $L$. non habuifle videtur, praecipue cum in Mantifla $9 \mathrm{r}$. 205. apud $C$. erratmon dicat, hunc a $C$. $C$. mino$r e$, praeter alia, etiam elytris truncatis difcrepare, quae tamen illi propria funt. Imo idem ibi defcriptus $C$. erratus, vel $C$. Andreat ftatim fecuturus (n. 543.), verus C. Crux minor L. videtur, omnes enim in illum competunt defcriptionis in Mant. datae notae. An vere C. C. minor Auctoris idem fit cum C. bipuffulato. F. E. S. difcernere non andeam.

Comparatio C. Crucis muioris ac minoris talem confufionem optime extricare poffe videtur. Hic $C$. C. maiore dimidio minor eft, omnino glaber, nitidus, antennis bafi rutis, capite maiore et aequa- 
li, thorace multo minore, paullulum latiore quam caput, nitido, fubcordato, in medio leuiter fulcato, rubro. Thorax in $C$. C. naiore orbicularis, quafi puluinatus, lateribuis marginatus, admodum punczatus, fufco-pubefcens. Elytra ciusdem thorace paullo latiora, pubercentia, veluti crenato - ftriata, vix nitida, apice integra: $C$. C. minovis contra thorace duplo latiora, pene' quadrata, laeuia, nitida, glabra, vix ftriata: ftriis fimplicibus, exterue marginata, apice truncata. Hinc nigredo lata apicis, quae in C. C. maiore confpicitur , deficit, et marginem fummum folum circumdat. Pedes maioris omnino nigri, minoris vero. teftaceo-rubri funt, geniculis nigris. Ambo eidem familiae nentiquam annumerari poffunt. C. C. mcior a reliquis feparandus videtur, quia forma fingulari gaudet, nullique cum eo communi.

Comparationem aliam $C$. C. minoris cum $C$. bipuftulato. $F . E . S$. adiungam, quorum pofterior gracilior et ratione craffitudinis anguftior eft, thorace maiore, capite crafio, elytris thorace paullo latioribus, haud quadratis, nec apice truncatis, absque macula fcutellari futuraque nigra; macula pone elytri finguli medium magna fubrotunda nigra, nec futuram, nec marginem attingente, ad marginem diducta, vt ifthmo lunulato cum apicis macula tenui cohaereat, elytrorum margine femper intacto. Sic in poftica parte fpatium includitur latum elytris commune, freto futurali cum fuperiore clytrorum parte connexum. Interdum vero id fnitium valde diminuitur, atque vix apparet, interdum ifthmus, maculas coniungens, omnino deeft; interdum maculae mediae cohaerere fafciamque fingere videntur. Color corporis totius minime nitidus eft, vt in $C$. Cruce minore, color 
color niger vero nitorem quemdam caeruleum im. mixtum habet. Magnitudo, antennarumque color variat. Saepius C. C. minore duplo maior eft, nonnumquam autem eiusdem magnitudinis. Icones optimas Panzeri Fn. Inf. Germ. XVI. apud modo dictos compares Carabos. $\mathrm{Hg}$.

548. C. Andreace thorace orbiculato nigro, nitido, elytris pallidis faficia media nigra. F. Sp. 3 II. 70 .

\& C. erratus. Rofii Mant. 9r. 205 . An C. Crux minor. F. E. S. I. I. I60. I59?

(Panz. F. I. G. XVI. 2.)

Statura omnino C. Crix minor. Elytra apice parum fufca. Pedes pallidi. Fafcia in medio elytrorum ad futuram dilata. Hab. cum priori nimus frequens. Long. $2{ }_{i}^{r}$. lat. I.

Vide, quae ad praecedentem animaduerfa funt. Hg.

$$
\text { (p. 222.) }
$$

* 549. C. cyathiger thorace elytrisque fuluis, poftice nigro-maculatis, maculaque nigra ad futuram caliciformi.

Statura et habitu fimilis $C$. Crux minor. Caput nigrum nitens. Antennae nigrae articulo primo et fecundo fermgineis. Thorax fuluus, aut faturate teftaceus, linea longitudinali exaratus. Elytra fulua, ftriata, nitida, pofticeque maculis duabus nigris rotundis; in fingulo elytro vna, quae intacto margine exteriore extenduntur vltra elytri dimidiun, et quas interiacet diftincta, et in futura pofita macula alia longitudinalis caliciformis nigra. Apex vero et bafis elytrorum immaculata. Subtus ater, pectore pedibusque fuluis. Tab. VII. f. 3 . Hab. in filuis rarus. Long. 2. lat. $I \frac{T}{5}$. 
550. C. azureus cyaneus, antennis pedibusque rubris. F. Sp. 308. 53.

$\odot$ Bergftr.

\& F. E. S. I. I. I55. I32.

C. Proteus. var. $\delta$. Rofil Mant. 86. 955 .

Statura C. lati; caeruleo colore nitens. Thorax fuborbiculatus. Elytra ftriata, antennae pedesque toti rubri. Hab. in locis arenofis filueftribus rarrs. Long. 5 . lat. 2.

Mire variat colore, nunc obfcure, nunc laete cyaneo nunc nigro, nunc viridi, nunc fufco-cupreo, nunc fufco, pedibus femper ferme rufis, rariflime piceis.

Auctor in Mantiîla fua ipfe dubitat, C. azureum varietatem $C$. Protei Paykull. effe. Id certe perfpeEtum habeo, Paykullium aut $C$. affinem Schrankii, quem $C$. ourati $P$ ayjk. varietatem coniiciam, au, $C$. azurenn verum non nouife, nullo modo enim varietates a fe inuicem haberi poffunt. $\mathrm{Hg}$.

55I. C. dentatus, cyaneus, thorace' cylindrico fubaequali, ore, antennis pedibusque rufis.

\& Roffi Mańt. 83. I89.

Cicindela enarginata. F. E. S. I. I. $177 \cdot 37$.

Olinier. Encyclop. method. V. 135. 39.

Olinier. Ent. ou hift. nat. des Inf. t. 3. f. 38 .

Magnitudo et habitus $C$.crepitantis. Totus cyaneus, nitidus. Os antennaeque rufae articulo primo praelonga, apice nigro. Mandibulae exfertae, elongatae, magis quam in C. Jjinibarbi ab hoc omnino diuerfo. Caput punctulatum. Thorax anguftus, fere cylindricus, et in medio canaliculatus. Elytra punćtato - ftriata, apice truncata. Pedes toti ruf. Tab. II. f. 1 I. Hab. fub arborum,corti- 
ce, et in truncis emortuis, hieme non rarus. Long, 3. lat. $I_{\bar{\sigma}}$.

Corpus pube, qnadam luce immifia, fufcefcente tectum. Elytra quafi crenato-ftriata, apice truncata, angulis lateralibus parum prominentibus, vt emarginata dici poffent. Celeb. Fabricius ad Cicindelarum genus noftrum infectum transtnlit, quibus quidem mandibularum forma appropinquatur, mihi tamen Carabus videtur, illi familiae annumerandus, vi $C$. crepitans, funcans etc. locum tenent. Ne mandibularum formam folam fpectes, fuadet fimilis earum fabrica $C$. roftrati $F$, ' cuius partes oris a reliquis recedere videntur Carabis. Fabricius hune $C$. dentatum $R$. illo nomine $C$. cmarginatae miffum accepiffe videtur, quia Olinierius idem nomen eidem dedit infecto l. c. addens, formam quodammodo Carabi habere. Hg.

$$
\text { (p. 223.) }
$$

* 552. C. mendax thorace fuborbiculato, nigro aeneo, elytris pedibusque teftaceis.

Statura omnino C. Cr.maioris. Antennae rufae. Caput nigro-aeneum, immaculatum. Thorax fuborbiculatus, nigro-aeneus, linea dorfali imprefra. Elytira teftacea ftriata integra. Abdomen lubtus nigro-aenemm. Pedes toti teftacei. Non confundendus cum $C$. tefacaco, qui multo minor eft, et dinerfus. Tab. II. f. Io. Hab. in ligno putrefcente prope terrain. Long. $3 \frac{x}{3}$. lat: $I \frac{1}{3}$.

* 553. C. firfciolatus capite thoraceque nigro. aeneis, antennis pedibus, elytrisque tefiaceis, ad apicem nigro - marginatis.

Statura fere praecedentis. Antennae ferrugineac. Caput et thorax nigro-aenea immaculata. 
Thorax obcordatus, anguftus; linea dorfali impreffa. Elytra faturate teftacea ftriata, abdomine paullo breuiora, et a dimidio ad totutn apicem late nigro - marginata, fafciaque longitudinali nigra communi futuram occupante, et elytrorum apicem non attingente. Pedes toti teftacei. Subtus niger abdomine elytris concolore. Tab. II. f. 8. Hab. fub arborum cortice in filuis non frequens. Long. 4. lat. $\mathrm{I} \frac{\mathrm{I}}{3}$.

Specimen huius Carabi a Dn. de Paykull, quem infecta multa a Dn. Roffio accepiffe fcimus, dono mihi datum defcribam. Statura C. Sefquiftriato Panzeri paullulum fimilis eft, fed anguftior. Capitis thoracisque colorem potius brunneum, quam nigroaeneum vocarem. Elytra apice fubtruncata cum reliquo corpore rufa funt, margine vndique brun. neo-nigro cincta, vt vitta a humero per elytrum decurrere videatur rufa, quae tamen apicem non attingit. Haec difcrepantia ab aetate minore deriuanda videntur, qua fpecimen meum forte captum eft. $\mathrm{Hg}$.

554. C. meridianus niger, elytris antice, pedibusque teftaceis. F.Sp. 3 I2. 7\%

o F. E. S. I. I. I64. I77.

Elytra ftriata bafi flauefcentia. Pedes teftacei. Antennae fufcae bafi rufae: nitidus. Hab. in terra. Long. $I_{2}^{\prime}$. lat. $\frac{1}{2}$.

Celeberrimus Paykullius recte indicafie videtur, $C$. dorfalem F. E. S. I. I. I65. I83. Roffi Mant. I9I. 204. varietatem huius effe, differentiam enim Specificam detegere nequeo, et tranfitus alterius ad alterum non deeft. Hg. 


$$
\text { ( } p \text {. 224.) }
$$

* 555. C. truncatellus niger, fupra fubaeneus, elytris apice fubtruncatis. Linm. Syft. Nat. 672. 43. Fil. Sv. 8I4.

\& F. E. S. I. I. 168.69 .

Totus niger, fupra fubaeneus. Elytra fubftriata, apice oblique truncata. Hab. in ligno putrido. Long. I. lat. $\frac{\pi}{2}$.

556. C. atricapillus thorace rotundato, rufo, elytris obtufis teitaceis, capite atro. F. Sp. 313.82.

\& F. E. S. I. I. I40. 69.

Geoffr. Inf. x. I 53. 25 .

Hab. fub corticibus. Long. $\frac{2}{3}$.

Longus effe folet $2 \frac{1}{2}$ lin. latus ${ }_{4}^{3}$. Sed interdum dimidio breuior occurrit. Color elytrorum variabilis eft folito luridus; nonnumquam eandem cum C. Cruce minore fignaturam nigram habent, apice excepto numquam atro. Interdum macula prope apicem communis reftat, nonnumquam fucura fufcefcens euadit, macula pone fcutellum communi fere deleta; faepius vero umnino pallide lurida funt. Statura quodammodo fimilis eft $C$. anguftato, velCicindelae anguftatae F. E.S I. x. 169.3., quem a Fabricio ad Cicindelas translatum aegre video, cum ne oris partes quidem, vt mandibulae in $C$. dentato Roff., locum illum approbent.

In opere Voetiano Tom. II. t. 47. f. x 2. infectum repraefentatum videmus, quod Voetius ad Donacias fuas, quo genere Malachios, Cantharides, Lampyrides etc. comprehendit; refert, ex Africa oriundum. Quisque, ibi depictum videns coleopteron, mecum fentiet, $C$. atricapillum noftrun melins depingi non poffe, quam ibidem factum eft, quamuis contendere non audearn, eundem effe cum noftrate. 
De citato Geoffroyi, a me addito, vide, quae ad C. tefuctenm 11. 546 . adnotaui. Hg.

* 557. C. pediftris niger, nitidus, thorace mfo, elytris punctis duobus pellucidis.

An C. arenariur. Scop. Ent. Carn. 277?

\& Notoxus pedeftris. Rofii Mant. 45. I r 4 . Apterus, capite antennisque nigris. Thorax rufus elongatus. Elytra nigra nitidifima, locis duobus pellucidis, altero ad bafin, altero ad apicem. Pedes et femora nigra, bafi rufa. Elytra, armato oculo, pilis albis pubefcunt. Hab. in terra et arena frequens. Long. $I_{\frac{1}{2}}$. lat. $\frac{1}{4}$.

Citatum $\in$ Scopolio auctor ipfe poftea omifit, hac minime fpectans. $\mathrm{Hg}$.

55S. C. 4 -guttatus thorace rotundato, atro, elytris nigits, punctis quatuor albis. F. Sp. 3 r 3 . 80.

2 F. E. S I. r. 165 . 18ז.

Difiert a C. 4 -maculato. Elytra haud obtufa. Thorax valde rotundatus, ater. "Tibiae pallidae. Hab. fub arborum cortice. Long. $\frac{3}{3}$.

Herbftius Arch. t. 29. f. 9. Carabum repraefentat, quem ipfe poffideo, qui vero nullo modo magnitudini cum C. truncatello comparatae conuenit, cuiusque caput et thorax haud nigra, fed obfcure viridia funt. Oliuierius eundem 1. c. p. 353. n. I53. citat, fed nomine deceptus videtur. $\mathrm{Hg}$.

$$
\text { (p.225) }
$$

* 559. C. lenipes thorace rotundato, ater nitidus elytris punctato-ftriatis, pedibus rufis.

An C. picipes F. Mant. 203. 98 ?

Statira C. profini. Agilis, paruns. Antennae fufcae, bafi ferrugineae. Thorax anguftus fere orbicu* 
orbiculațıs, laeuis, nitidus. Elytra punctato-ftriata, nitida. Pedes longiufculi rufi, femoribus antica parte nigris. Color atro-caeruleus. Hab. fub arborun corticibus rarior. Long. $2 \frac{1}{2}$. lat. I.

560. C. 4-maculitus thorace ferrugineo, glabro, elytris obtufifimis fufcis, maculis duabus albis. F. Sp. $313 \cdot 79$.

$\odot$ Pontopp.

\&'F. E. S. I. I. 165 . 180.

Abdomen et femora nigra, tibiae pallidae. Hab. cum praecedentibus. Long. I $\frac{2}{3}$. lat. $\frac{2}{3}$.

Pedes in fpeciminibus meis pallidi funt. Elytrorum fignatura admodum variabilis eft. Maculae raro confpiciuntur integrae. Elytra nune atra funt, nunc fufca, nunc difeo communi teftaceo, nune teftacea tota, absque vllo ferme marginis fufci rudimento. Thorax nuric rufus, nunc brunneus margine pallefcente. $\mathrm{Hg}$.

$56 \mathrm{I}$. C. bipunitatus aeneus, antennis nigris, tibiis pallidis. F. Sp. 313.86 .

\& F. E. S. I. x. 167.189 .

Caput thorax, elytra fubaenea. Elytra punctis duobus impreffa, altero antice, altero poftice. Hab. in ligno putrido. Long. $\frac{2}{3}$.

Pẹdes omnino nigri funt. $\mathrm{Hg}$ 。

\section{(p. 226.)}

- 562. C. exfanguis totus pallidus oblongus elytris in medio nigro - guttatis.

Elytra quafi albida macuiis nigris đuabus ob. foletis, in fingulo vna. Alatus. Hab. in putridis arborum truncis. Long. $2 \frac{\pi}{2}$. lat. $\frac{x}{4}$.

* 563. C. minutis' totus niger laeuis.

Geoffr. Inf. I. I53. 26. 
Minor C. truncatello Linn. Elytra non ftriata, neque oblique truncata. Totus colore nigro-aeneo nitido. Thorax obcordatus in medio linea exaratus et anguftior, quam in C. truncatello. Hab. in ligno putrido. Long. I. lat. $\frac{1}{4}$.

* 564. C. Sigma fubflauus, thorace cordato, elytris fafcia undata nigra, capite nigro.

Thorax fubflauus. Elytra pallida. Hab. cum aliis exefas arbores. Long. 1. lat. $\frac{1}{4}$.

* 565. C.pufillus capite thoraceque nigris, elytris fufcis.

Vere exiguus et vix pediculo aequalis. Antennae fubferrugineae longitudine corporis. Caput et thorax nigra, obfcura. Elytra integra, fordide teftacea. Abdonen nigrum. Pedes fubrufi. Hab. in ligno putrefcente Ilicis.

$$
\text { ( } p .2=\text {. })
$$

* 569. C. Non plus vitra flaums, oculis nigris, thorace obcordato, elytris antennisque pilofis fub. clauatis.

Pediculo minor. Facies Carabi, at palpi fubclauati, antennaeque ad apicem craffiores articulis fere rotundis approximatis, pilofis, vltimo máiore acuminato. Alatus. Hab. in quifquiliis.

\section{SCARITES.}

Palpi fex filiformes. Labium corneum dentatum. Antennae moniliformes.

567. S. Gigis ater, pedibus anticis palmatodigitatis, mandibulis fulcatis, elytris fubftriatis. F. Sp. 314. I. 


\section{Olinier N. 36. pl. I. f. I. b. f. 1. c.}

Magnitudine admodum variat. Maiores apud nos, longi I5 $_{5}$ lati 6 lin. Apterus. Caput antice fulcatum, poftice rotundatum. Maxillae porrectae, quadridendatae. Labium inferius punctis duobus excauatis contiguis. Thorax antice lunatus, margine vnidentato, elytris fuperius vix late et obfolete ftriatis, fubtus quafi argentatis lucidis, et manifefte punctato - ftriatis. Pedes antici palmato-digitati, poftici quatuor pilis rufis ciliati. $\mathrm{Hab}$. in campis fabulofis, fub ftercore bouino frequens. Long. 9, I2, I5. lat. 4, 5, 6 .

Fabricii Sc. Gigas Africae aequinoctiali indigenus eft, et cum Herbftii. Arch. t. 49. f. 4. citet, confiteor, figura ibi data admodum a noftro difirepante, dubium meum de identitate Scaritis Fabriciani et Roffiani valde augeri; cum abdomen ibi ouatum fit, quod in noftro fubquadratum, bafi recta truncatum, verfus apicem dilatatum ibidemque rotundatum eff. Thorax quoque in eadem figura pro portione multo longior eft. Tibiae Sc, Gigantis $\boldsymbol{R}$., praefertim externe, ciliatae funt pilis tenuibus villofis longioribus. Specimen itaque defcribere in animo eft, quod a Dn. de Paykullio mecum benigniter communicatum pofideo, vt ii, qui Sc. Gigantem verum Africanum habent, coniecturam meam de diuerfitate vtriusque aut confirment, aut reiiciant.

Statura depreffa admodum cum $S$. fubterraneo $F$. conuenit, fed quadruplo maior eft. Specimen meum enim a maxillarum, forcipitis in modum fefe tegentium, apice ad anum vsque ${ }_{5}$ lin. longum eft. Caput cum ore conforme cum eisdem Sc. Subterrinei partibus. Thorax capite paullu- 
lum latior, fed aliquanto breuiòr, quafi femicircularis, ita, vt apex diametrum conftituat, quem linea imprefla legit; fpatiumque inter hanc lineam marginemque anticum ipfum leuiter ftriatum eft. Superficies leuis, conuexa, linea media impreffa. Latera marginata funt, et verfus tiloracis bafin, quae valde angufta eft et partem tertiam apicis vix aequat, denticulus eminet. Eiytra thorace anguftiora, et capite thoraceque vna funtis, paullnlum breuiora, poftice latiora, leuia, nec ftriata, nec punctata, angulo parvo acuto in humero prominulo. Tibiae anticae palmato-lobatae, latere interiore pilis fufcis brenibus, reliquae omnes vero pilis longioribus denfis rutilis ciliatae funt., Thorax latus $5 \frac{3}{4}$ lin. bafi $2 \frac{7}{2}$; longus 3 lin. Elytra bafi lata $4 \frac{\pi}{2}$, pone medium vero $5 \frac{\mathrm{l}}{2} \mathrm{lin}$. $\mathrm{Hg}$.

568. S. thoracicus thorace ouato, elytris aeneis, pedibus ferrugineis. .

o S. gibbus. F. E. S. I. I. 96. I0. Panz. F. I. G. V. I.

Carabus globofus. Herbft. Arch. p. I42. n. 58 . t. I9. f. I7. K.

Minor S. arenario. Caput obfcurum. Antennae, mandibulae et palpi ferruginei. Thorax valde gibbofus, rotundatus, obfcure nitens. Elytra aurato - nitentia, ftriata ftriis punctatis. Pedes ferrnginei, antici folforii. D. D'Antic. Soc. Linn.

Hab. in arenofis fub terra primo vere frequens. Long. $1 \frac{3}{4}$. lat. $\frac{2}{3}$.

Magnitudine admodum variat, colore femper fere metallico, rarifime piceo. Truncus in meis non ita globofus, vt Panzerus f. c. exhibet. Hg. 


\section{(p. 228.)}

569. S. arenarius piceus, tibiis anticis palmato-digitatis. F. Sp. 314.4 .

q F. E. S. I. I. 96. 9:

Noftra fpecimina conueniunt cum defcriptione Linnaei. Hab. in campis arenofis minus frequens.

* 570. S. clypeatus ater, clypeo rotundato, enarginato, elytris ftriatis, ftriis punctatis.

Fuesly. Arch. t. 29. f. 4 .

Medius, ater. Caput duplo maius thorace. Clypeus rotundatus, emarginatus, pilofus, mandibuJas tegens. Antemnae piceae pilofae. Thorax lunatus, punctatus, antice acutus. Elytra frriata, ftriis punctatis. Pedes nigri pilofi, antici fofforii. Tarfi onmes fufci.

Valde affinis C. interrupto F. D. D'Antic. Soc. Linn.

Hab. in pratis filuaticis arenofis. Long. 5. lat. 2.

Conferantur adnotata ad C. interruptum n. 520, rbi idem Herbfiii citatum male allegatum eft. , Hg.

* 5 ? I. S. Calydonius alatus niger, thorace obcordato, capite tricorni.

\& Curabus Calydonins. F. E. S. I. I. r44. 89. Confer Sccrit. Demam. Roffi Miant. 92. 206. Statura C. intermupti: S.clypeato maior. Antennae fufcac pilofae. Caput nigrum exfertum punctatum pilofun. Clypeus apice emarginatus, tricornis, duobus lateralibus recuruis acutis, altero frontis crafio, antrorfum retorto truncato. Thorax niger obcordatus, punctatus, pilis raris afperfus. Elytra nigra punctato - ftriata ftriis in fingulo octo.

$$
\text { S } 3
$$

Pedes 
Pedes nigri pilofi, antici fofforii. Femina inermis. Variat colore interdum piceo, vel fubrufo, et magnitudine duplo ninori, maxillis cornutis, fronte mutica, pedibus fubferrugineis. - Differt a $S$. clypeato capite non ita magno, neque ita depreffo, et in mafculis cornuto; thorace obcordato, non lunato, neque antice acuto. Tab. VIII. f. 8 . mas. f. 9. femina. - Hab. in arenofis. Long. 8 ; lat. $2 \frac{\pi}{2}$.

Statura fingularis, et nulli mihi noto fimilis eft. Icon fatis bene expreffa illam optime monftrabit. $C$. intterruptun nullo modo autem cum illo comparaffem, cum vterque admodum a fe inuicem difcrepent. Statura craffa, conuexa, dorfo tamen depreffo. Color niger nitidus quidem eft. fed ob pilos breues vix apparentes languidus euadit. De varietate $a b$ auctore indicata vide ipfum in Mantiffa. l. c.

Multi Carabi nunc ad Scaritum genus transferuntur, qui quidem Carabis non bene annumerati fuere; qui tamen non magis Scaritis fpeciem repraefentant. Scarites veros Sc. Gigantem Roff. Sc. Gigantem, fubterraneum, arenarium et gibbum Fabr., Sc. collarem Herbft. etc. habeo, quibus etiam Carab. cephalotes. et Scarit depreffus. F. adiungi poffent. - Hicce Sc. Calydonius autem, cum aliis genus intermedium inter Scarites et Carabos conStituere videtur optimum. Hg.

\section{(p. 220.)}

* 572. S. rufus rufus, pilolus, thorace ouato, elytris punctato - ftriatis.

Statura omnino $S$. thoracici. Totus rufus pilofus. Palpi anteriores praelongi. Thorax valde gibbus, rotundatus, laeuis, nitidus. Elytra pun- 
ctato - ftriata. Pedes antici fofforii. Tab. IV. f. 3. Hab. cum $S$. thoracico eodem tempore, et loco frequens. Long. $1 \frac{4}{5}$. lat. $\frac{x}{3}$.

\section{PIMELIA.}

Palpi filiformes. Maxilla vnidentata. Labium menbranaceum, rotundatum, fubemarginatum. Antennae filiformes.

573. P. muricata nigra, coleoptris obtufis ftriis muricatis. F. Sp. 316.5 .

$\odot$ Linnei Muf.

D Pet. Sp. I. C. f. XIV. Confer. p. $25.11 . x 23$.

o F. E. S. I. I. I00. 8.

Aptera, tota nigra, corpore fphaerico globofo. Oculi femilunares. Thorax vbique emarginatus, orbiculatus, laeuis. Elytra connata, obtula, filcis tribus eleuatis, fpatiisque interiectis punctatis. Pedes antici brelles; tibiarum fpinae porrectae. Thorax absque punctis duobus impreffis. Hab. filuas arenofas, frequens in arena prope Formicarum aceruos. Long. ?. lat. 4.

Quid Fabricius citato: "Pallas Ic. x. 706. 5 x. Tenebrio echinatus" fibi velit, nefcio. Icon Petagnae citata fatis bona eft, ftaturam certe optime exprimit. Quod Geoffroyns aliique ftriarum elenatarum puncta retrorfum fpectantia, quae ftrias muricatas reddunt, haud animaduerterint; inde deducendum videtur, quod femper a tergo, nec 2 latete easdem luftrauerint. Hg.

$$
(p .230 .)
$$

574. P. carinnta nigra, thorace orbiculato, elytris lineis eleuatis tribus laeuibus. F:Sp. 3 I9. 20.

D Petagna. Sp. Inf. Cal. ז25. f. xg.

$\mathrm{S}_{4}$ 
을. E. S. I. I. $104.2 \%$

Tota atra, femoribus anticis craffioribus fubdentatis. Hab. in campis filuaticis minus frequens: Long. ?. lat. 2:.

Forskaolii infectum, quod, vti e nomine concludere licet, Aegypto indigenum eft, huc non referrem, cum Pimeliarum fpecies verbis folis difficulter diftinguantur, hinc patria tam longinqua fufpicionem fufficientem praebere poffit, infectum vtrumque diuerfum coniiciendi. $\mathrm{Hg}$.

575. $P$. reflexa thorace marginato, marginibus, reflexis, elytris muricatis, linea laterali carinata, lacui. F. Sp. 3 I8. I6.

D Tęu. Pterygodes, Pallas. Icon. Inf. x. 4 I. 6. t. 6. (C.) f. 6 ,

8 F. E. S.I. I. I04. 24.

Affinis $P$. Spinofae. Tota atra. Thorax antice lunatus, lateribus valde marginatus. Elytra connata foriis duabus punctorum eleuatorum, ct linea laterali valde carinata, pone quam altera linea punctorum fimilium. Apex acuminatus. Hab. in ligno cariofo Cuprefli, frequens in Colle Fefolano, loco vulgo dicto Bellevante. Long. 8. lat, 4 .

Pimeliarum genus admodum dubium eft, et in plura diftribuendum videtur. Quis enim, $P$. firiatam et reflexam, anguftatam èt variolofan intuens, eidem generi annumeratas crederet? In Infectis tamen, terris tam remotis, vt plura horum, indigenis, genera difficulter inftituuntur, cum folum ferme habitum, nec victum cultumque, qui faepius optimam in generibus condendis demonAtrant viam, confulere licet. Hg. 


\section{LXXVIII. (p. BLAPS.}

Palpi quatuor clauati. Maxilla recta bifida. La

bium membranaceum fifum. Antemane apice moniliformes.

576 . B. mortifuga atra; coleoptris mucronatis, laenibus. F.Sp. 321.3 .

$\odot$ Raj., Lift.; Pet., Mouff., Frifch., Schaeff. Ic. t. 6. f. I3,

J) Scop. E. C. 252 . Schrank. E. I. 4 I 5.

\& F. E. S. I. I. 107. 3 .

Schaeft. Ic. t. 60. f. 3 .

Panz. F. I. G. III. 3. (male piaia).

Variat admodum magnitudine. Hab. in vmbrofis humidis frequens. Long. 9, I 1,15 . lat. 4, 5.

Mas femina admodum gracilior eft, et elytrorum acumine praelongo gaudet, quod in femina vix apparet. Ille: Schaeff. Ic. t. 60. f. 3. (ita in F. E. S. loco t. 6. f. r3. legendum eft); haec: $t_{0}$ 37. f. 6. repraefentata eft. $\mathrm{Hg}$.

\section{LXXIX: TENEBRIO,}

Palpi quatuor inaequales, anteriores fubclanati, pofteriores filiformes. Maxilla bifida. Labiuin

truncatum, corneum, integrum.'Antemiae

\section{moniliformes.}

577. T. molitor oblongus piceus, elytris ftria. tis. F. Sp. 322. 2.

$\odot$ Raj., Frifch., Sulz.

q F. E. S. I. I. III. 6 。

Variat fernoribus anticis fimplicibus, et femoribus anticis craffioribus, tibiis incuruis; vti etiam magnitudine. Hab. plerumque in filuis. Saepe in. 
uentus in ligno putrido et fub cortice Quercus. Long. 7. lat. $2 \frac{1}{2}$.

Color nunc niger, nunc rufefcens. - Tibiae anticae incurlae huic generi propriae ridentur. De citato Schaeff. Ic. t. 66. f. r. vide adnotatum ad n. 580. Hg.

\section{(p. 232.)}

* 578. T. affinis oblongus, niger, elytris punEtato - ftriatis.

T. molitori fimillimus, at dimidio minor, et ely'tra punctato-ftriata. Tibiae anticae incuruae. Etfi $T$. molitor admodum variare videatur, hic tamen diftinctus. Hab. in truncis cariofis Quercus frequens. Long. $5^{\frac{1}{2}}$. lat. $I^{\frac{1}{2}}$.

Ipfe Tenebrionem pofideo, T. molitori admodum fimilem, qui, eiusdem ferme longitudinis, dimidio anguftior eft, et elytra profunde punetatoItriata gerit. Quem nomine T. cylindrici Schneideri in mufeo afferuo. Hg.

* 579. T. Mauritunicus? alatıs, niger, fubtus piceus, thoracis marginibus antice, polticeque dente angulatis. Linn. Syít. Nat. 674. 4.

An Tenebroides complanatus Piller It. per Pol.Scl. t. 9. f. 9 ?

D Rofii Mant. 93. 208

Trogofita Caraboïdes. F. E. S. I. I. II5. 2.

- Panz. F. I. G. III. 4.

Tenebrio picens. Schall. Act. Hallenf. r. 3 rg.

Carabus Bucephalus. Herbft. Arch. IV. I4I.' 57. t. 29 . f. $\mathrm{r} 6$. $\mathrm{i}$.

Lucanus dubius. Scriba. Ephem. Ent. I. 42. 6. Herbft. Kaeff. III. p. 3 r6. I 8 .

L. fufcus. Preislers. Boehm. Inf. I. 6. 3. t. I. I. A. B. 
Habitus fere $L$. interrupti. Scar. arenario maior. Niger, glaber, oblongus. Caput punctis impreflis. Maxillae brenes, arcuatae, dentatae. Palpi lex? Antennae moniliformes, apice extrorlum crafliori. Thorax marginatus, antice pilis breuiffimis flauis ciliatus, dente obfoleto. Elytra marginata, ftriata. Abdomen et pedes fibbrufi. Femora latiufcula, tarfi minimi. Tab. VII. f. 15. et Tab. III. f. 12. at haee paullo maior. Hab. fub arborum cortice in filuis

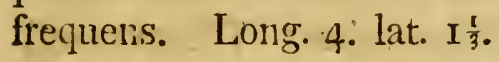

De citato e Piller etc. vix dubito, quoad ex epitome illa, in Scriba. Entom. Iourn. II. ror. exhibita, quae defcriptiones, iconibus omifis, continet, concludere licet. Infectum hocce dubium femper fuit. Fabricius, in Oliuierii veftigium, nouum ex illo aliisque adiectis condidit genus, cui nomen Trogofitae dedit. Infectorum, ibidem enumeratorum, nulla alia poffideo, quam hanc $T$. caraboïdem et $T$. fermingeam, hinc de genere iudicare nequeo. Plures Entomologi celeberrimi, e. g. Schneiderus, ac Panzerus Paffcilum inter ruptum Trogofitae genus fubire iubent, fed, ne alia commemorem, pedes antici admodum difcre. pant, et vietum alienum indicant.

Tenebr. Mauritunicum Linn. alium efle, iam patria fuadet, et fi Degeeri $L$. manrum, quem ex Suriname accepit, quemque eundem cum $T$. Mauritanico Limn. habet, re vera eundem credamus, figura eiusdem data t. I3. f. 9. Tomi V. admodum differt.

Alii, quorum vnus Celeb. Fabricins eft, T. caraboidem Linn. allegant, at Linnaeus apterum dicit, cum hic alatus fit, et coleoptera carinata, ab eodem commemorata, afpicere nequeo. 
Fabricii citatum: Cavabus cocdunatus. Degeer. 4. 97. 7. t. 3. f. I3. ad.C. roftratun translocetur. Hg.

$$
\text { ( } p .233 .)
$$

580. T. culinaris? alatus, ferrugineus; elytris itriatis, clypeo emarginato. F. Mant. 2 I 2. I 5.

D Tenebrio rufus. Piller. It. per Por. Scl. t. $z^{\circ}$ f. 4 ?

\& F. E. S. I. I. II3. I6.

Pinz. F. I. G. IX. I.

Per totum concolor, fufco-rubens. Thorax marginatus, fubplanus, nitens. Elytra lineata. Latitudo fere rbique eadem. Noftra fpecimina exacte conueniunt cum figura Piller. 1. c. parum, aut nihil cum figuris Ten. culinaris a Schaeffero exhibitis Icon. t. 66. f. I. t. I30. f. 5. Plerumque totus fufco - fanguineus, at colore et magnitudine variat. Hab. filuis fub arborum cortice frequens. Long. 4. lat. $I_{2}$.

In eisdem Schaefferi figuris, quarum $t, 66$. f. I. Auctor nofter apud T. Molitoven iterum alleganit, nec liunc nec Tenebrionem mihi notum agnofco. Forte Helopis fpeciem repraefentat. $T$. culinarem Auftoruni ipfum non poffideo, fed alium, illi admodum fimilem, quem Celeberr. Panzerus Fn. Inf. Germ. IX. t. 2, nomine $T$. ferruginei depinxit atque defcripfit, et quem in trunco quercus vetuftae decifae reperieram. Qui praecipue thorace antice retufo ac depreffo infignis eft, quod cuim a Linnaeo in Fn. Su. $8 \times 6$. in $T$. culinaris fpeciminibus maioribus, quae femitras coniisit, commemoratum fit, hurc $T$. clllinarcm $L$. eundem cum $T$. fermgineo meo 1 . c. coniicere licet. Cum eiusdem vnicum tantum fpecinen pofldeam, quod ipfe reperi, difcernere nequeo, 
nequeo, vtrum Linnaeus minores, thorace antice imprefio carentes recte mafculos coniecerit, an diuerfae fpeciei fint. $\mathrm{Hg}$.

* 58 1. T.? fufcus alatus niger, obfcurus, capite depreffo, thorace angulato margine ferrulato, elytris crenato - ftriatis.

Totus niger obfcurus, fubtus piceus. Antemae moniliformes, thorace breuiores. Caput exfertum, depreffum, apice dilatato, fubmarginato. Thorax antice valde angulatus, pofticeque rotundatus, dente vtrinque obfoleto, margineque prominulo, ferrulato. Elytra rugofa, crenato-ftriata margine reflexa. Alae fulcefcentes. De genere dubito: an Pimelia? - Hab. in filuis, femel inuentus. Long. 3. lat. $I \frac{x}{2}$.

* 582. T. chryfomelinus teftudineus, glaber, nitidus, elytris obfolete ftriatis, in mèdio obfcurioribus.

An Tenebrio teftudinetss Piller. It. per Pofeg. p. 83.t. ?. f. 6.?

Corpus ouato - oblongum. Antennae breues moniliformes. Thorax et elytra glabra, nitida te. ftudinei coloris. Pedes ferruginei. Alatus. Hab. fub arborum cortice, praefertim Quercus, frequens. Long. I $\frac{3}{4}$. lat. $\frac{3}{4}$. I.

* 583. T.? dubius.

$$
\text { (p. 234.) }
$$

+ Cebrio brelicornis. Roffi Mant. 34. 84 . Infectum hoc antifyftematicum adeo fingulare ef, vit nciciam tuto referre ad genus: determinandum propono ac relinquo Entomologis peritioribus.

Totam 
Totum teftaceum, praeter oculos et apices maxillarum, qui nigri funt. Apterum. Mágnitudo M. Profcarabaei, habitus Tenebrionis. Primo intuitu videtur Meloe : attentius cunfiderantibus, dubium fuboritur de Elatere, deindeque Tenebrionis character quodammodo impreflus, confufionem, et perplexitatem non facile fine face Metamorphofeos extricandam adducit. Antennae breuiffimae, lineam longae, fubclauatae, articulis lentiformibus, compreffis, pilofis, vix extrorfum craffioribus articulo primo inaiore et clauae aequali, ante oculos pofitae. Palpi quatuor inaequales, filiformes, guorum antici craffiores, et longiores fuperant fongitudine antennas. Caput fi non exfertum, certe non deflexum. Frons veluti imprefla nota quadam femilunari. Os clypeo truncato, labio emarginato, bifido? mandibulis duabus porrectis, annuli inftar arcuatis, acuminatillimis, fimplicibus, capite fere longioribus. Thorax conuexus, itrinque punctatus, fubmarginatus, fubtus mucrone et foneola; poftice ad latera acute angulatus vti in Elatere. Elytra gibba, flexilia, pone delifcentia, late ftriata, valde abbreuiata, fubmarginata $\mathrm{Ab}$. domen ouatum pilofum, molle, terminatum ftiIo, vel cauda recta, breui, cornea, apice bifida, quac manifefte apparet deftinata ad oua ponenda. Femora poftica bafi lamina magna elliptica, feu quid finile trochanteri Caraborum, commune tamen elateribus aliisque. Tibiae compreffae, apice fpinis duabus. Tarfi quinque tam in pedibus allticis, quam pofticis, admodum breues, vltimo hamulato. An Tenebrio? Tarforum numerus remo. nere videtur.

De- 
Defcriptio haec is non prolixa nimis videbitur, qui veritatem amant, et fane intelligunt, quantam dinerforum characterum varietatem in hoc infecto natura coniunxerit. Tab. I. f. 2. - Hab. Florentiae et Pifis: intentum in locis humentibus fordidis. Long. 8. lat. 4.

Hoc infectum nondum vidi; fingulare quidem videtur, fed, quoad ex icone adiecta iudicare, Tenebrionis et Elateris notas afpicere nequeo, et ob elytra, et ob habitum reliquum potius ad Meloes amandafiem. Oliuierius, vid. Mand. cit. cum Cebrione Gigante coniunxit. Proprium tamen genus conftituere videtur. $\mathrm{Hg}$.

\section{(p.235.) \\ LXXX. HELOPS.}

Palpi quatuor anteriores fecuriformes, pofteriores clauati. Labium corneum, integrum. Antenuae moniliformes.

584. H. lanipes aeneus, elytris ftriatis, acuminatis. F. Sp. 324. 2.

- D Schrank. E. I. 4 I2. Schaeff. Ic. t. 5I. f. 3.

\& F. E. S. I. I. II8. 4 .

Palpi fecuriformes. Antemnae pedesque nigra. Thorax fubquadratus, denfe punctatus. Elytra conuexa, punctato - ftriata, elytro fingulo ftriis octo apice coeuntibus, mucrone tereti terminatu. Abdomen nigrum. Plantae rufo - villofae. Nitens, apteius rudimentis tantum alarum. Hab. in ligno putrefcente minime rarus. Long. 5. lat. $2_{4}^{\frac{\pi}{4}}$.

$$
\text { (p. } 236 .)
$$

585. H. dentipes ater, elytris punctato - fulcatis, femoribus anticis acute fulcatis. F.Sp. 326. I 2. 
Caput et thorax atra, laenia, obfcura. Elytra fulcata, et in fingulo fulco linea punctorum imprefforum. Pedes nigri femoribús anticis fubtus carinatis, compreflis, et medio interiore acute dentatis, plantis fubhirfutis flauicantibus.

Petagna Sp. Inf. Cal. Nat. I29. f. I5. exhibet iconem ab eo affignatain Ten. Caraboidi, quae huic Helopi omnio conuenire videtur; fed antennae filiformes, palpi antici fecuriformes, plantae fubhirtae, flauicantes, tibiae anticae ante apicem non dentatae (character a Fabricio aflignatus T. Caraboidi) mea fpecimina huc referenda effe declarant. Hab. in truncis arborum cariofis praefertim Alni. Menf. Febr. faepe lectus. Long. 4. lat. $2 \frac{1}{3}$.

T. carabö̈dem $F$. Auctor nofter, vt fupra n. 579 . videre licet, ipfo ignaro, iam habet, hinc nil de identitate huius Helopis, imo, quoad ex figura Petagnae 1. c. indicarti poteft, nil fimilitudinis cum illo furpicari aliquis poffit.

Helopum fpecies, ob colorem femper fere atrum, e deferiptionibus folis diffculter extricantur, cum admodum paucis datum fit, differentiam ftaturae ac fculpturae, quae folae fpecies diuerfas (vti quidem in omnibus reliquis infectis, vbi tamen colores adiumentum haud leue praebent, ) diftinguunt, verbis fatis bene determinare. Fabricii H. dentipes ex ora Coromandeliana oriundus eft, contendere itaque audeo, eiusdem et Auctoris H. dentipedem dinerfas fpecies effe. Hg.

* 586. H. triftis niger, thorace fubquadrato, elytris obfolete punctato-ftriatis. 
Statura fere $H$. lamipedis, fed latior. Totus niger: glaber, fubnitidus, Antennae nigrae filiformes. Caput planum punctulatum clypeo antice emarginato, Thorax fubquadratus, conue. xu!s, lateribus angulatis. Elytra fere comnata, conuexa, obfolete punctato-ftriata. Plantae fubtus hirfutae rufae, Apterus, Tab. V. f, I, Occurrit etiam alius valde minor, qui tantum differre videtur elytris minus obfolete itriatis, Habit. in truncis cariofis frequens, Long. 6. lat, 2 :.

Specimen habeo, a Dn. de Paykull dono mihi miffum, quod eandem fere magnitudinem habet cum icone data. Thoracis forma quodammodo cum $H$. linipede conueniens eft, fed caetera fatis difcrepant. Tutum corpus nigrum eft et nitorem quemdam fericeum habet. Caput cum thorace punctis creberrimis excauatis notatum eft. Capitis clypeus (vel frons prolongata) emarginatus eft, vti et labium fuperius. Thorax in içone non bene expreffus eft, latitudine enim paullo longiorem, quam bretiorem dicerem, quod tamen in figura repraefentatum videmus. Puluinatus eft, feu conuexus, pro conuexitate vero fatis deprefo fus. Vndique margine laeui circumdatur, antice paullo anguftior eff, ibidemque fubemarginatus, vt anguli laterales, eodem modo, quo anguli bafeos, promineant, id, quod auctor latẹre angilata vocat, latera enim proprie fic dicta rotundata funt. Elytra thoracem longitudine, dimidia parte fuperant, vix autem latiora funt, et pone attenuantur. Ad latera inflectuntur, ef carinam lateralem prominentem efficiunt, $p t i$ in Pimeliis multis. Parum conuexa fint, ve potius deprefla dicantur, frriasque gerunt punçtakas longițdinales, quarum interualla punctis excauatis confer. T tiffimis 
tiffimis afperguntur, praecipue latera verfus, ad futuram enim haec puncta rariora, certe minora funt. Margo elytrorum, qui fupra confpici nequit, latus eft. Pectus, abdomen, pedes punctata funt. Tarfi pilis fuluis infra obducti.

$\mathrm{Ob}$ defcriptionem breuiorem decernere non audeo, num Fabricii $H$. Hottentotta E. S. I. I. I 22. 23 . idem fit cum modo defcripto, quod fufpicari facile vellem. Hg.

* 58 7. H. chalybaeus violaceus, thorace fubquadrato, elytris punctato-ftriatis.

Statura et magnitudo omnino $H$. ir iftis, fed clypeus et labium fuperius in hoc rotundatum, integrum; in illo emarginatum. Elytra quae in praecedente fere comnata funt, in hoc nullo modo: praetereaque color conftanter fipra violacens, fubtus niger, ftriae elytrorm magis puncratae magisque impreffae; thorax fubquadratus angulis anticis rotundatis margine magis eleuato hunc optime ab alio diftingumut. Hab. in Alno emortua rarior.

$$
\text { (p. } 237 .)
$$

588. H. quilquilius niger, antennis pedibusque ferrugineis. F. Sp. 326. I3.

I F. E. S. I. I. 122.26 .

Paruus; fubtus totus ferrngineus. Hab. in quifquiliiis.

* 589. H. ovatus fufco-niger, fubaeneus, thorace rotundato, elytris gibbis, punctato - ftriatis, ore antennis pedibusque rufis.

Corpus partum, globofum, onatum. Caput acneo-nigrum ore antemnisque rufis. Thorax aeneo-niger, laenis, nitidus, rotmndatus conue- 
xus, emarginatus, punctatus. Elytra nigroaenea, nitida, conuexa, punctato-ftriata. Abdomen, pectus, pedesque rufa. Tab. IV. f. r. Hab. in truncis arborum cariofis non rarus. Long. $2 \frac{\pi}{5}$. lat. $\frac{3}{3}$.

\section{MELOE.}

Palpi fubfiliformes, Maxilla recta, bifida. Labium corneum, emarginatum. Antennae moniliformes.

59o. MT. Profcarabacus corpore violaceo. F. Sp. $327 . \mathbf{I}$.

○ Lift., Mouff., Ionft., Clut., Frifch., Hoefn., Bergftr. . Sulz.

D Scop. E. C. 194. Schrank. E. I. 424.

\& F. E.S. I. I. 5 I . I.

Panz. F. I. G. X. I 2.

Meyeri Tentam. Monogr. Gen. Meloes. Goetting. I793. p. II. N. I.

Intermedius antennarum articulus maris dimidiatus. Tacta oleum limpidiffimum flanicans e geniculis pedum emittit. M. maialis numquam inuenta. M. Vralenfis Pall. Iter. 2. 722. 56. triplo minor, vix fpecie differt. Elytra in hac corpore longiora, magis laeuia, et punctis rarioribus afperfa. Hab. in locis apricis. Long. 7, ro. lat. $3,4 \frac{\mathrm{T}}{3}$.

De antennarum forma interdum fingulariter variante vide Celeb. Meyerum l. c. Citatum Geoffroyi I. 377. t. 7 . f. 4. celeb. Panzerus ad M. tectam meam (Fn. Inf. G. X. r 4.) referendum cenfet, et fi figuram refpiciamus, bene monitum videtur. Sed et magnitudo, et color caeruleus totus, in defcri- 
defcriptione commemoratus, fuadere videntur, id M. profcarabaco tamen annumerandum effe.

Panzerus primus fere in Fauna Inf. Germ. locum, Meloum fpecies viterius inquirendi aperuit, et $\mathrm{fpe}-$ cies ibi notificatae quibusdam infuper nouis algeri poffent. $\mathrm{Hg}$.

\section{(p. 238.)}

* 59 r. MT. Tuccir aptera, nigra, capite, thorace lobato, elytrisque cribri inftar impreflis.

\& Meyer. 1. c. p. 30. n. X.

Tota nigra, obfcura, violaceo colore minime nitens, corpore fubtus molli pilofo. Antennae nigrae breues, rectae, articulis omnibus (fecundo excepto) aequalibus, rotundis et magis approximatis quam in $M$. profcarabaeo. Elytra coriacea variolofa, pone dehifcentia, abbreuiata et abdomen cingentia, hoc eft, non eleuata vti in Profcarabaeo. Caput magnum punctatum. Thorax foueolis maioribus, rotundis, late disiunctis, irregulariter excauatus, et cute crafla duriore te. ctus vti in Profcarabaeo; fed haec omnia maiora et magis diftincta. Sulcus longitudinalis profunde exaratur; fpatiaque interiecta inter caput, et thoracem, thoracen et elytra, circum[cribunt thoracem quafi in duos lobos. Pedes apice quadrungulato, feu digito duplici bifido. Magnitudo $M$. Profcarabaei, at ftatura brenior et facies diuerfa. Grauida tamen admodum ventricofa euadit. An' femina M. Profcarabaei? Figura Ionftoni. Inf.p. 74. f. I 4. cum hac nullo modo connenit. Tab. IV. f. 5. Hab. in locis apricis filueftribus rariffima. Long. 8 , Io. lat. 4,5 . 
Celeberrimus Fabricius hancce Neloem auctoris noItri apud M. punctatsm fuam allegat. Quain Fabricii Meloem me nondum nouiffe, confiteor, fed auctoris M. punctatam meae pundiatae (Panż. F. I. G. X. ז6. M. Brmfuicen/. Meyer. 1. c. p. 25. n. VII.) affinem coniicio, inio eandem cum illa putarem, nifi thoracis defcriptio vetaret. $\mathrm{Hg}$.

\section{LXXXlli. I. I.YTTA.}

Palpi quatuor poftice clanati. Mluxillu bifida. Litbiun truncatum. Antennae filiformes.

592. L. veficatoria viridis, antemis nigris. F. Sp. 328. I.

$\odot$ Raj., Schaeff., Sulz., Mouff., Ionft.

D Schrank. E. I. 4 I8.

\& L. veficatoria ("L. rufficollis") F. E. S. I. 2. 83. I.

Variat admodum magnitudine ct colore nagis minusuc viridi-aurato. Hab. in Fraxino Orno. Long. 7. lat. 2.

In Ent. Syft. Fabr. id vitium calami, in citato fupra iam commemoratum, corrigatur. Hg.

593. L. erythrocpphala atra, capite teftaceo, thorace elytrisque cinereo - lineatis. F.Sp. 329.8 .

안. F. S. I. 2: 86. 13. Antennae nigrae. Caput tetaceum, fronte linea media nigra. Thorax niger fubrotundus, emarginatus filco dorfali, lateribusque cinereis. Elytra nigra margine cinereo. Subtus tota nigra albo tomento pubefcens, pedibus pofticis praelongis. Alac albae. Magnitudine admodum vaT. 3 riat, 
riat, fed plerumque aequat L. veficatoriam. Hab. in floribus Viciae fatiuae, aliisque non rara. Long. 5 , 7. lat. $\mathbf{I}, \mathbf{I} \frac{\mathbf{r}}{2}$.

Lyttam poffideo, quae erythrocephalae nomen fine meretur, cui tamen margo elytrorum thoracisque liturae cinereae umnino defunt, quam itaque nouam fpeciem interim habeo, donec reram L. erythrocephalam comparare liceat. Eadem cum $L$. dubia Fabricii E. S. I. 2. 86. I5. videtur, praecipue cum Fabricius Sulzeri Meloem Algiricun Hift. Inf. t. 7. f. I 2., qui infectum fuum ex Heluetia accepit, alleget, quae figura exacte cum Lytta mea quadrat. Idem de figuris Pallas Icon. Inf. t. E. f. 29. a. b. contendam, quamuis defcriptionem, quae ad hanc figuram pertinet, adhibere non licuit. $\mathrm{Hg}$.

$$
\text { (p.240.) }
$$

594. L. Afra nigra, thorace rufu. F. Sp. 330. 10.

Atra, capite thorace ano femoribusque rufis.

of Confer Mylabrem teftaceam Roffi Mant. 93. 209. Nitens, alata. Antennae, palpi, oculi, elytra, pectus, abdominis bafis, tarfique nigri coloris: reliqua rufa.

Mas Mylab. teftaceae. F. Sp. 33 г. 7. quae cum hac coniungenda, et a praecedente differt elytris teftaceis apice nigris, fed facies, et alia omnia finilia, nempe teftacea pectore, elytrorumque apicibus nigris. Antennac in vtroque fexu nigrae filiformes, palpi quatuor filiformes. Femina raro variat elytris immaculatis. Cum apud nos frequenter occurrant facpius apud nos copula iunEtas reperi, attamen quod nobis vifum, vt $a b$ 
omni erroris fufpicione vindicetur, feueriori ipfiusmet Cl. Fabricii examini atque iudicio lubentiflime fubiicimus. Tab. III. f. 3. mas. Tab. III. f. r. femina. Hab. in floribus. Long. 4. lat. $\mathbf{I}_{4}^{\frac{1}{4}}$.

Meum de his Lyttis aliquis fi requirat iudicium, auEtorem nec L. Afram, nec Mylabrems teftaceans habuiffe credo, ferl cum illius patria Africa, huius vero Sibiria a Fabricio vocaretur, hancce auctoris Lyttum peculiarem fpeciem habeo, quae cum illis modo commemoratis quibusdam non magis conueniat, quam forte $a b$ illis recedat, quod vero difcernere nequeo, cum et $L$. Afrace et $M$. teftaceac $F$. fpecimina milhi defint. I. Afroce Roff. illam varietatem, quam $M$. teflacean $F$. coniecerat, a Dn. Paykull mecum commúnicâtam, folam poffideo, et breuibus indicare volo.

Primo ftatim intuitu coniectura auctoris reiici, sidetur, qua $M$. teftacecam putat, cum nullam Mylabris notam liabeat. Infectum, ex Lyttarum more, anguftum eft et caeteros Lyttae characteres gerit. Attamen vix Lyttarum fpeciem credo. Infectum quafi medium inter Cantharides, Lyttas et Necydalum fpecies, e. g. N. vflulatim. Caput rubroluteum, palpis, oculis, antennisque nigris. Thorax, capitis latitudine, eiusdemque colore, fere quadratus et quafi puluinatus, in medio paullulum transuerfe impreffus, poftice marginatus. Scutellum magnum luteo-luridum. Elytra mollia, luteo-teftacea, futura apiceque late atra, qui color ater fenfim cum teftaceo coit. Alae fufcefcentes. Pectus antice teftaceum, pone nigrum. Abdomen elytris brenius, bafi nigrum, apice margineque teftaceum. Pedes rubello-luted tibiartim apice tarfisque fufco-atris. Magnitudinem L. atratae circiter aeq̨uat. Figura repraefentata ftaturam I.ytta

non 
non fatis bene exprimit, et in fpecimine meo nigredo apicis latior, etiam futuram et marginem externum inferiorem occupat. $\mathrm{Hg}$.

\section{MYLABRIS.}

Palpi filiformes. Maxilla conieà, compreffa, bifida. Lubium membranaceum, fubmargi-

- natum. Antennace moniliformes.

505. MI. Cichorei nigra, elytris flauis, fafciis tribus nigris. F. Sp. 330. 2.

$\odot$ Haffelq., Imperat., Linn. Muf.

D Meloe variabilis, Pallas Ic. Inf. Roff. 8r. 7. t. E. f. $7-14$.

M. fafciatius. Fuesl. Helv. 399.

Ad M. variabilem Pallas. I. c. quae varietas minor videtur. "M. Sichorei, noftra fpecimina referenda fint. Caput thorexque nigra, hirfuta, Elytra flaua; fafciis tribus nigris flexuofis, prima et fecunda interdum interrupta, reliqua ommia nigra. Hab. in Cichoriis et carduis praefertim Mariano, frequens menf. Iul. Long. $6 \frac{1}{2}$ lat. $1_{4}^{3}$.

Mylabrum fpecies dubio non leui iam dudum fubies Étae erant, et Pallas in Icon. Inf. primus aliquam fpecierum confufarum feparationem conatus eft. Signatułae fimiles multis Entomologis easdem fpecies confrituunt, nec patriam, nec magnitudinem, imo nec rationem partium inter fefe computantibus. Praecipue id in M. Cichorei patet. Infectum, quod Orienti indigenum eft, et quod Europaei magnitudinem ter fuperat, tamen idem iudicatur, quia eandem elytrorum lituram gerit.

Linnáei Meloem Cichorei, quem in Mut. Loud. Vlr. I03. defcriplit, noh noui, eiusdem enim elytrosum defcriptio nulli mihi noto conuenit. Meloe Cichorei 
Cichorei Sulz. Hift. Inf. abbr. t. ?. f. I r. a. (vbi duae fpecies, admodnm a $\mathrm{fe}$ inuicem differentes, mas feminaque eiusdem fpeciei temere iudicantur) idem eft cum $M$. phalerato. Pallas Ic. Inf. t. E. f. 3. b.; eiusdem vero f. II. ipfa, vel mas prio. ris ab eo putatus, idem cum $M$. luncto. Pallas. 1. c. t. E. f. 5. et Myl. Americana. Herbft. Arch. 65. t: 30 . f. 5. a.

Noftras Mylabris Cichorei a Schrankio Ent. Inf. 4 ro. optime defcripta, et a Schaeffero Ic. t. 15 r. f. 3 . depicta eft, et nullo modo cum illa confundi poteft. In Pallafii Iconib. nullam illi fimilem video, et cum auctor dicat, eiusdem $M$. Cichorii Pallafii M. variabili annumerandam efle, nil certi de eius Mylabri determinare pollum. Id addere placet, etiam a Pallafio in hac fpecie plures coniunctas efie, quas differre, mibi perfuafum eft, partium intes fefe portionem refpicienti. Hö.

$$
\text { (p. 24I.) }
$$

506. $M$. Algirica atra, elytris teftaceis immaculatis. F. Sp. 330.3 .

Tota atra, elytris folis tefaccis, immaculatis. Antennae moniliformes articulis cordiformibus. Caput et thorax nigra hirfuta. Elytra teftacea, " flexilia. Alae nigrae. Subtus etiam villolula. Hab. Pifis. Long. 7. lat. $\mathbf{I}_{\frac{\mathrm{t}}{2}}$

Aliud rurfus infectum, quod ob patriam a Fabricio commemoratam, defcriptionemque eius nimis breuem, admodum incertum eft. Cum rero id teneam, patriam fatis remotam infectorum etiam differentiae fpecificae fufpicionem iuftam praebere, de hac regula in hoc quoque infecto non recedam. Fabricius Indiam patriam vocat, nec tamen determinauit, vtrum orientalem, an vccidentalem fpeEaret. Herbitius Lyttac Indicte, quam Fabricius 
apud hanc allegat, patriam Americam vel Indian occidentalem exhibet. Linnaeus Algiriae eam indigenam appellat. Wulfenius, etiam citatus, in Capite bonde fpei infectum fuum reperit. Cyrilli Neapolit. r: t. 2. f. Io. ibidem a Fabricio allegatus, eandem certo Mylabrem cum auctore noftro habuit, quam a caeteris diuerfam habeo, donec Indicam et Italicam fimul videre, easque comparare liceat, vt re ipfa conuictus, fententiam retractem.

Italicae fpecimen mutilatum pofideo, ex Sardinia allatum, cui antenne defunt. Caput thorace latius. Thorax fupra planiufculus, antice leuiter transuerfe impreffus. Scutellum nigrum. Elytra rubro - teftacea, pone admodum flexilia, coftis, vt in congeneribus, eleuatis, longitudinaliter decurrentibus, quarum prima et fecunda, iuxta futuram, prae reliquis eminent. Alae colore nigrefcente funt. Abdomen comprefium, et fubtus veluti carinatum. $\mathrm{Hg}$.

\section{CEROCONA.}

Palpi aequales filiformes. Waxilla inferior linearis integra. Labium inferius membranaceum, bifidum. Antennae moniliformes, irregulares.

597. C. Schaefferi viridis, antennis, pedibusque luteis. F. Sp. 33I. I. Nas.

$\odot$ Sulz.

오 C. Schreberi. F. E. S. I. 2.82. 3 .

In mafculo tarfi tibiaeque anticae dilatatae; et in medio exterius late appendiculatae, ab aliis valde dilierfae: quod in fig. 9. t. VI. Geoffroy deeft, nec a Limnaev, Fabricio notatum, nequidem a Scopoli Del. Fl. et Fin. Inf. A. Mas etiam differt a fe- 
mina forma antennarim fingulari. In mafculo antennae irregulares rufae, pedes femper rufi. In femina antemnac clauatae nigrae, pedes femper nigri. Igni admota, aut folis radiis potelltioribus expofita diu refiftit, vitam non facile deferens. Hab. Pifis, loco vulgo dicto Cimipalto in Anthemi, et Paftinacae floribus menf.'Iun. et Iul. adeo copiofa, vt congiorum menfyra lcgi po-

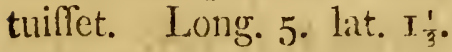

Aufurus quidem videor, auctorem infectum, quod tanta copia in illis regionibus exftat, et quod a multis fufe lateque defcriptum eft, cum alio confudifie, contendens. Sed qui defcriptionis notis attente comparauerit, mecum confentiet. Cerocoma vtraque, $C$. Schaefferi nempe et $L$. Schreberi, admodum fibi fimilis eft, et difliculter e defcriptionibus diftinguitur. In $C$. Sclveberi abdomen luteum eft, ano nigricante, in C. Schaefferi vero caeruleo-aut viridi-metallicum. Huius thorax magis rotundatus, prioris autem panllo longior, foueoliscue duabus, quae in antica thoracis parte confpiciuntur, minus infignibus, quam in $C$. Schaefferi. In mafculo $C$. Schatferi tibiae non dilatatae, vt in $L$. Schreberi, et feminae prioris antennis pedibusque luteis, pofterioris vero nigris vtuntur.

Inde facile apparet, vnde D. Roffius figuras et defcriptiones $\boldsymbol{C}$. Silveberi ab infecto fuo difcrepantes adnotat.

Fabricii defcriptio breuis atque diagnofis $C$. Vahlii exacte cum femina $C$. Schrebcri congruit; alias itaque et certas differentiale notas addere debuiffet, quia vere dinerfam credimus, cum patriam eius Barbarian vocet. $\mathrm{Hg}$. 


\section{(p. 242.) \\ LXXXVI. MORDELLA.}

Palpi quatuor, anteriores clauati, pofteriores filiformes. Maxilla bifida. Labium membranaceum, bifidm. Antennae moniliformes.

598. M. aculeata ano aculeato, corpore atro, immaculato. F. Sp. 333. ?.

$\odot$ Sulz.

D Scop. E. C. I 92. Schrank. E. I. $42 \%$.

\& F. E. S. I. 2. II3. I.

Habitat in floribus femiflofculofis et Vmbellatis. Long. 2. lat. $\frac{3}{3}$.

Mas duplo, interdum triplo minor femina. Citatum Schaeff. Ic. t. I27. f. 7. ab auctore et Fabricio allegatum, ad $I M$. friciatan omnino referendum eft. Celeb. Schneiderus in Citatis Fabr. c Schacff. Iconib. Futes! Mag. III. 2. p. 138. recenfitis: "Hoc, inquit, citatum, quamuis aculeus ani"depictus fit, tamen fafciatam fpectare videtur. - Harrerus cum Fabricio confentit." At in omnibus rpeciminibus meis M. fafciatıre aculeus ani, non fane minor, quam $M$. aculectucie, confpici poteft. Harrerus, vti e fynonymis et verbis defcriptioni adiectis patet, cum Linnaeo $M$. fnJciatam - $M$. aculeatue varietatem habet, et fic a Fabricio recedit. M. fascintia femper M. aculeata maior eft, et pro portione angultior ac longior. $\mathrm{Hg}$.

$$
\text { (p. } 243 \text {.) }
$$

599. M. fafciatr, ano aculeato, nigra, elytris fatciis diabus cinereis. F. Sp. 333.8.

D Schaef. Ic. t. $1 \geq 7$ f. $\%$.

T. F. E. S.I. 2. II3. 2.

Simillima praecedenti, cum qua etiam coniunxit Linnaeus, v. Fn. Sv. 832. Difiert tamen elytris bafi, 
bafi, fafciaque media, villofo-cinereis nitentibus. Hab. in floribus, faepe inuenta cum praecedente. Long. $2 \frac{r}{2}$. lat. 1 .

Confer adnotata ad priorem, vbi idem Schaefferi citatum iterum exhibitum eft. $\mathrm{Hg}$.

* 600. M. Sexpunctati ano aculeato atra, elytris punctis fex albidis.

Statura M. aculeatae, fed paullo maior. Antennae nigrae, dentatae. Palpi anteriores clauati claua oblique truncata. Caput et thorax nigra, laenia. Elytra punctis fex albo pilofis ad futuram pofitis, quorum antica minutiffina, media fubcontigua, poftica maiora. Subtus tota nigra abcominis lateribus ferico albo punctatis. Videtur varietas $M$. I2-punctatae. Hab. in pratis.

* 6or. M. i2 - punctata ano aculeato, nigra, capite, thorace, elytrisque albo-maculatis.

o $M$. perlata. Sulz. Hift. Inf. t. 7. f. $x_{4}$ ? Statura $M$. aculeatae fed duplo maior. Antennae ferratae nigrae. Caput deflexum nigrum albopubefcens fronte puncto impreffo. Thorax niger albo-marginatus, punctis binis albis. Elytra nigra albo-maculata punctis in fingulo fex, primo ad bafin fere lunato. Subtus tota nigra, abdominis lateribus albo-punctatis. Femora poftica dilatata comprefla. Anus valde aculeatus. Tab. IV. f. 4. Hab. ruri in Althaea. Long. 4. lat. $I_{4}$.

$$
\text { ( } p \text {. 244.) }
$$

602. M. flabellata antennis pectinatis, tefta cea, ore pectore abdominisque dorfo atris. F: App. ad Sp. 50 I. 
\& Ripipluorus flabellatus. F. E. S. I. 2. I I 6. Delicriptio F. iterum exhibetur. Hab. frequens in Origani floribus, in Paftinaca et Refeda. Long. 2. lat. $\frac{3}{4}$.

Caput cum thorace rutilum, thoracis bafi tefacea. Elytra teftacea. Alae fumatae. Pedes quatuor antici, et femora pofticorum nigra. Tibiae horum, fingulique tarforum articuli bafi teftacei, apice nigri funt. Meum fpecimen a capite dependente ad anum 3 lin. longum, medio vero vitra I lin. latum eft. Fabricius, quod etiam ab auctore iteratum eft, duplo minorem $M$. fervuginea dicit, in Ent. Syft. autem duplo maiorem. Comparationes cum infectis externis raro iuuant. $\mathrm{Hg}$.

603. M. paradoxa antemnis pectinatis, thoracis lateribus, elytrisque teftaceis. F. Sp. 332. 2.

of Ripiphorus paradoxus. F. E. S. I. 2. III. 5 . Mordella paradoxa. Frölich Naturf. XXVI. I64. 92.

Statura omnino IV. flabellatae, cui elytrorum colore etiam fimillima, at triplo aut quadruplo maior. Caput paruum, nigrum, fubpilofum, palpis anticis longioribus porrectis. Antennae longitudine thoracis vtrinque admodum pectinata, atrae. Thorax gibbus poftice trilobus lobis lateralibus teftaceis, dorfo lacuna profunda lata nigra, excauatus. Elytra teftacea, poftice attenuata, apice nigra. Pectus nigrum. Abdomen integrum teftaceum primo fegmento, ano, punctisque lateralibus nigris. Pedes nigri longiufculi. Alae fubhyalini flanefcentes. Hab. in plantis rariffima. Long. 4. lat. It. 


$$
\text { (p. =45.) }
$$

604. M. flau flaua, elytrorum apicibus nigris. F. Sp. 334. 14.

\& F.E. S. I. 2. II5. I3.

Panz. F. I. G. XIII. I 4 .

Caput, thorax, antemne et elytra flaua, folis elytrorum apicibus nigris. Abdomen totum atrum. Variat antennis nigris bafi tantum flaua. Hab. in Vinbellatis frequens. Long. $\mathbf{I}_{\mathbf{4}}$. lat. $\frac{1}{4}$.

Anus nunc aculeatus, aculeo longo, nunc fere inermis. Abdomen cum pectore nigrum, faepius autem capiti thoracique concolorum luteum eft. Interdum pectus folum nigrum, abdomen vero rubrum, apice nigricante. Elytra e luteo in nigricantem vergunt colorem, apice rariffime diftincte nigro. Vereor ne Cel. l'anzeri Mordella dorfalis Fn. Inf. Germ. XIII. I5 varietas tantum fit. Hg.

* 605. MI. bimaculata flaua, elytris macula media nigra, apice immaculato.

Praecedenti affinis et forte mera varietas: magnitudo ct ftatura eadem; difiert tantum elytris apice immaculato, et macula in medio nigra ub. foleta. Hab. cum priore.

\section{STAPHYLINVS.}

Palpi filiformes. Mlaxillu vnidentata. Labium membranaceum, trifidum. Antenuae moniliformes.

Staphylinorum magna copia exfat, tam magnorum, quam paruorum, et cum infuper colore ant notis aliis, vt in caeteris efle folet infeetis, parum difrincti fint, fpecies difficulter ermuntur. Haec difficultas effecit, vt in pluribus operibus fere omit- 
terentur, et in aliis leuiter, nec fufficienter defcriberentur. Cuae omnia Celeb. Paykullium, virum de Entomologia admodum meritum. atque hiftoriae naturali enixe deditum, perpulere, vt in Monographia, Staphylinis folis dicata, (Mon. Staphylinorum Sueciae a Guftauo de Paykull, Vpfaliae litt. I. Edmann. x.789. 8 mai.) omnes eorum (pecies, in Suecia repertas, enumeraret, et accurate defcriberet, in fummam omnium Entomologorum gratiam. Ibi, vti in reliquis Monographiis folet, omnia infecta comprehendit, quae Linnaeus genere Staphylinorum comprehendebat; ideo Staphylinos, Oxyporos, Paederos Fabricii coniunxit. Celeb. auftor haud fane absque bona ratione id fecit; fed cum praecipua ratio illa videtur. quo plura infecta vno nomine comprehendere poffet, talis diftributio in genera, vt opinor, operis pretium admodum auxiffet. Minime vero his modo dictis indicare vo1o, vt genera Fabriciana fupra commemorata ftricte feruaret, fed potius noua genera condere debuiffet. Illis enim paucis, quos celeberrimus Herbftius in genus optimum Plelaphus redegit, redemtis, ifta tria genera tamen nondum fufficiunt. In generibus his nouis conftituendis, ftatura quidem, vti femper, praecipue fpectanda eft, fed victum infuper confulendum puto, quia horum infectorum habitus et antennae, et quod per fe patet, oris partes pro victu diuerfi videntur.

Adiiciamus, quod faepius experti fumus, ftaphylinos plures aut illis affines morte abdomen contrahere, vt dimidio, imo triplo breuius fiat, quam viuentibus. Abdominis itaque portio in refpectu caeterarum partium, non femper characterem certum 'fuppetit, cui foli fides haberi poffit. Et caeteris defcriptionis notis congruenti, fola tantum abdominis longitudine non conueniente, tamen infe- 
Etum noftrum 'ftaphylinus: defcriptus effe poteit. $\mathrm{Hg}$.

606. St. politus niger, thorace, elytrisque nitidis..... Sp. 335.7 .

\& F. E. S. I. 2. $524 \cdot 20$.

Caput et thorax polita nitida. Thorax plerumque ater antice truncatius, poftice rotundatus. Elytra atra nitida thorace breuiora. Corpns clongatum. Pedes quandoque rufefcentes. Dificrentia fuecifica effentialis Limnaema confiftit in thorace decem punctis excauato vir absque lente confpiciendis, led haec puncta mumero faepe variant, vti etiam color elytrorum. Recens odorem Ambrofiacum fpargit. Hab. in terra. Long. 9. lat. $1 \frac{x}{3}$.

Fabricils, quem anctor nofter fequitur, Schaefferi Icon. t. 39. f. I2. allegat, quae figura autem caput thorace latius gerit. Hinc a Paykullio ad St. fimilem translata eft, vbi quoque Fabricius iterum citare voluit, quia ibideni Schaceff. Ic. t. zo. f. 12. legendum eft Schaeff. Ic. t. 39. f. 12. Fabricius forfan alium Staphylinum deferipfit. qram Linuaeus et Paykullius, cum St. nitidun illi fimillinum dicat, et cum colorem aeneum taceat. Ille enim, quem St. nitidum $F$. et Payk. habemus, faepius elytris nigris occurrit, quam varietatem St. politun $F$. et St. nigrmm nitidun, anterinis obsure-fufcis, abiomine elongato Degeerii I $T$. p. 22. n. $z$. credimus. Hinc patet, nos citatum Degeerii e citatis ad St. politum L. et Payk. allegatis, tollendum velle. Idem de Geoffroyano cenfemus. Praeterquam quod null:ım coloris aenei capitis, thoracis et elytrorum mentionem faciat, antennarum articulum fupremum fubglobofum, nec 
apice lunulato-emarginatum defcribit, qui accurate contemplatus, e defcriptione fe non praebet.

$\mathrm{Hg}$.

607. St. maxillofus pubefcens niger fafciis cinereis. F. Sp. 334.3 .

$\odot$ Raj., Lift., Ionft., Bocc, Geoffr.

\& F. E. S. T. 2. 52 . 9.

et St. olens. F. E. S. I. 2, 520. 6. (St. vnicolor. Herbft. Arch. p. I 49. n. 4.)

Videtur Fabricus fub nomine S. maxilloji conjumxille fpecies, quas Degeer et Geoffroy voluere diftinctas.

Adultior totus ater glaber encudit F. Cuin id afferat $C$. Fabricius, innixus etiam auctoritati Linriaci, in dubium reucare non licet: at conferendus tamen Degeer t. 4 p. 20. qui Linnaeum confutans, ab eo diffentit, et St. buitentum diuidit a faphylino toto atro: in hac difrinctione fecutus etiain a Geoffroy Inf. 1. 362.5. Apud nos quidem St. baltentus, feu St. fafcia transuerfali cinerea, ii non adultus, tamen iandiu declaratus, differt femper magnitudine minori tribus, vel quatıor lineis a St. toto atro, et forma capitis, ac antennarum etiam diuerfa. Colorem, praefertim fi a pubefcentia procedat, attritu et lumiditate variabilem effe, facile concedimus, fed forma et magnitudo poft declarationem párum fubiecta mutationi videtur. S. balteatus in Suecia frequens; alter a Degeer numquam inuentus. Ego quiden lunc labui faepius in aetate minime adulta femper totum atrum. *

Hab.

* Placet obferuationern noftram nunc confirmatam videre 2 
Hab. vterque in filuis, et hortis, etiam in fter. core bouino, rapina victitans. Long. 8 , Ir. lat. $2,2\}$.

Saepius a nobis iam adnotatum eft, auctorem ante fe diEta anxie fequi, et hic quoque locus dicta noftra probat. Ipfi enim de differentia St. maxillofi et olentis fatis perfuafum eft, tamen non audet, illos feparare, vt ex olente fpeciem peculiarem conftitueret, quóniam Linnaeus et Fabricius illos coniunxerant. Ifta verecundia mala ị auctoribus non raro occurrit. Viros magnos et celebres infallibiles credunt. Sẹd id vnicus Faunarum ac Monographiarum vfus eft, quod tales magnorum virorùm lapfus corrigant. Illós enin viros, rem yniuerfam comprehendentes, leue vitium facile fallit, ille vero, cuius oculis res exiguae magnitudinis fubiecta eft, nùmquạm fic falli debet.

In Entom. fyftem. Fabr. 1s. cts. citatum Geoffroy. Inf. 1. 360. 1. fub St. maxillofo deleatur, cum fub olente bene iam exhibitum fit. $\mathrm{Hg}$ :

$$
\text { (p. 247) }
$$

608. St. murinus pubefcens, cinereus, nigronebulofus. F. Sp. 334. 2.

- D Scop. E. C. 302. Schrank. E. I. 433.

I F. E. S. I. 2. 520.4 .

Apud nos faepe cinerafcens punctis nigris lucidis. Elytra longitudine thoracis fubtus caerulea. Hab, in fino frequens. Long. 5. lat. $I_{2}^{1}$.

Sçopoliị

Ct. Fabricio in Mantißa, adiunto nomine St. olentis. Mal ler. F. Frid. p. 23. N. 28 . Geoffr. Inf. 1. 360, 1. t. 7. fo 1. Rai. Inf. 109. 1. Adnotat. Auat ijifizs.

Inde magis fingulare nobis videtur, cur auktor illam $f_{\uparrow}$ parationem non faceret, cum, vt ipfe adnotat; celeberrimum Fabricium fequi potuiffet. Vid. fupra adiecta. Hs. 
Scopolii St. murinus tam obfcure defcriptus eft, vt melius praetermittatur. Degeerius fpecimen magnum defcripfit atque depinxit, quale raro occurrit, et quod Fabricius in Ent. fyft. 520. 3. nomine St. nebulofi diftinxifie videtur. Idem de Geoffroyi defuriptione conuenit.

St. murinzis a pubefcente difcrepat ftatpra anguftiore, antennis apicem verfus minus incrafintis, thorace pone magis anguftato, ac rotundato; angulis lateralibus futurne anterioris prominentibus acutis, elytris, refpectu thoracis, paullulum breuioribus, abdomine fubtus nigro, bafi tantum annulorum argenteo-pilufa. - Elytra in pube/cente quoque fubtus caerulea funt. Cornicula duo, quorum mentionem Linnaeus ac Degeerius fecere, in aliis etiam e. g. in St. olente vidi. $\mathrm{Hg}$.

609. St. erythropterus ater, elytris, antennarum bafi, pedibusque rufis. F. Sp. 335.5 .

$\odot$ Frifch., Stroem.

D Scop. E. C. 306 . Pod. m. g. p. 48. Schrank. E. I. 435 .

q F. E. S. I. 2. $522 . x 4$.

Antennae rufac articulis vltimis fufcis. Palpi rufi. Caput latitudine thoracis, nigrum margine poftico aureo lucido. Thorax antice fubtruncatus puncto vtrinque aureo, pone rotundatus. Scutellum atrum. Elytra teftaceo - rufa pilis concoloribus ad apicem praefertin ciliata. Abdomen nigrum punctis lateralibus tam fuperne, quam inferne aurea pube nitidis. Pedes rufi femoribus bafi nigris. Variat magnitudine minori, thorace, abdomineque nullibi aureo colore lucidis. Raro inuentus in filuis fub arborum truncis cariofis. Long. 8. lat. 2. 
Specimina habeo, quae abdomen ita contraxere, it vix elytrorum longitudinem fuperet. $\mathrm{Hg}$.

$$
\text { (p. }=48 .)
$$

6ro. St. fermigincus ater, nitens, capite thorace maiore, elytris ferrugineis.

Ater nitidifinuss. Caput magnum, exfertum, punctatum, poitice dilatatum fuperans longitudine et latitudine thoracem. Elytra brenia, fubpunctata, laete fernginca. Hab. in terra Florentiae. Long. 5 . lat. ${ }^{3}, 4$.

* 6r r. St. Vlimi ater, nitidas, elytris, abdo. minisque feginento penultimo rufis.

Antennae nigrae bafi ferrugineac. Palpi fubrufi. Thorax ater, laeuis, nitidus. Elytra ru$\mathrm{fa}$, punctulata, linea duplici punctorum longitudinalium. Abdomen atrum fegmento penultimo fere toto rufo, vltimoque corniculis duobus inftructo. Pedes nigri tibiis fufcis. - Tab. V. f. 6. Saepins inueni fub cortice Vlmi primo rere. Long. 5 . lat. 2.

*612. St. cumreus obfcure cupreus, antennis pedibusque fufcis.

Pracccdenti paullo maior. Antennae fufcae fibpilofue. Thorax laeuis elytro nitidior, fubcarimatus. Pedes fufci tibiis fpinulofis. Tab. VII. [. I3. - Hab. in arena. Long. $6 \frac{1}{2}$. lat. $I_{4}^{\frac{1}{4}}$

\section{(p. 249.)}

*613. St. apneus ater, aenens, nitidus, fcutello amplo, elytris aeneis.

St. niger, elytris nigro-aeneis. Geoffr. Inf. I. $367.1 \%$.

$$
\text { V } 3 \text { Sta- }
$$


Statura, et habitus $S$. politi. Antennae nigrae computata bafi articulis vindecim extremo lunulato. Caput, et thorax atra, laeuia nitidifima. Elytra laete aenea, fubpunctata thorace maiora bafi late in fenicirculum excauata pro receptione fcutelli concoloris. Abdomen'atrum àpice nonnihil pilcfo. Pedes nigri. Hab. in ftercore bouino: faepe inuentus menf. Febr. Long. 4,5 . lat. I.

* 6r 4. St. morfatans nigro - aeneis, capite, thorace, elytrisque junctatis, pedibus fuluis.

Diftincus a praecedenti colore magis fufco, et corpore magis elongato. Antennae fuccae bafi rufa, apice acuto, cinereo. Caput nigro-acneum, compreffum, punctatum, fubcarinatum, maxillis forcipatis magnis acutis. Palpi nigri. Thorax nigro - aeneus, punctatus, carinatus. Elytra thoraci concoloria, eodemque modo punctata. Abdomen nigrum. Pedes toti fului, tarfis anticis breuibus, dilatatis, pilofis. Reperi vagum in arena. Long. $6 \frac{\mathrm{r}}{4}$. lat. I.

\section{(p. 250.)}

* 6 I5. St. faxatitis niger, nitidus, fubpube-. fcens, elytris, pedibuts, abconinisque apice tefaceis. Schrank. E. I. 442.

St. niger nitens, pedibus, coleoptrisque teftaceis, elytris punctatis. Geoffr. InI. I. 365 . I I.

St. fuluoptesus. Fourcroy. Ent. Inf. Par. 16 7. I I. Niger. Caput et thorax nitida. Elytra caftanea. Abdominis apex, et pedes obficure teftacei, poftici vero nigri. Hab. in terra. Long. $3 \frac{1}{4}$. lat. $\frac{2}{3}$. 
6r6. St. chrifomelinns niger, thorace, elytris, pedibusque teitaceis. F. Sp. 337. 2 I.

\& Oxyportus cluryfonelinus. F. E. S. I. 2. 534. I5. Panz. F. I. G. IX. I 4.

Hab. in arena. Long. $\mathbf{I}_{4}^{\prime}$. lat. $\frac{2}{3}$.

Hoc infectum admudum variat, et Paykullio confentio, qui confitetur, fe, colore excepto, nullum characterem 'pecificum detegere poffe, quo varietates diftingueret, quarum illas, quas noui, breui profpectu lecturo fubiiciam

I. Thorace toto rubro

a. capite nigro

se. elyt ris rubicundis, bafi nigra: $O$. chryfonelinus $F$.

3. elytris atris, interdum extremo apice obfcure brunneis. Hanc fingularem varietatem a nullo obferuatam vidi: Paykullius certe eam nondum nouit.

b. capite rubro - abdomine rubro, apice nigro: Paykullii St. difparis varietas eft

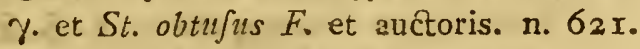
confer ibi adnotata.

2. Thorace nigro margine laterali rubicundo.

Eft Oxyporas brunneus. F. E.S I. 2. 535. I9. An St. littorcus. Payk. Mon. 80. 55? aut St. difparis var. ?. p. 79 ? - Elytra obfcure rutila, bafi obfcuriora, margine exteriore nigricante. $\mathrm{Hg}$.

* 617. St. fubzerraneus niger, elytris antice extrorfum flauis, pedibus nigris. Linn. Syft. Nat. 684. I2. Fn. Sv. 849.

o Oxyporus fubterroneus. F. E. S. I. 2. 535. Corpus atrum, nitidum. Antemnae filifornes, corpore dimidio breniores. Elytra truncata nigra a $\mathrm{V}_{4}$ medie- 
medietate anteriore, fed latere exteriore macula magna flauefcente. Thorax lacuis, conuexus. Abdomen glabrum, elytris duplo longius, apice fetis duabus paruis fubulatis. Long. $I_{\frac{2}{3}}^{2}$. lat: $\frac{1}{3}$.

6 Is. St. rufipes ater, glaber, pedibus rufis. F. Sp. 337. 22.

옿 Oxyporus rufipes. F. E. S. I. 2. 535. 2 r. Trtus ater, glaber, exceptis pedibus rufis. Colore variat. Hab. in plantis putridis. Long. $I \frac{1}{2}$ lat. $\frac{1}{2}$.

Citatum e Geoffroyo Inf. $367 . r_{5}$. delendum eft, hunc enim non defignat Oxyporum, vti e defcriptione fatis patet. Forte eiusdem St. niger elytris fufcis margine ficuso 368 . r9. huc fpectat? - In Entom fyftem. 1. c. Degeerii citatum a Fabricio omiffum eft.

Infeetum admodum variat, quod ad colorem attinet. Elytra nunc rufa, nunc caftanea, nunc obfcura margine externo et poftico rubicundis. Interdum minor, thoracis lateribus et bafi rubro marginatis occurrit, an O. marginellins. F. E. S. I. 2. 536. 23 ? - Hg.

$$
\text { (p. 25r.) }
$$

6r9. St. fufcipes ater, elytris pedibusque teftaceis. F. Sp. 336 . I5.

오․ E. S. I. $2.527 \cdot 38$.

$\mathrm{Hab}$. in fimo. Long. I⿳.

In his et aliis decernere non audeo, vtrum Auctor verum haberet Staphylinum, an non. $\mathrm{Hg}$.

620. St. bip!ffulatus niger, elytris puncto ferrugineo. F. Sp. $33^{6}$. 12.

\& F. E. S. I. 2. $526.34 \cdot$ Panz. F. I. G. XVI. 2 I. Antennae rufae in medio fufcae, fubclauatae, longitudine thoracis. Thorax ater, laeuiffimus, gibbus. 
gibbus. Elytra nigra rotundata, in medio finguli elytri ad bafin macula rufa. Abdomen breue, nigrum lateribus hifpidum ano acuminato, fubrufo. Lecti plures fub cortice Fraxini Orni. d. 27 . Febr. Long. $I_{\frac{3}{3}}$. lat. $\frac{1}{2}$.

621. St. obtufus teftaccus, elytris antice, ano. que nigris. F. Sp. 336. I I.

\section{\& Schrank. E I. 237. 45 r.}

Antennac teftaceae apice nigrae. Caput, et thorax teltacea. Oculi nigri. Elytra teftacea antice nigra. Abdomen nigrum annulo teftaceo ante apicem. Pedes teftacei. Hab. fub corticibus. Long. I 1 . lat. $\frac{1}{3}$.

Vide, quae fupra ad St. chrofomelinun adnotatimus. O. obtufus ibidem varictatem $\mathrm{I}$. b. conftituit. Fabricius et auctor nofter cum Paykullio St. outufum Limnciei allegant, qui tamen alium defcripfit, quamnis defcriptio eins nimis breuis fit. Fabricius St. obtufum fuum quidem ad St. chrysonelimum transtulit, fed, quoad e defcriptione iudicare pofium, O. cnalis E.S. I. 2. 533. 10. idem St. obtufus eft. Hg.

$$
\text { (p. =5:.) }
$$

622. St. piccus niger, thorace depreffo, lineis eleuatis tribus, elytris piceis. F. Sp. 338. 25. o F. E.S. $\dot{i} .2$. 530.55 .

Corpus nigrum magnitudine Pediculi, fed oblongius. Thorax fubrotundus planus, depreffus, in medio ftriis tribus. Elytra breuiffima, truncata, picca. Pedes pallidi. Hab. in ligno putrido. Long. I. lat. $\frac{\mathrm{T}}{5}$.

* 623. St. Buphthalmos niger, immaculatus oculis magnis exfertis. Schrank. E. I. 445.

V 5 \& St. 
오. St. cluicornis. Roffi Mant. 98. 220.

F. E. S. I. 2. $52 \% \cdot 37$.

Ater, oblongus, oculis magnis prominulis. Antennate peciesque flaui. Thorax elongatus, antice attenuatus, capite angufior.: Elytra pulchre punctata lougitudine thoracis. Magnitudo minoris Formicae. Hab. fub arborum cortice frequens. Long. 2. lat. $\frac{1}{4}$

Tres fpecies omnino diftinctae $a b$ auktoribus confunduntur, quas diftinctas, et fynonymis fuis inftruCas exhibeo.

I. St. clauiconis. F. E. S. I. 2. 527.37. antennis flauis, primo articulo et clana atris, thorace fere orbiculato, elytris immaculatis, fatura breuiore, colore obfcuriore, quam biguttati, pedibus nigris. St. buphthalmos. Schrank. E. I. 445 .

2. St. biguttatus. F. et L. anguftior, thorace quodammodo fufformi: medio incraffato, elytris longioribus, puncto medio flauo, antennis pedibusque nigris, colore magis aeneo.

Panz. F. I. G. XI. I\%.

St. Iuno ß. Payk. Moin 33: 25.

3. St. Cicindeloides. Schaller. A E. Hallenf. I. 324 . St. finilis. Herblt. Arch. 25 I. I5.

St. buphthalmos. auctoris noftri?

Omnium breuifimus, thorace fubglobofo, antennis flanis, apice obfcuriore, pedibus flauis, geniculis nigris. Color niger, nitidus.

St. claticornem n. T. numquam vidi pedibus flauis inftructum, et, cum auctor pedes Staphylini fui eos defcripfit, $S_{t}$. buphthalmum eius, eundem cum n. 3. St. cicindelnide Schall. coniicio. Hg. 


\section{OXYPORVS.}

Palpi inaequales, anteriures filiformes, pofteriores fecuriformes. Labiun emarginatum cum mucrone. Antennae moniliformes.

624. O. rufus rufus, capite elytrorum abdominisque pofticis nigris. F. Sp. 33 S. I.

$\odot$ Gad., Schàeff. Ic. t. 35, f. 3 .

D Scop. E. C. 307. Sclirank. E. I. 438.

Schaeff. Ic. t. 85 . f. 3 .

옥. E. S. I. A. 53 I. I.

Panz F. I. G. XVI. Ig.

Antennae breues, fere clauatae, rufae, apice nigro. Palpi rufi, pofteriores fecuriformes lunati. Hab. in Boleto, et plantis putrefcentibus. Long. 3. lat. $\mathrm{t}$.

Celeberrimus Fabricius in Ent. Fyft. 53ix. 2. alinm exhibet, fimillimum, cuius ftatura minime differt, quem olim varietatem O. mufi putauere. Caput huius et thorax niger, elytra liuida: angulo extetiore pofteriore nigro, abdomine nunc rufo, sunc prope anum nigro, nunc toto nigro, pedibus linidis, nonnumquam bafi nigricantibus. Variat raro capite thoraceque rufis, temere nigro variegatis. O. rufus interdum thotace obfure rufo, nigromaculato, elytrorumque bafi hon, vt folet, rufa, fed liuida obuian fit. Anceps fane infectum eft, attamen aliquando St. maxillofí decem fpecimina in eodem loco reperi.

o. rufus, et, fi diftinctus eft, O. maxillofus a caeteris omnino feparandi videntur. Habitus eorum genus peculiare porcit, quod forfan Oxyporis exoticis augeri poffet, in noftratibus enim, quod fciam, nullum fibi fimilem habent. Hg. 


\section{(p. 253.)}

- 625. O. bicolor niger, elytris, pedibus, palpis, antennarum bafi, et apice pallide-rufis.

Caput paruum nigrum. Palpi pallide-rufi. Antennae fubpilofae, in medio nigrae, bafi et apice flauefcentes, articulo vltimo maximo onato. Thorax ater, laeuiflimus. Elytra rufi. Abdomen nigrum, apice rufo - villofo. Pedes pallide - rufi. Hab. in Fraxino Orno fub cortice. Long. $3 \frac{1}{4}$. lat. \%. vix.

Forte idem cum St. merdario $F$ ? aut St. ancti $\beta$. Paykull.? Hg.

\section{PAEDERVS.}

Palpi inaequales, anteriores clauati, pofteriores filiformes. Labium lineare integrum. Antennae moniliformes.

626. P. riparius rufus, elytris cacruleis, - capite abdominisque apice nigris. F. Sp. 339. I.

D Schrank. E. I. 44 T.

S.E.S.I. 2.536. I.

Panz. F. I. G. IX. Ix.

Hab. in terra ct in plantis obuius. Long. 3 . lat. $\frac{\mathrm{I}}{\mathrm{r}}$.

Schaeff. Ic. t. 7 s. f. 3. apud hunc allegari folet, quamuis peffima fit. Celeb. Patykuilius ad St. collarem fuum transfert, et quidem, vti apparet, optime. Sed ex iconibus Schaefferi nil certi determinari poteft, et cum Harrerus hanc figuram in $P$. ripario alleget, res magis anceps fit. $\mathrm{Hg}$.

* 627. P. impreffus pallide - fufcus, thoracis dorfo concauo canaliculato. 
온 Staphylinus canaliculatus. Roffi Mant. 98. 219 . F. E. S. I. 2.523 .42 .

Antennae nigrae, longiufculae craffae, bafi ferrugineae. Caput et abdomen nigra. Thorax, elytra, pedes pallide - fufca. Thoracis dorfum concanum fulco medio longitudinali, Inuenti duo in ligno putrido. .. Long. 2. lat. $\frac{1}{4}$.
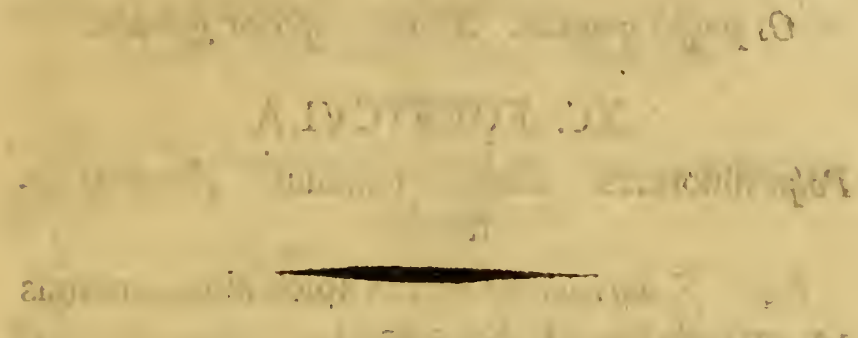


\section{(p.25.5.)}

\section{CLASSIS SECVNDA. VLONATA.}

Os palpis quatuor. Maxilla inferior galeata.

\section{FORFICVLA.}

Palpi filiformes. Labium trifidum. Antennae fetaceae.

628. F. auricularia elytris apice albis, antennis 14 - articulatis. F. Sp. 340. I.

(7) Mouff, , Petiv., Lift., Frifch., Ionft., Merian., Sulz.

D Schrank. E. I. 455 .

o F. E. S. II. T. I.

Hab. in plantis vbique notifimum animal. Long. 5, 6, 7. lat, I, 2 .

Saepius fub cortice arborum antiquarum magna copia reperitur. Prima iuuentute fere totus albus eft. Specimen habeo, cuitus forceps intra vltimum abdominis fegmentum ita retractum eft, vt apices tantum promineant, qui fere recti funt, In eodem fpecimine id fegmentum minus angulatum eft, quam folet. Aliud fpecimen mihi eft, e cuius ore vermis inteftinalis Afcaridum generis pendet, longa, teres, glabra, alba, admodum inuoluta. $\mathrm{Hg}$. 629. F. minor elytris teftaceis immaculatis, antenuis 10-articulatis, F. Sp. 340.3.

D) Schrank. E. I: 456 .

Q F. E. S. II. 3. \% 
Antennac compntata bafi articulis indecim, apice albicante. Hab. in plantis rarior. Long. $2 \frac{x}{2}$. lat. $\frac{\pi}{2}$

\section{(p. 256.) \\ XCI. BLATTA.}

Palpi filiformes. Mlaxilla inferior apice fiffa. Labium: meinbranaceum bifidum, laciniis fiffis, pinna exteriore maiori. Antennae

fetaceac.

630. B. Americana ferruginea, thoracis clypeo poftice exalbido. F. Sp. 342. 5 .

$\therefore$ B. Aurelianenfis. Fourcroy. Ent. Par. 2..2.

Q.F. E. S. II. 7.6 .

Frons imprefla punctis duobus albis. $\mathrm{Hab}$. in of ficina piltoria non rara. " Long. I 9. lat. 6 .

63 I. $B$. orientalis ferrugine -fufa immacula. ta, elytris fulco oblongo. F. Sp. 343. I4.

○ Mouff., Rai,, Column., Ionft, , Frifch., Sulz.

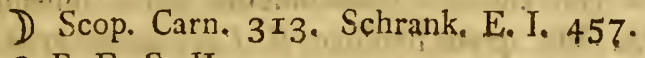

\& F. E. S. II. 9: I7:

Statim ac declarata alba, mox fit rufa, demum ferruginco - fufca euadit. Pellitur odore Cupreffi. Hab. paffim in hypocauftis. Long. 9. lat. $4 \frac{x}{2}$.

\section{(p. 257.)}

632. B. Lappionica flauefcens, elytris nigromaculatis. F. Sp. 344. 16.

$\odot$ Sulz.

D Scop. E. C. $3 \times 4$. B. filueftris.

Schrank. E. I. 458.

\& F. E.'S. II. TO. 2 r.

Variat thorace toto flauefcente, et difca tantum nigro. Subtus tota nigra. Antennae modo nigrae, 
grae, modo flauae, vti pedes. Elytra in mafculis abdomine nulto latiora. Hab. folitaria: apud nos frequens in fepibus. Long. $3 \frac{x}{2}, 4 \frac{1}{2}$. lat. 2 ; $2 \frac{1}{2}$.

Blattae duae exftant, quarum aitera $B$. Lapponiclz efr, 1ti in Schaefferi Icon. t. 88. f. 2. 3. et Sulz. Hitt. Inf. t. 8. f. 3. depicta eft, coleoptris tho race quinquies longioribus, abdomine angufto; sltera eandem cum illa latitudinem.habet, vix tamen dimidian eius longitudinem fuperat. . Thorace maicre, elytris vix anguftiore: difco luteo, margine diaphano; antennis longioribus fubfufcis, pedibusque pellucidis thauis praedita eft. Prioris. fjecimina omnia, quae vidi, difcum thoracis nigrum, antennas pedesque nigricantes habent. Thorax multo minor eft. - A Alteram pofteriorem a celeb. Herbftio nomine Blattac perfpiciliaris (Arch. 186. 12. t. 49. f. Ir.) diftinctam video, et B. Germanicum Panz. F. I. G. II. I7. illi ad. modum affinem habeo. Vnum vero fpecimei prioris vidi, thoracis difco obfcure luteo, tibiisque pallidis. Illa, quam feminam $B$. Lapponicae credo, fimilis quidem $B$. perfpicillari eft, 'fed thoracis difcum nigrum, elytraque abdomine breuiora habet, quae in perfpicillari abdomine paullo longiora funt.

Fabricius in Spec. 1. c.: "variat, inquit, rarius corpore multo breuiori et thorace toto flauefcente." Vereor, ne B. perfpicillarem Herbft. illis verbis Spectet. In Ent. fyft. id annotatum omifit, et fub n. 23. Blattam linidam habet, quam fere eandem cum $B$. perfpicillari puto. Sub eo Geoffr. Inf. I. 38 r. 3. allegat, qui tamen veram B. lapponicam defcripfit, quamuis vtramque fpeciem mifcuiffe videtur. Anne ab auctore adnotata varietas eadeut 
eadem fit, difcernere nequeo; nullam enim faturae breuis mentionem fecit, quae tamen adeo infignis eft. Caeterum confilium Entomologorum de his iamiam commemoratis fpeciebus rogo, et interea diuerfas habere liceat, quod tam graues rationes pofcere videntur.

Schaefferus t. I 58. f. 2. aliam Blattam repraefentauit, quam Harrerus in Defcript. Schaeff. Icon. ed. prior. p. 255 . n. 4 I9. varietatem 'B. Lapponicae habet." Equidem Blattan ibi depictam veram B. "maculatam. F. E. S. II. II. 26. credo. " $B$. hemipteran F. E. S. II. I2. 30 . ex fpecimine Mufei mei-cum defcriptione congruente, $B$. $m a$ culatce feni. varietatem habed. A qua B. lapponicae femina differt elytris eodem modo, vt in mafculo ; firriatis nec laeuibus. " $\mathrm{Hg}$.'

633: B. merginata nigra, thorace rufo albomarginato, elytris nigris limbo albo. F. Sp. 502. 17 . 18. App.

와 F. E. S. II. Ir. 27 .

Raro inueni.

\section{..tif i (p.258) \\ XCII. MANTIS,}

Palpi fubaequales. Maxilla inferior compreffa acutifima. Labium bifidum laciniis fiflis, pinnis aequalibus. Antennae fetaceae.

634. $M$. religiofa thorace laeui fubcarinato, elytrisque viridibus, immaculatis. F. Sp. 348 . I $9 .[\beta]$

○ Roef. Inf. 2. Gryll. t. x. 2. Sulz., Seeligm., Seb.

D Mantes Geoffr, Inf. x. 399. I. t. 8. f. 4 . $\mathrm{X}$ Roef. 
Roef. t. 4. t. I2. f. 8.

\&. M.oratoric $\beta$. F. E. S. II. 20. 3I. $\beta$.

Noftraifpecimina habent ad bafin femorum anticorum maculam magnam nigrafi, quae in medio alba eft; aliamque minoren trapetziformem fupra bafin femorum pofticorum, ab elytris occultatam. Variat admodum niagnitudine et colore. Fateor me non intelligere, quia de cauffa Geoffioy apponat pro fynonymo $M$. Religiofae definitionem Linnaeanam M. Gongylodis, et Fourcroy in Entom. Parif nominet pro religiofa Gongylodern, quam Geoffroyus non defcripfit. Hab. frequens in aruis et hortis. Long. 29, 30. lat: 4,6 .

- M. religiofan L Fabricius $M$. oratorice varietatem habet, fed prorfus diftinguendae. funt. Geoffroyus $\mathrm{ab}$ auctore bene ad M. veligiofam relatus eft. Roefelii t. $x, 2$. Fabricius et ad orcitoricm, et ad religiofam refert, fed t. I. f. 2 : et t.: 2 . f. 6 . (fic legendum eft) ad religiofam pertirent, cun tabula 12. tom. 4. ab auctore exhibita. Quomodo vero citata: Degeer. Inf. 3. 4 I O. 5. t. 37. f. 2. Seba Thefaur. 4. t. 67. f. 9, 10. ad $M$. oratoriam $L$. a Fabr. transferri poffint, non video. Macula in bafi femorum anticorum in meo quoque fpecimine adeft, et apud Roefel. t. 4. t. 12. in fig. 8. et Io. videri poteft.-

Quod ad Geoffroyun attinet, infeeta fua optime defcripfit, fpecies bene diftinxit, et in genera redegit, fed in fynonymia faepius fallebatur, quod vtique in eius fcriptis apparet. Fourcroyus in Entom. Parif. caeteros Entomologos non curauit, nomina noua infectis dedit, vbi placuit, aut falfa appofuit, quod minus confilio feciffe, quam quod nomina vera ignoraffe videtur. Miror itaque, auEtorem nutec demum hoc mirari. $\mathrm{H}_{\mathrm{S}}$ 
635. $M$. pauperata thorace lineari fpinulofo, femoribus anticis fpina terminatis, reliquis lobo. F. Sp. 346. 9 .

D Thunberg. N. S. 36 I. Drury. Inf. t. 50. f. 2. varietas?

\& F. E. S. II. I\%. I8, et confer. I8. 25, M. petti. nicornem.

Defcribit F. l. c. Corpus viridi alboque varium, abdominis margine in medio, et vtrinque eleuato lobato.

Noftra fpecimina interdum variant antennis pectinatis cornu fere triplo longioribus, et omnino conueniunt cum Drury. Inf. 1. t. 56. f. 2. a Fabricio citata, fed nullo modo cum figuris Roefel. Inf. 2. Gryll. t. 7. quae exhibent tibias lóbo bafeos multo maiori. Defcriptio accuratiffimá Gronouii. Zooph. 639. indicat adeffe plerumque in alis M. Gongylodis maculam ferrugineam, ' quae in nuftris deeft, et nihil ait de pedun colore pallido, viridique vario, a Fabricio in $M$. pouperata adnotato; ad hanc igitur fueciem referenda effe videtur haec noftra: fed ftatuendùm etiam, quod variat antennis pectinatis, "vti figura a Drury allata indicat.

Inuenta Pifis in horto Academico in Lathyro heterophyllo d. I5. Iul. Vt retro afpiciat, eleganter collum reflećtit. Manu apprehenfa exhibens ei flores Lathyri tamquan cicur pluries comedit. Vixit paucos dies. Mox faepe lecta etiam larua et pupa Flórentiae. Long. a capitis vertice ad elytr. apicem 32 . lat. 4 .

Auktoris verba, obfcura, fic intelligenda funt: Fabricius ad $M$. Gongylodem fuarn et Roefel. 2. Gryll.

$\mathrm{X} 2$

t. $z$. 
t. 7., et Drury. Inf. 2. t. 50. f. 2. refert. Roefelii icon cum infecto auctoris nullo modo, Drurii vero optime conuenit; cum itaque Fabricii quoque defcriptio $M$. Gongylodis non in omnibus congruat, Dirurium ad $M$." prinperntam translocat Roffus, quia infectum eius cum deferiptione huius Mantis et fimul cum icone Drurii optime conuenit. $\mathrm{Hg}$.

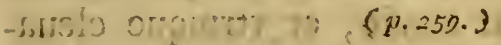

*636. MI. Roffra baculiformes, aptera. pirpo Roff Maint. 102. 225:

F. E. S. II. I3. 4.

Mágnitudo iuxta aetatem diuerfa. Tota dilıte viridis, glabra, corpore admodum tenu, et praelongo; paullulum compreffo, baculi inftar vibique aequali, et caudan verfis anmlis dífincto. Caput deflexum, teres, poftice anguftatum, thoraci connatum. Antennae bremes, cylindraceae, Ocelli exigui, prominuli fuaerici. Os tentaculis quatuor fubaequalibus, labio inferiore bifido. Tibiac et femora longifima, introrfum denticulata, quorum antica crafiora, prope caput inhaerent. Alae mullae, Tarfi quisque. Tab. VIII. $f$. I.

Inuenitur figura et hiftoria a me exhibita etiam in Actis focipt. Itaticre tom. IV. et in lonfono, qui figurain folummodo habet t. XI. f. 2. et t. XII. f. 26 :

Habitat in fepibus virentibus ad foflas aquarum frequeitior fed et in ericetis, et hortis quoque inuenta.

- Hic non diffunilandum, fed ingenue fatendum eft hose infectum iamdiu quodammodo notum $\because . J$

S. 25 Kir - 
Kirkero, Ionftono, Stenoni, Rhedio fuiffe. Neminem ante me rebar de eo praecipuc verba feciffe; eaque in opinione confirmatus fireram a celeberrimis Entomologis, praefertim a Scopoli. Tractu temporis forte mihi contigit, vt oculos coniicerem in exemplar quoddam Rhedionum a meo, aliisque non paucis diuerfum, nempe magis integrum, nec fraudatum tabula vltima, quae huins inlecti iconem exhibet, licet in aliarum numerum non referatur. Sane difficillimum, ac pene impoffibile eft, absque figurae infpectione, non dicam e verbis colligeie, fed ne fufpicari quidem de fpecie infeeti ab co per iocum, vt ait, et in animi remiffione obfertati. Verum nifi placuit cognofcere me deffitutum primaria laude, placuit tamen, et id ine folatur, quod auctoritate Rhedii, ac Ster:onis confirmatum deprehendo huinsmodi infectum reucra effe perfectum animal, non laruam, vti vulgo afferitur, et vti videre eft in Experincratis Rhedianis circa Infectorun generationen jagr. 88. Edit. Florentiae. 1\%67. Quid vero habeat de hoc Infecto Kïrkerus in libro XII. Mnundi fubferranci pag. 37 . recreandi animi gratia non omittendum. "Nirum et hoc addan ex Liburni, quam Vitem albam rocant, ramulis faepe faepius me inneniłe Zoophytum fex pedibus, et finbtilifimo corpore conftans cum capite Encae progreffiuo moru Arancarnm ad inftar incedens, et cum diligentius originem huius infecti fcrutarer, inueni tandem id ex dicti Liburni fruticis ramulis putredine quadan infectis'natum: fiquidem non femel deprehendid illud ramo adhuc viridi adnatum, vnde

$$
\text { X } 3
$$

non 
non immerito Xylophytum dixi: Inueni et nonnulla, quae ex pofteriore parte lignum, ex anteriori vero iam animam obtinuerant, quae hinc inde agitabatur: Vidi et quaedam quae intermedio corpore quod pedes fuftinebant lignum, prorfus idem cum ramulo Liburni erat, pedes vero et caput iam animata mouebantur. Statim tamen ac tota ramuli medulla cum exfertis fex ramulis anima informabatur, tum etiam veluti a trunco recifum animal humi reliquorum infectorum है $\pi \circ \delta \omega v$ more gradiebatur, quam ftupendam metamorphofin, dum compluribus oftenderem ex.noftris, vix dici poteft, quantopere adeo rarum, et monftruofum partum mirati fint, praefertim cum anteriorem partem vna cum pedibus inotum affectantem pofteriori ftipiti adhuc annexa viderent." Figura habet hunc titulum: "Xylophyton ex ramulis Libumi in Mufeo Auctoris." - Long. $\max$ 42. lat. 2.

\section{(p.26x)}

637. M. pagana alis reticulatis, albis; macula laterali ferruginea, manibus chelatis. F. Mant. 230. 37 .

D Mantis pufill r. Schrank. E. I. 460.

Pallas. fpicil. Fafcic. 9. I5. t. r.

An $M$. brenticornis Degeer. tom. VIr. 620. IS. t. 46 . f. 9. Io?

\& F. E. S. II. $24 \cdot 49$.

Facies et habitus Mantis. Oculi magni prominuli, viridi-aurati. Os palpis quatuor. Antennae filiformes, thorace breuiores, ferrugineac. Thurax valde elongatus cylindricus, flauefcens. Abdomen oblongum, ouatum, albidum, dorfo et lateri- 
bus fuluo - maculatis. Pedes antici tibiis exterius decoloribus; intus fufcis; latis, craffis, dentatis, denticulo vltimo pone bafin longiore, et vinco ad apicem armati. Alae omnes venis tenuiffimis nigris reticulatae, hyalinae, inacula marginali oblonga ferruginea. Lecta in Fraxino Orino d. 25. Imn., dein ctiam in Rubo, et facpius in Ziziplio. Long. ?. lat. I.

\section{(p. 262.) \\ XCIII. ACRYDIVM.}

Palpi filiformes. Labium cylindricun apice fiffum. Antennate filiformes.

638. A. bipunctatum thoracis fcutello abdominis longitudine. F. Sp. 35 I. I.

$\odot$ Rai., Lepech., Sulz.

ᄋ F. E. S. II. 26. 2. Panz. F. I. G. V. I8.

Habitat in pratis et circa hortos frequens. Long. $3 \frac{1}{2}$. lat. $\mathrm{I} \frac{1}{3}$.

Breue et craffum, fcutelli thoracis carina admodum cleuata. Interdum carina rutila eft, et in vtroque thoracis latere fignatura nigra $S$ fere referente, vt \$2 fiat, confpicitur. $\mathrm{Hg}$.

639. A. futinlatumn thoracis fcutello abdomine longiore. F. Sp. 35I. 2.

() Sulz.

I) Goetze. E. B. 2.478 .

운 F. E. S. II. 263 .

Praecedenti nimis affinis, et forte mera cius varictas. Hab. in locis herbofis frequens ad foffas et aquas ftagnaites. Long. 5 . lat. $\mathbf{I} \frac{\mathrm{T}}{2}$. 
Priore longius ac gracilius et carina in medio thoracis vix eleuata. Femora quoque pedum pofticorum minus valida funt.

Plures forte fpecies $a b$ auetoribus confunduntur. Nobis certe perfuafum eft, fola obferuata ratione longitudinis fcutelli, fpecies diftingui non pofle. $\mathrm{Hg}$.

\section{(p. 263.$)$}

XCIV. TRVXALIS.

Palpi inaequales filiformes. Galea truncata fornicata. Labium inferius bifidum. Anteninae enfiformes.

640. T. nafutus capite conico, corpore viridi. F. Sp. $35^{2}: \mathrm{I}$.

$\odot$ Linn. Muf., Sulz., Drury, Seb.

641. B. Gryllus turritus Linn. Syft. Nat. 42\%. Ed. X.

$\odot$ Seb.

\& F. E. S. II. 26. I.

Singulare infectum capite conico, thorace iplo longiore, apice antennis, bafi ore inftructo. Anternae, in multis, quos vidi et habui, plenmque grifeae, modo fufcae, in prouectioribus fibrufae, femper latae, enfiformes, triquetrae, articulatae. Caput antice flauefcens, interdum nigro - punctatum, maxillis nigris, oculis teftaceis oblongis. Thorax breuis, fubcarinatus, margineque laterali eleuato. Elytra linea alba interrupta. Abdomen dorfo purpurafcente albo punctato. Femora poftica apice fpinis duabus. Magnitudo admodum variat. 
$T$. turritus differre videtur a $T$. nafuto colore elytrorum, qui in nomm!lis iuxta actatem,- aut albidus cofta nigra albo-interrupte lineata; aut fufcus colta baleos viridi; ant viridis elytrorum cofta, thoracisque margine laterali rufefcentibus. - T. conicur anne varietas praecedentium?

Hab. vterque frequens in filnis Pifanis, et alibi menf. Aitg. et Sept. Long. a cap. vertice ad apicem elytri 28 . lat. $\max .2$.

\section{(p. $=64$. \\ XCV. ACHETA.}

Palpi inaequales, filiformes. Labium qnadrifidum. Antenuce fetaceae.

642. A. Gryllotalpa alis caudatis elytro longioribus, pedibus anticis palmatis. F. Sp. 353. I.

$\odot$ Rai., Ionft., Catesb., Frifch., Sulz., Barth., Séb.

I Schrank. E. I. 463. Goed. Inf. I. pl. 76.

S. F. E. S. II. 28. I.

Hab. humum recentem horticolis infefta. Long. I8. lat. 4.

- 643. A. domeftica alis caudatis elytro longioribus corpore glanco. F. Sp. 353. 3 .

$\odot$ Rai., Mlouff., Iqnft., Hoefn., Seb.

오.F. E. S. II. 29.3 .

Infectum plerisque notum, cuius accuratifimam hiftoriam dedit Degeer. 3.509. r. Hofpitatur in muris domefticis. In ftatı adulto perfecto non frequens. Mihi raro datum eft colligere declaratum in immenfa laruarum et puparum copia, quae in

$$
\mathrm{X}_{5}
$$

loco 
loco calido humenti, horto meo annexo, continuo obuerfabatur. Long. 8. lat. $\mathrm{I}_{\frac{2}{3}}$.

\section{(p. 265.)}

644. A. campeftris alis elytro breuioribus, corpore nigro, ttilo lineari. F. Sp. 355. Io.

$\odot$ Rai., Frifch., Seb.

q F. E. S. II. 3 I. II.

Coniunxit Geoffroyus hanc cum $A$. domeftica, at vix vllo iure. Hab. in aritis et pratis frequens. Long. II. lat. 4 .

645. A. Italica capite thoraceque flauefcentibus, elytriśs aqueis longitudine alae. F. Sp. 355 . I2.

오․ F. E. S. II. 32. 16 .

Defcribit eam F. l.c. Hab. ruri. Long. 6.

\section{(p. 266.) \\ XCVI. LOC VSTA.}

Palpi inaequales obtufi. Maxilla apice tridentata. Labium bifidum, laciniis interne feta fubulata inftructis. Antennate fetaceae.

646. L. viridiffema alis viridibus immaculatis, antennis longiffimis.

- $\odot$ Lirt., Robert., Frifch., Schaeff. Ic. t. I39. f. 3. Goed., Zinann.

D) Schrank. E. I. 466.

P. F. E. S. 11. 41. 32 .

$\mathrm{Hab}$. in campis et hortis frequens, noctu ftridens. Long. 22. lat. 3 .

Harrerus in libro cit. infuper Schaeff. Ic. t. 9I. f. 6. allegat, varietatem coloris flauefcentis, cui libenter affentio; fed etiam t. 247. f. 4. 5. citat, quam vix eandem cum viridiffina credere poffum. 
Eodem modo fub Gryllo biguttulo innuneras varietates comprehendit, quarum plures fpecies peculiares videntur. $\mathrm{Hg}$.

647. L. verrucinorc alis viridibus fufco-maculatis, antennis longitudine corporis. F. Sp. 359. 24.

$\odot$ Geoffr., Rai., Frifch., Ionft., Merian., Sulz.

D Schrank. E. I. 468.

\& F. E. S. II. 42.33 .

Rultici Sueciac huic Locuftae verrucas manus admonent, quas praemordet, et enomit in vuluns liquorem, vnde rerruca contabelcit. Linn. Narrat Degeer. vnam habuille, cuius e corpore tres prodiernint vermes Gordio aquatico fimiles. Infeltatur A. Gymopterorum alis plerumque inhacrente. Hab. in pratis filuaticis, apud nos copiofifima. Long. 18. lat. $4 \frac{1}{6}$.

Geoffroyus confilio omifius, et L. falcatae n. $65^{\circ}$. annumeratus eft. $\mathrm{Hg}$.

$$
\text { (p. 267.) }
$$

648. L. varia thorace viridi-flauo, lineato, fronte acuminata, antennis longiffmis. F. Sp.360. 25.

$\odot$ Sulz.

\& F. E. S. II. 42.35 .

Defcripta eft a Fabricio 1. c. Habit. in hortis. Long. 7 . lat. $I \frac{1}{4}$.

* 649. L. perforata viridis, aptera, capite poftice nigro, thorace fellae ad inftar concano.

\& L. ephippiger F. E. S. II. 44. 42 . Haec, quam olim admiratione quadam nuncupstam vidi nomine $G$. pedeftris $E$. et quam dubitaui efie 
efle poffe L. grifeo-ayteran Degeer. Tnf. 3. 436. 3. ni fallor, forte eft L. Ephippiger F. Mant. 235. 33 ?

Color aetate admodum variat: nunc viridis, nuic flauefcens. Antennae corpore fefquilongiores. Oculi rotmodi prominuli. Caput laene teres occipite nigro absque ocellis. Thorax coriaceus fcaber, valde excauatus, et fellae ad inftar concauts, rudimentis alarum fub futo thoracis poftice dilatato, porrecto reconditis, et vix in dorfo apparentibus cartilagincis, rotundis, conuexis, punctatis margine crafliori, et tenui quafi vitrea membrana ad lonitum edendum intructis, quorum vnum fere alteri fuperincumbit. Corpus viride, (in cadatere fufcum) glabrum, obefum. Cauda feminae enfifera falcata afcendens abdomine longior. Inter primum ac fecuncum par pedum anticorum retringue ad thoracis margines, foramen nuagnum onale caunm in. vtroque fexu, criius vius mihi non fatis bene adhuc cognitus: praetercaque puncta. tria minutifima impreffa, fub thorace inter fingulum par pedum. Femora pofica aliquantulun incraflata, vnde faltus grauis. In hac fpecie femina etiam fridule cantillat, quod mihi noum, mutuo attritu duarum cartilagimum. Cun frequens fit apud nos fupra Filices in pratis filuaticis, non femel datum mihi videre copula inctam cum mafcilo ei fimillino: et oua matura faepe a me in abdomine feminae inuenta animal perfectum declarant. Tab. VIII. f. 3. 4. - Long. comput. enfe feminae 24. a capite vero ad anum Is. lat. $4 \frac{\mathrm{T}}{2}$. 


$$
(p .268 .)
$$

* 65o, A. falcuta.

Gryllus canda enffifera recurtata. Iinn. Fn. Sv. 2283.

Gryllus falcatus. Pod: M. p. 52.

Locufte cauda eniffera curua. Geoffr:Inf. I. 397. It Gryjlits falcatus. Scop. E. C. 32z? Schrank. E: I: 467 . Schaeff. Ic. t. 138 . f. 1. 2. mas. f. 3 . fcm,

Aittennae longifimae. Elytrum viride alis brenius. Abdomen femincum têrminátuni enile furfim inflexo ferrigineo quadinplici. Hab. Hin hortis frequens. Long. Ir. lat. 2."

Citatum e Geofiroyo, Fabricius ad L. verrucinorm refert. Hg.

$$
\text { (p. 269.) }
$$

* 65 r. L. tuberculata thorace planiufculo, vertice tuberculato, laeni, elytris elongatis, angnftis, antemis teftaceis longitudine elytronn. Degeer. In 3 . 444.5 . t. 38 . f. $\mathrm{x}$.

of Rolli Mant. IO3. $\approx 27$.

Flano - viridis. Antennae, pédesque poftici elytro aequales. Caput vertice laeniter tuberculato. Thórax planiufculus. Elytra angufta, elongata apice obtufo. In feminis cauda enfiféra recta vbique aequalis decem fere lineas longa. Mihi vifae tantum duae feminae. Hab. in pratis apricis filuaticis. Long. '9. Iat. 2. 


\section{XCVII.' GRYLLVS.}

Labium bifidum, ouatum laciniis fornicatis. Antennae filiformes.

652. G. Lineola thorace fubcarinato fufco, $\mathrm{li}$ nea dorfali rufa, femoribus pofticis intus fanguineis, tibiis caeruleis. F. Sp. 365. 20.

o. F. E. S.II. 54.29.

Fabricii optima defcriptio exaćte conuenit cum noftris. Forte G. migratorius Scop. communicatus a R. P. Wulfen huc referendus? Habit. frequentillimus apud nos, et hibernat in hortis. Long. 24,32 . lat. 4,5 .

$$
\text { (p. 270.) }
$$

653. G. flauns thorace carinato, alis flauis, fafcia nigra, apice cinereis, F. Sp. 369. 40.

오 F. E. S. II. $59 \cdot 53$.

A Fabricio optime defcriptus eft: Apud nos coTor capitis et elytrorum variat, tibiaeque pofticae rubrae. Degeerius Inf. 3. 493. 9. an huc pertinet? vix credo. - Hab. fteriles colles frequens. Long. I 5. lat. 4 .

654. G. thalafjenus thorace laeui, viridi, alis hyalinis, latere tenuiori viridibus, apice fufcis. F. Sp. $367 \cdot 31$.

\& F. E. S. II. $57 \cdot 43$.

Defcripfit illum Fabricius. Variat magnitudine. Hab. in pratis. Long. I 7. lat. 2.

655. G. Italicus fufcus, thorace carinato, alis rubris apice hyalinis. F. Sp. $367 \cdot 29$.

$\odot$ Linn.: Muf.

D) Schrank. E. I. 472 . 
\& F. E. S. II. 5: 4 .

Alae bafi rubrae. Fennora poftica lata craffa nigra transuerfin ftriata, tibiis rubris, bafi tantum liigro-punctatis. Hab. in montuofis. Long. i 3. lat. 2 .

$$
(p, z>1)
$$

656. G. carulefieis thorace fubcarinato, alis virefcenti-caerulcis fafcia nigra. F. Sp. 369. 37.

○-Linn. Muf., Frifch., Sulz., Seb., Rai.

D Schrank. E. I. $47^{\circ}$.

i F. E. S. 11. $59 \cdot 5^{\circ}$.

Hab. frequens in locis faxofis apricis. Long. I2. lat. $2 \frac{2}{3}$.

657. G. caemulans thorace laeuiufculo, elytris pallidis, nigro-maculatis, alis latere tenuiori caerulefcentibus. F. Sp. $368 \cdot 33$.

\& F. E. S. II. $58 \cdot 45$.

Corpus medium, trifte. Elytra hyalina reticulata, afperfa confufe maculis nigris. Alae colore caeruleo absque fafcia nigra ad latus tenuius. Hab. in locis laxofis. Long. I 1 . lat. 2.

658. G. viridulus thorace cruciato, corpore fupra viridi, elytrorum margine albido. F. Sp. 370.44 .

$\odot$ Linn. It., Frifch.

D Schrank. E. I. 473 .

\& F. E. S. II. 6I. 59 .

Hab. in aruis, Long. 9. lat. $1 \frac{\mathrm{T}}{2}$.

$$
\text { (p. 2z2.) }
$$

659. G. rufus fufcus abdomine rufo, antennis fubclauatis. F. Sp. 3 ? r. 48.

D Schrank. 
D Schrank. E. I. 474.

3. F. E. S. IJ. 62.63.

Hab. in pratis frequens. Long. I0. lat. $I \frac{2}{3}$.

Gryllos eorumque affines absque vllis fere annotationibus et obferuationibus adiectis praetermifi, quamuis multa ibi monenda effent; cum, quia mufeum meum huius ordinis infectorum pauca tantum comprehendit, quem defectum correcturus - fum, tum, 'quia fallax ac periculofum eft, ex iconibus, aut vel defcriptionibus folis iudicandi.

Hg.

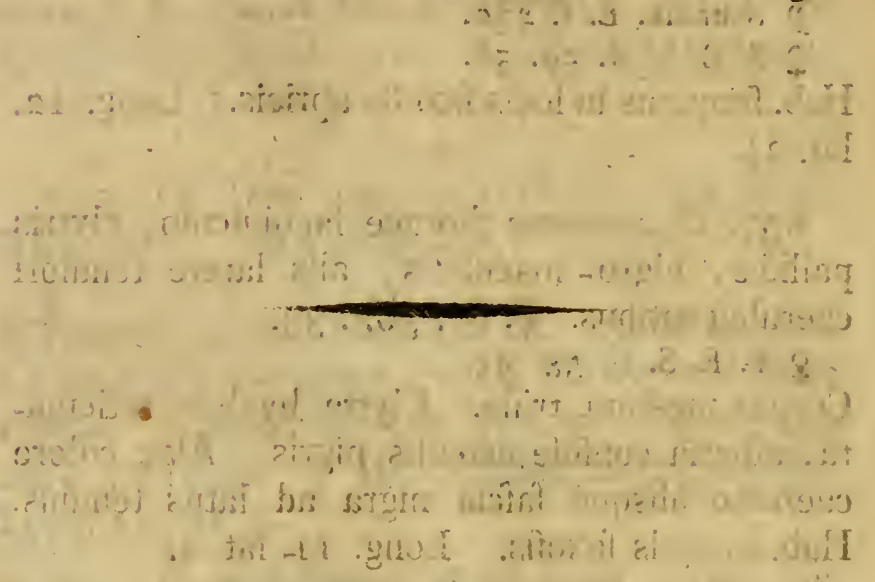

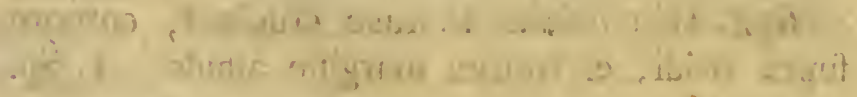




\section{MANTISSA INSECTORVM}

EX HIBENS

SPECIES NVPER IN ETRVRIA COLLECTAS

A

PETRO ROS SIO.

ADIECTIS FAVNAE ETRVSCAE ILLVSTRATIONIBVS AC EMENDATIONIBVS.

PAR S PRIOR

INSECTA EX ORDINE ELEVTHERATORVM ET VLONATORVM C O II L ECTENS.

$\therefore$ COMPLECTEN




\section{LECTORI.}

En tibi, ENTOMOPHILE, infectorum fpecies a me nuper in Etruria collectas, quas oro excipias animo ipfo indulgenti quo fuperiores, labores complexus es. Illuftrationes ac emendationes fpecierum in Fauna Etrufca editarum addidi, easque partim occafione data, fuo loco, partim in Operis calce, quemadmodum et Icones praeftantiora infecta exhibentes ibidem indicatas inuenies. Vale.

Dabam Pifis. Prid. Kal. Mart. I792.

AVCTOR. 


\section{CLASSIS PRIMA \\ ELEVTHERATA.}

\section{(p.5.) \\ I. LVCANVS.}

I. L? virefcens viridi - aeneus, maxillis tridentatis. F. Sylt. App. 8 I 7 .

D Trogofita virefcens. Oliu. Ent. n. I9. 8. t. I. f. 5 . Maior L. Caraboüde, et oblongior. Antennae aeneo-nigrae, capite longiores, vndecim-articulatae, articulis tribus vltimis extrorfum craffioribus. Maxillae craflae, prominentes, capite breuiores, tridentatae. Caput viridi-aeneum punctulatum depreffum, antice canaliculatum clypeo apice obtufe quadridentato. Thorax viridi-aeneus depreffus, poftice rotundatus. Scutellum inconficuum. Elytra viridi - aenea punctatofubftriata. Subtus omnino concolor. Lectus in filuis Pifanis. Long. 7. lat. 2.

$T$. vire $\int e n t e m ~ F$. non allego, quia Americae indigena eft, et cum e defcriptione Fabricii, ob breuitatem, nil determinari poffit, $T$. vivefcentem Auct. et $\mathrm{Fabr}$. diuerfas habere liceat, quod praecipue in illis fpeciebus, quae verbis aegre diftinguuntur, fuadendum eft. De Oliuierii Trogofita nihil decernere pofium, quia eiusdem opus adire nondum mihi licuit. Hg.

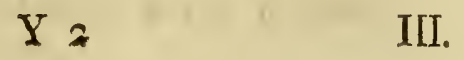




\section{SCARABAEVS.}

2. S. punctulatus fcutellatus, muticus, mandibulis laciniatis, clypeo triangulari, thorace punCtato, elytris fublineatis, punctisque maiusculis inordinate excauatis. Fn. Etr. I. 9. Ig. t. $x$. f. I.

$\odot$ Sc. punctulatus. Villers. Ent. I. 40. 88. t. I. f. 3 .

o Sc. Talpa Panz. Beytr. t. 6. f. x. 2 .

Sc. punfatus F. E. S. I. x. 2 I. 64. mas.

Sc. Monodoin. F. E. S. I. I. 20. 5\%. femina.

Statura et magnitudo feminae Sc. Sileni. Elytra late lineata, lineis laenibus, vix eleuatis, obliquis, conuergentibus. Femora poftica crafra. Niger immaculatus. Magnit. variat. Hab. filuas et hortos frequentifimus.

In Fn. Etr. 1. c. tum dubitabam, an Sc. punctulatus Auctoris, Sc. Monodon' F. et Idiota Herbft. effet, nunc vero nullum dubium reftat, et fignum interrogationis, illis citatis adieftum, deleatur. $\mathrm{Hg}$.

\section{(p. 6.)}

3. Sc. Foffor fcutellatus, thorace inermi retufo, capite tuberculis tribus, medio fubcomuto. F. Sp. $15 \cdot 59$.

(- Act. Nidrof. Voet.

D Villers. Ent. $x$. I . 15 .

q F. E. S. I. I. $23 \cdot 72$.

Habitat in montuofis. Long. 4. lat. 2?.

Fabricius in Sp. Inf. lapfu, calami habet Schaeff. Ic. t. r 44, f. 78. quod poftea bene ita emendauit, vt fieret, f. 7.8 . Auctor nofter habet f. $z$ ? nobis vero nullum dubium de vtraque figura reftat. Id quidem confitendum eft, f. 8., quae varietatem elytris caftaneis, repraefentat, brevio- 
rem effe, quam f. 7., fed in Schacferi figuris, effigies ftriče fequi non oportet. $\mathrm{Hg}$.

4. Sc. Fordidus fcutellatus, capite tuberculato, thorace nigro, margine pallido puncto nigro, elytris grifeis. F. Sp. $x \approx .68$.

o F. E. S. I. I. 29.90.

A Fabricio in Syft. Ent. I6. 55. defcriptus. eft. Hab. cum congeneribus. Long. $2 \frac{1}{2}$. lat. $1 \frac{x}{3}$.

*5. Sc. rubidis niger abdomine elytrisque ferrugincis, thorace antice vix excauato, capite tuberculis tribus. Olin. Ent. 3. 81. 77. t. 26 . f. 224 .

o Si. Scrutator F. E. S. I. I. $24 \cdot 73$. Similis $S_{c}$. funeturio, at triplo maior. Caput nigrum antice fubemarginatum, lateribus angulatum, fupra tuberculis tribus, intermedio clenato acuto. Thorax inermis, punctatus, antice vix excauatus, niger, lateribus fermgincis. Scutellum nigrum. Elytra ferruginea frriata. Corpus fubtus, pedesque nigra, tarfis abdomineque obfcure ferrugineis. Oliz. - Hab. Florentiae.

Fabricius 1. c. dicit: ftatura et fumma affinitas Sc. finctarii et celeb. Herbftius Sc. Jordidum vix ab eo diftinguere audet, fed triplo maior eft, et eaden cum Sc. Foffore magnitudine gandet, a quo ftatura longiore et anguftiore differt. Elytrorum dorfum depreffum eft, qua re infuper a Sc. fimbtario difcrepat. $\mathrm{Hg}$.

$$
\text { (p. z.) }
$$

6. Sc. irroratus fcutellatus, thorace rugofo foffula vtrinque impreffa, capite fubcomuto, elytris rugofo - fulcatis nigris, rufo - fubmaculatis. Fin. Etr. I. 7. I6.

$$
\text { Y } 3 \quad \text { Clypeus }
$$


Clypeus marginatus, carinatus, et in medio corniculo breuiffimo armatus. Antennae nigrae. Thorax fubrugofus margine crenulato. Scutellum paruum. Elytra antice coarctata, late rugolofulcata, maculis, feu punctis plurimis obfoletis rufis, vix diftinguendis afperfa. Femora antica duobus denticulis interne infructa. Tibiae anticae quadridentatae. Hab. Florentiae et Pifis ra. rus. Long. 8. lat, 4.

* 7. Sc. furcifer fcutellatus, muticus, niger, elytris rugofis, femoribus anticis lamina et dente valido inftructis, pectore bifurcato.

Statura et magnitudo Sc. irrorati, Totus niger obfcurus, Clypeus marginatus, carinatus, corniculo feu potius lineola vix apparente. Thorax magnus, circularis, conuexus, fubrịgofus foffula laterali. Elytra fubrugofa ad latera angutata. Scutellum paruum fere nullum. Tibiae anticae longae, arcuatae, quadridentatae, absque tarfis. Femora antica intus, et inferne appendice, feu lamina cuneiformi porrecta, vtrinque dentata; denteque altero acuto fuperne inftructa. Pectus fpinis duabus validis inftar furcae armatum. Cum a praecedente differat tantum pectore bifurcato, et lamina cuneiformi femorum anticorum, alterum effe fexum afferere poffe videtur. Raro lectus. Long. 8. lat. 4 .

$$
\text { (p. 8.) }
$$

8. Sc.rufipes fcutellatus, muticus, ater, thorace glabro nitido, elytris ftriatis, F. Sp. 20.84.

D Geoffr. Inf, I, 83. 21. Schrank. E. I. 30. Habitat in ftercore, infrequens, Long. 4. lat. 2. 
Fabricii Sc. rufipedem non allegamus, quia AuEtor Sc. oblongm Scop. et Herblt. K. t. I 8. f. 2., qui verus rufipes $F$. eft, non habuifle videtur. Menfura enim potius $S_{c}$. rufipeden Linn. vel $S_{c}$. nigripedem F. E. S. I. I. 35. 14. defignat, et. Geofr. citatum huic annumerandum eft. $\mathrm{Hg}$.

9. Si. plagiatus fcutellatus, muticus, niger, elytris plaga rufefcente. F.Sp, 2 I. 87.

D Schrank. E, 1. 28 .

Affinis $S_{c}$. jubterraneo, et forte mera varietas, In fingulo elytio macula oblonga: $\{$ rufefcens iuxta fituram, Hab. in ftercore bouino. Long, 2。 lat. $\mathbf{~}$.

Sc. fubterranens interdum variat elytrorum macula longitudinali obfolete rubra, et hanc varietatem

- Auctor habuifie videtur. Verus enim plagiatus Sc. fubterraneo triplo minor', et granario paullo longior eft, reliquas fi taceamus differentiae notas. Schrankii $S_{c}$. feneftralis. E. I. 28. ab Auctore citatus, huc non fpectat, vt e defcriptione eius fatis apparet. $\mathrm{Hg}$.

* 10, Sc. fufcus fcutellatus fupra fufcus, elytris tomentofis ftriatis.

Paullo minor $S c$. quisquilio, et totus fupra obfcure fufcus, pube fubferruginea; fubtus niger nitidus. Clypeus rotundatus, marginatus, tuberculo folitario vix apparente. Antennac capitulo trifido. Tibiae anticae tridentatae. Elytra fubtiliffime, atque obfolete punctato-ftriata. Tarfi fubrufi. Hab. fub corticibus.

I I. Sc. lunaris exfcutellatus, thorace tricomi intermedio obtufo bifido, capitis cormu erecto, clypeo emarginato, F. Sp. 24. 108. mas.

$$
\mathrm{Y} 4
$$


Copris capitis clypeo lunulato, margine elenato, corniculo denticulato Geoffr. Inf. I. 88: I. femina. Hunc Cl. D. Olinier fpeciem diuerfam effe a praecedente credit, et diftinguit fub nomine Sc. emarginati. t.8. f. 64. a. b. Mihi tamen liceat, venia Celebris Viri, in priftina manere fententia, donec res aliter commonfret ac vifa pluries. Confer Fn. Etr. I. I I. 24.

Iunior variat colore caftaneo. Hab. vterque fimul in ftercore bouino pafcuorum.

Celeberrimus Fabricius Oliuierinm in diftinguenda illa fexus varietate fecutus eft, vti in Fn. Etr. 1. c. videre eft. Mea experientia me cum Auctore confentire iubet. $\mathrm{Hg}$.

\section{IV. '(p.p.) $\mathrm{TROX}$.}

12. T. niger niger, thorace marginato inaequali, elytris punctato - ftriatis, lineis eleuatis fpinofis.

Infectum idem quod dedimus in Fin. Etrufca. I ?. 39. fub dubio nomine $T$. Luridi qui diuerfus.

Corpus totum obfcure nigrum. Thorax marginatus, inaequalis, villo breui rufefcente ciliatus, late dorfo fulcatus, at haud fpinofus. Elytra marginata, punctato - ftriata, lineis tantum. quinque in fingulo elytro clenato-fpinofis. Videtur nouus. Hab. Florentiac.

\section{MELOLONTHA.}

I3. $M$. ruficornis villofa teftacea abdomine albicante. F. Sp. $37 \cdot 8$. 
ㅇ F. E. S. I. 2. I59. I9.

A Fabricio defcripta eft. Variare faepe videtur magnitudine et colore obfcuriore. Hab. in plantis rarior.

AuEtor nofter, Fabricium fecutus, Voet. t. 6. f. 50. allegat, fed in hac figura bonam $M$. villofae $\mathrm{F}$. E. S. $5_{56.4}$. effigiem agnofcimus. Herbftii itaque figura, quae Voetiana initata videtur, ad $M$. villofan $F$. referatur. IV. villofal Herbft. t. 22 . f. 8. enim $M$. pilofa F. E. S. I. 2. I56. 5. eft. Ex icone fi iudicemus, M. Anketeri Herbft. K: t. 22. f. 4. M. villofae $F$. admodum afinis videtur, et cum noftris fpeciminibus exacte conuenit. $\mathrm{Hg}$.

14. M. abdominalis capite thoraceque caeruleopilofis, elytris teltaceis, abdomine albo-villofo. F. Sp. App. 496.

\& F. E. S. I. 2. x I I. 69 .

Paullo minor $M$. horticola, et thorax rotundatus. Species diftincta. Hab. Pifis rarior. Long. 4. lat. $2 \frac{T}{2}$. vix.

I5. MI. argentea clypeo marginato nigra, fubtus argenteo - nitens, elytris teftaceis. F. Sp. 44. $48 ?$
( ) Voet.
D Sc. farinofus. Villers. Ent. I. 30. 46.
\&. M. Squamofa F. E. S. I. 2. I74. 78 .

Statura et magnitudo fere $S$. Squrmo/i. Vill. Thorax et elytra fquammis, feu polline luteo - virefcente tecta, nonnihil abbreuiata. Abdomen fupra nigrum, fubtus viridi - argenteum. Tibiae anticae bidentatae. Pedes plerumque teftacei, fed interdum nigri. Variat magnitudine duplo

$$
\text { Y } 5 \text { minori, }
$$


minori, vnde forfan in hac fpecie determinanda orta confufio. Tibiae anticaebidentatae, et dorfum fub elytris nignum vifae funt notae conftantes. Varietas minor forte eft alter fexus. Hab. in plantis et floribus. Long. 4. lat. 2. et vltra.

- In hac et fequentibus confer, quae in Fn. Etr. 2 I. 48. adnotata funt. Hg.

16. M. fquamofa,

$$
\text { (p. II.) }
$$

Scarab. Squamofus fcutellatus, violaceus, fquammis fubtus argenteis, fupra violaceis, tibiis anticis tridentatis. Villers. Ent. I. 30. 47.

M. farinofa. F, Mant. 23. 60.

\& $M$. farinofa. F. E. S. I, 2. I73. 77 .

M. caevuleq. Herbft. K, III, I2I, t. 25. f. 5 .

Statura praecedentis. Color elytrorum laete violaceus, fplendidus. Abdomen fubtus argenteum, fupra lutefcens. Tibiae anticae tridentatae. Tarf nigri. Long.

Fabricius M. farinofan Linn, ad Forinofam fuam refert, fed nullo inre. Herbftius $M$. graminicolam $F$. $M$. farinofom Linn, credit, et omnia optime congruerent, nifi Linnaeus eam $M$. horticolae magnitudinem fere aequantem diceret. $\mathrm{Li}$ benter itaque $M$. argenteanz $F_{0}$, quae Sueciae potius indigena videtur, quam $\int q u a m o \int a F_{\text {, }}$, ean- dem cum farinofa Linn, habeo. Hg.

* I 7. M. dubia nigra, cinereo hirta, corpore virefcenti-argenteo, elytris teftaceis.

Defcriptio $M$. puluerulentae $F$, huic nequaquam competere videtur. Statura praecedentium, fed duplo minor. Caput, et thorax nigra, fquammis viridi - argenteis vix afperfa, et pilis denfis cinereis 
longioribus hirta. Elytra teftacea, abdomine breuiora, pilofa, Corpus fubtus viridi - argenteum pilofum, fupra nignum. Tibiae anticae bidentatae. Pedes nigri, Obf. In hac antennac et palpi omnino nigri; in praecedentibus rufi, antemaeque ad bafin rufae. Hab. in plantis.

\section{HISTER,}

I8. H. maior ater, elytris fubftriatis, thoracis marginibus ciliatis. F. Sylt. 52. I.

Simillimus $H$. vnicolori, at duplo maior, et thorax pilis ferrugineis denfis ciliatus, $\mathrm{F}$.

Gaudeo hanc fpeciem indigenam effe etiam Etruriae, fic enim magis magisque afferere polfum quod manifefte differt ab H. laeuo Fn. Etr. I. 28.63. forma corporis magis fubquadrata, minusque deprefla; capite minus exferto, imo fere toto in thoracem retracto, labio fuperiore, feu clypei apice emarginato, excanato, neque elongato vti in $H$. lacuo, mandibulis diuerfis, et aequalibus; claua antemarum fubrufa, thorace antice, et lateribus pilis ferrugineis denfe ciliato, absque foueola vtrinque imprefla; fterno antice acuto, aliisque notis, quas referre fuperuacuum. Hab. in ftercore bouino rarus.

Fabricii $H$. maior Africanus eft, ideo dubitare licet, an Auctoriș Hifter idem fit, idque eo magis in ge-nere, cuius fpecies verbis aegre diftinguntur.

$\mathrm{Hg}$.

$$
(p, 72 .)
$$

19. H. cyaneus thorace aeneo, elytris caerulefcentibus. F. Sp. 6o. 3 .

q Herbft. K. IV. p. 50, t. $3^{6,}$ f. II, a, b.

Defcri- 
Defcriptio Fabricii Syft. Ent. competit noftris fpeciminibus, qlae omnino conueniunt cum is e Suecia a D. de Paykul. acceptis. Color aeneus thoracis, quadam tantum incidentia lucis confpicuus. Hab. Pifis in ftercore bouino non rarus. Long. 4. lat. 2.

Celeb. Herbftius $H$. cycnetun fuum ex Hungaria accepit, itaque idem fane cum noftro eft. An Fabricii Hifter huc quoque pertineat, decernere non audeo. $\mathrm{Hg}$.

* 20. H. maculatus niger, nitidus, elytris pallide rubris, frriis curuis, anterioribus obfoletis, macula magna bafeos nigra ad futuram communi. - Defcriptio H. bipuftulati Schrankii. En. Inf. 67. cum noftris minime conuenire videtur.

Statura $H .4$-maculati, a quo tamen differt clypeo capitis magis prominulo, magisque ad apicem coanguftato acuminato. Differt antennis clana rufa, non nigra; thorace linea ante margincm delituto; elytrisque omnino pallide rubris, praeter maculam et futuram, quae nigra funt. Abdomen in hoc magis globofum, et tibiae anticae quadridentatae in $H$. quadriniaulato rarius et profundius tricientatac. Macula baleos elytiorum inaequalis, vnica, et ita ad futuram pofita, vt imctis clytris foman reierat fubquadratam. $\mathrm{Ab}$ H. bimaculato magis etiam differt, cuins, practer alias, nota conftans eft foucola feu puncum impreffum ad latera antica thoracis. Habitat cum congeneribus in ftercore bo!nino.

$$
\text { ( } \left.{ }^{\prime} . r_{3}\right)
$$

* zr. H. minor ater nitens, elytris fubdepreffis ftriatis, ftriis tribus integris, binisque interio- 
rioribus abbreuiatis, claua antennarum ferruginea.

Statura et magnitudine fere conuenit cum figura a Villers Ent. t. I. f. ?. exhibita ad indicandum H. I2-ftriatum Schrankii, fcilicet $H$. deprefjo paullo maior, magisque conuexus. Caput parum prominens, ironte impreffi, apice attenuatum. Antemae claua ferruginea. Thorax laeuis. Elytra abdomine panllo breuiora, ftriata, ftriis in fingulo tribus vix obliquis, integris; et duabus fitulrae proximis abbreniatis $a b$ apice vsque ad medium diductis. Tibiae anticae quadridentatae. Differt ab $H$. depreffo, praefertim magnitudine, et corpore clytrisque minus depreffis. Hab. fub ligno putrido, et fub corticibus frequens. Long. $\mathrm{I}_{3}^{2}$. lat. I.

* 22. H. decemfriatus ater nitens, elytris punctato-ftriatis, ftriis futurae proximis, antice coenntibus.

Accedere videtur ad $H$. abbreniatum $F$. ftatura rotundo-globofa: Magnitudo fere $H$. fulcati, at faepe maior. Ater nitidus. Antennae totae nigrae. Caput poft mortem intra thoracem retractum. Thorax omnino laeuis. Elytra apice truncata, punctato - ftriata ftriis in fingulo quinque, prima marginis exterioris obliqua abbreniata, fecunda tertir et quarta parmm obliquis, et ad apicem productis, quinta futurae proxime recta, et cum praecedente antice adeo iuncta, quafi ac vina eademque eflet ftria recurrens. Nota haec conftans et fingularis, etfi quid fimile in $H$. acneo interdum obfernetur. Inter quartam vero et viti- 
mam ftriam, alia interior abbreuiata ab apice elytri extenditur vique ad dimidium. Pofteriora $a b$ dominis elytris paullo longiora. Hab. cum congeneribus frequens.

$$
\text { (j. 14.) }
$$

* 23. H. duodecim-ftriatus ater, nitens, elytris duodecimftriatis, anteinis ferrugineis.

Duae obferuantur difficultates ne mihi fuadeam hunc effe verum $H$. I2-ftriatum Schrankii E.I. 7o. Villeis Ent.t. f. 7. nempe magnitudo minor, et color antennartum a Sihrankio praetermilfus, qui in noftris fpeciminibus ferrugineus eft: at cum nullum alium nouerim, cui magis $\mathrm{Cl}$. Viri defcriptio competat, nomen idem feruandum effe duxi.

Statura omnino praecedentis, fed paullo minor. Antennae ferrugineae. Caput retraetum fronte non impreffa. Thorax laeuis. Elytra abdomine breuiora, conuexa, ftriata ftriis minutiffime punctatis. In jingulo fex a bafi ad apicem decurrunt ita $v t$, aequalibus a fe diftuntes jpatiis, difcuin totum occupent. Pecies nigri interdum picei. Hab. in truncis arborum emort. non rarus. Long. I. lat. $\frac{2}{3}$.

Antennarum color nunc nigricans, nune ferrugineus. Mea fpecimina numquam ita parua funt, vt fulcatum aequent, fed interdum plus duplo maiora. Nihil itaque de Hiftero Auctoris determinare porfum, quoniam ftriae fex integrae pluribus communes videntur. Id enim fatendum eft, ftriam futurae proximam in $H$. 12-friato Schrconk. faepius interruptam effe. $\mathrm{Hg}$.

24. H. Julcatus ater, thorace elytrisque punctato-fulcatis, apice retufis. Fu. Etr. I. 31. 70. t. II. f. 3 . 
H. friatus. Forfter. N. I. C. I I.

H. globulofus. Oliuier. Ent. t. 2. f. I5. a. b.

Hab. in ftercore equino pafcuorum, primo vere.

Hifter Auctoris, quem Dn. Langins mecum communicauit, ab Hiftero Fabricii et Herbftii, in Fn. Etr. 1. c. citato, multum differt, quamuis inter Hifteros adhuc notos, illi maxime affinis fit. Differentiam praecipuam adiiciamus :

H. Sulcato Fabr. plus dimidia parte minor, eoque breuior. Thorax lineis eleuatis fex, extimis duabus abbreuiatis, reliquis quatuor integris, in medio pofitis. Elytrum fingulum lineas eleuatas fex, et $\mathrm{fi}$ lineam eleuatam futurae et alteram carinae, inflexura laterali elytri effectale, adiicias, octo habet. Interftitia inter has lineas tenuiter ftriata, et in medio vnica tanțum punctorum imprefforum ferie notata funt.

Hinc Synonyma ita diftribuenda funt:

I. H. Julcatus. Roffi Fn. Etr. I. 3 r. \%o. t. 2. f. 3. H. Julcatis. Moll Neues Ent. Mag. vo Fuesl. II. İे̆. 105 .

H. friatus. Forfter. N. Sp. Inf. Cent. I I.

H. globulofus. Oliuier. Ent. t. 2. f. I 5. a. b, (ex auctoritate Rofiii.)

II. H. fulcatus. F. E. S. I. T. 74. I2。

H. friatus. Herbft. K. t. 36. f. т. a. b. A.

H. fulcatus. Olinier. Inf. x. 8. I - 20. t. I. f. 6. (ex auctoritate Fabricii). Hg.

* 25. H. parmulus ater, elongatus, elytris laeuiffimis nitidis, antennis pedibusque ferrugineis.

\& H. parallelepipedus et picipes. Herbft. K. t. 35 . f. $x$ I. t. 36 . f. 13 ?

Minor femine Oryzae. Statura corporis elongata. Laeuis, nitidifimus, ater, pedibus exceptis, 
ceptis, antennisque a fractura ad clauam ferrugineis. Elytra admodum abbreuiata, conuexa. Abdomen apice attenuatum. Hab. in ligno putrefcente, hieme non rarus. Long. $\frac{3}{4}$. lat. $\frac{1}{3}$.

$$
\text { (p. } \text {. 5.) }
$$

* 26. H. puffllus niger, fubnitidus, elytris punctatis; thorace vtrinque ad marginem fulco longitudinali imprefio.

Minor pediculo, eodemque modo longiufculus. Niger fubnitidus. Antennarum claua ferruginea. Thorax fulco vtrinque ad marginem longitudinali profunde excauato. Elytra abbreuiata, vndique punctulata punctis impreffis, armato oculo manifefte confpicuis, vude nitoris defectus. Hab. fub arborum cortice cum congeneribus rarior.

Eundem cum flawicorni Herbft. K. t. 36. f. 2. coniicerem, qui ftriis elytrorum omnino caret, et fub cortice quercus habitat, fed is non fine nitore eft.

$\mathrm{Hg}$.

* 27. H. atomos ater laeuiffimus, nitidus, elytris fubintegris, capite toto exferto.

Vix maior femine Papaueris. Cum $H$. minimum in Fn. Etr. I. 32. ? I. dedimus, co minorem nullum inueniri poffe vifum, at reuera hic duplo minor, et ftatura diuerius. Corpus fubrotundum, globofum, atrum, laeue, nitidum; in $H$. minimo ouatum. Differt etiam antennis nigris claua concolori, capite paruo toto exferto, elytris vix abbreuiatis. Habitat fub cortice Vlmi menf. Febr. *

$\mathrm{X}$.

* Characteres indicati in fpeciebus a me defcriptis conftanter obferuati in pluribus indiuiduis. H. pyginaesm, qui apud 
(p. I6.)

\section{DERMESTES.}

28. D. vulpinus? oblongus laenis niger, thoracis lateribus cinereo - villofis, fubtus albidus. F. Sp. 64. 9.

D. cadaucrinus Fn. Etr. I. 34. 77. videtur huc referendus.

Mea fpecimina conueniunt cum defcriptione Fabricii. Femora poftica etiam bafi cinereo - fáfciata. Magnitudo variat. Speciem e defcriptionibus tnto determinare non potui. Hab. cadautera. Long. $4 \frac{1}{3}$. lat. $\mathrm{I}_{2}^{\prime}$.

Fabricium non allego, eandem ob cauffam, quae pro-

hituit, ne $D$. cadautinum $F$. citem. vid. Fn. Etr.

1. c. $\mathrm{Hg}$.

20. D. trifúfcintus ouatus niger, elytris fafciis tribus undatis cinereis. F. Mant. 34. 7 .

D) Villers Illuftr. $4.2 \pm 9$.

ㅇ F. E. S. I. r. 228. 7•

Defcribit illum Fabricius. Elytrorum fafciae interdum obliteratae. De genere dubitat D. Villers. 1. c. Hab. in plantis rarus. Hong. $I \frac{1}{3}$. lat. $\frac{\pi}{2}$.

30. $D$ ? dentatus elongatus hirtus, niger, elytris teftaceis. Fn. Etr. 37. 82. t. 3. f. 2. et add.

Infectum forte noui generis.

An Cantharis ferraticomis. Scop. E. C. I35? Sed antennae differunt paullulum a defcript. Scopolina.

nos etiam habitare videtur, cum authores in eo defcribendo nimis diffentiant, erroris periculo praetermifimus, iten. que alios, quorum differentiam oculis magis diftinguere, quam verbis exprimere datum. Adiot. Aint. ipf. 
Variat magnitudine. Nidulatur in ligno antiquo. Menf. Iun. et Jul. faepe inueni in poltibus feneftrarum, et ianuis domefticis exeuntem e factis foraninibus; fed ruri etiam lectus in floribus.

Citatum ex Scopolio omnino delendum eft, eiusden Cantharis Ciftelarum fpecies videtur.

Ex fpecimine, quod comparari licuit, figura noftra, praecipue elytra, nimis lata funt. Infectum fingulare, quod nulla ratione, ne antennarum quidem, Dermeftum generi annumerari poteft. Potius Lagriis adiungatur, antennis tamen alienis, quadriarticulis, articulis tribus vltimis compreffis, duobus triangularibus, vltimo ouali. An eiden generi, cui Ptilinus teftaceus, annumerandum?

Hg.

$$
\text { (p. } x_{r} \cdot \text {.) }
$$

3I. D. fornicatus fufcus obfcurus, corpore quato laeui fornicato, antennis longiffimis: claua fubulata. De Geer. 4. 2I6. 9. t. 8. I. I5. Villers Ent. I. 57. 46.

Genus incertum, at facies Dermeftis. Antennae claua elongata apice fubulato, capite thoraceque paullo longiores. Hab. in truncis cariofis. Long. 2. lat. I. et vltra.

* 32. D. fuliginofus ouatus ater, antennis ferrugineis.

Statura et magnitudo D. lardarii. Totus ater, et veluti fuligine obductus. Antennae fnbrufae. Alae fufcefcentes. Accedere videtur ad $D$. atrum Degeer. Inf. t. 4. 223. I. t. I 8. f. 7. at differt colore aterrimo et ftatura breuiori. Hab. in arborum carie frequens. 
33. D. bicolor oblongus niger, fubtus teftaceus, elytris ftriatis. F. Sp. 64. 8 .

\& F. E. S. I. I. 229 . IO.

Herbft. K. IV. p. I 25. 6. t. 40. f. 6.

Statura D. murini, fed paullo maior. Totus fuperne niger, capite thoraceque praefertim ad latera villis fuluo luteis tectus; inferne teftaceus. Elytra fubftriata. Hab. in truncis arborum exficcatis.

* 34. D. dubius cyaneo-niger fubhirtus, elytris rubris.

Infectum forte noui generis. Primo intuitu Cantharis, aut Lagria, aut Clerus videtur. Corpus totum cyaneo-nigrum, elytris exceptis rubris. Statura $D$. fanguinicollis, at paullo maior. Caput exfertum hifpidulum. Palpi quatuor inaequales fubfiliformes. Antennae porrectae, thorace breuiores, clauatae claua ex articulis tribus compofita, primo et fecundo apice oblique truncatis, extimo acuminato. Thorax rotundatus, emarginatus, punctulatus, fubhirtus, lateribus pofticis paullulum reflexis. Elytra integra tenuiffime punctulata, rubra, nitida, eiusdemque coloris elytrorum, Boftrichi Capucini. Alae nigrae. Tarfi quatuor. Lectus iuxta fepes in filuis April. m. Long. $4 \frac{\mathrm{x}}{2}$. lat. I.

\section{(p. $1 \mathcal{8}$.}

\section{BOSTRICHVS.}

35. B. chalcographus ater, elytris praemorfodentatis rufis. F. Sp. 68. 4.

\& F. E. S. I. 2. $3^{6} 5 \cdot 5 \cdot$ Panz. F. I. G. XV. 4.

Z2

C. $H$. 
C. H. v. Sierftorpff iiber cinige Infektenarien, welche den Fichten vorziiglich fihiiddich find. etc. Helmft. ap. Fleckeifen. I794. p. 56. \$. 46-48. f. I2. et II.

Duplo aut triplo minor B. typographo. Antennae teftaceae. Corpus glabrum. Colore variat. $\mathrm{Hab}$. fub arborum cortice.

36. B. $m y$ gmaeus ater nitidus, elytris integris rufis, abdomine retufo. F. Mant. 37. I I.

q F. E. S. I. 2.367 .13 .

Eccoptogafter pygmaens. Herbft. K. t. 49. f. 2. b. . Habitat intra cortices arborum emortuarum.

37. B. crenatus glaber atcr, elytris integris $1 \mathrm{i}$ ceis, crenato-ftriatis. F. Mant. 37.10.

q F. E. S. I. 2. 366.10. Panz. F. I. G. XV. $7 \cdot$ Habitat in truncis arborum.

Primo vere magnam copiam huius' infecti in truncis Fraxini excelfioris reperi, quorum pleraque iam mortua erant. Aetate pronectis elytra nigra funt.

A caeterorum habitu non parum recedit, et, ni fallor, Boftrichorum genus etiam nunc dirimendum et in plura diftribuendum eft, quamuis celeb. Fabricius Apates, et celeb. Herbftius Eccoptogaftri gentem feparauere. Habitus enim plurium a veris Boftrichis, e. g. B. typographo, nimis diuerfus eft. Hunc $B$. crenatnm interea cum $A n$ thribo vario, fcabrofoque, et Boftricho vario proprio genere, cui nomen Anthribo relictum eft, coniunxi. Anthribum enim Albinum, latiroftrem etc., vti iam in Fn. Etr. I4I. 336. adnot. relatum eft, genere Platyftomo fociaui. De reliquis, fi locus milhi dabitur, videbo. Hg. 


$$
\text { (p. 19.) }
$$

38. B. ligriperda villofus teftaceus, corpore nigricante, tibiis quatuor pofticis ferratis. F. Mant. 37. 12.

\&. F. E. S. I. 2.367 .14$.

C. H. v. Sierfiorpff 1. c. p. 59. S. 5r. 52. f. 14.15 .

Fabricius defcribit. Hab. in ligno.

39. B. varius fubuillofus, corpore nigro, cinereoque vario. F. Sp. 64. 8 .

Statura et magnitudo $B$. pimiperdae, fed paullo - latior, et breuior. Antennarum claua acuminata. Hab. cum praccedentibus.

In Entomol. fyftem. Fabricii hunc Boftrichum reperire nequeo, et haud dubie plane ea de caufa omiffus videtur, quia in Mantifia 38 . I5. pro eius nomine vitio typographico nomen teftacents exhibitus erat.

Caeterum confer, quae fupra ad $B$. crenatum n. 37 . monui. $\mathrm{Hg}$.

* 40. B. ater glaber niger nitidus, elytris integris, antennis pedibusque rufis.

Statura $B$.piniperdae, at minor, et ab eo difinctus etiam colore magis atio, magisque glabro, laeui, nitido. Hab. in ligno.

\section{ANOBIVM.}

* 4r. A. punctatum grifeo-fufcum, elytris ftriatis, punctisque excauatis, thorace in medio gibbofo.

Ptinus punctatus grifeo-fufcus, elytris ftriatis punctisque excauatis. Degeer. Inf. 4. 230. 3 . 
Statura omnino A.pertinacis, cuius forte varietas. Antennae fufcae, tres articuli vltimi maiores. Thorax in medio gibbus, margineque poftico parum reflexo. Elytra punctatoftriata. Hab. in ligno.

$$
\text { (p.20) }
$$

\section{PTINVS.}

* 42. P. longijes teftaceo - flauus pilofus, antennis pedibusque longiffimis.

Statura et magnitudo P. Furis. Totus teftaceo - flauus pilofus, alatus. Antennae corpure fere duplo longiores. Oculi nigri. Thorax eleuatus, tuberculofus. Elytra laeuia. Pedes longiffimi. Hab. Florentiae.

* 43. $P$. variegatus thorace bituberculato, elytris nigris albo-fafciatis, antennis pedibusque cinereo nigroque variis.

Statura $P$. Furis, at fere duplo maior. Antennae corpore breuiores fufcae, articulis apice cinereo-tomentofis. Thorax bituberculatus, cinereo rufefcens. Scutellum cinereum. Elytra nigra laeuia, nitida, punctato-ftriata fafciis duabus, altera bafeos, altera apicis vndulatis candidis. Pectus, abdomen, pedes cinerei femoribus nigro - anmulatis. Hab. Florentiae.

44. P. Latro thorace bidentato teftaceus, immaculatus. F. Sp. 73. 6.

\& F. E. S. I. I. 240 . 7 .

Statura et magnitudo P. Furis. Antennae longitudine corporis. Thorax anguftus dentibus dorfalibus 
falibus binis. Elytra punctato - ftriata. Hab. in lignio.

Minime ftatura eadem $P$. Furis eft. Fur enim globofus, Latro contra oblongus eft. $\mathrm{Hg}$.

\section{(p. 2r.) \\ XIX. SPHAERIDIVM.}

45. S. fmetariun atrum immaculatum, elytris laeuiffimis. F. Sp. 79. 9.

\& F. E. S. I. I. $240.7^{\circ}$

Partum et vix magnitudine feminis Oryzae. Statura oblongior quam in congeneribus. Totum atrum nitidifimunim. Species quidem diftincta, non varietas vti errore dicttim in Fn. Etrufca. Hab. in ftercore bouino rarius.

46. S. pulicarium nigrum oblongum, elytris abbreuiatis, abdomine acuto. F. Sp. 79. I2.

$\odot$ Linn. 2. 574. 33. et 2. 564. 24 . Fn. Sv. 37 o. [435.] Geoffr. Rai.

q F. E. S. I. x. $83 \cdot 30$.

Synonymia Authorum errata ${ }^{1}$ Derm. pulicarius Linn. et Anthribus Geoflir. Inf. I. 308. 4. Sph.pulicarium non indicant. Corpus oblongo - globofum, atrum, laeue, nitidiffimum. Antennae tennes longitudine thoracis claua perfoliata. Elytra armato oculo vix marginata, abbreuiata. Abdomen apice acutum. Pedes fubrufi. Saepe lectum hieme in truncis arborum emort. Long. $\frac{3}{4}$. lat. $\frac{7}{3}$.

47. S. luteun luteum, elytris pubefcentibus laeuibus. F. Mant. App. 378. 3-4.

N. Veris. Fn. Etr: I 63. I47. t. 2. f. I2. nomen delendum et huc referenda defcriptio. Hab. medio vere in floribus Crataegi. 
Sph. Colon. F. E. S. I. I. 78. 4. mera varietas S. lutei videtur, ex fpeciminibus, quae Dn. Paykull aliique benigniter mecum commmunicauere, Hoc $S p h$. Colon idem eft, quod celeberrimus Herbftius K. t. 43. f. 5. e. E. nomine Strongylus 4-punctatus depinxit. Hg.

\section{TRITOMA.}

\section{( $p .22$.}

48. T. bipuftulata atra, elytris macula laterali coccinea. F. Syft. 68. I.

Tritoma incerta. Fn. Etr. I. 5 I. I I 9.

Synonymia Geoffroyana falfa, occafionem in Fn. Etr. dubitandi praebuit. ,Hab. fub arborum cor. iice. Mox lecta frequentior.

49. $T$. fericea nigricans holofericea, pedibus teftaceis. F. Sp. 44. 6.

q F. E. S. I. 2. $507 \cdot 8$.

Statura et magnitudo Cocc. minutiffmae. Corpus totum cinereo-holofericum. Antennae, pedesque teftacei. Hab. in arborum truncis.

Hanc cum aliis huius generis, de quibus inferendis apto loco celeb. Fabricius 1. c. ipfe dubitat, clariffim. Knochius in proprium genus redegit, cui nomen Ptomaphagus dedit, quod a $\pi \tau \tilde{\omega} \mu \alpha$ (lapfum quid h. l. corpus mortuum) et $\varphi a^{\prime} \gamma \omega$ (comedo) ortum ducit, quia haec infesta in cadaueribus reprehenduntur.

An Auctoris infectum verum Fabricianum fit, non decernam. $\mathrm{Hg}$.

\section{IPS.}

- 50. I. fallax picea laeuis nitida: antennis pedibusque rufis.

An 


\section{An I. ruficornis Olinier.?}

\& Lyctus nitidus. F. E. S. I. 2. 505. 12 ?

Statura et facies onmino I. taxicomis. Fn. Etr. Herbft. Arch. 2I. fed dimidio minor, et color elytrorum obfcurior. Antennae ad apicem tantum clauatae claua perfoliata, diuerfae $a b$ antennis I. taxicomis, eam ab illa plane difinguunt. Hab. fub corticibus.

* 51. I. teftacea oblonga teftacea immaculata.

Statura I. crenatae, et paullo oblongior. Corpus depreflum, immaculatum. Antennae teftaceae. Oculi nigri. Thorax fubquadratus, teftaceus fulco medio impreffo longitudinali obfoleto. Elytra laeuiffima, et abdomen nigrum praecipue diftinguun $\kappa^{\circ}$ a $N$. ferruginea. Lecta in arculis infectorum.

$$
\text { (p. 23.) }
$$

* 52. I. atra atra, oblonga, nitida, elytris ftriatis, thorace puncto vtrimque impreffo, antennarum claua pedibusque fubrufis.

Minor I. crenatu et minus depreffa. Antennae longitudine thoracis claua rufa pilofa. Thorax ater nitidus, latitudine elytrorum, emarginatus, punctulatus, punctoque vtrinque maiore excauato. Elytra atra nitida, fubdepreffa, laeuiter ftriata. Pedes fubrufi. Hab. in ligno putrido.

\section{SILPHA.}

53. S. laenigata atra, elytris laeuibus, fub. punctatis. F. Sp. 8, . I3.

() Rai. 90. 9.

P F. E. S. I. I. 252. I4.

$\mathrm{Hab}$. in hortis et filuis frequens. Long. 6. lat. 3 .

$$
\mathrm{Z}_{5}
$$

In 
In F. E. S. 1. c. vitio typographico Sulz, t. 2. f. 6. pro t. 2. f. I6. politum ef.

Raii citata pleraque inutilia funt, nec infecti notitiae inferuiunt. Raius tamen a pluribus citatur, et h. 1. etiam ab Auctore, qui antea numquam folebat. Sed eius verba apponamus ipfa: "9. Scarabaeus inter cadauera frequens, depreflus, parum ranaliculatus, vndique niger. Scapulae magnae funt, fub quibus caput retrahit. Cauda parum vltra elytra extenditur. Iratus humorem atrum euomit. 9. Scarabaeus praecedenti fimilis, fed paullo maior, elytris laeuibus." Hunc pofteriorem Auctor fane fpectauit. Hg.

\section{NITIDVLA.}

* 54. N. maculata teftacea pubefcens, elytris maculis tribus fufcis, pone medium transuerfim pofitis.

Antennae longiufculae. Oculi nigri. Thorax fubquadratus, angulis pofticis acutis. Elytrorum maculae oblongae, obfoletae; in fingulo elytro vna, et media futurae communi. Femora craffuccula. Hab. fub corticibus rara. Long. $I_{3}^{\frac{2}{3}}$ lat. I.

\section{(p.24.)}

55. N. Arigata ouata fufca, thoracis margine, elytris margine, lineola bafeos, ftrigaque apicis fuluis. F. Mant. $5^{\text {I. }}$ ?

\& F. E. S. I. I. $257 \cdot 9$.

Strongylus ftrigatus. Herbft. K. t. 43. f. ?. g. G.

A Fabricio defcripta eft. Color elytrorum fufconiger lineolis fuluis circumfcripins, fi illum oculi tantum aduertant, eleganter pictus apparet. Lecta Pifis in horti pariete. 
56. N. difcoiden thorace marginato nigra, elytrorum difco ferrugineo. F. Sp. 92. I I.

\& F. E. S. I, I. 259 . I9. Herbft. K. t. 53. f. ?: g. G.

Defcribit eam Fabricius, Antennae ferrugineae claua fufca. Pedes picei. Lectae duae in equi cadauere infepulto.

* 57. N. chryfomeloides obfcure teftacea fubglobofa pubefcens, elytris laeuibus, thorace vix obfcurioribus.

Duplo maior $N$. aeftiua. Corpus totum obfcure teftacem. Antennae breuiffimae admodum clauatae. Oculi nigri. Thorax laeuis, gibbus, latitudine elytrorum vix marginatus, et pube tenuiffima, certa fitu, ferrugineo micanti. Elytra gibba laeuia, vix pubefcentia, thorace obfcuriora. Habit. in ligno putrido. Long. I. lat. $\frac{1}{2}$.

$$
\text { (p. 25.) }
$$

58. N. hnemorhoidalis? nigra elytris apice ferrugineis. F. Mant. 52. 12.

\section{o F. E. S. I. I. 259. 18 ?}

Statura N. Colon, at vix minor. Caput, antennae, palpique rufi. Antennae huius generis, claua folida, breues. Palpi antici apice fubclauato, et oblique truncato. Thorax niger margine antico et laterali rufo. Elytra laeuia, nigra, apice late rufa. Abdomen obfcure nigrum. Pedes omnes rufi. Lecta fub corticibus menf. Mart.

\section{COCCINELLA.}

59. C. tripunitata coleoptris rubris, punctis nigris tribus. F. Mant. 54. 1 \%. Linn. Fn. 472? Villers Ent. 1. 75.5 . 
Ea, quae rariffime occurrit apud nos, certe mera varietas eft $C$. 5 -punctatae, cui fortuito in elytris deeft punctum pofticum marginale: reliqua omnia fimilia. In Fil. Etrufca dubitatum, at errore calami, aut Typograph. irrepfit praecedentis pro jequentis. Hab. in plantis.

Confer, quae ad C. tripunctatam Fn. Etr. p. 65. monui. $\mathrm{Hg}$.

60. C. nigrofafciata coleoptris rubris punctis nigris octo, fafcia media atra. Fn. Etr. I.66. I55.

Femina C. 9-punctatae, cum qua copula iunctam faepius reperi. Hab. in plantis.

61. C.23-punctata coleoptris rubris, punctis nigris viginti tribus diftinctis. F. Sp. I01. 46.

o Villers Ent. I. ro4. 25 .

7 F. E. S. I. I. 28 r. 7 r.

C. impunctata var. 6. Herbft. K. V. p. 3. 6r.

Parua. Thorax ruber punctis tribus nigris. Elytra rubra. Puncta 3 nigra, dein 4 , tum 3 , vltimo, punctum transuerfum ex duobus connatum. Accedit punctum fcutellare commune. Puncta omnia rotunda, diftincta. Subtus etiam rufa vna cum pedibus. Hab. in plantis.

$$
\text { (p.26.) }
$$

62. C. 12 - punctata coleoptris flauis, punctis nigris duodecim, extimis linearibus repandis. F. Sp. 98. 32.

D Villers Ent. r. 100. I7. Fig. Schaefferi. non rite citata.

q F. E. S. I. I. $278 \cdot 55$.

Minuta. Thorax flauus, punctis maculisque duabus nigris. Elytra flaua. Puncta quinque nigra 
praeter exteriorem quafi ex quatuor punctis connatum, lineare, repandum ad marginem exteriorem, inter primum et vltimum punctum. Sutura longitudinalis nigra. Subtus nigra tibiis flauis. Puncta thoracis variare videntur. In noltris faepe duo fint bafeos compofita ex aliis duobus. Hab. in hortis minus frequens.

63. C. If-muculata coleoptris luteis: fitura, punćtisque quatuordecim nigris diftinctis. F. Mant. 58.57 .

\& F. E. S. I. I. 279.63.

Praecedente maior. Caput albidum. Thorax albidus, nigro-punctatus, fere vti in C. 12 -punftatn, fed punctis plerumque diftinctis. Elytra lutea punctis 3,3 , 1 anterioribus in femicirculo pofitis, diftinctis, oblongis, praeter penultimum rotundum futurae proximun. Pedes lutei femoribus pofticis apice nigris. Hab. nuri fat frequens.

64. C. 4 - guttata coleoptris rufis, punctis albis quatuordecim. F. Sp. 102. 52.

$\odot$ Rai., Lift.

D Scop. E. C. 248 .

o F. E. S. I. I. 284.85 . Herbft. K. t. 59: f. 3 。 Dantur aliae dimidio minores huic omnino fimiles, quae fane referendae funt ad $C$. I 2 - guttatam Geoffr. Pod. Schrank. E. I. $3 \mathrm{I}$ 1. at quamuis elytrorum puncta fint tantum duodecim, de varietate praecedentis dubito. Hab. in Salice frequens. Long. $2 \frac{\mathrm{x}}{4}$. lat. $I_{\frac{\mathrm{T}}{4}}$.

Thorax in C. 1q-guttata proportione minor ef quam in $12-$ guttata. Guttae in C. 14 - guttata, maiore, pofitae funt vtrinque hoc ordine: 3 . ad fcutellum, 3. transuerfim pofitae, marginali paul- 
lulum promota et maiore, 2, r. - in $12-$ guttata, minore: $x$. ad fcutellum, 2 , cum fequentibus 2, transuerfim oblique pofitae, vt a margine defcendant futuram verfus, tandem. I. - Magnitudo vtriusque ita variat, vt 12 -guttatcie fpecimina interdum 14-guttatae fpecimina minuta fuperent. $\mathrm{Hg}$.

$$
\text { (p. 27.) }
$$

65. C. I4-puftulata coleoptris nigris, punctis albis quatuordecim. F. Sp. 106. 69.

D Schrank. E. I. I22. Villers Ent. I. II 4. 58.

I F. E. S. I. I. 290. III.

Statura et magnitudo $C .2$-punctatae. Thorax niger puncto vtrinque marginali arcuato flauo; faepius tamen margo etiam anticus totus flauefcit. Puncta elytrorum 2, 2, 2, I interdun flaua, interdum rubra. Hab. in floribus frequens.

\section{CASSIDA.}

66. C.margaritacea virens, elytris viridi - argenteis nitidis, capite pectore nigris. F. Mant. 63.22. o F. E. S. I. I. 309. 8 .

Statura C. nebulofue, at paullo minor. Caput et peçus nigra. Abdomen, peđes antemnaeque pallide viridia. Color elytrorum viridi-argenteus perit cum vita. Hab. apud nos fat frequens.

Caffidarum fere omnes colorem plus minusue, vita exuta, mutant, virides grifeo-luteae fieri, nobiles vero, qui ftria metallica fuperbiunt, hoc infigne amittere folent. Id antem quum in his, tum in aliis eleutheratis, e. g. in Crioceride merdigera obferuaui, colorem minus mutari, fi acu transfixa tempore fubinde morirentur, quam fi igne 
aut aqua feruida necarentur. Sed illa methodus animum durum pofcit. $\mathrm{Hg}$.

\section{(p. 28.) \\ XXX. CHRYSOMELA.}

6?. C. Goettingenfis ouata, atra, pedibus violaceis. F. Sp. I 16.2.

D Laichart. Inf. Tyrol. r. I43. 2. C. corictric.

S F.E. S. I. I. 309. 8.

C. haemoptera paullo maior, globofior, obfcurior. Plantae fubrufae. Hab. in Gramine frequens. Long. 4. lat. $2 \frac{1}{2}$.

Roefelii citatum Inf. 2. Scar. 3. t. 5. Anctor nofter dubium exhibet, cum tamen omnino delendum fuiffet. In Fn. Etr. 80. 190. ad C. Tinnaceti bene iam translatum erat. - Quomodo Laichartin. gii C. coriaria citari potuit, non video. Furte C. haemoptera Auct. eft Goettingenfis F., et $C$. Goettingenfis illa C. coriaria? Hg.

68. C. faftuofa ouata aurea, coleoptris lineis tribus caẹiuleis. F. Sp. I 24.48.

D Schrank. E. I. I75. Gronou. Zooph. 56r.

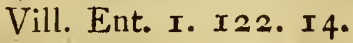

of F. E. S. I. I. 324.80 .

Aurato - virens fulgida. Linea caerulea longitudinalis in medio elytrorum, et vnica communis in futura. Alae rubrae. Colore admodum variat. Hab. in lamio albo, et vrticis frequens. Long. 3 . ât. $I_{\frac{1}{2}}^{\frac{1}{2}}{ }^{*}$

Variat

- Citatio Geoffroyana, quam authoritate Linnaei in fynonymia C. crneae dedimus, v. Fn. Etr. I. 196. ad hanc referenda effe videtur: $C$. aenea enim hac maior eft, et caret fafciis caeruleis: In $C$. foultsofa alae rubrae, in $C$. aessea nigrae: qua nota laec etiam diftinguitur a $C$. Graminis, cui funt rubellae. Adis. Anit. iys. 
Variat colore cupreo aut viridi: fafciis femper caeruleis; interdum autem viridi-aenea eft, absque vllo fere fafciarum rudimento. $\mathrm{Hg}$.

$$
\text { (p. 29.) }
$$

* 69. C. violacea ouata violacea, antennis plantisque fufcis.

C. violacea. Panzer. Journ. Allem.

\& F. E. S. I. I. 3I5. 39.

Statura et magnitudo fere $C$. politae. Tota laete violacea nitida, vnicolor. Thorax ac elytra punctulata punctis inordinate fparfis. Antennae et plantae fufcae. Raro inueni. Long. $3 \frac{1}{3}$. lat. $I \frac{2}{3}$.

Conferantur adnotata ad C. Eamopteram. Fn. Etr. $\mathbf{I}$. 79. $187 . \mathrm{Hg}$.

* 7o. C. nigricomis oblongo-ouata violacea, nitens; thoracis lateribus incraftatis, prominulis, antennis nigris.

An C. nitens. Muf. Lesk. p. I3. n. 26I?

Statura ouatá oblongior, quam in noftratibus omnibus. Tota violncea nitida colore $C$. Alnis Antennae tantum nigrae et longiufculae. Thorax fere latitudine elytrorum, antice excauatus, poftice rotundatus, in medio conuexus, margine laterali craflo prominulo. Elytra laeuia, vix fubpunctata.

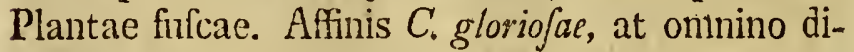

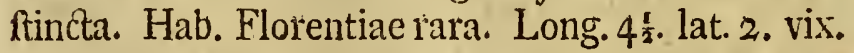

71. C. gloriofa ouata viridis nitida, elytris lined caerulea. F. Sp. App. 497.

\& F. E. S. I. I. $324 \cdot 82$.

Duplo maior $C$. faftuofa. Color viridis nitens, linea vnica caertillea in medio elytrorum. Frons puncto imprefla. Thorax latus. Elytra nonnihil vtrin- 
que anguftata, poftice latiora. Antennarum articulus ultimus, plantaeque grifeo-fufcae. Hab. apud nos rarior.

\section{(p. 30)}

* 72. C. gemellata viridis, punctis elytronm per ftrias gemellas digeftis. Ent. Parif. I I 0. 22. La Cluryom. it fries jumelles.

Villers Ent. I. I38. 68.

Statura et magnitudo fere $C$. faftuofac. Supra aeneo-viridis. Antennae nigrae. Thorax breuis laeuiffimus lineola abbreuiata poftica marginali. Elytra laeuia punctis duplici ordine quafi in ftrias gemellas inordinate digeftis. Subtus aeneo - caerulefcens plantis fufcis. Alae albae. Hab. apud nos rarior. Long. $2 \frac{1}{5}$. lat. $I_{\frac{T}{2}}$.

Variat magnitudine, vt nunc $C$. laminam $F$, nune C. variantem $F$. aequet; colore nunc fere nigro, nunc cyaneo, nunc orichalcico. Striarum in fingulo elytro paria quatuor, atque ftriam folitariam futuram legentem, in omnibus fpeciminibus meis obferuo, punctis nunc maioribus et rarioribus, nunc minutis ac denfis, quae ftrias magis regulares fingunt. Celeb. Herbftii Cliryfomelam orychalcam Arch. p. 60. n. 43. t. 23. f. 22. allegarem, nifi feptem tantum ftrias punctorum numeraret. Abdomen quoque numquam fufcum, fed, vti auktor monet, caeruleo-nigrum vidi. $\mathrm{Hg}$.

73. C. vulgatiffma oblonga caerulea, antennis bafi ferrugineis. F. Sp. I30. 79 .

D Schrank. E. I. I26.

$\$$ Galeruca vitellinae. F. E. S. I. 2. 18. 22. var. Corpus magis oblongum, quan ouatum. Antennae nigrae longiusculae. "Thorax fubquadra- 
tus margine laterali faepius in medio nomnihil contracto, angulis pofticis acutis. Elytra punctis longitudinaliter digeftis. Hab. in falicibus frequentifima cum $C$. vitellinae.

74. C. Armoracide onata caerulefcens nitida, fubtus nigra. F. Sp. 127.66.

\& F. E. S. I. I. 330. I 12.

Similis $C$. Betulae, at corpore magis globofo. Abdominis apex obfcure flauefcit. Hab. in Armoracia, Plantagine, aliisque. 'Long. $I_{3}^{1}$. lat. $I$. vix.

Citatum Degeerii. Inf: 5.322. 25. falfum eft, ad $C$. Cochleariae $F$. pertinens, quod elytra ab eo defcripta punctato-ftriata probant. Forte eiusdem C. Betulae n. 24 , quae a Fabricio et Auctore fub C. Betulce perperam citata erat, huc fpectat. Hg. (p. 3r.)

* 75. C. pyyritofa futco-aenea nitida, antemis craffucculis, thorace laeui, elytris punctatoftriatis.

Statura et magnitudo praecedentis. Antennac pone medium craflufculae. Subtus etiam toto fufco-aenea. Differt a $C$. Armoraciate colore dinerfiffimo, et a $C$. metallica Fu. Etr. colore minus nitido, et forma thoracis antice lationi. Habit. in pratis filuaticis, menf. Mai. faepe lecta.

- 76. C. ruficollis oblonga viridi-aenea, thorace pedibusque rufis. F. Syft. Ent. App. 820.

of Galeruca ruficollis. F. E. S. I. 2. I3. 4 . Fabricius eam defcripfit. Valde aftinis C. Polygoni. Nonnulla indiuidua poffideo capite toto rufo, et alia, quae forte referenda funt ad $B . r u f-$ collem. F. Mant 55. Hab. in plantis non rara. 
E defcriptione Geoffroyi Inf. x. 263. I6, quod ad C. ruficollem allegari folet, C. Polygoni agnofco, omnia enim quadrant. Mirari itaque poffum, qua de cauffa Auetor hoc citatum, quod ipfe iam fub C. Polygoni exhibuerat, (Fn. Etr. I. 87. 209.) h. 1. iterum enumerat. $\mathrm{Hg}$.

\section{ALTICAE}

faltatoriae femoribus pofticis incraffatis.

77. C. nemorum faltatoria, atra, elytris flauis margine omni nigto. F. Sp. I36. I I4.

D Scop. E. C. 2 I5. Schrank. E. 1. I54.

o Galernca nemorum. F. E. S. I. 2. 34. 104.

Magnitudo maximi Pulicis. Thiorax niger. Elytra flaua vndique margine nigro cincta. Pedes pallidi. Hab. in Pulmonaria, in Lepidio latifolio fre. quens, copiofa menf. Mai.

$$
\text { (p. 32.) }
$$

* 78. C. Pulex faltatoria, nigro-aenea nitens, antennarum bafi tibiisque rufis.

An C.-Pulex. Schrank. E. I. I60? Magnitudo praecedentis et facies veri Pulicis. $\mathrm{Ob}$. longa, tota aeneo-nigra, nitens. Thorax, elytraque fubtiliffime, ac inordinate punctata. Tibiae tarfique rufi. Hab. in plantis minus frequens.

79. C. violaceo-punctata faltatoria, ouata, thorace punctato virefcenti caeruleo, elytris violaceis punctato-ftriatis, pedibus rufis. Degeer. Inf. 5. 343.48 .

Magnitudo praecedentis, fupra laete violacea, fubtus nigra; antennae, pedesque rufi. Hab. in plantis.

* 80. C. dorfalis faltatoria, luteo-liuida, elytris futura nigra.

Statura 
Statura C. atricillae. Antennae fubluteae. Oculi nigri. Caput, thorax, ac elytra pallide - flaua. Elytra fubpellucida laeuia, futura nigra. Abdomen et femora poftica nigra. Tarfi pallidi. Variat pedibus anticis -interdum totis pallidis. Hab. in Verbafco non rarạ,

\section{(p. 33.)}

\section{CRYPTOCEPHALVS.}

81. C. Mornei ater, elytris maculis duabus flanis marginalibus. F. Sp. 144. 37 .

Fn. Etr. T. 96. 235 .

Habitat in Hyperico perforato. Menf. Iun. fub vefperum frequens copiofus, et faepe copula iunctus. Long. $I \frac{1}{2}, 2$. lat. $\frac{3}{4}$.

Auctor obferuata fua eisdem verbis, quibus in Fn. Etr. 1. c., iterum exhibet, fed hunc Cryptocephalum copula iunctum ipfe cepi, at vtrinsque fexus maculae flauae erant. Schaeff. Ic. t. 30 . f. 5. , in Fn. Etr. plane omifla, h. l. rurfus citatur, figno interrogationis adiecto. Harrerus 1. c. ed. 2. p. I35. ñ. I58. ad C. bipulfulatum refert. Cum vero in mufeo Schnefferiano fpecimen deeffet, e quo effigies fucta erat, certo indicare non poterat. Sufpicatur, eam figuram ad Chryf. bigzttatum Schaller. A\&. Hall. pertinere. Haec eadem eft cum $C$. Hiibneri $F$, vti iam in Fn. Etr. IO2. in adn. ad 247 . monuimusts. Sed potius tigura, quae nullum maculae flauicantis veftigium gerit, omnino praetermitțatur. Schaeff. t. 238. f. 2. quoque allegata, ab Hạrrero l. c. ad Crypt. 4-guttatum Müll. relata eft. Ab eodem Schaeff. Ic. t. 30 . f. 7 ad C. Morcei refertur, quae omnium optime conmenit. $\mathrm{Hg}$. 
* S2. C. tricolor niger, thorace atro, maculis fex inaequalibus luteis, elytris rufis, punctis tribus nigris.

Statura et magnitudo C. bipunitati. Antennae nigrae thorace longiores. Caput nigrum intra thoracen recondituni. Thorax ater laeniffimus nitidus, maculis duabus in medio dorfi poftice, obliquis maioribus; itemque duabus vtrinque ad marginem exteriorem fere contiguis, eademque parte firiniliter emarginatis, luteis. Elytra rubra inordinate, ac fubtilifime punctulata. In fingulo ad bafin puncta duo rotunda, et alind fere in medio elytri quafi ex binis compofitum nigra. Subtus totus niger. Miffus ab illo ac egregio: Viro Com. Leopoldo Galli, qui Entomologiae etiam anantiffimus, Infecta multá in Etruriá collegit, et beneuole mihi communicauit. Hab. Florentiae in montuofis; frequens loco vulgo dicto Montefpertoli.

Cryptocephali fpecies, quae C. cordato. F., diftinguendo. Schmeid. et variegato Hybno affinis eft. Specimen, quod habeo, in capite puncta duo flaua minuta habet. Caetérum defrriptio bene con* gruit. Hg.

\section{(p. 34.) \\ XXXII. CISTELA.}

Ciffela Gigas, et Tenebrio dubius Fu. Etrufare ad nouum genus retulit $\mathrm{Cl}$. Olinierus fub nomine Ceb. longicornis et Ceb. breuicomis.

\section{CEBRIO.}

Antennae filiformes fubferratae corpore paullo breniorcs, I I - articulatae, articulo 2.3-9. breuifA a 3 timis. 
fimis. Labium fuperius corneum breue, antice ciliatum. Miandibulae porrectae, corneae, arcuatae, fimplices, rotundatae, ciliatae. Labiun inferius breue, fubconeum, integrum. Palpi quatuor filiformes inaequales; anteriores longiores, quadriarticulati, articulo primo breuiori, aliis aequalibus, etc.

83. C. longicornis pubefcens fufcus, elytris, abdomine femoribusque teftaceis: Olin, Ent. p. 5. n. I. t. I. f. I. a. b. c.

Ciffela Gigas, Fn. Etr. r. 107. 256. Hab. Pifis et Florentiae non rarus,

84. C. breuicornis apterus teftaceus mandibulis exfertis nigricantibus, antennis breuiffmis. Oliu. Ent. 5, I2. 2. t. I. f, 2. a. b, c.

Tenebrio? dubius Fn. Etr. I. 583. t. I. f. 2. Hab. Florentiae et Pifis rarifimus.

\section{( $\mu .35$. \\ XXXIII. CRIOCERIS,}

85. C. Capreae thorace nigro maculato, elytrisque grifeis, antennis nigris, F. Sp. I 50.4.

of Galerucr Caprone. F. E. S. I. 2. 2 I. 40. Abdomen et femora ante apicem nigra, tibiis tarfisque concoloribus, Hab, in Salice. Long, $2 \frac{1}{2}$. lat. $I \frac{1}{2}$.

Citatum e Degeerio Inf. 5. 325. 30. tollendum videtur, quia cum fpeciminibus noftris $C$. Capreae $F$. non conuenit. Hg.

\section{LAGRIA.}

86. $L$. aenea antennis ferratis, hirta obfcure. aenea. F. Mant. 94. 23 . 
Defcribit eam Fabricius. Hab. in floribus fiequells.

In Mantifia Fabricii, quae vitiis typographicis abundat, in Lagriarum genere duae fpecies funt, quae ainene vocantur, quarum prior p. 93. n. I. Dryops aenea, Ent. Syft; pofterior p. 94. n. 20 . L. metallica Ent. Syft. I. 2. 8 I. r 5. facta eft. Quam cum Fabricius Barbariae indigenam dicat, aliam credere licet. $\mathrm{E}$ breni certe defcriptione, quam Fabricius exhibet, iudicare nolo, quamuis illa Lagrià, quan Auctor fpectat, etiam in noftris regionibus, licet rariffime, occurrat. In Fn. Etr. 38. 86. ad Melyrin bimaculatum iam monui, Lagrias huic metallicae fimiles a $L$. hivta dirimendas effe, quod Oliuierius genere Melyrinn fecifie videtur. Hg.

* 87. L. viridis aeneo-viridis hirta, thorace marginato, antennis aeneis.

Praecedente minor. Tota aenea viridis nitida ad cyaneum vergens. Antennae aeneo - nitidae longitudine thoracis. Thorax lateribus, pofticeque marginatus, planiufculus, feu minus quam in fuperiore conuexus, hirtus. Elytra vti thorax aeneo - viridia punctulata marginata, minus hirta. Abdomen, et pedes toti elytris concolores. Variat magnitudine duplo minori, colore viridi-fubaurato. Hab. in floribus frequens.

\section{(p. $3^{6 .)}$ \\ XL. CVRCVLIO.}

* Longiroftres femoribus jimplicibus.

88. C. Bardanae longiroftris cylindricus, grifeotomentofus, pedibus anticis elongatis. F. Mant. 104. 84 . 
车 F. E. S. I. 2.418 .107$.

C. cylindricus. Herbft. Arch. IV. ZI. 20.

Statura et magnitudo fere $C$. paraplectici. Iunior totus tomento-grifeo tectas, adultior niger, et glaber euadit. Elytra rotundata, obtufa. Pedes anteriores parum elongati. F.

In hoc elytia fubtilifime ac laeuiter punctatoftriato; in $C$. puluerulenio Scop. qui forte eft $C$. anguftatus $F$. transierfim rugola. Habit. in Arctio Lappa, aliisque plantis non rarus.

C. Bardance $F$. qui magnitudine variat, faepins cum $C$. anguftato $F$. confunditur, a quo tamen, vti Auctor bene monuit elytrorum fculptura plane differt. Sed Dn. Roffius ipfe Dn. Langio $C$. Bardanae fpecimen nomine $C$. angufati mifit. Elytra $C$. angufiati quidem transuerfim rugofa funt, fed nihilominus punctorum, nullo modo vero conniuentium, feries longitudinales obfcurae afpiciuntür.

c. Birdande faepius cinereo afperfus occurrit, interdum vero flauicans eft; imo fpecimina vidi, cinerea quider, fed fupra puluere ferrugineo tecta. Roftrum longitudine variat. $\mathrm{Hg}$.

* 89. C. fanguineus longiroftris cylindricus, lruber, oculorum fupercilio, thoracisque lateribus flauefcentibus.

Statura et magnitıdo C. Afcanii, at elytra apice obtufa. Roftrum tloracis longitudine, totum fupêrne fanguineum, inferne cinereum. Antennae breues; fractae, ciriereo - fufcae. Caput fanguineum, quod vbique inflectit. Oculi nigri fuperciJio flauo. Thorax láeüis fanguineus, margine laterali flauo - lineato. Elytra laeuia, fanguinea, apice 
obtufo. Stemum et abdomen cinerea. Pedes cinerei femoribus dilute fanguineis. Color idem, qui in Call. Sanguineo, et cuin in tenuifinna pube confiftat, facile attritu deletur; et tunc niger eua: dit. Lectus Pilis in Verbalci foliis d. 24. Mart. Long. a bafi roftri ad apicem elytrorum 4. lat. I.

C. Barlanae quidem eodem fere modo variat, vt hic Curculio defcribitur; fed vix coniicere auderem, AuEturem hanc varietatem feparafie, èt fpeciem peculiarem ex ea feciffe. Hg.

$$
\text { ( } p .37 .)
$$

90. C. cupriroftris longiroftris, oblongus, viridiaeneus, elytris firiatis; roftro cupreo. F. Mant. 100: 43 .

\& Attelabus cupriroftris. F. E. S. I. 2. 289. 25? Magnitudo C. Graturii. Corpus oblongum nitidum: Roftrum arcuatum cupreum. Thorax viridi-aereus, laeuiffumus nitidus. Elytra fubtilifine ftriata, viridi - aenea nitida. Abdomen viride. Pedes fulci. Habit. in Alno non rarus.

91. C. caierulefens longiroftris, obfcure caeruleus; elytro fingulo decemftriato, roftro deflexo. Schrank. E. I. 202.
C. cierulefiens. Scop. E. C. 87 .

ㅇ C. caeruleo - viriciis nitens, thorace punctato, elytris ftriatis. Geoffi. Inf. I. 284. 16.

C. viridis. Fourcroy Ent. Parif. x. I20. I6.

Statura praecedentis, at latior. Roftrum tenue longum, deflexum. Thorax excauito-punctatus, etiam inferius. Elytra fnbtilifime friati friis decem inpunctatis. Femora mutica. Raro lectus. Long. $I \frac{1}{2}$. lat. $\frac{2}{3}$.

Variat colore viridi et cyaneo. Statura $C$. gronctrio nonnihil fimilis. $\mathrm{Hg}$.

$$
\text { Á } 5 \quad * 92 \text {. }
$$


* 92. C. timidus longiroftris ater laeuis, nitidus: elytris fubitriatis, roftro craflufculo incuruo.

Magnitudo fere $C$. Betulae, at paullo oblongior, et corpore ouato globofo. Tutus ater nitidus. Roftrum craffufculum incuruatum antennis nigris. Thorax latus. Elytra fubtilifime punctato - ftriata. Fcmora inermia. Alatus. Hab. in pratis filuaticis frequens.

$$
\text { (p. 38.) }
$$

* 93. C. perpenfus longiroftris, grifeo - fufcus, thorace, elytrisque hifpidis, cinereo nigroque obfolete punctatis.

Statura C. Antimhini Fn. Etr. Roftum tenue, porrectum. Elytra ftriata. Feinora mutica. Tibiac teftaceae. Hab. in filuis. Long. x. lat. ${ }_{2}^{\mathrm{r}}$.

94. C. Rumicis longiroftris, grifeus, nigro-nebulofus, antennis fufcis. F. Sp. I 7०. 55 .

$\odot$ Rai.

\& F. E. S. I. 2. $4 \times 3 \cdot 86$.

Magnitudo variat. Habit. in Rumice frequens. Long. 3. lat. I.

Limnaei C. Rumicis Syit. Nat. 2. 6ז3. 60. Fn. Sv. 590 . ob femiora dentata huc non fpectat. Hg.

* C. infidus longiroftris, piceus, nitidus; thorace punctato, femoribus clauatis, compreffis.

- Statura $C$. grantrii, cui valde affinis, at maior, et diftinctifimus. Difiert a $C$.granario praeter magnitudinem quae fere dupla, colore piceo nitidiore; roftro magis porrecto; thorace diuerfimode punctato punctis rotundioribus. Elytra in hoc thorace longiora nitida, manifefte punctato-ftriata. Femora mutica, clauata claua compreffa, imprimis 
primis antica. Tibiae brenes, comprefrae. Sirbtus concolor nitidus punctulatus. Hab. Pifis.

96. C. cintins longiroftris globofus rufus, elytris ftriatis fafcia transuerfa alba, Geoilr, Inf, I.2. 89. 28 .

C. Erythrolencos. Ed. Linn. I3.

Magnitudo C. rufiroftris. Roftrum tenue nigrum. Antenuae ferrugineac claua apice cinereo. Hab. in plantis non frequens.

$$
\text { (p. 30.) }
$$

* Longiroftres femoribus dentatis.

* 97. C. binotatus longiroftris, fufcus, elytris. fubdepreffis, pone medium puncto albo notatis.

Statura C. Colon, at maior. Roftrum mediorre craffulculum carinatum longitudine thoracis. Tho-. rax fufcus, fubcarinutus, linea laterali cinerea obfoleta. Elytra bafi fubdepreffa, obfolete punctatoftriata, fufca, pone medium anguftata, gibba, puncto vtrinque albo. Antennae pedesque fufci femoribus fubdentatis. Apterus. Color fufcus in tefaceum vergit. Habiat in filueftribus paludofis; hieme in truncis arborum putridis minime rarus.

Hic Curculio idem videtur, quem Schallerus. Aet. Hall. I. p. 283 . C. copucinnen vocat, et vbi Schaeff. Ic. t. I 60 . allegat, quae certe potius hunc, quam $C$. Colon $F$. repraefentat, ad quem ab Harrero relata eft. - Forte C. binotatus F. E.

S. I. $2.484 \cdot 379$. huc referendus eft? $\mathrm{Hg}$.

* 98. C. tigratus longiroftris? femoribus dentatis, brunneus fuluo - maculatus, thorace depreffo carinato, elytris fulcatis punctatisque.

Magni- 
Magnitudo C. Abietis. Totus brunneis maculis laete ferrugineis afperfus. Antennae fractae, pilofae, claua fubrotunda craffufcula. Roftrum mediocre carinatim fulcis tomento fuluo contectis. Thorax rotundatus, depreffus, carinatus maculis fafciisque ferrugineis ordine difpolitis. Elytra fulcata lineis eleuatis, abdominis latera admodum inuoluentia, @àlita, fuluioque vindique macilata. Femora omnia dentata annulo fuluo. 'Inter fingulos fulcos ftriae duae, punctoriun imprefforum. Hab. in filuis non frequens.

* 99. C. bipuftulatus longiroftris, femoribus dentatis niger, fubpilofis, elytris friatis, plaga vtrinque rufefcente.

Statura C. V̈erbafci quo paullo minor. Roftrum tenue antennis nigris. Elytra late ftriata, fubpilofa, maculan in medio gerentia infignem rotündam rufefcentem. Femora dentata, craflufcula. Lecas raro.

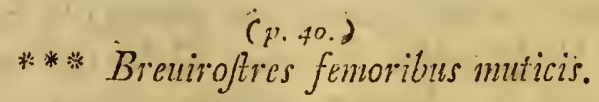

* roo. C. caniefcens breuiroltris, cinerens nitidus fubpilofizs, elytris nigro maculatis.

Minor C. incano, et corpus magis onatum, magisque globofum. Caput cinereum roftro craffo breuiffimo. Antennac bienifimae fractae cinereae claua fubrufa. Thorax cinereus in medio oblcurior. Elytra cinerca fubpilofa, leuiter punctatoftriata, punctisque inaequalibus nigris afperfa, coalita, âpiceque parum acuminato. Color totius corporis albido - cinereus nonnihil in aeneün vergens. Lectus pluriés fub arborum cortice tempore hiberno. Long. 4: lat, 1s. 
* Iо I. C. crudutus ouato-oblongus, thorace elytrisque fcabris, apice acuminatis pilofis.

Naior C. Arinadillo, et oblongior. "Totus niger fubobfcurus. Roftrum brene, craf̣um, bifulcatum, apice dilatatum. Antemne in medio fractae longiores. Thorax ac elytra punctis eleuatis fcabra, eaque magnam partem abdominis obtcgentia, minime ftriata, apice acuminato, pilisque fubferrugineis hirto, quafi penicillato. Abdominis bafis inferue compreffa, retufia. Femora omnia clauata inermia. Alae mullae. Apex elytrorum nunc magis nunc minus pilofus. Dubitaui de C. Nigrita, ac defcriptio minime conuenit, et fpecies noua vi: detur. Hab. Florentiac. Long. 6. lat.2. et vltra.

\section{(p. 4r.)}

I02. C. coftatus breniroftris cinereus, thorace nigro: lineis quatuor cinereis. F. Mant. I I 5 . 194. C. bilineatus Fn. Etr. I. 1 16.28 I.

Defcriptio Fabriciana $C$. coftati iterum exhibetur. Hab. in pratis fiequens.

Confer adnotata ad C. bilineatzmm. Fn. Etr. 1. c. Hg.

* I03. C.Vintor breniroftris? niger, oblongus, thorace elytrisque fquamofo - aeneis fubnitidis.

Mágnitudo fere C. pyri, at ftatura diltinctus, et colore etiam minus nitido. Roftrum mediocre craffulculum longitudine thoracis. Antennae breues fractae fufcae claun fubcinerea. Thorax lateribus fquamofo-aeneịs-obfcuris. Elytra mutica. Ademta pube aeneo - rufefcente totus niger apparet. Lectus Pifis. Long. $2 \frac{1}{3}$. lat. I.

I04. C. Scabriculus breniroftris, corpore cines reo hilpido. F. Sp. I89. 159. 
\& F. E. S. I. 2. 460.3 เ3.

C. Viuerra. Herbft. Arch. 4. 83. $77^{\circ}$

Magnitudo $C$. cernini. Totus cinereus, Roftrum breue canaliculatum. Antennae craffuffculae vix longitudine roftri. Elytra ftriata hifpida, praefertim poftice. Femora inermia. Raro lectus in locis arenofis Pifani nemoris. Long. $I_{2}$. lat. I. vix.

In Fauna Etrufca I. 138.334 . Herbftii Arch. $t$. 45. f. 7. allegaui, quod citatum tamen delendum eft, cum ad hirfutulum F. E. S. I. 2. 468. 312. pertineat. C. Viucrra. Herbft. 1. c. contra huic annumerandus eft. $\mathrm{Hg}$.

(p. 42.)

* I05. C. breuiroftris breuiroftris, ater, fubnitidus, elytris punctato - fulcatis, roftro latitudine capitis.

Paullo maior $C$. granario, a quo praecipue differt thorace elytris breuiore, roftro breuiffino crallo latitudine capitis. Ater nitidus, fed minime lucens, glaberrimus. Antennae breuiffimae, nigrae apice cinereo. Caput punctulatun, at refpectu thoracis laeue. Thorax excauato - punctatus. Elytra punctato - fulcata, connata. Femora fubclauata inermia. Hab. in agro Pifano.

* Io6. C. limofus breuiroftris, niger obfcurus, thorace elytrisque limo exficcato obductis, antennis rufis.

An C. lutofus. Mur. Lesk. p. 22. 11. 482 ?

Statura $C$. lineati, quo duplo maior. Niger, fed totus femper crufta terrea contectus. Roftrum breue, craffum, nigrum. Antennae nufae claua fufco-nigra. Thorax fubglobofis. Elytra ademto limo nigra ftriata. Femora mutica: antica fubclauata. 
Abdomen cinerafcens. Hab. Florentiae et Pifis fat frequens.

* Io7. C.vifus breuiroftris, fufus, elytris fafciis duabus marginalibus cinereis, antennis, pedibusque teltaceis.

Nedius inter $C$. lineatum et $C$. argentatum. Antennae fractae teftaceae claua obfcuriori, apice cinereo. Roftum breuifimum. Caput et thorax tomento flano lucido fubaureo - tećta. Elytra contuexa ftriata, flauo - tomentofa durfo fufco, fafciis duabus cinereis, prima obliqua prorfus marginali; altera apicis vsque ad futuram producta. Abdomen fufco nitiaum, Pectus macula vtrinque cinerea. Pedes teftacei tenues, femoribus muticis, et verfus apicem cinereo-annulatis. Alatus. Hab. in plantis non rarus.

$$
\text { (p.43.) }
$$

* I08. C.Amandillo breniroftris, femoribus fub. dentatis, niger, elytris crenato-ftriatis, friarum forlulis cinereo - flauefcentibus.

Corpus ouato - globofum nigrum. Antennae fractae articulo primo praelongo. Roftrum brene craffum bifulcatum. Thorax fubrotundus punctis eleuatis fcaber. Elytra connata, admodum conuexa, crenato - ftriata pilis fordide cinereis breuiffinis in ftriarum foueolis hinc inde afperfa, vnde cinereo veluti puluere inquinata apparent. Fe. mora clauata fubdentata. Alae nullae.

Defcriptio C. Nigritae Fn. Etr. I4I. 340. ad hunc, male pro $C$. Nigrita affumtum, referenda. Hab. in filuis frequens. Long. $4 \frac{1}{2}$. lat. $3 \frac{1}{4}$. 
199. C. Sulcatus breuiroftris, femoribus dentatis ater, elytris ftriatis, ferrugineo-maculatis. F. Sp.

- 197.212.

+ F. E. S. I. 2. $485 \cdot 382$.

C. grifeopunEtatils. Degeer. 5. 350.9?

Femora omnia apice clauata, poftice etiam acute dentata. Differt a C. Armadillo praefertim ftatura oblongiori, et macularum colore non fordide cinereo, fed ferruginco-flauefcente. Elytra crenato - ftriata. Magnitudine variat. Rarolectus hieme fub arborum cortice.

\section{RHINÓMACER.}

I Io. R. Brentoides glaber piceus, thorace punctato, elytris punctato - frriatis. D. D'Antic Fn. Etr. I. I44. 347. t. 3. f. I4.

Pimelin anguftata. F. Sp. 320. 24. Mant. 209. 28 ? Hab. fub arborum cortice obulus.

Citatum e Fabricio, in Fn. Etr. 1. c. a me quoque, probatum, delendum eft, quia aliud omnino diuerfum infectum defignat, quod, in Tranquebaria captum, amicus meus Dalidorfius mecum communicauit. Comparationem amborum, primo intuitul fimilium, adiiciam.

Habitus idem quidem in vtroque, hinc eidem annumeranda funt generi; $P$. angufata $F$. autem, vt e multis fpeciminibus iudicare licet, paullo longior, femper vero duplo craffior eft. In $R h$. Brentoide Roff. caput latitudine longins eft, in $P$. anguftuta $F$. autem tertia parte latius. Idem de thorace obferuatur, qui in pofteriore multo latior et depreffus, in priore longior atque fubcylindricus eft. Caeterum caput et thorax vtriusque punctatus eft, punctis in Rh. Brent. multo maioribus et ráriori- 
bus, in $P$. anguft. autem minimis atque contignis. $P$. anguftutae thorax antice fere eandem latitudinem habet cum elytris, in $R h$. Brent. autem dimidia parte anguftior eft. Elytra huius gibba; friarumque puncta admodum profunda funt, quae in $P$. anguftata vix oculo armato confpiciuntur. Pedes in hac nigri, nec picei funt,

Hic locus dictum noftrum, faepius vfitatum, confirmat: Ex patria admodum diuerfa, infecta in figuris et deferiptionibus fimilia, tamen diuerfa iudicanda effe. $\mathrm{Hg}$.

\section{(p. 44.) \\ XLII. ATTELABVS.}

* I I I. A. bicolor cupreus, hirtus, elytris faturate teftaceis.

Statura et magnitudo A. pubefcentis, at paullo latior. Roftrum temue elongatum longitudine fere dupla thoracis, fubrufum, apice dilatato, bifido. Antennae fubrufae, recte moniliformes, medio roftri infidentes, claua fufca. Oculi prominuli nigri. Caput, thorax, abdomen, femora cuprea, quafi fubaurata hirta. Thorax punctulatıs. Elytra punctato - ftriata hirta faturate teftacea. Tibiae fubrufae. Hab. Florentiae ra. rus.

\section{CLERVS.}

* I1 2. C. vniuittatus niger, elytris fafcia media vilica alba.

Statura C. vinifafciati, at paullo minor. Antennae nigrae bafi ferrugineae. Caput thoraxque nigra pilofa, minus tamen quam in $C$. vnifafciato. $\mathrm{Bb}$ 
Elytra nigra, nitida, ftriato-punctata, fafcia media recta vnica alba. Abdomen nigrum. Pedes ferruginei femoribus nigris. Alae fuicefcentes. Hab. Pifis rarus.

\section{(p. 45.) \\ XLIV. NOTOXVS.}

* I 13. $N$. trifrifciatus thorace in cornu fupra caput protenfo, elytris trifafciatis. Fn. Etr. I. 149. 354. B.t. 2. f. r. 4 .

Notoxus, quem habet Io. Moraeus Vpl. Nou. Sp. Inf. I 789. ab hoc dinerfus. Nofter in Suecia non habitat.

Statura et lrabitu omnino fimilis $N$. Monocero, at fpecies fane diftincta. Differt magnitudine paullo minori; elytris nigro trifufciatis; abdomine fufco. Elytrorum apex fafcia nigra terminatus. - Habitat menf. Iun. et Iul. in Salice, Bignomia radic. aliisque plantis frequens copiofus. Amare videtur folia glabra rmbraque tecta, quae - velociter curfitando huc, illuc percurrit.

* I 14. N. pedffris niger thorace elongato rufo, elytris pubelcentibus, puncto vtrinque bafeos fubrufo.

Coralus pedefris. Fn. Etr. I. $270.55 \%$.

\& $N$. thoracicus. Schneideri ap. Panz. Fn. Inf. Germ. XXIII. 6.

Proximus Cantharidi Geoffr. I. 344. 8. La Cantharide fourmi, quae forte huius generis.

Magnitudo N. trifufciati. Antennae nigrae filiformes longitudine dimidii corporis, articulis breuibus vltimo oblongo acuminato. Palpi quatuor fecuri- 
fecuriformes nigri. Caput paullulum a thorace remotum collo tenuiffimo, nigrum nitidum deflexum, et conformatum vt in $N$. monocero, ac trifafiato. Thorax elongatus rotundatus, antice latior latitudine capitis, poftice anguftatus, ' punctulatus rufus. Elytra integra conuexa, thorace duplo latiora, poftice praefertim albo pubefcentia, nigra, puncto bafeos vtrinque fubrufo. Pedes nigri femorum bafi tenui ferruginea. Alae albae. Hab. in terra, arena, ligno putefcente frequens. Long. I $\frac{1}{3}$. lat. $\frac{1}{3}$.

Citatum e Geoffroyo exhibitum, ad $N$. floralem $F$. pertinet. Hic adnotandum eft, quod in Fn. Etr. Ir 5. 279. apud Lagricm floralem omifimus, N. florilem Fabr. numquan maculis duabus ouatis transuerfis parallelis occurrere, vt ibi defcribitur, fed elytra nigricantia, bafin verfus rufa effe, qualia Geoffroyus 1. c. defcripfit. N. floralis Aut., N. antherini eiusdem varietas iunior videtur, coiore nigro adhuc fufco? Meloe floralis Linno, eadem videtur cum $L$. florali Ro/f. Celeberrimus Fabricius itaque in Ent. Syft. I. x. 212. 10. optime id citatum incertum exhibet; plane potius tollendum videtur. Caeterum $N$. floralis $F$. citatis Schrankii Meloe pedicullarius. En. Inf. Auftr. 422. adiici poteft. De citato e Panzeri Fr. Inf. Germ. adiecto, non dubirc, ipfe enim e Vindobona Notoxum accepi, qui non magis cum figura data, quam cum defcriptione Roffii quadrat. - Rečte quaerere videor", cur Auctor Lugriam cutherinam atque floralem . Fau nae, his Notoxis non annumeraret, quibus tamen ftatura proximae funt. Hg. 
* I $5 . N$. cinctellis niger, thorace elongato, elytris macula vtrinque bafeos; cruceque poftica futurae conmuni fubrufis.

Statura magis, quam in praecedente cylindrica, et paullo ninor. Caput nigrum thorace vix latior. Palpi antennaeque nigrae. Thorax niger antice rotundatus, poftice nonnihil anguftatus, emarginatus, vix latitudine longior. Elytra thorace duplo laticra, minutifime punctata, integia comexa, nigra, macula ad bafin vitrinque fere trigona, poficcque cruce ad futuram commuii faturate teftaceis notata. Bafis ad fcuteilum, atque apex nigra. Abdomen nigrum. Pedes nigri tibiis pallide ferrugineis. Elytra paullo minus quam in praecedente conuexa. Hab. fub corticibus rarus.

Huius Notoxi deferiptio exalte cum $N$. aniherino $F$. congrnere videretur, nifi hic iam in Fn. Etr. exhibitus effet. Hic adnotandum eft; figuram Panzeri Fn. Inf. Germ. XI. I 4. in elytrorum pietura non fatis conuenire, certe varietatem defignare, cum fafcia pofterior rubra tam lata fit, vt elytro'rum apicem totum occupet, et in fingulo elytro maculam nigram mediam circumdet. Hg.

* I 6. N. hifpirius niger hifpiảus, tibiis antennisque teftaceo-pallidis, elytris macula bafeos flaua.

Facies N. pedeftris, at paullo minor. Antennae teftaceo-fufcae moniliformes. Caput nigrum, nitidum, deflexum, punctulatum, latitudine thoracis., Oculi prominuli. Thorax niger hifpidus, admodum conuexus, fubglobofus. Elytra obfcu- 
re nigra hirfitifima, integra, macula magna bafeos flaucfeente, Subtus totuis niger hifpidus, demtis tibiis pallide teltaceis. Alatus. Lectus fub foliis disiectis.

* I 1 z. N. Myrmicocephahus fermgineus, laeuis, glaber, capite thorace latiore, elytris apice obtufis.

Statura et magnitudo $N$. peneftris. Caput maghum obfcurc ferrugineum. Palpi, antennaeque ferrugineae apice craffiori vti in praecedente, Thorax ferrugineus, elongatus, capite angutior, connexus, fubpunctatus. Elytra ferruginea lacuia connexa integra, apice obtufo. Subtus ferrugineus abdomine obfcuriori. Alae albac. Habitat ...

$$
\text { (p. 4r.) }
$$

* ${ }^{-}$i \&. N. hifpidulus hifpidus, thorace, elytrisque obfcure teftaceis, capite punculato nigerrimo.

Statuma et magnitudo N. Non plus vitra. Antennae, palpique teftacei. Caput nigerrimum punctulatum, latitudine thoracis. Thorax anguftus, elongatus, conuexus, fere cylindricus, immarginatus, punctulatus, obfcure teftaceus, pilofus. Elytra teftacea pilofa, integra, punctulata. Subtus omnino obfcure teftacens. Differt a $N$. Non plus vitra, colore, forma thoracis anguftiori, magisque elongata, capite nigro, ac hirfutia magis confpicua. Hab. fub arborum cortice hieme.

* I 9. N. Non phus vitra flauus, oculis nigtis, elytris antennisque pilofis fubclauatis.

Carabus Non plus vitva. Fn. Etr. I. 272. 566. Pediculo aequalis corpore toto flauo. Statura et habitus praecedentium. Antennae ad apicem crafflores articulis fere rotundis, approximatis pilofis, $\mathrm{Bb} 3$

vltimo 
vltimo maiore acuminato. Thorax minus quam in fuperioribus elongatus, conuexus pracfertim bafi. Elytra punctulata, pilofa. Hab, in quisquiliis non: frequens.

Libenter N. minutum. Panz, Fn, Inf. Germ. XXIII. 5. allegarem, et huic citato PSeluphum Hellu'igii. Herbft. Kaef. t. 39. f. I2. a, adiungerem. Hg.

* 120. N. tenuicollis laeuis, capite thoraceque nigris, antennis elytrisque obfcure teftaceis, pedibus pallidis.

Duplo minor $N$. peedeftre. Corpus totum tenuiffima pube fubobfcurum. Antennae longiusculae filiformes, apice vix craffiores obfcure teltaceae. $\mathrm{Ca}$ put nigrum thorace fere maius. Thorax conuexus rotundatus, poftice paullo anguftior, niger. Elytra integra, laenia, capite thoraceque longiora teftaceo-fufca. Abdomen nigrum. Pedes pallidi. Alae albae. Lectus tempore hiberno fub corticibus, at aeftate etiam in plantis.

\section{$(p .48$.}

* 121. N. 4-guttatus hifpidus, antennis pedibusque teftaceis, elytris maculis quatuor obfcure flauis.

Statura praecedentis, magnitudo $N$. trifafciati. Palpi, antennaeque obfcure teitaceac. Caput nigrum hirtum. Thorax fubglobofus, minus elongatus, poftice vix anguftior, latitudine capitis, punctulatus, niger, hirtus. Elytra integra hirta, tenuiffime punctulata, bafi nigra ${ }_{2}$ mox macula vtrinque obfcure flaua fere marginem attingente, dein falcia nigra, tum macula altera minori obfcure flana rotunda, apice denique nigro. Abdomen nigrum. Pedes teftacei.

Aliud 
Aliud fpecimen habeo quod differt a praecedente palpis, antemnis pedibusque nigris; elytris maculis quatuor fubrufis, paullo maioribus, quafi in fafciam connatis, corpore non hifpido, fed pubefcente. An fatis diftinctus?

Occurrit cum aliis.

- 122. N. bifuficiatus glaber, nitidus, thorace antice nigro, poftice ferrugineo, elytris nigris, fâfciis duabus luteis in medio interruptis.

Habitus ac ftatura fere $N$. cinctelli, at dimidio minor. Caput nigrum laeue, nitidiffinum. Palpi, antemneqque ferrugineas. Thorax capite paullo minor, elongatus, conuexus, poftice nomihil anguftatus, forma magis cylindrica, laeuis, nitidus, antice' niger, poftice ferrugineus. Elytra laeuia, nitida, punctulata, nigra, fafciis duabus luteis in medio late interruptis, feu maculis quatuor luteis; .binis prope bafill, et binis pone medium notata: hae maculae tamen marginem exteriorem attingunt, et apex fummus ad fitturam flanefcit. Pedes ferruginei femoribus apice nigris. Lectus fub arbornm cortice Mart. m.

N. neftarino. Panz. Fn. Inf. Germ. XXII. 7. affinis videtur. $\mathrm{Hg}$.

$\mathrm{Bb} 4 \quad$ XLVII.

* Ad fpecies Notoxi quod attinet fupra relatas, monendum, fumman differentiam confiftere in antennis articulis tribus vltimis crafioribus; reliquum, palpi in omnibus fecuriformes, omniumque facies, ac habitus idem, q:ii in $N$. pedeflire, peritiorum etiam fententia habito pro vero Notoxo. Etfi tamen de affinitate inter $\cos$ maxime dubitari nequeat, fi in aliquem ex defcriptis, fortaffe generis error irrepferit, gratia praefertim impotentiae eos rectius examinandi, (cum nonnulli in Sueciam miff ad confulendum D. de Paykull. cafu perierint,) indulgeant mihi Entomologi. Adiroor. Antit. ipf. 


\section{(p. 49.) \\ XLVII. CERAMBYX.}

I23. C. fafciculatus thorace fpinofo, elytris integris, punctis tribus hifpidis, antennis mediocribus hirtis. F. Mant. I 34. 37 .

क F. E. S. I. 2. 262. 39. Panz. F. I. G. XIV. 15. Degeer. Inf. 5. 7x. 9. t. 3. f. I7.

Paullo maior C. hifpido. Antennae longitudine corporis, articulis bafi cinereis, apice fufcis, hirtae. Caput et thorax fufca. hirta. Elytra fufca hirta lineolis eleuatis; verfus balin pallidiora, quafi fafciata fafcia ad marginem exteriorem diducta vsque ad elytri bafin, fafciculis, feu punctis tribus nigris hifpidis, apice omnino integra. Pectus ct abdomen cinerea. Pedes grifei. Raro lectus. Long. $2 \frac{1}{4}$. lat. $\frac{2}{3}$ vix.

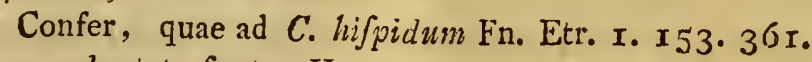
adnotata funt. $\mathrm{Hg}$.

$$
\begin{gathered}
\left(p . \text { so.) }^{\circ}\right. \\
\text { XLVIII. LAMIA. }
\end{gathered}
$$

124. L. vnifufciata thorace inermi ouato, fordide nigro, elytris fufcis nigro - punctatis, fafcia media atra flexnofa flatno-marginata. Fn. Etr. r. 147. 370 . t. r.f. 5 .

Cerambyx fafciatus. Villers Ent. x. 239. 38 . videtur omnino diuerits, vti figura Schaefferi ab eo citata apertiffime oftendit. Thorax in meis fpeciminibus nullo modo fpinofus. Elytrorum fafcia non fufca, fed atra et flexuofa. Hab. fub arborum cortice rara.

Schaefferi Ic. t. 192. f. 9., a Villers citata, C. griSeum F. E. S. I. 2. 26r. 37. defignare videtur, 
qui in mufeis quoque nornine C. longicomis Schrseideri occurrit, et cuius effigiem optimam Celeb. Panzerus in Fn. Inf. Germ. XIV. I 4 . dedit. $C$. fafiatus Villers itaque, ex citato, C. grifens $F_{\text {. }}$ videtur. Hg.

\section{SAPERDA.}

125. S. erytirocephata thorace rotundato villofo, rufa, antemnis, pectore elytrisque nigris: $F$. Mant. I48. I3.

D Herblt. ap. Fuesly. Arch. Inf, 5. t, 26, f, 3. Vil= lers Ent. I. 24.3 .5 I. t. I, f. 28 .

\& F. F. S. I. 2. 3Ir. I9.

Media inter $S$. oculatan ct cylindricam; fatura eadem. Caput rufum maxillis, antennisque nigris meaiocribus. Thorax cylindricus villofus rufus, margine antico ct poftico nigris. Elytra glabra cinereo-nigra culore elytrorum S. oculatae, codemque modo punctulata. Pectus nigricans. Abdomen rufum macula magna bafeos nigra. Pedes rufi. Alae nigrac. Lécta in fepibus menf. Iun.

Thoracem interdum totum fere nigrum efle, iam Schrankius et Herbftius adnotanere. Minores thorace nigro efie folent, macula pofteriore rufa. $\mathrm{Hg}$.

\section{(p. 5r.)}

126. S. linearis cylindrica nigra, pedibus luteis, antennis mediocribus. F. Sp. 232. 6 .

. $)$ Herbft. ap. Fuesly. Arch. Inf. 5. t. 26 . f. I.

Schaeff. Ic. t. 55 . f. 6.

q F.E. S. I. 2. 309. I2. Panz. F. I. G. VI. I4. Roefel. Inf. 2. Scar. 2: t. 3 .

Paullo maior $S$. cylindrica, et magis nigra. $A b$ dominis dorfum fupra teftaceum. Saepe lecta ruri in floribus.

$\mathrm{Bb} 5$

Duplo 
Duplo maior eft S. cylindrica. De citato Roefelii confer Fn. Etr. I. I64. 374 , adnot. Hg.

I27. S. lineola nigra, thoracis linea dorfali, femoribusque apice rufis. F. Sp. $235 \cdot 26$.

1) Herbft. ap Fuesly. Arch. Inf, 5. t. $26, \mathrm{f}, 4$.

ㅇ․ F. S. I. 2. 3 r $8 \cdot 53$.

Statura $S$. cylindricae, at paullo minor, Nigra villofula: Antennae lungitudine corporis, apice non attenuatae nigrae. Linea rubra in dorfo thoracis, Elytra concoloria. Abdominis apex, femora omnia apice, vti et tibiae anticae rubra. Variat abdomine, pedibus fecundi et tertii paris totis nigris: femina. Hab. in pratis filuaticis menf. Iunl. non frequens,

\section{CALLIDIVM.}

128. C. Salicis thorace tuberculato fpinofoque rufum, pectore elytrisque nigris. F. Mant. 154 . 38 .

Generis in hoc Infecto incertitudo, fummaque apuid nos raritas, clim vnum tantum fpecimen habuerin colore antennarum, elytrorumg̨ue paullulum diuerfum a $C$. Sutlicis F. ofcafionem dedit mihi affumendi in Fin. Etrufca I. I63. 373. t. I. f. 4. pro noua fpecie. Ad hoc igitur referantur defcriptio, et figura 1.c. exhibitae, et fallum $R$. Eirufici nomen deleatur. Videtur Stenocorns. Lectum Florentiae.

Infecta quaedam fingulari fortuna vtuntur, quod eadem fere tempeliate a pluribus deferibuntur et nomine donantur. E quorum numero etiam hoc infectum elt. Fabricius, Herbftius, Schrankius, 
Laichartingius, Roffius, fingulus alio nomine hoc infectum defignauere. Commodum imo nouum citatum, a nullo, quod fciam, adhibitum, vidico: Cerambyx gloucoptcrus. Schaller. Act. Hallenf. I. p. 294. t, I. f, 4., qui varietatem illam defcripfit, cui elytra grifeo-teflicea funt, cuius mentionem in Fn. Eir. 1. c. feci, fed et elytra interdum nigra efle commemorat, $\mathrm{Hg}$.

\section{(p. 52.)}

129, C. clauppes nigrum onacum, femoribus omnibus, clauatis, antennis longioribus. F. Sp. 236.4 .

D Herbf. ap. Fuesly, Arch. Inf. 5, t. 26. f. 9.

of F, E, S. I. 2.320 .

Statura et magnitudo C. Bainli. Nigrum opacum. Antennae in noftris (fortaflo feminis) corpore paullo breniores, craffae, articulis apice interno fpina terminatis. Thorax fubrotundus, depreffus, vti totum corpus. Femora omnia valde clauata. Alae fufcac. Hab, in finis rarum. Long. 8. lat. $2 \frac{1}{2}$.

Antennarum longitudo variat. Specimen maius, quod feminam habeo, antennis aliquanto longioribus eft, quam aliud, multo minus. $\mathrm{Hg}$.

I30. C.variabile thorace glabro, corpore fuicoaeneo, antennis pedibusque fufcis.

Simile $C$. violaceo, fed minus. Variat colore: alterum violaceum; thorax fubferrugineus, pedes ferruginei, femora clauata, nigra. Alterum thorace elytrisque linidis, femoribus fimilibus. Fn. Sv. 669. Hab. in Pinetis. Long. $4 \frac{1}{3}$. lat. I.

Conferantur adnotata mea ad C. Fennicum. Fn. Etr. r. 170. 384. Confiteor, me difecrnere non poffe, vtrum Auctor fub C. variabili fuo, C. Fennicum 
$F$. an $C$. aeneun Herbftii fpectet. Prius tamen potius credo, quia Linnaei defcriptionem $C$. variabilis $L$. appofuit, quod tamen idem cum $C$. Fennico $F$. videtur. $\mathrm{Hg}$.

13r. C. gibbofun thorace rotundato nigrum, elytris cinereo - fafciatis, bafi bituberculatis, apice aciminatis. F. Mant. I56. 62 ,

Noftum Infectum variare in multis videtur a C. gibbofo $F$. Vtrum fit noul fpecies, a defcriptione periti indicabunt. Statura C. myjfici, at fere dimidio minus. Antennae corpore paullo breuiores, fubpilofae, articulis bafi obfolete cinereis, apice ferrugineo - fufcis, fpinaque laterali parmm perfpicua inftructis. Caput et thorax nigra obfcura immaculata tomentofa, vti totum corpus. Thorax non tantum rotundatus, fed globofus, et in medio valde. connexus. Elytra bafi obfcure nigra, et prope futuram vtrinque valde tuberculofa fere vti etịam in C. myffico obferuantur. In medio elytrorum fiafcia terminat lata cinerea, cui fuperius incumbit ftriga linearis arcuata nigra: mox fequitur fafcia atra latitudine praecedentis cincreae: et demum aequale fpatium terminat elytra apice cbtruncato, et in fpinam validam margine cxter- . no excurrente. Pedes fufco- nigri tarís libbferrugineis. Lećtum in montuofis. Long. 4. lat. $\frac{3}{4}$.

Fabricii C. gibbofum, e defcriptione Oliuierii, tamen iden videtur. Varietatem C. Majflienfis $F$., feu Achilleae Schneideri, habeo, quae, omnibas fere huic defcriptioni refpondet, fed elytra bafi non magis bituberculata funt, quam in aliis. Nulla alia autem nota a $C$. Majjlitienfi differt, quam quod elytri fafcia prima et fecunda fere connatae fint, interuallo leuiter cano. Hg. 
132. C. pufillin thorace rotundato nigrum, antemis tibiisque teltaceis. F. Mant. I $_{55} 39$.

\section{. F F. E. S. I. 2. 330. 53 .}

Corpus $C$. Alni minus, obfcure nigrum. Antennae corpore longiores teftaceae pilofae. 'Thorax rotundatus, fubdepreffis, muticus. Pedes temoribus clauatis fufcis, tibiis tarfisque teftaceis." Alae al. bae. Hab. Pifis。

\section{(p. 54.) \\ LV. LEPTVRA.}

* I33. L. 'elongata nigra, elytris teftaceis: punctis duobus, cruce, fafciisque nigris. Fn. Sv. Ed. 2. 2275 .

De Geer. Inf. 5. 134. 10.

Geoffr. Inf. I. 224 . 5 .

Schaeff. Icon. t. 58. f. Io.

\& L. calcarate. F. E. S. I. 2: $347 \cdot 4 \mathrm{~T}$ 。

L. armata. Herbft. Arch. t. 26. f. 24 .

T. Subspinofa. F. E. S. I. 2. $347 \cdot 42$.

L. Scopoliana. Laichart. Tyrol. II. I $45 \cdot-$ -

Defcriptiones Degeeriana et Geoffroyann optimae. Magnitudo $L$. quadrifafciatae, at corpus magis elongatum, magisque apice attenuatum. Antennae corpore fubbreniores nigrae articulis bafi flauefcentibus. Caput nigrum. Palpi flaui apice nigri. Thorax niger flauo-pubefcens, antice anguftatus, vtrinque tuberculofus angulis pofticis acutis. Elytra flaua, antice nigro-punctata, poltice fafciis tribus nigris, prima late interrupta, apice attenuato truncato. Pedes primi et fecundi paris flaui taris nigris: poftici femoribus, tibiisque bafi dimiriato - flanis. 'Tibiac pofticae faepitis, at non femper: 
- Temper latere interno verfus apicem deficientes, quaî mutilatae, dentatae; forte diftinctio fexus. Hab. flores in filuis non rara. Long. $7 \frac{1}{2}$. lat. 2 .

Confer Fn. Etr. 1. I 82. 40r. adnot. Puneta ely... trorum fimul fuintorum antica nigra quinque effe esci, fnlent, nunc magna, atque inter fefe confluentia, junc minora, duobus lateralibus conflatis, vt tria conftituant, elytris communi, medio، Interdum fex minutifima confpiciuntur, "interduni aütem eorim quatuor, futurali deficiente. Saepius autem omnino fere defunt, et vix rudimentum corum fupereft. Fafcia, puncta fequens, nonnumquam cum futura, femper niçra, coit, nonnumquam, vero $a b$ ea diuifa eff. Fafciae omnes autem plus minusue latae funt. $\mathrm{Hg}$.

\section{IVII. PYROCHROA.}

134. $P$. coccinea nigra, thorace elytrisque fanguineis immaculatis. F. Sp. 254. I.

$\odot$ Geoffr., Linn:, Schaeft.

D Villers Ent. t. 2. f. 3.5. Schrank. E. I. 323.

S. F. E. S. I. 2. 104. I. Panz. F. I. G. XIII. Ix. Frifch. Inf. XII. Pl. III. t. 6. f. 7 .

In noftis caput femper nigrum; fronte foueola ampliuscula impreffa. Elytra nullo modo ftriata. Hab. Florentiae et Pifis in fepibus non frequens. Lecta ineunte Aprili. Long; 8. lat. vnius elytri ad apicem defunta $I_{2}^{\prime}$.

Geoffroyus capat quoque rubrum defcribit. Id quidem fufpectum videretur, cum $P$. rubens $F$, quae eadem $P$. rubers. Schrank. E. I. 324., capite rubro gaudeat, praecipue vero, cum haec $P$. rubens frequens circa Parifios occurrat. Sed color ruber $P$. rubentis minime illam elegantiam habet, quam Geoffro- 
Geoffroyus deferibit. 'Mihi itaque perfuafum eft, Geoffroyi citatum huc fpeetaue. Imo caput $P$. coccinecie, quodam lucis illapfu, vellere coccineo, quamuis raro, obtectum videtur. - Linnaeus in fyltemate Naturae elytra ftriata vocat, in Fi. Si. proprie non ftriata dicit. Id autem confiteri me oportet, ea quodammodo levifíme ftriata effe, - Figura Schaefferi t. 90. f. 4., fi fola fpectetur, fatis bona eft, et absque dubio citari potuilet; cum figura quidem a Panzero 1. c. data, comparari nequit.

Fabricius in Entom. Syftem. $P$. Pettinicomem rpecie difinxit, quam in fpeciebus cum $P$. coccinea eandem fufpicabatur. Aliquando hanc $P$. Peftinicor$n e m$ Fabr, Linnaei $L$. Sanguineam coniiciebam, cum huíns elytra ftriata diceret. Plura tamen huic coniecturae obftare videntur. Id antem fingulare eft, qua de caufla Linnaeus $P$. pettinicor. nem et $P$. coccineam alienis generibus fepararet, et cur elytra prioris expreffe non firiata diceret, cum tamen quodammodo fic vocari pofient, quia lineas quasdem eleuatas gerunt. $\mathrm{Hg}$.$$
\text { (p. } 55 \text { ) }
$$$$
\text { LXI. CVCVIVS. }
$$

I35. C. teftacus thorace fubquadrato mutico. teftaceus, femoribus compreffis. F. Mant. I66. 7 . \& F. E. S. I. 2. 96. II.

Triplo aut quadruplo minor C. flauipede, ftatura fere eadem. Antemac longitudine fere corporis filiformes, teftaceac, graues. Corpus elongatum depreflum, teftacem inmaculatum. Pedes breues teftacei femoribus compreffis. Hab. fub corticibus. Long. $I_{\frac{3}{3}}$ lat. $\frac{1}{4}$. 
Variat magnitudine et antennarum longitudine, quae nunc corpori aequales, nunc dimidia fere parte breuiores funt. Corpus glabrum, nitidum. Equidem hanc Cucuii fpeciem fub cortice Aefculi Hippocoftani et ni fallor, Quercus reprehendi. Hg.

\section{CANTHARIS.}

I36. C. pallida thorace marginato, atra, elytris pedibusque pallide teitaceis. F. Mant. I67. I2. Q F. E.S. I. 2. 217.16 .

Defcribit eam Fabricius. Fenora nigra. Alae albae. Hab. in plantis frequens. Long. $2 \frac{1}{2}$. lat. $\frac{1}{2}$.

Confer adnotata ad $C$. pallipedem. Fn. Etr. I. I92. 417. Femora nigra numquam vidi. Hg.

\section{(p. 56.)}

I37. C. plumbed plumbeo-nigra. Ent. Par.63. 15.

q An C. atra. F. E. S. I. 2. 2I5. II?

Antennae maris vix thoracem aequant, in femina dimidii corporis longitudinem. Nigra nitida. Villers Ent. 4. 320. 20. Hab.in floribus. Long.2. lat. $\frac{\pi}{2}$. vix.

\section{NECYDALIS.}

* 138. C.hybrida thorace teretiufculo rufo, elytris fubulatis, bafi futuraque teftaceis margine et apice nigris.

Tota determinare haud potui. Sub hoc nomine peritioribus iudicandam relinquo.

Statura et magnitudo omnino $N$. caeruleae. Caput antennaeque nigrae. Thorax punctis duobus impreflis, teretiufculus rufus. Elytra lineis tribus eleuatis ftriata, fubulata, bafi futuraque vsque ad medium 
medium teftacea, toto margine externo, apiceque nigra. Pectus atrum. Femora poftica clauata, arcuata, tota atra nitida. Alae nigrae. Lecta in Paftinacae floribus.

Equitem e fpecimine, ex Italia accepto, quod defcriptioni optime congruit, libenter iudico, hanc fpeciem efie nouam. Tibiae et femora, vbi coniunguntur, flauicant. Elytra fupra fericea funt.

Eabricius in Ent. Syft. I. 2. 352. I 2. N. humerolem habet, quam, licet thorax et abdomen nigra fint, libenter huic $N$. hybridae annumerarem, quia caetera optime conuenire videntur. Ipfe Necydalem habeo, huic hybridae fimillimam, thorace abdomineque nigro, quae autern elytra medio anguItata habet, quorum ftriae admodum eleuatae funt. Haec quoque prohibent, quo minus eam eandem cum $N$. .hnmerali $F$. habeam, cum proprie diceret 1. c.: Elytra absque lineis ant margine elenatis. Ad $N$. humeralem $F$. autem Cantharis nigra elytris attenuatis, antice luteis; La cantharide à bande jaune. Geoffr. Inf. I. 342. 2. pertinet. $\mathrm{Hg}$.

39. N. flauefcens elytris fubulatis teftaceis, corpore nigro, femoribus pofticis clauatis arcuatis. Linn. Syft. Nat. 642. 8.

De Geer. Inf. 5. I $55 \cdot 4$.

C. femorata. Scop. E. C. 145 .

C. fulua. Geoffr. Inf. I 343. 4 .

C. flatefcens. Geoffr. Inf. x. 343. 3. 5.

Schrank. E. I. $3^{x} 5$ ?

Hanc vnam eamdemque fpeciem effe cum $N$. Podagraria Fabr. Sp. 264. 8. afferere haud dubitamus. Saepe eodem tempore, et loco in filuefribus tantum, plura huius fpeciei lecta indiui$\mathrm{C} c$ dua, 
dua, femperque obferuatum variare femoribus pofticis munc clanatis, nunc fimplicibus, clana modo ad bafin tefracea, modo tota nigra. In iis, quibus pedes onmes fimplicibus funt, color pedum femper teftace - flauus, femorum anticonum apice, tibiis, tarfisque fubnigris; thorax femper teftaceo-flanus, elytrisque concolor, ad fuluum quandoque vergens, vti etiam maxima abdominis pars. $\mathrm{Ca}$ put nigrum. Antennae nigrae bafi teftaceae. Alae fufcre. Quae vero femoribus gaudent clauatis, corpus totum aeneo-nigrum, primi ct fecundi paris pecibus tantum, elytrisque exceptis teftaceo - flains. In omuibus thorax et elytra pubefcunt. Apex elytrorum interdum niger. In hac Necydali femori. poftica clanata marcm defignare videntur.

Necydalis Podagrarizie elytris fubulatis teftaceis corpore nigro femoribus pofticis clanatis, bafi teftaceis. F. Sp. 264. 8. Si fpecies ommino diftincta eft a $N$. flanefcente, vti indicat $\mathrm{Cl}$. Villers Ent. 1. p. 281 . ego etiam fateor, me numquam vidille. Certum tamen eft Necydales admodum variare. Valde variat $N$. rufa, et ex obferuationibus noftris (conf. Fn. Etrufcam) conftat effe mafculum $N$. atrae: $N$. ruficollis forte vna eadenque fpecies eft cum $N$. caerulefcente: $N$. viritiffina et caerulea etiam variant faepe colore, et occurrunt apud nos frequenter femoribus pofticis nunc fimplicibus, nunc clauatis. Erraret ne igitur Entomologus, qui diceret in hoc genere fpecies nimis auctas? Hab. in fepibus, vbi Podagraria crefcit.

Anctor N. Podagraviae non liabuifie videtur, fed $N$. femoratam Scopol. Id quidem fingulare, hanc pofteriorem eandem effe cum $N$. flawe $c$ cente, quam 
N. prateranom Schrankii E. I. 3 r6. habeo. Illi libenter afientio, N. Podagrcriae $F$. a $N$. flauefente non fpecie diuerfam effe, cum ipfe vtramque in eodem tractu reperierem. Sed $N$. femorat Scop. nimis differt, nec $N$. Podagraviae, nec $N$. praeteranae Schrank. varietas effe poteft. Infuper varietatem eius pofiideo, fufficienti copia repertam, cui, caeteris omnibus paribus, pedes fimplices funt. Si autem Auctoris $N$. flaue $c e n s$, Schrankii $N$. pratcrana eft, repetendum eft, Linnaei flaue centem plane diuerfam effe. $\mathrm{Hg}$.

\section{(p. 58.) \\ LXV. ELATER.}

* I 40. E. litigiofus teftaceo-rufus, oculis folis nigris, antennis vix thorace longioribus.

Magnitudo et ftatura E. ineati Vill. Elytra laeniter ftiata. Alae fufcae. Videtur noua fpecies affinis E. Auftriaco Schrankii, et difficillime diftinguenda. Magnitudine variat minori. Habit. in plantis obuius. Long. 6. lat. I. et vitra.

I4 I. E. lineatus niger elytris obfcure-liuidis, fufco-fublineatis. Villers Ent. I. 304. 6.

Corpus mediocre fufcum. Antennae fubferrugineae. Elytra obfcure-liuida, lineis duabus nigricantibus verfus futuram dorfalem pofitis. Vill.

In noftris fpeciminibus fane multis pedes teftacei, et elytrorum lineae omnes fufcae quafi in ftrias gemellas digeftae. Hab. in plantis frequens. Long. 4. lat. I.

* I42. E. cylindricus aeneo-niger tomentolus, elytris laeuiter punctato - ftriatis.

E. cylindricus. D. de Paykull. 
Magnitudo praecedentis. Totus nigro-aeneus pubefcens. Antennae thorace longiores. Hab. ruri frequens.

$$
\text { (p. 59.) }
$$

143. E. thoracicus niger, thotace toto rufo. F. Sp. 270.32 .

D E. graninzens. Scop. E. C. 290 .

q F. E. S. I. 2. 227. 51. Panz. F. I. G. VI. $x 2$.

In E. ruficolli Fn. Etr. fynonymia Scopolina delenda, hunc enim fub nomine E.graminei indicare videtur Scopoli:

Statura E. ruficollis, at duplo maior. Antennae nigrae thorace fubbreuiores. Caput nigrum nitidun. Thorax latitudine elytrorum admodum connexus, globofus, fuperne totus rufus nitidus, inferne fepto, feu fafcia longitudinali inigra ab ore diducta. Elytra fubtilifime ftriata, nigra, nitida, fcutello valde excauato, nigro. Abdomen pedesque nigra. Alae fufcae. Differt ab E. ruficolii magnitudine duplo maiori, antennis breuioribus, minusque ferratis, thorace fupra toto rufo, fubtus dinerfimode maculato, pedibus totis nigris (in E.ruficolli rufis) femoribus aliter conformatis breuioribus, et non ita, vti in illo depreffis. Habit. Florentiae.

Confer adnotata ad $E$. ruficollem Fn. Etr. I. 206. 444. E. ruficollis Schrankii En. 355. quoque fufpectus et $E$. thoracico annumerandus videtur. Dicit enim: Totus ater, thorax conuexus ruber. Voet. II. t. 42. f. 6 . a celeberrimo Fabricio hinic $E$. thoracico annumeratur. Sed figura effigiem optimam $E$. fanguinicollis mei repraefentat, quem Panzerus in Faun. Inf. Germ. fua VI. r2. depinxit, et defcripfit. Hic Elater admodum a thora- 
cico differt, et, colore atque Elateris characteribus exceptis, nil commune cum eo habet. Statura omnino alia eft, quod figura Panzeri fat bene oftendit. Hic locus autem ịdoneus eft, adnotandi me Elaterem cepifie, qui ne minima quidem nota ab $E$. Sanguinicolle differt, excepto thorace toto nigro. Hunc varietatem labeo, quia eiusmodi varietates in infectis faepins occurrunt. $\mathrm{Hg}$.

* I 44. E. binotatus niger, ferrugineo - pubefcens, clytris ad apicem macula pallide flaua.

Affinis $E$. bimaculato $F$ r. Etr. Totus niger furcus, pube fubferinginea tomentofus. Antennae fufco-nigrae longitudine thoracis. Thorax angulis pofticis, feu dentibus admodım acutis, elytris nitidior. Elytra ftriatạ macula ad apicem pallide flaua. Pedes teftacei femoribus fufcis. Alae albae. Occurrit fed rariffme elytris alio puncto flauo anteriore obfolete notatis. Hap. fub arborum cortice. . Long. $I \frac{1}{2}$. lat $\frac{x}{28}$

\section{(p.60.) \\ LXVI. BVPRESTIS,}

145. B. nouemnaculata el ytris ferratis, nigra, fronte puncto vnico, thorace quatuor, elytris tribus flauis. F. Mant. I 79. 36.

Fn. Etr. I. 2x6, 463 .

Variat thorace maculis 2,4 , nullis, fronte immaculata, elytris femper maculis tribus. F.

- Numquam vidi thorace quadrimaculato. Inter mea fpecimina fane plura, nam frequens eft apud nos, habeo varietates fronte thoraceque immaculatis, elytris maculis tantum quatuor pofticis: aliasque, quae praeter nouem maculas ordinarias 
vlterius habent punctum flauum in elytronum apice, Abdomen immaculatum. Elytra fubtilifime ftriata. Habitat etiam in hortis.

Confer ea, quae in Fanna Etrufca monui, vbi citatum Linnaei, quod h. 1. Auctor iterauit, delendum fuadeo. Varietates, quas obferuare potui, tabella propofiturus fum. Occurrunt:

a) fronte macula vnica, thorace maculis duabus dorfalibus, bafi propioribus, nunc minoribus et a futura poftica $f$. bafi feiunktis, nunc maioribus, et cum illa connexis,

a) elytro fingulo maculis tribus: humerali ita lunata, vt finus thoracen fpectet.

B) - - maculis quatuor, cum in apice infuper macula aditit.

b) fronte thoraccque immaculato

a) elytris maculis iisdem, vt in variet. a., aut macula illa lunata admodum parua, et punctum tantum repraefentante. In vno fpecimine lunulae cornua tantum reftauere, medio interrupto, vt: quaeque in puncto duo diuifa fit.

B) macula lunata tòta deficicute, vt in ambobus elytris maculac quatuor tantum adfint.

$\gamma$ ) macula nunc fecunda, nunc vltima in vtroque elytro diuifi, vt aluo puncta inde fiant.

Haec omnia macularum variabilem inủolem indicant, quae praecipue ex eo patet, quod in vno fpecimine in dextro thoracis latere infuper punctum minutum flauum adfit, quod vero in latere finiltro deficit, vt tres tantum maculae in thorace fint.; Specimina talia fi occurrerent, vbi in vtroque thoracis latere id punctum effet, probaretur, thoracem interdum quadrimaculatum effe. In altero fpecimine fafcia, feu macula, intermedia elytri finiftri bipartita eft, in elytro autem dextro integra, imo medio latior. $\mathrm{Hg}$. 
* I 46. B. cyaniconis elytris integris, viridis, antennis cyaneis, femoribus anticis fubtus aureoignitis, pofticisque clanatis.

An B. femorata. Villers Ent. I. 338. 34. t.. I. f. a. quae forte non rite fatis defcripta?

- Paullo maior B. manca. 'Antennae thoraci fub.' aequales bafi, feu articulo primo longiore, clanato, viridi, reliquis cordato - orbiculatis, compreffis, cyanicis. Caput viride. Oculi nigri. Thorax planus lateribus rotundatis, dilatatis, antice pofticeque truncatus viridis. Elytra viridia, fubpunctata, ad latera coarctata, ad apicem attenuata, apice ipfo obtufo. Abdomen fub elytris caeruleum. Subtus tota viridi-aurea nitidior. Femorim anticorum apophyfes, ac latus internum fere totum alureoignitum, fplendidum. Femora poftica vix pilofa, admodum incraflata, vnde ampla cauitas vtrinque in abdomine ad ea recipienda idonea. Alae obfcurae cofta obfcuriori. Habitat in plantis rara. Long. 5. lat. $\mathrm{I}^{3}$.

$$
\text { (j. ox.) }
$$

* I47. B. deaurata fupra obfcure viridis, tho. racis, elytrorumcue margine aureo.

An $B$. aurulentae, feu potius deauratas varietas. F. Mant. 182. 6־?

Magnitudo praccedentis. Antennae nigrae. Caput obfcure viridi - acneum, pilofum villo albicante tenniffimo. Thorax fere planus, fubpilofus, lateribus rotundatis, in medio viridis margine exteriore late, et obfcure viridi - aeneo. Elytra integra viridia, nitidiora margine exteriore aureo - rubro, nitidilimo, anguftiori. Subtus tota cuprea fplendida. Lecta in filuis.

$$
\mathrm{Cc} 4
$$


148. B. rutilans elytris tridentatis, viridibus, nigro-maculatis, margine aureo. F. Mant. 17 ?: I6.

D. B. rutilans. Villers Ent: r; 336. 22.

\& F. E. S. I. $2 \cdot 192.27$.

- Statura et magnitudo fere praecedentis. Anten. nae ferratae, nigrae. Caput, thoraxque viridia. Thorax auro late marginatus punctatifimus. Elytra ftriata, ad fituram viridifima, ad marginem ignea, punctis nigris lucidis afperfa. Corpus aurato-viride. Elytra apice ferrata. Hab. Florentiae rara.

Fabricius allegat. Scharff. Ic. t. 35. f. 6 , quod citatum primo ftatim afpectu reiiciendum eft. Harrerus contra quoque fallitur, $B$. aun ilentam Lima. ad hanc figuram referens. Idem fecit nofter $\mathrm{Au}$ ctor in Fn. Etr. I. 215.462 . Illi autem affentio, quod Harterus B. Quercus. Herbft. Arch:?

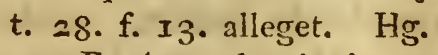

I49. B. elata elytris integerrimis, linearibus, corpore elongato aeneo nitido. F. Mant. 184. 84.

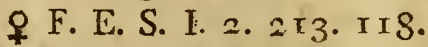

Statura $B$. atrac oblonga, at minor, et tota aenea nitida. F. Antennae thorace breniores ferratae. Thurax antice valde conuexus, poftice depreflis, finuatus, marginatus. Elytra ante medium ad latera anguftata, punctata, futura proininula. Corpus elongatum. Hab. in filuis. Long. $2 \frac{1}{2}, 3$ : $^{\circ}$ lat. $\frac{3}{4}$.

B. viridi breuior eft, eiusdem autem fere ftaturae et coloris fufco-metallici. $\mathrm{Hg}$.

$$
\text { (p. 6.2) }
$$

150. B. laeta elytris integris viridibus, capite thoraceque ameis. F. Sp. 282.59. 


\section{아 F. E. S. I. $2.214 \cdot 124$ :}

Statura et magnitudo $B$. Nitululae. Caput, tharax, pectus, abdomen aurea nitida. Elytra, pedesque viridia. Thorax aureo-ignitus, vtrinque depreffus vti in B. Nitidula. Defcriptio F. Syft. Ent. nullo modó cum meo fpecimine consenit; cơpus enim noin lineare, non totura $B$. viridis, pedies demum non rufefcentes; Hab. Florentiae rata.

Varietas B. mitidulce eft. Confer, quae ibi adnotauimus. Fn. Etr. I. $220.470 .-$ Srhaeff. Ic t. 67. f. 4. quidem huc pertinere dicitur, tamen ob formam male expreflam, cum figuris fequentibus, quas $B$. vitidulqun repraefentare ferunt, non citanda ẹf. $\mathrm{Hg}$,

* I 51. B.caerulea elytris integerrimis laeuibus, corpore fupra cyaneo, fubtus plumbeo, oculis uigris.

Primo intuitu videtur varictas B.cyoneare, fratura enim et color fupenius idem, at difincța. Corpus fublineare nitidun. Differt a B. cyaner magnitudine interdum plus quam duplo minori, oculis nigris, elytris non rugofis, thorace conuexiore, poficeque magis lato, corpore fubtus plumbeo. Lectae quatuor in plinta filuae Pifunae.

I 52. B. pygmaca clytris integris cyaneis, capite thoraceque aeneis nitidis. F. Mant. 183. 78 .

\section{오 F. E. S. I. 2. 2 II. I ro.}

Statura et magnitudo $B$. minutae. Caput et thorax laenia, aenea, nitidifima. Elytra cyanea nitida, inmaculata. Corpus obfcure nitidum. Habit. in Vmbellatis frequens. 


$$
\text { (p. } \left.\sigma_{3}\right)
$$

153. B. minuta clytris integerimis transuerle rugolis, thorace fubtrilubo laeui, corpore ouato nigro. Vill. Ent. 1. 334. I5.

In Ed. XIII. Linu. praetermifTa.

Minor altera $B$. minuta. Geoffi. Inf, I. I28. 6. Cucuius fufco-cupreus triangularis etc. cui finilis; fed elytra fine fafciis vndulatis, villofo-albidis. Hanc Villers credit veram $B$. minutam Linn. Hab. in plantis rarior.

Vide, quae in Fn. Etr. r. 22I. 472, monui. Hg.

* 154: B. nitida elytris integerrimis, viridis, nitens, thorace foueola poftica vtrinque profinda excauato.

Statura $B$. Salicis, at fere dimidio minor. Caput viride. Frons imprefla. Antennae nigrae thorace: fubbreniores. Oculi fufci. Thorax viridis, nitens, antice conuexus, poltice marginatus, et foffula magna vtrinque profunde excauatus. Elytra viridia, nitida, integerrima, fcabriufcula, acute-marginata, anticeque parun coarciata. Subtus tota fubaeneo - viridis.

ß. Variat elytris aureo - rubris.

Differt a $B$. Salicis magnitudine, qua vix aequat $B$. sitidulam, foflula thoracis valde profundiore, coleoptrisque (etiam in varietate $\beta$ ) numquain aureis bafi viridibus, fed totis aureis. A $B$. nitidula vero difert praecipue forma thoracis oininino diuerfa. Hahitat in floribus compofitis frequens. long. 2. vix, lat. $\frac{x}{4}$.

\section{ELAPHRVS.}

I55. E. Curaboides. 
Cicindela Caraboides thorace cordato hemifphaerico, marginato, elytris nigro - acneis, punctis copiofis impreffis. Schrank. E. I. 193.360 .

Villers Ent. I. 326. 14. t. x. f. 39 .

Caput et thorax nigro-aenea. Oculi exferti, et maxillae infignes. Thorax perfecte cordatus, marginatus vti in Carabis, fed margo anguftus linea longitudinali excauata. Elytra nigro-aenea maculis viridi-aeneis variegata, minutifime punctata; ad vtrumque marginem puncta excalıta lata in lineam longitudinalem difpofita. Pedes fufci. Pécus nigro-aencum. Abdomen atrum nitens. Schir. Hab. in fapulofis non raro. Long. $2 \frac{1}{2}$. lat. $I \frac{r}{2}$.

$$
\text { (p. 64.) }
$$

- 156. E. nebulofis oblcure aeneo - viridis, elytris cinereo-nebulofis.

Statura E. flatipedis, at triplo maior. Antennae fufcae. Thorax cordatus linea longitudinali impreffa. Elytra obfoure aeneo - viridia, laenia, ad fituram interdum bipunctata, et frarfim cinerea pube nebuilofa. Stibtus a eneo-niger; tibiis tarisque ferrugineis. Hab. in locis arenofis frequens.

* i 57 . E.pyritofus aeneus, elytris punctis duobus maiufculis profunde imprefís, pedibus atroaeneis.

Proximus E. alpino D. de Paykull. et praecedente paullo minor. Caput aeneum. Thorax aeneus, conuexus, vix marginatus. Elytra aenea, fubtilifime friata, punctis duobus valde confpicuis pone nedium prope futuram, inter ftrian fe. cundam, et tertiam imprefis, altero anterius, al- 
tero pofterius. Color fupra prorfus aeneus, fubtus atro - viridi - aeneus, nitidus. Pedes concolores nitidi. Hab. cun praecedentibus rarior.

\section{(p. 65.) \\ LXIX. HYDROPHILVS.}

158. H. Caraboides niger nitidus, elytris fubftriatis. F. Sp: 289.3.

- D Fn. Etr. $x, 227 \cdot 48 z$,

Niger laeuis conuexus, elytris punctato-ftriatis, capitulo antennarum perfoliato nigro, capite thoraceque friis punctorum abbreuiatis recuruis.

Mas pedibus anticis absque paimulis, omnino feminae fimilis, menf. Mart. et April. coire folet. Penis infidet medius inter duo cornicula, feu potius appendices corneas, lanıeliformes; apice at-; tenuatas, quae ad introductionem, firmioremque copilaun fuifentandam accommodatae videntur: Oua magnitudine vix cedunt is $B_{\text {. Pauoniat ma- }}$ ioris. Datur alius, mihi varietạs, qui magnitudine excepta triplo, aut quadruplo minori, nulla alia nota ditinguitur a prạecedente, fimuique frequens occurrit, nempe H. picipes. F. Mlailt. 188. 6.

Hab. in aquis fiequens.

159. H. orbiculatus fubrotundus, corpore glabro. F. Sp. 290. 6.

\& F. E. S. I. I. I84. IO.

Defcribit eum Fabricius. Hab, in aquofis.

$$
\text { (p. ६6.) }
$$

* 160. H. pallidus pallidus la euis, abdomine, oculis femoribusque fubnigris. 
Statura et magnitudo omnino $H$. Luriti, fed caput, thorax et elytra la euia; pallida, fubdiaphana, interdum tamen fufcedine quadam afperfa. $\mathrm{Ab}$ domen et femora obfcure nigra. Tibiae, tarfi, antenuarum bafis, et palpi pallide rufi, hiçue porrecti, longiufculi. Variat quandoque elytrorum colore obfcuriori. Hab: in aquis ftagnantibus.

\section{DYTISCVS.}

I6I. D. diffmilis niger, depreffus; thoiacis elytrorumque margine exteriore ferrugineo-flauefcente, elytris, in femina obfcurioribus, ninus laeuibus fibrofis, neque ftriato - punctatis. Fn. Etr. I. 230. 489 .

Cum in determinando pro $D$. cofali varia fit Entomologorum fententia, nomen $D$. dijparis iam viurpatum mutaui.

Valde affinis $D$. coftali, cui femina huits praefertin fimillima. Differt tantum magnitudine paullo minore, corpore toto fubtus ferrugineo, ftriaque coftali ferruginea apicem haud attingente, caetera aequalia. Obf Fafcia futpra os puncto vtrinque impreffa, flatuefcens, fupra quam aliud punctum maius adelt fimiliter vtrinque impreffum. Mas variat colore te?tudineo - nigro; at rarifime. Hab. in Arni aquis, etiam in follis.

I62. D. bipuftulatus ater la euis, capite poftice punctis duobus rubris. F. Sp. 294. I5.

q. Mull. Linn. Nat. Cl. V. p. 340.

D. immaculatus. Schrank. E. I. $377^{\circ}$

D. InEtuofus. Ent. Par. x. $6 ! \cdot 6$.

D. Gronosii. Gron. Muf. 2. p. 164, n. 555. an?

Schaeff. 
Schaeff. Ic. t. 8. f. 9 ?

q Fn. Etr. I. 233. 492 .

Dytifcus ater etc. De Geer. Inf. 4. 40r. 8. et variet. . maior elytris rufo bipunctatis. Confer.

Anceps diu haefi an varietas a $D_{e}$ Geerio indicata, quae gaudet elytris rufo bipunctatis, diftinguenda foret a $D$. bipufulato $F$. Vt veritas, quain fequimur, clarius certiusque inmotefcat, liceat obferuationes noftras in medium afferre, et peritioribus Entomologis fubiicere.

Hi duo Dytifci, qui eodem temporie et loco apud nos etiam frequenter occurrunt, primo int-' tuitu fere fimiles funt, toti atri, vinis altero minoi, et in vtroque caput poftice punctis duobus rubris, vix oculo inermi confpicuis, notatur.

Minor: D. ater laeuis capite pofterius pinctis duobus nubris; fane eft $D$. bipuftulutus Fabricii, vti magnitudine ab anctoribus allata, aliisque notis conftare videtur.

Maior: Varietas eft elytris rufo-bipunctatis a $D e$ Geerio tantum 1. c. fupra indicata, filicet $D$. ater corpore comuexo nigro toto, pedibus antennisque rufo-fulcis; Vurietas.

Obferuatum igitur $D$. minorem, fcilicet bipufulutum, conftanter differre a $D$. maiore, feut val. $D_{e-}$ geerii praeter magnitudinem, corpore elytrisque minus conuexis; immaculatis; apice magis depreffo et obtufo; pedibus quatuor anticis nigris, raro piceis. In $D$.maiore palpi, antenna eque rufae, et caput poftice punctis duobus rubris vti in $D$. minori notatum, fed elytra magis conuexa, apice magis elongato, et punctis in fingulo binis rufis; altero 
altero pone medium verfus marginem exteriorem oblongo; altero ad apicem rotundo, quae etiam nudis oculis in Infecto praefertim viuo afpicimntur, et anulfis elytris fi inferna parte obfernentur, eo quod pellucida, manifeftifime apparent. Pedes quatuor antici fubrufi, tarfis tribus primis pilis canis hifpidis, quafi fafciculatis. Alae in hoc vti in praecedente albohyalinae, ad coftam marginemque temuiorem fufco-nebulofae.

Hae notae, cum nemo adhuc afferat copula iunctum cum $D$. maiori, fufficientes videri poffent ad declarandos eos fpecie diftinctos. Attamen nec, rebus omnibus bene perpenfis, cum Dégeerio creảimus vnam eandemque fpeciem effe, quae variat magnitudine maiori, elytrisque magis conuexis, rufo-bipunctatis. Hab. in aquis frequens. Long. 3, 4, 5. lat. $I \frac{1}{2}, 2,2 \frac{1}{2}$.

Dytifcus a Degeerio defcriptus omnino dinerfus eft. Duplo maior, magis conuexus, et pone magis anguftatus. D. bipuftulatus frequenter apud nos occurrit, numquam autem $D$. ater Degeerii vifus eft. Confer Adnot. in Fn. Etr. l. c. Hg.

$$
\text { (p.68.) }
$$

163. D. transuerfolis ater, thorace antice ferrugineo, elytrorum margine ftrigaque bafeos abbreniata flauis. F. Mant. $19 \mathrm{I}, 23$.

오․ E. S. I. x. $192,26$. Hab. in aquis.

* I64. D. pufulatus niger laenis, macula frontis lunulata rufa.

Statura et magnitudo D. friati. Os, antennae, palpique iufi. Caput nigrum macula magna fion- 
tis rufa, in Infecto viuo, praefertim natante maxime confpicua, poft nortem certo tantum fitu vifibili. Thorax imo margine laterali fubrufo. Elytra nigra läeuia immaculata. Subtus piceus pedibus anticis fubrufis. Capitis clypeus antice' vtrinque punctis duobus impreffis, vti in D. friato, fed in hoc disiunctis, in illo contiguis. Aqua extractus, digitisque ad examinandum leuiter contrectatus, vitam quae in aliis diu permanet, deferit breui. Hab. cum praecedente.

I65. D. Hybneri la euis ater, ore, thoracisque margine ferrugineis, elytris linea marginali flaud. F. Mant. I90. $2 \mathrm{I}$.

q F. E.S. I. I. 192024 。

Magnitudo et fratura D. transiterfalis. Caput nigrum, ore, antennis palpisque pallide ferrugineis. Thorax niger margine imprimis antice ferrugi11eo. Elytra la euia, atra, ftriata, ftriis in fingulo tribus punctatis vix oculo inermi confpicuis, lineaque flaua marginali, quae tamen apicem haud attingit. Subtus niger pedibus quatuor anticis flauis; et prini paris in mafculo patellatis. Habitat in aquis fat frequens.

$$
\text { ( }
$$

* I66. D.tamiatus fupra niger, la enis, nitidus, fubtus ferrugineus, ore, verticiș punctis duobus, thoracis margine fafciaque media flauis.

\& D. cincrens. Oliuier. Encycl. Method. VI. p. 309. n. ro.

D. bilincatus. Ibidem p. 3 I9. $\mathrm{x}$.

D. bilineatiss. Degeer. Inf. IV. 400. 6.

Statura $D$. cincrei, magnitudo D. transuergalis. Caput nigrum, ore, lineola trigrona media, puinctisque 
ctisque verticis duobus transuerfis flauis, fere vti in D. cinereo. Antennae palpique ruf. Thorax antice, pofticeque niger nitidus margine laterali, fafciaque transuerfa media marginem vtrinque attingente flauis. Elytra glabra, laenia, nigra nitida, punctis minutifimis flauis vndique irrorata, fed oculo tantum armato confpiciendis. Subtus totus flauo-ferrugineus. Caput antice vtrinque punctis duobus impreflis, diftantibus. Habitat in aquis rarus. .

Absque dubio idem eft cum $D$. cinereo. Fabr. Sp. 293. Ir. Vide, quae D. cinereo. Fn. Etr. I. 232. 490. addidimus, vbi tamen nondum fufpicabar, noftrum Auctorem eundem Dytifcum quoque defcripfifie.

Sutura thoracis antica et poftica interdum quoque 110 teo-marginatae funt. Elytrorum margo exterior quoque lutefcit. Hg.

I6 7. D. bipunctatus? ater, thorace flauo, punctis duobus nigris, elytris flauo-fufcoque variis. F. Mant. I90. I9.

\& F. E. S. I. I. I92. 22.

Statura $D$. feneftrati, et vix maior. Caput nigrum ore fubferrugineo, fronte, armato oculo, rufo-bipunctata. Thorax pallide-flauus punctis duobus dorfalibus atris. . Elytra laeuia, glabra, pallideflaua, nigro variegata. Pectus nigrum. Abdomen, pedes antennae teftaceae. Anceps diu haef, anne potius referendus effet ad $D$. irroratum $F$. Color abdominis magis conuenit, at magnitudo remouet. Lectus fuper herbam in prato pạluttri.

Defcriptio, ab Auctore data, omnino conuenit cum fpeciminibus meis, colore abdominis excepto, Dd quod 
quod nigrum eft, fegmentorum marginibus eo htius rufis, quo apice propiora funt. Haec differentia autem vix confideranda eft. $D$. irroratus $F$. ex America oriundus eft, hinc idem cum $D_{\text {I }}$ tifco Italico effe non poteft. $\mathrm{Hg}$.

I6 ${ }^{2}{ }^{2}$ D. viiginofus ater nitidus, antennis, pedibus elytrorumque latere exteriore ferrugineis. F. Sp. 295. 2 I.

D Mull. Linn. Nat. cl. V. p. 350 .

Scop. E. C. 296. Schrank. E. I. 378.

\& F. E. S. J. T. $194 \cdot 3 \mathrm{x}$.

Magnitudo G. Natatoris. Caput, palpi, antennae, pedesque rufi. Thorax rufus margine antico obfcuriori. Elytra nigricantia punctulis excanata, latere extcriore rufo - flanefcente. Abdomen nigrum. Hab. in aqua ftagnante. Long. $2 \frac{\pi}{3}, 3$. lat. $\mathrm{I}_{\frac{1}{4}}$.

Anktoris Dytifum verum Fabricii vliginofum effe, non dubito; corpus tamen $G$. Natatore longius eff. Elytra, oculo armato inuifa, ftrias punctorum imprefiorum vix confpicuas habent, quod plerisque Dytifcis commune eft.

Linnaei 1 . vliginofus huc non fpectat, quod e defcriptione eins fatis patet. Scopolii D. viliginofus nec idem cum Fabriciano, nec cum I,innaeano eft. Sic quoque D. vliginofus Schrankii e citatis tollendus eft. Idem ab Auctoribus inditum nomen non femper idem defignat infečum, Hg.

\section{(p. $\% 0$.}

I68. D. minutus flauefcens, elytris fufcis, margine flauo-maculato. F. Sp. $297 \cdot 36$.

D Geoffr. Inf. x. I9I. I2.

\& D. obliquns. F. E, S. I. I. I98. 56. Panz. F. I. G, XIV, 6. 
Ad bafin abdominis lamina magna mobilis femorum partem obtegens. Variat capite nigro, elytris modo maculis nigris, modo immaculatis. Hab. in aquofis.

D. minutum. F. E. S. I. 200. 63. non allego, quia Auctoris Dytifcus idem eft cum D. obliquo F. 1.c. vix enim putare licet, Fabricium eundem Dytifcum bis defcripfifie. Citatum itaque e Degeerio: Inf. 4. 404. I8. t. I6. f. 9. Io., quod auctorem duxifle videtur, vt fuum Ditifcum, D. minutum $F$. haberet, ad $D$. obliqunm transferendum eft.

Magnitudo et pictura huius infecti mire variant. Nunc rufum, nunc grifeum elytris aut nigro-, aut immaculatis, nunc nigricans occurrit. Thorax tamen flauus, aut certe lucidior eft, quam reliquum corpus. Caput nunc ferrugineum, oculis folis nigris, nunc nigrum ore ferrugineo eft. Nonnulla fpecimina mihi funt, eodem modo picta, vt Dyt. marginepuntatus. Panzeri. F. I. G. XIV. xo. reliquis quoque congruentibus. $\mathrm{Hg}$.

\section{CARABVS.}

I69. C. purpurafcens niger, thoracis elytrorumque marginibus violaceis, elytris rugofe punctatofriatis.

Paykull. Mon. Car. n. 4.

C. purpurafcens. F. Mant. I95. 6.

Schaeff. Ic. t. 88. f. I.

\& F. E. S. I. x. $x 25.6$ ?

Defcriptio eadem, quam Paykullius dedit. Hab. apud nos rarior: bis tantum inuentum cadauer in filuis.

Celeberrimus Schneiderus in optimo eius fcripto: Neneftos Magazin fiir die Entonsologie III. Heft. Dd 2 p. 
p. 357. aflerit, C. purpurafcentem Paykullii eundem efie cum $C$. catenulato $F$. Idque in verba amici mei acutiffimi credo, quamuis defcriptio $C$. catenulatum $F$. haud bene defignat. Fatendum tamen eft, huius noniulla fpecimina illis tribus feriebus punctorum eleuatorum carere. Vtrum tamen Auctoris $C$. purpurafcens, Fabricii $C$. purpurafcens, an eiusdem $C$. cateriulcitus fit, decernere non pofium:

Caeterum de his et reliquis Carabis, aliisque infectis, quae celeberrimus Fabricius in parte prima Entomologiae fyftematicae defcripfit, Entomologos, interius eorum hiftoriam atque criticam exploraturos, fupra commemoratum opus amici Schneideri adire iubeo: $\mathrm{Hg}$ 。

\section{(p. $; \dot{i}$.}

I 7o. C. hortenfis * apterus, niger, elytris fubrugofis, punctis aeneis excauatis triplici ferie, margine cyaneo. F. Mant. I96. I6.

Linn. Syft. Nat. 668. 3. Fn. Sv. 783.

Paykull. Mon. Car. n. 7 .

C. violaceus. Degeer. Inf. 4. 89. 3.

Schaeff. Ic. t. II. f. 2.

\& F. E. S. 1. 1. 12\%. 13. Panz. F. I. G. V. 2. Defcriptio Mon. Car. Hab. in putridis. Apud nos minime frequens.

$$
\text { - (p. } 72 .)
$$

I 7 I. $C$. coniuexiss apterus, conuexus, ater, laeuis, thorace poftice emarginato. F. Mant. I 97.22. Paykull. Mon. Car. n. x3. Schaeff. Icon. t. 3. f. 2. of F. E. S. I. I. I $29.2 \mathrm{I}$. Defcriptio Mon. Carab. Lectus in Pifano nemore. * 172 .

- In Fn. Etr. 1. 237. 506. deleatur nomen C. hortenfis, et ad C. auropunitatum referantur defcriptio et figura 1. c. exhibitae. Adv. Autt. ipfo 
- I 72. C. Paykullii niger nitidus, capite maximo exferto, thorace obcordato, elytris laeuibus, tenuifime punctato - ftriatis.

Magnitudo $C$. Spinigeri... Caput nigrum maximun latitudine fere thoracis, vti in $C$. interrupto, at magis exfertum. Maxillae, ocilique nigri: Palpi picei. Antennae craffufculac, capite thoraceque breuiores fufcefcentes, primis quatuor articulis nigris nitidis. Thorax niger cordatus, marginatus, conuexus, antice latitudine elytrorum; poftice paullo anguftior, linea longitudinali, ftriaque magna poftica vtrinque late, ac profunde excanata. Elytra nigra laeuia, connata, conuexa, tenniffime punctato - ftriata, punctis difcoïdalibus maioribus nullis, fummoque margine exteriore impunctato. Sternum nigrum. A idomen nigrum, interdum piceum: Pedes nigri, breniares femoribus incraffatis. Alae nullae. Forma thoracis latior, quam in C. Jjinigero, magisque fubquadrata.

Infectum dicatum Nobil ac celeberrimo viro Guftcuro de Paykull ad vninerfam naturalem hiftoriam locupletandam nato Entomologo fumino, et in ordinandis, ilhntrandis defcribendisque miro fucceffu variis Infectorum generibus nulli fecundo. Hab. Florentiae rarus,

\section{(p. $\div 3$.}

I73. C. granulatus apterus, nigricans, elytris aeneis friatis, interiectis punctis eleuatis longitudinalibus. F. Mant. I97. 25. Sp.301. I ?.

$\odot$ Sulz. Bergftr.

D Payk. Mon. Car. n. 9. Villers Ent. I. 357. in calce Obferu.

Schaeff. Ic. t. 4 6. f. 4. parua et male picta. 
Q. Fn. Err. I. 239. 508.

Defcriptio Mon. Car. vnde quoque varietates cum fynonymis defumtae funt.

- Schiefi. Ic. t. 46. f. 4. aperte legendum eft t. I56. f. 4. Caeterum confer, quae in Fn. Etr. 1. c. adiecimus. $\mathrm{Hg}$.

$$
\text { (p. 7.4.) }
$$

I 24. C. Lencophthalmus apterus, niger, elytris decemftriatis, difco quadripunctatis, thorace femirotundato, poftice vtrinque biftriato. Mon. Car. 11. 16 .

Fn. Etr. r. 24. 5 I r.

Defcriptio et obferuata Paykull. Mionogr. iterum exhibentur.

In meis peciminibus, quae aptera funt, et omnino conucuint cum allata Monographine defcriptione, thorax non tantum poftice vtrinque biftriatus (nota confantifima, qua a plerisque fuae gentis optime diftinguitur;) fed etiam fulco longitudinali profunde exaratus, et margo elytrorum exterior confpicue punctatus. Hab. in putridis et fub lapidibus frequens.

Auctoris C. Leutophthalmus idem videtur cum $C$. Frifchii Herbft. Fuesly. Arch. p. 138. 43. Caeterum confer, quae in Fn. Etr. 1. c. monuiinus. $\mathrm{Hg}$.

\section{(p. 75.)}

I 75. C. auropunitutus alatus, niger, laeuis, thorace poftice emarginato, elytris fubtiliffime punctatoftriatis, punctisque paullo maioribus triplici ferie excauatis. Payk. Mon. Car. n. 4 I.

C. auto-punitatus. Herbft. Arch. V. I3 I. I5.

Fn. Etr. I. 237 . 506. t. I. f. 3. ad hunc Carabum pertinet vide C. liortiverem. 
C. Indagator F. Mant. $197 \cdot 35$ ? affinis videtur, fed apterus ille, hic alatus; an a celeb. Auctore oculo modo fugitiuo defcriptus?

Defcriptio Mon. Car. In Suecia magnitudine paullo minore. Hab. in pratis filuaticis, frequens m. lul. polt foenifecium.

Puneta impreffa nunc lucida, nunc obfcura funt. $C$. Indagator $F$. alius eft, quod iam patria fuadet. $\mathrm{Hg}$.

I 76. C. Terricoli niger, abdominc longiore, elytris libuiolaccis, friatis, difco impunctatis, thorace obcordato, vtrinque poftice impunctato. Mon. Car. 11. I 7 .

C. Terricola. Herbft. ap. Fuesl. Arch. Inf, 6. p. I 40 , 5 I. t. 29. f. I 4 .

Delicriptio Non. Car. Paykullii. Inter $C_{\text {. apte- }}$ ros a Cl. Auctore numeratus, at mea fpecimina femper' alata. Hab. cum aliis frequens.

Statura fere eadem cum $C$. plaño $F$. i. objolato and. at dimidio minor. $\mathrm{Hg}$.

\section{(p.7\%.)}

177. C. punftulatus niger, thorace quadrato, elytris ftriatis punctis plurimis longitudinalibus impreffis. Fn. Etr. I. 254. 530.

Bupreftis ater laeuis, pedibus antennarumque bafi ferrugineis. Geoffir. Inf. 1. 16 r. 39.

C. flauipes niger, fubtus picens, pedibus nufis, thorace fubquadrato, elytris arcte iuncto, elytris nouemftriatis: friis quatuor punctatis. Apterus. Mon. Car. n. 2 r.

† C. Ciftelöides. Hellw. ap. Panzer. Fn. Inf. Germ, XI. I 2.

Habitus et ftatura C.melanocephali, at duplo ma ior. Defcriptio Monographiae optima. Pedes in 
noftris plerumque rufo fulcefcentes, - Habitat fub terra, et in ligno putrido obuius,

Hic adnotandum mihi videtur C. pum iulatum F. E. S. I. I. I50. x I4 alium, valde diụerfum efle, cui citatum C. punczulatus Schaller. Act. Hallenf. I. p. 3 r8. adiungatur. Vide animaduerfa ad C. infidum n, I98. $\mathrm{Hg}$.

I78. C. Ariatus niger, thorace poftice foueola vtrinque biloba, elytris ftriis nouem profundioribus: punctis tribus difcoidalibus. Paykull. Mon. Car. n. 26.

- C. nigrofriatus. De Geer. Inf. 4, 96. 12.

C. Leucophthalmus F. Mint. I98. $4 \mathbf{I}$.

Defcriptio et obferuatio Mon. Carab. Thorax in medio nonnihil complanatus, et corpus ac elytra minus, quam in $C$. Leucophthalmo conuexa: Noftrates tamen vix eum magnitudine fuperant; et pedes habent, forte ratione aetatis, fubrufos, in reliquis conueniunt omnino cum Suecis. Habit. fub terra Martio m. frequens.

De citato e Paykullio leue tamen dubium mihi exfiftit, quia pedes quoque in meis fpeciminibus, cum Carabo Roffiano omnino conuenientibus, femper piceo-rufi funt. Hic Carabus, quem cum amicis nomine $C$. Monticolae communicaui, fcaritum fpeciem prae fe fert. Specimina mea in monte Affe fub lapidibus deprehendi.

Fabricius C. friatum Paykullii apud C. Nigritan E. S. I. I. I 58. I 49. allegat. Inde concludimus, eiusdem C. Leucophthalnum Mant. huc non fpeहtare. Hg.

$$
\text { (p. z7.) }
$$

179. C. cinctus fufcus, capite thoraceque viridiaeneis, elytrorum margine pedibusque pallidis. F. Sp. 3 10. 62. 
Fn. Etr. I. $250.523 \cdot$ t. 4 . f. 9 :

Color capitis thoracisque viridi-aeneus, elytrorum profunde cyaneus. Magnitudo femper eadem, Confer, Fn. Etr. 1, c. Hab. hieme fub cortice roboris; mox faepe lectus etiam in Vlino.

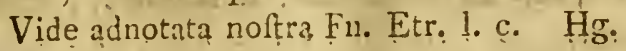

I80. C. ruficornis alatus, niger, antẹnnis pedībusque ferrugineis, thorace bafi finbretufo, fubrugofo, elytris ftriatis, fubtilifịime punctațis, fubuillofis. Payk. Mon. Car. n. 74.

C. ruficornis. F. . Mant. I99: 48: Geoffr. Inf. I. 160. 38: Le Buprefte noir velouté.

C. puluerulentus. Fn. Etr. I. 25.3.526. t. 3! f. 7 . Defcriptio Mon. Car. Hab. in ligno putrefcen. te, et liub lapidibus rarior:

\section{(p. 78.$)$}

18 I. C. veftitus fubtus niger, fuprą viridi = aeneus, antennis pedibusque pallidis, elytris pubefcentibus, punctato-ftriatis, punctis maioribus nullis, limbo exteriore pallido poftice latiore. Mon, Car. n. 44 .

Linn. Syft. Nat. 6\%0, I6.

Geoffr. Inf. I. I62. 41. Le Buprefte vert ì boudure. C. marginatus. F. Mant. 202. 84. ab hoc diuerfus, et a Linnaeo in Fn. Sv. 804 . defcriptus.

Defcriptio et obfer!atio Mon, Car.

Carabus hic, cui competit.omnino allata delcriptio, et magnitudo $C . \sigma$ - punctati rarior habitat apud nos. At contra occumit alius frequens, in Fn. Etrufca fitb nomine $C$. marginati non recte defcriptus, qui mihi modo erit varietas 3. C. veftiti, nam fi fpecies (vti videtur) diftincta, fane admodum proxima eft $C$. veftito, cum differat tantum ina- 
gnitıdine duplo maiore, thorace magis fubquadrato, elytrisque limbo exteriore pallido, poftice vix latiore.

Hab. fub lapidibus et in truncis arborum cariofis. Var. $\beta$. quae in Mon. Car. defcribitur, mihi prorfus ignota.

Ille Carabus. quem Auctor varietatem C. veffiti habet, C. marginatus. Fn. Etr. I. 25I. 524., peculiarem fiftit fpeciem. $C$. veffito duplo maior eft. $\mathrm{Ca}$. put et thorax in fpecimine, quod vidi, cuprea erant, nec adeo denfe punctata, vt in C. veftito. Thoracis, pro portione longioris, margo extimus nullo modo flauefcebat, vt in $C$. vefito. Elytra minus lata, minusque punctata in maiore, in quo limbus elytrorum flauus pone vix dilatatus eft. Multo magis, quam $C$. veflito, hic Carabus $C$. cinm Eio $F$. affinis eft, fed hic breuior, vix pubefcens, thoraceque magis rotundato eft. $\mathrm{Hg}$.

\section{( $p . ; 9$.}

* I82. C. Jpoliatus capite thoraceque aeneis, elytris vinidi - cupreis, limbo exteriore pallido, rndique aequali, futura aene-lucida.

Magnitudo $C$. veftiti var. maior. $\beta$., eiusdemque ftaturae, at thorax paullo anguftior, fere obcordatus. Caput aeneum nitens. Palpi nufi. Antennae in viuo piceae tribus primis articulis pallide rufis. Thorax aeneus nitens fere obcordatus, linea longitndinali, punctisque oblongis maiufculis pofticis profmdius excauatis. Elytra viridi-cuprea, ftriata, punctis difcoidalibus nullis, limbo exteriore pallido vndique aequali, et ad imum futurae marginem aeneo-Incida. Stermum et abdomen etiam iub elytris nigrum. Pedes pallidi. Alae albae. 
Differt a $C$. veftito var. maior. $\beta$., antennis piceis prininis articulis pallide rufis; in $C$. veftito totae pallidae rufae. Differt thorace paullo anguftiore, inagis obcordato, elytris non pubefcentibus, non viridi - holofericeis, magis conuexis, magisque ad cupreum vergentibus, fitura aeneo-lucida, thoracique concolori; demum differt abdomine etiam in dorfo toto nigro; in C. vefito var. maior. $\beta$. abdomen nigrum margine tamen fub elytris late pallido. Praeterea in C. Spoliato elytrorum limbus exterior, pedesque paullo pallidiores; et fere albidi. Coloris dinerfitas, thorax longior et anguffior, et abdominis dorfum omnino nigrem, praeter alias notas, difierentiam conftantem fpecificam, ftatnere videntur. Addendum quod C. Spoliatus rarior, et uunquam inuentus cohabitare cum $C$. veftito. Hab. in tiuncis putrefcentibus minime frequens. Long. ?. lat. $2 \frac{2}{3}$.

\section{(p. so.)}

I83. C. nigricomis niger, thorace cupreo, ely: tris ftriatis, viridibus, pedibus piceis. F. Nant. 202. 82.

Faykull. Mon. Car. n. 7o. cuius defcriptio itetum exhibetur. Statura et labitus fere C. holofericei, fed apud nos paullo minor, lacuis, elytrisque vti in $C$. veftito certo fitu ferrugineo micantibus. Hab. in pratis humentibus fat frequens. Long. $4^{\frac{1}{2}}$. lat. 2.

C. nigricornis, quem Panzerus Fn. Inf. Germ. VI. 4. optime depinxit, quocunque lucis illapfu, numquam ferrugineo micat, quod apud C. holofericei varietatem, quae a permultis cum C. nigviconi confunditur, omnino ita fe exlibet. Hine Auetoris 
C. nigricornem C. holofericei varietatem habere mihi liceat. Haec maiorem fidem habent, cum in defcriptione Paykullii Monographiae, ab Auctore noftro integra repetita, antennarum articulus priL. mus rufus defcribatur; antennis $C$. nigricomis,
quod nomen iam fuadet, femper ynicoloribus nigris. Ex his modo dictis patet, me quoque Paykullii C. nigricomnen C. holofèricei varietati annumerare. Magnitudo dicta minor nullum offendat, quia fpecimina illius yarietatis $C$. holofericei minora effe folent.

Celeberrimus Schneiderus Nenft. Magaz. p. 362. C. nitidulum Schrank. En. n. 401. forte allegandum putat ; quam coniecturam tamen probare nequeo, yt e defcriptione fatis patet. Hoc citatum ad àlium fpectat Carabum, C. veftito fimilem, linbo flauicante vero deficiente. $\mathrm{Hg}$.

* 184. C. rufus obfcure rufus, thorace obcordato, elytris fubftriatis.

Magnitudo C. Germani var. mai. Totus obfcure rufus, vnicolor. Thorax obcordatus, conuexus, pofice coarctatus, truncatus, vix marginatus, lineola longitudinali, angulisque pofticis impunctatis. Elytra conuexa, fubftriata, non nitida, latitudine fere thoracis absque punctis marginalibus. A Car. ferrugineo dinerfiffimus. Hab, in filuis, raro lectus.

I85. C. humeralis niger, thorace obcordato, elytris ftriato - punctatis, truncatis, antice rufis, pedibus rufoflanis. Mon. Car, n. 23 .

- Defcriptio et var. $\beta$. indidem defumtae funt. Noftrum fpecimen differt tantum magnitudine paullo maiori, at in reliquis conuenit. Lectus in ligno putrefcente. 


\section{(p.81.)}

* I 66. C. Jmardgdulus viridis, nitidus, thorace fibquadrato, elytris ftriatis.

Magnitudo C. aterrimi. Caput viride nitidum, laene, punctis duobus impreffis. Palpi antennaeque fufcae primo articulo rufo. Thorax latitudine elytrorum, viridis, nitidus, laeuis ; fubquadratus, poftice fubdepreffus, lineola longititdinali vix apparente, ftriaque, feu potius pimcto vtrinque excanato. Elytra viridia, nitida, la enia, fubtilifime friata, impunctata, contiexa. Subtus totus ater, nitidus, plantis exceptis ferrigineo pilolis. Alatus. Variat magnitudine pedibusque interdun rufis. Differt a C. lepido, femper aptero, alis thorace vna tantum ftria poftica impreffa; et colore elytrorum lacte viridi: qui in $C_{\text {: lepido ad cupreum }}$ vergit. Hab. fub lapidibus, et in ligno putrido frequens:

\section{(p. \&.)}

187. C. 6 -punctatus capite thoraceque viridibus, elytris cupreis. F. Sp. 309. 60.

() Goed. Schaeff. Ic. t. 66. f. 4 .

D Schrank. E. I. 402. Vill. Ent。 1. 372. 6. De Paykull. Mon. Car. n. 42 .

of F. E. S. I. I. $157 \cdot x 45$.

Defcriptio Payk. Mon. var. $\beta$. viridi - a eneus, elytris concoloribus apud nos frequentior. Hab. in locis apricis. Inuentus etiam fub arbonum cortice tempore hiberno. Long. 4. lat. $I_{\frac{2}{3}}$.

* I88. C. Tencbrioides ater, thorace lato, ely. trorum ftriis punctatis. Geoffr. Inf. x. I59. 34 . Le Buprefte pareffeux.

C. Tenebrioüdes. Linn. Ed. XIII. 1986. 2 I3. 
C. piger. Fourcroy Ent. Parif. 42. Villers Ent. I. $3 \% 7 \cdot 63$.

Statura lata vndique fere aequalis, et magis conuexa quam in plerisque fuae gentis. Caput nigrum, nitidum, minus exfertum. Palpi antenna eque breues, fubrufae. 'Thorax niger, nitidus, latitudine elytrormm valde conuexus, fubquadratus, marginatus, antice laeuis, poftice punctula tus, foueola vtrinque leuiter impreffa. Elytra nigra, admodum et aequaliter conuexa, ftriata, ftriis nouem minutifime punctatis, extremo apice emarginato. Steinum et abdomen nigra nitida. Pedes nigri, breues, praefertim antici tibiis apice latius. culo, fininisque craffis inftructo. Alatus. Variat magnitudine, abdomine, tibiis, tarfisque fubrufis. Hab. in arenolis et fub lapidibus frequens. Long. 7. lat. 3 .

Iuem Carabus, qui in Mufeis Germaniae nomine $C_{\text {. }}$ Tenebrioides Schneideri occurrit; res admodum rara, sbi eidem infecto idem nomen a duobus Auctoribus datum eft. Hg.

\section{(ק. 83.)}

x 89. C. dentatus cyaneus, thorace cylindrico îbacquali, ore, antenuis pedibusque rufis. Fn. Etr. 1. 260. 55 I. t. 2.f. 1 I.

Carabun hunc ad Cicindelae genus referendum efle credant alii; mihi eft, femperque erit $C_{u r}$ bus; nam facies, liabitatio, viuendi modus, incelfis, aliaque inter $C$ arabos naturaliter fatuere videntur. Hab. fub Vlmi cortice in truncis emortuis.

* $\mathbf{9 0}$. C. diftinclus teftaceus fubpilofus, àbdomine, elytrorumque fafcia longitudinali futurae communi atro-caeruleis. 
Statura et magnitudo $C$. dentati. Caput téftaceum. Oculi nigri. Palpi antennaeque teftaceae articulo primo praelongo apice nigro vt in $C$.dentato. Mandibulae pariter vt in illo exfertae, elongatae, teftaceac. Thorax anguftatus elongatus, fere cylindricus, emarginatus, teftaceus. Elytra punctato-ftriata, integrạ, teftacea, fafcia longitudinali futurae communi atro-caerulea. Pectus pedesque teltace: Abdomen atro-caeruleum. Alac albae. Etfi habitu et magnitudine fere fimilis C. crepitanti, Caraboque dentato; attamen ab is omnino diftinctus. Differt enim a $C$. crepitante forna capitis, antennarumque articulo primo longiffimo, thorace emarginato, elytris teftaceis, fafcia etc. A C. dentato differt colore prorfus diuerfo, thorace $110 n$ canaliculato, elytris integris. Hab. cum aliis exefas arbores. Raro lectus hieme.

\section{(p.sa.)}

I9 . C. Germanus cyaneus, capite, elytris pedibusque teftaceis, elytris apice violaceis. F.Sp. 3I2. ? I.

Faun. Etrufca. I. 249. 5:2.

D Poda M. G. p. 273.

Sclia efferi Icon. t. 3 I. f. I 3. citata a Fab. aliisque pertinct ad C.viritamm. Defcriptio Scopolina optima, at in noftris pedes non thoraci, fed fomper elytris concolores, fcilicet tefracei. Diximus in Fn. Etr. 1. c. variare interdum capite nigro; muc autem, re nelius confiderata, non omittendum dubium, fere ad certitudinem redastum, hunc $C_{l \neq}$ rabun hucusque a nobis pro varietate $C$. Germani habitum, fpeciem fortaffe ab co efle difinctam: hic eniu, quamuis habitu et ftatura C. Germano 
fimillimus, attameñ retiera conftanter differt magnitndine, capite atro, thoracis lateribus pofticis non extrorfum, neque acute angulatis, vti in $C$. Germano, fed obtufis; rinacula elytrorum violacea non aciminata; maximamque eorum partem occupante. Praeterea C. Germanus frèquens hieme fub arborum cortice, lignoquie putrido; lic eo tempore raro, aut numquain inuentus, etfi alio occurrat faepiffime; addendumque quod in hac fpecie, aut varietate vidi et afferuo fimile cum fimili coptala iunctum.

Hab. in filuis frequens. Long. $3 \frac{1}{2}$. lat. $I_{\frac{1}{2}}$.

Haec de C. Germani putata varietate enumerata abunde probant, eam omnino fpecie diftinguendam effe. Hg.

* 192. C. exhalans capite, thorace pedibusque rufis, elytris truncatis, nigro-caeruleis, maculis duabus flauis.

Statura et magnitudo omnino C. crepitantis, cuius videtur effe varietas elegantifina, ac fingularis: differt enim tantum elytris maculatis, maculis in fingulo elytro binis, altera pone bafin, altera pone nedium fubrotundis flauis. In C. crepitante, qui apud nos frequens copiofus occurrit, abdomer obferuatur in viuo modo obfcure nigrum, modo rufum. In indiuiduis magnitudine minoribus, rufum, in maioribus (fortaffe feminis) opacum nigrum. In hac noftra varietate tamen, quamuis minores aequiparet, abdomen opacum nigrum. Hab. fub terra cum C. crepitante Mart.' menf. rarus. 


\section{(p. 85.)}

* r 93. C. Pfanmodes niger, fubdepreffus, capite, thorace, limbo elytrorum, antennis pedibusque liuide rufis.

Statura $C$. arenarii, at minor. Caput rufum. Oculi nigri. Palpi antennaeque rufae longiores. Thorax luperne et inferne rufus, obcordatus, connexus, fubmarginatus, lineola obfoleta longitudinali punctisque pofticis ordinariis impreffis. Elytra nigra, fubdeprefla, ftriata, ftriis oculo armato punctatis, litubo exteriore, pofticoque rufo. Abdomen nigrum. Pedes rufi. Alatus. Limbus rufus poftice latior. Differt a $C$. fabulofo F. Mant. 47. a Car. liwido Linn. Fn. Sv. 79 1. et a $C$. Jabulojo Mon Car. n. 28. cum in noftris caput, thoraxque fint omnino rufa, et elytra nullo modo dici poffint fubtilifime ftriato - punctata, punctis quibusdam difcoidalibus, vti in Mon. Car. l. c. afferitur. Hab. Florentiae rarus.

r 94. C. atcrimus niger, nitidiffimus, thorace conuexo, elytris leuiffime punctato-ftriatis: punctis vtrinque tribus difcoidalibus maioribus. Mon. Car. n. 78 .

Herbft ap. Fuesly Arch. 6. 140. 50. t. 29. f. I3.

C. oblongo-punctatus. Fn. Etr. T. 254.528 .

Defcriptio et obferuatio Paykullii l. c. repetitae. $\mathrm{Hab}$. in filuis primo vere non rarus. Long. 6. lat. $I \frac{3}{4}$.

195. C. Proteus laeuis, cylindricus, thorace punctis ordinariis laeuioribus, elytris ftriatis, difco impunctatis. Mon. Car. n. 72.

$C$. aeneus F. Mant. 202. $8 \mathrm{I}$.

Ee

Defcriptio 
Defcriptio Mon. iterata. Hab. in Suecia auftrali. Mihi numquam lectus.

Hic Carabus admodum variat, vti videre eft in Mon. Car. 1. c. Varietates in ea indicatae, quac apud nos occurrunt, et quas ego inueni, funt fequentes.

$V a r . \delta$. fubtus niger, fupra cyaneus, antennis pedibusque rufis. M. C.

C. azurcits.F. Mant. 20 I. 7 r. Fn. Etr r. 266. 550.

Pedes et antemae interdum fufcefcentes. De hoc tamen licetúe mihi dubitare, an reucra fit $C$. Protei varietas? Forma thoracis paullulum diner1a, punctaque poftica magis excauata. Hab. in locis arenofis non frequens.

$V a r . \varepsilon$. Niger, pedibus antennisque rufis. M.C.

C. latus.. De Geer. Inf. 4. 100. Geoffr. Inf. I. 160. 37. Schrank. E. I. 2 I I. 397. C. latus, Sed maior.

Communis.

$V a r: \eta$. Niger, thorace fipra, clytrisque viridiaeneis, pedibus antennisque rufis. $\mathrm{M}$. C.

C. affinis. Schrank. E. I. 212.399.

Variat pedibus antennisque piceis. Long. $5 \%$ lat. $I^{\frac{2}{3}}$. Hab. fub lapidibus, et in truncis arborum fat frequens.

Confer, quae de his varietatibus apud $C$. azureum.

'Fn. Etr. 266. 550. monui. Hg.

196. C. affimilis niger, thorace capite longiore, poftice impunctato, elytris frriatis, margine punctato. Mon. Car. n. 32 .

Defcriptio Monographiae iterum exhibita. In noftris thorax lineola longitudinali inpreffi. Hab. cum praecedentibus frequens. Long 7 . lat. 2 . vix. 
197. C. obfirus niger, fupra obfcure nigro-fubaeneus, elytris, difco vtrinque tripunctato. Vur. Mon. Cir. n. 45. fule, optimeque defcriptus. Conf.

Statura et magnitudo $C$. mendacis. Fn. Etr. et fere $C$. picipcdis. Thorax laeuifimus longitudine paullo latior, conuexus, vix marginatus, lineola longitudinali, punctisque pofticis excauatis. Inter ftriam fecundam et tertian elytrorum puncta tria minutiffime excauata, margine etiam exteno punctato. Pedes nigri. Variat colore interdum magis nigro, interdum magis cyaneo. Hab. cum C. crepitunte, viridano, aliisque iuxta fepes tempore hiemali fub terra degens.

\section{(p. 8s.)}

* I98. C. infidus niger nitidus, thorace femirotundato, elytris fubdepreffis, punctato-1triatis, antemnis tibiisque ferrugineis.

An $C$. depreffus. Mon. Car.?

C. pilicomis. Fn. Ftr. I. $255 \cdot 53 \mathrm{~T}$., qui omnino delendus.

\& C. brenicollis. F. E. S. I. I. I50. I I3.

Panz. Fn. Inf. Germ. XI. \&.

Facies C. .jinibarbis, fed duplo maior. Palpi antennaeque ferrugineae, bafi nitidiores, pilis longionibus hirtae. Thorax fufco-niger nitidus, orbiculatus antice pofticeque anguftatus, marginatus punctulatus, fulco longitudinali conuexitatem quafi in duos lobos diuidente. Elytra fufconigra nitida, fubdepreffa, 9 -ftriata, ftriis confertiflime punctatis, punctis quatuor vtrinque in ftria fecunda paullo maioribus longitudinaliter excauatis, interdum vix perfpicuis. Pedes fubrufi. Femora plerumque nigra. Alae albae. Iunior va- 
riat colore fufco - rufo. Variat etiam magnitudine minori. Hab. fub corticibus, et in truncis cariofis primo vere frequens. Long. $5 \div$. lat. $24^{1}$.

De citato e Paykullii Monographia tamen dubito, quia plura non congruunt. Id praecipuum obftaculum eft, quod Carabus depreffus $P$. apterus eft. In thoracis defcripticne lineae mediae longitudinalis impreffae mentio nulla facta eft. Porro ibidem in angulo linea obliqua fatis profunde itiprefia eft. Elytrorum ftriae punctatae funt, punctis:haud leniffimis. - Hic Carabus in mufeis non paucis nomen $C$. picens gerit.

Fabricius Ent. Sytt. x. I50. I 14 . apud C. punfulatum illud citatum Paykullianum allegat, culi tamen dubium non minus obftat; cum, quia Paykullius apterum dicit Carabum fuum, tum, quia in angulo pofteriore thoracis, qui etiam inepte fubrotundatus vocatus eflet, puncta maiora impreffa omnino adfunt. Hg.

199. C. agilis thorace rotundato rufus, elytris abdomineque nigris. F. Mant. 204. 99.

payk. Non. Car. n. 64.

q F. E. S. I. I. I39. 67 .

Defcriptio Monographiae, eiusdemque Var. $\gamma$. fufcus, fubtis, abdomine excepto, maxillis, antennis pedibusque rufo - flauis, elytris puncto medio pallido; apur nos frequens. Hab: in foliis arborum conuolutis.

\section{(p.89.)}

200. C. ouatus ouatus, thorace antice capitis, poftice elytrorum latitudine, vtrisque adnato, elytris ftriatis, punctis difcoïdalibus nullis. Mon. Car. n. $8 \mathrm{I}$. 
C. vulgaris. Fn. Etr. I. 244. 5r5. Linn. Fn. Sv. 799. $\beta$.

Defcriptio Nonographiae.

Var. f. Supra obfcure aeneus, fubtus cum antemnis pedibusque piceus. C. vulgaris var. a. Linn. Syit. N. 675.27. Fil. Su. 799.

Var. $\gamma$. Supra viridi-aeneus, fubtus niger.

$V a r$. $\delta$. Supra nigro - caerulefcens, fubtus niger nitidus. C. amenes. De Geèr. Inf. 4. 98. $\mathrm{r}_{5}$.

Sequitur obferuatio Non. iterum exhibita.

C. metallicus. Scop. E. Carn. 270. videtur referendus ad varietatem $\delta$. Hab. fib lipidibus frequens.

Confer adnotata mea ad $\dot{C}$. vulgarem Fn. Etr, $x$. 244. 5'5. Hg.

\section{(p. 90.)}

* 201. C. votiuns niger nitidus, thorace vix marginato, elytris ftriatis, punctis difcuidalibus nullis, antennis ferrugineo - flauis.

C. nigritae affinis, fed minor, Caput nigrum nitidum. Antennae flanefcentes. Thorax niger, nitidus, vix marginatus, fubquadratus, lineola longitulinali, punctisque pofticis ordinariis imprelfis. Elytra nigra nitida, ftriata; punctis discoidalibus mullis. Sternum et abdomen nigra. Fomora nigra. Tibiae tarfique ferruginei. \& $C$. Nigrita diltinguitul thorace vix marginato, p.inctisque difcoidalibus nullis. Hab, in truncis artorum cariofis.

* 202, C. velocipes ater fubaeneus, elytris punctato-ftriatis, antennis nigris, pedibus pallide rufis. 
C. rufipedi Mon. Car. affinis, cuius forte varietas. Antemnae nigrae primis duobus articulis rufis. Caput atrum fubaeneum nitidiffimum. Thorax ater fubaeneus, nitidus, cordatus, lineola longitudinali, punctoque poftico vtrinque excauato, latitudine dimidia elytrorum. Elytra atra fubaenea, nitida, punctato-ftriata. Femora atra fubaenea. Tibiae tarfique pallide rufi. Alae nigrae. Hab. fub corticibus. Long. 2. lat. $\frac{3}{4}$.

203. C. rufipes fupra obfure-fubaeneus, fubtus niger pedibus rufis, thorace glaberrimo, elytris integris, fubtilifime punctato-ftriatis. Mon. Car. 11. 63 .

Defcriptio loci citati abbreuiata; eadem tamen. Hab. cum praecedentibus.

$$
\text { (p. 21.) }
$$

204. C. dorfalis thorace rotundato nigro, coleoptris pallidis, macula magna dorfali nigra. F. Mant. 205. I 16.

\& F. E. S. I. x. $165 \cdot 183$.

Paruus. Caput atrum nitidum. Thorax ater nitidus, orbiculatus, margine tenuiffime pallefcente. Elytra fubtiliffime ftriata, nitida, pallida, macula dorfali nigra fere commuii. Corpus nigrum. Pedes ferruginei. Antennae, in noftris, fufcae, bafi ferruginea. Confer C.meridianum var: $\gamma$. Mon. Cai. Hab. fub foliis putridis frequens.

Confer, quae aptud C. meridianum. Fn. Etr. 268 . 554. monui. $\mathrm{Hg}$.

* 205. C. erratus niger, thorace orbiculato rufo, coleoptrisque flauis, fafcia media nigra ad vtrumque marginem dilatata. 
\& C. Andreare. Fn. Etr. $x .265 \cdot 548$.

C. Ciux minor. Fabr. E. S. I. I. I60. I59.

Panz. F. I. G. XVI. 2.

Statura C. Turciri, at maior. Caput nigrum nitens. Antennae fufcefcentes articulo primo, et fecundo ferrugineis. Thorax rufus, orbiculatus, marginatus, angulis pofticis paullulum productis, lineolaque dorfali vix impreffa. Elytra minus connexa, tenuifime fubfriata, apiceque oblique truncata, flaua; fafcia media transuerfa nigra, quae ad futuram, et marginem exteriorem, apicenque adeo extenditur vt fpatium margine nigro cinctum, feu maculam fubrotundam flanam vtrinque conftituat. Abdomen nigrum. Pedes faepius rufi, interdum nigri. Differt a $C$. Turcico magnitudine maiori, colore, macula poftica apicis flana. A C. C. minori, differt etiam ftatura, capite anguftiore, thorace magis orbiculato, fafcia minus lata, elytris truncatis. etc.

Heic autem fatendum, hunc Carabım male a me affumtum fuiffe in Fin. Etrufia pro C. Andretie; cui thorax iuxta $C$. Fabricii diagnofin niger eft, non rufus; proindeque mirum videri, me nec poffidere, uec rmquam vidille hanc fpeciem, quae pro Italica habetur. Hab. cum aliis rarior.

Illo tempore, quo Auktor nofter fcribebat, C. Crux minor idem Carabus erat, quem nunc C. bipufululatum vocamus. C. Crux minor Auctoris Fn. Etr. x. 263.547 . itaque idem cum C. bipufullato videtur. De hoc certe $C$. errato nullum dubium reftat, quo minus verus C. Crux minor Linn., et nune quoque Fabr., fit. Vide animaduerfa ad C. Crucem minorem. Fn. Etr. l. c. Hg.

LXXIII. 


\section{LXXIII. (p. pa.) SCARITES.}

* 206. S. Dama niger, thorace obcordato, capitis clypeo bafi bituberculofo, apice vtrinque cornuto, cornibus erectis, excauatis, compreffis, biramofis.

Diximus in Fn. Etr. 1. 275. 57 I. Sc. Calydoninum variare magnitudine minori, maxillis cornutis; at re melius perpenfa, varictas ibi indicata modo fpecies diftincta videtur, nam etfi ftatura eadem, magnitudo tamen quadruplo minor, et caput, vti in diagnofi, omnino diuerfum. Reliqua omria in vtraque fpecie fimilia. Hab. in arenofis.

\section{TENEBRIO.}

* 20\%. T. Cornifronis oblongus, ruber, nitidus, elytris nigris: capite bicorni.

\& Ips haemorrhoidatis. F. E. S. I. 2. $5^{\text {I3. II. }}$ Panz. Fn, Inf. Germ. XII. I6.

Statura et magnitudo omnino $S$. Rufjece Fn. Etr. I. I36. cui primo intuitu adeo fimilis, vt facillime cum eo confundatur. Conf. fig. 6. tab. A. conf. etiam A. rubrum. De Geer. Inf. 5. 285. I. t. 8. f. 12. Caput rufum fronte admodum excauata, et corniculis duobus eleuatis rufis inftructa. Palpi quatuor inaequales, antici fubclauati, poftici filiformes, rufi. Antennae brenes, rufae, moniliformes. Oculi nigri. Thorax rufus, nitidus, laeuis, conuexus, fubnuarginatus. Elytra nigra, nitida, extremo apice rufa, conuexa, fubtiliffme punctatoftriata, friis in fingulo elytro nouem. Sternum, abdomen, pedes rufa. Alae albae. Frons in altero 
fexu inermis, Differt a S.Ruffica, capite cornuto, antennis brenioribus, apice minus clauato, ftriis elytrorum diftinctionibus, abdomine toto rufo etc. $\mathrm{Hab}$, in quifquiliis et truncis arbonm cariofis, rariffimus. Long. $2 \frac{1}{2}$. lat, $1 \frac{1}{5}$.

Statura huius infecti, vt mihi videtur, adeo non affinis eft ftaturae $I$. nigripennis, it libenter illi anonymo afientiam, hoc infectum ali generi, Dirperibus fcilicet, annumeraturo, (vid. Schneid. Magazin. V, Heft. p. 559. adnot ad. n. 2.) Sed forte melius peculiare genus conftituit, cui plura infecta Americana adiungerem, quae, ftaturae quoque eiusdem, infuper capitis corniculis gaudent.

Celeberrimus Fabricius 1. c, quaerit, an Hifpa cormigeva antennis ferratis, thorace rufo, elytris caeruleis, capite bicorni. Mant, x. 47. 5. huc fpeEtet, Cum hoc infectum celeberrimo viro ipfi ignotum fit, me idem non noffe libenter confiteor. Sed in Fn. Etr, I. 36, 80. apud Dermefiidem fanguinicollem figurae 4. t. 220. Scháefferi Icamum mentionem feci, quae $a b$ Harrero ad noftram 1. haemorrhoidalcm relata eft, licet et figura et defcriptio valde difcrepent, $S i$ ibi depictum infeEtum reuera caput bicorne haberet, forte commemorata Hifpa cornigera Fabr. eflet? Hg.

\section{(p. 9.3.)}

208. T. Mauritanicus alatus nige:; fubtus piceus, thoracis marginibus antice pofticeque dente angulatis. Linn. Syft. Nat. 674.4.

Trogofita Manritanica. Oliu, Ent, Ig. 2. I. t. I. f. 2. A. B.

Geoffr. Inf. I. 64. 5.

Dorth. Mem, d'Agric. a. x787. p. 64, f. I. 2. Fn. Etr. I. 280. 579. t. 7. f. I5.

$$
\text { Ee } 5
$$


Hab. fub arborum cortice frequens.

Vide Fn. Etr. 1. c. Hg.

\section{MYLABRIS.}

209. M. teftacea teftacea, pectore, elytrorumque apicibus nigris. F. Mant. 217.8.

Praeter ea, quae diximus de $L$. Afra et $M$. teftacea in Fn. Etr. r. 292. 594. et.in Appendice, addendum varietatem elytris totis nigris variare interdum etiam capite, pedibusque nigris. Hab. in floribus Dipfaci Fullomm frequentifima.

\section{(p. .4.)}

* 2 Io. M. fulua nigra, thorace rufo-flauefcente, elytris faturate teftaceis.

Statura $M$. teftaceae, at dimidio maior, ac diftinCiffima. Caput atrum nitidum, glabrum, tenuifime punculatum, inflexum. Palpi quatuor inaequales, filiformes nigri. Antennae nigrae, filiformes I $i$ - articulatae. Thorax rufo-flaueicens nitidus, glaber, ominino conformatus vti in $M$. teftacea. Scutellun nigrum. Elytra faturate teftacea, lacuia, flexilia, vix pubefcentia, integra, fed abdomine paullo breuiora. Sternum, abdomen, pedes atra. Alae obfcurae. Abdomen craflum ventricolum; forte femina?

Non leuiter admiranti mihi prodiit e pupa obtecta putamine proprio oniformi ferrugineo, ftigmatibus vtrinque longitudinaliter impreffo, quam inueni abrupto nido ip. varimntis (conf.) in eius medio fitan prope cellnlas, vbi neficio quomodo introducta larua, veluti in apto fibi domicilio vna cum Apibus l. c. defcriptis, metamorphofin fubierat. Long. $5^{\frac{\pi}{2}}$. lat. $\mathbf{I}_{\frac{3}{4}}^{\frac{3}{4}}$.

LXXXVI. 
LXXXVI. MORDELLA.

2 I I. M. abiominalis ano aculeato, nigra, thorace abdomineque fuluis. F. Sp. 333. IO.

\section{오 F. E. S. I. 2. II 4. 9 .}

Stitura $M$. aculectae. Caput, pectus, elytra, fpina analis, pedes átra. Thorax et abdomen fulua, immaculata, niticia. Lecta in floribus.

\section{(p.05.)}

212. M. frontalis atra, fronte pedibusque flauefcentibus. F. Sp. 333. 12.

\& F. E. S. I. 2. II 4. 9. Panz. F. I. G. XIII. I3.

Parua. Frons flaua, et pedes praefertim antici flaui. Hab. in floribus.

* 213. M. nigra nigra, vniculor, antennis bafi flauefcentibus.

Statura et magnitudo M. finucae. Caput et thorax minora, miuusque gibba guam in $M$. aculeata. Thorax fuborbiculatus. Antennae prorfus moniliformes, nigrae primis articulis flanefcentibus. Auus absque aculeo. Hab. in floribus.

\section{STAPHYLINVS.}

2 I 4. St. hirtus hirfutus, niger, thorace abdomineque poftice flatis. F. Sp. 334. I.

( ) Sulz. Hift. Inf. t. 7. f. 16.

Paykull. Mon. Staph. n. x. defcriptio optima.

q F.E. S. I. 2. 519. 2. Panz. F. I. G. IV. 19.

Inter maximos numerandus, facillimeque dignofcendus. Lanugo aureo - flana interdum a etate deteritur. Hab. in fimo, et frercore bonino fat frequens in locis filuaticis arenofis. 


\section{(p. o6.)}

2 I.5. St. cyaneus niger, capite, thorace elytrisque cyaneis. Mon. Staphl. n. ?

St. airocaerulefcens. Geoffr. I. 36 r. 2 .

․․․ E. S. I. 2. 52I. II.

Defcriptio Monograph. Hab. apud nos rarior. Long. 7. lat, $1 \frac{\mathrm{T}}{2}$.

* 2 เ6. St. bicinctus niger, capite, thorace elytrisque profunde cyaneis, abdomine atro, vltimis duobus fegmentis bafi late aureo holofericeis.

Praecedenti proximus, eiusdemque maguitudinis ac ftaturae, at lllagis affinis St, morfitanti. Fn. Etr. I. 308. 6 r.

Caput bati latitudine fere thoracis, ad latera porica fubangulatum fufco-cyaneum. Pali rufi. Antennae filiformes, nigrae bafi rufa, apice acuto cinereo-rufo, capite vix duplo longiores. Thorax fufco-cyaneus, punctulatus, capite longior, et paulo latior, antice truncatus, angulatus, poftice rotumdatus. Elytra fucco - cyanea, punculata, thorace paulo longiora. Scutellum nigrum. Abdomen elytris plus duplo longius, atrum, vltimis duobus fegmentis bafi, lanugine aurea nitente late fafciatis. Subtus totus niger pedibus exceptis omnino rufis. Fafciae vix marginem exteriorem attingunt, et in mortuo, fegmentis contractis, maximam parten occultantur. Lectus in Fraxini Orui ligno putrefcente. Long, $7 \frac{\pi}{3}$. lat. $x_{\frac{\pi}{2}}$.

$$
\text { (p. 97.) }
$$

21 7. St. pubefcens pubefcens, capite flauo, thorace elytrisque fufco - nigroque nebulofis; punctis impreflis. Geoffr. Inf. I. 363.8.

Paykull. 
Paykull. Mon. Staph. n. 9. Degeer. Inf. 4. I \%. 2. St. ocltrocephalus. Ed. XIII. Linn. 2034. 85 . St. chryfocephalus. Ent. Par. I. 166.3. 아 F. E.S. I. 2. 520.5 .

Defcript. in Mon. Capitis collum pariter flaumm. Antennae bafi flatae, 6 vltimis articulis nigris. Thorax et elytra fufco nigroque nebulofa. Abdomen ad latera lanuginofim, fubtus cinereo-pubefcens, nigro - punctulatum. Hab. in fimo rarus.

2IS. St. tomentofus fufcus pubefcens, pedibus, thora cis lateribus, elytrorumque bali pallidis, penultimo fegnento abdoninis reliquis haud longiore. Mon. Car. Append. p. 138. 11.53. 54.

Defcribitur ibidem. Var. $\beta$. futcus, pubefcens, pedibus pallidis. Mon. - Habit, vterque apud nos in putridis.

$$
\text { (p. 08.) }
$$

'219. St. canaliculatus flaums, capite, abdominisque cingulo atris, thorace canaliculato. F. Mant. 221.20.

Paccicus inprefJus. Fn. Etr. t. 314.62\%.

Defcriptio Monogr. Staph. n. 23. Hab. in ligno putrido frequens.

220. St.clauicomis niger immaculatus, thorace rotundato leui, antennis extrorlim craffioribus. F. Mant. 221. 2I.

St. Imno. Geofir. In C. I. 37 r. $2+$.P Pyk, Mr. St. n. 25 .

St. Buplithalmos. Schrank. E. I. 445 .

Fn. Etr. I. 3 Ir. 623.

Defcriptio Monogr. Magnitudo praecedentis, at paullo latior. Hab. fub arborum cortice frequens. 
Conferantur animaduerfa ad St. Buphthalmum. Fn. Etr. 'т. 3 I r. 6ะ3. Hg.

* 22r. St. palitius pallide flauus, pilofus, capite elytrorumque pofticis nigris.

Statura et magnitudo St. tomentofi. Caput nigrum nitidun, thorace angutius. Antennae nigrae, bafi flauae, ad apicem craffiores, longitudine capitis atque thoracis. Thorax pallide flaums, parum conuexus, rotundatus, emarginatus angulis obtufis, poftice latior, fed magnitudine cedit thoraci St. tomentofi. Elytra pallide flaua, la euia, pilofa, capicis thoracisque longitudine; apice macula magna nigra, quae tamen marginem interiorem haud attingit. Abdomen rufoflaumm, pilofum, incurmum, apice obtufo. Subtus via cum pedibus omnino pallide flauus. Hab. in putridis, et fub arborm foliis disicctis.

222. St. orbiculatus niger, thorace conuexo, orbiculato, antemnis palpis pedibusque fufco rufefcentibus. Mon. Staph. 11. 26.

\& Paederits orbiculatus. F. E. S. I. 2. 539.9. Defcr. Mon. Hab. cum aliis rarior.

\section{(p. roo.)}

223. St? fanguincus niger, elytris fanguineis, abdomine gibbo antennis longioribus. Mon.' Staph. 11. 29.

St. fangaineus. Limn. Syft. Nat. 689. I9. Fn. Sv. 853.

\& F. E. S. I. 2. 529. 5 I. Panz. F. I. G. XI. I9. Statura, ac habitu a Staphiglinorum genere diuerfus, magisque capitis forma, atque thoracis $N o$ toxis proximus. Iure ad nouum genus fub nomi- 
ne Pfelaphi a Cl. D. Herlyt. relatus; Hiftor. Inr. p. 106. t. 39. f. 9. Minor St. fitcato, quo magis gibbus et globofior. Caput thoracis latitudine, inflexum nigrum, interảum picenm, in medio tuberculofum, vix rugofum. Palpi flanefientes. Antennae fulcae, longitndine dimidii corporis, moniliformes, articulis tribus vltimis craflioribus, vltimoque clanato rufo. Thorax rotundatus, connexus, niger nitidus, puncto in medio bafeos interdum imprello, abdomine multo anguftior. Elytra teftaceo - rufa, admodum connexa, valde abbreuiata, et veluti dorfo arctiffime adnexa, mediun tantum abdomen obtegentia. Reliqua pars abdominis nuda, magis etiam gibba, g!obofa nigra. Pedes longinfculi rufi femoribus a pice craffioribus, bafi tenui nigra. Occurrunt alii praecedenti fimiles, qui variant magnitudine, et colore corporis interdum toto faturate tefraceg. Hab. in terra arenofa, et in arborum carie non rarus.

Cel. Herbftinm non allegarem, quia hanc fpeciem optimi generis non nouifie videtur. Sed idem de Panzeró 1. c. diceren, quia infectum eius totum fanguineum eft, cum meis fpeciminibus ornnibus elytra tantum rrbbra fint. Libenter equidem credam, iuniora fpecimina teftacea eife, fed cum plures fpecies, valde fibi fimiles, quamuis fatis diturfae, tali colore fint, has cum St. fang grinci varieta te confufas putare liceat. $\mathrm{Hg}$. 


\section{(p. Tor) \\ LXXXIX. PAEDERVS.}

224. $P$. elongatus niger elytris poftice pedibusque fuluis. F. Sp. 339. 3 .

q F. E. S. I. 2. 537· 3. Panz, Fn. Inf. Germ. IX. 12.

Defcriptio Mon. Staph. n. I7. Magnitudo media: Hab. in ftercore et ligno putrido obuius. 


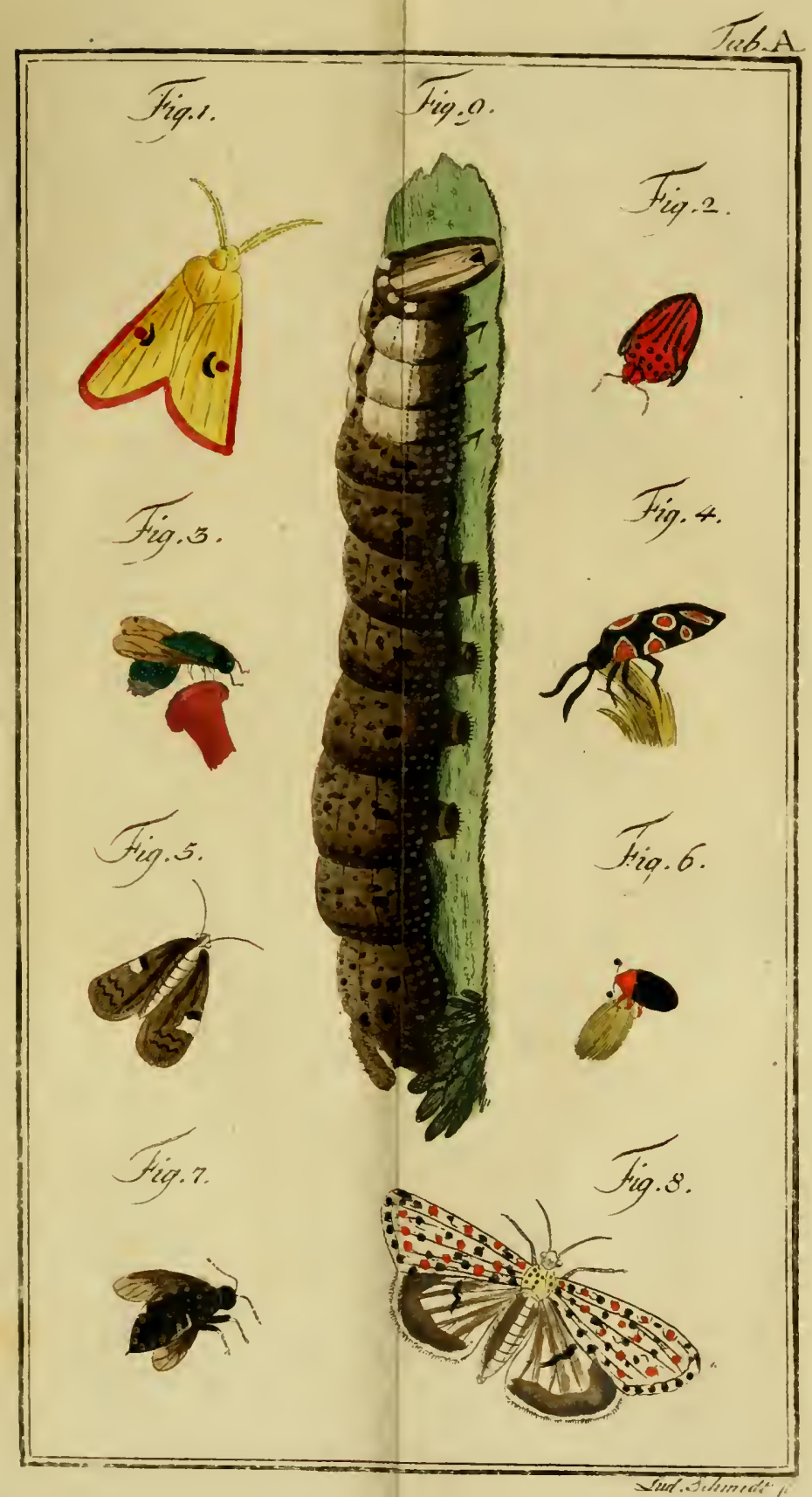


Tab.I.

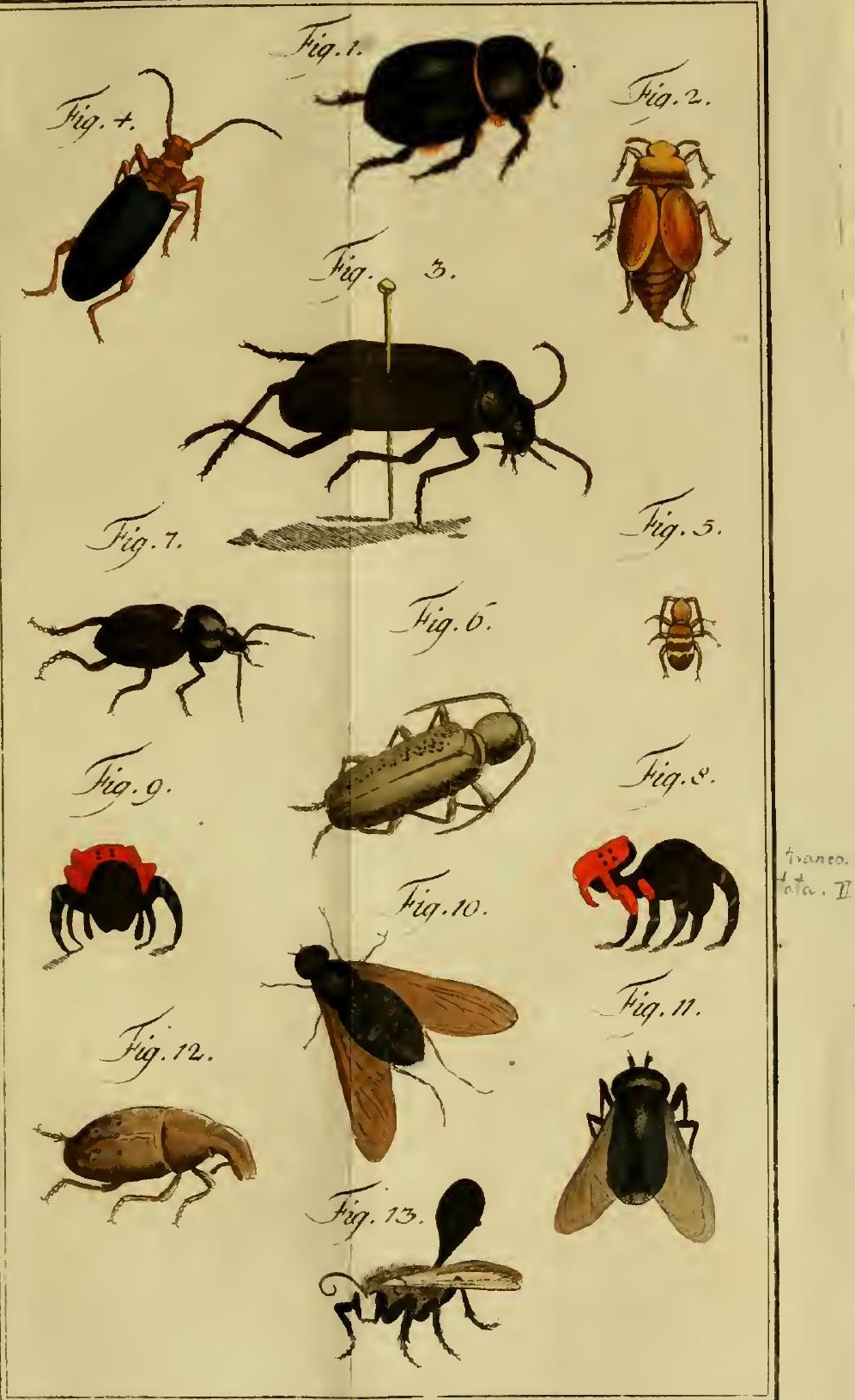



Tat II

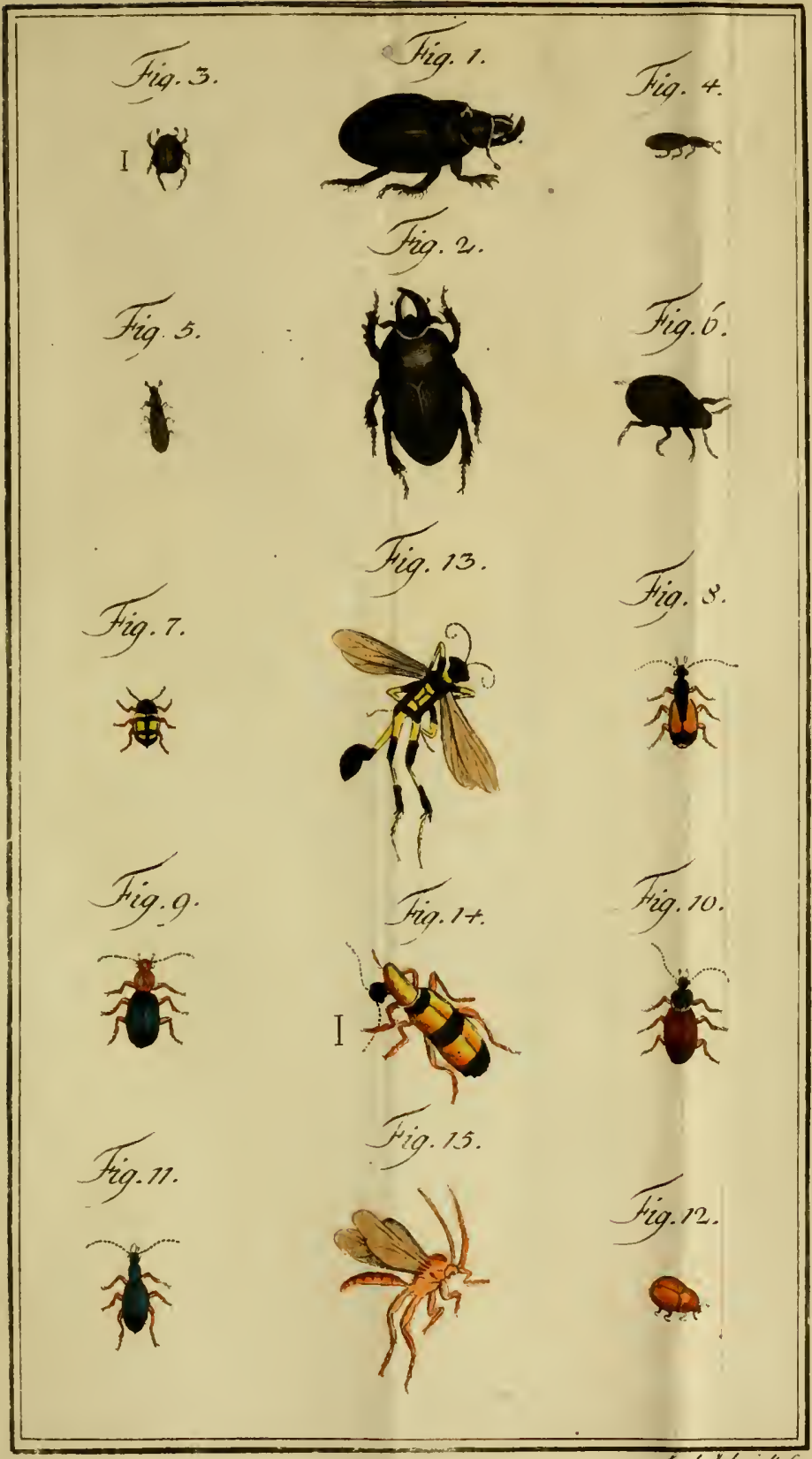

Iud thmial /c. 

Tox' ni

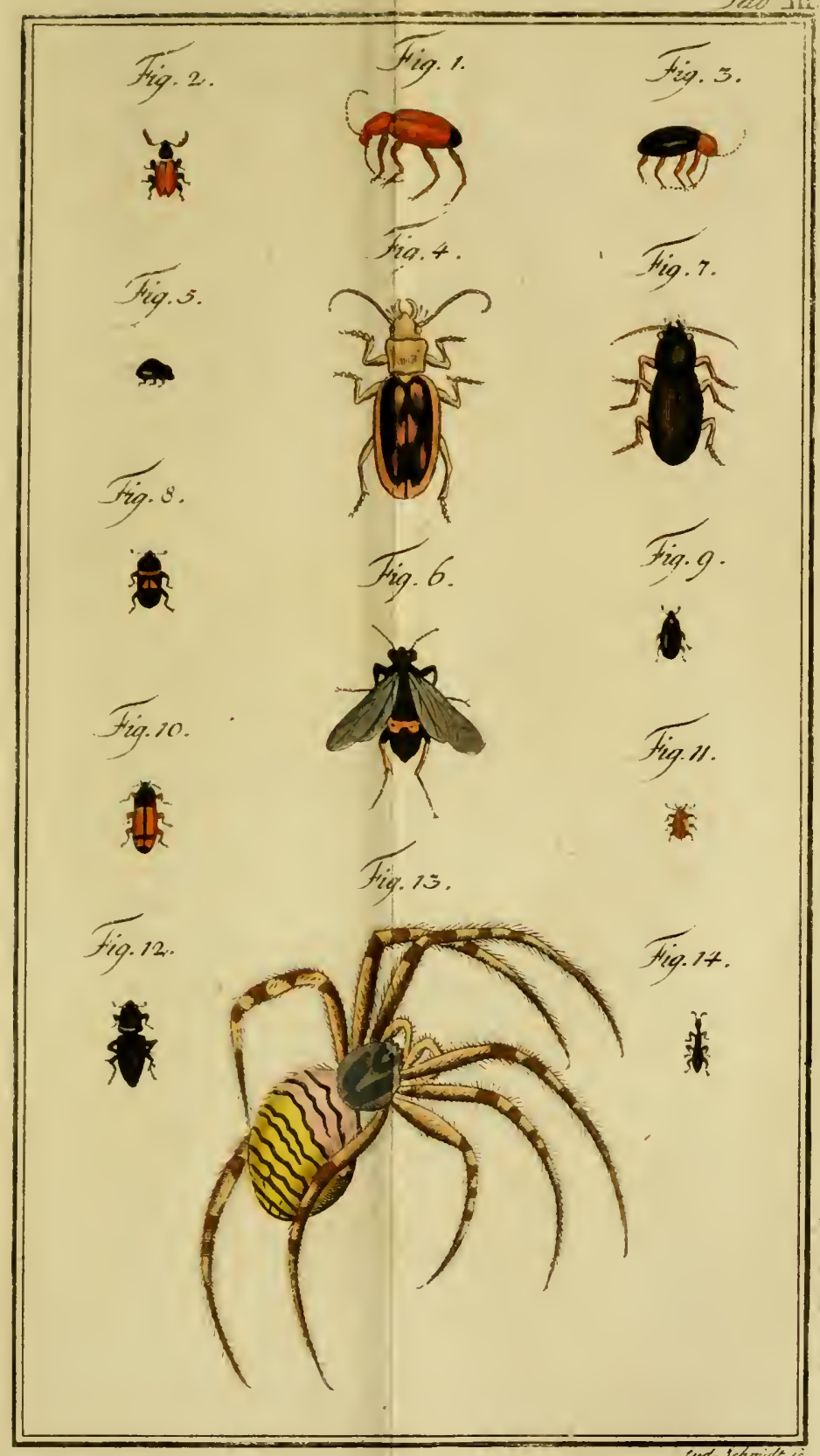

Lind tchmidt 0 

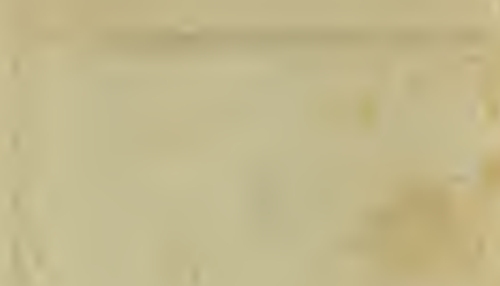

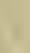

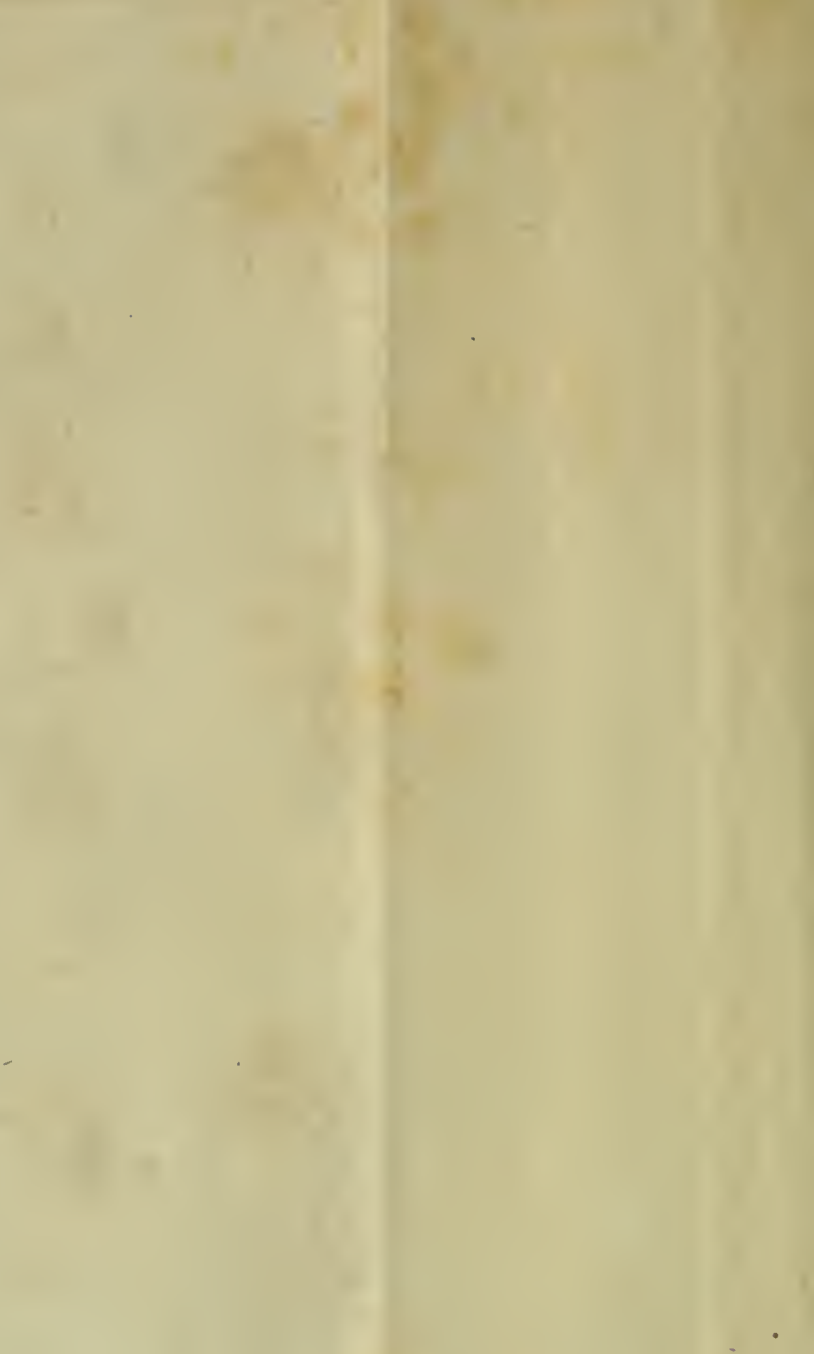


Tab. IV.

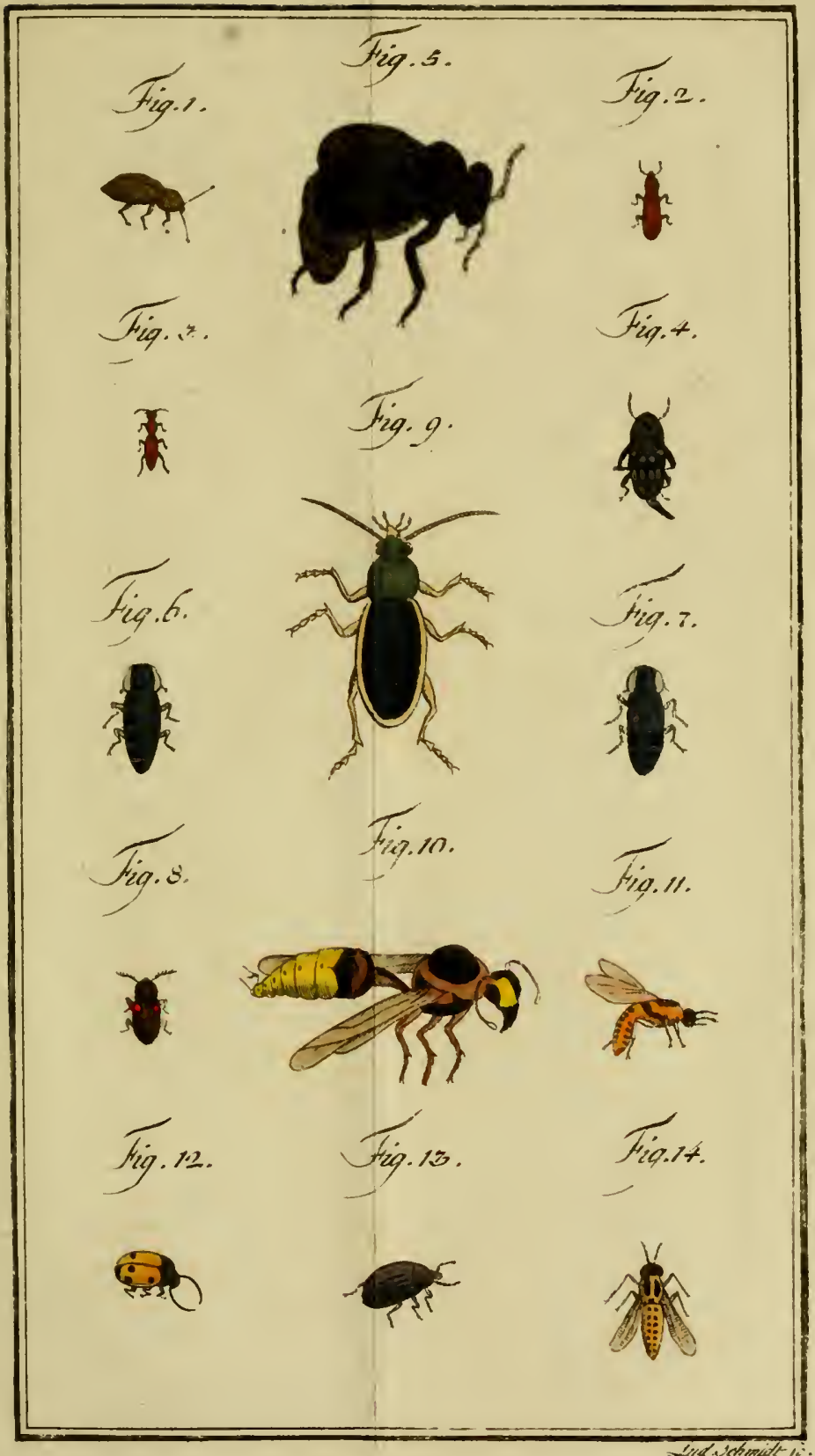

Ind estercits $/ 4$. 

Tab.V.
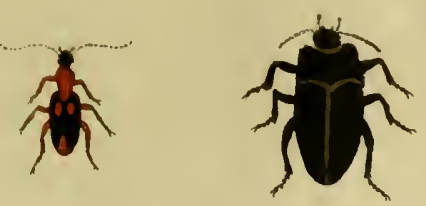

Hiq. 3.

Fig.7.
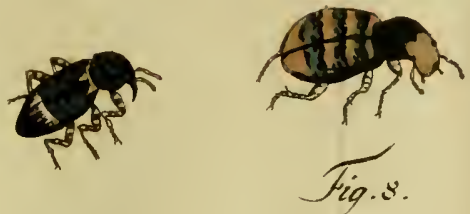

Fig.7.

Fig.s.

Fig. 6.

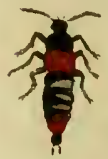

ax
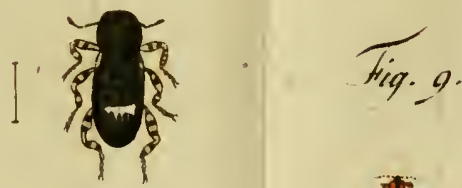

剩

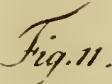

Fiq.10.
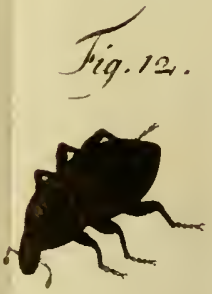

stud.- romide is. 

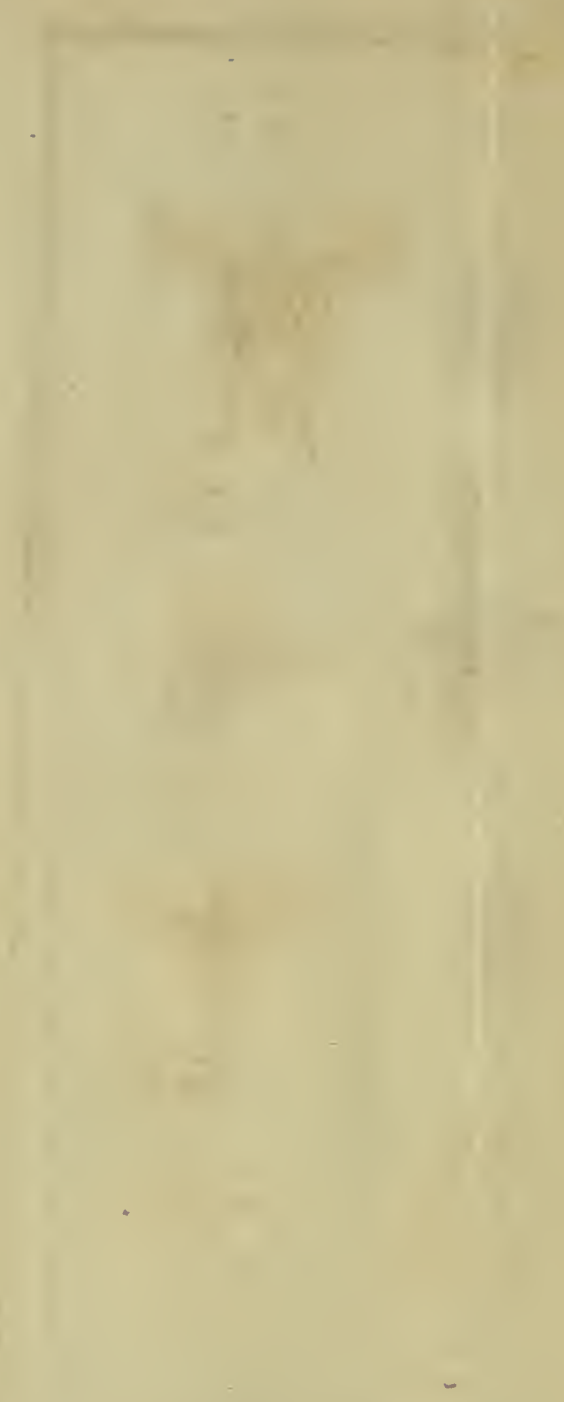

-

+3 
Tab. TI.

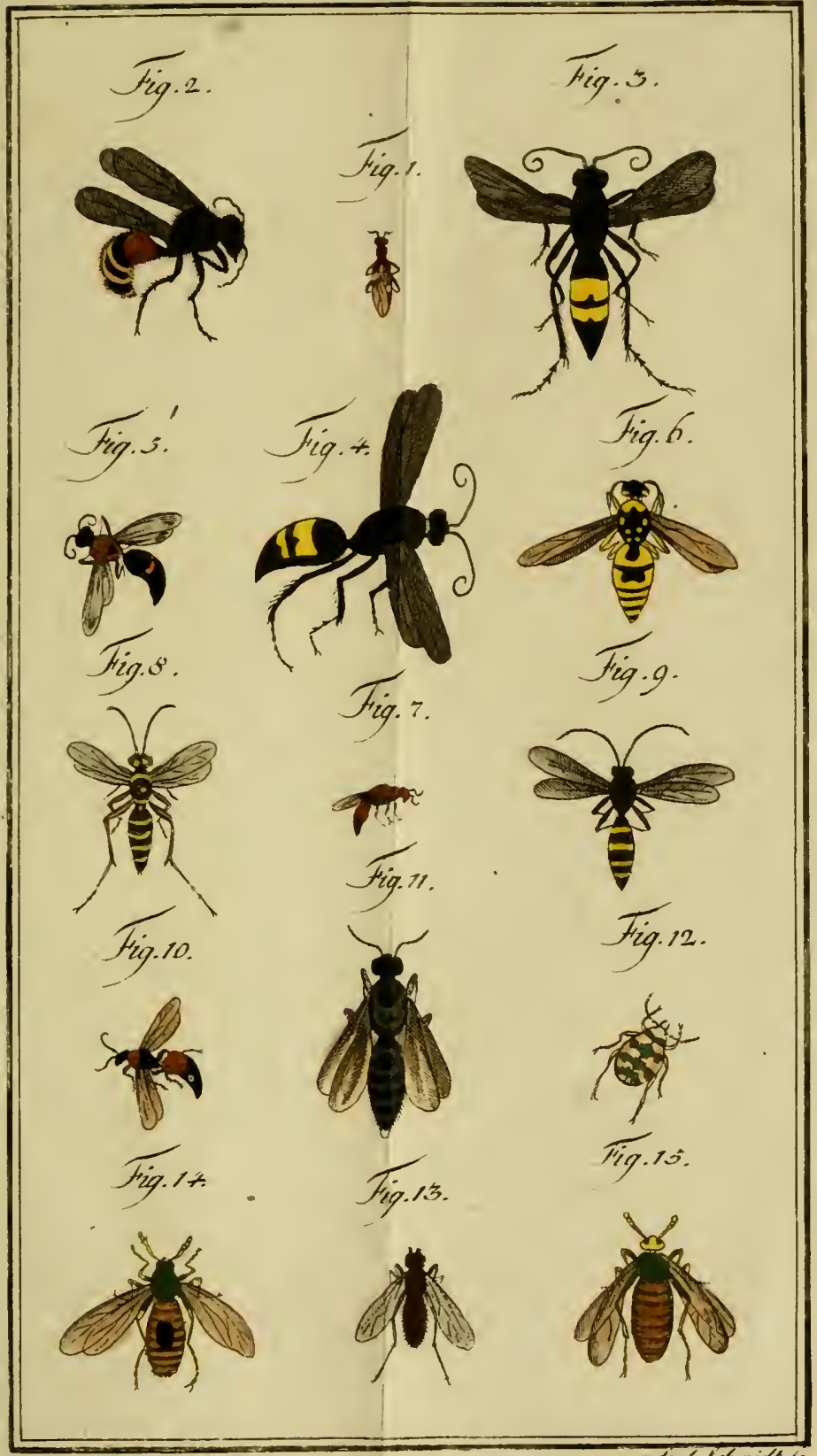



Tert.VII

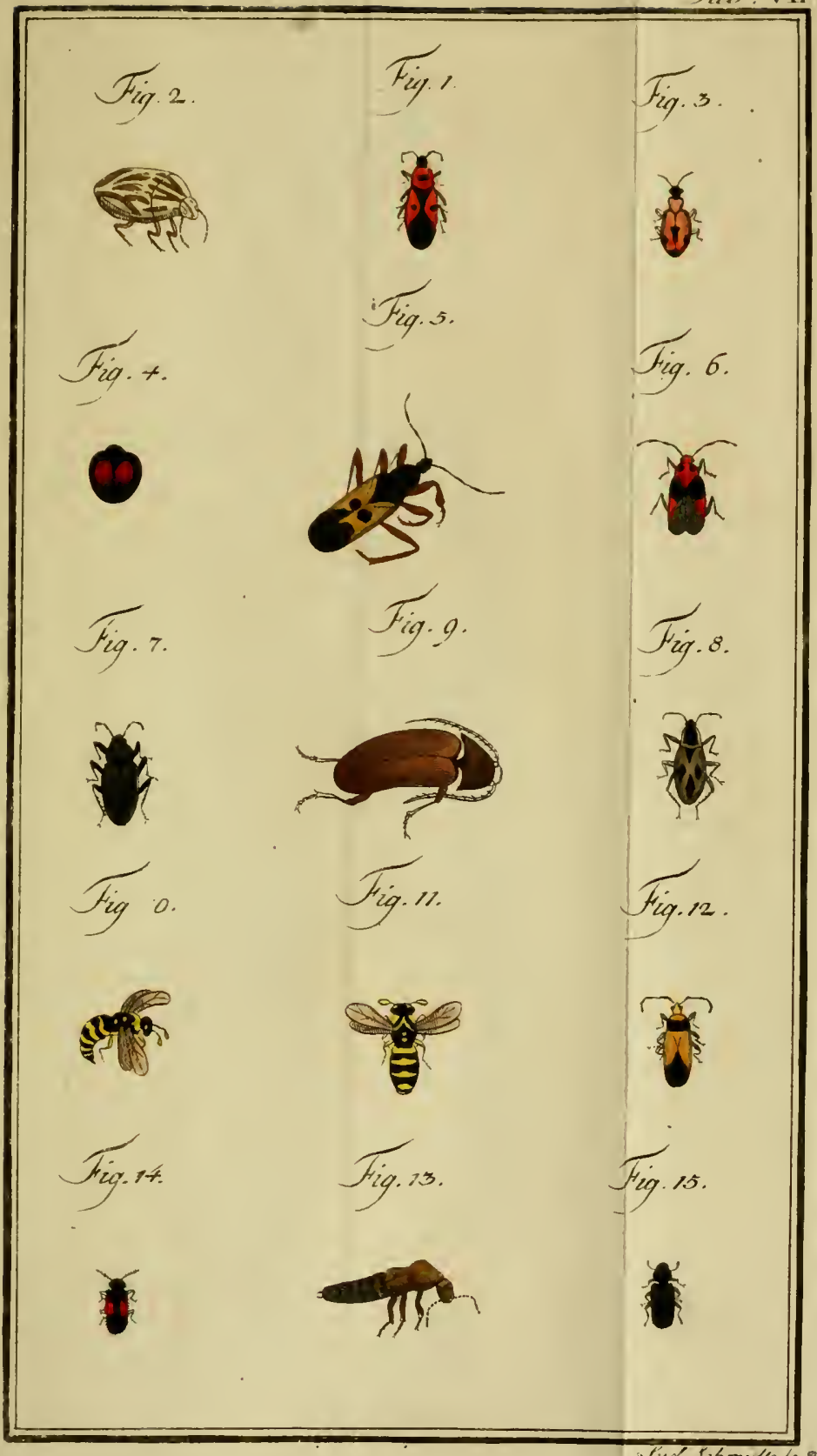



Tab. vाl

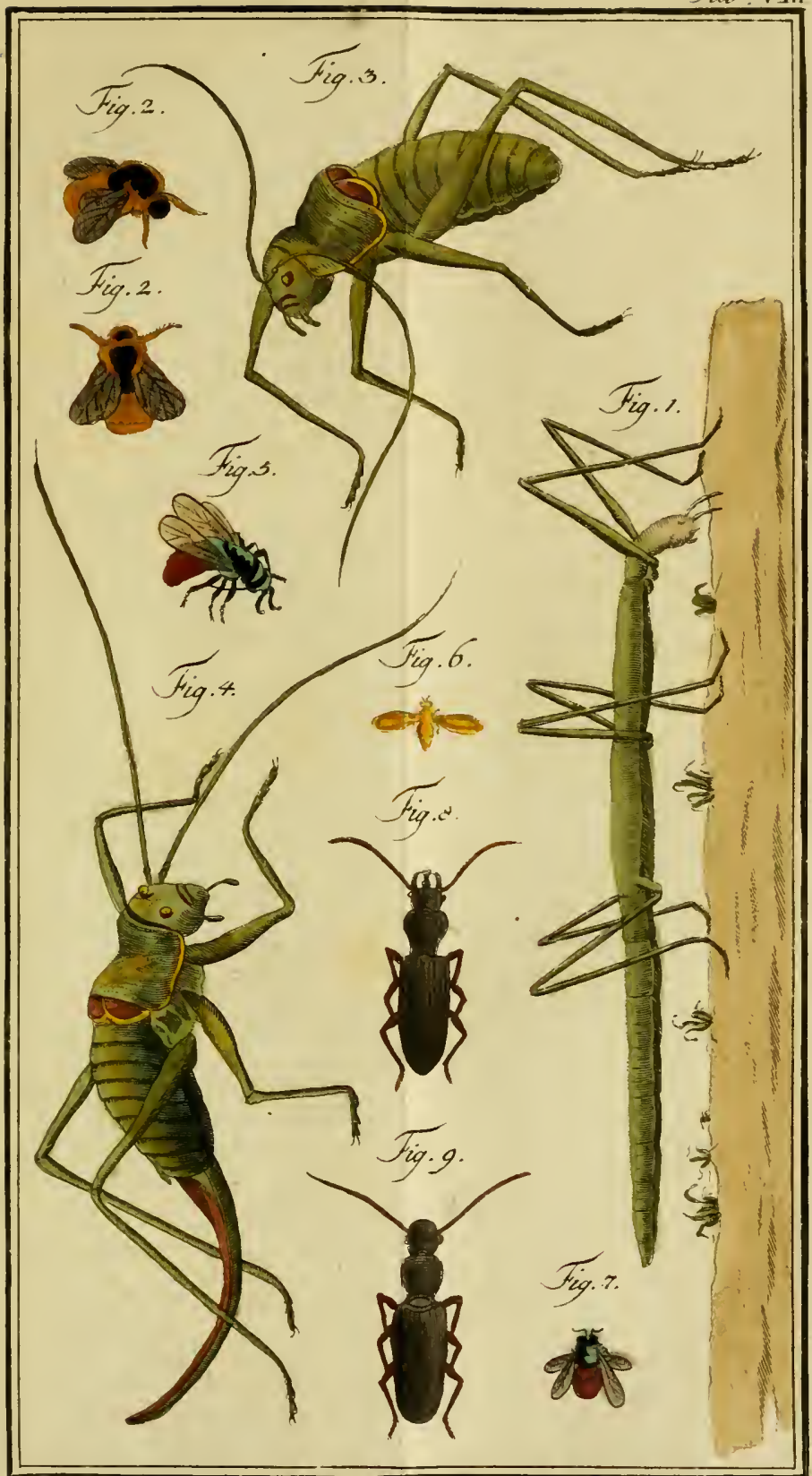

"ient. Schmidt " 


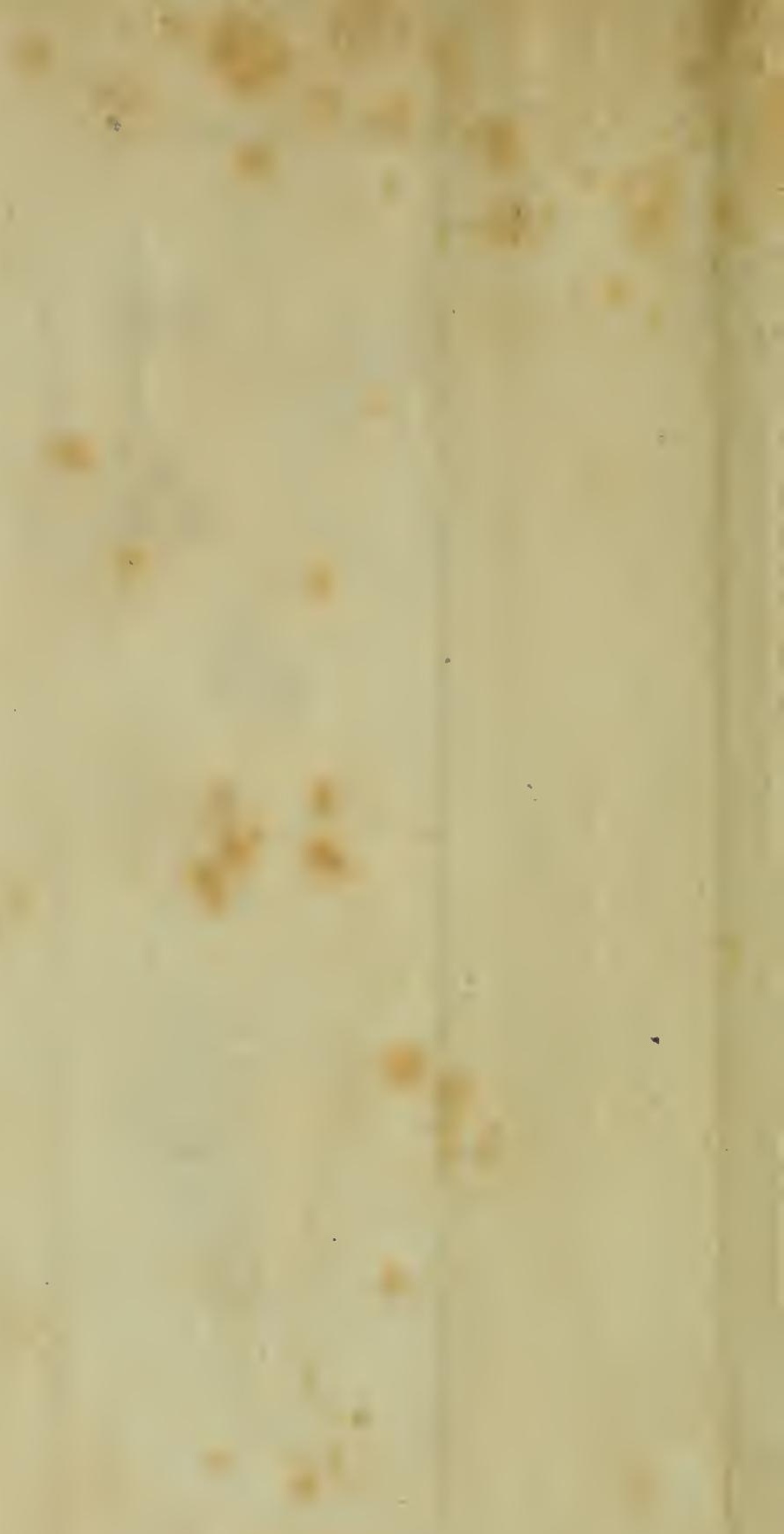




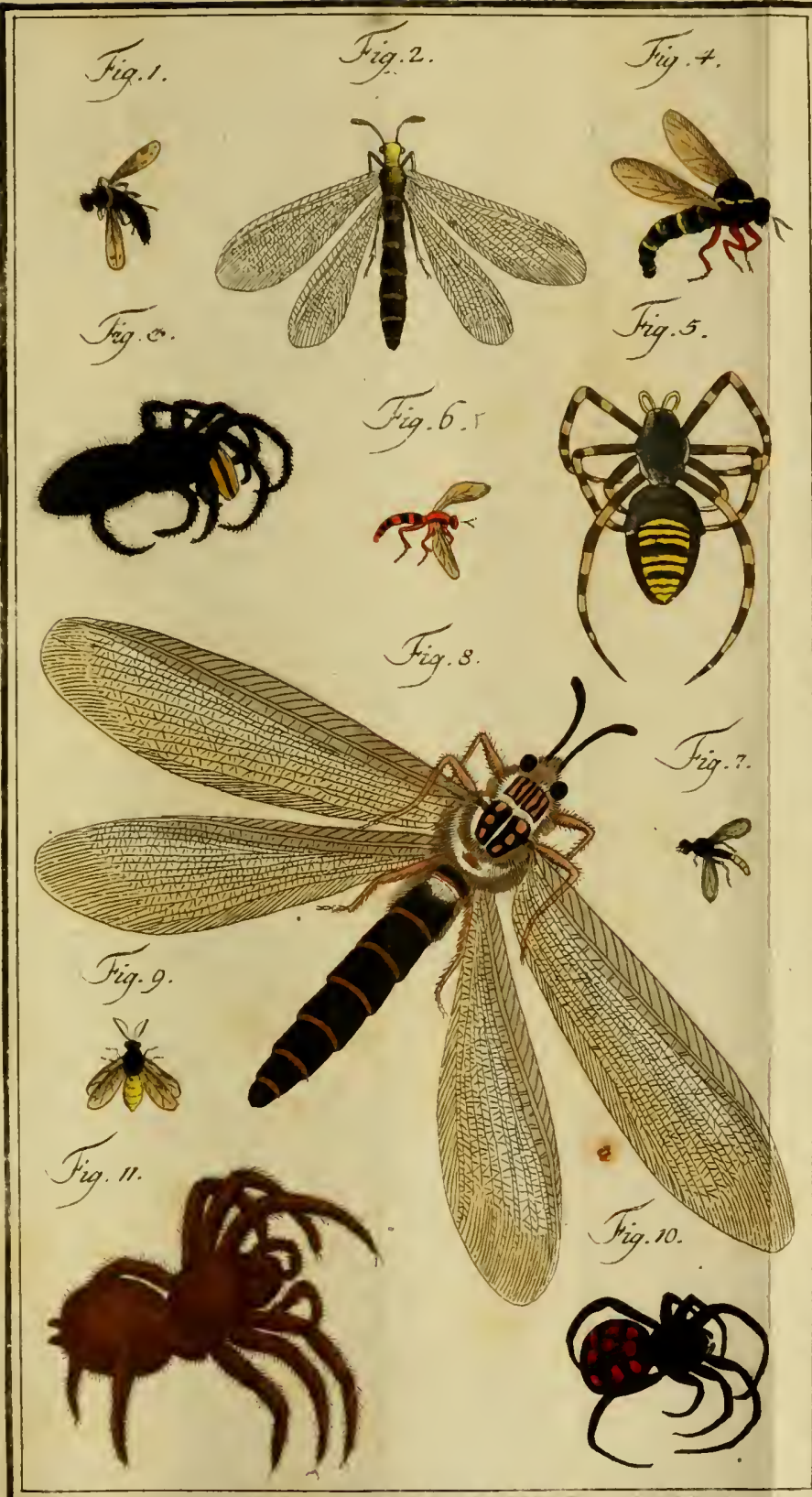

Ind. Jchmidt 


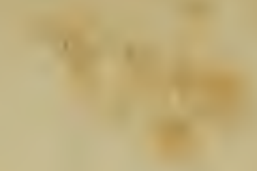

(n)

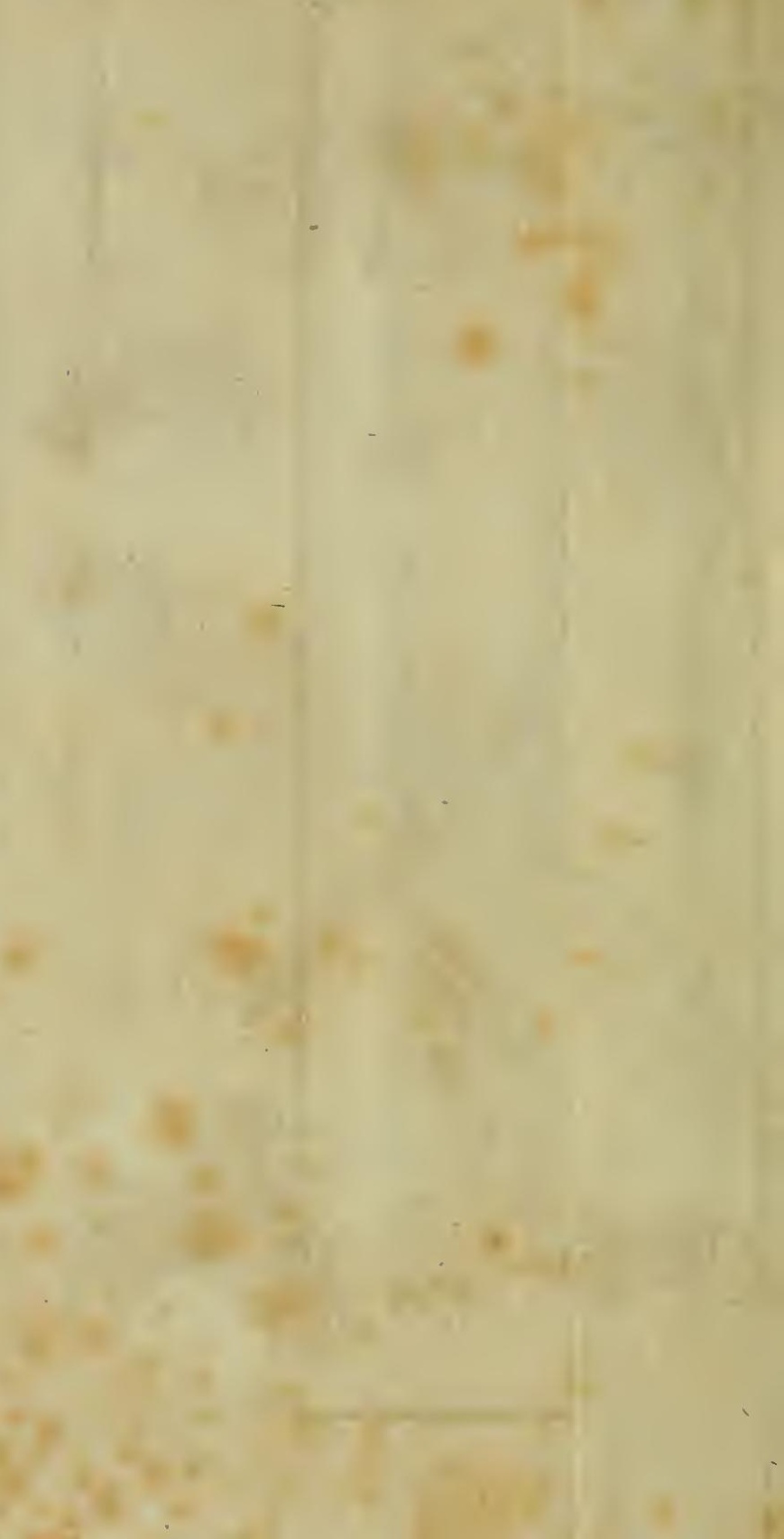


Juth...

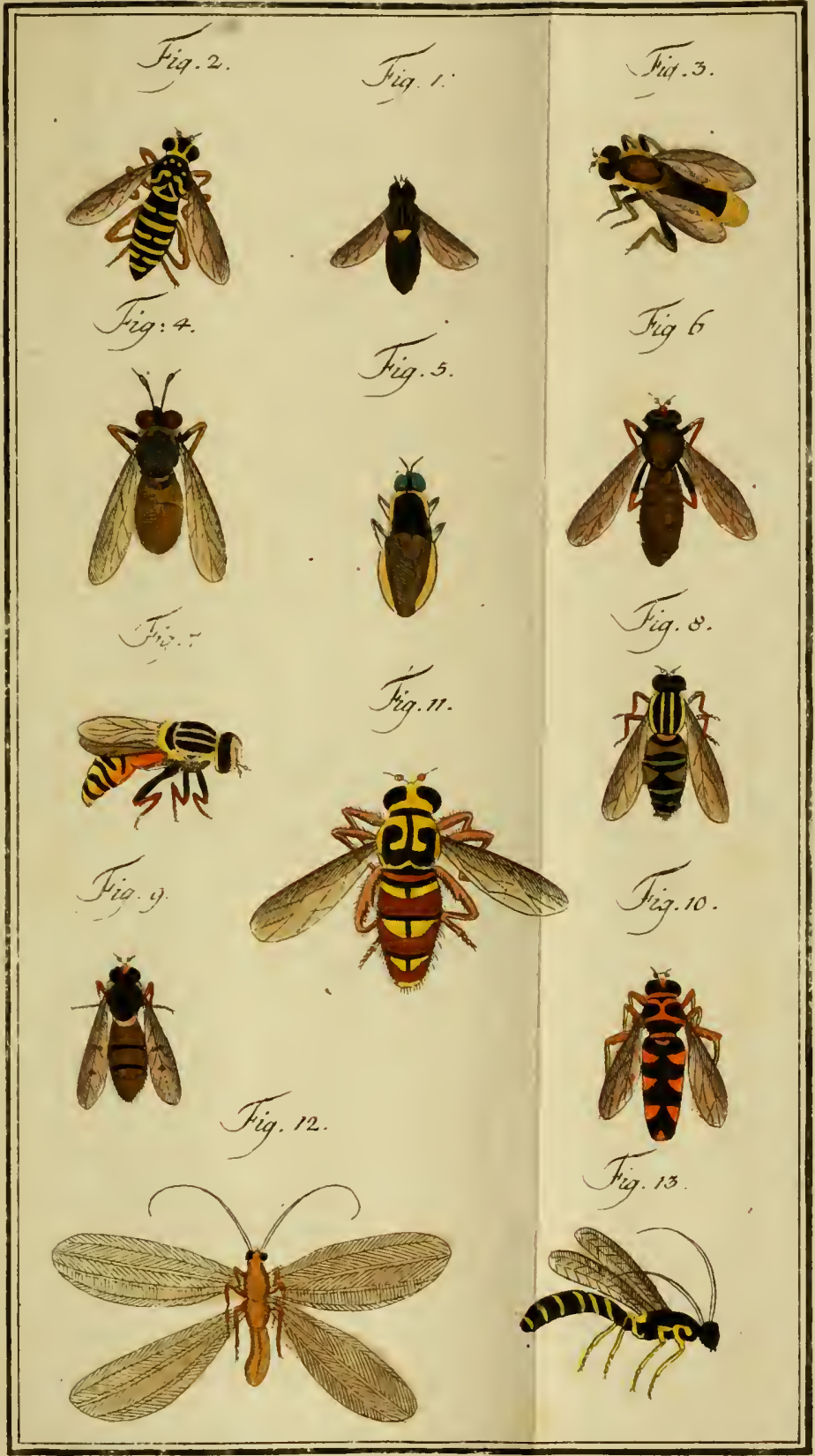





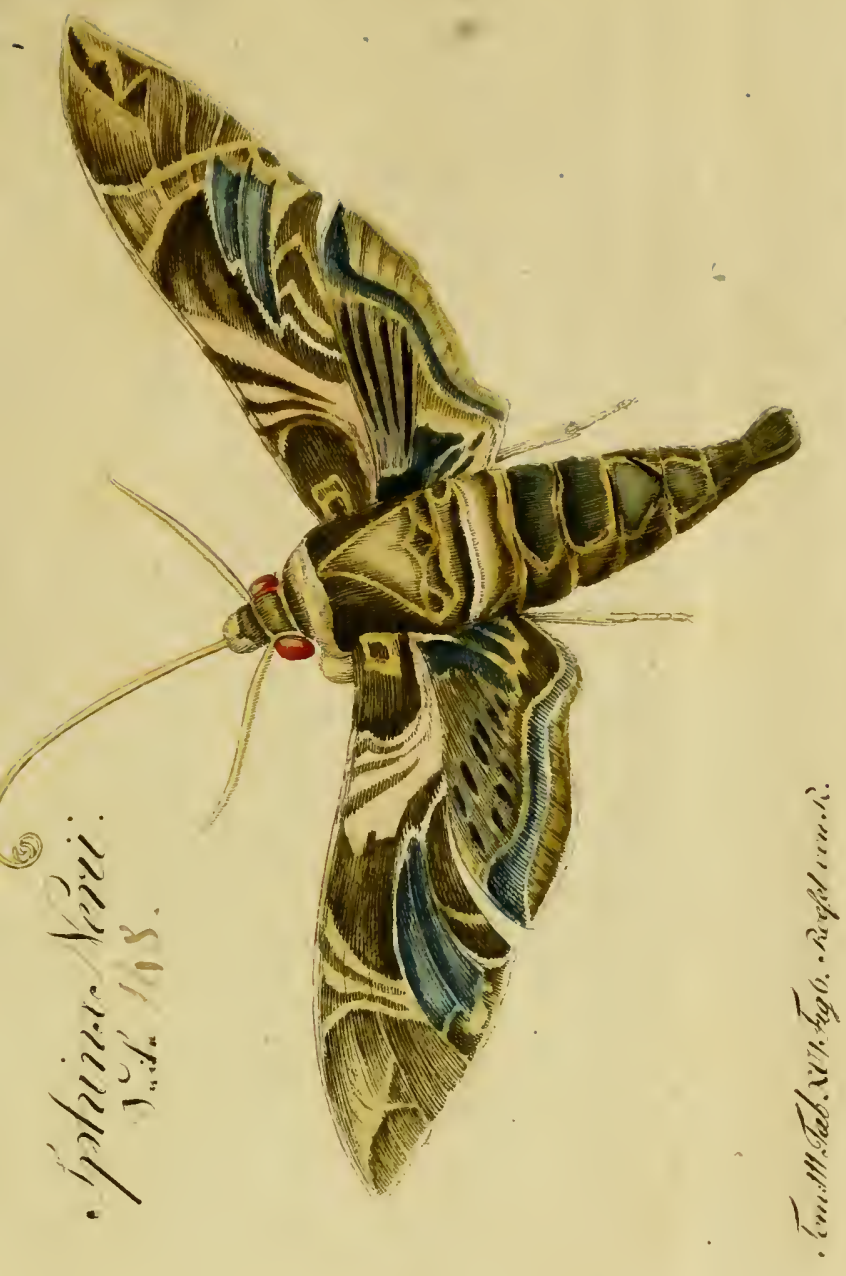





0 
